\title{
Scopariusicides, Novel Unsymmetrical Cyclobutanes: Structural Elucidation and Concise Synthesis by a Combination of Intermolecular [2 + 2] Cycloaddition and C-H Functionalization
}

Min Zhou, ${ }^{\dagger, \dagger, \|}$ Xing-Ren Li, ${ }^{\dagger, \S, \|}$ Jian-Wei Tang, ${ }^{\dagger,}$ Yang Liu, ${ }^{\Delta}$ Xiao-Nian Li, ${ }^{\dagger}$ Bin Wu, ${ }^{\dagger}$ Hong-Bo Qin,${ }^{\dagger}$ Xue Du,${ }^{\dagger}$ Li-Mei Li, ${ }^{\Delta}$ Wei-Guang Wang, ${ }^{\dagger}$ Jian-Xin Pu, ${ }^{\dagger} * *$ and Han-Dong Sun ${ }^{\dagger}$

† State Key Laboratory of Phytochemistry and Plant Resources in West China, Kunming Institute of Botany, Chinese Academy of Sciences, Kunming 650201, PR China

Key Laboratory of Chemistry in Ethnic Medicinal Resources, State Ethnic Affairs Commission and Ministry of Education, Yunnan Minzu University, Kunming 650031, PR China

${ }^{\S}$ University of Chinese Academy of Sciences, Beijing 100049, PR China

${ }^{\Delta}$ Department of Immunology, School of Basic Medical Sciences, Chengdu Medical College, Chengdu 610083, PR China

* Corresponding author Tel.: +86-871-65223616;

E-mail: pujianxin@mail.kib.ac.cn. 
Contents of Supporting Information

\begin{tabular}{|c|c|c|}
\hline No. & Contents & Pages \\
\hline 1. & Detailed experimental procedures & $5-42$ \\
\hline 2. & Figure S1. The key 2D NMR correlations of compound $\mathbf{1}$ & 8 \\
\hline 3. & Table S1. ${ }^{1} \mathrm{H}$ NMR, ${ }^{13} \mathrm{C}$ NMR, and HMBC spectroscopic data of $\mathbf{1}$ and $\mathbf{2}$ & 10 \\
\hline 4. & Scheme S1. Synthesis of scopariusicide A (1) & 12 \\
\hline 5. & Table S2. Data comparison between synthetic and natural 1 & 18 \\
\hline 6. & Scheme S2. Attempts to irradiation of biosynthetic precursors or analogues & 19 \\
\hline 7. & Scheme S3. Model reaction & 22 \\
\hline 8. & Scheme S4. Several attempts for modified [2+2] cycloaddition & 24 \\
\hline 9. & Scheme S5. Selected optimization for Pd-catalyzed C-H arylation & 26 \\
\hline 10. & Scheme S6. Scopariusicides analogs of $\mathbf{1 0 a}-\mathbf{1 0 w}$ & 33 \\
\hline 11. & $\begin{array}{l}\text { Table S3. The effect of natural and synthetic molecules on human } \mathrm{T} \text { cell } \\
\text { proliferation and cytotoxicity on PBMC viability }\end{array}$ & 43 \\
\hline 12. & Figures S2-S3. ${ }^{1} \mathrm{H}$ and ${ }^{13} \mathrm{C}$ NMR spectra of $\mathbf{1}$ & 44 \\
\hline 13. & Figures S4-S7. HSQC, HMBC, ${ }^{1} \mathrm{H}-{ }^{1} \mathrm{H}$ COSY, and ROESY spectra of $\mathbf{1}$ & $45-46$ \\
\hline 14. & Figure $\mathrm{S} 8 . \mathrm{CD}$ spectrum of $\mathbf{1}$ & 47 \\
\hline 15. & Figure S9. X-ray structure of $\mathbf{1}$ & 48 \\
\hline 16. & Figures $\mathrm{S} 10-\mathrm{S} 11 .{ }^{1} \mathrm{H}$ and ${ }^{13} \mathrm{C}$ NMR spectra of $\mathbf{2}$ & 49 \\
\hline 17. & Figures S12-S15. HSQC, HMBC, ${ }^{1} \mathrm{H}-{ }^{1} \mathrm{H}$ COSY, and ROESY spectra of 2 & $50-51$ \\
\hline 18. & Figure S16. CD spectrum of 2 & 52 \\
\hline 19. & Figures S17-S18. ${ }^{1} \mathrm{H}$ and ${ }^{13} \mathrm{C}$ NMR spectra of $\mathbf{3}$ & 53 \\
\hline 20. & Figures S19-S22. HSQC, HMBC, ${ }^{1} \mathrm{H}-{ }^{1} \mathrm{H}$ COSY, and ROESY spectra of $\mathbf{3}$ & $54-55$ \\
\hline 21. & Figure S23. X-ray structure of $\mathbf{3}$ & 56 \\
\hline 22. & Figures S24-S25. ${ }^{1} \mathrm{H}$ and ${ }^{13} \mathrm{C}$ NMR spectra of $6 \mathbf{a}$ & 57 \\
\hline 23. & Figures S26-S29. HSQC, HMBC, ${ }^{1} \mathrm{H}-{ }^{1} \mathrm{H}$ COSY, and ROESY spectra of $\mathbf{6 a}$ & $58-59$ \\
\hline 24. & Figure S30. X-ray structure of $\mathbf{6 a}$ & 60 \\
\hline 25. & Figures S31-S32. ${ }^{1} \mathrm{H}$ and ${ }^{13} \mathrm{C}$ NMR spectra of $\mathbf{6 b}$ & 61 \\
\hline 26. & Figures S33-S36. HSQC, HMBC, ${ }^{1} \mathrm{H}^{-1} \mathrm{H}$ COSY, and ROESY spectra of $\mathbf{6 b}$ & $62-63$ \\
\hline 27. & Figure S37. X-ray structure of $\mathbf{6 b}$ & 64 \\
\hline 28. & Figures S38-S49. ${ }^{1} \mathrm{H}$ and ${ }^{13} \mathrm{C}$ NMR spectra of $\mathbf{6 c}$ & 65 \\
\hline 29. & Figures $\mathrm{S} 40-\mathrm{S} 43$. HSQC, $\mathrm{HMBC},{ }^{1} \mathrm{H}-{ }^{1} \mathrm{H}$ COSY, and ROESY spectra of $\mathbf{6 c}$ & $66-67$ \\
\hline 30. & Figure S44. X-ray structure of $\mathbf{6 c}$ & 68 \\
\hline 31. & Figures S45-S46. ${ }^{1} \mathrm{H}$ and ${ }^{13} \mathrm{C}$ NMR spectra of $\mathbf{6 d}$ & 69 \\
\hline 32. & Figures S47-S50. HSQC, HMBC, ${ }^{1} \mathrm{H}-{ }^{1} \mathrm{H}$ COSY, and ROESY spectra of $\mathbf{6 d}$ & $70-71$ \\
\hline 33. & Figures S51-S52. ${ }^{1} \mathrm{H}$ and ${ }^{13} \mathrm{C}$ NMR spectra of $6 \mathbf{e}$ & 72 \\
\hline 34. & Figures S53-S56. HSQC, HMBC, ${ }^{1} \mathrm{H}^{1}{ }^{1} \mathrm{H}$ COSY, and ROESY spectra of $6 \mathbf{e}$ & $73-74$ \\
\hline 35. & Figures S57-S58. ${ }^{1} \mathrm{H}$ and ${ }^{13} \mathrm{C}$ NMR spectra of $7 \mathbf{a}$ & 75 \\
\hline 36. & Figures S59-S62. HSQC, HMBC, ${ }^{1} \mathrm{H}-{ }^{1} \mathrm{H}$ COSY, and ROESY spectra of $7 \mathbf{a}$ & $76-77$ \\
\hline 37. & Figures S63-S64. ${ }^{1} \mathrm{H}$ and ${ }^{13} \mathrm{C}$ NMR spectra of 8 & 78 \\
\hline 38. & Figures S65-S68. HSQC, HMBC, ${ }^{1} \mathrm{H}-{ }^{1} \mathrm{H}$ COSY, and ROESY spectra of 8 & $79-80$ \\
\hline 39. & Figures S69-S70. ${ }^{1} \mathrm{H}$ and ${ }^{13} \mathrm{C}$ NMR spectra of synthetic $\mathbf{1}$ & 81 \\
\hline 40. & Figures S71-S72. ${ }^{1} \mathrm{H}$ and ${ }^{13} \mathrm{C}$ NMR spectra of $\mathbf{1 0}$ & 82 \\
\hline 41. & Figures S73-S74. ${ }^{1} \mathrm{H}$ and ${ }^{13} \mathrm{C}$ NMR spectra of $\mathbf{1 2}$ & 83 \\
\hline 42. & Figures S75-S76. ${ }^{1} \mathrm{H}$ and ${ }^{13} \mathrm{C}$ NMR spectra of $\mathbf{1 5}$ & 84 \\
\hline
\end{tabular}




\begin{tabular}{|c|c|c|}
\hline No. & Contents & Pages \\
\hline 43. & Figures S77-S80. HSQC, HMBC, ${ }^{1} \mathrm{H}-{ }^{1} \mathrm{H}$ COSY, and ROESY spectra of $\mathbf{1 5}$ & $85-86$ \\
\hline 44. & Figures $\mathrm{S} 81-\mathrm{S} 82 .{ }^{1} \mathrm{H}$ and ${ }^{13} \mathrm{C}$ NMR spectra of $\mathbf{1 6}$ & 87 \\
\hline 45. & Figures S83-S86. HSQC, HMBC, ${ }^{1} \mathrm{H}-{ }^{1} \mathrm{H}$ COSY, and ROESY spectra of 16 & $88-89$ \\
\hline 46. & Figures S87-S88. ${ }^{1} \mathrm{H}$ and ${ }^{13} \mathrm{C}$ NMR spectra of 21 & 90 \\
\hline 47. & Figures S89-S90. ${ }^{1} \mathrm{H}$ and ${ }^{13} \mathrm{C}$ NMR spectra of 22 & 91 \\
\hline 48. & Figures S91-S94. HSQC, HMBC, ${ }^{1} \mathrm{H}-{ }^{1} \mathrm{H}$ COSY, and ROESY spectra of 22 & $92-93$ \\
\hline 49. & Figures S95-S96. ${ }^{1} \mathrm{H}$ and ${ }^{13} \mathrm{C}$ NMR spectra of $\mathbf{2 3}$ & 94 \\
\hline 50. & Figures S97-S100. HSQC, HMBC, ${ }^{1} \mathrm{H}-{ }^{1} \mathrm{H}$ COSY, and ROESY spectra of $\mathbf{2 3}$ & $95-96$ \\
\hline 51. & Figures S101-S102. ${ }^{1} \mathrm{H}$ and ${ }^{13} \mathrm{C}$ NMR spectra of $7 \mathbf{b}$ & 97 \\
\hline 52. & Figures S103-S106. HSQC, HMBC, ${ }^{1} \mathrm{H}^{-1} \mathrm{H}$ COSY, and ROESY spectra of $\mathbf{7 b}$ & 98-99 \\
\hline 53. & Figures S107-S108. ${ }^{1} \mathrm{H}$ and ${ }^{13} \mathrm{C}$ NMR spectra of $7 \mathbf{c}$ & 100 \\
\hline 54. & Figures S109-S112. HSQC, HMBC, ${ }^{1} \mathrm{H}^{-1} \mathrm{H}$ COSY, and ROESY spectra of $7 \mathbf{c}$ & $101-102$ \\
\hline 55. & Figures S113-S114. ${ }^{1} \mathrm{H}$ and ${ }^{13} \mathrm{C}$ NMR spectra of $\mathbf{7 d}$ & 103 \\
\hline 56. & Figures S115-S118. HSQC, HMBC, ${ }^{1} \mathrm{H}^{-1} \mathrm{H}$ COSY, and ROESY spectra of $7 \mathbf{d}$ & $104-105$ \\
\hline 57. & Figures S119-S120. ${ }^{1} \mathrm{H}$ and ${ }^{13} \mathrm{C}$ NMR spectra of $7 \mathbf{e}$ & 106 \\
\hline 58. & Figures S121-S124. HSQC, HMBC, ${ }^{1} \mathrm{H}^{-1} \mathrm{H}$ COSY, and ROESY spectra of $7 \mathbf{e}$ & $107-108$ \\
\hline 59. & Figures S125-S126. ${ }^{1} \mathrm{H}$ and ${ }^{13} \mathrm{C}$ NMR spectra of $\mathbf{7 f}$ & 109 \\
\hline 60. & Figures S127-S130. HSQC, HMBC, ${ }^{1} \mathrm{H}^{-1} \mathrm{H}$ COSY, and ROESY spectra of $\mathbf{7 f}$ & $110-111$ \\
\hline 61. & Figures S131-S132. ${ }^{1} \mathrm{H}$ and ${ }^{13} \mathrm{C}$ NMR spectra of $\mathbf{7 g}$ & 112 \\
\hline 62. & Figures S133-S136. HSQC, HMBC, ${ }^{1} \mathrm{H}^{-1} \mathrm{H}$ COSY, and ROESY spectra of $\mathbf{7 g}$ & $113-114$ \\
\hline 63. & Figures S137-S138. ${ }^{1} \mathrm{H}$ and ${ }^{13} \mathrm{C}$ NMR spectra of $\mathbf{7 h}$ & 115 \\
\hline 64. & Figures S139-S142. HSQC, HMBC, ${ }^{1} \mathrm{H}^{-1} \mathrm{H}$ COSY, and ROESY spectra of $\mathbf{7 h}$ & $116-117$ \\
\hline 65. & Figures S143-S144. ${ }^{1} \mathrm{H}$ and ${ }^{13} \mathrm{C}$ NMR spectra of $7 \mathbf{i}$ & 118 \\
\hline 66. & Figures S145-S148. HSQC, HMBC, ${ }^{1} \mathrm{H}^{-1} \mathrm{H}$ COSY, and ROESY spectra of $7 \mathbf{i}$ & $119-120$ \\
\hline 67. & Figures S149-S150. ${ }^{1} \mathrm{H}$ and ${ }^{13} \mathrm{C}$ NMR spectra of $\mathbf{7} \mathbf{j}$ & 121 \\
\hline 68. & Figures S151-S154. HSQC, HMBC, ${ }^{1} \mathrm{H}^{-1} \mathrm{H}$ COSY, and ROESY spectra of $\mathbf{7} \mathbf{j}$ & $122-123$ \\
\hline 69. & Figures S155-S156. ${ }^{1} \mathrm{H}$ and ${ }^{13} \mathrm{C}$ NMR spectra of $7 \mathbf{k}$ & 124 \\
\hline 70. & Figures S157-S160. HSQC, HMBC, ${ }^{1} \mathrm{H}^{-1} \mathrm{H}$ COSY, and ROESY spectra of 7k & $125-126$ \\
\hline 71. & Figures S161-S162. ${ }^{1} \mathrm{H}$ and ${ }^{13} \mathrm{C}$ NMR spectra of 10a & 127 \\
\hline 68. & Figures S163-S164. ${ }^{1} \mathrm{H}$ and ${ }^{13} \mathrm{C}$ NMR spectra of $\mathbf{1 0 b}$ & 128 \\
\hline 69. & Figures S165-S166. ${ }^{1} \mathrm{H}$ and ${ }^{13} \mathrm{C}$ NMR spectra of $\mathbf{1 0 c}$ & 129 \\
\hline 70. & Figures S167-S168. ${ }^{1} \mathrm{H}$ and ${ }^{13} \mathrm{C}$ NMR spectra of $\mathbf{1 0 d}$ & 130 \\
\hline 71. & Figures S169-S170. ${ }^{1} \mathrm{H}$ and ${ }^{13} \mathrm{C}$ NMR spectra of $\mathbf{1 0 e}$ & 131 \\
\hline 72. & Figures S171-S172. ${ }^{1} \mathrm{H}$ and ${ }^{13} \mathrm{C}$ NMR spectra of $\mathbf{1 0 f}$ & 132 \\
\hline 73. & Figures S173-S174. ${ }^{1} \mathrm{H}$ and ${ }^{13} \mathrm{C}$ NMR spectra of $\mathbf{1 0 g}$ & 133 \\
\hline 74. & Figures S175-S176. ${ }^{1} \mathrm{H}$ and ${ }^{13} \mathrm{C}$ NMR spectra of $\mathbf{1 0 h}$ & 134 \\
\hline 75. & Figures S177-S178. ${ }^{1} \mathrm{H}$ and ${ }^{13} \mathrm{C}$ NMR spectra of $\mathbf{1 0 i}$ & 135 \\
\hline 76. & Figures S179-S180. ${ }^{1} \mathrm{H}$ and ${ }^{13} \mathrm{C}$ NMR spectra of $\mathbf{1 0 j}$ & 136 \\
\hline 77. & Figures S181-S182. ${ }^{1} \mathrm{H}$ and ${ }^{13} \mathrm{C}$ NMR spectra of $\mathbf{1 0 k}$ & 137 \\
\hline 78. & Figures S183-S184. ${ }^{1} \mathrm{H}$ and ${ }^{13} \mathrm{C}$ NMR spectra of $\mathbf{1 0 I}$ & 138 \\
\hline 79. & Figures S185-S186. ${ }^{1} \mathrm{H}$ and ${ }^{13} \mathrm{C}$ NMR spectra of $\mathbf{1 0 m}$ & 139 \\
\hline 80. & Figures S187-S188. ${ }^{1} \mathrm{H}$ and ${ }^{13} \mathrm{C}$ NMR spectra of $\mathbf{1 0 n}$ & 140 \\
\hline 81. & Figures S189-S190. ${ }^{1} \mathrm{H}$ and ${ }^{13} \mathrm{C}$ NMR spectra of $\mathbf{1 0 o}$ & 141 \\
\hline 82. & Figures S191-S192. ${ }^{1} \mathrm{H}$ and ${ }^{13} \mathrm{C}$ NMR spectra of 10p & 142 \\
\hline 83. & Figures S193-S194. ${ }^{1} \mathrm{H}$ and ${ }^{13} \mathrm{C}$ NMR spectra of 10q & 143 \\
\hline 84. & Figures S195-S196. ${ }^{1} \mathrm{H}$ and ${ }^{13} \mathrm{C}$ NMR spectra of $\mathbf{1 0 r}$ & 144 \\
\hline
\end{tabular}




\begin{tabular}{clc}
\hline No. & \multicolumn{1}{c}{ Contents } & Pages \\
\hline 85. & Figures S197-S198. ${ }^{1} \mathrm{H}$ and ${ }^{13} \mathrm{C}$ NMR spectra of 10s & 145 \\
86. & Figures S199-S200. ${ }^{1} \mathrm{H}$ and ${ }^{13} \mathrm{C}$ NMR spectra of 10t & 146 \\
87. & Figures S201-S202. ${ }^{1} \mathrm{H}$ and ${ }^{13} \mathrm{C}$ NMR spectra of 10u & 147 \\
88. & Figures S203-S204. ${ }^{1} \mathrm{H}$ and ${ }^{13} \mathrm{C}$ NMR spectra of 10v & 148 \\
89. & Figures S205-S208 HSQC, HMBC, ${ }^{1} \mathrm{H}-{ }^{-1} \mathrm{H}$ COSY, and ROESY spectra of 10v & $149-150$ \\
90. & Figures S209-S210. ${ }^{1} \mathrm{H}$ and ${ }^{13} \mathrm{C}$ NMR spectra of 10w & 151 \\
91. & Figures S211-S214. HSQC, HMBC, ${ }^{1} \mathrm{H}-{ }^{1} \mathrm{H}$ COSY, and ROESY spectra of 10w & $152-153$ \\
92. & Figures S215-S216. ${ }^{1} \mathrm{H}$ and ${ }^{13} \mathrm{C}$ NMR spectra of $\mathbf{6 f}$ & 154 \\
\hline
\end{tabular}




\section{Detailed experimental procedures}

\section{General experimental procedures}

Optical rotations were measured with a JASCO DIP-370 digital polarimeter. UV data were obtained on a Shimadzu UV-2401A spectrophotometer. A BioRad FtS-135 spectrophotometer was used for scanning IR spectroscopy with $\mathrm{KBr}$ pellets. 1D and 2D NMR spectra were recorded on DRX-400 or Avance III 600 spectrometers. Unless otherwise specified, chemical shifts $(\delta)$ were expressed in ppm with reference to the solvent signals. Mass spectra were recorded on a VG-Auto-Spec-3000 spectrometer. X-ray diffraction was realized on a Bruker SMART APEX CCD crystallography system. [2 + 2] cycloaddition were realized on a Matrix 313-10 photoreactor $(16 \times$ $60 \mathrm{~W}$ mercury lamps, $h v=313$ or 360 nm, Peking Perfectlight, Inc., Peking, People's Republic of China). Dry dichloromethane (DCM), acetonitrile $\left(\mathrm{CH}_{3} \mathrm{CN}\right)$, toluene $(\mathrm{PhMe}), t$ - $\mathrm{BuOH}$, methanol $(\mathrm{MeOH})$ and acetone were obtained by passing these previously degassed solvents through activated alumina columns. Reagents were purchased at the highest commercial quality. Column chromatography was performed with silica gel (100-200 mesh; Qingdao Marine Chemical, Inc., Qingdao, People's Republic of China). Semi-preparative HPLC was performed on an Agilent 1100 liquid chromatograph with a Zorbax SB-C $18,9.4 \mathrm{~mm} \times 25 \mathrm{~cm}$ column. Preparative HPLC was performed on a Shimadzu LC-8A preparative liquid chromatograph with a Shimadzu PRC-ODS (K) column. Fractions were monitored by TLC and spots were visualized by heating silica gel plates sprayed with $8 \% \mathrm{H}_{2} \mathrm{SO}_{4}$ in EtOH. All solvents including petroleum ether $\left(60-90{ }^{\circ} \mathrm{C}\right)$ were distilled prior to use.

\section{Plant material}

The leaves of Isodon scoparius were collected from Shangri-La, Yunnan province, People's Republic of China, in September 2008. Voucher specimens (KIB20080812) were deposited at the State Key Laboratory of Phytochemistry and Plant Resources in West China, Kunming Institute of Botany, Chinese Academy of Sciences, and were identified by Prof. Xi-Wen Li.

\section{Extraction and isolation}

The air-dried leaves of Isodon scoparius $(10.2 \mathrm{Kg})$ were extracted with $70 \%$ aqueous acetone $(3 \times$ $40 \mathrm{~L}, 2$ days each) at room temperature. The solvent was evaporated in vacuo to afford a crude extract $(1.0 \mathrm{~kg})$, which was suspended in $\mathrm{H}_{2} \mathrm{O}$, and then extracted successively with EtOAc and $n$ - $\mathrm{BuOH}$. The EtOAc-soluble part (400 g) was decolorized on $\mathrm{MCI}$ gel with $90 \% \mathrm{MeOH} / \mathrm{H}_{2} \mathrm{O}$ to 
obtain a yellow gum $(310 \mathrm{~g})$. The gum was purified by $\mathrm{CC}$ (column chromatography on $\mathrm{SiO}_{2}$ with $\mathrm{CHCl}_{3}-\mathrm{Me}_{2} \mathrm{CO}$ gradient system 1:0, 9:1, 8:2, 7:3, 6:4 and 1:1) to yield six main fractions, Fr. A-F. Fr. $\mathrm{A}\left(\mathrm{CHCl}_{3}\right.$ /acetone 1:0,10.8 g) eluting with $\mathrm{PE} / \mathrm{CHCl}_{3}(10: 1,5: 1,2: 1$, and $0: 1)$ and yield subfractions A1-A4. Subfraction A3 (4.5 g, $\left.\mathrm{PE} / \mathrm{CHCl}_{3} 2: 1\right)$ was fragmented by repeated $\mathrm{CC}$, first on $\mathrm{RP}-18$ with a gradien elution of $\mathrm{MeOH} / \mathrm{H}_{2} \mathrm{O}$ (30:70 to 1:0) to yield fractions A31-A36, Subsequently fraction A35 $(1.5 \mathrm{~g})$ was purified by a silica gel column $\left(\mathrm{PE} / \mathrm{CHCl}_{3} 2: 1\right.$ to $\left.0: 1\right)$ to yield 3 (820 $\left.\mathrm{mg}\right)$ and $\mathrm{A} 352-354$. Subfraction A352 (120 mg) was purified by semipreparative HPLC ( $3 \mathrm{~mL} / \mathrm{min}$, detector UV $\lambda_{\max }$ $202 \mathrm{~nm}, \mathrm{MeCN} / \mathrm{H}_{2} \mathrm{O}$ 87:13) to yield 1 (32 mg, $\left.22.5 \mathrm{~min}\right)$ and 2 (5 mg, $\left.24.5 \mathrm{~min}\right)$. Fraction A4 (1.3 g) was also purified by a silica gel column $\left(\mathrm{PE} / \mathrm{CHCl}_{3} 2: 1\right.$ to $0: 1$, and $\mathrm{CHCl}_{3} /$ acetone $10: 1$ to $\left.2: 1\right)$ to yield 3 (195 mg). Scopariusicide A (1): White power; $[\alpha]_{\mathrm{D}}^{21}-35.51(c$ 0.12, MeOH); UV (MeOH) $\lambda_{\max }(\log \varepsilon) 200$ (0.32); IR (KBr) $v_{\max } 3447,3431,2930,2972,1740,1723,1516,1253,1189,1035$, 996, $921 \mathrm{~cm}^{-1}$; Positive HRESIMS $[\mathrm{M}+\mathrm{Na}]^{+} \mathrm{m} / z, 613.3872$ (calcd for $\mathrm{C}_{38} \mathrm{H}_{54} \mathrm{O}_{5} \mathrm{Na}, 613.3869$ ); ${ }^{1} \mathrm{H}$ and ${ }^{13} \mathrm{C}$ NMR see Table 1.X-ray Crystallographic Analysis of scopariusicide A (1): $\mathrm{C}_{38} \mathrm{H}_{54} \mathrm{O}_{5}, M=$ 590.81, orthorhombic, $a=10.0955(6) \AA, b=13.4185(8) \AA, c=24.9270(15) \AA, \alpha=90.00^{\circ}, \beta=$ $90.00^{\circ}, \gamma=90.00^{\circ}, V=3376.8(3) \AA^{3}, T=100(2) \mathrm{K}$, space group $P 212121, Z=4, \mu(\mathrm{CuK} \alpha)=0.589$ $\mathrm{mm}^{-1}, 24836$ reflections measured, 5017 independent reflections $\left(R_{i n t}=0.1013\right)$. The final $R_{l}$ values were $0.0500(I>2 \sigma(I))$. The final $w R\left(F^{2}\right)$ values were $0.1464(I>2 \sigma(I))$. The final $R_{l}$ values were 0.0662 (all data). The final $w R\left(F^{2}\right)$ values were 0.1552 (all data). The goodness of fit on $F^{2}$ was 1.104. Flack parameter $=0.1(2)$. The Hooft parameter is 0.01(7) for 2161 BiJvoet pairs.

Scopariusicide B (2): White power; $[\alpha]_{\mathrm{D}}^{22}-84.17\left(c\right.$ 0.07, MeOH); UV (MeOH) $\lambda_{\max }(\log \varepsilon) 200$ (0.43); IR (KBr) $v_{\max } 3439,2956,2930,1734,1613,1514,1459,1251,1180,1034 \mathrm{~cm}^{-1}$; Positive HRESIMS $[\mathrm{M}+\mathrm{Na}]^{+} \mathrm{m} / z 613.3866$ (calcd for $\left.\mathrm{C}_{38} \mathrm{H}_{54} \mathrm{O}_{5} \mathrm{Na}, 613.3869\right) ;{ }^{1} \mathrm{H}$ and ${ }^{13} \mathrm{C}$ NMR see Table 1.

Isoscoparin Q (3): White power; $[\alpha]_{\mathrm{D}}^{22}-138.35(c 0.08, \mathrm{MeOH}) ; \mathrm{UV}(\mathrm{MeOH}) \lambda_{\max }(\log \varepsilon) 205$ (0.57); Positive HRESIMS [M + Na $]^{+} m / z, 325.2144$ (calcd for $\mathrm{C}_{20} \mathrm{H}_{30} \mathrm{O}_{2} \mathrm{Na}, 325.2143$ ). ${ }^{1} \mathrm{H}$ NMR $\left(400 \mathrm{MHz}, \mathrm{CDCl}_{3}\right) \delta_{\mathrm{H}}: 5.91(1 \mathrm{H}, \mathrm{s}, \mathrm{H}-14), 4.63\left(2 \mathrm{H}\right.$, overlapped, $\left.\mathrm{H}_{2}-18\right), 4.38(1 \mathrm{H}, \mathrm{dd}, J=13.2$ and $2.9 \mathrm{~Hz}, \mathrm{H}-11)$, 2.35-2.06 (5H, overlapped, H-1a, $\mathrm{H}_{2}-3$, and $\left.\mathrm{H}_{2}-12\right), 1.84$ (3H, s, H-16), 1.71-1.22 (8H, overlapped, H-1b, $\mathrm{H}_{2}-2, \mathrm{H}-8, \mathrm{H}_{2}-6$, and $\left.\mathrm{H}_{2}-7\right), 1.03$ (3H, s, $\left.\mathrm{H}_{3}-20\right), 1.01$ (3H, s, $\left.\mathrm{H}_{3}-19\right), 0.74$ (3H, d, $\left.J=6.5 \mathrm{~Hz}, \mathrm{H}_{3}-17\right) ;{ }^{13} \mathrm{C}$ NMR (101 MHz, Pyr) $\delta_{\mathrm{C}}: 165.3$ (s, C-15), 160.0 (s, C-4), 159.0 (s, C-13), 116.7 (d, C-14), 103.5 (t, C-18), 84.0 (d, C-11), 49.1 (d, C-10), 43.1 (s, C-9), 40.6 (s, C-5), 37.3 (t, C-6), 37.3 (d, C-8), 33.3 (t, C-3), 29.8 (t, C-12), 28.4 (t, C-2), 28.0 (t, C-7), 23.8 (t, C-1), 22.8 (q, 
C-16), 20.8 (q, C-19), 16.7 (q, C-17), 13.9 (q, C-20). X-ray Crystallographic Analysis of 3: $\mathrm{C}_{20} \mathrm{H}_{30} \mathrm{O}_{2}$, $M=302.44$, monoclinic, $a=20.0748(7) \AA, b=6.8584(3) \AA, c=25.9078(9) \AA, \alpha=90.00^{\circ}, \beta=$ $106.3990(10)^{\circ}, \gamma=90.00^{\circ}, V=3421.9(2) \AA^{3}, T=100(2) \mathrm{K}$, space group $C 2, Z=8, \mu(\mathrm{CuK} \alpha)=0.567$ $\mathrm{mm}^{-1}, 14077$ reflections measured, 4870 independent reflections $\left(R_{\text {int }}=0.0444\right)$. The final $R_{1}$ values were $0.0936(I>2 \sigma(I))$. The final $w R\left(F^{2}\right)$ values were $0.2628(I>2 \sigma(I))$. The final $R_{l}$ values were 0.0948 (all data). The final $w R\left(F^{2}\right)$ values were 0.2745 (all data). The goodness of fit on $F^{2}$ was 1.387. Flack parameter $=0.3(3)$. The Hooft parameter is 0.20(8) for 1713 BiJvoet pairs.

\section{X-ray Crystal Structure Analysis.}

The intensity data for scopariusicide A (1), isoscoparin Q (3), and synthetic compounds $\mathbf{6 a}-\mathbf{6 c}$ were collected on a Bruker diffractometer using graphite-monochromated $\mathrm{Cu}$ or $\mathrm{Mo} \mathrm{K} \alpha$ radiation. The structures of these compounds were solved by direct methods (SHELXS97), expanded using difference Fourier techniques, and refined by the program and full-matrix least-squares calculations. The non-hydrogen atoms were refined anisotropically, and hydrogen atoms were fixed at calculated positions. Crystallographic data for the structures of scopariusicide A (1), isoscoparin Q (3), and synthetic compounds $\mathbf{6 a - 6 c}$ have been deposited in the Cambridge Crystallographic Data Centre database (deposition number CCDC 1039339 for $\mathbf{1}, 1039335$ for 3, 1039341 for $\mathbf{6 a}, 1039342$ for $\mathbf{6 b}$, and 1039343 for $\mathbf{6 c}$ ). Copies of the data can be obtained free of charge from the CCDC at www.ccdc.cam.ac.uk.

\section{Structural elucidation of scopariusicides A and B and their hypothetical biosynthetic pathway.}

An $70 \%$ acetone extract from the aerial parts of I. scoparius was subjected to repeated column chromatography over silica gel followed by semi-preparative and preparative HPLC, leading to the isolation of scopariusicides A (1) and B (2), the first two members of a new class of unsymmetrical cyclobutanes, together with their possible biosynthetic precursor $\mathbf{3}$. The structures were elucidated by interpretation of the NMR, X-ray crystallographic, and CD data.

Scopariusicide A (1) was isolated as colorless needles. Its molecular formula of $\mathrm{C}_{38} \mathrm{H}_{54} \mathrm{O}_{5}$ was determined by NMR spectroscopy and the observation of a HREIMS peak at $\mathrm{m} / z 613.3872[\mathrm{M}+$ $\mathrm{Na}^{+}$(calcd. 613.3869), indicating 12 degrees of unsaturation. Its ${ }^{13} \mathrm{C}$ NMR spectrum featured 38 carbon resonances (Table S1). On the basis of DEPT data, 6 of these 38 carbons were identified as methyl groups (one being oxygenated), 12 as methylenes (including two $\mathrm{sp}^{2}$ methylenes), 12 as methines (of which five were $\mathrm{sp}^{2}$ methines and two were oxygenated), and 8 as quaternary carbons 
(including three $\mathrm{sp}^{2}$ carbons and two carbonyls). Extensive analysis of 2D NMR data (HSQC, HMBC, and ROESY) indicated a clear similarity between $\mathbf{1}$ and scopariusic acid, an ent-clerodane-based head-to-tail cyclobutane derivative also isolated from I. scoparius. Interestingly, the NMR data for compound $\mathbf{1}$ indicate that it can be subdivided into three distinct structural fragments: a diterpenoid nucleus (part A), a phenylpropanoid derivative (part B), and a $\mathrm{C}_{8}$ fatty alcohol (part C).
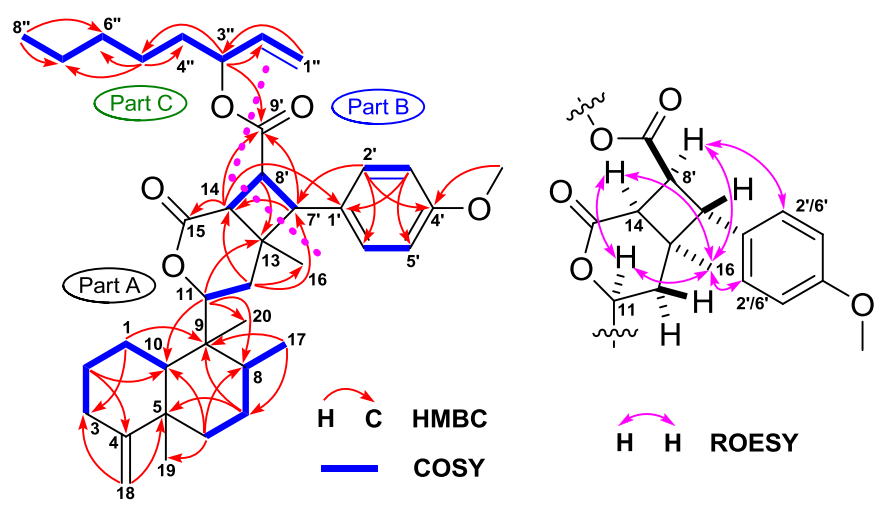

Figure S1. The key 2D NMR correlations of compound 1.

The connectivities of fragments A-C and the relative configuration of $\mathbf{1}$ were established by ${ }^{1} \mathrm{H}-{ }^{1} \mathrm{H}$ COSY, HMBC, and ROESY experiments (Figure S1), and verified by single-crystal X-ray diffraction (Figure S2). Firstly, the substructure of part A in $\mathbf{1}$ was similar to the diterpenoid nucleus of scopariusic acid. ${ }^{4 \mathrm{~b}}$ The main difference was the C-11 and C-15 peaks of 1 were downshifted and upshifted, respectively, relative to their equivalents in scopariusic acid, indicating the linkage of these carbons to form a six-membered lactone. Additionally, the key HMBC correlations from H-11 to $\mathrm{C}-13$ and $\mathrm{H}-14$ to $\mathrm{C}-12$ indicated that the pre-existing $\mathrm{C}-13 / \mathrm{C}-14$ double bond on the lactone had become saturated in 1, suggesting that part A was linked to part B or C via C-13 and/or C-14. Secondly, the presence of a phenylpropanoid moiety (part B) was indicated by the $\mathrm{H}-8 / \mathrm{H}-7^{\prime}{ }^{1} \mathrm{H}-{ }^{1} \mathrm{H}$ COSY correlation together with the HMBC correlations from $\mathrm{H}-8$ ' to C-9' and C-1', and from $\mathrm{H}-\mathrm{7}^{\prime}$ to C-9', C-2', and C-6'. This phenylpropanoid moiety was determined to possess a methoxy group at the C-4' position based on $\mathrm{HMBC}$ correlations from the 4'-OMe group to $\mathrm{C}-4$ '. The remaining ${ }^{13} \mathrm{C}$ NMR signals were assigned to a $\mathrm{C}_{8}$ fatty alcohol (part $\mathrm{C}$ ), which was identified as 1-octen-3-ol on the basis of ${ }^{1} \mathrm{H}-{ }^{1} \mathrm{H}$ COSY correlations $\left(\mathrm{H}_{2}-1 " / \mathrm{H}-2 " / \mathrm{H}-3 " / \mathrm{H}_{2}-4 " / \mathrm{H}_{2}-5 " / \mathrm{H}_{2}-6 " / \mathrm{H}_{2}-7 " / \mathrm{H}_{3}-8 "\right)$, combined with HMBC correlations from H-3" to C-1" and C-5", H-4" to C-2" and C-6", and H-8" to C-5" and C-6". Having elucidated the structures of these subunits, their connectivity remained to be determined. Parts A and B of compound $\mathbf{1}$ were suggested to be linked by a cyclobutane ring similar to that of 
scopariusic acid. However, the NMR data for carbons C-13, C-14, C-7', and C-8' of 1 were quite different to those for the equivalent carbons of scopariusic acid, indicating that the substitution patterns of the cyclobutane rings in these two compounds differ substantially. The HMBC correlations of $\mathrm{H}-7$ ' with $\mathrm{C}-12, \mathrm{C}-13, \mathrm{C}-14$, and $\mathrm{C}-16$, of $\mathrm{H}-8$ ' with $\mathrm{C}-13, \mathrm{C}-14$, and $\mathrm{C}-15$, and of H-14 with C-8', C-7', and C-9', combined with the $\mathrm{H}-8 / \mathrm{H}-14{ }^{1} \mathrm{H}-{ }^{1} \mathrm{H}$ COSY correlation, suggested that C-7' is directly bonded to C-13 and that C-8' is directly bonded to C-14 to form a 'head-to-head' cyclobutane ring. In addition, the HMBC correlation of H-3" with C-9' suggested that parts B and C are connected via C-9'. The planar structure of 1 was thus established as a novel ent-clerodane-based head-to-head cyclobutane derivative with an unusual 1-octen-3-ol substituent. The partial relative configuration of $\mathbf{1}$ was determined from its ROESY spectrum. The ROESY cross-peaks of $\mathrm{H}-11 / \mathrm{H}_{3}-16 / \mathrm{H}-14, \mathrm{H}-16 / \mathrm{H}-2^{\prime} / \mathrm{H}-6$ ', and $\mathrm{H}-8^{\prime} / \mathrm{H}-16 / \mathrm{H}-2^{\prime} / \mathrm{H}-6^{\prime}$ suggested that $\mathrm{H}-11, \mathrm{H}-14, \mathrm{H}-16$, and $\mathrm{H}-8^{\prime}$ all have an $\alpha$-orientation, while $\mathrm{H}-7^{\prime}$ is $\beta$-oriented. However, the relative stereochemistry of C-3" was not readily determined from its ROESY spectrum. The assigned structure and configuration of compound $\mathbf{1}$ were ultimately verified by single-crystal X-ray diffraction, which unequivocally established the absolute configuration of all its stereocenters and confirmed the presence of the unusual cyclobutane ring described above. The absolute configuration of $\mathbf{1}$ was further confirmed by synthesis.

Scopariusicide B was isolated as a colorless gum and was assigned the same molecular formula $\mathrm{C}_{38} \mathrm{H}_{54} \mathrm{O}_{5}$ as 1 based on HREIMS results $(m / z \text { 613.3872 [M }+\mathrm{Na}]^{+}$, calcd 613.3869) and NMR data. The ${ }^{1} \mathrm{H}$ and ${ }^{13} \mathrm{C}$ NMR spectra of $\mathbf{2}$ were very similar to those of $\mathbf{1}$; the main differences were that the ${ }^{1} \mathrm{H}$ NMR spectrum of 2 contained a peak at $\delta_{\mathrm{H}} 5.19$ (br s) in place of the C-3 methylene group of 1 [which was responsible for the $\mathrm{H}_{2}-3$ peaks at $\delta_{\mathrm{H}} 2.27(\mathrm{~m})$ and $2.11(\mathrm{~m})$ ], and a methyl group peak at $\delta_{\mathrm{H}} 1.58(\mathrm{~s})$ in place of the $\mathrm{C}-18$ exomethylene functionality of $\mathbf{1}$ [which was responsible for the $\mathrm{H}_{2}-18$ peaks at $\left.\delta_{\mathrm{H}} 4.64(2 \mathrm{H}, \mathrm{s})\right]$. These differences indicated that the exocyclic C-4/C-18 double bond of $\mathbf{1}$ is replaced with an endocyclic C-3/C-4 double bond in $\mathbf{2}$. The relative configuration of $\mathbf{2}$ was deduced to be identical to that of $\mathbf{1}$ from the assignments of the cross-peaks in its ROESY spectrum. In addition, the CD spectrum of 2 revealed a negative Cotton effect at $294 \mathrm{~nm}$, similar to that observed for $\mathbf{1}$. These results suggest that $\mathbf{1}$ and $\mathbf{2}$ have the same absolute configuration.

Biosynthetically, scopariusicides might be formed via a crossed head-to-head intermolecular $[2+$ 2] cycloaddition between an ent-clerodane diterpenoid (part A or 3) and an unusual ester (1a) of trans-4-hydroxycinnamic acid (part B) and (3R)-1-octen-3-ol (part C) (Scheme 1). 
Table S1. ${ }^{1} \mathrm{H}(500 \mathrm{MHz}) \mathrm{NMR},{ }^{13} \mathrm{C}(125 \mathrm{MHz}) \mathrm{NMR}$, and HMBC Spectroscopic Data of 1 and 2 in $\mathrm{C}_{5} \mathrm{D}_{5} \mathrm{~N}(\delta \mathrm{ppm})$

\begin{tabular}{|c|c|c|c|c|c|c|}
\hline \multirow{2}{*}{ no. } & \multicolumn{3}{|c|}{1} & \multicolumn{3}{|c|}{2} \\
\hline & $\delta_{\mathrm{C}}$ & $\delta_{\mathrm{H}}$ & $\operatorname{HMBC}\left({ }^{1} \mathrm{H} \rightarrow{ }^{13} \mathrm{C}\right)$ & $\delta_{\mathrm{C}}$ & $\delta_{\mathrm{H}}$ & $\operatorname{HMBC}\left({ }^{1} \mathrm{H} \rightarrow{ }^{13} \mathrm{C}\right)$ \\
\hline 1 & $24.3 \mathrm{t}$ & $2.34, \mathrm{~d}(13.5) ; 1.62, \mathrm{~m}$ & $2,3,9,10$ & $20.5 \mathrm{t}$ & $2.43, \mathrm{~m} ; 1.62, \mathrm{~m}$ & $2,3,9,10$ \\
\hline 2 & $28.9 \mathrm{t}$ & $1.74, \mathrm{~m} ; 1.34, \mathrm{~m}$ & $1,3,4,10$ & $27.0 \mathrm{t}$ & $2.11, \mathrm{~m} ; 1.95, \mathrm{~m}$ & $1,3,4,10$ \\
\hline 3 & $33.8 \mathrm{t}$ & $2.27, \mathrm{~m} ; 2.11, \mathrm{~m}$ & $1,2,4,5,18$ & $121.7 \mathrm{~d}$ & 5.19, br s & $1,2,4,5,18$ \\
\hline 4 & $160.4 \mathrm{~s}$ & & & $143.8 \mathrm{~s}$ & & \\
\hline 5 & $41.0 \mathrm{~s}$ & & & $38.9 \mathrm{~s}$ & & \\
\hline 6 & $37.7 \mathrm{t}$ & $1.57, \mathrm{~m}$ & $4,5,7,8,10,19$ & $36.8 \mathrm{t}$ & $1.32, \mathrm{~m} ; 1.13, \mathrm{~m}$ & $4,5,7,8,10,19$ \\
\hline 7 & $28.5 \mathrm{t}$ & $1.48, \mathrm{~m} ; 1.41, \mathrm{~m}$ & $5,6,8,9$ & $28.2 \mathrm{t}$ & $1.40, \mathrm{~m} ; 1.35, \mathrm{~m}$ & $5,6,8,9$ \\
\hline 8 & $37.4 \mathrm{~d}$ & $1.32, \mathrm{~m}$ & $7,9,10,17,20$ & $36.9 \mathrm{~d}$ & $1.61, \mathrm{~m}$ & $7,9,10,17,20$ \\
\hline 9 & $43.5 \mathrm{~s}$ & & & $42.7 \mathrm{~s}$ & & \\
\hline 10 & $49.3 \mathrm{~d}$ & $1.41, \mathrm{~m}$ & overlapped & $46.9 \mathrm{~d}$ & $1.60, \mathrm{~m}$ & overlapped \\
\hline 11 & $85.3 \mathrm{~d}$ & $4.29, \mathrm{~d}(11.0)$ & $8,9,10,12,13,20$ & $85.3 \mathrm{~d}$ & $4.33, \mathrm{~d}(11.0)$ & $8,9,10,12,13,20$ \\
\hline 12 & $40.5 \mathrm{t}$ & $2.19, \mathrm{t}(11.4,) ; 2.14, \mathrm{~d}(13.9)$ & $9,11,13,14,16,7^{\prime}$ & $40.4 \mathrm{t}$ & $2.27, \mathrm{dd}(11.5,13.4) ; 2.17, \mathrm{~d}(13.6)$ & $9,11,12,13,14,16,7^{\prime}$ \\
\hline 13 & $40.0 \mathrm{~s}$ & & & $39.9 \mathrm{~s}$ & & \\
\hline 14 & $44.3 \mathrm{~d}$ & $3.41, \mathrm{~d}(10.4)$ & $13,14,15,16,7^{\prime}, 8^{\prime}, 9^{\prime}, 1^{\prime}$ & $44.2 \mathrm{~d}$ & $3.45, \mathrm{~d}(10.5)$ & $13,14,15,16,7^{\prime}, 8^{\prime}, 9^{\prime}, 1^{\prime}$ \\
\hline 15 & $172.3 \mathrm{~s}$ & & & $172.3 \mathrm{~s}$ & & \\
\hline 16 & $22.8 \mathrm{q}$ & $1.07, \mathrm{~s}$ & $12,13,14,7^{\prime}$ & $22.6 \mathrm{q}$ & $1.08, \mathrm{~s}$ & $12,13,14,7^{\prime}$ \\
\hline 17 & $17.3 \mathrm{q}$ & $0.79, \mathrm{~d}(6.6)$ & $7,8,9$ & $16.9 \mathrm{q}$ & $0.77, \mathrm{~d}(6.4)$ & $7,8,9$ \\
\hline 18 & $103.9 \mathrm{t}$ & $4.64, \mathrm{~s}$ & $3,5,4,19$ & $18.7 \mathrm{q}$ & $1.58, \mathrm{~s}$ & $3,5,4,19$ \\
\hline 19 & $21.2 \mathrm{q}$ & $1.03, \mathrm{~s}$ & $4,5,6,10$ & $20.1 \mathrm{q}$ & $0.97, \mathrm{~s}$ & $4,5,6,10$ \\
\hline 20 & $14.5 \mathrm{q}$ & $1.02, \mathrm{~s}$ & $8,9,10,11$ & $14.5 \mathrm{q}$ & $1.00, \mathrm{~s}$ & $8,9,10,11$ \\
\hline $1^{\prime}$ & $131.5 \mathrm{~s}$ & & & $131.4 \mathrm{~s}$ & & \\
\hline $2^{\prime}$ & $129.5 \mathrm{~d}$ & $7.27, \mathrm{~d}(8.5)$ & $1^{\prime}, 3^{\prime}, 4^{\prime}, 7^{\prime}$ & $129.4 \mathrm{~d}$ & $7.33, \mathrm{~d}(8.5)$ & 1', 3', 4', 7' \\
\hline $3^{\prime}$ & $114.8 \mathrm{~d}$ & $7.04, \mathrm{~d}(8.5)$ & $1^{\prime}, 2^{\prime}, 4^{\prime}$ & $114.7 \mathrm{~d}$ & $7.06, \mathrm{~d}(8.5)$ & $1^{\prime}, 2^{\prime}, 4^{\prime}$ \\
\hline $4^{\prime}$ & $159.5 \mathrm{~s}$ & & & $159.5 \mathrm{~s}$ & & \\
\hline $5^{\prime}$ & $114.8 \mathrm{~d}$ & $7.04, \mathrm{~d}(8.5)$ & $1^{\prime}, 2^{\prime}, 4^{\prime}$ & $114.7 \mathrm{~d}$ & $7.06, \mathrm{~d}(8.5)$ & $1^{\prime}, 2^{\prime}, 4^{\prime}$ \\
\hline $6^{\prime}$ & $129.5 \mathrm{~d}$ & $7.27, \mathrm{~d}(8.5)$ & $1^{\prime}, 4^{\prime}, 5^{\prime}, 7^{\prime}$ & $129.4 \mathrm{~d}$ & $7.33, \mathrm{~d}(8.5)$ & $1^{\prime}, 4^{\prime}, 5^{\prime}, 7^{\prime}$ \\
\hline $7^{\prime}$ & $51.9 \mathrm{~d}$ & $4.10, \mathrm{~d}(9.7)$ & $12,13,16,1^{\prime}, 2^{\prime}, 6^{\prime}, 8^{\prime}, 9^{\prime}$ & $52.0 \mathrm{~d}$ & $4.13, \mathrm{~d}(9.6)$ & $12,13,16,1^{\prime}, 2^{\prime}, 6^{\prime}, 8^{\prime}, 9^{\prime}$ \\
\hline $8^{\prime}$ & $41.8 \mathrm{~d}$ & $4.18, \mathrm{t}(10.1)$ & $13,14,16,1^{\prime}, 7^{\prime}, 8^{\prime}, 9^{\prime}$ & $41.7 \mathrm{~d}$ & $4.23, \mathrm{t}(10.1)$ & $13,14,16,1^{\prime}, 7^{\prime}, 8^{\prime}, 9^{\prime}$ \\
\hline $9^{\prime}$ & $172.5 \mathrm{~s}$ & & & $172.5 \mathrm{~s}$ & & \\
\hline $1 "$ & $116.8 \mathrm{t}$ & $5.36,(\mathrm{~d}, 17.3) ; 5.18,(\mathrm{~d}, 10.6)$ & $2^{\prime \prime}, 3 "$ & $116.6 \mathrm{t}$ & $5.37, \mathrm{~d}(17.2) ; 5.17, \mathrm{~d}(10.6)$ & $2 ", 3 "$ \\
\hline $2^{\prime \prime}$ & $138.1 \mathrm{~d}$ & $5.97,(\mathrm{ddd}, 17.0,10.5,6.2)$ & 1", 3", 4" & $138.0 \mathrm{~d}$ & $5.97, \mathrm{~m}$ & 1", 3", 4" \\
\hline $3^{\prime \prime}$ & $76.1 \mathrm{~d}$ & $5.61, \mathrm{q}(6.4)$ & 9', 1", 2", 4", 5" & $76.0 \mathrm{~d}$ & $5.62, \mathrm{q}(6.4)$ & 9', 1", 2", 4", 5" \\
\hline 4" & $34.7 \mathrm{t}$ & $1.93, \mathrm{~m} ; 1.76, \mathrm{~m}$ & 2", 3", 5", 6" & $34.6 \mathrm{t}$ & $1.92, \mathrm{~m} ; 1.75, \mathrm{~m}$ & 2", 3", 5", 6" \\
\hline $5 "$ & $25.4 \mathrm{t}$ & $1.46, \mathrm{~m}$ & 3", 4", 6", 7" & $25.3 \mathrm{t}$ & $1.47, \mathrm{~m}$ & 3", 4", 6", 7" \\
\hline 6" & $32.4 \mathrm{t}$ & $1.27, \mathrm{~m}$ & 4", 5", 7", 8" & $32.2 \mathrm{t}$ & $1.26, \mathrm{~m}$ & 4", 5", 7", 8" \\
\hline 7" & $23.3 \mathrm{t}$ & $1.26, \mathrm{~m}$ & $5 ", 6 ", 8^{\prime \prime}$ & $23.2 \mathrm{t}$ & $1.24, \mathrm{~m}$ & $5^{\prime \prime}, 6 ", 8^{\prime \prime}$ \\
\hline $8 "$ & $14.7 \mathrm{q}$ & $0.83, \mathrm{t}(6.9)$ & 6", 7" & $14.5 \mathrm{q}$ & $0.81, \mathrm{t}(7.0)$ & 6", 7" \\
\hline $1 " '$ & $55.7 \mathrm{q}$ & $3.71, \mathrm{~s}$ & $4^{\prime}$ & $55.5 \mathrm{q}$ & $3.70, \mathrm{~s}$ & $4^{\prime}$ \\
\hline
\end{tabular}




\section{Immunosuppressive activity bioassay.}

$T$ cell isolation and culture: Human peripheral blood mononuclear cells (PBMC) were isolated from three healthy donors by density-gradient centrifugation using Lymphoprep and re-suspended in the complete medium of RP1640 supplemented by 10\% fetal bovine serum (FBS, Invitrogen, Carlsbad, CA, USA). Similarly, mouse splenic mononuclear cells were isolated and $\mathrm{CD}^{+} \mathrm{T}$ cells were also purified by immunobeads by negative selection. Human $\mathrm{CD}^{+} \mathrm{T}$ cells were purified by negative selection using the Pan T cell Isolation Kit II Human, according to the manufacturer's instructions (Miltenyi Biotec, Bergisch Gladbach, Germany). The purity of the resulting $\mathrm{T}$ cell populations was examined by flow cytometry using PE-anti-CD3 (BD PharMingen, San Diego, CA, USA) and a T cell population with purity of $95 \%$ was used for the following experiments.

Proliferation assay: The level of cell proliferation was determined by flow cytometry analysis with CFSE labelling. Briefly, the purified human $\mathrm{T}$ cells $\left(10^{6}\right.$ cells $\left.\mathrm{ml}^{-1}\right)$ were stained with $2.5 \mu \mathrm{M}$ 5-carboxyfluorescein diacetate succinimide ester (CFSE, Molecular Probes, Eugene, OR, USA) at $37^{\circ} \mathrm{C}$ for $10 \mathrm{~min}$, washed twice with PBS and resuspended in complete medium. Subsequently, the CFSE-labeled human T cells $\left(10^{6}\right.$ cells $\left.\mathrm{ml}^{-1}\right)$ were stimulated by plate-bound anti-CD3 $\left(2 \mu \mathrm{g} \mathrm{m}{ }^{-1}\right.$, HIT3a clone) and soluble anti-CD28 (1 $\mu \mathrm{g} \mathrm{ml}^{-1}, \mathrm{CD} 28.2$ clone, BD PharMingen $)$ in the presence of increased concentrations of each test compound dissolved in DMSO that was less than $0.025 \%$ or vehicle alone for $72 \mathrm{~h}$. The proliferation of CFSE-labelled cells was analysed by flow cytometry on a FACSCalibur (Becton Dickinson, San Jose, CA, USA) using CellQuest acquisition and ModFit analysis software (Becton Dickinson).

CCK-8 assay: The effect of each test compound on the survival of human PBMC was determined using the CCK-8 assay, according to the manufacturer's instruction. Briefly, PBMC was incubated in the presence of different concentrations of each test compound or vehicle alone for $72 \mathrm{~h}$. The viability of cells was evaluated by the CCK- 8 assay and detection of absorbance at $450 \mathrm{~nm}$ on a SpectraMax M5 microplate reader (Molecular Devices, Sunnyvale, CA, USA). 


\section{Synthesis procedures}

Scheme S1. Synthesis of scopariusicide A (1) starting from main compound 4 from the title plant

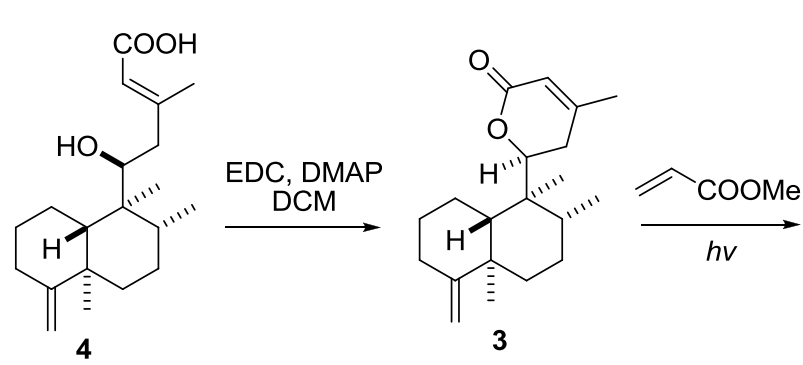

3

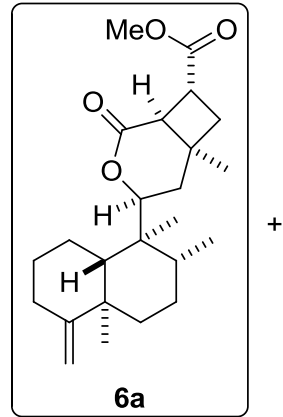

$6 a$

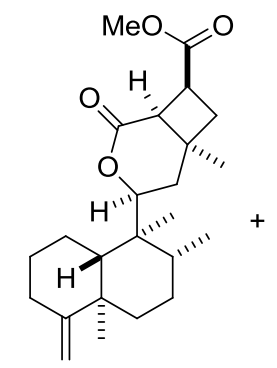

$6 b$

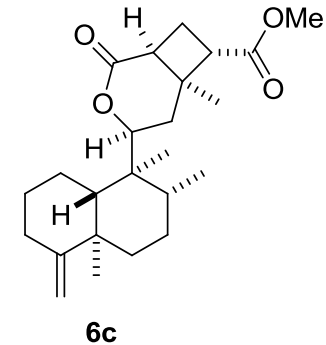

$6 c$

LiOH, MeCN
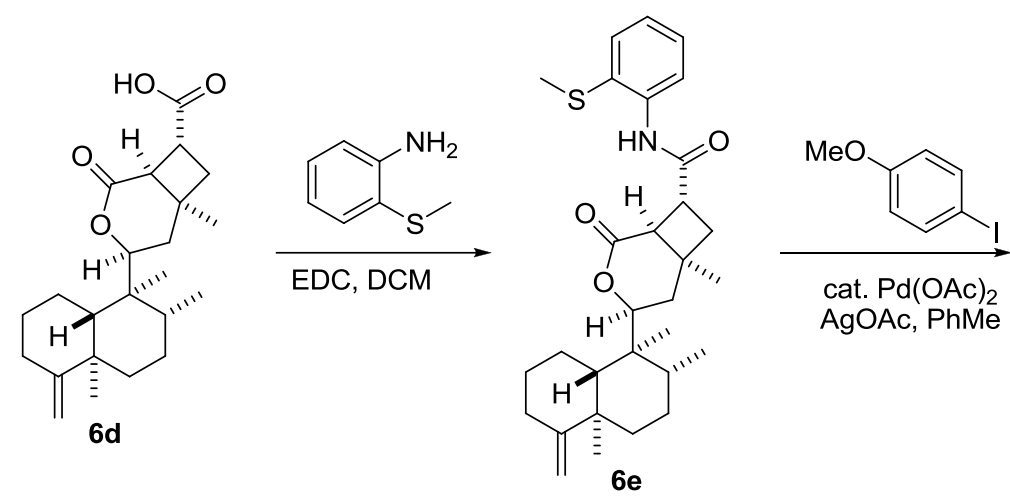

急

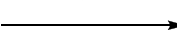

$6 d$

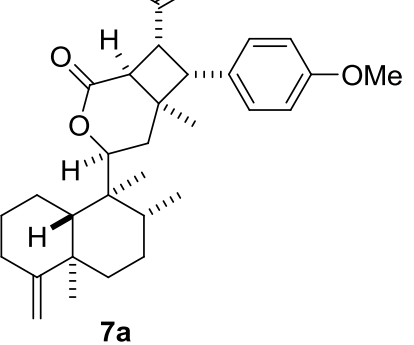

$7 a$

$\mathrm{NaOH}, \mathrm{EtOH}$<smiles>COc1ccc([C@@H]2C(C(=O)O)[C@H](C(=O)O)C2(C)C)cc1</smiles>

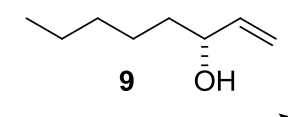

DCC, DMAP, DCM

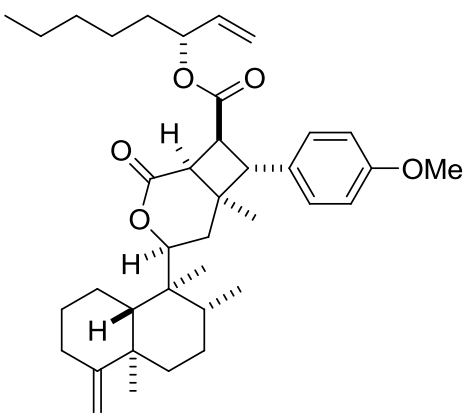

1 (synthetic compound)

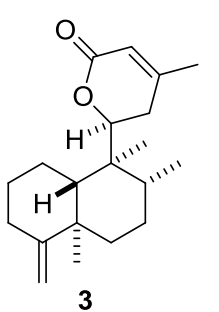

Synthesis of 3: compound 4 (10.0 g, $31.25 \mathrm{mmol})$ was dissolved in dry DCM (250 $\mathrm{mL})$ and the flask was cooled to $0{ }^{\circ} \mathrm{C}$ in an ice bath. EDC $(12.0 \mathrm{~g}, 62.5 \mathrm{mmol}, 2$ equiv) and DMAP (1.91 g, $15.63 \mathrm{mmol}, 0.5$ equiv) were added in a single portion and the reaction mixture was warmed to room temperature and stirred for an additional $4 \mathrm{~h}$. After filtering the reaction mixture through a pad of celite, it was washed with saturated aqueous $\mathrm{NaHCO}_{3}(200 \mathrm{~mL})$ and $1 \mathrm{M} \mathrm{HCl}(200 \mathrm{~mL})$. The combined extracts were washed with brine $(250 \mathrm{~mL})$, dried over $\mathrm{Na}_{2} \mathrm{SO}_{4}$ and concentrated in vacuo. The resulting 
white solid was purified by column chromatography $\left(\mathrm{CHCl}_{3}\right)$ to give $3(9.25 \mathrm{~g}, 30.63 \mathrm{mmol}, 98 \%$ yield) as a white solid. This process is repeated three times.
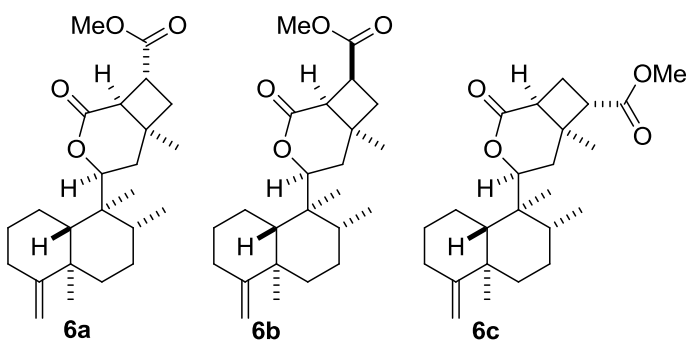

Synthesis of $\mathbf{6} \boldsymbol{a}-\boldsymbol{6} \boldsymbol{c}$ : compounds $\mathbf{3}(40.0 \mathrm{mg}, 0.13 \mathrm{mmol})$ and 5 (171.0 $\mathrm{mg}, 1.99 \mathrm{mmol}, 15.0$ equiv) were dissolved in acetonitrile and acetone $(5: 1,12 \mathrm{~mL})$ in a quartz tube. This process is repeated six times. The reaction vessels were carefully sealed and irradiated using a photoreactor $(313 \mathrm{~nm})$ at room temperature for $5 \mathrm{~h}$. The resulting mixture was concentrated in vacuo and then directly subJected to flash column chromatography (silica gel, $\mathrm{CHCl}_{3}$ :EtOAc, 20:1) and preparative HPLC to give two maJor head-to-head cyclobutanes 6a (205.8 mg, $0.54 \mathrm{mmol}, 68 \%$ ) and $6 \mathbf{b}$ (101.4 mg, $0.26 \mathrm{mmol}, 27 \%)$, and a minor head-to-tail cyclobutane $6 \mathbf{c}(10.1 \mathrm{mg}, 0.05 \mathrm{mmol}$, $3 \%$ ) in $98 \%$ combined yield with a 5:2:0.2 ratio. Compound 6a: White power; Positive ESIMS [M + $\mathrm{Na}]^{+} m / z$ 411; ${ }^{1} \mathrm{H}$ NMR (600 MHz, $\left.\mathrm{CDCl}_{3}\right) \delta_{\mathrm{H}}: 4.49(1 \mathrm{H}, \mathrm{s}, \mathrm{H}-18 \mathrm{a}), 4.47(1 \mathrm{H}, \mathrm{s}, \mathrm{H}-18 \mathrm{~b}), 4.11(1 \mathrm{H}, \mathrm{d}$, $J=11.1 \mathrm{~Hz}, \mathrm{H}-11), 3.70(3 \mathrm{H}, \mathrm{s}, \mathrm{OMe}), 3.20\left(1 \mathrm{H}, \mathrm{dt}, J=10.1\right.$ and $\left.7.0 \mathrm{~Hz}, \mathrm{H}-8^{\prime}\right), 3.08(1 \mathrm{H}, \mathrm{d}, J=6.8$ Hz, H-14), 2.26 (1H, dt, $J=13.7$, and $6.8 \mathrm{~Hz}, \mathrm{H}-3 \mathrm{a}), 2.15$ (1H, t, $J=6.5, \mathrm{H}-7 \mathrm{a}$ ), $2.10-2.03$ (2H, m, overlapped, H-7a' and H-3b), 1.88-1.78 (3H, m, overlapped, H-1a, H-2a, and H-12a), 1.63-1.49 (4H, m, overlapped, H-1b, H-6a, H-7a, and H-12b), 1.47-1.43 (2H, m, overlapped, H-6b and H-7b), 1.28 (1H, m, H-8), 1.23 (3H, s, H3-16), 1.18 (1 H, m, H-10), 1.03 (3H, s, H3-19), 0.98 (3H, s, H3-20), 0.82 $\left(3 \mathrm{H}, \mathrm{d}, \mathrm{J}=6.4 \mathrm{~Hz}, \mathrm{H}_{3}-17\right) ;{ }^{13} \mathrm{C}$ NMR $(151 \mathrm{MHz}, \mathrm{CDCl} 3) \delta_{\mathrm{C}}: 174.3$ (s, C-9'), 173.7 (s, C-15), 160.0 (s, C-4), 103.0 (t, C-18), 84.9 (d, C-11), 52.4 (q, OMe), 48.6 (d, C-10), 44.2 (d, C-14), 42.9 (s, C-9), 40.3 (s, C-5), 37.5 (t, C-12), 37.0 (d, C-8'), 36.9 (t, C-6), 36.8 (d, C-8), 36.3 (t, C-7'), 34.4 (s, C-13), 33.1 (t, C-3), 28.4 (t, C-2), 27.8 (s, C-7), 26.5 (q, C-16), 23.7 (t, C-1), 20.6 (q, C-19), 16.7 (q, C-17), 13.8 (q, C-20). X-ray Crystallographic Analysis of 6a: $\mathrm{C}_{24} \mathrm{H}_{36} \mathrm{O}_{4}, M=388.53$, monoclinic, $a=$ $31.775(5) \AA, b=7.2610(11) \AA, c=19.184(3) \AA, \alpha=90.00^{\circ}, \beta=104.026(2)^{\circ}, \gamma=90.00^{\circ}, V=$ 4294.1(12) $\AA^{3}, T=100(2) \mathrm{K}$, space group $C 2, Z=8, \mu(\mathrm{MoK} \alpha)=0.080 \mathrm{~mm}^{-1}, 22283$ reflections measured, 11041 independent reflections $\left(R_{\text {int }}=0.0501\right)$. The final $R_{l}$ values were $0.0668(I>2 \sigma(I))$. The final $w R\left(F^{2}\right)$ values were $0.1889(I>2 \sigma(I))$. The final $R_{l}$ values were 0.0770 (all data). The final $w R\left(F^{2}\right)$ values were 0.1943 (all data). The goodness of fit on $F^{2}$ was 1.103 . Flack parameter $=$ 0.0(12). Compound 6b: White power; Positive ESIMS $[\mathrm{M}+\mathrm{Na}]^{+} m / z$ 411; ${ }^{1} \mathrm{H}$ NMR $(600 \mathrm{MHz}$, $\left.\mathrm{CDCl}_{3}\right) \delta_{\mathrm{H}}: 4.49(1 \mathrm{H}, \mathrm{s}, \mathrm{H}-18 \mathrm{a}), 4.47(1 \mathrm{H}, \mathrm{s}, \mathrm{H}-18 \mathrm{~b}), 4.07(1 \mathrm{H}, \mathrm{d}, J=11.3 \mathrm{~Hz}, \mathrm{H}-11), 3.65$ (3H, s, OMe), $3.51(1 \mathrm{H}$, ddd, $J=16.1,8.0$, and $4.5 \mathrm{~Hz}, \mathrm{H}-8$ '), $3.00(1 \mathrm{H}, \mathrm{d}, J=11.1 \mathrm{~Hz}, \mathrm{H}-14), 2.28(1 \mathrm{H}$, td, 
$J=13.6$ and $5.1 \mathrm{~Hz}, \mathrm{H}-3 \mathrm{a}), 2.18\left(1 \mathrm{H}, \mathrm{dd}, J=12.4\right.$ and $\left.6.2 \mathrm{~Hz}, \mathrm{H}-7 \mathrm{a}^{\prime}\right), 2.12-2.06$ (3H, m, overlapped, H-12a, H-7b', and H-3b), 1.98 (1H, m, H-1a),1.87 (1H, m, H-2a), 1.76 (1H, d, J = 13.9 Hz, H-12b), 1.60-1.52 (3H, m, overlapped, H-1b, H-6a, and H-7a), 1.49-1.43 (2H, m, overlapped, H-6b and H-7b), 1.36-1.24 (3H, m, overlapped, H-2b, H-8, and H-10), 1.23 (3H, s, $\left.\mathrm{H}_{3}-16\right), 1.04$ (3H, s, $\left.\mathrm{H}_{3}-19\right)$, $0.97\left(3 \mathrm{H}, \mathrm{s}, \mathrm{H}_{3}-20\right), 0.82\left(3 \mathrm{H}, \mathrm{d}, J=6.7 \mathrm{~Hz}, \mathrm{H}_{3}-17\right)$; ${ }^{13} \mathrm{C}$ NMR $(151 \mathrm{MHz}, \mathrm{CDCl} 3) \delta_{\mathrm{C}}: 173.8$ (s, C-9'), 172.4 (s, C-15), 160.3 (s, C-4), 102.9 (t, C-18), 84.9 (d, C-11), 52.1 (q, OMe), 48.6 (d, C-10), 44.3 (d, C-14), 42.9 (s, C-9), 40.3 (s, C-5), 37.0 (t, C-12), 36.9 (d, C-8), 36.3 (d, C-8'), 36.3 (t, C-6), 35.1 (t, C-7'), 34.7 (s, C-13), 33.2 (t, C-3), 28.4 (s, C-7), 27.8 (t, C-2), 27.2 (q, C-16), 23.4 (t, C-1), 20.6 (q, C-19), 16.7 (q, C-17), 13.8 (q, C-20). X-ray Crystallographic Analysis of $\mathbf{6 b}: \mathrm{C}_{24} \mathrm{H}_{36} \mathrm{O}_{4}, M=388.53$, monoclinic, $a=10.270(2) \AA, b=7.2570(16) \AA, c=14.424(3) \AA, \alpha=90.00^{\circ}, \beta=92.479(3)^{\circ}, \gamma=$ $90.00^{\circ}, V=1074.0(4) \AA^{3}, T=100(2) \mathrm{K}$, space group $P 21, Z=2, \mu(\mathrm{MoK} \alpha)=0.080 \mathrm{~mm}^{-1}, 10531$ reflections measured, 5114 independent reflections $\left(R_{\text {int }}=0.0343\right)$. The final $R_{1}$ values were 0.0399 $(I>2 \sigma(I))$. The final $w R\left(F^{2}\right)$ values were $0.0969(I>2 \sigma(I))$. The final $R_{l}$ values were 0.0485 (all data). The final $w R\left(F^{2}\right)$ values were 0.1018 (all data). The goodness of fit on $F^{2}$ was 1.054 . Flack parameter $=-0.2(8)$. Compound 6c: White power; Positive ESIMS $[\mathrm{M}+\mathrm{Na}]^{+} \mathrm{m} / z, 411 ;{ }^{1} \mathrm{H}$ NMR $(600$ MHz, $\left.\mathrm{CDCl}_{3}\right) \delta_{\mathrm{H}}: 4.51(1 \mathrm{H}, \mathrm{s}, \mathrm{H}-18 \mathrm{a}), 4.49(1 \mathrm{H}, \mathrm{s}, \mathrm{H}-18 \mathrm{~b}), 4.04(1 \mathrm{H}, \mathrm{d}, J=11.1 \mathrm{~Hz}, \mathrm{H}-11), 3.70(3 \mathrm{H}$, s, OMe), $2.92(1 \mathrm{H}, \mathrm{t}, J=8.8 \mathrm{~Hz}, \mathrm{H}-14), 2.78(1 \mathrm{H}, \mathrm{ddd}, J=12.4,10.6$, and $8.9 \mathrm{~Hz}, \mathrm{H}-7 \mathrm{a}), 2.59$ (1H, dd, $J=10.7$ and $3.9 \mathrm{~Hz}, \mathrm{H}-8$ '), 2.39 (1H, ddd, $J=12.9,9.1$, and $\left.4.0 \mathrm{~Hz} \mathrm{H}-7 \mathrm{~b}^{\prime}\right), 2.29$ (1H, m, H-3a), 2.10 (1H, m, H-3b), 1.99 (1H, d, $J=14.0$ Hz, H-12a), 1.93 (1H, d, $J=14.0$ Hz, H-1a), 1.93 (1H, d, $J$ $=12.3 \mathrm{~Hz}, \mathrm{H}-2 \mathrm{a}), 1.93(1 \mathrm{H}, \mathrm{br} \mathrm{t}, J=14.0 \mathrm{~Hz}, \mathrm{H}-12 \mathrm{~b}), 1.66-1.52$ (3H, m, overlapped, H-1b, H-6a, and H-7a), 1.49-1.45 (2H, m, overlapped, H-6b and H-7b), 1.30-1.17 (3H, m, overlapped, H-2b, H-8, and H-10), 1.17 (3H, s, H-16), 1.05 (3H, s, H-19), 0.99 (3H, s, H-20), 0.84 (3H, d, J = 6.7 Hz, H-17); ${ }^{13} \mathrm{C}$ NMR (151 MHz, CDCl3) $\delta_{\mathrm{C}}: 175.2$ (s, C-9'), 172.6 (s, C-15), 160.1 (s, C-4), 103.1 (t, C-18), 84.9 (d, C-11), 51.8 (q, OMe), 48.7 (d, C-10), 47.7 (d, C-14), 42.8 (s, C-9), 40.5 (s, C-5), 40.4 (s, C-13), 40.3(d, C-8'), 40.0 (t, C-12), 37.0 (d, C-8), 37.0 (t, C-6), 33.1 (t, C-3), 28.4 (t, C-2), 27.8 (s, C-7), 23.7 (t, C-1), 22.6 (t, C-7'), 22.2 (q, C-16), 20.6 (q, C-19), 16.8 (q, C-17), 13.8 (q, C-20). X-ray Crystallographic Analysis of 6c: $\mathrm{C}_{24} \mathrm{H}_{36} \mathrm{O}_{4}, M=388.53$, monoclinic, $a=11.623(3) \AA$, $b=7.3026(16)$ $\AA, c=12.673(3) \AA, \alpha=90.00^{\circ}, \beta=95.271(3)^{\circ}, \gamma=90.00^{\circ}, V=1071.1(4) \AA^{3}, T=100(2) \mathrm{K}$, space group $P 21, Z=2, \mu(\mathrm{MoK} \alpha)=0.080 \mathrm{~mm}^{-1}, 10516$ reflections measured, 5237 independent reflections $\left(R_{\text {int }}=0.0388\right)$. The final $R_{l}$ values were $0.0462(I>2 \sigma(I))$. The final $w R\left(F^{2}\right)$ values were $0.1050(I>$ $2 \sigma(I)$ ). The final $R_{1}$ values were 0.0630 (all data). The final $w R\left(F^{2}\right)$ values were 0.1162 (all data). 
The goodness of fit on $F^{2}$ was 1.033. Flack parameter $=-0.2(11)$.

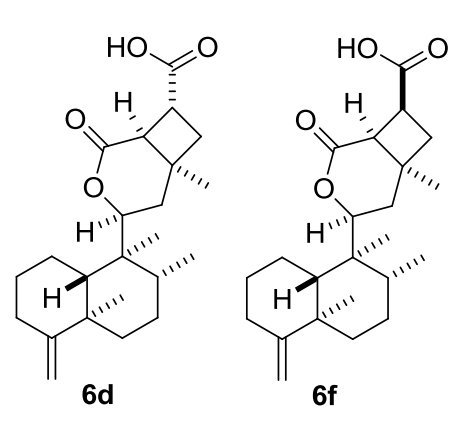

Synthesis of $\mathbf{6 d}$ : compound $\mathbf{6 a}(100.4 \mathrm{mg}, 0.26 \mathrm{mmol})$ was dissolved in $\mathrm{MeCN}(10 \mathrm{~mL})$, and then $1 \mathrm{M} \mathrm{LiOH}(10 \mathrm{~mL})$ was added and stirred for $24 \mathrm{~h}$ at room temperature. The resulting aqueous solution was acidified with $1 \mathrm{M} \mathrm{HCl}$, exhaustively extracted with $\mathrm{CHCl}_{3}(3 \times 20 \mathrm{~mL})$ and dried over sodium sulfate. Concentration in vacuo gave 6d (92.4 $\mathrm{mg}, 0.25 \mathrm{mmol}, 95 \%$ ) as a white solid which was further purified by preparative HPLC. Compound 6d, White power; ${ }^{1} \mathrm{H}$ NMR $\left(600 \mathrm{MHz}, \mathrm{CDCl}_{3}\right) \delta_{\mathrm{H}}: 4.49(1 \mathrm{H}, \mathrm{s}$, H-18a), 4.47 (1H, s, H-18b), 4.15 (1H, d, $J=11.2$ Hz, H-11), 3.05 (2H, overlapped, H-14 and H-8'), $2.26(1 \mathrm{H}, \mathrm{dt}, J=13.4$, and $4.5 \mathrm{~Hz}, \mathrm{H}-3 \mathrm{a}), 2.10-2.05$ (3H, m, overlapped, $\mathrm{H}_{2}-7^{\prime}$ and $\left.\mathrm{H}-3 \mathrm{~b}\right), 1.82-1.80$ (2H, overlapped, H-2a and H-12a), $4.15(1 \mathrm{H}, \mathrm{d}, J=11.2 \mathrm{~Hz}, \mathrm{H}-11), 1.75(1 \mathrm{H}, \mathrm{br} \mathrm{d}, J=13.2 \mathrm{~Hz}$, $\mathrm{H}-1 \mathrm{~b}), 1.61-1.44$ (6H, m, overlapped, $\mathrm{H}-1 \mathrm{~b}, \mathrm{H}-12 \mathrm{~b}, \mathrm{H}_{2}-6$, and $\left.\mathrm{H}_{2}-7\right), 1.29-1.18$ (3H, m, overlapped, H-2b, H-8, and H-10), $1.16\left(3 \mathrm{H}, \mathrm{s}, \mathrm{H}_{3}-16\right), 1.03\left(3 \mathrm{H}, \mathrm{s}, \mathrm{H}_{3}-19\right), 0.95\left(3 \mathrm{H}, \mathrm{s}, \mathrm{H}_{3}-20\right), 0.82(3 \mathrm{H}, \mathrm{d}, J=$ $\left.6.6 \mathrm{~Hz}, \mathrm{H}_{3}-17\right) ;{ }^{13} \mathrm{C}$ NMR (151 MHz, CDCl3) $\delta_{\mathrm{C}}: 179.7$ (s, C-9'), 176.5 (s, C-15), 160.1 (s, C-4), 103.1 (t, C-18), 85.2 (d, C-11), 48.7 (d, C-10), 45.0 (d, C-14), 42.9 (s, C-9), 40.3 (s, C-5), 38.5 (t, C-12), 37.0 (d, C-8'), 36.9 (t, C-6), 36.6 (d, C-8), 36.1 (t, C-7'), 33.3 (s, C-13), 33.1 (t, C-3), 28.4 (t, C-2), 27.9 (s, C-7), 26.5 (q, C-16), 23.8 (t, C-1), 20.7 (q, C-19), 16.8 (q, C-17), 13.8 (q, C-20). And, compound $\mathbf{6 b}(15 \mathrm{mg}, 0.039 \mathrm{mmol})$ was dissolved in EtOH $(2 \mathrm{~mL})$, and then 10 equiv $\mathrm{NaOH}$ was added and stirred for $8 \mathrm{~h}$ at room temperature to give $\mathbf{6 f}(10.0 \mathrm{mg}, 0.027 \mathrm{mmol}, 69 \%)$.

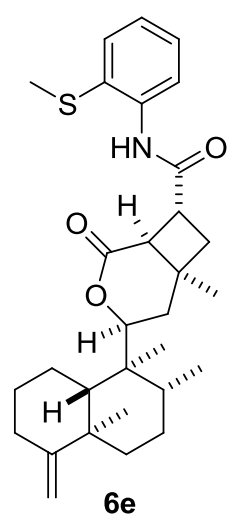

Synthesis of $\boldsymbol{6} \boldsymbol{e}$ : compound $\mathbf{6 d}(80.0 \mathrm{mg}, 0.21 \mathrm{mmol})$ was dissolved in dry DCM (10 $\mathrm{mL})$ and the flask was cooled to $0{ }^{\circ} \mathrm{C}$ in an ice bath. EDC $(80.2 \mathrm{mg}, 0.42 \mathrm{mmol}, 2$ equiv) and 2-aminothioanisole ( $40 \mu \mathrm{L}, 0.32 \mathrm{mmol}, 1.5$ equiv) were added in a single portion and the reaction mixture was warmed to room temperature and stirred for an additional $2 \mathrm{~h}$. After filtering the reaction mixture through a pad of celite, it was washed with saturated aqueous $\mathrm{NaHCO}_{3}(10 \mathrm{~mL})$ and $1 \mathrm{M} \mathrm{HCl}(20 \mathrm{~mL})$. The combined extracts were washed with brine $(20 \mathrm{~mL})$, dried over $\mathrm{Na}_{2} \mathrm{SO}_{4}$ and concentrated in vacuo. The resulting yellow solid was purified by preparative HPLC to give 6e (99 $\mathrm{mg}, 0.20 \mathrm{mmol}, 95 \%$ ) as a yellow solid. Compound $6 \mathbf{e}$, White power; Positive ESIMS $[\mathrm{M}+\mathrm{Na}]^{+} \mathrm{m} / \mathrm{z}$ 518; ${ }^{1} \mathrm{H}$ NMR $\left(600 \mathrm{MHz}, \mathrm{CDCl}_{3}\right) \delta_{\mathrm{H}} 9.79(1 \mathrm{H}, \mathrm{s}, \mathrm{NH}), 8.40(1 \mathrm{H}, \mathrm{d}, J=8.0 \mathrm{~Hz}, \mathrm{H}-3 "), 7.44(1 \mathrm{H}, \mathrm{d}, J$ = $7.8 \mathrm{~Hz}, \mathrm{H}-6 "), 7.28$ (1H, t, J = 8.0 Hz, H-5"), 7.13 (1H, t, J=8.0 Hz, H-4"), 4.66 (1H, s, H-18a), $4.65(1 \mathrm{H}, \mathrm{s}, \mathrm{H}-18 \mathrm{~b}), 4.31(1 \mathrm{H}, \mathrm{d}, J=9.6 \mathrm{~Hz}, \mathrm{H}-11), 3.75\left(1 \mathrm{H}, \mathrm{dd}, J=17.4\right.$ and $\left.8.3 \mathrm{~Hz}, \mathrm{H}-8^{\prime}\right), 4.47$ 
(1H, d, $J=7.9 \mathrm{~Hz}, \mathrm{H}-14), 2.53$ (1H, dd, $J=11.8,8.2 \mathrm{~Hz}, \mathrm{H}-7 \mathrm{a}), 2.30$ (3H, s, SMe), 2.27-2.19 (2H, overlapped, H-7b' and H-3a), 2.08-2.05 (2H, overlapped, H-3b and $\mathrm{H}-1 \mathrm{a}), 1.78(2 \mathrm{H}$, overlapped, H-12a and H-12b), 1.62-1.45 (5H, m, overlapped, H-1b, H-2b, H-6a, H-6b, and H-7a), 1.39 (1H, m, H-7b), 1.33-1.21 (3H, m, overlapped, H-2b, H-8, and H-10), 1.26 (3H, s, H-16), 1.03 (3H, s, H-19), $1.02(3 \mathrm{H}, \mathrm{s}, \mathrm{H}-20), 0.79$ (3H, d, $J=6.7 \mathrm{~Hz}, \mathrm{H}-17) .{ }^{13} \mathrm{C}$ NMR (151 MHz, Pyr) $\delta_{\mathrm{H}}: 175.3$ (s, C-9'), 172.7 (s, C-15), 160.4 (s, C-4), 138.7 (s, C-1"), 131.2 (d, C-6"), 130.3 (s, C-2"), 127.9 (d, C-5"), 125.9 (d, C-4"), 124.3 (d, C-3"), 103.9 (t, C-18), 85.2 (d, C-11), 49.1 (d, C-10), 46.1 (d, C-14), 43.6 (s, C-9), 40.9 (s, C-5), 40.2 (d, C-8'), 37.7 (t, C-6), 37.4 (d, C-8), 37.2 (t, C-12), 35.4 (t, C-7'), 34.3 (s, C-13), 33.7 (t, C-3), 28.8 (t, C-2), 28.4 (s, C-7), 26.7(q, C-16), 24.4 (t, C-1), 21.1 (q, C-17), 17.8 (SMe), 17.2 (q, C-19), 14.4 (q, C-20).

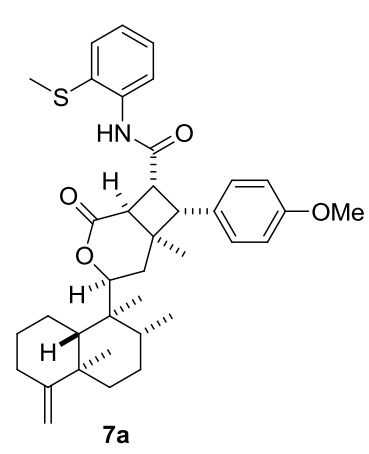

Synthesis of 7a: compound $6 \mathbf{e}(80.0 \mathrm{mg}, 0.16 \mathrm{mmol}), \mathrm{Pd}(\mathrm{OAc})_{2}(4 \mathrm{mg}, 0.02$ mmol, 0.1 eq), AgOAc (58.5 mg, $0.35 \mathrm{mmol}, 2.2$ equiv), and p-methoxyiodobenzene ( $112.3 \mathrm{mg}, 0.48 \mathrm{mmol}, 3$ equiv) were placed in a sealed tube and $6 \mathrm{~mL}$ of toluene was added under ambient conditions. The tube was sealed and placed into a $115{ }^{\circ} \mathrm{C}$ oil bath for $5 \mathrm{~h}$. The reaction was cooled to room temperature, diluted with DCM $(10 \mathrm{~mL})$ and filtered through a pad of celite. The combined extracts were washed with brine $(20 \mathrm{~mL})$, dried over $\mathrm{Na}_{2} \mathrm{SO}_{4}$ and concentrated in vacuo. The resulting black oil was purified by silica gel chromatography (4:1 $\mathrm{CHCl}_{3}$ : EtOAc) and preparative HPLC to give a yellow oil 7a (78.9 mg, $\left.0.13 \mathrm{mmol}, 82 \%\right)$. Compound 7a, White power; Positive ESIMS $[\mathrm{M}+\mathrm{Na}]^{+} m / z$ 624; ${ }^{1} \mathrm{H}$ NMR $(600 \mathrm{MHz}, \mathrm{Pyr}) \delta_{\mathrm{H}:} 9.83$ $(1 \mathrm{H}, \mathrm{s}, \mathrm{NH}), 8.22(1 \mathrm{H}, \mathrm{d}, J=8.0 \mathrm{~Hz}, \mathrm{H}-3 "), 7.56\left(2 \mathrm{H}, \mathrm{d}, J=8.6 \mathrm{~Hz}, \mathrm{H}-2^{\prime}\right.$ and H-6'), $7.35(1 \mathrm{H}, \mathrm{d}, J=$ $7.6 \mathrm{~Hz}, \mathrm{H}-6 "), 7.23$ (1H, t, J = 7.4 Hz, H-5"), 7.08 (1H, t, J = 7.4 Hz, H-4"), 7.00 (2H, d, J = 8.6 Hz, H-3' and H-5'), 4.67 (1H, s, H-18a), 4.66 (1H, s, H-18b), 4.49 (1H, dd, J = 10.7 and 7.4 Hz, H-11), $4.46\left(1 \mathrm{H}, \mathrm{dd}, J=8.0\right.$ and $\left.3.8 \mathrm{~Hz}, \mathrm{H}-8^{\prime}\right), 4.06(1 \mathrm{H}, \mathrm{d}, J=7.3 \mathrm{~Hz}, \mathrm{H}-14), 3.98(1 \mathrm{H}, \mathrm{d}, J=10.8 \mathrm{~Hz}$, H-7'), $3.64(3 \mathrm{H}, \mathrm{s}, \mathrm{OMe}), 2.28(1 \mathrm{H}, \mathrm{m}, \mathrm{H}-3 \mathrm{a}), 2.22(1 \mathrm{H}, \mathrm{m}, \mathrm{H}-1 \mathrm{a}), 2.09$ (3H, s, SMe), $2.28(1 \mathrm{H}$, overlapped, H-3b), 2.02-1.98 (2H, overlapped, $\left.\mathrm{H}_{2}-12\right)$, 1.42-1.37 (2H, m, overlapped, H-7b, and H-10), 1.31-1.25 (2H, m, overlapped, H-2b and H-8), 1.25 (3H, s, H3-16), 1.08 (3H, s, H3-20), 1.05 (3H, s, $\left.\mathrm{H}_{3}-19\right), 0.81\left(3 \mathrm{H}, \mathrm{d}, J=6.7 \mathrm{~Hz}, \mathrm{H}_{3}-17\right) ;{ }^{13} \mathrm{C}$ NMR (151 MHz, Pyr) $\delta_{\mathrm{C}}: 175.0$ (s, C-9'), 170.7 (s, C-15), 160.4 (s, C-4), 159.7 (s, C-4'), 138.3 (s, C-1"), 131.8 (d, C-3' and C-5'), 130.9 (s, C-1'), 130.8 (s, C-2"), 130.8 (s, C-6"), 127.7 (d, C-5"), 126.0 (d, C-4"), 124.6 (d, C-3"), 114.5 (d, C-2' and C-6'), 104.0 (t, C-18), 85.5 (d, C-11), 55.6 (q, OMe), 53.4 (d, C-7'), 49.2 (d, C-10), 46.8 (d, C-8'), 
43.7 (s, C-9), 43.4 (d, C-14), 41.0 (s, C-5), 40.6 (t, C-12), 40.3 (s, C-13), 37.7 (t, C-6), 37.3 (d, C-8), 33.8 (t, C-3), 28.9 (t, C-2), 28.4 (s, C-7), 24.5 (t, C-1), 22.7 (q, C-16), 21.2 (q, C-19), 17.5 (q, SMe), 17.3 (q, C-17), 14.5 (q, C-20).

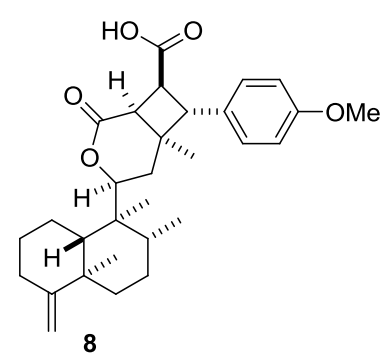

Synthesis of 8: compound 7a (60 mg, $0.10 \mathrm{mmol})$ and $\mathrm{NaOH}(20 \mathrm{mg}$, $0.50 \mathrm{mmol}, 5$ equiv) was dissolved in dry $\mathrm{EtOH}(5 \mathrm{~mL})$ and placed in a sealed tube under ambient conditions. The tube was sealed and placed into a $100{ }^{\circ} \mathrm{C}$ oil bath for $2 \mathrm{~h}$. The resulting solution was acidified with $1 \mathrm{M} \mathrm{HCl}$, exhaustively extracted with EtOAc $(3 \times 20 \mathrm{~mL})$ and dried over sodium sulfate. Concentration in vacuo gave $8(43.2 \mathrm{mg}, 0.09 \mathrm{mmol}, 85 \%)$ as a white solid which was further purified by preparative HPLC. Compound 8, ${ }^{1} \mathrm{H}$ NMR $\left(600 \mathrm{MHz}, \mathrm{CDCl}_{3}\right) \delta_{\mathrm{H}}$ : $7.35\left(2 \mathrm{H}, \mathrm{d}, J=8.6 \mathrm{~Hz}, \mathrm{H}-2^{\prime}\right.$ and H-6'), 7.07 (2H, d, $J=8.6 \mathrm{~Hz}, \mathrm{H}-3^{\prime}$ and H-5'), 4.45 (1H, s, H-18a), 4.43 (1H, s, H-18b), 4.34 (1H, d, $J=11.2 \mathrm{~Hz}, \mathrm{H}-11), 4.29$ (1H, dd, $J=10.6$ and $\left.9.2 \mathrm{~Hz}, \mathrm{H}-8^{\prime}\right), 4.17$ $(1 \mathrm{H}, \mathrm{d}, J=9.1 \mathrm{~Hz}, \mathrm{H}-7$ '), $3.72(3 \mathrm{H}, \mathrm{s}, \mathrm{OMe}), 4.17(1 \mathrm{H}, \mathrm{d}, J=9.1 \mathrm{~Hz}, \mathrm{H}-14), 2.47(1 \mathrm{H}, \mathrm{d}, J=13.0 \mathrm{~Hz}$, H-1a), $2.38(1 \mathrm{H}, \mathrm{t}, J=11.7 \mathrm{~Hz}, \mathrm{H}-12 \mathrm{a}), 2.26(1 \mathrm{H}, \mathrm{td}, J=13.3$ and $4.8 \mathrm{~Hz}, \mathrm{H}-3 \mathrm{a}), 2.13(1 \mathrm{H}, \mathrm{d}, J=$ $11.7 \mathrm{~Hz}, \mathrm{H}-12 \mathrm{~b}), 2.08$ (1H, br d, $J=13.0 \mathrm{~Hz}, \mathrm{H}-3 \mathrm{~b}), 1.74(1 \mathrm{H}, \mathrm{br} \mathrm{d}, J=10.3 \mathrm{~Hz}, \mathrm{H}-7 \mathrm{a}), 1.63-1.57$ (3H, m, overlapped, $\mathrm{H}-1 \mathrm{~b}$ and $\left.\mathrm{H}_{2}-6\right), 1.53-1.31$ (5H, m, overlapped, $\mathrm{H}_{2}-2, \mathrm{H}-7 \mathrm{~b}, \mathrm{H}-8$, and $\mathrm{H}-10$ ), $1.07\left(3 \mathrm{H}, \mathrm{s}, \mathrm{H}_{3}-16\right), 1.05$ (3H, s, H $\left.\mathrm{H}_{3}-20\right), 1.02\left(3 \mathrm{H}, \mathrm{s}, \mathrm{H}_{3}-19\right), 0.79\left(3 \mathrm{H}, \mathrm{d}, J=6.6 \mathrm{~Hz}, \mathrm{H}_{3}-17\right) ;{ }^{13} \mathrm{C}$ NMR (151 MHz, Pyr) $\delta_{\mathrm{C}} 175.5$ (s, C-9'), 172.9 (s, C-15), 160.6 (s, C-4), 159.5 (s, C-4'), 132.2 (s, C-1'), 129.8 (d, C-2' and C-6'), 114.8 (d, C-3' and C-5'), 104.0 (t, C-18), 85.2 (d, C-11), 55.7 (q, OMe), 52.2 (d, C-7'), 49.4 (d, C-10), 44.1 (d, C-14), 43.6 (s, C-9), 42.8 (d, C-8'), 41.0 (s, C-5), 40.1 (s, C-13), 39.9 (t, C-12), 37.8 (t, C-6), 37.4 (d, C-8), 33.9 (t, C-3), 29.0 (t, C-2), 28.5 (s, C-7), 24.4 (t, C-1), 23.0 (q, C-16), 21.2 (q, C-19), 17.3 (q, C-17), 14.6 (q, C-20).

Synthesis of 1: compound 8 (43.2 $\mathrm{mg}, 0.09 \mathrm{mmol})$ and $9(11.5 \mathrm{mg}, 0.09 \mathrm{mmol})$ were dissolved in dry $\operatorname{DCM}(5 \mathrm{~mL})$ and the flask was cooled to $0{ }^{\circ} \mathrm{C}$ in an ice bath. DCC (35.6 mg, $0.18 \mathrm{mmol}, 2$ equiv) and DMAP (3 mg, $0.02 \mathrm{mmol}, 0.2$ equiv) were added in a single portion and the reaction mixture was warmed to room temperature and stirred for an additional $4 \mathrm{~h}$. After filtering the reaction mixture through a pad of celite, it was diluted with DCM $(10 \mathrm{~mL})$, and then washed with saturated aqueous $\mathrm{NaHCO}_{3}(10 \mathrm{~mL})$ and $1 \mathrm{~N} \mathrm{HCl}(10 \mathrm{~mL})$. The combined extracts were washed with brine $(10 \mathrm{~mL})$, dried over $\mathrm{Na}_{2} \mathrm{SO}_{4}$ and concentrated in vacuo. The resulting white solid was purified by preparative HPLC to give synthetic $1(45.1 \mathrm{mg}, 0.08 \mathrm{mmol}, 85 \%$ yield) as a white solid. NMR data comparison between synthetic and natural scopariusicide A (1) see Table S2. 
Table S2. ${ }^{1} \mathrm{H}(500 \mathrm{MHz})$ and ${ }^{13} \mathrm{C}$ NMR (125 MHz) data comparison between synthetic and natural scopariusicide $\mathrm{A}(\mathbf{1})$ in $\mathrm{C}_{5} \mathrm{D}_{5} \mathrm{~N}(\delta \mathrm{ppm})$

\begin{tabular}{|c|c|c|c|c|}
\hline \multirow{2}{*}{ no. } & \multicolumn{2}{|r|}{ synthetic 1} & \multicolumn{2}{|r|}{ natural 1} \\
\hline & $\delta_{\mathrm{C}}$ & $\delta_{\mathrm{H}}$ & $\delta_{\mathrm{C}}$ & $\delta_{\mathrm{H}}$ \\
\hline 1 & $24.3 \mathrm{t}$ & $2.34, \mathrm{~d}(13.5) ; 1.62, \mathrm{~m}$ & $24.3 \mathrm{t}$ & $2.35, \mathrm{~d}(13.5) ; 1.62, \mathrm{~m}$ \\
\hline 2 & $28.9 \mathrm{t}$ & $1.74, \mathrm{~m} ; 1.34, \mathrm{~m}$ & $28.9 \mathrm{t}$ & $1.75, \mathrm{~m} ; 1.34, \mathrm{~m}$ \\
\hline 3 & $33.8 \mathrm{t}$ & $2.27, \mathrm{~m} ; 2.11, \mathrm{~m}$ & $33.8 \mathrm{t}$ & $2.27, \mathrm{~m} ; 2.11, \mathrm{~m}$ \\
\hline 4 & $160.4 \mathrm{~s}$ & & $160.4 \mathrm{~s}$ & \\
\hline 5 & $41.0 \mathrm{~s}$ & & $41.0 \mathrm{~s}$ & \\
\hline 6 & $37.7 \mathrm{t}$ & $1.57, \mathrm{~m}$ & $37.7 \mathrm{t}$ & $1.57, \mathrm{~m}$ \\
\hline 7 & $28.5 \mathrm{t}$ & $1.48, \mathrm{~m} ; 1.41, \mathrm{~m}$ & $28.4 \mathrm{t}$ & $1.49, \mathrm{~m} ; 1.42, \mathrm{~m}$ \\
\hline 8 & $37.4 \mathrm{~d}$ & $1.32, \mathrm{~m}$ & $37.4 \mathrm{~d}$ & $1.32, \mathrm{~m}$ \\
\hline 9 & $43.5 \mathrm{~s}$ & & $43.4 \mathrm{~s}$ & \\
\hline 10 & $49.3 \mathrm{~d}$ & $1.41, \mathrm{~m}$ & $49.3 \mathrm{~d}$ & $1.41, \mathrm{~m}$ \\
\hline 11 & $85.3 \mathrm{~d}$ & $4.29, \mathrm{~d}(11.0)$ & $85.3 \mathrm{~d}$ & $4.29, \mathrm{~d}(11.0)$ \\
\hline 12 & $40.5 \mathrm{t}$ & $2.19, \mathrm{t}(11.4,) ; 2.14, \mathrm{~d}(13.9)$ & $40.4 \mathrm{t}$ & $2.19, \mathrm{t}(11.4,) ; 2.15, \mathrm{~d}(13.9)$ \\
\hline 13 & $40.0 \mathrm{~s}$ & & $40.0 \mathrm{~s}$ & \\
\hline 14 & $44.3 \mathrm{~d}$ & $3.41, \mathrm{~d}(10.4)$ & $44.3 \mathrm{~d}$ & 3.43, d (10.4) \\
\hline 15 & $172.3 \mathrm{~s}$ & & $172.3 \mathrm{~s}$ & \\
\hline 16 & $22.8 \mathrm{q}$ & $1.07, \mathrm{~s}$ & $22.7 \mathrm{q}$ & $1.07, \mathrm{~s}$ \\
\hline 17 & $17.3 \mathrm{q}$ & $0.79, \mathrm{~d}(6.6)$ & $17.2 \mathrm{q}$ & $0.78, \mathrm{~d}(6.6)$ \\
\hline 18 & $103.9 \mathrm{t}$ & $4.64, \mathrm{~s}$ & $103.9 \mathrm{t}$ & $4.64, \mathrm{~s}$ \\
\hline 19 & $21.2 \mathrm{q}$ & $1.03, \mathrm{~s}$ & $21.1 \mathrm{q}$ & $1.01, \mathrm{~s}$ \\
\hline 20 & $14.5 \mathrm{q}$ & $1.02, \mathrm{~s}$ & $14.4 \mathrm{q}$ & $1.01, \mathrm{~s}$ \\
\hline $1^{\prime}$ & $131.5 \mathrm{~s}$ & & $131.5 \mathrm{~s}$ & \\
\hline $2^{\prime}$ & $129.5 \mathrm{~d}$ & $7.27, \mathrm{~d}(8.5)$ & $129.5 \mathrm{~d}$ & $7.28, \mathrm{~d}(8.6)$ \\
\hline $3^{\prime}$ & $114.8 \mathrm{~d}$ & $7.04, \mathrm{~d}(8.5)$ & $114.8 \mathrm{~d}$ & 7.04, d (8.6) \\
\hline $4^{\prime}$ & $159.5 \mathrm{~s}$ & & $159.5 \mathrm{~s}$ & \\
\hline $5^{\prime}$ & $114.8 \mathrm{~d}$ & $7.04, \mathrm{~d}(8.5)$ & $114.8 \mathrm{~d}$ & 7.04, d (8.6) \\
\hline $6^{\prime}$ & $129.5 \mathrm{~d}$ & $7.27, \mathrm{~d}(8.5)$ & $129.5 \mathrm{~d}$ & $7.28, \mathrm{~d}(8.6)$ \\
\hline $7^{\prime}$ & $51.9 \mathrm{~d}$ & $4.10, \mathrm{~d}(9.7)$ & $51.9 \mathrm{~d}$ & $4.12, \mathrm{~d}(9.8)$ \\
\hline $8^{\prime}$ & $41.8 \mathrm{~d}$ & $4.18, \mathrm{t}(10.1)$ & $41.8 \mathrm{~d}$ & $4.21, \mathrm{t}(10.1)$ \\
\hline $9^{\prime}$ & $172.5 \mathrm{~s}$ & & $172.6 \mathrm{~s}$ & \\
\hline $1 "$ & $116.8 \mathrm{t}$ & $5.36,(\mathrm{~d}, 17.3) ; 5.18,(\mathrm{~d}, 10.6)$ & $116.8 \mathrm{t}$ & $5.36,(\mathrm{~d}, 17.3) ; 5.17,(\mathrm{~d}, 10.6)$ \\
\hline $2^{\prime \prime}$ & $138.1 \mathrm{~d}$ & $5.97,(\mathrm{ddd}, 17.0,10.5,6.2)$ & $138.1 \mathrm{~d}$ & $5.97,(\mathrm{ddd}, 17.0,10.5,6.2)$ \\
\hline $3 "$ & $76.1 \mathrm{~d}$ & $5.61, \mathrm{q}(6.4)$ & $76.2 \mathrm{~d}$ & $5.62, \mathrm{q}(6.4)$ \\
\hline 4" & $34.7 \mathrm{t}$ & $1.93, \mathrm{~m} ; 1.76, \mathrm{~m}$ & $34.7 \mathrm{t}$ & $1.93, \mathrm{~m} ; 1.76, \mathrm{~m}$ \\
\hline $5^{\prime \prime}$ & $25.4 \mathrm{t}$ & $1.46, \mathrm{~m}$ & $25.4 \mathrm{t}$ & $1.46, \mathrm{~m}$ \\
\hline $6^{\prime \prime}$ & $32.4 \mathrm{t}$ & $1.27, \mathrm{~m}$ & $32.3 \mathrm{t}$ & $1.27, \mathrm{~m}$ \\
\hline 7" & $23.3 \mathrm{t}$ & $1.26, \mathrm{~m}$ & $23.3 \mathrm{t}$ & $1.26, \mathrm{~m}$ \\
\hline $8 "$ & $14.7 \mathrm{q}$ & $0.83, \mathrm{t}(6.9)$ & $14.7 \mathrm{q}$ & $0.81, \mathrm{t}(7.0)$ \\
\hline $\mathrm{OMe}$ & $55.7 \mathrm{q}$ & $3.71, \mathrm{~s}$ & $55.6 \mathrm{q}$ & $3.71, \mathrm{~s}$ \\
\hline
\end{tabular}


Scheme S2. Attempts to irradiation of biosynthetic precursors or modified precursors-like analogues failed to constructure the desired unsymmetrical cyclobutanes
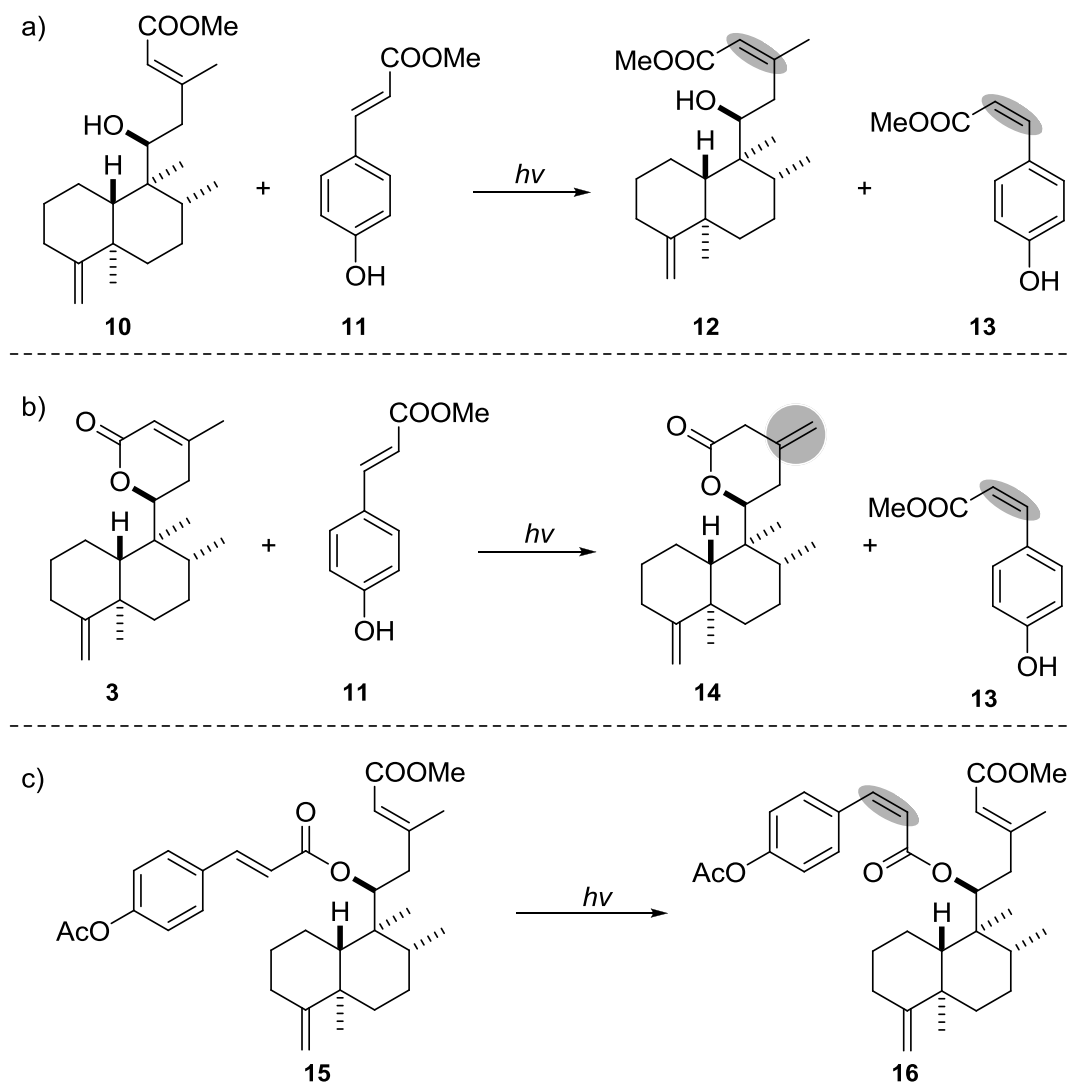

Reagents and conditions: photoreactor with $16 \times 60 \mathrm{~W}$ mercury lamps, $h v=313$ or $360 \mathrm{~nm}, 10 \mathrm{~mL}$ quartz tube, solvents $\left(\mathrm{MeCN}, \mathrm{PhMe},(\mathrm{CH})_{2} \mathrm{CO}\right.$, or $\mathrm{DCM}$ ), additives (none, 2'-acetonaphthone, $\mathrm{CuOTf}$, or $\mathrm{BF}_{3} \cdot \mathrm{Et}_{2} \mathrm{O}$ ).

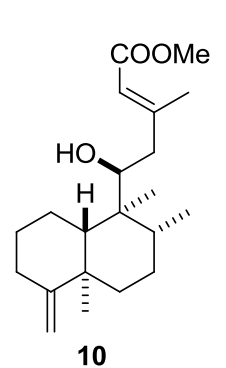

Synthesis of 10: compound $4(320 \mathrm{mg}, 1.0 \mathrm{mmol})$ was dissolved in dry DCM:MeOH $(1: 1,50 \mathrm{~mL})$ and the flask was cooled to $0{ }^{\circ} \mathrm{C}$ in an ice bath. DCC (412 mg, 2.0 mmol, 2 equiv) and DMAP (122 mg, $1.0 \mathrm{mmol}, 1$ equiv) were added in a single portion and the reaction mixture was warmed to room temperature and stirred for an additional $12 \mathrm{~h}$. After filtering the reaction mixture through a pad of celite, it was washed with saturated aqueous $\mathrm{NaHCO}_{3}(100 \mathrm{~mL})$ and $1 \mathrm{~N} \mathrm{HCl}(100 \mathrm{~mL})$. The combined extracts were washed with brine $(200 \mathrm{~mL})$, dried over $\mathrm{Na}_{2} \mathrm{SO} 4$ and concentrated in vacuo. The resulting white solid was purified by column chromatography $\left(\mathrm{CHCl}_{3}\right)$ to give $10(327 \mathrm{mg}, 1.0$ mmol, 98\%) as a white solid CCompound 10, ${ }^{1} \mathrm{H}$ NMR $\left(600 \mathrm{MHz}, \mathrm{CDCl}_{3}\right) \delta_{\mathrm{H}}: 5.76(1 \mathrm{H}$, br s, H-14), 4.50 (1H, br s, H-18a), 4.48 (1H, br s, H-18b), 3.73 (1H, d, J = 10.3 Hz, H-11), 3.67 (3H, s, OMe), $2.18\left(3 \mathrm{H}, \mathrm{s}, \mathrm{H}_{3}-16\right), 1.05\left(3 \mathrm{H}, \mathrm{s}, \mathrm{H}_{3}-19\right), 0.95\left(3 \mathrm{H}, \mathrm{s}, \mathrm{H}_{3}-20\right), 0.85\left(3 \mathrm{H}, \mathrm{d}, J=6.6 \mathrm{~Hz}, \mathrm{H}_{3}-17\right) ;{ }^{13} \mathrm{C}$ NMR (150 MHz, CDCl $\left.{ }_{3}\right) \delta_{\mathrm{C}}: 167.1$ (s, C-15), 160.6 (s, C-4), 158.8 (s, C-13), 117.7 (d, C-14), 102.9 (t, C-18), 76.0 (d, C-11), 51.1 (q, OMe), 48.8 (d, C-10), 44.0 (s, C-9), 43.49 (t, C-12), 40.6 (s, C-5), 
37.4 (d, C-8), 37.2 (t, C-6), 33.4 (t, C-3), 28.6 (t, C-7), 28.4 (t, C-2), 24.0 (t, C-1), 20.8 (q, C-19), 19.3 (q, C-16), 17.0 (q, C-17), 14.1 (q, C-20).

Synthesis of 12: compounds 10 (100 mg, $0.30 \mathrm{mmol})$ and $11(53 \mathrm{mg}, 0.30 \mathrm{mmol})$

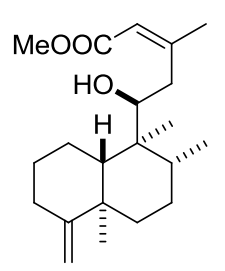

12 were dissolved in acetonitrile $(10 \mathrm{~mL})$ in a quartz tube. The reaction vessels were carefully sealed and irradiated using a photoreactor $(313 \mathrm{~nm})$ at room temperature for $12 \mathrm{~h}$. The resulting mixture was concentrated in vacuo and then directly subJected to flash column chromatography (silica gel, $\mathrm{CHCl}_{3}: \mathrm{EtOAc}, 20: 1$ ) and preparative HPLC to give 12 (45 mg, $0.13 \mathrm{mmol}, 45 \%)$. Compound 12, ${ }^{1} \mathrm{H} \mathrm{NMR}\left(600 \mathrm{MHz}, \mathrm{CDCl}_{3}\right)$ $\delta_{\mathrm{H}}: 5.83(1 \mathrm{H}$, br s, H-14), $4.51(1 \mathrm{H}$, br s, H-18a), 4.49 (1H, br s, H-18b), 3.69 (1H, overlapped, H-11), 3.68 (3H, s, OMe) 1.92 (3H, s, H3-16), 1.06 (3H, s, $\left.\mathrm{H}_{3}-19\right), 0.95$ (3H, s, $\left.\mathrm{H}_{3}-20\right), 0.86$ (3H, d, $J$ $\left.=6.6 \mathrm{~Hz}, \mathrm{H}_{3}-17\right) ;{ }^{13} \mathrm{C}$ NMR $\left(150 \mathrm{MHz}, \mathrm{CDCl}_{3}\right) \delta_{\mathrm{C}}: 168.6$ (s, C-15), 160.7 (s, C-4), 156.2 (s, C-13), 118.1 (d, C-14), 102.7 (t, C-18), 76.9 (d, C-11), 51.6 (q, OMe), 48.6 (d, C-10), 44.4 (s, C-9), 40.6 (s, C-5), 37.2 (d, C-8), 37.0 (t, C-6), 36.3 (t, C-12), 33.3 (t, C-3), 28.5 (t, C-7), 28.5 (t, C-2), 25.8 (q, C-16), 24.0 (t, C-1), 20.7 (q, C-19), 17.2 (q, C-17), 13.9 (q, C-20).

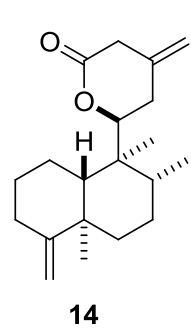

Synthesis of 14: compound 3 (100 mg, $0.33 \mathrm{mmol})$ and 11 (53 mg, $0.30 \mathrm{mmol})$ were dissolved in acetonitrile $(10 \mathrm{~mL})$ in a quartz tube. The reaction vessels were carefully sealed and irradiated using a photoreactor $(313 \mathrm{~nm})$ at room temperature for $12 \mathrm{~h}$. The resulting mixture was concentrated in vacuo and then directly subJected to flash column chromatography (silica gel, $\mathrm{CHCl}_{3}:$ EtOAc, 20:1) and preparative HPLC to give 16 (21 mg, $0.07 \mathrm{mmol}, 21 \%)$. Spectral data see previous works (Organic Letters, 2013, 15, 4446-4449).

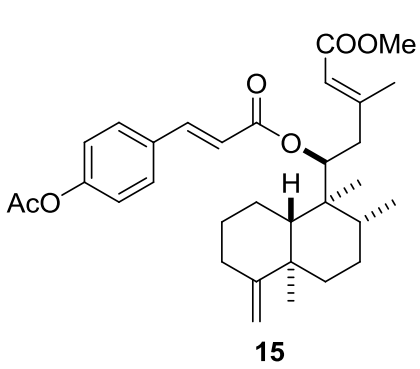

Synthesis of 15: 10 (300 mg, $0.9 \mathrm{mmol})$ and $p$-acetylcinnamic acid (556 $\mathrm{mg}, 2.7 \mathrm{mmol})$ were dissolved in dry DCM $(50 \mathrm{~mL})$ and the flask was cooled to $0{ }^{\circ} \mathrm{C}$ in an ice bath. EDC $(380 \mathrm{mg}, 2.0 \mathrm{mmol})$ and DMAP (110 $\mathrm{mg}, 0.9 \mathrm{mmol})$ were added in a single portion and the reaction mixture was warmed to room temperature and stirred for an additional

$15 \mathrm{~h}$. After filtering the reaction mixture through a pad of celite, it was washed with saturated aqueous $\mathrm{NaHCO}_{3}(200 \mathrm{~mL})$ and $1 \mathrm{M} \mathrm{HCl}(200 \mathrm{~mL})$. The combined extracts were washed with brine $(250 \mathrm{~mL})$, dried over $\mathrm{Na}_{2} \mathrm{SO}_{4}$ and concentrated in vacuo. The resulting white solid was purified by column chromatography $\left(\mathrm{CHCl}_{3}\right)$ to give $15(282 \mathrm{mg}, 0.54 \mathrm{mmol}, 60 \%)$ as a white solid. Compound 15, White power; Positive ESIMS $[\mathrm{M}+\mathrm{Na}]^{+} \mathrm{m} / z$ 545; ${ }^{1} \mathrm{H} \mathrm{NMR}\left(400 \mathrm{MHz}, \mathrm{CDCl}_{3}\right) \delta_{\mathrm{H}}: 7.54(1 \mathrm{H}, \mathrm{d}$, 
$\left.J=16.2 \mathrm{~Hz}, \mathrm{H}-3^{\prime}\right), 7.52\left(2 \mathrm{H}, \mathrm{d}, J=8.2 \mathrm{~Hz}, \mathrm{H}-5^{\prime}\right.$ and 9'), 7.09 (2H, d, $J=8.2 \mathrm{~Hz}, \mathrm{H}-6^{\prime}$ and 8'), 6.30 $(1 \mathrm{H}, \mathrm{d}, J=16.2 \mathrm{~Hz}, \mathrm{H}-2 '), 5.61$ (1H, br s, H-14), 5.44 (1H, d, $J=9.6 \mathrm{~Hz}, \mathrm{H}-11), 3.57$ (3H, s, OMe), $2.43(1 \mathrm{H}, \mathrm{d}, J=11.2 \mathrm{~Hz}, \mathrm{H}-12 \mathrm{a}), 2.30$ (1H, m, H-3a), 2.30 (3H, s, OAc), 2.18 (3H, s, H $3-16), 2.18$ (1H, overlapped, H-12b), 2.15 (1H, overlapped, H-1a), $2.13(1 \mathrm{H}$, overlapped, H-3b), 1.93 (1H, m, H-7a), 1.62 (1H, overlapped, H-6a), 1.63 (1H, overlapped, H-1b), 1.54 (1H, m, H-6b), 1.53 (2H, m, $\left.\mathrm{H}_{2}-2\right), 1.43$ (1H, m, H-8), 1.35 (1H, m, H-7b), 1.22 (1H, m, H-10), 1.05 (3H, s, H3-19), 1.01 (3H, d, $\left.J=6.6 \mathrm{~Hz}, \mathrm{H}_{3}-17\right), 0.80\left(3 \mathrm{H}, \mathrm{s}, \mathrm{H}_{3}-20\right) ;{ }^{13} \mathrm{C} \mathrm{NMR}\left(100 \mathrm{MHz}, \mathrm{CDCl}_{3}\right) \delta_{\mathrm{C}}: 169.4$ (s, OAc), 166.9 (s, C-15), 166.7 (s, C-1'), 160.2 (s, C-4), 156.9 (s, C-13), 152.2 (s, C-5'), 143.9 (d, C-3'), 132.4 (s, C-4'), 129.5 (d, C-5' and 9'), 122.3 (d, C-6' and 8'), 118.4 (d, C-2'), 118.3 (d, C-14), 103.2 (t, C-18), 76.8 (d, C-11), 51.1 (q, OMe), 48.6 (d, C-10), 44.6 (s, C-9), 42.0 (t, C-12), 40.5 (s, C-5), 37.9 (d, C-8), 37.1 (t, C-6), 33.3 (t, C-3), 28.6 (t, C-7), 28.2 (t, C-2), 23.9 (t, C-1), 21.4 (q, OAc), 20.8 (q, C-19), 18.8 (q, C-16), 16.9 (q, C-17), 13.5 (q, C-20).

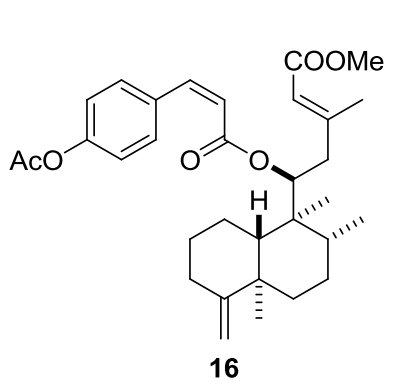

Synthesis of 16: compound 15 (100 mg, $0.19 \mathrm{mmol})$ was dissolved in acetonitrile $(10 \mathrm{~mL})$ in a quartz tube. The reaction vessels were carefully sealed and irradiated using a photoreactor $(313 \mathrm{~nm})$ at room temperature for $12 \mathrm{~h}$. The resulting mixture was concentrated in vacuo and then directly subJected to flash column chromatography (silica gel, $\mathrm{CHCl}_{3}$ :EtOAc, 20:1) and preparative HPLC to give $\mathbf{1 6}(100 \mathrm{mg}, 0.10$ mmol, 50\%). Compound 16, White power; Positive ESIMS $[\mathrm{M}+\mathrm{Na}]^{+} \mathrm{m} / z$ 545; ${ }^{1} \mathrm{H}$ NMR $(400 \mathrm{MHz}$, $\left.\mathrm{CDCl}_{3}\right) \delta_{\mathrm{H}}: 7.50\left(2 \mathrm{H}, \mathrm{d}, J=8.2 \mathrm{~Hz}, \mathrm{H}-5^{\prime}, 9^{\prime}\right), 7.03\left(2 \mathrm{H}, \mathrm{d}, J=8.2 \mathrm{~Hz}, \mathrm{H}-6^{\prime}\right.$ and $\left.8^{\prime}\right), 6.84(1 \mathrm{H}, \mathrm{d}, J=$ $\left.12.6 \mathrm{~Hz}, \mathrm{H}-3^{\prime}\right), 5.85$ (1H, d, J = 12.6 Hz, H-2'), 5.63 (1H, br s, H-14), 5.45 (1H, d, J=9.6 Hz, H-11), 3.58 (3H, s, OMe), 2.43 (1H, d, $J=11.2 \mathrm{~Hz}, \mathrm{H}-12 \mathrm{a}), 2.31$ (1H, m, H-3a), 2.30 (3H, s, OAc), 2.19 (3H, s, H3-16), 2.18 (1H, overlap, H-12b), 2.13 (1H, overlap, H-1a), 2.09 (1H, overlap, H-3b), 1.93 (1H, m, H-7a), 1.59 (1H, overlap, H-6a), 1.57 (1H, overlap, H-1b), 1.54 (1H, m, H-6b), 1.53 (2H, m, $\left.\mathrm{H}_{2}-2\right), 1.40$ (1H, m, H-8), 1.28 (1H, m, H-7b), 1.17 (1H, m, H-10), 1.03 (3H, s, H3-19), 0.97 (3H, d, $\left.J=6.6 \mathrm{~Hz}, \mathrm{H}_{3}-17\right), 0.78\left(3 \mathrm{H}, \mathrm{s}, \mathrm{H}_{3}-20\right) ;{ }^{13} \mathrm{C} \mathrm{NMR}\left(100 \mathrm{MHz}, \mathrm{CDCl}_{3}\right) \delta_{\mathrm{C}}: 169.4$ (s, OAc), 166.7 (s, C-15), 165.9 (s, C-1'), 160.2 (s, C-4), 157.1 (s, C-13), 151.2 (s, C-5'), 143.0 (d, C-3'), 132.4 (s, C-4'), 131.3 (d, C-5' and 9'), 121.5 (d, C-6' and 8'), 119.6 (d, C-2'), 118.2 (d, C-14), 103.1 (t, C-18), 76.4 (d, C-11), 51.1 (q, OMe), 48.5 (d, C-10), 44.4 (s, C-9), 41.9 (t, C-12), 40.4 (s, C-5), 37.8 (d, C-8), 37.1 (t, C-6), 33.3 (t, C-3), 28.5 (t, C-7), 28.2 (t, C-2), 23.7 (t, C-1), 21.4 (q, OAc), 20.7 (q, C-19), 18.9 (q, C-16), 16.9 (q, C-17), 13.5 (q, C-20). 
Scheme S3. For model reaction

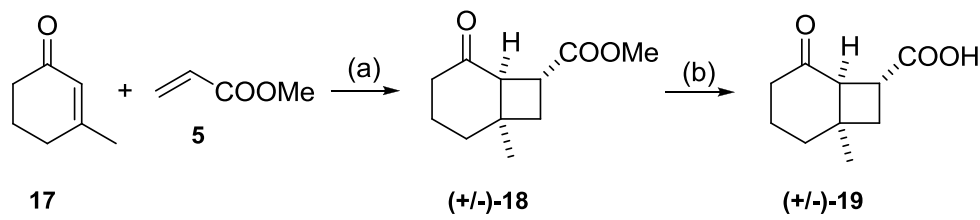

$\stackrel{(\mathrm{c})}{\longrightarrow}$

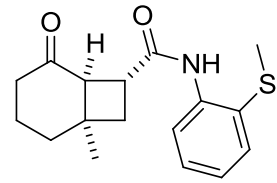

$(+/-)-20$ $\stackrel{(d)}{\longrightarrow}$

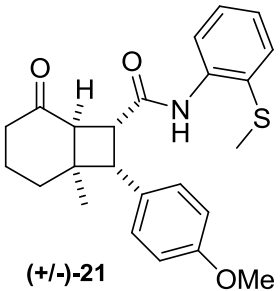

Reagents and conditions: (a) photoreactor with $16 \times 60 \mathrm{~W}$ mercury lamps, $h v=313 \mathrm{~nm}, 10 \mathrm{~mL}$ quartz tube, MeCN, 17 (1.0 equiv), 5 (15.0 equiv), rt, $10 \mathrm{~h}$; (b) $1 \mathrm{M} \mathrm{NaOH}: \mathrm{MeCN}=1: 1, \mathrm{rt}, 20 \mathrm{~h}$; (c) (+/-)-19 (1 equiv), 2-aminothioanisole (1.5 equiv), EDC (2.0 equiv), $0{ }^{\circ} \mathrm{C}$ to rt, DCM, $2 \mathrm{~h}, 43 \%$ yield from 17 and 5; (d) palladium(II)acetate ( 0.15 equiv), $p$-methoxyiodobenzene (3.0 equiv), $\mathrm{Ag}_{2} \mathrm{CO}_{3}\left(2.2\right.$ equiv), $t-\mathrm{BuOH}, 120{ }^{\circ} \mathrm{C}, 10 \mathrm{~h}, 75 \%(+/-)-21$.

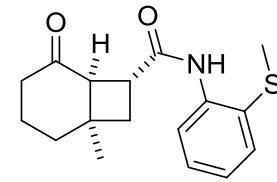

20

Synthesis of 20: $17(110 \mathrm{mg}, 1.0 \mathrm{mmol})$ and $5(2 \mathrm{~mL})$ were dissolved in acetonitrile $(5 \mathrm{~mL})$ in a quartz tube. The reaction vessels were carefully sealed and irradiated using a photoreactor $(313 \mathrm{~nm})$ at room temperature for $10 \mathrm{~h}$. A white solid (105 mg) which was used directly in the following step without further purification. Then, the white solid (105 mg) was dissolved in MeCN (5 mL), and then $1 \mathrm{M}$ $\mathrm{NaOH}(5 \mathrm{~mL})$ was added and stirred for $20 \mathrm{~h}$ at room temperature. The resulting aqueous solution was acidified with $1 \mathrm{M} \mathrm{HCl}(\mathrm{PH}=3)$, exhaustively extracted with $\mathrm{CHCl}_{3}(3 \times 20 \mathrm{~mL})$ and dried over sodium sulfate. The resulting mixture $(91 \mathrm{mg})$ was further dissolved in dry DCM $(10 \mathrm{~mL})$ and the flask was cooled to $0{ }^{\circ} \mathrm{C}$ in an ice bath. EDC $(139 \mathrm{mg}, 1.0 \mathrm{mmol})$ and 2-aminothioanisole $(70 \mu \mathrm{L})$ were added in a single portion and the reaction mixture was warmed to room temperature and stirred for an additional $12 \mathrm{~h}$. After filtering the reaction mixture through a pad of celite, it was washed with saturated aqueous $\mathrm{NaHCO}_{3}(10 \mathrm{~mL})$ and $1 \mathrm{M} \mathrm{HCl}(20 \mathrm{~mL})$. The combined extracts were washed with brine $(20 \mathrm{~mL})$, dried over $\mathrm{Na}_{2} \mathrm{SO}_{4}$ and concentrated in vacuo. The resulting mixture was concentrated in vacuo and then directly subJected to flash column chromatography (silica gel, $\mathrm{CHCl}_{3}$ :EtOAc, 20:1) and preparative HPLC to give 20 (129 mg, $\left.0.43 \mathrm{mmol}, 43 \%\right)$. Compound 20, ${ }^{1} \mathrm{H}$ NMR $\left(400 \mathrm{MHz}, \mathrm{CDCl}_{3}\right) \delta_{\mathrm{H}}: 8.59(1 \mathrm{H}, \mathrm{br} \mathrm{s}, \mathrm{NH}), 8.23(1 \mathrm{H}, \mathrm{d}, J=8.2 \mathrm{~Hz}, \mathrm{H}-3 "), 7.46(1 \mathrm{H}, \mathrm{d}, J=$ $8.4 \mathrm{~Hz}, \mathrm{H}-6 "), 7.28$ (1H, br t, $J=8.4 \mathrm{~Hz}, \mathrm{H}-4 "), 7.04$ (1H, br t, $J=8.4 \mathrm{~Hz}, \mathrm{H}-5 "), 3.15$ (1H, q, $J=8.2$ Hz, H-2'), 2.87 (1H, d, J = 8.4 Hz, H-2), 2.30-2.42 (2H, m, H-6), 2.42 (3H, s, SMe), 2.31 (1H, dd, $J$ $\left.=12.0,9.1 \mathrm{~Hz}, \mathrm{H}-3 \mathrm{a}^{\prime}\right), 2.03\left(1 \mathrm{H}, \mathrm{t}, J=12.0 \mathrm{~Hz}, \mathrm{H}-3 \mathrm{~b}^{\prime}\right), 1.96\left(2 \mathrm{H}, \mathrm{m}, \mathrm{H}_{2}-5\right), 1.71\left(2 \mathrm{H}, \mathrm{m}, \mathrm{H}_{2}-4\right), 1.24$ $\left(3 \mathrm{H}, \mathrm{s}, \mathrm{H}_{3}-7\right) ;{ }^{13} \mathrm{C}$ NMR (100 MHz, $\left.\mathrm{CDCl}_{3}\right) \delta_{\mathrm{C}}: 214.6$ (s, C-1), 171.7 (s, C-1'), 138.1 (s, C-1"), 132.3 (d, C-4"), 128.3 (s, C-2"), 126.4 (d, C-5"), 124.4 (d, C-3"), 121.1 (d, C-6"), 54.0 (d, C-2'), 39.0 (d, 
C-2), 38.4 (t, C-6), 36.6 (s, C-3), 34.6 (t, C-4), 34.2 (t, C-3'), 27.9 (q, C-7), 20.2 (t, C-5), 14.2 (q, $\mathrm{SMe})$.

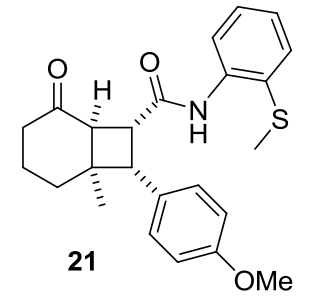

Synthesis of 21: 20 (76 mg, $0.25 \mathrm{mmol}), \mathrm{Pd}(\mathrm{OAc})_{2}(8.4 \mathrm{mg}, 0.038 \mathrm{mmol}, 0.15$ equiv), $\mathrm{Ag}_{2} \mathrm{CO}_{3}$ (151 mg, $0.55 \mathrm{mmol}, 2.2$ equiv), and $p$-methoxyiodobenzene (175 mg, $0.75 \mathrm{mmol}, 3$ equiv) were placed in a sealed tube and $5 \mathrm{~mL}$ of $t$ - $\mathrm{BuOH}$ was added under ambient conditions. The tube was sealed and placed into a $110{ }^{\circ} \mathrm{C}$ oil bath for $10 \mathrm{~h}$. The reaction was cooled to room temperature, diluted with DCM (10 mL) and filtered through a pad of celite. The combined extracts were washed with brine $(20 \mathrm{~mL})$, dried over $\mathrm{Na}_{2} \mathrm{SO}_{4}$ and concentrated in vacuo. The resulting black oil was purified by preparative HPLC to give a yellow oil 21 (78 mg, $0.19 \mathrm{mmol}, 75 \%$ ). Compound 21, Positive ESIMS $[\mathrm{M}+\mathrm{Na}]^{+} \mathrm{m} / z$ 432; ${ }^{1} \mathrm{H}$ NMR $\left(400 \mathrm{MHz}, \mathrm{CDCl}_{3}\right) \delta_{\mathrm{H}}: 8.48(1 \mathrm{H}, \mathrm{br}, \mathrm{NH}), 8.00(1 \mathrm{H}$, d, $J=8.2 \mathrm{~Hz}, \mathrm{H}-3 "), 7.38$ (1H, dd, $J=8.4,1.1 \mathrm{~Hz}, \mathrm{H}-6 "), 7.28$ (1H, overlap, H-4"), 7.28 (2H, overlap, H-5' and 9'), $7.04(1 \mathrm{H}$, br t, $J=8.4 \mathrm{~Hz}, \mathrm{H}-5 "), 6.78\left(2 \mathrm{H}, \mathrm{d}, J=8.8 \mathrm{~Hz}, \mathrm{H}-6{ }^{\prime}\right.$ and 8'), 4.04 $\left(1 \mathrm{H}\right.$, br t, $\left.J=9.3 \mathrm{~Hz}, \mathrm{H}-2^{\prime}\right), 3.73$ (1H, overlap, H-3'), $3.70(3 \mathrm{H}, \mathrm{s}, \mathrm{OMe}), 3.33(1 \mathrm{H}, \mathrm{d}, J=9.3 \mathrm{~Hz}$, H-2), 2.50 (1H, m, H-5a), 2.36 (1H, m, H-5b), 2.14 (3H, s, SMe), 2.04 (1H, m, H-5a), 1.95 (1H, m, $\mathrm{H}-5 \mathrm{~b}), 1.95$ (1H, m, H-4a), 1.74 (1H, m, H-4b), $0.92\left(3 \mathrm{H}, \mathrm{s}, \mathrm{H}_{3}-7\right) ;{ }^{13} \mathrm{C}$ NMR $\left(100 \mathrm{MHz}, \mathrm{CDCl}_{3}\right) \delta_{\mathrm{C}}$ : 212.4 (s, C-1), 170.3 (s, C-1'), 159.4 (s, C-7'), 138.8 (S, C-1"), 132.3 (d, C-4"), 131.3 (d, C-5' and 9'), 131.0 (s, C-2"), 128.4 (d, C-5"), 128.3 (s, C-15), 125.2 (d, C-3"), 122.5 (d, C-6"), 114.1 (d, C-6' and 8'), 55.3 (q, OMe), 52.2 (d, C-3'), 52.0 (d, C-2), 45.2 (d, C-2'), 43.3 (s, C-3), 39.3 (t, C-6), 37.2 (t, C-4), 29.2 (q, C-7), 21.8 (t, C-5), 17.9 (q, SMe). 
Scheme S4. Several attempts for modified intermolecular [2+2] cycloaddition

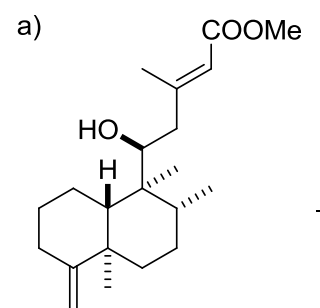

10

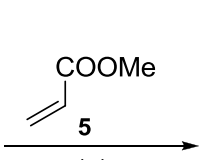

(a)

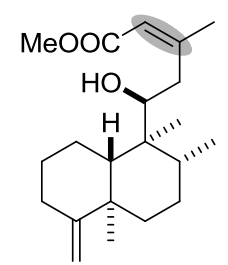

12

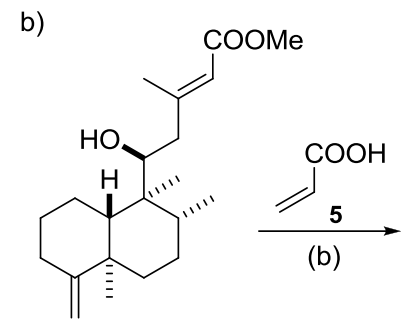

10

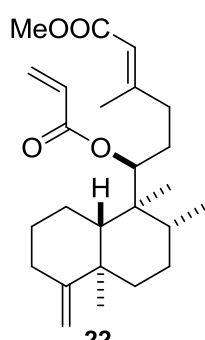

22
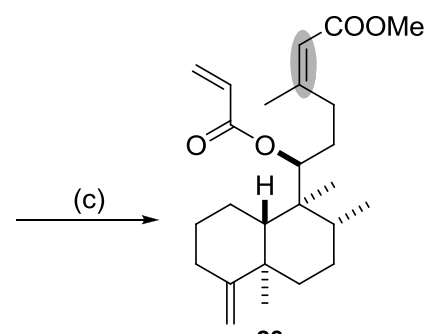

23

Reagents and conditions: (a) photoreactor with $16 \times 60 \mathrm{~W}$ mercury lamps, $h v=313$ or $360 \mathrm{~nm}, 10 \mathrm{~mL}$ quartz tube, MeCN, 10 (1.0 equiv), $\mathbf{5}$ (15.0 equiv), rt, 10 h, $60 \%$ 12; (b) 7a (1 equiv), $7 \mathbf{b}$ (1 equiv), EDC (2.0 equiv), DMAP ( 0.4 equiv), DCM, $0{ }^{\circ} \mathrm{C}$ to rt, 15 h, $70 \%$; (c) photoreactor with $16 \times 60$ W mercury lamps, $h v=313 \mathrm{~nm}, 10 \mathrm{~mL}$ quartz tube, MeCN, , rt, 8 h, $50 \% 23$.

Synthesis of 22: compound 10 (300 $\mathrm{mg}, 0.9 \mathrm{mmol})$ and $\mathbf{5}(1 \mathrm{~mL})$ were dissolved in<smiles>C=CC(=O)O[C@H](CC/C(C)=C/C(=O)OC)[C@@H]1[C@@H](C)CC[C@]2(C)C(=C)CCC[C@@H]12</smiles>

22 dry DCM (25 mL) and the flask was cooled to $0{ }^{\circ} \mathrm{C}$ in an ice bath. EDC (380 $\mathrm{mg}$, $2.0 \mathrm{mmol})$ and DMAP (110 $\mathrm{mg}, 0.9 \mathrm{mmol})$ were added in a single portion and the reaction mixture was warmed to room temperature and stirred for an additional 15 h. After filtering the reaction mixture through a pad of celite, it was washed with saturated aqueous $\mathrm{NaHCO}_{3}(200 \mathrm{~mL})$ and $1 \mathrm{M} \mathrm{HCl}(200 \mathrm{~mL})$. The combined extracts were washed with brine $(250 \mathrm{~mL})$, dried over $\mathrm{Na}_{2} \mathrm{SO}_{4}$ and concentrated in vacuo. The resulting white solid was purified by column chromatography $\left(\mathrm{CHCl}_{3}\right)$ to give $22(253 \mathrm{mg}, 0.63$ mmol, 70\%) as a white solid. Compound 22, Positive ESIMS $[\mathrm{M}+\mathrm{Na}]^{+} m / z$ 411; ${ }^{1} \mathrm{H}$ NMR $(600$ $\left.\mathrm{MHz}, \mathrm{CDCl}_{3}\right) \delta_{\mathrm{H}}: 6.29\left(1 \mathrm{H}, \mathrm{d}, J=17.4 \mathrm{~Hz}, \mathrm{H}-2 \mathrm{a}^{\prime}\right), 6.03(1 \mathrm{H}, \mathrm{dd}, J=17.4,10.2 \mathrm{~Hz}, \mathrm{H}-1$ '), $5.77(1 \mathrm{H}$, d, $J=10.2$ Hz, H-2b'), 5.58 (1H, br s, H-14), 5.44 (1H, d, $J=10.8$ Hz, H-11), 4.48 (1H, br s, H-18a), 4.49 (1H, br s, H-18b), 3.61 (3H, s, OMe), 2.39 (1H, d, $J=13.3 \mathrm{~Hz}, \mathrm{H}-12 \mathrm{a}), 2.37$ (1H, m, H-3a), 2.13 (3H, s, H3-16), 2.18 (2H, overlapped, H-3b and H-12b), 2.02 (1H, m, H-1a), 1.87 (1H, m, H-7a), 1.59-1.44 (5H, overlapped, $\mathrm{H}-1 \mathrm{~b}, \mathrm{H}_{2}-2$, and $\left.\mathrm{H}_{2}-6\right), 1.39$ (1H, m, H-8), 1.29 (1H, m, H-7b), $1.18(1 \mathrm{H}$, m, H-10), 1.01 (3H, s, H3-19), $0.96\left(3 \mathrm{H}, \mathrm{d}, J=6.6 \mathrm{~Hz}, \mathrm{H}_{3}-17\right), 0.75\left(3 \mathrm{H}, \mathrm{s}, \mathrm{H}_{3}-20\right) ;{ }^{13} \mathrm{C} \mathrm{NMR}(150$ $\left.\mathrm{MHz}, \mathrm{CDCl}_{3}\right) \delta_{\mathrm{C}}: 166.7$ (s, C-15), 166.2 (s, C-1'), 160.2 (S, C-4), 156.8 (s, C-13), 130.8 (t, C-3'), 128.6 (d, C-2'), 118.3 (d, C-14), 103.2 (t, C-18), 76.8 (d, C-11), 51.1 (q, OMe), 48.5 (d, C-10), 44.5 
(s, C-9), 41.9 (t, C-12), 40.4 (s, C-5), 37.8 (d, C-8), 37.1 (t, C-6), 33.3 (t, C-3), 28.5 (t, C-7), 28.2 (t, C-2), 23.8 (t, C-1), 20.7 (q, C-19), 18.8 (q, C-16), 16.9 (q, C-17), 13.4 (q, C-20).

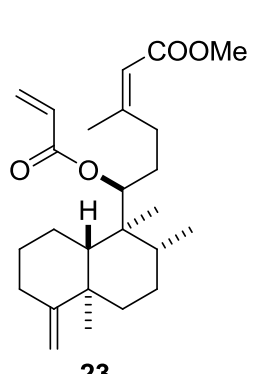

Synthesis of 23: 22 (100 mg, $0.25 \mathrm{mmol})$ was dissolved in acetonitrile (5 mL) in a quartz tube. The reaction vessels were carefully sealed and irradiated using a photoreactor $(313 \mathrm{~nm})$ at room temperature for $8 \mathrm{~h}$. The resulting mixture was concentrated in vacuo and then directly subJected to preparative HPLC to give 23 (52 mg, $0.13 \mathrm{mmol}, 50 \%$ ). Compound 23, Positive ESIMS $[\mathrm{M}+\mathrm{Na}]^{+} \mathrm{m} / \mathrm{z}$ 411; ${ }^{1} \mathrm{H}$ NMR $\left(600 \mathrm{MHz}, \mathrm{CDCl}_{3}\right) \delta_{\mathrm{H}}: 6.28\left(1 \mathrm{H}, \mathrm{d}, J=17.4 \mathrm{~Hz}, \mathrm{H}-3 \mathrm{a}^{\prime}\right), 6.00(1 \mathrm{H}, \mathrm{dd}, J=17.4,9.6 \mathrm{~Hz}$, H-2'), 5.75 (1H, d, $\left.J=9.6 \mathrm{~Hz}, \mathrm{H}-3 \mathrm{~b}^{\prime}\right), 5.64(1 \mathrm{H}$, br s, H-14), $5.38(1 \mathrm{H}, \mathrm{d}, J=10.2 \mathrm{~Hz}, \mathrm{H}-11), 4.48$ (1H, br s, H-18a), 4.49 (1H, br s, H-18b), 3.61 (3H, s, OMe); ${ }^{13} \mathrm{C}$ NMR (150 MHz, CDCl 3 ) $\delta_{\mathrm{C}}: 166.7$ (s, C-15), 166.3 (s, C-1'), 160.4 (s, C-4), 156.4 (s, C-13), 130.6 (t, C-3'), 128.9 (d, C-2'), 118.8 (d, C-14), 103.0 (t, C-18), 78.2 (d, C-11), 51.0 (q, OMe), 48.5 (d, C-10), 44.7 (s, C-9), 40.4 (s, C-5), 37.7 (d, C-8), 37.2 (t, C-6), 33.3 (t, C-12), 33.1 (t, C-3), 28.5 (t, C-7), 28.2 (t, C-2), 25.7 (q, C-16), 23.6 (t, C-1), 20.8 (q, C-19), 17.0 (q, C-17), 13.4 (q, C-20). 
Scheme S5. Selected optimization for Pd-catalyzed C-H arylation
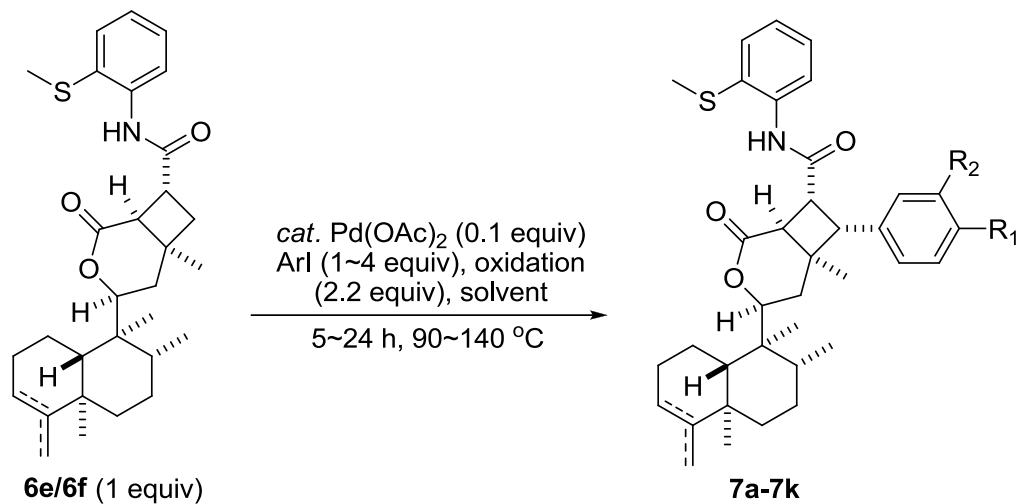

\begin{tabular}{|c|c|c|c|c|c|c|c|}
\hline entry & aryl iodide & ratio of substrates & oxidation & solvent & temp $\left({ }^{\circ} \mathrm{C}\right)$ & time $(\mathrm{h})$ & products/yield $(\%)^{b}$ \\
\hline 1 & $\mathrm{R}_{1}=\mathrm{OMe}, \mathrm{R}_{2}=\mathrm{H}$ & 6e:ArI (1:3) & $\mathrm{AgOAc}$ & $t-\mathrm{BuOH}$ & 90 & 5 & $7 \mathbf{a} / 68$ \\
\hline 2 & $\mathrm{R}_{1}=\mathrm{OMe}, \mathrm{R}_{2}=\mathrm{H}$ & 6f:ArI (1:3) & $\mathrm{AgOAc}$ & $t-\mathrm{BuOH}$ & 90 & 5 & $7 \mathrm{j} / 65$ \\
\hline 3 & $\mathrm{R}_{1}=\mathrm{OMe}, \mathrm{R}_{2}=\mathrm{H}$ & 6e:ArI (1:3) & $\mathrm{AgOAc}$ & $t$-BuOH & 120 & 5 & $7 \mathbf{a} / 42$ \\
\hline 4 & $\mathrm{R}_{1}=\mathrm{OMe}, \mathrm{R}_{2}=\mathrm{H}$ & 6e:ArI (1:3) & $\mathrm{AgOAc}$ & $t-\mathrm{BuOH}$ & 90 & 24 & $7 \mathbf{a} / 28$ \\
\hline 5 & $\mathrm{R}_{1}=\mathrm{OMe}, \mathrm{R}_{2}=\mathrm{H}$ & 6e:ArI (1:3) & $\mathrm{AgOAc}$ & $\mathrm{PhMe}$ & 115 & 5 & $7 \mathbf{a} / 82$ \\
\hline 6 & $\mathrm{R}_{1}=\mathrm{OMe}, \mathrm{R}_{2}=\mathrm{H}$ & 6f:ArI (1:4) & $\mathrm{AgOAc}$ & $\mathrm{PhMe}$ & 115 & 5 & $7 \mathbf{j} / 80$ \\
\hline 7 & $\mathrm{R}_{1}=\mathrm{OMe}, \mathrm{R}_{2}=\mathrm{H}$ & 6e:ArI (1:3) & $\mathrm{AgOAc}$ & $\mathrm{PhMe}$ & 115 & 5 & $7 \mathbf{a} / 75$ \\
\hline 8 & $\mathrm{R}_{1}=\mathrm{OMe}, \mathrm{R}_{2}=\mathrm{H}$ & 6e:ArI (1:2) & $\mathrm{AgOAc}$ & $\mathrm{PhMe}$ & 115 & 10 & $7 \mathbf{a} / 64$ \\
\hline 9 & $\mathrm{R}_{1}=\mathrm{OMe}, \mathrm{R}_{2}=\mathrm{H}$ & 6e:ArI (1:1) & $\mathrm{AgOAc}$ & $\mathrm{PhMe}$ & 115 & 5 & $7 \mathbf{a} / 52$ \\
\hline 10 & $\mathrm{R}_{1}=\mathrm{OMe}, \mathrm{R}_{2}=\mathrm{H}$ & 6e:ArI (1:3) & $\mathrm{Ag}_{2} \mathrm{CO}_{3}$ & $t$-BuOH & 100 & 5 & $7 \mathbf{a} / 58$ \\
\hline 11 & $\mathrm{R}_{1}=\mathrm{OMe}, \mathrm{R}_{2}=\mathrm{H}$ & 6e:ArI (1:3) & none & $t-\mathrm{BuOH}$ & 100 & 5 & $7 \mathbf{a} / 0$ \\
\hline 12 & $\mathrm{R}_{1}=\mathrm{OMe}, \mathrm{R}_{2}=\mathrm{H}$ & 6e:ArI (1:3) & $\mathrm{Ag}_{2} \mathrm{CO}_{3}$ & $\mathrm{PhMe}$ & 115 & 5 & $7 \mathbf{a} / 62$ \\
\hline 13 & $\mathrm{R}_{1}=\mathrm{OMe}, \mathrm{R}_{2}=\mathrm{H}$ & 6e:ArI (1:3) & $\mathrm{K}_{2} \mathrm{CO}_{3}$ & $t$-BuOH & 120 & 24 & $7 \mathbf{a} / 0$ \\
\hline 14 & $\mathrm{R}_{1}=\mathrm{OMe}, \mathrm{R}_{2}=\mathrm{H}$ & 6e:ArI (1:3) & $\mathrm{CsOAc}$ & $t$-BuOH & 120 & 24 & $7 \mathbf{a} / 0$ \\
\hline 15 & $\mathrm{R}_{1}=\mathrm{OH}, \mathrm{R}_{2}=\mathrm{H}$ & 6e:ArI (1:3) & $\mathrm{AgOAc}$ & $t-\mathrm{BuOH}$ & 120 & 24 & $7 \mathrm{c} / 32$ \\
\hline 16 & $\mathrm{R}_{1}=\mathrm{OH}, \mathrm{R}_{2}=\mathrm{H}$ & 6e:ArI (1:3) & $\mathrm{AgOAc}$ & $t-\mathrm{BuOH}$ & 110 & 12 & $7 \mathrm{c} / 48$ \\
\hline 17 & $\mathrm{R}_{1}=\mathrm{F}, \mathrm{R}_{2}=\mathrm{F}$ & 6e:ArI (1:3) & $\mathrm{AgOAc}$ & $t-\mathrm{BuOH}$ & 120 & 24 & $7 d / 60$ \\
\hline 18 & $\mathrm{R}_{1}=\mathrm{NO}_{2}, \mathrm{R}_{2}=\mathrm{H}$ & 6e:ArI (1:3) & $\mathrm{AgOAc}$ & $t-\mathrm{BuOH}$ & 100 & 24 & $7 \mathrm{e} / 82$ \\
\hline 19 & $\mathrm{R}_{1}=\mathrm{Br}, \mathrm{R}_{2}=\mathrm{H}$ & 6e:ArI (1:3) & $\mathrm{AgOAc}$ & $t-\mathrm{BuOH}$ & 140 & 24 & $7 f / 75$ \\
\hline 20 & $\mathrm{R}_{1}=\mathrm{Br}, \mathrm{R}_{2}=\mathrm{H}$ & 6f:ArI (1:3) & $\mathrm{AgOAc}$ & $t$-BuOH & 140 & 24 & $7 \mathrm{~g} / 70$ \\
\hline 21 & $\mathrm{R}_{1}=\mathrm{Br}, \mathrm{R}_{2}=\mathrm{H}$ & 6e:ArI (1:3) & $\mathrm{AgOAc}$ & $\mathrm{PhMe}$ & 140 & 24 & $7 f / 85$ \\
\hline 22 & $\mathrm{R}_{1}=\mathrm{F}, \mathrm{R}_{2}=\mathrm{H}$ & 6e:ArI (1:3) & $\mathrm{AgOAc}$ & $\mathrm{PhMe}$ & 120 & 24 & $7 \mathbf{h} / 78$ \\
\hline 23 & $\mathrm{R}_{1}=\mathrm{F}, \mathrm{R}_{2}=\mathrm{H}$ & 6f:ArI (1:3) & $\mathrm{AgOAc}$ & $\mathrm{PhMe}$ & 120 & 24 & $7 i / 69$ \\
\hline 24 & $\mathrm{R}_{1}=\mathrm{F}, \mathrm{R}_{2}=\mathrm{H}$ & 6e:ArI (1:3) & $\mathrm{AgOAc}$ & $t-\mathrm{BuOH}$ & 120 & 24 & $7 \mathbf{h} / 58$ \\
\hline 25 & $\mathrm{R}_{1}=\mathrm{OCF}_{3}, \mathrm{R}_{2}=\mathrm{H}$ & 6e:ArI (1:3) & $\mathrm{AgOAc}$ & $t-\mathrm{BuOH}$ & 120 & 24 & $7 \mathbf{b} / 77$ \\
\hline 26 & $\mathrm{R}_{1}=\mathrm{OCF}_{3}, \mathrm{R}_{2}=\mathrm{H}$ & 6f:ArI (1:3) & $\mathrm{AgOAc}$ & $t$-BuOH & 120 & 24 & $7 \mathbf{k} / 70$ \\
\hline 27 & $\mathrm{R}_{1}=\mathrm{OCF}_{3}, \mathrm{R}_{2}=\mathrm{H}$ & 6e:ArI (1:3) & $\mathrm{AgOAc}$ & $\mathrm{PhMe}$ & 120 & 24 & $7 b / 75$ \\
\hline
\end{tabular}

${ }^{a} \mathbf{6 e}: \Delta^{4,18}, \mathbf{6 f}: \Delta^{3,4},{ }^{b}$ The yields denoted here were calculated based on the starting compounds $6 \mathbf{e}$ or $\mathbf{6 f}$. 
General procedure for the synthesis of $7 \boldsymbol{b}-7 \boldsymbol{k}$ from $\boldsymbol{6} \boldsymbol{e}$ and $\boldsymbol{6} f$ : A sealed tube containing the corresponding 6e or $\mathbf{6 f}, \mathrm{Pd}(\mathrm{OAc})_{2}$ (0.1 equiv), oxidation (2.2 equiv), aryl iodide (1 3 equiv) and solvent $(6 \mathrm{~mL})$ was sealed and placed into a $90 \sim 140{ }^{\circ} \mathrm{C}$ oil bath for $5 \sim 24 \mathrm{~h}$. The reaction was cooled to room temperature, diluted with DCM $(10 \mathrm{~mL})$ and filtered through a pad of celite. The combined extracts were washed with brine $(20 \mathrm{~mL})$, dried over $\mathrm{Na}_{2} \mathrm{SO}_{4}$ and concentrated in vacuo. The resulting black oil was purified by silica gel chromatography $\left(4: 1 \mathrm{CHCl}_{3}\right.$ :EtOAc) and preparative HPLC to give the corresponding $\mathbf{7 a}-\mathbf{7 k}$.

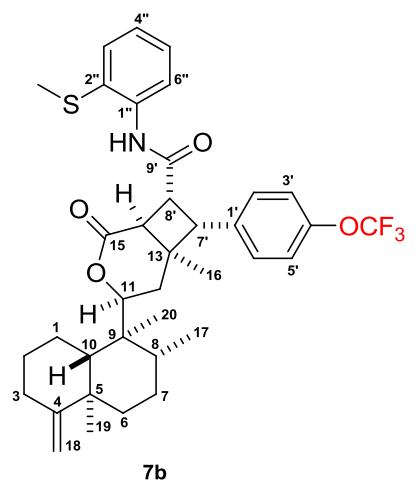

Compound 7b: Yield: $52 \mathrm{mg}, 77 \%$; Positive ESIMS $[\mathrm{M}+\mathrm{Na}]^{+} \mathrm{m} / z$ 678; ${ }^{1} \mathrm{H}$ NMR $\left(600 \mathrm{MHz}, \mathrm{CDCl}_{3}\right) \delta_{\mathrm{H}}: 8.56(1 \mathrm{H}, \mathrm{s}, \mathrm{NH}), 8.16(1 \mathrm{H}, \mathrm{d}, J=8.0$ Hz, H-3"), 7.38 (1H, d, J = 7.7 Hz, H-6"), 7.23 (1H, overlapped, H-5"), $7.20\left(2 \mathrm{H}\right.$, overlapped, H-2' and H-6'), $7.05\left(2 \mathrm{H}, \mathrm{d}, J=8.6 \mathrm{~Hz}, \mathrm{H}-3^{\prime}\right.$ and H-5'), 7.03 (1H, t, $J=7.4 \mathrm{~Hz}, \mathrm{H}-4 "), 4.53(1 \mathrm{H}, \mathrm{s}, \mathrm{H}-18 \mathrm{a}), 4.50(1 \mathrm{H}, \mathrm{s}$, $\mathrm{H}-18 \mathrm{~b}), 4.28(1 \mathrm{H}, \mathrm{d}, J=11.1 \mathrm{~Hz}, \mathrm{H}-11), 3.87(1 \mathrm{H}, \mathrm{dd}, J=10.4$ and 7.8 Hz, H-8'), $3.71(1 \mathrm{H}, \mathrm{d}, J=10.5 \mathrm{~Hz}, \mathrm{H}-7 '), 3.50(1 \mathrm{H}, \mathrm{d}, J=7.7 \mathrm{~Hz}, \mathrm{H}-14)$, $2.31(1 \mathrm{H}, \mathrm{m}, \mathrm{H}-3 \mathrm{a}), 2.12(1 \mathrm{H}, \mathrm{m}, \mathrm{H}-3 \mathrm{~b}), 2.09$ (3H, s, SMe), 2.01 (1H, d, J = $13.9 \mathrm{~Hz}, \mathrm{H}-12 \mathrm{a})$, 1.91-1.88 (2H, overlapped, H-1a and H-2a), $1.80(1 \mathrm{H}$, br t, $J=13.9 \mathrm{~Hz}, \mathrm{H}-12 \mathrm{~b}), 1.67-1.48(5 \mathrm{H}$, overlapped, $\mathrm{H}-1 \mathrm{~b}, \mathrm{H}_{2}-6$, and $\mathrm{H}_{2}-7$ ), 1.30-1.24 (3H, m, overlapped, H-2b, H-8, and H-10), 1.07 (3H, s, $\left.\mathrm{H}_{3}-19\right), 1.03$ (3H, s, H3-20), 0.99 (3H, s, H3-16), 0.85 (3H, d, $\left.J=6.6 \mathrm{~Hz}, \mathrm{H}_{3}-17\right) ;{ }^{13} \mathrm{C} \mathrm{NMR}(151$ $\left.\mathrm{MHz}, \mathrm{CDCl}_{3}\right) \delta_{\mathrm{C}}: 174.5$ (s, C-9'), 169.0 (s, C-15), 160.1 (s, C-4), 148.6 (s, C-1'), 137.8 (s, C-4'), 135.7 (s, C-1"), 132.9 (s, C-6"), 130.7 (d, C-2' and C-6'), 128.8 (d, C-5"), 126.4 (s, C-2"), 125.0 (d,

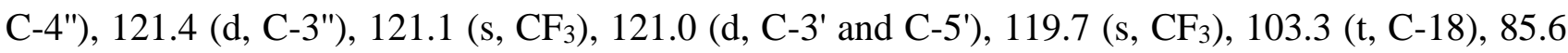
(d, C-11), 52.8 (d, C-7'), 48.9 (d, C-10), 45.8 (d, C-8'), 43.2 (d, C-14), 43.28 (s, C-9), 40.5 (s, C-5), 39.9 (t, C-12), 39.7 (s, C-13), 37.1 (d, C-6), 37.1 (d, C-8), 33.2 (t, C-3), 28.6 (t, C-2), 27.9 (s, C-7), 24.0 (t, C-1), 22.2 (q, C-16), 20.8 (q, C-19), 18.7 (q, SMe), 16.9 (q, C-17), 14.0 (q, C-20).

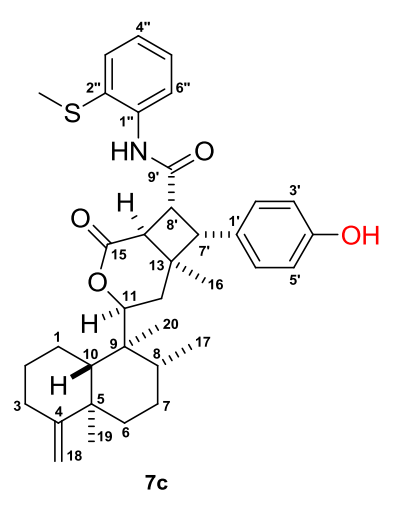

Compound 7c: Yield: $12 \mathrm{mg}, 48 \%$; Positive ESIMS $[\mathrm{M}+\mathrm{Na}]^{+} \mathrm{m} / z 610 ;{ }^{1} \mathrm{H}$ NMR $\left(600 \mathrm{MHz}, \mathrm{CDCl}_{3}\right) \delta_{\mathrm{H}}: 8.62(1 \mathrm{H}, \mathrm{s}, \mathrm{NH}), 8.11(1 \mathrm{H}, \mathrm{d}, J=8.0 \mathrm{~Hz}$, H-3"), 7.37 (1H, d, $J=7.6$ Hz, H-6"), 7.20 (1H, t, $J=7.4$ Hz, H-5"), 7.05 $\left(1 \mathrm{H}, \mathrm{t}, J=7.4 \mathrm{~Hz}, \mathrm{H}-44^{\prime}\right), 6.94$ (2H, d, $J=8.6 \mathrm{~Hz}, \mathrm{H}-2^{\prime}$ and H-6'), $6.52(2 \mathrm{H}$, $\mathrm{d}, J=8.6 \mathrm{~Hz}, \mathrm{H}-3^{\prime}$ and H-5'), 4.53 (1H, s, H-18a), 4.50 (1H, s, H-18b), $4.28(1 \mathrm{H}, \mathrm{d}, J=11.1 \mathrm{~Hz}, \mathrm{H}-11), 3.83\left(1 \mathrm{H}, \mathrm{dd}, J=10.4\right.$ and $\left.7.8 \mathrm{~Hz}, \mathrm{H}-8^{\prime}\right)$, $3.57(1 \mathrm{H}, \mathrm{d}, J=10.5 \mathrm{~Hz}, \mathrm{H}-7$ '), $3.50(1 \mathrm{H}, \mathrm{d}, J=7.7 \mathrm{~Hz}, \mathrm{H}-14), 2.30(1 \mathrm{H}$, 
m, H-3a), 2.11 (1H, m, H-3b), 2.09 (3H, s, SMe), 1.98 (1H, d, J=13.9 Hz, H-12a), 1.87-1.85 (2H, overlapped, H-1a and H-2a), 1.77 (1H, br t, $J=13.9 \mathrm{~Hz}, \mathrm{H}-12 \mathrm{~b}), 1.70-1.46$ (5H, overlapped, H-1b, $\mathrm{H}_{2}-6$, and $\mathrm{H}_{2}-7$ ), 1.30-1.23 (3H, m, overlapped, $\mathrm{H}-2 \mathrm{~b}, \mathrm{H}-8$, and $\left.\mathrm{H}-10\right), 1.06$ (3H, s, $\left.\mathrm{H}_{3}-19\right), 1.02$ (3H, s, $\left.\mathrm{H}_{3}-20\right), 0.94\left(3 \mathrm{H}, \mathrm{s}, \mathrm{H}_{3}-16\right), 0.84\left(3 \mathrm{H}, \mathrm{d}, J=6.7 \mathrm{~Hz}, \mathrm{H}_{3}-17\right) ;{ }^{13} \mathrm{C}$ NMR $\left(151 \mathrm{MHz}, \mathrm{CDCl}_{3}\right) \delta_{\mathrm{C}}$ : 174.9 (s, C-9'), 169.7 (s, C-15), 160.1 (s, C-4'), 155.4 (s, C-4), 137.7 (s, C-1"), 132.8 (s, C-6"), 130.4 (d, C-2' and C-6'), 128.6 (d, C-5"), 128.3 (s, C-1'), 126.8 (s, C-2"), 125.0 (d, C-4"), 121.4 (d, C-3"), 115.5 (d, C-3' and C-5'), 103.2 (t, C-18), 85.6 (d, C-11), 52.8 (d, C-7'), 48.8 (d, C-10), 45.9 (d, C-8'), 43.3 (d, C-14), 43.1 (s, C-9), 40.4 (s, C-5), 39.5 (t, C-12), 39.2 (s, C-13), 37.0 (t, C-6), 37.0 (d, C-8), 33.2 (t, C-3), 28.5 (t, C-2), 27.9 (s, C-7), 23.9 (t, C-1), 22.1 (q, C-16), 20.7 (q, C-19), 18.7 (q, SMe), 16.8 (q, C-17), 13.9 (q, C-20).

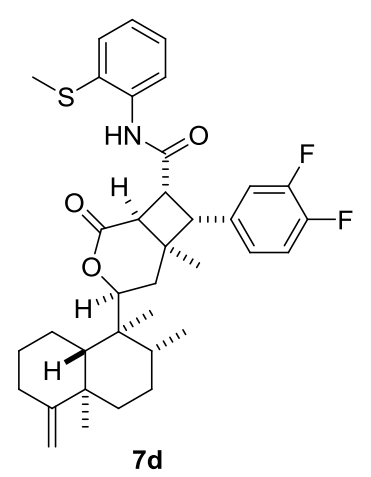

Compound 7d: Yield: $14 \mathrm{mg}, 60 \%$; Positive ESIMS $[\mathrm{M}+\mathrm{Na}]^{+} \mathrm{m} / z 630 ;{ }^{1} \mathrm{H}$ NMR $\left(600 \mathrm{MHz}, \mathrm{CDCl}_{3}\right) \delta_{\mathrm{H}}: 8.62(1 \mathrm{H}, \mathrm{s}, \mathrm{NH}), 8.12(1 \mathrm{H}, \mathrm{d}, J=8.0 \mathrm{~Hz}$, H-3"), 7.40 (1H, d, $J=7.7$ Hz, H-6"), 7.23 (1H, t, $J=7.4$ Hz, H-5"), 7.04 (1H, t, $J=7.4 \mathrm{~Hz}, \mathrm{H}-4 "), 7.06-7.02$ (2H, overlapped, H-2' and H-5'), 6.90 (1H, m, H-6'), 4.53 (1H, s, H-18a), 4.50 (1H, s, H-18b), 4.29 (1H, d, J = 11.1 Hz, H-11), 3.85 (1H, dd, $J=10.4$ and $7.8 \mathrm{~Hz}, \mathrm{H}-8$ '), 3.63 (1H, d, J = 10.5 Hz, H-7'), 3.48 (1H, d, J = 7.7 Hz, H-14), 2.31 (1H, m, H-3a), 2.21 (3H, s, SMe), $2.16(1 \mathrm{H}, \mathrm{m}, \mathrm{H}-3 \mathrm{~b}), 2.01(1 \mathrm{H}, \mathrm{d}, J=13.9 \mathrm{~Hz}, \mathrm{H}-12 \mathrm{a}), 1.90-1.88$ (2H, overlapped, H-1a and H-2a), $1.79(1 \mathrm{H}$, br t, $J=13.9 \mathrm{~Hz}, \mathrm{H}-12 \mathrm{~b}), 1.65-1.48$ (5H, overlapped, $\mathrm{H}-1 \mathrm{~b}, \mathrm{H}_{2}-6$, and $\mathrm{H}_{2}-7$ ), 1.30-1.24 (3H, m, overlapped, H-2b, H-8, and H-10), 1.07 (3H, s, H3-19), 1.03 (3H, s, H3-20), 0.99 (3H, s, $\left.\mathrm{H}_{3}-16\right), 0.86\left(3 \mathrm{H}, \mathrm{d}, J=6.6 \mathrm{~Hz}, \mathrm{H}_{3}-17\right) ;{ }^{13} \mathrm{C} \mathrm{NMR}\left(151 \mathrm{MHz}, \mathrm{CDCl}_{3}\right) \delta_{\mathrm{C}}: 174.3$ (s, C-9'), 168.8 (s, C-15), 160.0 (s, C-4), 150.9 (s, C-3'), 150.5 (s, C-3'), 150.4 (s, C-3'), 149.3 (s, C-3'), 149.2 (s, C-4'), 148.9 (s, C-4'), 148.8 (s, C-4'), 137.5 (s, C-1"), 134.1 (s, C-1'), 132.5 (s, C-6"), 128.6 (d, C-5"), 126.7 (s, C-2"), 125.2 (d, C-6'), 125.1 (d, C-4"), 121.3 (d, C-3"), 118.4 (d, C-2'), 118.3 (d, C-2'), 117.3 (d, C-5'), 117.2 (d, C-5'), 103.3 (t, C-18), 85.5 (d, C-11), 52.6 (d, C-7'), 48.8 (d, C-10), 45.6 (d, C-8'),

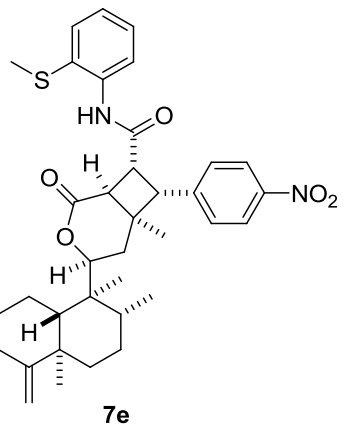
43.3 (d, C-14), 43.2 (s, C-9), 40.4 (s, C-5), 39.6 (t, C-12), 39.4 (s, C-13), 37.0 (d, C-8), 33.2 (t, C-3), 28.5 (t, C-2), 27.8 (s, C-7), 23.9 (t, C-1), 22.0 (q, C-16), 20.7 (q, C-19), 18.7 (q, SMe), 16.9 (q, C-17), 13.9 (q, C-20).

Compound 7e: Yield: $28 \mathrm{mg}, 82 \%$; ${ }^{1} \mathrm{H}$ NMR $\left(600 \mathrm{MHz}, \mathrm{CDCl}_{3}\right) \delta_{\mathrm{H}}: 8.79$ $(1 \mathrm{H}, \mathrm{s}, \mathrm{NH}), 8.09$ (2H, d, $J=8.6 \mathrm{~Hz}, \mathrm{H}-3^{\prime}$ and H-5'), $8.03(1 \mathrm{H}, \mathrm{d}, J=8.0 \mathrm{~Hz}$, H-3"), 7.38 (1H, d, $J=7.6 \mathrm{~Hz}, \mathrm{H}-6 "), 7.34$ (2H, d, $J=8.6 \mathrm{~Hz}, \mathrm{H}-2^{\prime}$ and H-6'), 
$7.21(1 \mathrm{H}, \mathrm{t}, J=7.4 \mathrm{~Hz}, \mathrm{H}-5 "), 7.07(1 \mathrm{H}, \mathrm{t}, J=7.4 \mathrm{~Hz}, \mathrm{H}-4 "), 4.54$ (1H, s, H-18a), 4.51 (1H, s, H-18b), $\left.4.31(1 \mathrm{H}, \mathrm{d}, J=11.1 \mathrm{~Hz}, \mathrm{H}-11), 3.90(1 \mathrm{H}, \mathrm{dd}, J=9.9 \text { and } 8.6 \mathrm{~Hz}, \mathrm{H}-8)^{\prime}\right), 3.77(1 \mathrm{H}, \mathrm{d}, J=$ $10.2 \mathrm{~Hz}, \mathrm{H}-7$ '), 3.53 (1H, d, J = 8.6 Hz, H-14), 2.29 (1H, m, H-3a), 2.26 (3H, s, SMe), 2.11 (1H, m, H-3b), 2.06 (1H, d, $J=13.9$ Hz, H-12a), 1.90-1.85 (3H, overlapped, H-1a, H-2a, and H-12b), 1.68-1.48 (5H, overlapped, $\mathrm{H}-1 \mathrm{~b}, \mathrm{H}_{2}-6$, and $\left.\mathrm{H}_{2}-7\right), 1.32-1.25$ (3H, m, overlapped, $\mathrm{H}-2 \mathrm{~b}, \mathrm{H}-8$, and H-10), 1.07 (3H, s, $\left.\mathrm{H}_{3}-19\right), 1.04$ (3H, s, $\left.\mathrm{H}_{3}-20\right), 0.95$ (3H, s, $\left.\mathrm{H}_{3}-16\right), 0.86$ (3H, d, J = $\left.6.7 \mathrm{~Hz}, \mathrm{H}_{3}-17\right)$; ${ }^{13} \mathrm{C}$ NMR (151 MHz, CDCl3) $\delta_{\mathrm{C}}: 174.0$ (s, C-9'), 168.6 (s, C-15), 159.9 (s, C-4), 147.1 (s, C-1'), 145.0 (s, C-4'), 137.1 (s, C-1"), 132.0 (s, C-6"), 130.1 (d, C-2' and C-6'), 128.3 (d, C-5"), 127.3 (s, C-2"), 125.4 (d, C-4"), 123.7 (d, C-3' and C-5'), 121.7 (d, C-3"), 103.3 (t, C-18), 85.4 (d, C-11), 52.8 (d, C-7'), 48.9 (d, C-10), 45.0 (d, C-8'), 43.9 (d, C-14), 43.2 (s, C-9), 40.4 (s, C-5), 39.3 (t, C-12), 38.9 (s, C-13), 37.0 (t, C-6), 37.0 (d, C-8), 33.1 (t, C-3), 28.5 (t, C-2), 27.8 (s, C-7), 23.9 (t, C-1), 22.2 (q, C-16), 20.7 (q, C-19), 18.5 (q, SMe), 16.8 (q, C-17), 13.9 (q, C-20).

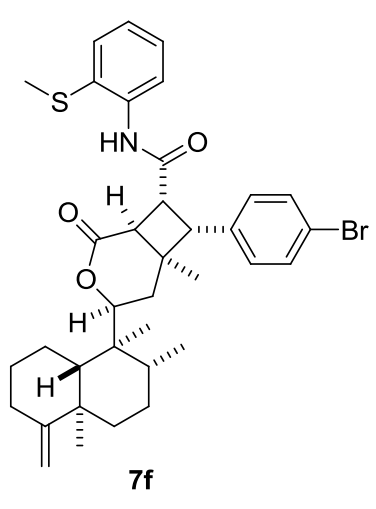

Compound 7f: Yield: $50 \mathrm{mg}, 85 \%$; Positive ESIMS $[\mathrm{M}+\mathrm{Na}]^{+} m / z$ 672; ${ }^{1} \mathrm{H}$ NMR $\left(600 \mathrm{MHz}, \mathrm{CDCl}_{3}\right) \delta_{\mathrm{H}}: 8.65(1 \mathrm{H}, \mathrm{s}, \mathrm{NH}), 8.17(1 \mathrm{H}, \mathrm{d}, J=8.0$ Hz, H-3"), 7.42 (1H, d, $J=7.6$ Hz, H-6"), 7.35 (2H, d, $J=8.6$ Hz, H-3' and $\left.\mathrm{H}^{-5}\right), 7.25$ (1H, t, $\left.J=7.4 \mathrm{~Hz}, \mathrm{H}-5 "\right), 7.06\left(2 \mathrm{H}, \mathrm{d}, J=8.6 \mathrm{~Hz}, \mathrm{H}-2^{\prime}\right.$ and H-6'), 7.05 (1H, t, $J=7.4 \mathrm{~Hz}, \mathrm{H}-4 "), 4.55(1 \mathrm{H}, \mathrm{s}, \mathrm{H}-18 \mathrm{a}), 4.52(1 \mathrm{H}$, s, H-18b), $4.30(1 \mathrm{H}, \mathrm{d}, J=11.1 \mathrm{~Hz}, \mathrm{H}-11), 3.87(1 \mathrm{H}, \mathrm{dd}, J=10.4$ and $\left.7.8 \mathrm{~Hz}, \mathrm{H}-8^{\prime}\right), 3.66\left(1 \mathrm{H}, \mathrm{d}, J=10.5 \mathrm{~Hz}, \mathrm{H}-7^{\prime}\right), 3.52(1 \mathrm{H}, \mathrm{d}, J=7.7 \mathrm{~Hz}$, H-14), 2.31 (1H, m, H-3a), 2.16 (1H, m, H-3b), 2.16 (3H, s, SMe), 2.02 (1H, d, J = 13.9 Hz, H-12a), 1.91-1.89 (2H, overlapped, H-1a and H-2a), $1.81(1 \mathrm{H}$, br t, $J=13.9 \mathrm{~Hz}, \mathrm{H}-12 \mathrm{~b}), 1.68-1.48(5 \mathrm{H}$, overlapped, $\mathrm{H}-1 \mathrm{~b}, \mathrm{H}_{2}-6$, and $\left.\mathrm{H}_{2}-7\right), 1.33-1.25$ (3H, m, overlapped, $\mathrm{H}-2 \mathrm{~b}, \mathrm{H}-8$, and H-10), 1.08 (3H, s, $\left.\mathrm{H}_{3}-19\right), 1.05$ (3H, s, $\left.\mathrm{H}_{3}-20\right), 0.98$ (3H, s, $\left.\mathrm{H}_{3}-16\right), 0.86\left(3 \mathrm{H}, \mathrm{d}, J=6.7 \mathrm{~Hz}, \mathrm{H}_{3}-17\right) ;{ }^{13} \mathrm{C} \mathrm{NMR}(151$ MHz, $\left.\mathrm{CDCl}_{3}\right) \delta_{\mathrm{C}}: 174.4$ (s, C-9'), 168.9 (s, C-15), 160.0 (s, C-4), 137.7 (s, C-1"), 136.0 (s, C-1'),

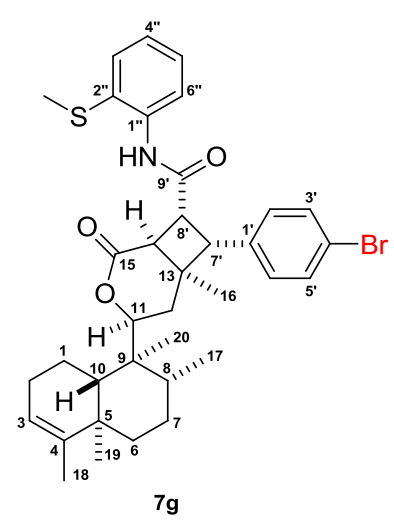
132.8 (s, C-6"), 131.6 (d, C-3' and C-5'), 130.9 (d, C-2' and C-6'), 128.7 (s, C-2"), 126.4 (d, C-5"), 124.9 (d, C-4"), 121.5 (s, C-4'), 121.1 (d, C-3"), 103.3 (t, C-18), 85.4 (d, C-11), 52.9 (d, C-7'), 48.8 (d, C-10), 45.5 (d, C-8'), 43.3 (d, C-14), 43.1 (s, C-9), 40.4 (s, C-5), 39.4 (t, C-12), 39.3 (s, C-13), 37.0 (t, C-6), 37.0 (d, C-8), 33.1 (t, C-3), 28.5 (t, C-2), 27.8 (s, C-7), 23.9 (t, C-1), 22.1 (q, C-16), 20.7 (q, C-19), 18.7 (q, SMe), 16.8 (q, C-17), 13.9 (q, C-20). 
Compound 7g: Yield: $18 \mathrm{mg}, 70 \% ;{ }^{1} \mathrm{H} \mathrm{NMR}\left(600 \mathrm{MHz}, \mathrm{CDCl}_{3}\right) \delta_{\mathrm{H}}: 8.67(1 \mathrm{H}, \mathrm{s}, \mathrm{NH}), 8.15(1 \mathrm{H}, \mathrm{d}, J$ $\left.=8.0 \mathrm{~Hz}, \mathrm{H}-3^{\prime \prime}\right), 7.40(1 \mathrm{H}, \mathrm{d}, J=7.6 \mathrm{~Hz}, \mathrm{H}-6 "), 7.35\left(2 \mathrm{H}, \mathrm{d}, J=8.2 \mathrm{~Hz}, \mathrm{H}-3^{\prime}\right.$ and H-5'), $7.25(1 \mathrm{H}, \mathrm{t}, J$ = $7.4 \mathrm{~Hz}, \mathrm{H}-5 "), 7.06$ (2H, d, $J=8.2 \mathrm{~Hz}, \mathrm{H}-2^{\prime}$ and H-6'), 7.05 (1H, t, $\left.J=7.4 \mathrm{~Hz}, \mathrm{H}-4 "\right), 5.21(1 \mathrm{H}, \mathrm{s}$, H-3), $4.32(1 \mathrm{H}, \mathrm{d}, J=11.1 \mathrm{~Hz}, \mathrm{H}-11), 3.86(1 \mathrm{H}, \mathrm{dd}, J=10.4$ and $7.8 \mathrm{~Hz}, \mathrm{H}-8$ '), $3.63(1 \mathrm{H}, \mathrm{d}, J=10.4$ Hz, H-7'), 3.50 (1H, d, J = 7.7 Hz, H-14), 2.16 (3H, s, SMe), 2.06-1.98 (3H, overlapped, H-1a, H-2a, and H-12a), 1.88 (1H, br t, $J=13.9 \mathrm{~Hz}, \mathrm{H}-12 \mathrm{~b}), 1.72-1.65$ (2H, overlapped, H-1b and H-6a), 1.57 (3H, s, H-18), 1.51-1.42 (3H, overlapped, $\mathrm{H}_{2}-7$ and $\left.\mathrm{H}-10\right), 1.30(1 \mathrm{H}, \mathrm{m}, \mathrm{H}-8), 1.13(1 \mathrm{H}, \mathrm{m}, \mathrm{H}-6 \mathrm{~b})$, 1.03 (3H, s, H $\left.{ }_{3}-19\right), 1.02$ (3H, s, $\left.\mathrm{H}_{3}-20\right), 0.97$ (3H, s, $\left.\mathrm{H}_{3}-16\right), 0.84\left(3 \mathrm{H}, \mathrm{d}, J=6.6 \mathrm{~Hz}, \mathrm{H}_{3}-17\right) ;{ }^{13} \mathrm{C}$ NMR (151 MHz, CDCl 3 ) $\delta_{\mathrm{C}}: 174.5$ (s, C-9'), 169.0 (s, C-15), 144.1 (s, C-4), 137.7 (s, C-1"), 136.0 (s, C-1'), 132.8 (s, C-6"), 131.6 (d, C-3' and C-5'), 130.9 (d, C-2' and C-6'), 128.7 (s, C-2"), 126.5 (d, C-5"), 124.9 (d, C-4"), 121.5 (s, C-4'), 121.2 (d, C-3"), 120.7 (d, C-3), 85.7 (d, C-11), 53.0 (d, C-7'), 46.6 (d, C-10), 45.6 (d, C-8'), 43.4 (d, C-14), 42.6 (s, C-9), 39.4 (t, C-12), 39.4 (s, C-13), 38.6 (s, C-5), 36.6 (t, C-6), 36.6 (d, C-8), 27.8 (t, C-2), 26.6 (s, C-7), 22.1 (q, C-16), 20.2 (t, C-1), 19.8 (q, C-19), 18.7 (q, SMe), 18.3 (q, C-18), 16.7 (q, C-17), 14.2 (q, C-20).

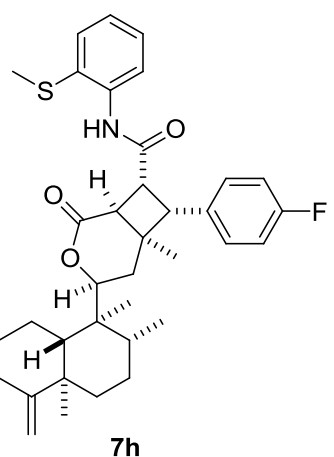

Compound 7h: Yield: $28 \mathrm{mg}, 78 \%$; Positive ESIMS $[\mathrm{M}+\mathrm{Na}]^{+} \mathrm{m} / z$ 612; ${ }^{1} \mathrm{H}$ NMR (600 MHz, CDCl3) $\delta_{\mathrm{H}}: 8.58(1 \mathrm{H}, \mathrm{s}, \mathrm{NH}), 8.20(1 \mathrm{H}, \mathrm{d}, J=8.2 \mathrm{~Hz}$, H-3"), 7.41 (1H, d, J = 7.6 Hz, H-6"), 7.25 (1H, t, $J=7.4$ Hz, H-5"), 7.16 (2H, dd, $J=8.4$ and $5.3 \mathrm{~Hz}, \mathrm{H}-2^{\prime}$ and H-6'), 7.05 (1H, t, $\left.J=7.4 \mathrm{~Hz}, \mathrm{H}-4 "\right)$, $6.92\left(2 \mathrm{H}, \mathrm{t}, J=8.4 \mathrm{~Hz}, \mathrm{H}-3^{\prime}\right.$ and H-5'), $4.55(1 \mathrm{H}, \mathrm{s}, \mathrm{H}-18 \mathrm{a}), 4.52(1 \mathrm{H}, \mathrm{s}$, H-18b), $4.30(1 \mathrm{H}, \mathrm{d}, J=11.1 \mathrm{~Hz}, \mathrm{H}-11), 3.88(1 \mathrm{H}, \mathrm{dd}, J=10.4$ and 7.8 Hz, H-8'), 3.69 (1H, d, J = 10.5 Hz, H-7'), 3.52 (1H, d, J = 7.7 Hz, H-14), $2.32(1 \mathrm{H}, \mathrm{m}, \mathrm{H}-3 \mathrm{a}), 2.14(1 \mathrm{H}, \mathrm{m}, \mathrm{H}-3 \mathrm{~b}), 2.13$ (3H, s, SMe), 2.01 (1H, d, $J=13.9 \mathrm{~Hz}, \mathrm{H}-12 \mathrm{a})$, 1.92-1.90 (2H, overlapped, H-1a and H-2a), $1.81(1 \mathrm{H}$, br t, $J=13.9 \mathrm{~Hz}, \mathrm{H}-12 \mathrm{~b}), 1.70-1.48(5 \mathrm{H}$, overlapped, $\mathrm{H}-1 \mathrm{~b}, \mathrm{H}_{2}-6$, and $\mathrm{H}_{2}-7$ ), 1.33-1.25 (3H, m, overlapped, H-2b, H-8, and H-10), 1.08 (3H, s, $\left.\mathrm{H}_{3}-19\right), 1.05$ (3H, s, $\left.\mathrm{H}_{3}-20\right), 1.00\left(3 \mathrm{H}, \mathrm{s}, \mathrm{H}_{3}-16\right), 0.87$ (3H, d, $\left.J=6.7 \mathrm{~Hz}, \mathrm{H}_{3}-17\right) ;{ }^{13} \mathrm{C} \mathrm{NMR}(151$ $\left.\mathrm{MHz}, \mathrm{CDCl}_{3}\right) \delta_{\mathrm{C}}: 174.5$ (s, C-9'), 169.1 (s, C-15), 162.9 (s, C-4'), 161.3 (s, C-4'), 160.1 (s, C-4), 137.9 (s, C-1"), 132.9 (s, C-6"), 132.7 (d, C-2' and C-6'), 130.9 (d, C-2' and C-6'), 130.8 (d, C-2' and C-6'), 128.8 (d, C-5"), 126.2 (s, C-2"), 124.8 (d, C-3"), 120.9 (d, C-4"), 115.5 (d, C-3' and C-5'), 115.3 (d, C-3' and C-5'), 103.2 (t, C-18), 85.5 (d, C-11), 52.8 (d, C-7'), 48.8 (d, C-10), 45.9 (d, C-8'), 43.1 (d, C-14), 43.1 (s, C-9), 40.4 (s, C-5), 39.7 (s, C-13), 39.6 (t, C-12), 37.0 (t, C-6), 37.0 (d, C-8), 
33.2 (t, C-3), 28.5 (t, C-2), 27.8 (s, C-7), 23.9 (t, C-1), 22.1 (q, C-16), 20.7 (q, C-19), 18.8 (q, SMe), 16.8 (q, C-17), 13.9 (q, C-20).

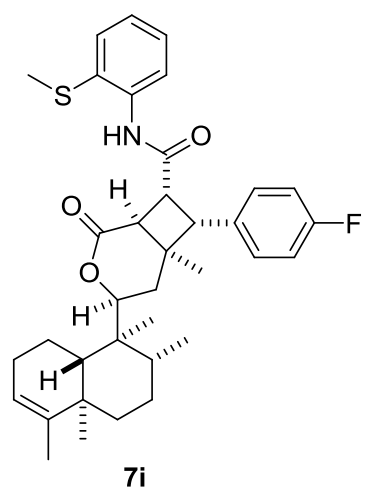

Compound 7i: Yield: $21 \mathrm{mg}, 69 \%$; Positive ESIMS $[\mathrm{M}+\mathrm{Na}]^{+} \mathrm{m} / z$ 612; ${ }^{1} \mathrm{H}$ NMR $\left(600 \mathrm{MHz}, \mathrm{CDCl}_{3}\right) \delta_{\mathrm{H}}: 8.60(1 \mathrm{H}, \mathrm{s}, \mathrm{NH}), 8.18(1 \mathrm{H}, \mathrm{d}, J=7.7$ Hz, H-3"), 7.40 (1H, d, J= 7.7 Hz, H-6"), 7.24 (1H, t, J= 7.4 Hz, H-5"), $7.14\left(2 \mathrm{H}, \mathrm{dd}, J=8.7\right.$ and $5.2 \mathrm{~Hz}, \mathrm{H}-2^{\prime}$ and $\left.\mathrm{H}-6^{\prime}\right), 7.04(1 \mathrm{H}, \mathrm{t}, J=7.4 \mathrm{~Hz}$, H-4"), 6.91 (2H, t, $J=8.6 \mathrm{~Hz}, \mathrm{H}-3^{\prime}$ and H-5'), $5.22(1 \mathrm{H}, \mathrm{s}, \mathrm{H}-3), 4.33$ $\left.(1 \mathrm{H}, \mathrm{d}, J=11.1 \mathrm{~Hz}, \mathrm{H}-11), 3.87(1 \mathrm{H}, \mathrm{dd}, J=10.4 \text { and } 7.8 \mathrm{~Hz}, \mathrm{H}-8)^{\prime}\right)$ $\left.3.68(1 \mathrm{H}, \mathrm{d}, J=10.4 \mathrm{~Hz}, \mathrm{H}-7)^{\prime}\right), 3.52(1 \mathrm{H}, \mathrm{d}, J=7.7 \mathrm{~Hz}, \mathrm{H}-14), 2.13(3 \mathrm{H}$, s, SMe), 2.07-1.96 (3H, overlapped, H-1a, H-2a, and H-12a), 1.87 (1H, br t, $J=13.9 \mathrm{~Hz}, \mathrm{H}-12 \mathrm{~b}$ ), 1.71-1.65 (2H, overlapped, H-1b and H-6a), 1.57 (3H, s, H-18), 1.53-1.42 (3H, overlapped, $\mathrm{H}_{2}-7$ and H-10), 1.29 (1H, m, H-8), 1.13 (1H, m, H-6b), 1.03 (3H, s, H3-19), 1.02 (3H, s, H3-20), 0.99 (3H, s, $\left.\mathrm{H}_{3}-16\right), 0.84\left(3 \mathrm{H}, \mathrm{d}, J=6.7 \mathrm{~Hz}, \mathrm{H}_{3}-17\right) ;{ }^{13} \mathrm{C} \mathrm{NMR}\left(151 \mathrm{MHz}, \mathrm{CDCl}_{3}\right) \delta_{\mathrm{C}} 174.6(\mathrm{~s}, \mathrm{C}-9$ '), $169.1(\mathrm{~s}$, C-15), 163.0 (s, C-4'), 161.3 (s, C-4'), 144.1 (s, C-4), 137.9 (s, C-1"), 132.9 (s, C-6"), 132.7 (s, C-1'), 130.9 (d, C-2' and C-6'), 130.8 (d, C-2' and C-6'), 128.8 (d, C-5"), 126.3 (s, C-2"), 124.8 (d, C-4"), 121.0 (d, C-3"), 120.7 (d, C-3), 115.5 (d, C-3' and C-5'), 115.4 (d, C-3' and C-5'), 85.7 (d, C-11), 52.9 (d, C-7'), 46.6 (d, C-10), 46.0 (d, C-8'), 43.2 (d, C-14), 42.6 (s, C-9), 39.8 (t, C-12), 39.6 (s, C-13), 38.6 (s, C-5), 36.6 (t, C-6), 36.6 (d, C-8), 27.8 (t, C-2), 26.6 (s, C-7), 22.1 (q, C-16), 20.2 (t, C-1), 19.8 (q, C-19), 18.8 (q, SMe), 18.3 (q, C-18), 16.7 (q, C-17), 14.2 (q, C-20).

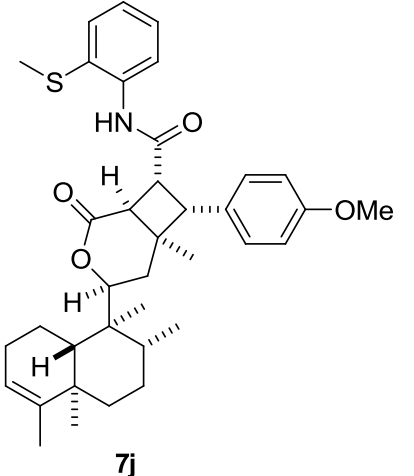

(3H, s, SMe), 2.08-1.87 (4H, overlapped, $\mathrm{H}_{2}-2$ and $\mathrm{H}_{2}-12$ ), 1.67-1.55 (3H, overlapped, H-1b, H-6a, and $\mathrm{H}-10), 1.61$ (3H, s, $\left.\mathrm{H}_{3}-18\right), 1.42-1.25$ (3H, overlapped, $\mathrm{H}_{2}-7$ and $\left.\mathrm{H}-8\right), 1.15$ (1H, s, H-6b), 1.25 (3H, s, H3-16), 1.06 (3H, s, H3-20), 1.00 (3H, s, H3-19), 0.78 (3H, d, J = 6.3 Hz, H3-17); ${ }^{13} \mathrm{C}$ NMR (151 MHz, Pyr) $\delta_{\mathrm{C}}: 175.1$ (s, C-9'), 170.7 (s, C-15), 159.7 (s, C-4'), 144.1 (s, C-4), 138.3 (s, C-1"), 131.8 (d, C-2' and C-6'), 131.1 (s, C-1'), 130.8 (s, C-2"), 130.8 (s, C-6"), 127.6 (d, C-5"), 126.1 (d, ${ }^{1} \mathrm{H}$ NMR $(600 \mathrm{MHz}, \mathrm{Pyr}) \delta_{\mathrm{H}} 9.92(1 \mathrm{H}, \mathrm{s}, \mathrm{NH}), 8.21(1 \mathrm{H}, \mathrm{d}, J=8.0 \mathrm{~Hz}$, H-3"), $7.60(2 \mathrm{H}, \mathrm{d}, J=8.4 \mathrm{~Hz}, \mathrm{H}-2 '$ and H-6'), $7.34(1 \mathrm{H}, \mathrm{d}, J=7.8 \mathrm{~Hz}$, H-6"), 7.22 (1H, t, $J=7.6 \mathrm{~Hz}, \mathrm{H}-5 "), 7.08$ (1H, t, $J=7.5 \mathrm{~Hz}, \mathrm{H}-4 ")$, $7.01\left(2 \mathrm{H}, \mathrm{d}, J=8.4 \mathrm{~Hz}, \mathrm{H}-3^{\prime}\right.$ and $\left.\mathrm{H}-5^{\prime}\right), 5.20(1 \mathrm{H}, \mathrm{s}, \mathrm{H}-3), 4.53-4.49$ (2H, overlapped, H-11 and H-8'), $4.10(1 \mathrm{H}, \mathrm{d}, J=7.3 \mathrm{~Hz}, \mathrm{H}-14), 4.00$ (1H, d, $J=10.7$ Hz, H-7'), 3.64 (3H, s, OMe), 2.29 (1H, m, H-1a), 2.10

Compound 7j: Yield: $21 \mathrm{mg}, 80 \%$; Positive ESIMS $[\mathrm{M}+\mathrm{Na}]^{+} m / z$ 624; 
C-4"), 124.8 (d, C-3"), 124.2 (d, C-3), 114.5 (d, C-3' and C-5'), 85.7 (d, C-11), 55.6 (q, OMe), 53.6 (d, C-7'), 47.0 (d, C-10), 46.8 (d, C-8'), 43.5 (d, C-14), 43.1 (s, C-9), 40.5 (t, C-12), 40.3 (s, C-13), 39.1 (s, C-5), 37.0 (t, C-6), 36.9 (d, C-8), 28.4 (s, C-7), 27.1 (t, C-2), 22.6 (q, C-16), 20.8 (t, C-1), 20.3 (q, C-19), 18.9 (q, C-18), 17.5 (q, SMe), 17.1 (q, C-17), 14.7 (q, C-20).

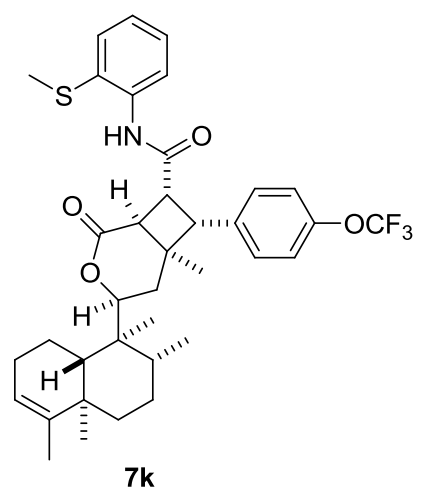

Compound 7k: Yield: $12 \mathrm{mg}, 70 \%$; Positive ESIMS $[\mathrm{M}+\mathrm{K}]^{+} \mathrm{m} / \mathrm{z}$ 694; ${ }^{1} \mathrm{H}$ NMR $\left(600 \mathrm{MHz}, \mathrm{CDCl}_{3}\right) \delta_{\mathrm{H}}: 8.60(1 \mathrm{H}, \mathrm{s}, \mathrm{NH}), 8.16(1 \mathrm{H}, \mathrm{d}, J$ $=7.7 \mathrm{~Hz}, \mathrm{H}-3 "), 7.39$ (1H, d, $J=7.7 \mathrm{~Hz}, \mathrm{H}-6 "), 7.24$ (1H, t, $J=7.4$ Hz, H-5"), 7.20 (2H, d, $J=8.6 \mathrm{~Hz}, \mathrm{H}-2^{\prime}$ and H-6'), $7.06(2 \mathrm{H}, \mathrm{t}, J=8.6$ Hz, H-3' and H-5'), 7.05 (1H, t, $J=7.4$ Hz, H-4"), 5.22 (1H, s, H-3), $4.33(1 \mathrm{H}, \mathrm{d}, J=11.1 \mathrm{~Hz}, \mathrm{H}-11), 3.88(1 \mathrm{H}, \mathrm{dd}, J=10.4$ and $7.8 \mathrm{~Hz}$, H-8'), $3.71\left(1 \mathrm{H}, \mathrm{d}, J=10.4 \mathrm{~Hz}, \mathrm{H}-7^{\prime}\right), 3.53(1 \mathrm{H}, \mathrm{d}, J=7.7 \mathrm{~Hz}, \mathrm{H}-14)$, $2.11(3 \mathrm{H}, \mathrm{s}, \mathrm{SMe}), 2.07-1.96$ (3H, overlapped, H-1a, H-2a, and H-12a), $1.88(1 \mathrm{H}$, br t, $J=13.9 \mathrm{~Hz}$, H-12b), 1.72-1.65 (2H, overlapped, H-1b and H-6a), 1.57 (3H, s, H-18), 1.53-1.42 (3H, overlapped, $\mathrm{H}_{2}-7$ and $\left.\mathrm{H}-10\right), 1.29$ (1H, m, H-8), 1.13 (1H, m, H-6b), 1.03 (3H, s, $\left.\mathrm{H}_{3}-19\right), 1.02$ (3H, s, $\left.\mathrm{H}_{3}-20\right)$, $1.00\left(3 \mathrm{H}, \mathrm{s}, \mathrm{H}_{3}-16\right), 0.85\left(3 \mathrm{H}, \mathrm{d}, J=6.7 \mathrm{~Hz}, \mathrm{H}_{3}-17\right) ;{ }^{13} \mathrm{C} \mathrm{NMR}\left(151 \mathrm{MHz}, \mathrm{CDCl}_{3}\right) \delta_{\mathrm{C}}: 174.5$ (s, C-9'), 169.0 (s, C-15), 148.5 (s, C-4'), 144.1 (s, C-4), 137.7 (s, C-1"), 135.6 (s, C-1'), 132.8 (s, C-6"), 130.7

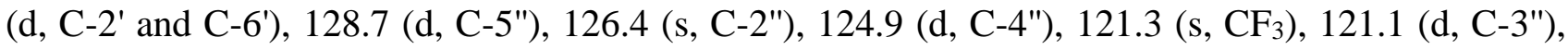
120.9 (d, C-3' and C-5'), 120.7 (d, C-3), 119.6 (s, CF 3 ), 85.7 (d, C-11), 52.9 (d, C-7'), 46.6 (d, C-10), 45.8 (d, C-8'), 43.2 (d, C-14), 42.6 (s, C-9), 39.8 (t, C-12), 39.6 (s, C-13), 38.6 (s, C-5), 36.6 (t, C-6), 36.6 (d, C-8), 27.8 (t, C-2), 26.6 (s, C-7), 22.1 (q, C-16), 20.2 (t, C-1), 19.8 (q, C-19), 18.6 (q, SMe), 18.3 (q, C-18), 16.7 (q, C-17), 14.2(q, C-20). 
Scheme S6. Synthesis of scopariusicides analogs 10a-10w

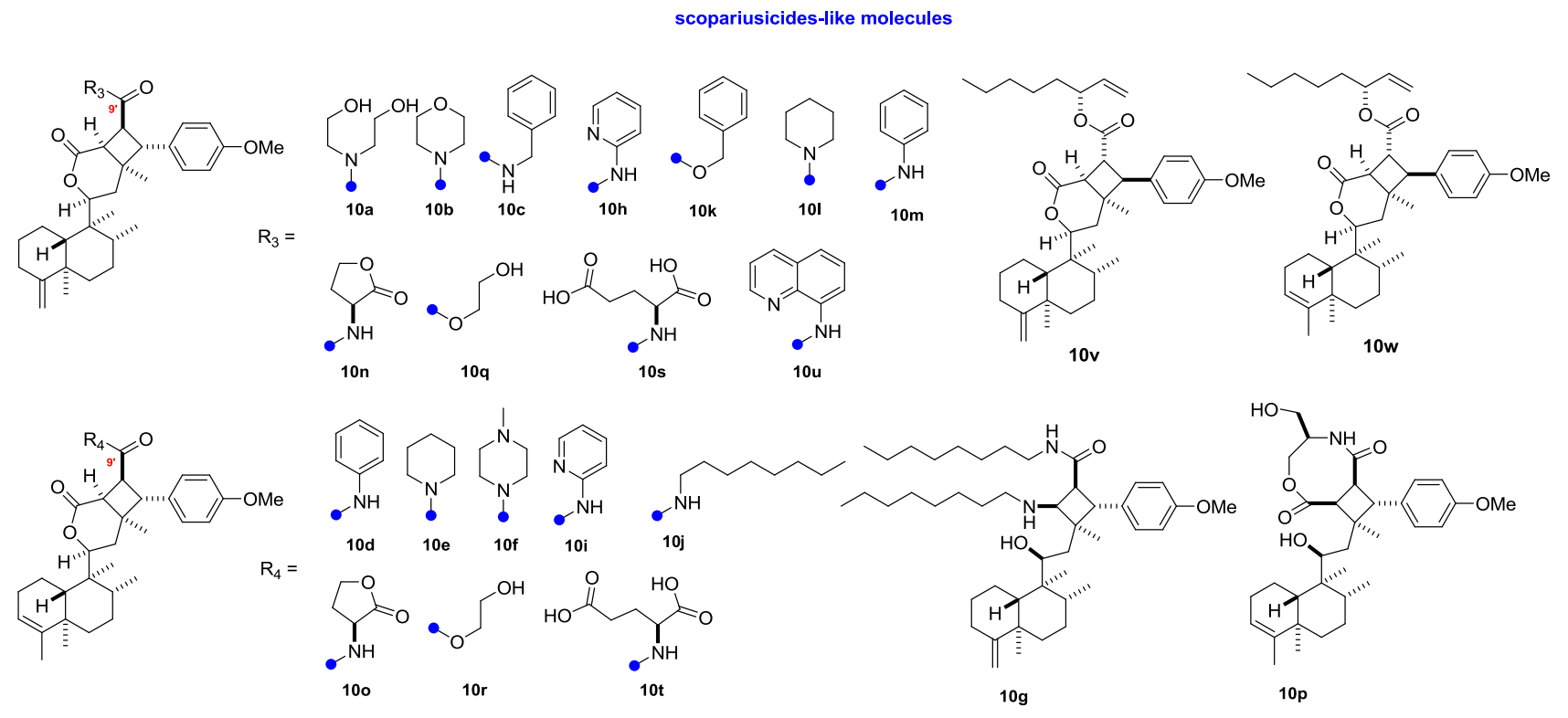

General procedure for the synthesis of compound 10a, 10d-10j, 10l-10p, 10s-10u: A mixture of 4 (1.0 equiv, each one), amine (1.5 2 equiv), HATU (1.2 equiv) and DIPEA (2.0 equiv) in dry DCM $(2 \sim 5 \mathrm{~mL})$ was stirred for 8 to $10 \mathrm{~h}$ at room temperature. Then the reaction mixture was diluted with DCM $(15 \mathrm{~mL})$, washed with water $(10 \mathrm{~mL} * 2)$ and brine $(10 \mathrm{~mL})$, dried over anhydrous $\mathrm{Na}_{2} \mathrm{SO}_{4}$, filtered and concentrated in vacuo. The crude product was purified by prep-HPLC to afford 10a, 10d-10j, 10l-10p, 10s-10u in 9-80\% yield, respectively.

Compound 10a: Yield: $28 \mathrm{mg}, 80 \%$; Negative ESIMS $\left[\mathrm{M}+\mathrm{H}_{2} \mathrm{O}-\mathrm{H}\right]^{-} \mathrm{m} / z, 584 ;{ }^{1} \mathrm{H}$ NMR (Pyridine- $d_{5}$, $600 \mathrm{MHz}) \delta_{\mathrm{H}}: 7.31\left(2 \mathrm{H}, \mathrm{d}, J=8.4 \mathrm{~Hz}, \mathrm{H}-2^{\prime}\right.$ and H-6'), $7.03\left(2 \mathrm{H}, \mathrm{d}, J=8.5 \mathrm{~Hz}, \mathrm{H}-3^{\prime}\right.$ and H-5'), 4.59 $(2 \mathrm{H}, \mathrm{d}, J=5.1 \mathrm{~Hz}, \mathrm{H}-18), 4.35$ (1H, d, J=9.8 Hz, H-7'), 4.28 (1H, d, J=10.0 Hz, H-8'), 4.26-4.22 (1H, m, H-11), 3.95-3.90 (1H, m, H-2"'a), 3.84-3.73 (3H, overlapped, H-1" and H-2"a), 3.67 (3H, s, OMe), 3.65-3.58 (3H, overlapped, H-1"' and H-2"'b), 3.56-3.51 (1H, m, H-2"b), 3.30 (1H, d, J=10.0 Hz, H-14), 2.30-2.16 (2H, overlapped, H-1a and H-3a), 2.13 (2H, m, H-12), 2.03 (1H, d, J=12.8 Hz, H-3b), 1.64 (1H, d, J=11.1 Hz, H-2a), 1.58-1.52 (4H, overlapped, H-1b, H-6a and H-8), 1.50-1.41 (1H, m, H-7a), 1.40-1.34 (2H, overlapped, H-7b and H-10), 1.34-1.21 (2H, overlapped, H-2b and H-6b), 1.12 (3H, s, H-16), 0.99 (3H, s, H-20), 0.98 (3H, s, H-19), 0.78 (3H, d, J=6.6 Hz, H-17); ${ }^{13} \mathrm{C}$ NMR (151 MHz, Pyr) $\delta_{\mathrm{C}}: 172.2$ (s, C-15), 170.4 (s, C-9'), 159.8 (s, C-4), 158.8 (s, C-4'), 131.7 (s, C-1'), 129.0 (d, C-2' and C-6'), 114.1 (d, C-3' and C-5'), 103.3 (t, C-18), 84.6 (d, C-11), 66.5 (t, C-2"), 66.2 (t, C-2'"), $55.0(\mathrm{q}$, OMe), 51.0 (d, C-7'), 48.7 (d, C-10), 45.9 (t, C-1"'), 44.3 (d, C-14), 42.8 (s, C-9), 42.8 (t, C-1"), 40.4 (s, C-5), 40.1 (t, C-12), 39.8 (d, C-8'), 39.2(s, C-13), 37.1 (t, C-6), 36.8 (d, 
C-8), 33.2 (t, C-3), 28.2 (t, C-2), 27.9 (t, C-7), 23.7 (t, C-1), 22.4 (q, C-16), 20.6 (q, C-19), 16.7 (q, C-17), 13.9 (q, C-20).

Compound 10b: Yield: 12mg, 40\%; Positive ESIMS $[\mathrm{M}+\mathrm{Na}]^{+} \mathrm{m} / z$ 549; ${ }^{1} \mathrm{H}$ NMR $(600 \mathrm{MHz}$, CDCl3) $\delta_{\mathrm{H}}: 7.39-7.26\left(3 \mathrm{H}\right.$, overlapped), $7.06\left(2 \mathrm{H}, \mathrm{t}, J=8.6 \mathrm{~Hz}, \mathrm{H}-2^{\prime}\right.$ and H-6'), $6.87(2 \mathrm{H}, \mathrm{t}, J=8.6$ Hz, H-3' and H-5'), $4.67(1 \mathrm{H}, \mathrm{q}, J=14.0), 4.53(1 \mathrm{H}, \mathrm{s}, \mathrm{H}-18 \mathrm{a}), 4.51(1 \mathrm{H}, \mathrm{s}, \mathrm{H}-18 \mathrm{~b}), 3.93(1 \mathrm{H}, \mathrm{d}, J=$ $9.2 \mathrm{~Hz}, \mathrm{H}-11), 3.79$ (3H, s, OMe), 3.67-3.62 (2H, overlapped), $3.15(1 \mathrm{H}, \mathrm{d}, J=7.8 \mathrm{~Hz}), 3.05$ (1H, d, $J=6.7 \mathrm{~Hz}), 2.32-1.91\left(4 \mathrm{H}\right.$, overlapped), 1.65-1.17 (10H, overlapped), 1.07 (3H, s, $\left.\mathrm{H}_{3}-19\right), 1.01(3 \mathrm{H}$, s, $\left.\mathrm{H}_{3}-20\right), 0.93\left(3 \mathrm{H}, \mathrm{s}, \mathrm{H}_{3}-16\right), 0.76\left(3 \mathrm{H}, \mathrm{d}, J=6.7 \mathrm{~Hz}, \mathrm{H}_{3}-17\right) ;{ }^{13} \mathrm{C} \mathrm{NMR}\left(151 \mathrm{MHz}, \mathrm{CDCl}_{3}\right) \delta_{\mathrm{C}}$ 180.0, 178.2, 160.8, 158.9, 135.7, 129.0, 128.9, 128.7, 128.6, 128.3, 114.0, 102.7, 74.1, 55.4, 53.6, 48.9, 48.2, 44.3, 43.8, 42.8, 40.7, 40.7, 39.1, 37.4, 37.2, 33.3, 28.7, 28.6, 24.0, 21.4, 20.9, 17.2, 13.9. Compound 10c: Yield: $41 \mathrm{mg}, 72 \%$; Positive ESIMS $[\mathrm{M}+\mathrm{Na}]^{+} \mathrm{m} / z$ 592; ${ }^{1} \mathrm{H} \mathrm{NMR}(600 \mathrm{MHz}$, $\left.\mathrm{CDCl}_{3}\right) \delta_{\mathrm{H}}: 6.97\left(2 \mathrm{H}, \mathrm{t}, J=8.6 \mathrm{~Hz}, \mathrm{H}-2^{\prime}\right.$ and H-6'), $6.82\left(2 \mathrm{H}, \mathrm{t}, J=8.6 \mathrm{~Hz}, \mathrm{H}-3^{\prime}\right.$ and H-5'), $4.52(1 \mathrm{H}$, s, H-18a), 4.49 (1H, s, H-18b), 4.03 (1H, d, J = 11.5 Hz), 3.80-3.44 (10H, overlapped), 3.76 (3H, s, OMe), 2.85 (1H, d, $J=10.0 \mathrm{~Hz}), 2.30-1.84$ (6H, overlapped), 1.60-1.17 (8H, overlapped), 1.04 (3H, s, $\left.\mathrm{H}_{3}-19\right), 0.97$ (3H, s, $\left.\mathrm{H}_{3}-20\right), 0.91$ (3H, s, $\left.\mathrm{H}_{3}-16\right), 0.81\left(3 \mathrm{H}, \mathrm{d}, J=6.7 \mathrm{~Hz}, \mathrm{H}_{3}-17\right) ;{ }^{13} \mathrm{C}$ NMR $(151$ $\left.\mathrm{MHz}, \mathrm{CDCl}_{3}\right) \delta_{\mathrm{C}}: 171.9,169.5,160.4,158.5,130.7,128.3,113.9,103.0,85.0,66.6,66.1,55.4,50.4$, $48.8,45.8,44.4,42.8,42.6,40.4,39.9,39.8,39.1$, 37.0, 37.0, 33.2, 28.5, 27.9, 23.6, 22.8, 20.7, 16.8, 13.9.

Compound 10d: Yield: $5 \mathrm{mg}, 28 \%$; Positive ESIMS $[\mathrm{M}+\mathrm{Na}]^{+} \mathrm{m} / z, 578 ;{ }^{1} \mathrm{H}$ NMR (Pyridine- $d 5,600$ MHz) $\delta_{\mathrm{H}}: 11.17(1 \mathrm{H}, \mathrm{s}, \mathrm{NH}), 8.03$ (2H, d, J=8.0 Hz, H-2" and H-6"), 7.27 (4H, overlapped, H-2', H-6', H-3" and H-5" ), 7.00 (3H, overlapped, H-3', H-5' and H-4"), 5.24 (1H, overlapped, H-3), 4.34 (1H, d, J=11.3 Hz, H-11), 4.29 (1H, dd, J=11.2, 7.3 Hz, H-8'), 4.18 (1H, d, J=7.2 Hz, H-7'), 3.69 (3H, s, OMe), 3.42 (1H, d, J=11.2 Hz, H-14), 2.82 (1H, t, J=12.5 Hz, H-12a), 2.67 (1H, dd, J=13.6, $6.8 \mathrm{~Hz}, \mathrm{H}-1 \mathrm{a}), 2.45$ (1H, m, H-2a), 2.07 (2H, m, H-2b and H-12b), 1.70 (1H, d, J=12.3 Hz, H-10), 1.64-1.54 (5H, overlapped, H-1b, H-8 and H-18), 1.48-1.35 (1H, m, H-7a), 1.28 (2H, overlapped, H-6a and H-7b), 1.18-1.10 (1H, m, H-6b), 1.02 (3H, s, H-20), 0.96 (3H, s, H-19), 0.95 (3H, s, H-16), $0.71(3 \mathrm{H}, \mathrm{d}, J=6.2 \mathrm{~Hz}, \mathrm{H}-17) ;{ }^{13} \mathrm{C}$ NMR $\left(151 \mathrm{MHz}\right.$, Pyridiene- $\left.d_{5}\right) \delta_{\mathrm{C}}: 172.2$ (s,C-15), 170.9 (s, C-9'), 158.8 (s, C-4'), 143.3 (s, C-4) , 140.5 (d, C-1"), 132.0 (s, C-1'), 129.6 (d, C-2' and C-6'), 129.0 (d, C-3" and C-5"), 123.2 (d, C-4"), 121.5 (d, C-3), 120.2 (d, C-2" and C-6"), 114.1 (d, C-3' and C-5'), 84.7 (d, C-11), 55.1 (q, OMe), 50.6 (d, C-7'), 46.6 (d, C-10), 44.8 (d, C-8'), 43.3 (d, C-14), 42.5 (s, 
C-9), 38.7 (s, C-13), 38.5 (s, C-5), 37.7 (t, C-12), 36.6 (t, C-6), 36.5 (d, C-8), 27.8 (t, C-7), 26.7 (t, C-2), 22.7 (q, C-16), 20.1 (t, C-1), 19.7 (q, C-19), 18.3 (q, C-18), 16.4 (q, C-17), 14.2 (q, C-20).

Compound 10e: Yield: $2 \mathrm{mg}, 26 \%$; Positive ESIMS $[\mathrm{M}+\mathrm{Na}]^{+} m / z$ 570; ${ }^{1} \mathrm{H}$ NMR (Pyridine- $d_{5}, 600$ MHz) $\delta_{\mathrm{H}}: 7.34\left(2 \mathrm{H}, \mathrm{d}, J=8.3 \mathrm{~Hz}, \mathrm{H}-2^{\prime}\right.$ and H-6'), $7.04\left(2 \mathrm{H}, \mathrm{d}, J=8.6 \mathrm{~Hz}, \mathrm{H}-3^{\prime}\right.$ and H-5'), $5.14(1 \mathrm{H}$, overlapped, H-3), 4.32 (1H, d, J=9.6 Hz, H-7'), 4.31-4.24 (2H, overlapped, H-11 and H-8'), 3.87-3.78 (1H, m, H-6"a), 3.67 (3H, s, OMe), 3.62-3.52 (2H, overlapped, H-6"b and H-2"a), 3.47 (1H, m, H-2"b), 3.30 (1H, d, J=9.9 Hz, H-14), 2.38 (1H, m, H-1a), 2.22 (1H, dd, J=13.6, $11.1 \mathrm{~Hz}$, H-12a), 2.14 (1H, d, J=13.6 Hz, H-12b), 2.02 (1H, m, H-2a), 1.86 (1H, m, H-2b), 1.67 (1H, m, H-5"a), 1.56 (6H, overlapped, H-1b, H-8, H-10, H-5"b and H-18), 1.46-1.36 (2H, overlapped, H-7a and H-2"a), 1.35-1.25 (3H, overlapped, H-7b, H-6a and H-2"b), 1.15-1.08 (4H,overlapped, H-6b and H-16), 0.99 (3H, s, H-20), 0.95 (3H, s, H-19), 0.78 (3H, d, J=6.2 Hz, H-17); ${ }^{13} \mathrm{C}$ NMR (151 MHz, Pyridine- $d_{5}$ ) $\delta_{\mathrm{C}}: 172.1$ (s,C-15), 169.6 (s, C-9'), 158.8 (s, C-4'), 143.3 (s, C-4), 132.0 (s, C-1'), 129.0 (d, C-2' and C-6'), 121.3 (d, C-3), 114.1 (d, C-3' and C-5'), 84.6 (d, C-11), 55.0 (q, OMe), 51.4 (d, C-7'), 46.5 (d, C-10), 46.3 (t, C-2"), 44.2 (d, C-14), 43.1 (t, C-6"), 42.2 (s, C-9), 40.2 (d, C-8'), 40.1 (t, C-12), 39.1 (s, C-13), 38.5 (s, C-5), 36.5 (t, C-6), 36.4 (d, C-8), 27.8 (t, C-7), 26.4 (t, C-2), 25.9 (t, C-5"), 25.3 (t, C-3"), 24.7 (t, C-4"), 22.5 (q, C-16), 20.0 (t, C-1), 19.7 (q, C-19), 18.2 (q, C-18), 16.5 (q, C-17), 14.1 (q, C-20).

Compound 10f: Yield: $4 \mathrm{mg}, 23 \%$; Positive ESIMS $[\mathrm{M}+\mathrm{H}]^{+} \mathrm{m} / z$ 563; ${ }^{1} \mathrm{H}$ NMR $(600 \mathrm{MHz}$, Pyridine- $\left.d_{5}\right) \delta_{\mathrm{H}}: 7.34\left(2 \mathrm{H}, \mathrm{d}, J=8.4 \mathrm{~Hz}, \mathrm{H}-2^{\prime}\right.$ and H-6'), 7.05 (2H, d, $J=8.4 \mathrm{~Hz}, \mathrm{H}-3^{\prime}$ and H-5'), 5.14 (1H, br s, H-3), 4.33 (2H, overlapped, H-7' and H-8'), 4.28 (1H, d, J = 9.2 Hz, H-11), 4.07 (1H, br s, H-3"a), 3.89 (3H, overlapped, H-5" and H-3"b), 3.67 (3H, s, OMe), 3.35 (1H, d, J=8.2 Hz, H-14), 3.05 (1H, m, H-6"a), 2.82 (1H, m, H-6"b), 2.76 (1H, m, H-2"a), 2.64 (1H, br s, H-2"a), 2.43 (3H, s, NMe), 2.33 (1H, t, J = 8.9 Hz, H-1a), 2.18 (2H, m, H-12), 2.00 (1H, m, H-2a), 1.84 (1H, m, H-2b), 1.61-1.52 (5H, overlapped, H-1b, H-8, H-10 and H-18), 1.43-1.35 (1H, m, H-7a), 1.35-1.28 (1H, m, H-6b), 1.27-1.20 (1H, m, H-7b), 1.16 -1.05 (4H, overlapped, H-16 and H-6b), 0.98 (3H, s, H-20), 0.95 (3H, s, H-19), 0.77 (3H, d, $J=6.3 \mathrm{~Hz}, \mathrm{H}-17) ;{ }^{13} \mathrm{C}$ NMR (151 MHz, Pyridine- $\left.d_{5}\right) \delta_{\mathrm{C}}: 172.5$ (s, C-15), 170.1 (s, C-9'), 158.9 (s, C-4'), 143.3 (s, C-4), 131.6 (s, C-1'), 129.0 (d, C-2' and C-6'), 121.2 (d, C-3), 114.1 (d, C-3' and C-5'), 84.8 (d, C-11), 55.0 (q, OMe), 53.6 (t, C-2"), 53.5 (t, C-6"), 51.2 (d, C-7'), 46.4 (d, C-10), 44.5 (q, NMe), 44.2 (d, C-14), 44.1 (t, C-5"), 42.2 (s, C-9), 41.0 (t, C-3"), 40.1 (t, C-12), 39.8 (d, C-8'), 39.2 (s, C-13), 38.4 (s, C-5), 36.4 (d, C-6), 36.4 (d, C-8), 27.7 (t, C-7), 26.4 (t, C-2), 22.4 (q, C-16), 20.0 (t, C-1), 19.6 (q, C-19), 18.2 (q, C-18), 16.4 (q, C-17), 14.0 
(q, C-20).

Compound 10g: Yield: $5 \mathrm{mg}, 16 \%$; Positive ESIMS $[\mathrm{M}+\mathrm{H}]^{+} m / z$ 722; ${ }^{1} \mathrm{H}$ NMR (Pyridine- $d_{5}, 600$ MHz) $\delta_{\mathrm{H}}: 8.00(1 \mathrm{H}, \mathrm{t}, J=5.8 \mathrm{~Hz}, \mathrm{NH}), 7.32\left(2 \mathrm{H}, \mathrm{d}, J=8.1 \mathrm{~Hz}, \mathrm{H}-2^{\prime}\right.$ and H-6'), $6.91(2 \mathrm{H}, \mathrm{d}, J=8.3 \mathrm{~Hz}$, H-3' and H-5'), $5.36(1 \mathrm{H}, \mathrm{d}, J=5.9 \mathrm{~Hz}, \mathrm{NH}), 4.66(2 \mathrm{H}, \mathrm{s}, \mathrm{H}-18), 4.51\left(1 \mathrm{H}, \mathrm{d}, J=11.5 \mathrm{~Hz}, \mathrm{H}-7^{\prime}\right), 4.05$ (1H, dd, J=11.6, 9.1 Hz, H-8'), 3.91 (1H, dd, J=11.4, 5.5 Hz, H-11), 3.62 (3H, s, OMe), 3.58-3.51 (2H, m, H-1"a and H-1'"a), 3.47-3.39 (3H, overlapped, H-1"b, H-1"'b and H-14), 2.72 (1H, d, J=12.1 Hz, H-1a), 2.53 (1H, dd, J=14.1, 11.5 Hz, H-12a), 2.37 (1H, dt, J=13.5, 6.9 Hz, H-3a), 2.20 (1H, d, $J=13.0 \mathrm{~Hz}, \mathrm{H}-3 \mathrm{~b}), 2.04-1.95$ (2H, overlapped, H-2b and H-12b), 1.76-1.44 (8H, overlapped, H-1b, H-2b, H-6, H-8, H-10, H-4" and H-4"'), 1.42-1.08 (22H, overlapped, H-16, H-19, H-3", H-3"', H-5", H-5"', H-6", H-6"', H-7" and H-7"'), 1.07 (3H, s, H-20), 0.86-0.78 (6H, m, H-8" and H-8"'), 0.76 (3H, d, $J=5.4 \mathrm{~Hz}, \mathrm{H}-17) ;{ }^{13} \mathrm{C}$ NMR (151 MHz, Pyridine- $\left.d_{5}\right) \delta_{\mathrm{C}}: 172.8$ (s, C-9' and C-15), 160.7 (s, C-4), 158.7 (s, C-4'), 132.0 (s, C-1'), 129.4 (d, C-2' and C-6'), 114.0 (d, C-3' and C-5'), 102.9 (t, C-18), 73.2 (d, C-11), 54.9 (q, OMe), 54.2 (d, C-14), 49.6 (d, C-7'), 48.9 (d, C-10), 44.5 (s, C-9), 43.2(s, C-5), 42.9 (d, C-8'), 40.7 (s, C-13), 39.9 (t, C-1"), 39.8 (t, C-1'"), 37.5 (t, C-6), 37.4 (d, C-8), 33.6 (t, C-3), 32.0(t, C-6"), 31.9 (t, C-6"'), 30.0 (t, C-2"), 29.9 (t, C-2"'), 29.5 (t, C-5"), 29.5(t, C-5"'), 29.1 (t, C-2), 28.7 (t, C-7), 27.4 (t, C-3"), 27.2 (t, C-3"'), 23.8 (t, C-1), 22.8 (t, C-7"), 22.7(t, C-7"'), 21.6 (q, C-16), 20.8 (q, C-19), 17.1 (q, C-17), 14.4 (q, C-20), 14.1 (q, C-8"), 14.1 (q, C-8"').

Compound 10h: Yield: $3 \mathrm{mg}, 23 \%$; Positive ESIMS $[\mathrm{M}+\mathrm{H}]^{+} \mathrm{m} / \mathrm{z} 557 ;{ }^{1} \mathrm{H}$ NMR $(600 \mathrm{MHz}$, Pyridine- $\left.d_{5}\right) \delta_{\mathrm{H}}: 11.65(1 \mathrm{H}, \mathrm{s}, \mathrm{NH}), 8.70$ (1H, overlapped, H-3"), 8.29 (1H, d, J=3.9 Hz, H-6"), 7.57 (1H, overlapped, H-4"), 7.29 (2H, d, $J=8.4 \mathrm{~Hz}, \mathrm{H}-2^{\prime}$ and $\left.\mathrm{H}-6^{\prime}\right), 7.01\left(2 \mathrm{H}, \mathrm{d}, J=8.5 \mathrm{~Hz}, \mathrm{H}-3^{\prime}\right.$ and H-5'), 6.85 (1H, m, H-5"), 4.62 (2H, s, H-18), 4.52 (1H, t, J=9.3 Hz, H-8'), 4.30 (1H, d, J = $11.3 \mathrm{~Hz}$, H-11), 4.25 (1H, d, J = 8.1 Hz, H-7'), 3.68 (3H, s, OMe), 3.50 (1H, d, J = 10.9 Hz, H-14), 2.59 (1H, t, $J=12.5 \mathrm{~Hz}, \mathrm{H}-12 \mathrm{a}), 2.51(1 \mathrm{H}, \mathrm{d}, J=12.8 \mathrm{~Hz}, \mathrm{H}-1 \mathrm{a}), 2.24(1 \mathrm{H}, \mathrm{dd}, J=13.3,4.8 \mathrm{~Hz}, \mathrm{H}-3 \mathrm{a}), 2.08$ (2H, overlapped, H-3b and H-12b), 1.85 (1H, m, H-2a), 1.65-1.41 (5H, ovrtlapped, H-1b, H-2b, H-6a, H-7a and H-8), 1.41-1.25 (2H, overlapped, H-6b and H-7b), 1.02 (3H, s, H-16), 0.99 (6H, s, H-19 and H-20), 0.73 (3H, d, $J=6.5 \mathrm{~Hz}, \mathrm{H}-17) ;{ }^{13} \mathrm{C}$ NMR (151 MHz, Pyridine- $\left.d_{5}\right) \delta_{\mathrm{C}}: 172.0$ (s, C-9'), 171.3 (s, C-15), 160.0 (s, C-4), 158.9 (s, C-4'), 153.3 (s, C-2"), 148.2 (d, C-6"), 137.8 (d, C-4"), 131.7 (s, C-1'), 129.3 (d, C-2' and C-6'), 119.3 (d, C-5"), 114.6 (d, C-3"), 114.1 (d, C-3' and C-5'), 103.2 (t, C-18), 84.5 (d, C-11), 55.0 (q, OMe), 50.3(d, C-7'), 48.8 (d, C-10), 44.2 (d, C-8'), 43.6 (d, C-14), 43.0 (s, C-9), 40.4 (s, C-5), 38.8 (s, C-13), 38.4 (t, C-12), 37.1 (t, C-6), 36.8 (d, C-8), 
33.3 (t, C-3), 28.4 (t, C-2), 27.8 (t, C-7), 23.7 (t, C-1), 22.7 (q, C-16), 20.5 (q, C-19), 16.6 (q, C-17), 13.9 (q, C-20).

Compound 10i: Yield: $8 \mathrm{mg}, 14 \%$; Positive ESIMS $[\mathrm{M}+\mathrm{H}]^{+} \mathrm{m} / \mathrm{z}$ 557; ${ }^{1} \mathrm{H}$ NMR $(600 \mathrm{MHz}$, Pyridine- $\left.d_{5}\right) \delta_{\mathrm{H}}: 11.67(1 \mathrm{H}, \mathrm{s}, \mathrm{H}-\mathrm{NH}), 8.72(1 \mathrm{H}$, overlapped, C-3"), 8.29 (1H, d, $J=3.8 \mathrm{~Hz}, \mathrm{H}-6 ")$, 7.55 (1H, overlapped, H-4"), 7.33 (2H, d, $J=8.4 \mathrm{~Hz}, \mathrm{H}-2^{\prime}$ and H-6'), 7.02 (2H, d, $J=8.5 \mathrm{~Hz}, \mathrm{H}-3^{\prime}$ and H-5'), 6.85 (1H, t, H-5"), 5.22 (1H, s, H-3), 4.55 (1H, t, $J=9.7 \mathrm{~Hz}, \mathrm{H}-8$ '), $4.34(1 \mathrm{H}, \mathrm{d}, J=11.3$ Hz, H-11), 4.27 (1H, d, J = 8.0 Hz, H-7'), 3.68 (3H, s, OMe), $3.51(1 \mathrm{H}, \mathrm{d}, J=10.9 \mathrm{~Hz}, \mathrm{H}-14), 2.66$ $(1 \mathrm{H}, \mathrm{t}, J=12.6 \mathrm{~Hz}, \mathrm{H}-12 \mathrm{a}), 2.59$ (1H, m, H-1a), $2.33(1 \mathrm{H}, \mathrm{m}, \mathrm{H}-2 \mathrm{a}), 2.12(1 \mathrm{H}, \mathrm{d}, J=13.6 \mathrm{~Hz}$, H-12b), 2.06 (1H, m, H-2b), 1.67 (1H, d, J = 12.2 Hz, H-10), 1.58 (5H, overlapped, H-1b, H-8 and H-18), 1.38 (1H, m, H-7a), 1.34 -1.25 (2H, overlapped, H-6a and H-7b), 1.13 (1H, m, H-6b), 1.04 (3H, s, H-16), 0.99 (3H, s, H-20), 0.95 (3H, s, H-19), 0.72 (3H, d, J = 6.1 Hz, H-17); ${ }^{13} \mathrm{C}$ NMR (151 MHz, Pyridine-d5) $\delta_{\mathrm{C}}: 172.1$ (s, C-9'), 171.4 (s, C-15), 158.9 (s, C-4'), 153.3 (s, C-2"), 148.2(d, C-6"), 143.3 (s,C-4), 137.9 (d, C-4"), 131.8 (s, C-1'), 129.4 (d, C-2' and C-6'), 121.4 (d, C-3), 119.3 (d, C-5"), 114.6 (d, C-3"), 114.2 (d, C-3' and C-5'), 84.7 (d, C-11), 55.1 (q, OMe), 50.5 (d, C-7'), 46.5 (d, C-10), 44.2 (d, C-8'), 43.6 (d, C-14), 42.4 (s, C-9), 38.8 (s, C-13), 38.5 (t, C-12), 38.4 (s, C-5), 36.5 (t, C-6), 36.4 (d, C-8), 27.8 (t, C-7), 26.6 (t, C-2), 22.7 (q, C-16), 20.1 (t, C-1), 19.6 (q, C-19), 18.3 (q, C-18), 16.4(q, C-17), 14.2 (q, C-20).

Compound 10j: Yield: $12 \mathrm{mg}, 14 \%$; Positive ESIMS $[\mathrm{M}+\mathrm{Na}]^{+} \mathrm{m} / z, 614 ;{ }^{1} \mathrm{H}$ NMR (Pyridine- $d_{5}, 600$ MHz) $\delta_{\mathrm{H}}: 8.67(1 \mathrm{H}, \mathrm{t}, J=5.8 \mathrm{~Hz}, \mathrm{NH}), 7.31\left(2 \mathrm{H}, \mathrm{d}, J=8.5 \mathrm{~Hz}, \mathrm{H}-2^{\prime}\right.$ and H-6'), $7.01(2 \mathrm{H}, \mathrm{d}, J=8.5 \mathrm{~Hz}$, H-3' and H-5'), 5.24 (1H, s, H-3), 4.32 (1H, d, J=11.3 Hz, H-11), 4.15-4.09 (2H, moverlapped, H-7' and $\left.\mathrm{H}^{-} 8^{\prime}\right), 3.68$ (3H, s, OMe), 3.54-3.45 (1H, m, H-1"a), 3.42-3.32 (2H, overlapped, H-1"b and H-14), 2.90 (1H, d, J=12.4 Hz, H-12a), 2.71 (1H, dd, J=13.6, 6.8 Hz, H-1a), 2.54-2.43 (1H, m, H-2a), 2.10 (1H, d, J=18.5 Hz, H-2b), 2.00 (1H, d, J=13.4 Hz, H-12b), 1.71 (1H, d, J=12.1 Hz, H-10), 1.66-1.50 (6H, overlapped, H-1b, H-8, H-18 and H-2"), 1.39 (1H, dd, J=14.1, 10.9 Hz, H-7a), 1.35-1.27 (2H, overlapped, H-6a and H-7b), 1.28-1.06 (11H, overlapped, H-6b, H-3", H-4", H-5", H-6" and H-7"), 1.03 (3H, s, H-20), 0.97 (3H, s, H-19), 0.93 (3H, s, H-16), 0.78 (3H, t, J=7.2 Hz, H-8"), 0.72 (3H, d, J=6.3 Hz, H-17); ${ }^{13} \mathrm{C}$ NMR (151 MHz, Pyridine-d5) $\delta_{\mathrm{C}}: 172.2$ (s, C-15), 171.8 (s, C-9'), 158.8 (s, C-4'), 143.3 (s, C-4), 132.5 (s, C-1'), 129.7 (d, C-2' and C-6'), 121.6 (d, C-3), 114.1 (d, C-3' and C-5'), 84.6 (d, C-11), 55.1 (q, OMe), 50.6 (d, C-7'), 46.6 (d, C-10), 44.3 (d, C-8'), 43.0 (d, C-14), 42.5 (s, C-9), 40.1 (t, C-1"), 38.7 (s, C-5), 38.5 (s, C-13), 37.3 (t, C-12), 36.6 (t, C-6), 36.5 (d, C-8), 31.8 (t, C-6"), 29.9 (t, C-2"), 29.4 (t, C-4"), 29.3 (t, C-5"), 27.9 (t, C-7), 27.2 (t, C-3"), 26.8 
(t, C-2), 22.8 (q, C-16), 22.7 (t, C-7"), 20.0 (t, C-1), 19.7 (q, C-19), 18.3 (q, C-18), 16.5 (q, C-17), 14.2 (q, C-20), 14.1 (q, C-8").

Compound 10l: Yield: $10 \mathrm{mg}, 23 \%$; Positive ESIMS $[\mathrm{M}+\mathrm{Na}]^{+} \mathrm{m} / z$ 570; ${ }^{1} \mathrm{H}$ NMR (Pyridine- $d_{5}, 600$ MHz) $\delta_{\mathrm{H}}: 7.30\left(2 \mathrm{H}, \mathrm{d}, J=8.3 \mathrm{~Hz}, \mathrm{H}-2^{\prime}\right.$ and H-6'), $7.02(2 \mathrm{H}, \mathrm{d}, J=8.6 \mathrm{~Hz}, \mathrm{H}-3$ ' and H-5'), 4.59 (2H, d, $J=6.0 \mathrm{~Hz}, \mathrm{H}-18), 4.32$ (1H, d, J=9.6 Hz, H-7'), 4.28-4.22 (2H, overlapped, H-8' and H-11), 3.85-3.77 (1H, m, H-6"a), 3.66 (3H, s, OMe), 3.62-3.52 (2H, overlapped, H-6"b and H-2"a), 3.50-3.42 (1H, m, H-2"b), 3.28 (1H, d, J=10.0 Hz, H-14), 2.30 (1H, d, J=13.6 Hz, H-1a), 2.24-2.07 (3H, overlapped, H-3a and H-12), 2.03 (1H, d, J=13.1 Hz, H-3b), 1.71-1.62 (2H, overlapped, H-2a and H-5"a), 1.58-1.51 (3H, overlapped, H-1b, H-5"b and H-7a), 1.50-1.38 (2H, overlapped, H-7b and H-3"a), 1.33-1.23 (2H, overlapped, H-3"b and H-2b), 1.11 (3H, s, H-16), 1.00 (3H, s, H-20), 0.98 (3H, s, H-19), 0.79 (3H, d, $J=6.6 \mathrm{~Hz}, \mathrm{H}-17) ;{ }^{13} \mathrm{C}$ NMR (151 MHz, Pyridine- $\left.d_{5}\right) \delta_{\mathrm{C}}: 172.0$ (s, C-15), 169.6 (s, C-9'), 159.9 (s, C-4), 158.8 (s, C-4'), 132.0 (s, C-1'), 129.0 (d, C-2' and C-6'), 114.1 (d, C-3' and C-5'), 103.3 (t, C-18), 84.4 (d, C-11), 55.0 (q, OMe), 51.2 (d, C-7'), 48.7 (d, C-10), 46.2 (t, C-2"), 44.2 (d, C-14), 43.1 (t, C-6"), 42.8 (s, C-9), 40.4 (s, C-5), 40.1 (d, C-8'), 40.0 (t, C-12), 39. 1 (s, C-13), 37.1 (t, C-6), 36.8 (d, C-8), 33.2 (t, C-3), 28.2 (t, C-2), 27.9 (t, C-7), 25.9 (t, C-5"), 25.3 (t, C-3"), 24.7 (t, C-4"), 23.7 (t, C-1), 22.5 (q, C-16), 20.6 (q, C-19), 16.7 (q, C-17), 13.9 (q, C-20).

Compound 10m: Yield: 5 mg, 27\%; Positive ESIMS $[\mathrm{M}+\mathrm{K}]^{+} \mathrm{m} / z$ 594; ${ }^{1} \mathrm{H}$ NMR (Pyridine- $d_{5}, 600$ MHz) $\delta_{\mathrm{H}}: 11.15(1 \mathrm{H}, \mathrm{s}, \mathrm{NH}), 8.02(2 \mathrm{H}, \mathrm{d}, J=8.1 \mathrm{~Hz}, \mathrm{H}-2 "$ and H-6"), 7.28 (2H, t, J=7.8 Hz, H-3" and H-5"), 7.23 (2H, d, J=8.3 Hz, H-2' and H-6'), 7.03 (1H, t, J=7.4 Hz, H-4"), 6.98 (2H, d, J=8.2 Hz, H-3' and H-5'), 4.63 (2H, br s, H-18), $4.30(1 \mathrm{H}, \mathrm{d}, J=11.4 \mathrm{~Hz}, \mathrm{H}-11), 4.26(1 \mathrm{H}, \mathrm{dd}, J=11.2,7.3 \mathrm{~Hz}$, H-8'), 4.15 (1H, d, J=7.3 Hz, H-7'), 3.68 (3H, s, OMe), 3.41 (1H, d, J=11.1 Hz, H-14), 2.75 (1H, t, $J=12.5 \mathrm{~Hz}, \mathrm{H}-12 \mathrm{a}), 2.60$ (1H, d, J=9.9 Hz, H-1a), 2.27 (1H, m, H-3a), 2.11 (1H, d, J=11.5 Hz, H-3b), $1.99(1 \mathrm{H}, \mathrm{d}, J=13.5 \mathrm{~Hz}, \mathrm{H}-12 \mathrm{~b}), 1.91$ (1H, m, H-2a), 1.57 (4H, overlapped, H-1b, H-2b, H-6a and H-8), 1.53-1.40 (2H, overlapped, H-10 and H-7a), 1.40-1.23 (2H, overlapped, H-6a and H-7b), 1.03 (3H, s, H-20), 1.00 (3H, s, H-19), 0.93 (3H, s, H-16), 0.72 (3H, d, J=6.5 Hz, H-17); ${ }^{13} \mathrm{C}$ NMR (151 MHz, Pyridine-d5) $\delta_{\mathrm{C}}: 172.1$ (s, C-15), 170.8 (s, C-9'), 160.2 (s, C-4), 158.8 (s, C-4'), 140.5 (d, C-1"), 132.0 (s, C-1'), 129.5 (d, C-2' and C-6'), 129.0 (d, C-3" and C-5"), 123.6 (d, C-4"), 120.2 (d, C-2" and C-6"), 114.1 (d, C-3' and C-5'), 103.2 (t, C-18), 84.5(d, C-11), 55.1 (q, OMe), 50.5 (d, C-7'), 48.8 (d, C-10), 44.7 (d, C-8'), 43.2 (d, C-14), 43.1 (s, C-9), 40.4 (s, C-5), 38.7 (s, C-13), 37.6 (t, C-12), 37.2 (t, C-6), 36.8 (d, C-8), 33.3 (t, C-3), 28.6 (t, C-2), 27.9 (t, C-7), 23.7 (t, C-1), 22.7 (q, C-16), 20.6 (q, C-19), 16.6 (q, C-17), 14.0 (q, C-20). 
Compound 10n: Yield: $15 \mathrm{mg}, 40 \%$; Positive ESIMS $[\mathrm{M}+\mathrm{Na}]^{+} m / z, 586 ;{ }^{1} \mathrm{H}$ NMR (Pyridine- $d_{5}, 600$ MHz) $\delta_{\mathrm{H}}: 9.89(1 \mathrm{H}, \mathrm{d}, J=6.7 \mathrm{~Hz}, \mathrm{NH}), 7.17\left(2 \mathrm{H}, \mathrm{d}, J=8.2 \mathrm{~Hz}, \mathrm{H}-2^{\prime}\right.$ and $\left.\mathrm{H}-6 '\right), 6.93(2 \mathrm{H}, \mathrm{d}, J=8.5 \mathrm{~Hz}$, H-3' and H-5'), 4.62 (2H, d, J=7.5 Hz, H-18), 4.49 (1H, td, J=9.4, 6.7 Hz, H-2"), 4.42 (1H, td, J=8.9, $3.1 \mathrm{~Hz}, \mathrm{H}-4 " \mathrm{a}), 4.27$ (1H, d, J=11.4 Hz, H-11), 4.16-4.07 (2H, overlapped, H-8' and H-4"b), 4.02 (1H, d, J=7.3 Hz, H-7'), 3.66 (3H, s, OMe), 3.37 (1H, d, J=11.1 Hz, H-14), 2.84-2.73 (1H, m, H-3"a), 2.68 (1H, t, J=12.5 Hz, H-12a), 2.56 (1H, d, J=12.0 Hz, H-1a), 2.46 (1H, m, H-3"b), 2.25 (1H, m, H-3a), $2.08(1 \mathrm{H}, \mathrm{d}, J=13.3 \mathrm{~Hz}, \mathrm{H}-3 \mathrm{~b}), 1.94(1 \mathrm{H}, \mathrm{d}, J=13.5 \mathrm{~Hz}, \mathrm{H}-12 \mathrm{~b}), 1.88(1 \mathrm{H}, \mathrm{m}, \mathrm{H}-2 \mathrm{a})$, 1.66-1.51 (4H, overlapped, H-1b, H-2b, H-6a and H-8), 1.48 (2H, overlapped, H-7b and H-10), 1.35 (1H, dd, J=13.7, 3.8 Hz, H-7b), 1.28 (1H, m, H-6b), 1.03 (3H, s, H-20), 1.01 (3H, s, H-19), 0.90 (3H, s, H-16), 0.72 (3H, d, J=6.6 Hz, H-17); ${ }^{13} \mathrm{C}$ NMR (151 MHz, Pyridine- $\left.d_{5}\right) \delta_{\mathrm{C}}: 175.6$ (s, C-1"), 172.4 (s, C-9'), 172.1 (s,C-15), 160.1 (s, C-4), 158.8 (s, C-4'), 131.9 (s, C-1'), 129.4 (d, C-2' and C-6'), 114.1 (d, C-3' and C-5'), 103.2 (t, C-18), 84.6 (d, C-11), 66.2 (t, C-4"), 55.0 (q, OMe), 50.3 (d, C-7'), 49.9 (d, C-2"), 48.8 (d, C-10), 43.2 (d, C-8'), 43.1 (s, C-9), 43.1 (d, C-14), 40.4 (s, C-5), 38.7 (s, C-13), 37.5 (t, C-12), 37.2 (t, C-6), 36.8(d, C-8), 33.3 (t, C-3), 28.5 (t, C-3"), 28.3 (t, C-2), 27.9 (t, C-7), 23.7 (t, C-1), 22.7 (q, C-16), 20.6 (q, C-19), 16.6 (q, C-17), 14.0 (q, C-20).

Compound 10o: Yield: $2 \mathrm{mg}, 14 \%$; Positive ESIMS $[\mathrm{M}+\mathrm{Na}]^{+} \mathrm{m} / z$ 586; ${ }^{1} \mathrm{H}$ NMR (Pyridine- $d_{5}, 600$ MHz) $\delta_{\mathrm{H}} 9.92(1 \mathrm{H}, \mathrm{d}, J=6.7 \mathrm{~Hz}, \mathrm{NH}), 7.22\left(2 \mathrm{H}, \mathrm{d}, J=8.3 \mathrm{~Hz}, \mathrm{H}-2^{\prime}\right.$ and H-6'), 6.95 (2H, d, J=8.4 Hz, H-3' and H-5'), $5.22(1 \mathrm{H}, \mathrm{d}, J=4.0 \mathrm{~Hz}, \mathrm{H}-3), 4.54-4.45(1 \mathrm{H}, \mathrm{m}, \mathrm{H}-2 " \mathrm{a}), 4.40(1 \mathrm{H}, \mathrm{dd}, J=8.9,3.2 \mathrm{~Hz}$, H-4"a), 4.31 (1H, d, J=11.4 Hz, H-11), 4.18-4.07 (2H, overlapped, H-8' and H-4"b), 4.05 (1H, d, $J=7.2 \mathrm{~Hz}, \mathrm{H}-7$ '), 3.67 (3H, s, OMe), 3.39 (1H, d, J=11.1 Hz, H-14), 2.86-2.73 (2H, overlapped, H-12a and H-3"a), 2.69-2.58 (1H, m, H-1a), 2.45 (2H, overlapped, H-2a and H-3"b), 2.10-2.02 (1H, m, H-2b), 1.99 (1H, d, J=13.5 Hz, H-12b), 1.68 (1H, d, J=12.4 Hz, H-10), 1.64-1.56 (5H, overlapped, $\mathrm{H}-1 \mathrm{~b}, \mathrm{H}-8$ and H-18), 1.47-1.36 (1H, m, H-7a), 1.34-1.26 (2H, overlapped, H-6a and H-7b), 1.16-1.09 (1H, m, H-6b), 1.02 (3H, s, H-20), 0.98 (3H, s, H-19), 0.91 (3H, s, H-16), 0.71 (3H, d, $J=6.4 \mathrm{~Hz}, \mathrm{H}-17) ;{ }^{13} \mathrm{C}$ NMR (151 MHz, Pyridine-d5) $\delta_{\mathrm{C}}: 175.6$ (s, C-1"), 172.5 (s, C-9'), 172.1 (s, C-15), 158.8 (s, C-4'), 143.2 (s, C-4), 132.0 (s, C-1'), 129.5 (d, C-2' and C-6'), 121.6 (d, C-3), 114.1 (d, C-3' and C-5'), 84.8 (d, C-11), 66.2 (t, C-4"), 55.1 (q, OMe), 50.4 (d, C-7'), 49.9(d, C-2"), 46.5 (d, C-10), 43.3 (d, C-8'), 43.0(d, C-14), 42.5 (s, C-9), 38.7 (s, C-13), 38.5 (s, C-5), 37.5 (t, C-12), 36.6 (t, C-6), 36.4 (d, C-8), 28.3 (t, C-3"), 27.8 (t, C-7), 26.6 (t, C-2), 22.7 (q, C-16), 20.0 (t, C-1), 19.7 (q, C-19), 18.3 (q, C-18), 16.4 (q, C-17), 14.2 (q, C-20).

Compound 10p: Yield: $1 \mathrm{mg}, 9 \%$; Negative ESIMS $[\mathrm{M}+\mathrm{Cl}]^{-} \mathrm{m} / z, 588 ;{ }^{1} \mathrm{H}$ NMR (Pyridine- $d_{5}, 600$ 
MHz) $\delta_{\mathrm{H}}: 7.32\left(1 \mathrm{H}, \mathrm{d}, J=8.6 \mathrm{~Hz}, \mathrm{H}-2^{\prime}\right.$ and H-6'), $6.91\left(1 \mathrm{H}, \mathrm{d}, J=8.5 \mathrm{~Hz}, \mathrm{H}-3^{\prime}\right.$ and H-5'), 5.07 (1H, overlapped, H-3 and H-2"), 4.59-4.53 (2H, overlapped, H-1"a and H-3"a), 4.41-4.32 (2H, overlapped, H-1"b amd H-3"'b), 4.03-3.95 (2H, overlapped, H-11 and H-7'), 3.88 (1H, t, J=7.1 Hz, H-8'), 3.64 (3H, s, OMe), 3.10 (1H, d, J=6.8 Hz, H-14), 2.62 (1H, dd, J=13.2, 6.3 Hz, H-1a), 2.49 (1H, m, H-2a), 2.37 (1H, dd, J=14.5, $11.3 \mathrm{~Hz}, \mathrm{H}-12 \mathrm{a}), 2.18$ (1H, d, J=14.2 Hz, H-12b), 2.14-2.07 (1H, m, H-2b), 1.84 (1H, d, J=12.1 Hz, H-10), 1.76-1.66 (1H, m, H-1b), 1.61-1.52 (2H, overlapped, H-6a and H-8), 1.51 (3H, s, H-18), 1.45-1.34 (1H, m, H-7a), 1.33-1.26 (1H, m, H-7b), 1.27-1.12 (3H, overlapped, H-6b and H-16), 1.11 (3H, s, H-20), 1.03 (3H, s, H-19), 0.84 (3H, d, J=6.5 Hz, H-17); ${ }^{13} \mathrm{C}$ NMR (151 MHz, Pyridine-d5) $\delta_{\mathrm{C}}: 181.0$ (s,C-15), 180.3 (s, C-9'), 159.0 (s, C-4'), 143.7 (s, C-4), 130.3 (s, C-1'), 129.4 (d, C-2' and C-6'), 121.3 (d, C-3), 114.1 (d, C-3' and C-5'), 73.7 (d, C-11), 59.2 (t, C-1"), 58.8 (t, C-3"), 58.3 (d, C-2"), 55.0 (q, OMe), 53.3 (d, C-7'), 48.4 (d, C-14), 46.5 (d, C-10), 44.3 (s, C-13), 43.9 (s, C-9), 40.8 (d, C-8'), 39.8 (t, C-12), 38.7(s, C-5), 36.6 (t, C-6 and C-8), 28.7 (t, C-7), 26.8 (t, C-2), 22.8 (q, C-16), 20.5 (t, C-1), 19.9 (q, C-19), 18.4 (q, C-18), 17.2 (q, C-17), 14.5 (q, C-20).

Compound 10q: Yield: $3 \mathrm{mg}, 19 \%$; Positive ESIMS $[\mathrm{M}+\mathrm{Na}]^{+} m / z$ 547; ${ }^{1} \mathrm{H}$ NMR (Pyridine- $d 5,600$ MHz) $\delta_{\mathrm{H}}: 7.22\left(2 \mathrm{H}, \mathrm{d}, J=8.6 \mathrm{~Hz}, \mathrm{H}-2^{\prime}\right.$ and H-6'), $7.01\left(2 \mathrm{H}, \mathrm{d}, J=8.6 \mathrm{~Hz}, \mathrm{H}-3^{\prime}\right.$ and H-5'), 4.65-4.55 (4H, overlapped, H-18 and H-2"), 4.26 (1H, d, J=10.9 Hz, H-11), 4.18-4.10 (3H, overlapped, H-7', H-8' and H-1"a), 4.10-4.05 (1H, m, H-1"b), 3.68 (3H, s, OMe), 3.36 (1H, d, J=9.4 Hz, H-14), 2.29 $(1 \mathrm{H}, \mathrm{d}, J=14.2 \mathrm{~Hz}, \mathrm{H}-1 \mathrm{a}), 2.25-2.14(2 \mathrm{H}$, overlapped, H-3a and H-12a), $2.10(1 \mathrm{H}, \mathrm{d}, J=13.7 \mathrm{~Hz}$, H-12b), 2.07-2.01 (1H, m, H-3b), 1.69 (1H, d, J=12.8 Hz, H-2b), 1.60-1.50 (3H, overlapped, H-1b, H-6a and H-8), 1.41-1.32 (2H, overlapped, H-7a and H-10), 1.32-1.25 (1H, m, H-7b), 1.25-1.12 (2H, overlapped, H-2b and H-6b), 1.02 (3H, s, H-16), 0.98 (6H, s, H-19 and H-20), 0.74 (3H, d, J=6.5 Hz, H-17); ${ }^{13} \mathrm{C}$ NMR (151 MHz, Pyridine- $\left.d_{5}\right) \delta_{\mathrm{C}}: 173.2$ (s, C-9'), 173.1 (s,C-15), 160.4 (s, C-4), 159.5 (s, C-4'), 131.4 (s, C-1'), 129.5 (d, C-2' and C-6'), 114.8 (d, C-3' and C-5'), 103.9 (t, C-18), 85.4 (d, C-11), 67.9 (t, C-2"), 60.8(t, C-1"), 55.6 (q, OMe), 51.7 (d, C-7'), 49.2 (d, C-10), 44.4 (d, C-14), 43.4 (s, C-9), 41.6 (d, C-8'), 40.9 (s, C-5), 40.4 (t, C-12), 40.1 (s, C-13), 37.6 (t, C-6), 37.3 (d, C-8), 33.7 (t, C-3), 28.9 (t, C-7), 28.4 (t, C-2), 24.3 (t, C-1), 22.7 (q, C-16), 21.1 (q, C-19), 17.2 (q, C-17), 14.4 (q, C-20).

Compound 10r: Yield: $1 \mathrm{mg}, 8 \%$; Positive ESIMS $[\mathrm{M}+\mathrm{Na}]^{+} m / z, 547 ;{ }^{1} \mathrm{H}$ NMR (Pyridine- $d_{5}, 600$ MHz) $\delta_{\mathrm{H}}: 7.26\left(2 \mathrm{H}, \mathrm{d}, J=8.3 \mathrm{~Hz}, \mathrm{H}-2^{\prime}\right.$ and H-6'), 7.03 (2H, d, J=8.4 Hz, H-3' and H-5'), $5.16(1 \mathrm{H}$, overlapped, H-3), 4.66-4.56 (2H, m, H-2"), 4.30 (1H, d, J=11.0 Hz, H-11), 4.18-4.10 (3H, 
overlapped, H-7', H-8' and H-1"a), 4.10-4.05 (1H, m, H-1"b), 3.68 (3H, s, OMe), 3.38 (1H, d, J=10.1 Hz, H-14), 2.40-2.33 (1H, m, H-1a), 2.24 (1H, dd, J=13.7, $11.2 \mathrm{~Hz}, \mathrm{H}-12 \mathrm{a}), 2.15$ (1H, d, J=13.7 Hz, H-12b), 2.15-2.06 (1H, m, H-2a), 1.90 (1H, d, J=18.0 Hz, H-2b), 1.62-1.49 (6H, overlapped, H-1b, H-10, H-8 and H-18), 1.41-1.26 (3H, overlapped, H-6a and H-7), 1.20-1.06 (1H, m, H-6b), 1.03 (3H, s, H-16), 0.97 (3H, s, H-20), 0.94 (3H, s, H-19), 0.73 (3H, d, J=6.4 Hz, H-17); ${ }^{13} \mathrm{C}$ NMR (151 MHz, Pyridine- $d_{5}$ ) $\delta_{\mathrm{C}}: 173.3$ (s, C-9'), 173.1 (s,C-15), 159.6 (s, C-4'), 143.9 (s, C-4), 131.5 (s, C-1'), 129.6 (d, C-2' and C-6'), 121.8 (d, C-3), 114.8 (d, C-3' and C-5'), 85.6 (d, C-11), 67.9 (t, C-2"), 60.8 (t, C-1"), 55.6 (q, OMe), 51.9 (d, C-7'), 47.0 (d, C-10), 44.4 (d, C-14), 42.8 (s, C-9), 41.6 (d, C-8'), 40.5 (t, C-12), 40.0 (s, C-13), 39.0 (s, C-5), 37.0 (t, C-6), 36.9 (d, C-8), 28.3 (t, C-7), 27.0 (t, C-2), 22.7 (q, C-16), 20.7 (t, C-1), 20.2 (q, C-19), 18.8 (q, C-18), 17.0 (q, C-17), 14.6 (q, C-20).

Compound 10u: Yield: $5 \mathrm{mg}, 28 \%$; Positive ESIMS $[\mathrm{M}+\mathrm{Na}]^{+} m / z$ 629; ${ }^{1} \mathrm{H} \mathrm{NMR}(600 \mathrm{MHz}, \mathrm{CDCl} 3)$ $\delta_{\mathrm{H}}: 8.80(1 \mathrm{H}, \mathrm{br} \mathrm{s}), 8.79(1 \mathrm{H}, \mathrm{br} \mathrm{s}), 8.27(1 \mathrm{H}, \mathrm{d}, J=7.0 \mathrm{~Hz}), 7.24(1 \mathrm{H}, \mathrm{t}, J=7.4 \mathrm{~Hz}, \mathrm{H}-5 "), 7.58-7.49$ (3H, overlapped), $7.12\left(2 \mathrm{H}, \mathrm{t}, J=8.6 \mathrm{~Hz}, \mathrm{H}-2^{\prime}\right.$ and $\left.\mathrm{H}-6{ }^{\prime}\right), 6.86\left(2 \mathrm{H}, \mathrm{t}, J=8.6 \mathrm{~Hz}, \mathrm{H}-3^{\prime}\right.$ and $\left.\mathrm{H}-5^{\prime}\right)$, $4.53(1 \mathrm{H}, \mathrm{s}, \mathrm{H}-18 \mathrm{a}), 4.51(1 \mathrm{H}, \mathrm{s}, \mathrm{H}-18 \mathrm{~b}), 4.12(1 \mathrm{H}, \mathrm{d}, J=11.1 \mathrm{~Hz}, \mathrm{H}-11), 4.07$ (1H, br t, $J=10.4$, H-8'), 3.88 (1H, d, $J=7.7$ Hz, H-14,), 3.26 (1H, d, $J=10.4$ Hz, H-7'), 2.32-2.07 (4H, overlapped), 1.94-1.91 (2H, overlapped), 1.60-1.22 (8H, overlapped), $1.06\left(3 \mathrm{H}, \mathrm{s}, \mathrm{H}_{3}-19\right), 0.97\left(3 \mathrm{H}, \mathrm{s}, \mathrm{H}_{3}-20\right)$, $0.91\left(3 \mathrm{H}, \mathrm{s}, \mathrm{H}_{3}-16\right), 0.83\left(3 \mathrm{H}, \mathrm{d}, J=6.7 \mathrm{~Hz}, \mathrm{H}_{3}-17\right)$.

Synthesis of $10 v$ and $10 w$ from $\mathbf{6} b$ : General procedure for the synthesis of $10 \mathrm{v}(18 \mathrm{mg}, 0.03 \mathrm{mmol}$, $10 \%$ total yield) and $\mathbf{1 0 w}(5 \mathrm{mg}, 0.01 \mathrm{mmol}, 12 \%$ total yield) from $\mathbf{6 b}$ (100 mg $0.3 \mathrm{mmol}$ for $\mathbf{1 0 v} ; 30$ $\mathrm{mg}, 0.01 \mathrm{mmol}$ for $10 \mathrm{w}$ ) were similar with the route for the synthesis of 1. Compound 10v: Positive ESIMS $[\mathrm{M}+\mathrm{Na}]^{+} m / z 613 ;{ }^{1} \mathrm{H}$ NMR $\left(600 \mathrm{MHz}, \mathrm{CDCl}_{3}\right) \delta_{\mathrm{H}}: 7.42\left(2 \mathrm{H}, \mathrm{d}, J=8.6 \mathrm{~Hz}, \mathrm{H}-2{ }^{\prime}\right.$ and H-6'), $7.04\left(2 \mathrm{H}, \mathrm{d}, J=8.6 \mathrm{~Hz}, \mathrm{H}-3^{\prime}\right.$ and $\left.\mathrm{H}-5^{\prime}\right), 5.88(1 \mathrm{H}, \mathrm{m}, \mathrm{H}-2 "), 5.51\left(1 \mathrm{H}, \mathrm{dd}, J=12.9\right.$ and $\left.5.8 \mathrm{~Hz}, \mathrm{H}-3^{\prime \prime}\right)$, 5.44 (1H, d, $J=17.3 \mathrm{~Hz}, \mathrm{H}-1 \mathrm{a} "), 5.15$ (1H, d, $J=10.6 \mathrm{~Hz}, \mathrm{H}-1 \mathrm{~b} "), 4.41$ (1H, overlapped, H-11), $4.41(1 \mathrm{H}, \mathrm{s}, \mathrm{H}-18 \mathrm{a}), 4.36(1 \mathrm{H}, \mathrm{s}, \mathrm{H}-18 \mathrm{~b}), 4.12(1 \mathrm{H}, \mathrm{dd}, J=10.4$ and $8.1 \mathrm{~Hz}, \mathrm{H}-8$ '), $3.84(1 \mathrm{H}, \mathrm{d}, J=$ $9.3 \mathrm{~Hz}, \mathrm{H}-7$ '), 3.61 (3H, s, OMe), 3.55 (1H, d, J = 8.1 Hz, H-14), 2.18 (1H, m, H-3a), 2.06 (1H, d, $J$ = 13.2 Hz, H-1a), $1.99(1 \mathrm{H}, \mathrm{d}, J=13.3 \mathrm{~Hz}, \mathrm{H}-3 \mathrm{~b}), 1.80(1 \mathrm{H}, \mathrm{m}, \mathrm{H}-12 \mathrm{a}), 1.72$ (1H, m, H-2a), 1.60-1.39 (6H, overlapped, H-1b, H-6a, H-7a, H-12b, and $\left.\mathrm{H}_{2}-4 "\right), 1.52$ (3H, s, H-16), 1.30-1.03 (11H, overlapped, H-6b, H-7b, H-8, H-10, H2-5", H2-6", and H2-7"), 1.01 (3H, s, H-19), 0.95 (3H, s, H-20), 0.78-0.75 (6H, overlapped, H-17 and H-8"); ${ }^{13} \mathrm{C}$ NMR (151 MHz, Pyr) $\delta_{\mathrm{C}}: 173.2$ (s, C-9'), 173.0 (s, C-15), 159.7 (s, C-4), 159.6 (s, C-4'), 137.3 (d, C-2"), 130.3 (s, C-1'), 129.3 (d, C-2' and C-6'), 116.6 (t, C-1"), 114.9 (d, C-3' and C-5'), 103.9 (t, C-18), 83.9 (d, C-11), 75.8 (d, C-3"), 55.4 (q, OMe), 51.3 
(d, C-7'), 49.2 (d, C-10), 43.6 (d, C-8'), 43.5 (d, C-14), 42.9 (s, C-9), 40.6 (s, C-5), 40.2 (s, C-13), 37.2 (t, C-6), 37.0 (d, C-8), 34.7 (t, C-4"), 33.6 (t, C-3), 31.9 (t, C-6"), 31.4 (t, C-12), 29.1 (t, C-2), 28.3 (s, C-7), 27.6 (q, C-16), 25.2 (t, C-5"), 24.4 (t, C-1), 23.0 (t, C-7"), 21.1 (q, C-19), 17.1 (q, C-17), 14.4 (q, C-8"), 14.4 (q, C-20). Compound 10w: ${ }^{1} \mathrm{H}$ NMR (600 MHz, $\left.\mathrm{CDCl}_{3}\right) \delta_{\mathrm{H}}: 7.46$ (2H, d, $J=8.5 \mathrm{~Hz}, \mathrm{H}-2^{\prime}$ and $\mathrm{H}-6$ '), 7.07 (2H, d, $J=8.6 \mathrm{~Hz}, \mathrm{H}-3^{\prime}$ and $\left.\mathrm{H}-5^{\prime}\right), 5.90$ (1H, m, H-2"), 5.53 (1H, dd, $J=12.9$ and $5.8 \mathrm{~Hz}, \mathrm{H}-3 "), 5.46$ (1H, d, $J=17.3 \mathrm{~Hz}, \mathrm{H}-1 \mathrm{a} "), 5.16$ (1H, d, $J=10.6 \mathrm{~Hz}, \mathrm{H}-1 \mathrm{~b} "), 5.04$ (1H, overlapped, H-3), 4.45 (1H, d, $J=11.3 \mathrm{~Hz}, \mathrm{H}-11), 4.14(1 \mathrm{H}, \mathrm{t}, J=8.7 \mathrm{~Hz}, \mathrm{H}-8$ '), 3.88 (1H, d, $J$ = 9.4 Hz, H-7'), 3.60 (3H, s, OMe), 3.58 (1H, overlapped, H-14), 2.14 (1H, m, H-1a), 1.94-1.84 (2H, overlapped, H-2a and H-12a), 1.60-1.48 (4H, overlapped, $\mathrm{H}-1 \mathrm{~b}, \mathrm{H}-12 \mathrm{~b}$, and $\left.\mathrm{H}_{2}-4 "\right), 1.54(3 \mathrm{H}, \mathrm{s}$, H-16), 1.39 (3H, s, H-18), 1.00 (3H, s, H-19), 0.91 (3H, s, H-20), 0.77 (1H, m, H-6b), 0.77-0.74 (6H, overlapped, H-17 and H-8"); ${ }^{13} \mathrm{C}$ NMR (151 MHz, Pyr) $\delta_{\mathrm{C}} 173.3$ (s, C-9'), 173.1 (s, C-15), 159.7 (s, C-4'), 143.7 (s, C-4), 137.3 (d, C-2"), 130.3 (s, C-1'), 129.3 (d, C-2' and C-6'), 121.3 (d, C-3), 116.7 (t, C-1"), 115.0 (d, C-3' and C-5'), 84.1 (d, C-11), 75.9 (d, C-3"), 55.4 (q, OMe), 51.2 (d, C-7'), 47.2 (d, C-10), 43.4 (d, C-8'), 43.0 (d, C-14), 42.9 (s, C-9), 40.2 (s, C-5), 38.8 (s, C-13), 36.7 (d, C-8), 36.5 (t, C-6), 34.7 (t, C-4"), 31.9 (t, C-6"), 31.4 (t, C-12), 28.1 (s, C-7), 27.6 (q, C-16), 27.2 (t, C-2), 25.2 (t, C-5"), 23.0 (t, C-7"), 20.7 (t, C-1), 20.0 (q, C-18), 18.6 (q, C-19), 16.9 (q, C-17), 14.7 (q, C-8"), 14.4 (q, C-20). 
Table S3. The effect of natural and synthetic molecules on human T cell proliferation and cytotoxicity on PBMC viability

\begin{tabular}{|c|c|c|c|c|c|c|c|c|}
\hline no. & $\mathrm{IC}_{50}(\mu \mathrm{M})^{\mathrm{a}}$ & $\mathrm{IC}_{50}(\mu \mathrm{M})^{\mathrm{b}}$ & no. & $\mathrm{IC}_{50}(\mu \mathrm{M})^{\mathrm{a}}$ & $\mathrm{IC}_{50}(\mu \mathrm{M})^{\mathrm{b}}$ & no. & $\mathrm{IC}_{50}(\mu \mathrm{M})^{\mathrm{a}}$ & $\mathrm{IC}_{50}(\mu \mathrm{M})^{\mathrm{b}}$ \\
\hline 1 & 20.7 & $>400$ & $10 \mathrm{a}$ & 5.6 & 183.2 & $10 \mathrm{o}$ & NA & NT \\
\hline 3 & 49.3 & $>400$ & $10 \mathrm{~b}$ & 5.0 & 63.1 & $10 p$ & NA & NT \\
\hline $6 e$ & 28.6 & 157.4 & $10 \mathrm{c}$ & 9.7 & 83.6 & $10 q$ & NA & NT \\
\hline $7 a$ & 57.9 & 166.3 & $10 d$ & 3.9 & 45.8 & $10 \mathrm{r}$ & 30.1 & 171.3 \\
\hline $7 b$ & 7.8 & 87.6 & $10 \mathrm{e}$ & 7.1 & 52.6 & $10 \mathrm{~s}$ & NA & NT \\
\hline $7 c$ & 6.2 & 56.7 & $10 f$ & 11.7 & 57.9 & $10 t$ & NA & NT \\
\hline $7 \mathrm{~d}$ & $\mathrm{NA}^{a}$ & $\mathrm{NT}^{\mathrm{b}}$ & $10 \mathrm{~g}$ & NA & NT & $10 u$ & 62.1 & 193.1 \\
\hline $7 e$ & 41.4 & 157.5 & $10 \mathrm{~h}$ & 15.3 & 178.1 & $10 \mathrm{v}$ & 37.6 & 161.9 \\
\hline $7 f$ & NA & NT & $10 \mathrm{i}$ & 33.7 & 165.5 & $10 w$ & 18.9 & 93.6 \\
\hline $7 \mathrm{~g}$ & 18.3 & 106.9 & $10 \mathrm{j}$ & 31.8 & 167.5 & & & \\
\hline $7 \mathrm{~h}$ & NA & NT & $10 \mathrm{k}$ & NA & NT & & & \\
\hline $7 \mathbf{i}$ & NA & NT & 101 & 21.8 & 171.3 & & & \\
\hline $7 \mathbf{j}$ & 29.8 & 100.5 & $10 \mathrm{~m}$ & 31.2 & 170.2 & & & \\
\hline $7 \mathrm{k}$ & 16.1 & 80.5 & $10 n$ & NA & NT & & & \\
\hline
\end{tabular}

${ }^{\mathrm{a}} \mathrm{IC}_{50}$ of inhibition on $\mathrm{T}$ cell proliferation; ${ }^{\mathrm{b}} \mathrm{IC}_{50}$ of cytotoxicity on PBMC viability; ${ }^{\mathrm{c}} \mathrm{NA}$ : no activity; ${ }^{\mathrm{d}} \mathrm{NT}$ : untested. 
Figure S2. ${ }^{1} \mathrm{H}$ NMR spectrum of natural scopariusicide A (1)

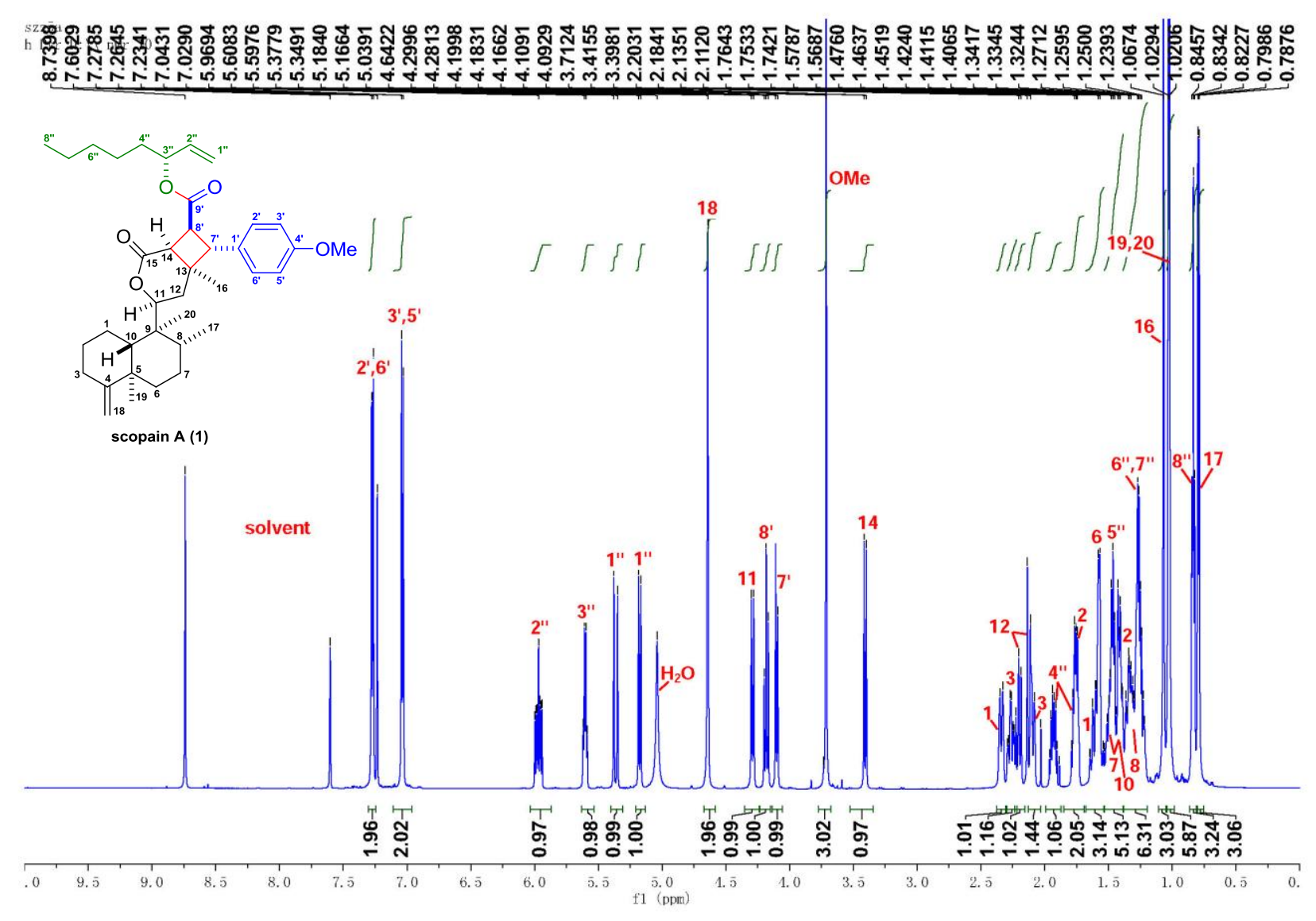

Figure S3. ${ }^{13} \mathrm{C}$ NMR spectrum of natural scopariusicide A (1)
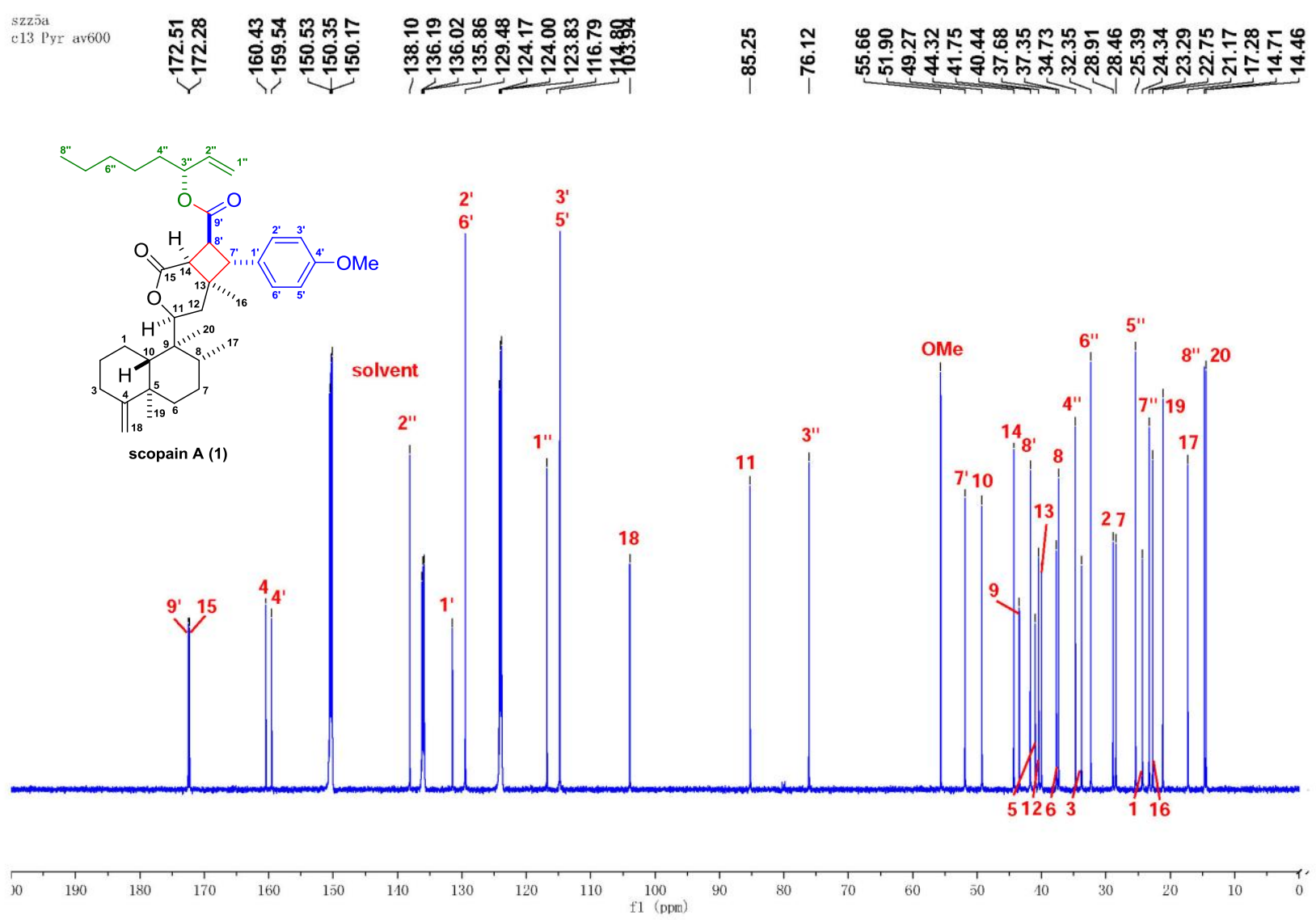
Figure S4. HSQC spectrum of natural scopariusicide A (1)

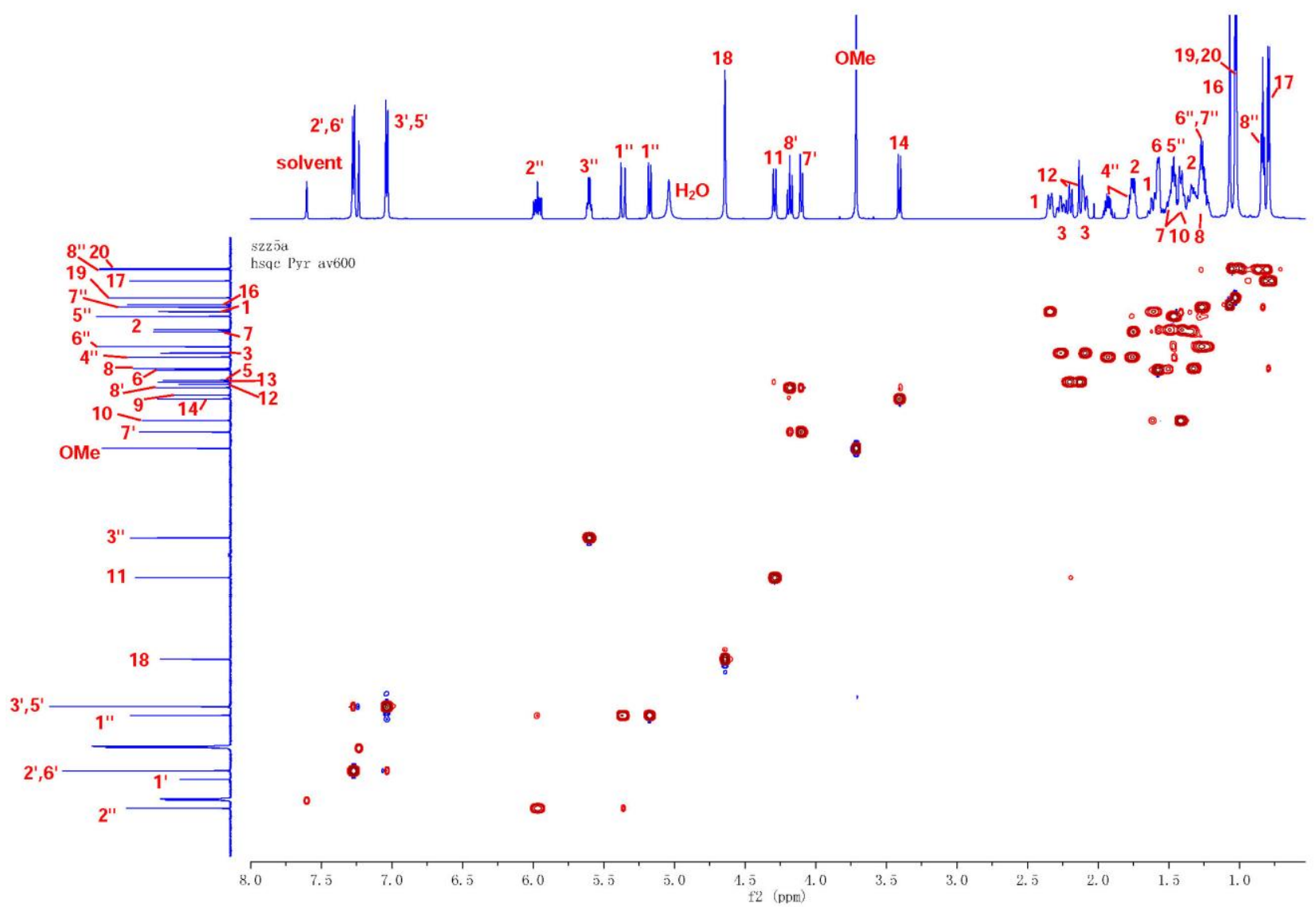

Figure S5. HMBC spectrum of natural scopariusicide A (1)

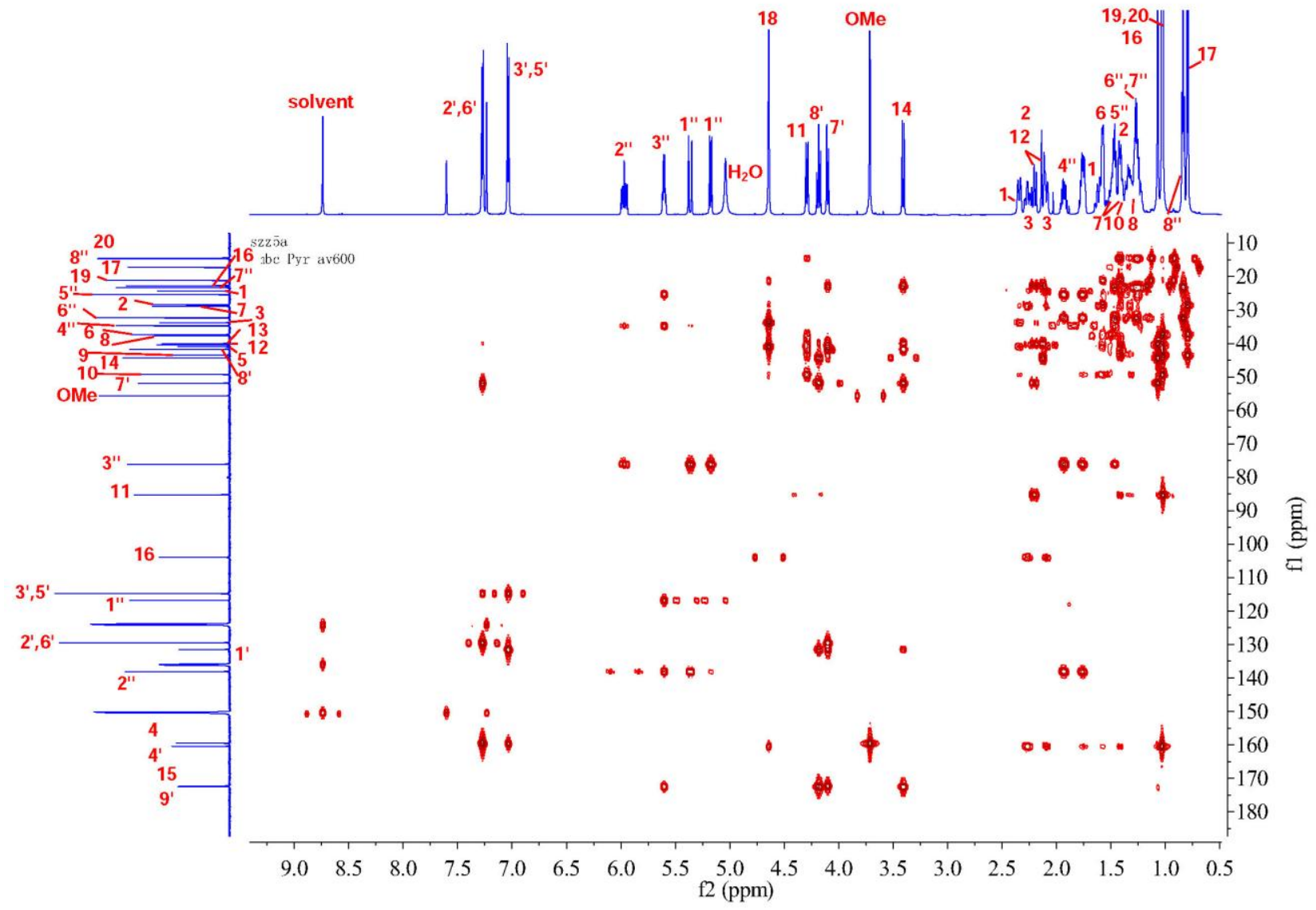


Figure S6. ${ }^{1} \mathrm{H}-{ }^{1} \mathrm{H}$ COSY spectrum of natural scopariusicide A (1)

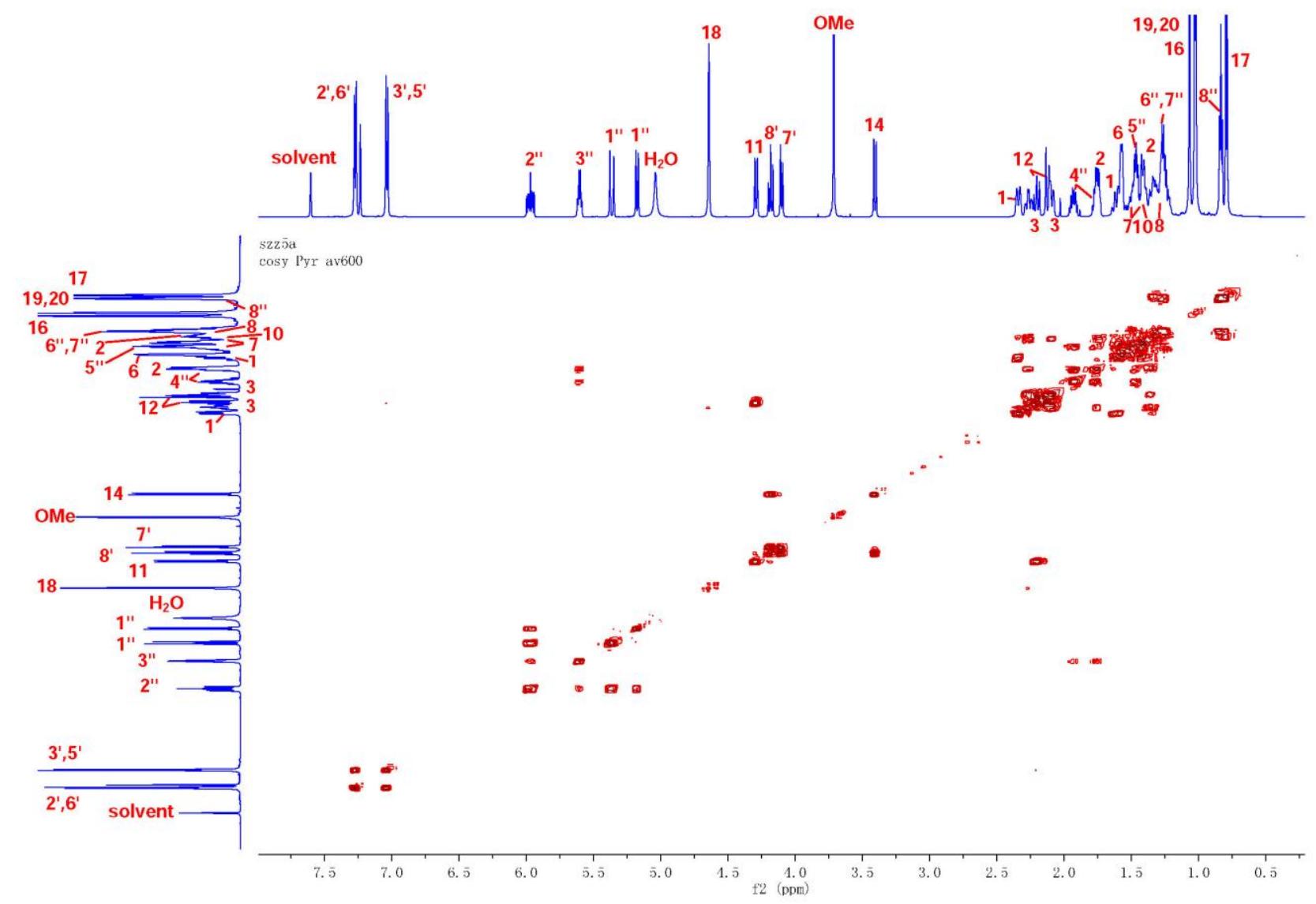

Figure S7. ROESY spectrum of natural scopariusicide A (1)

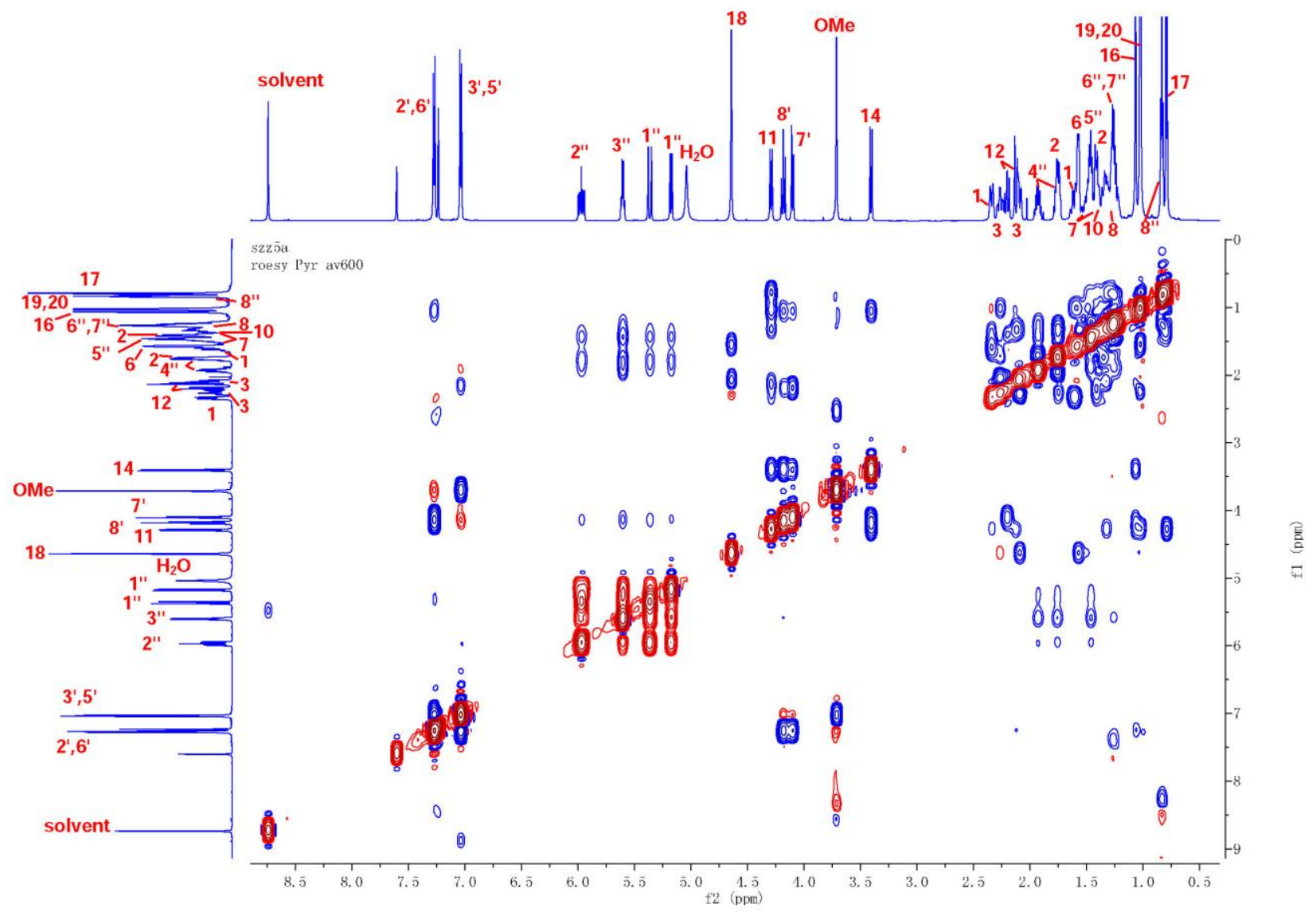


Figure S8. CD of natural scopariusicide A (1)
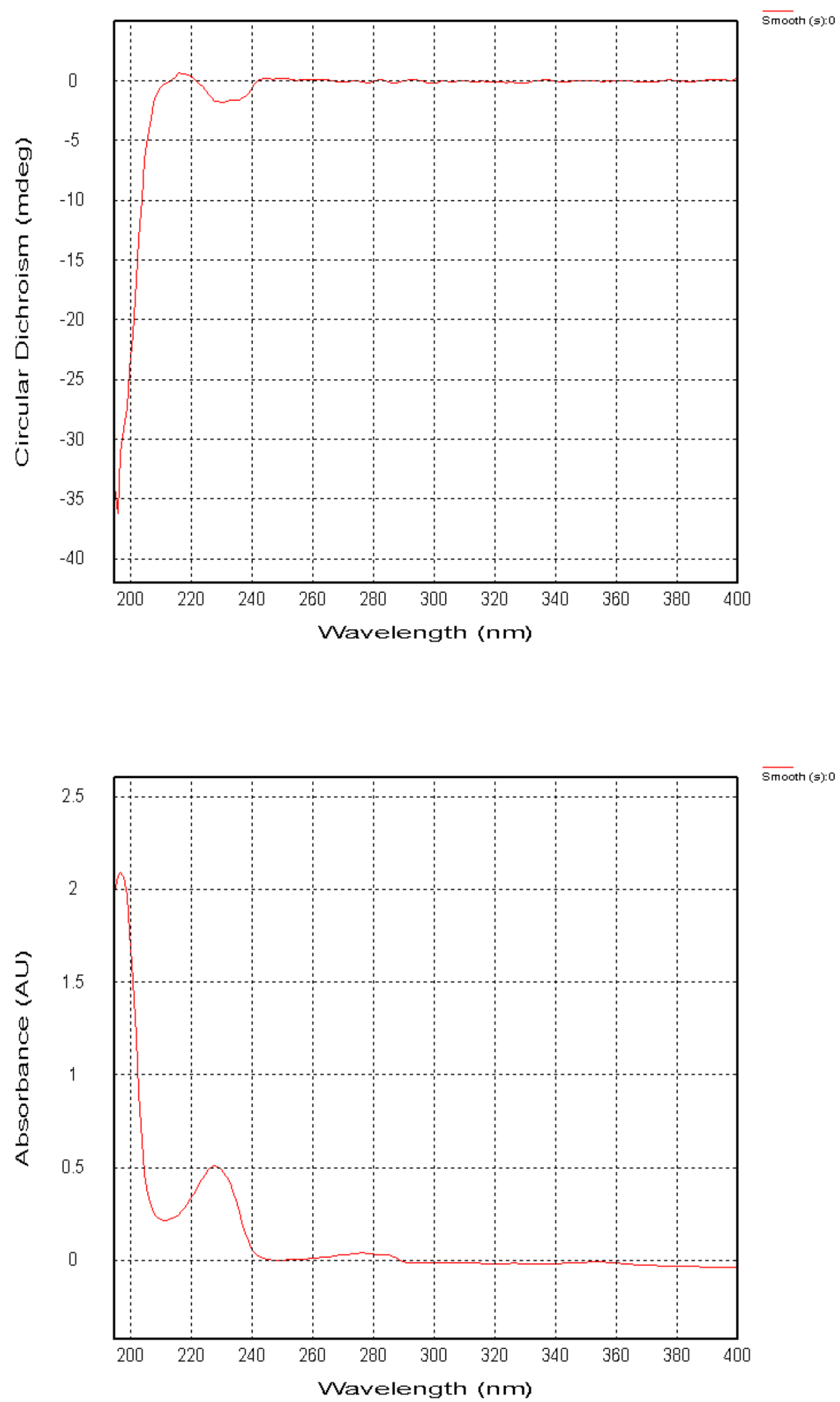
Figure S9. X-ray structure of natural scopariusicide A (1)
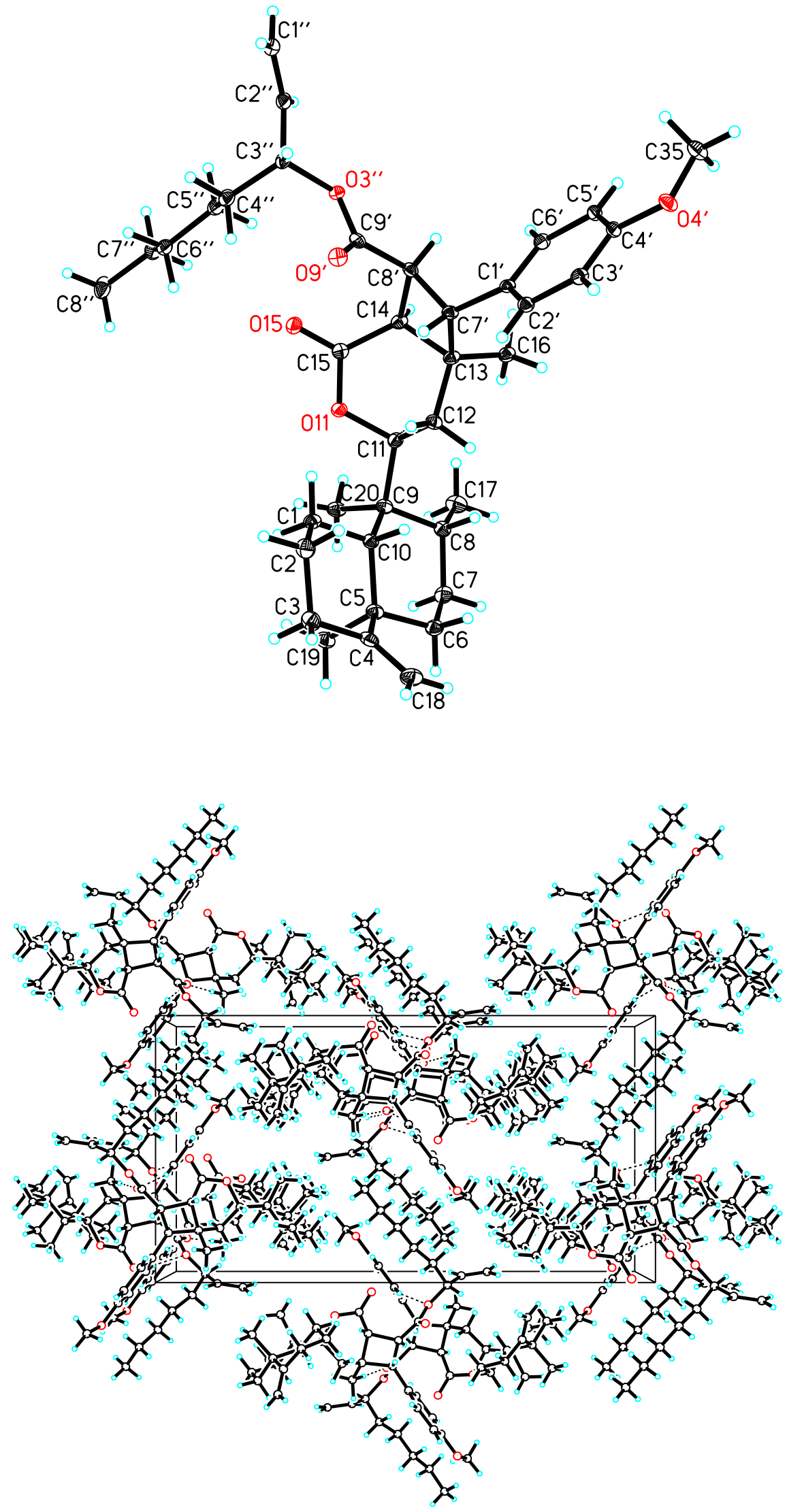
Figure S10. ${ }^{1} \mathrm{H}$ NMR spectrum of scopariusicide B (2)

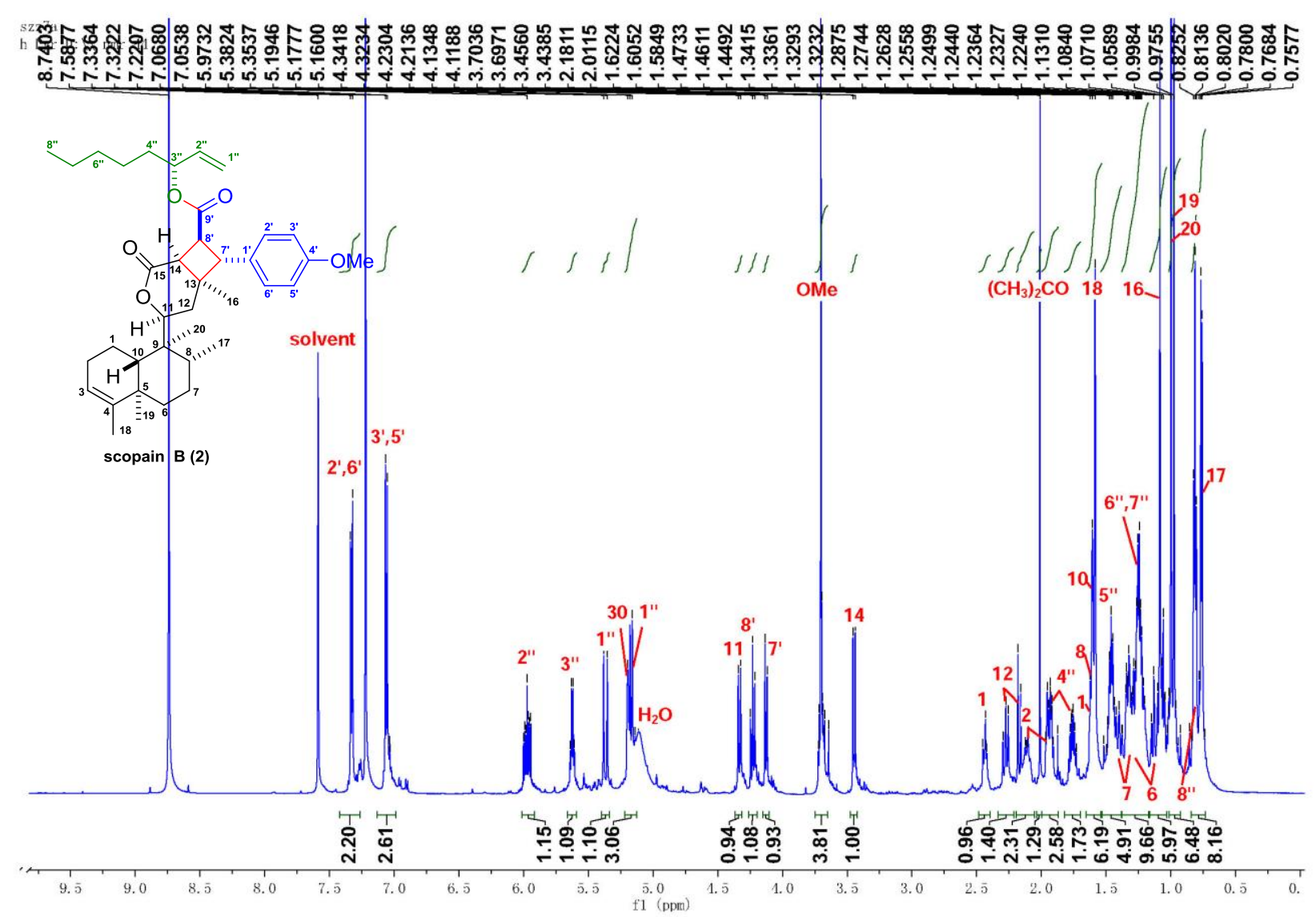

Figure S11. ${ }^{13} \mathrm{C}$ NMR spectrum of scopariusicide B (2)
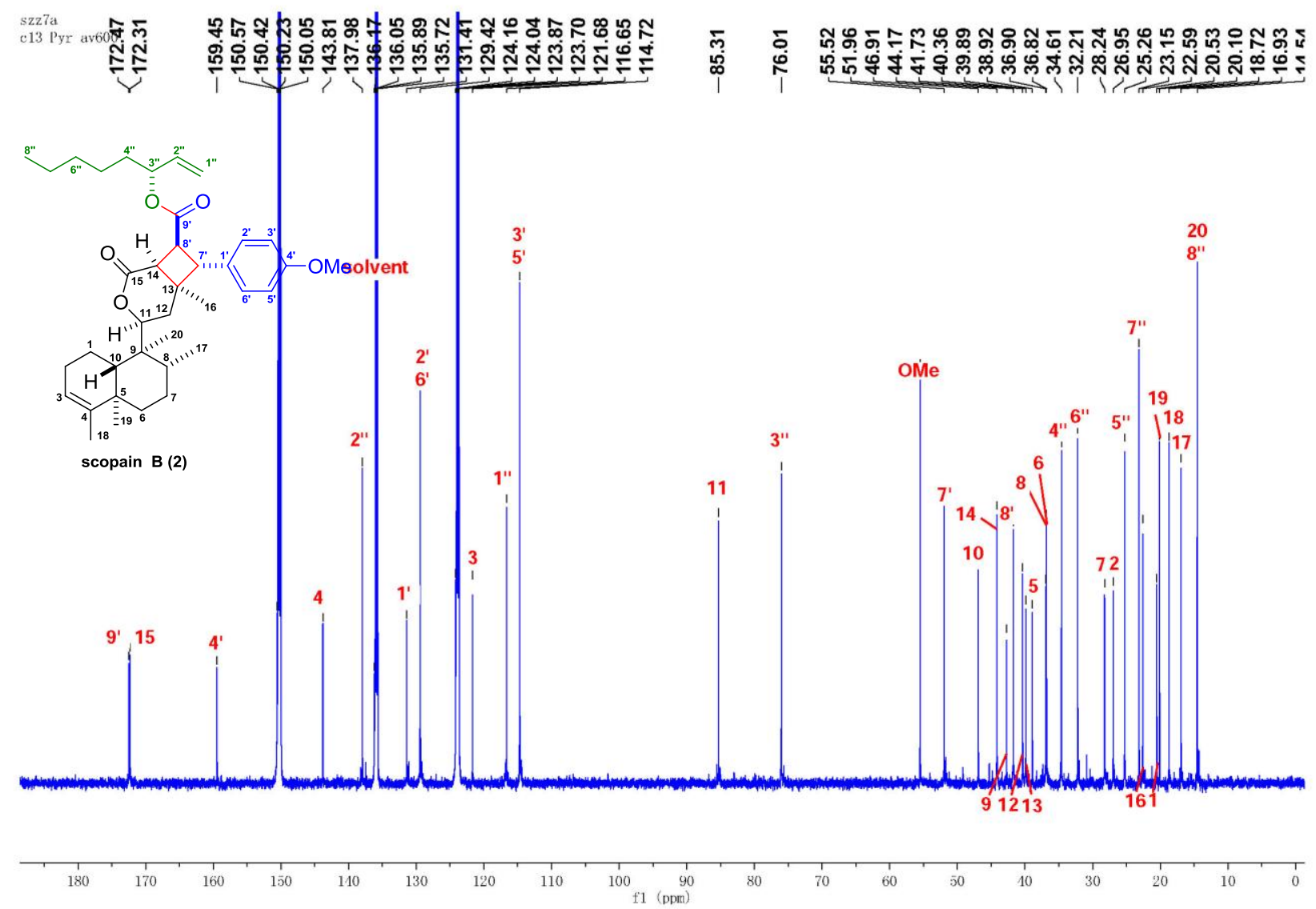
Figure S12. HSQC spectrum of scopariusicide B (2)

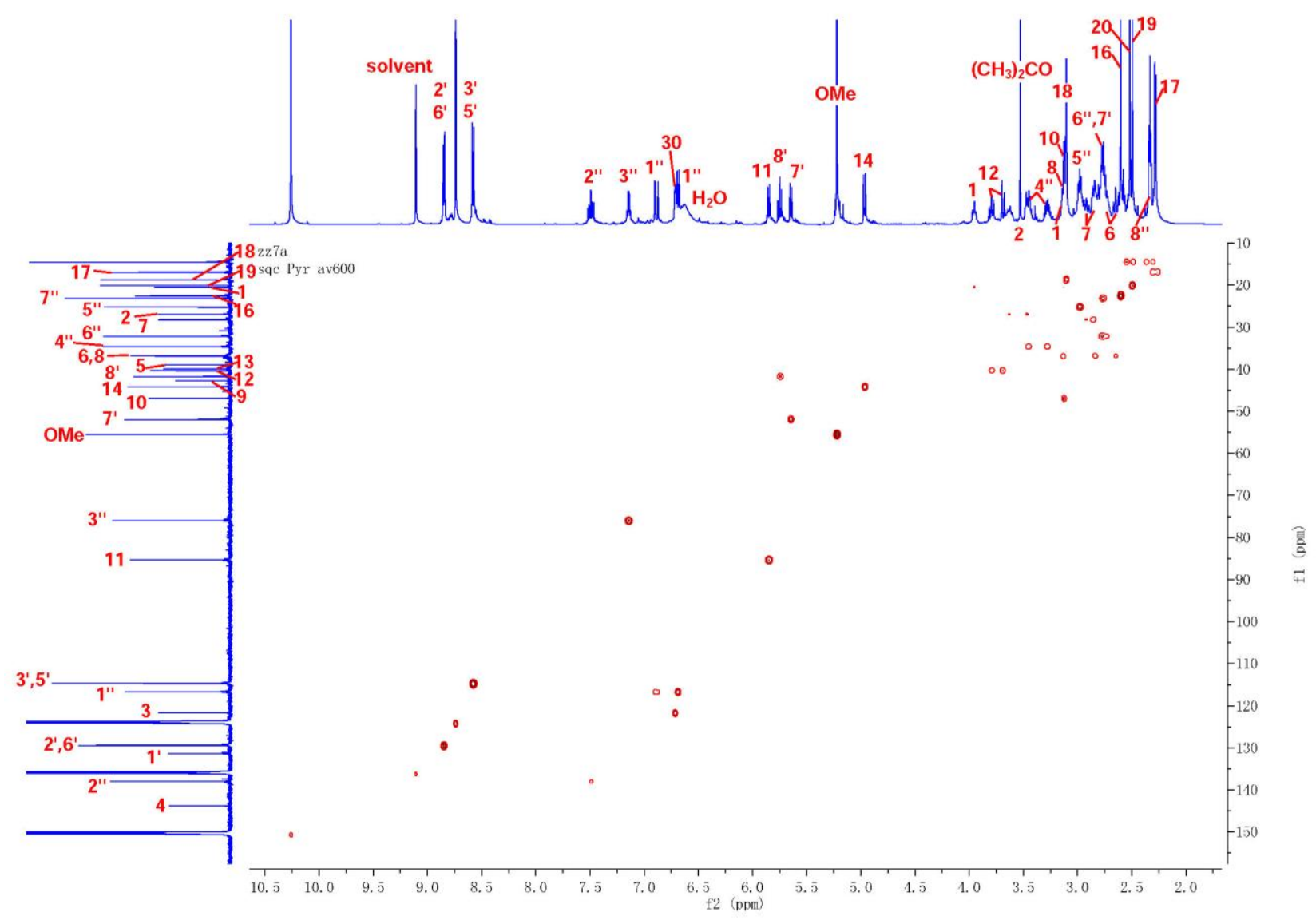

Figure S13. HMBC spectrum of scopariusicide B (2)

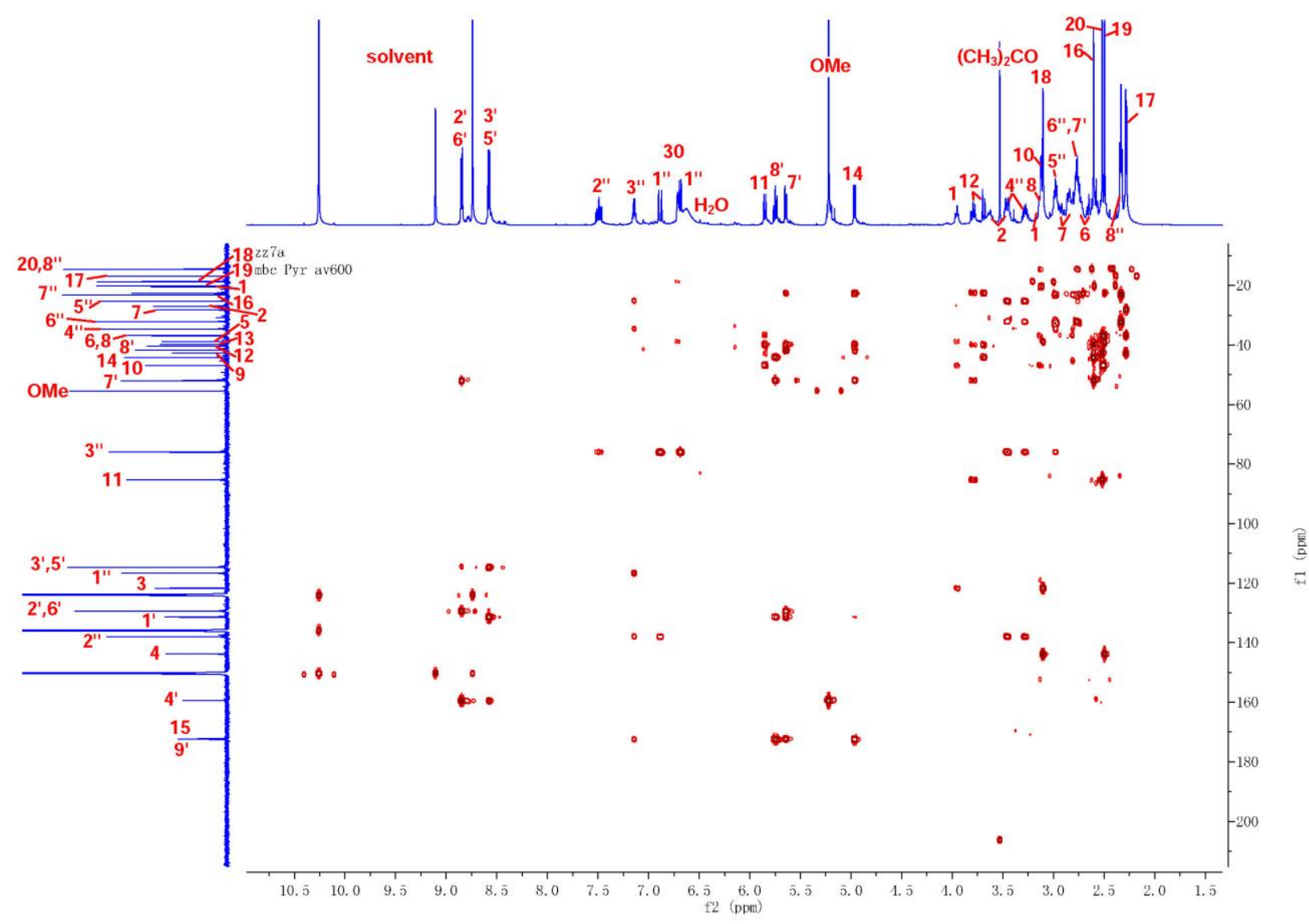


Figure S14. ${ }^{1} \mathrm{H}-{ }^{1} \mathrm{H}$ COSY spectrum of scopariusicide B (2)

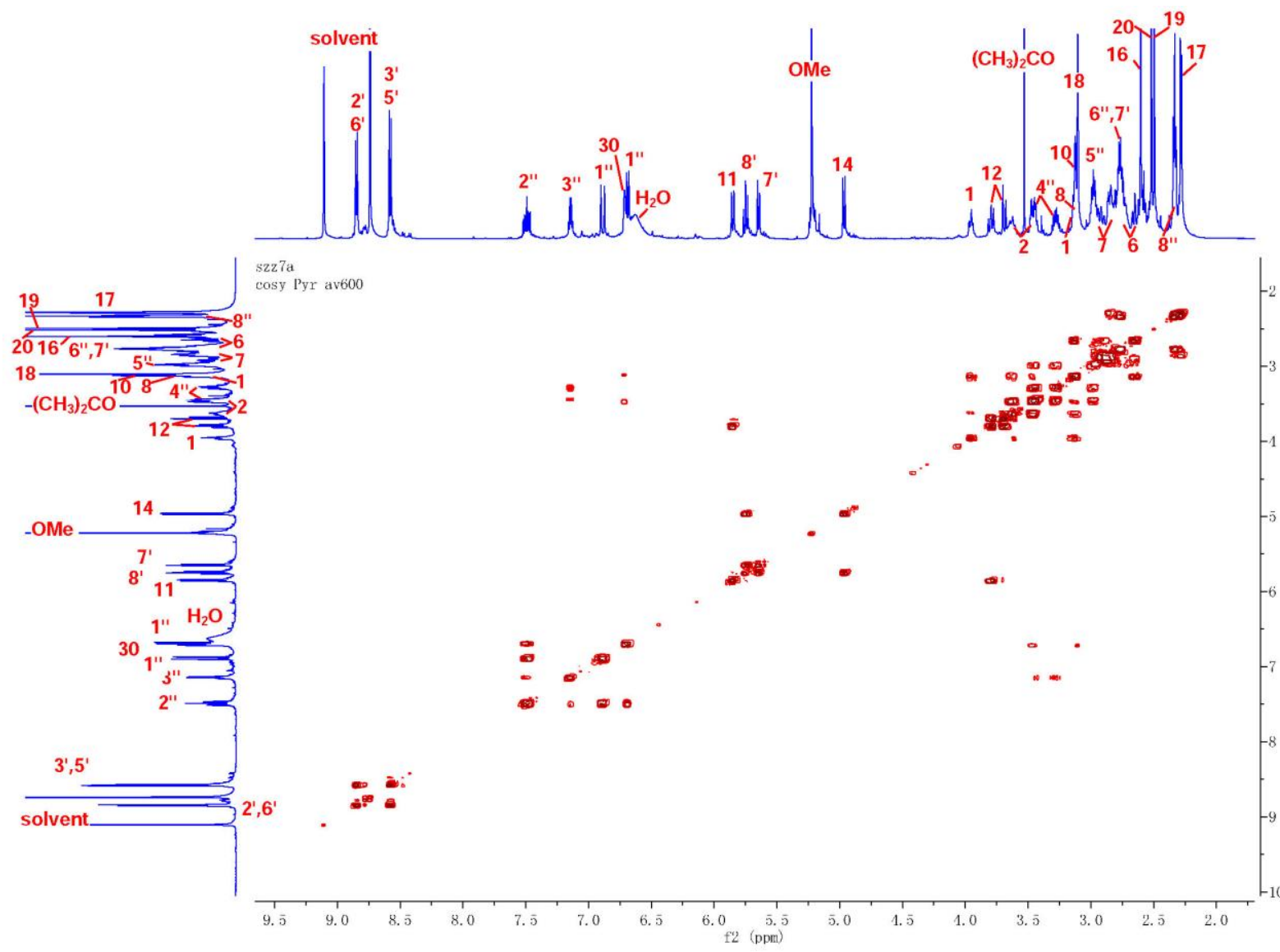

Figure S15. ROESY spectrum of scopariusicide B (2)

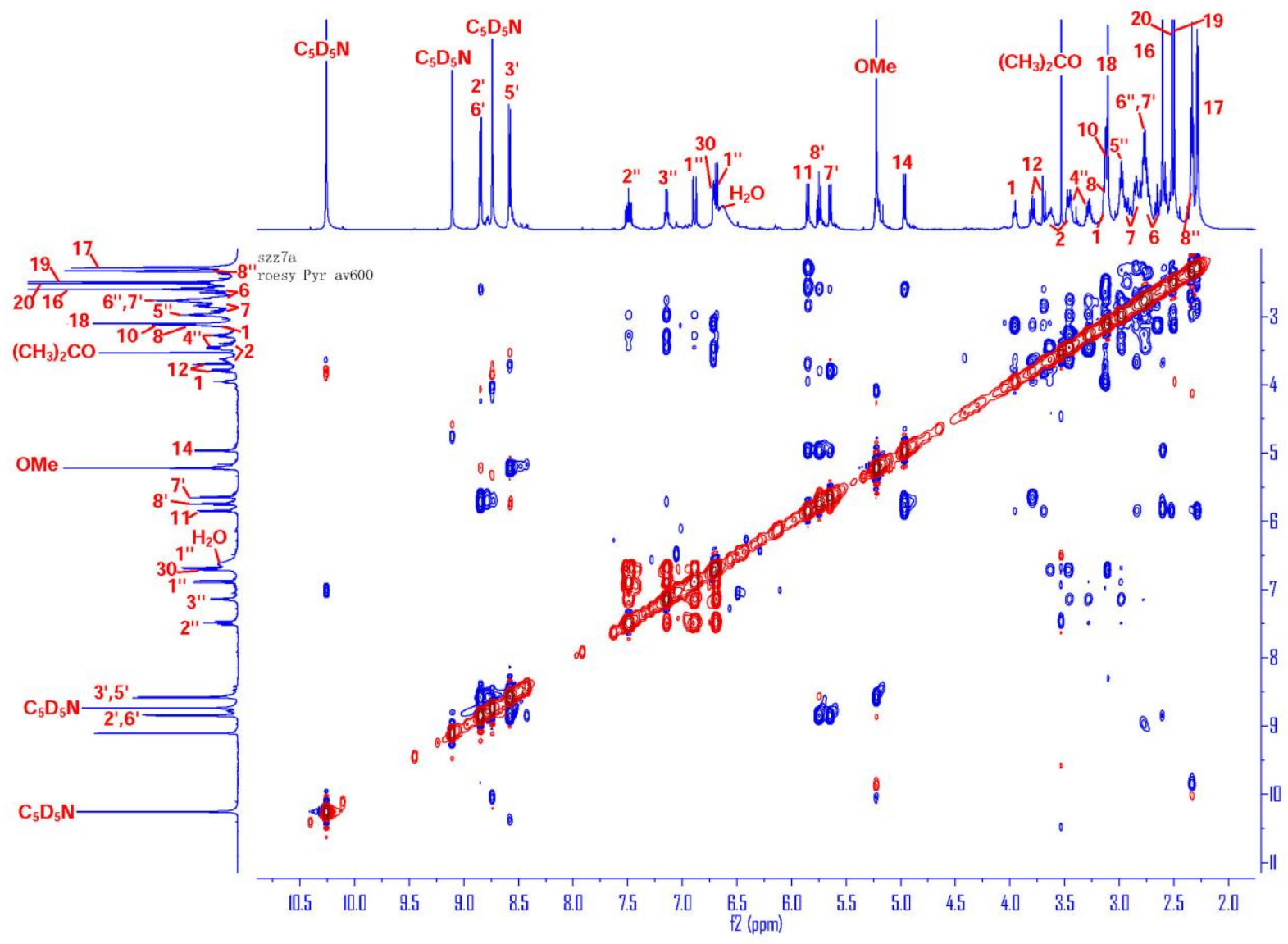


Figure S16. CD of scopariusicide B (2)
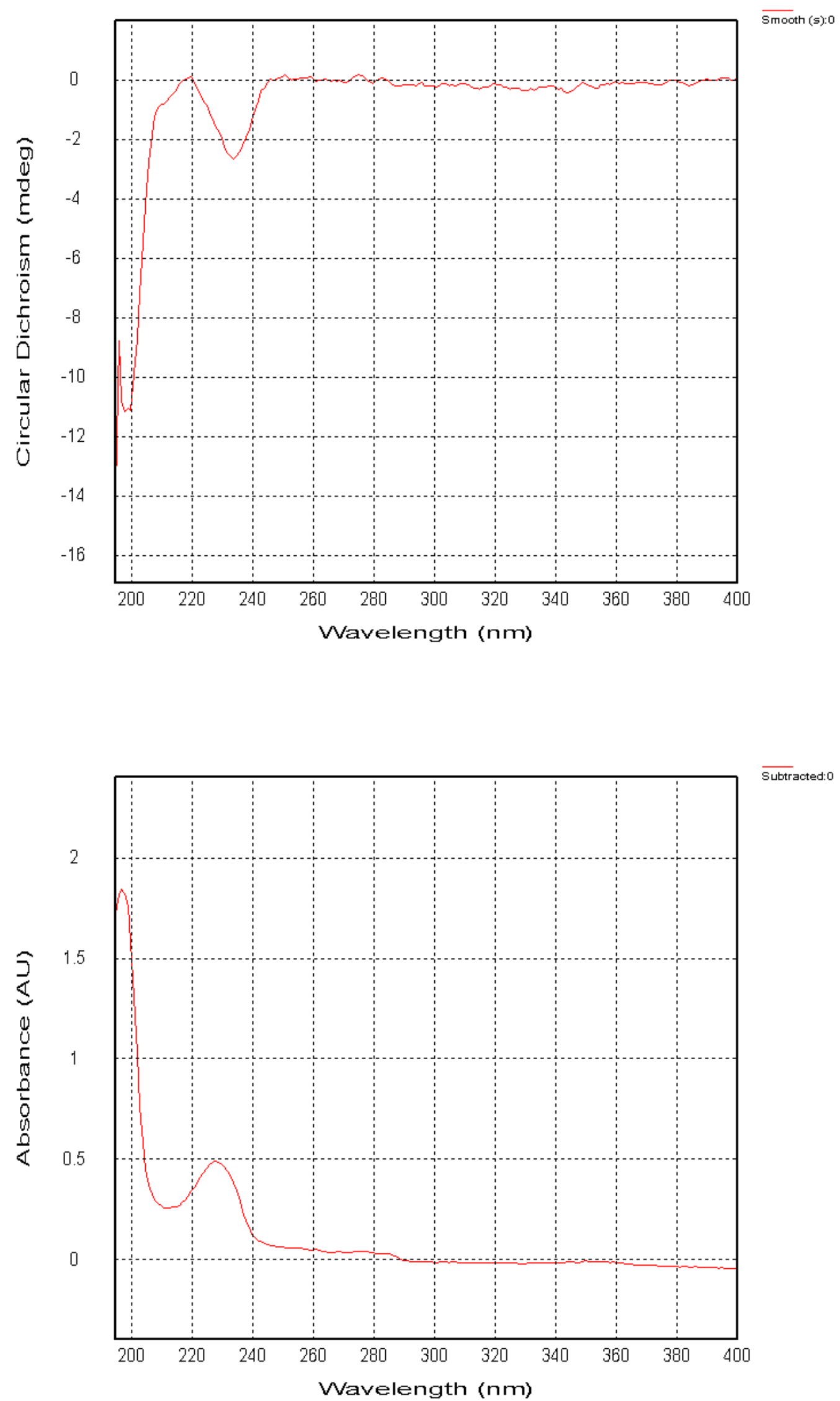
Figure S17. ${ }^{1} \mathrm{H}$ NMR spectrum of isoscoparin Q (3)

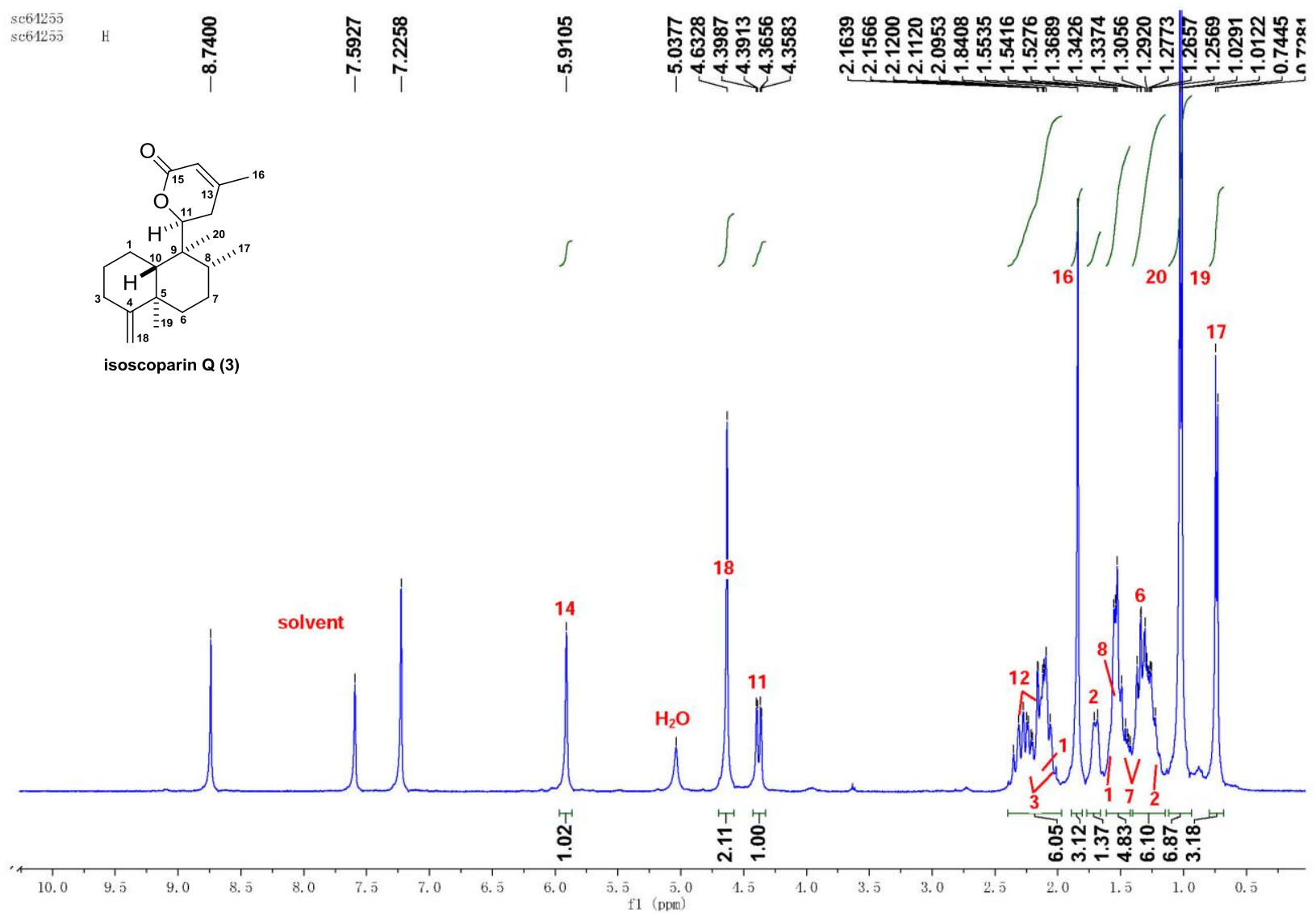

Figure S18. ${ }^{13} \mathrm{C}$ NMR spctrum of isoscoparin Q (3)
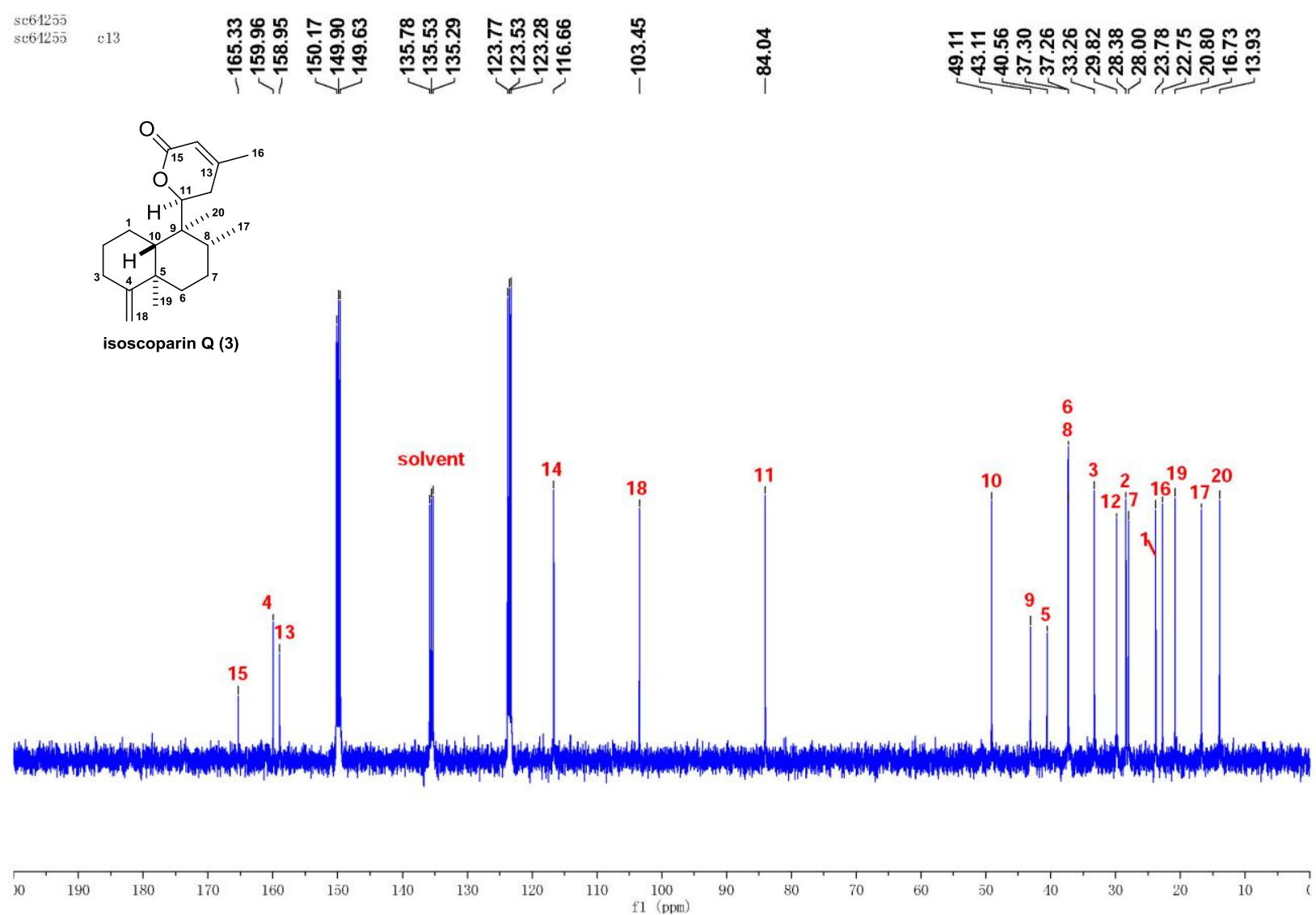
Figure S19. HSQC spectrum of isoscoparin Q (3)

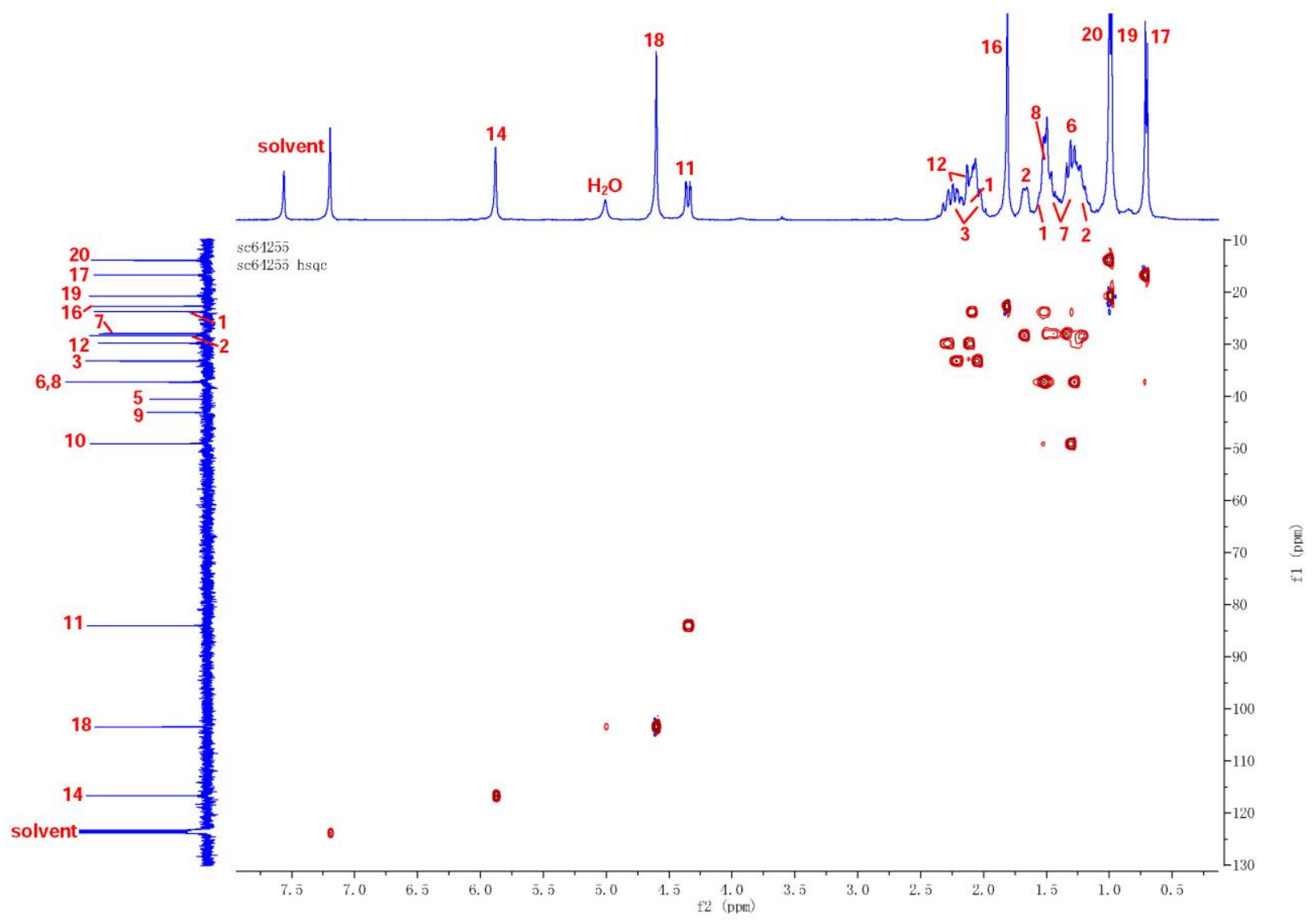

Figure S20. HMBC spectrum of isoscoparin Q (3)

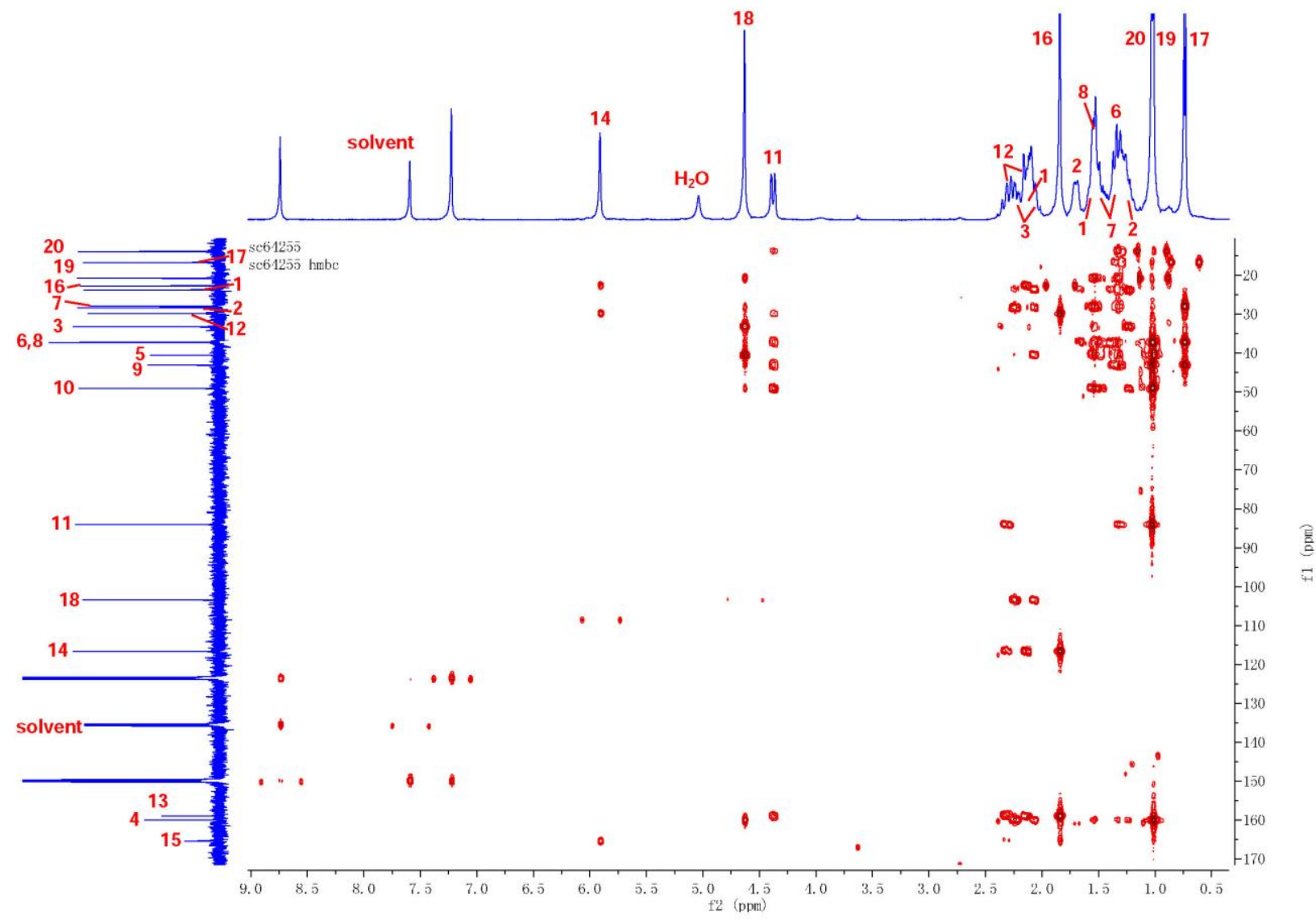


Figure S21. ${ }^{1} \mathrm{H}-{ }^{1} \mathrm{H}$ COSY spectrum of isoscoparin Q (3)

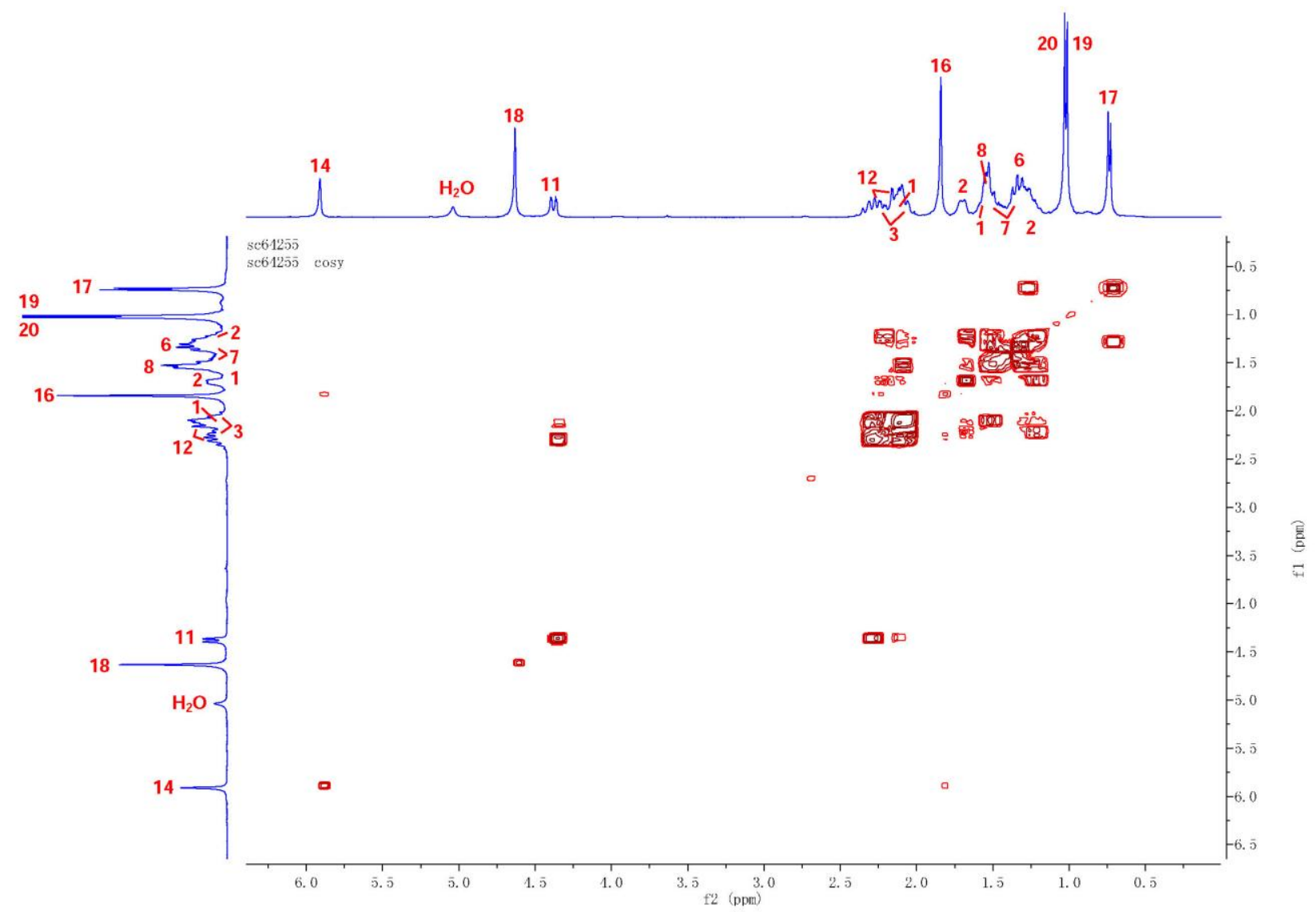

Figure S22. ROESY spectrum of isoscoparin Q (3)

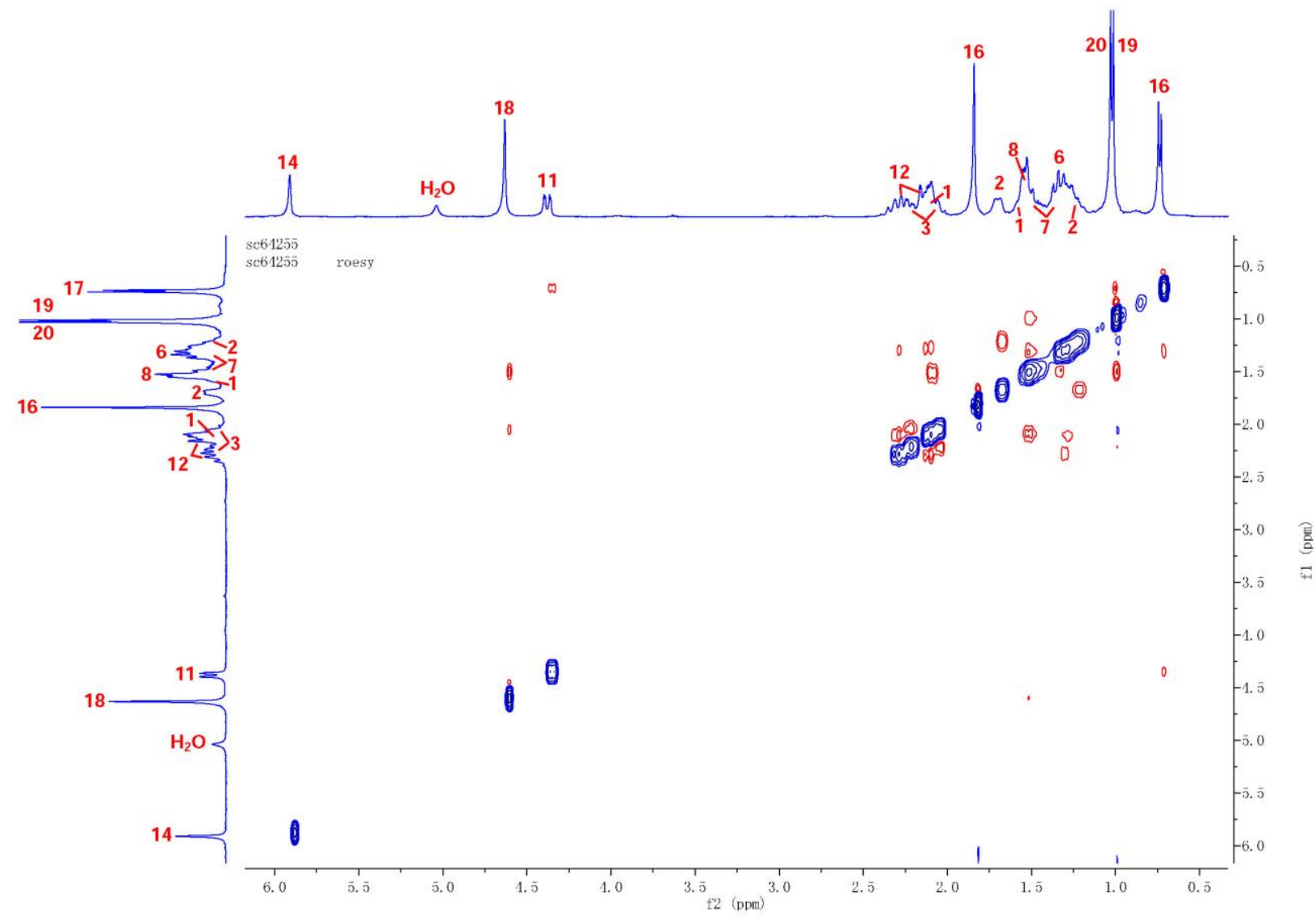


Figure S23. X-ray structure of isoscoparin Q (3)
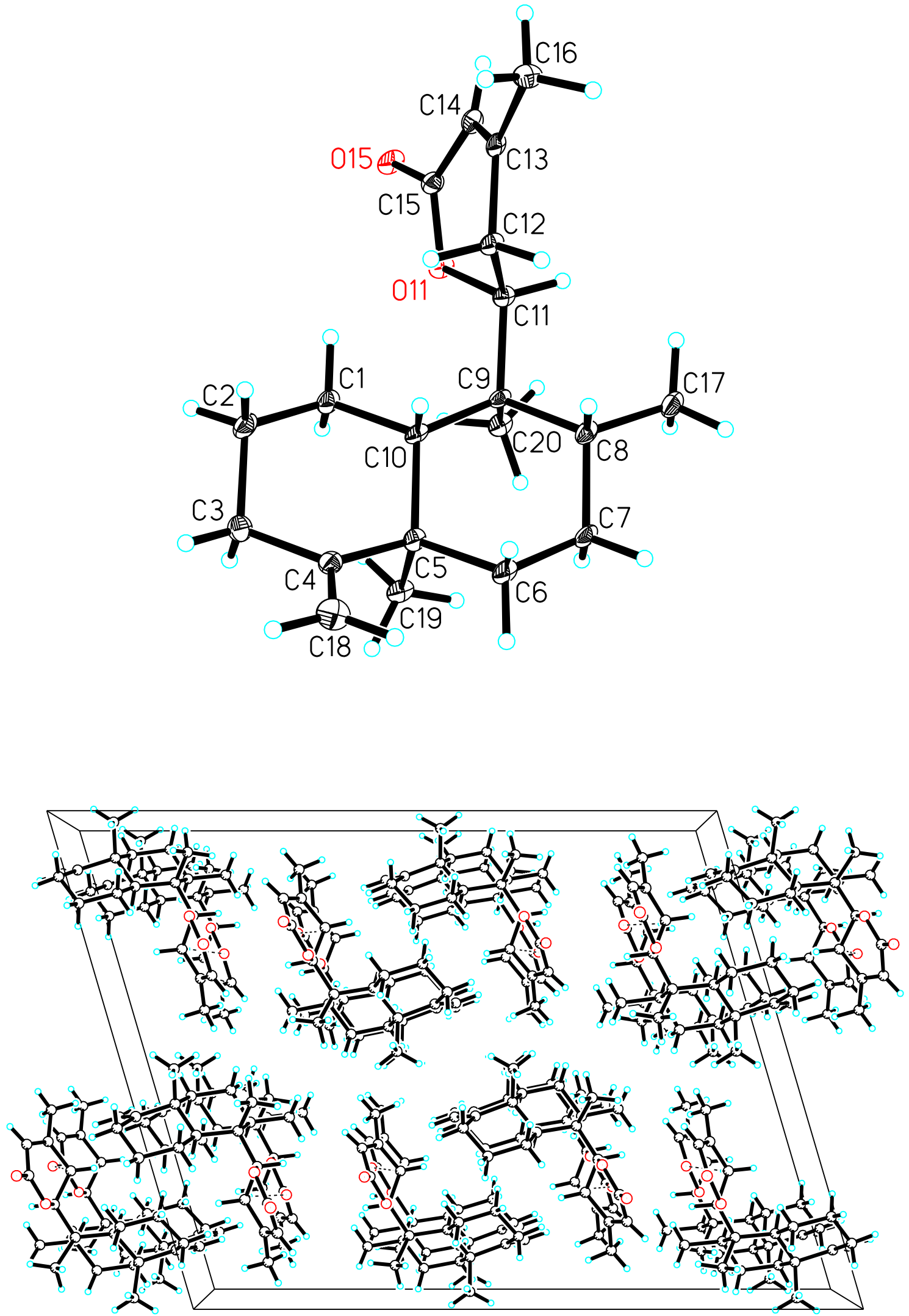
Figure S24. ${ }^{1} \mathrm{H}$ NMR spectrum of $\mathbf{6 a}$

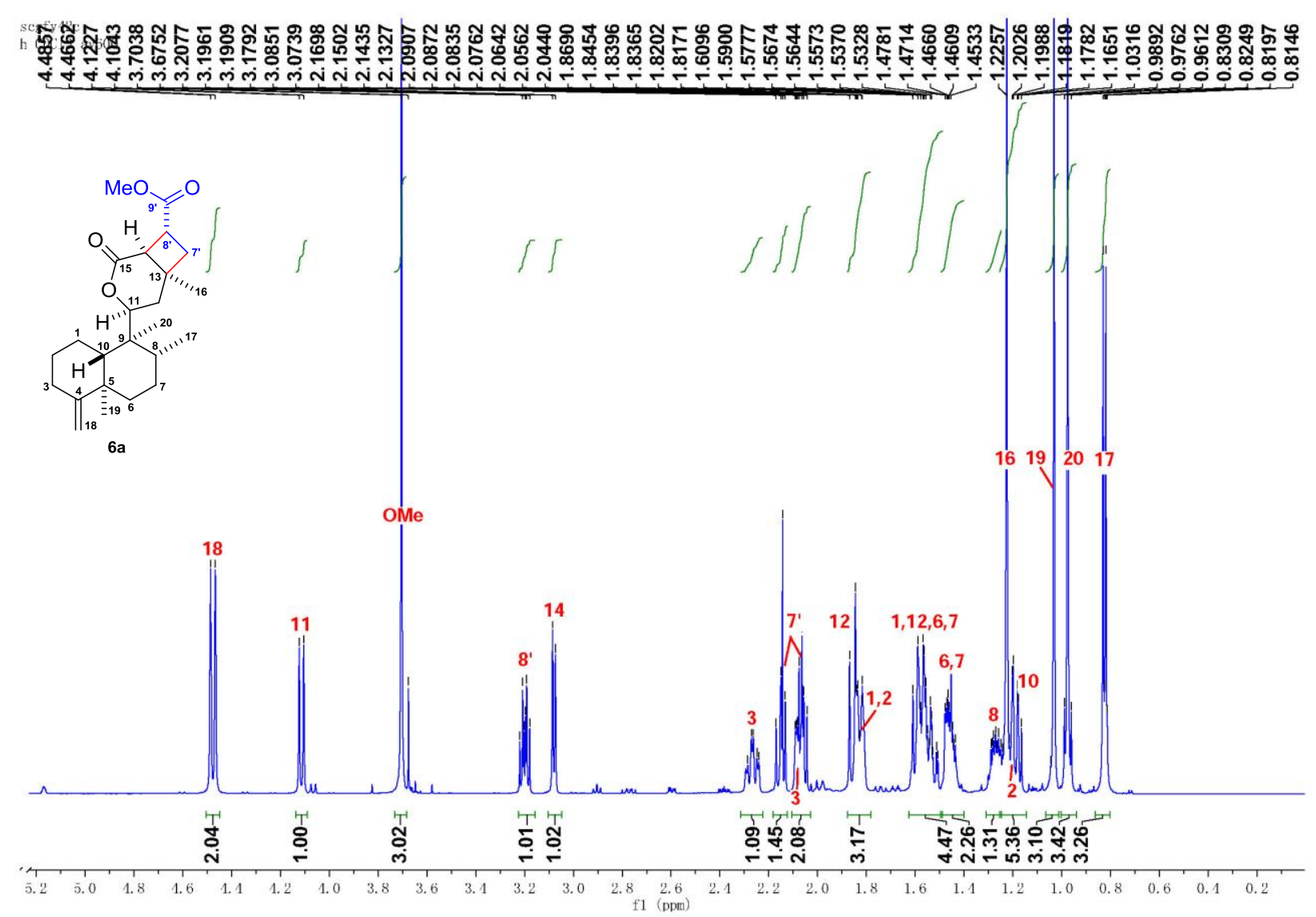

Figure S25. ${ }^{13} \mathrm{C}$ NMR spectrum of $\mathbf{6 a}$
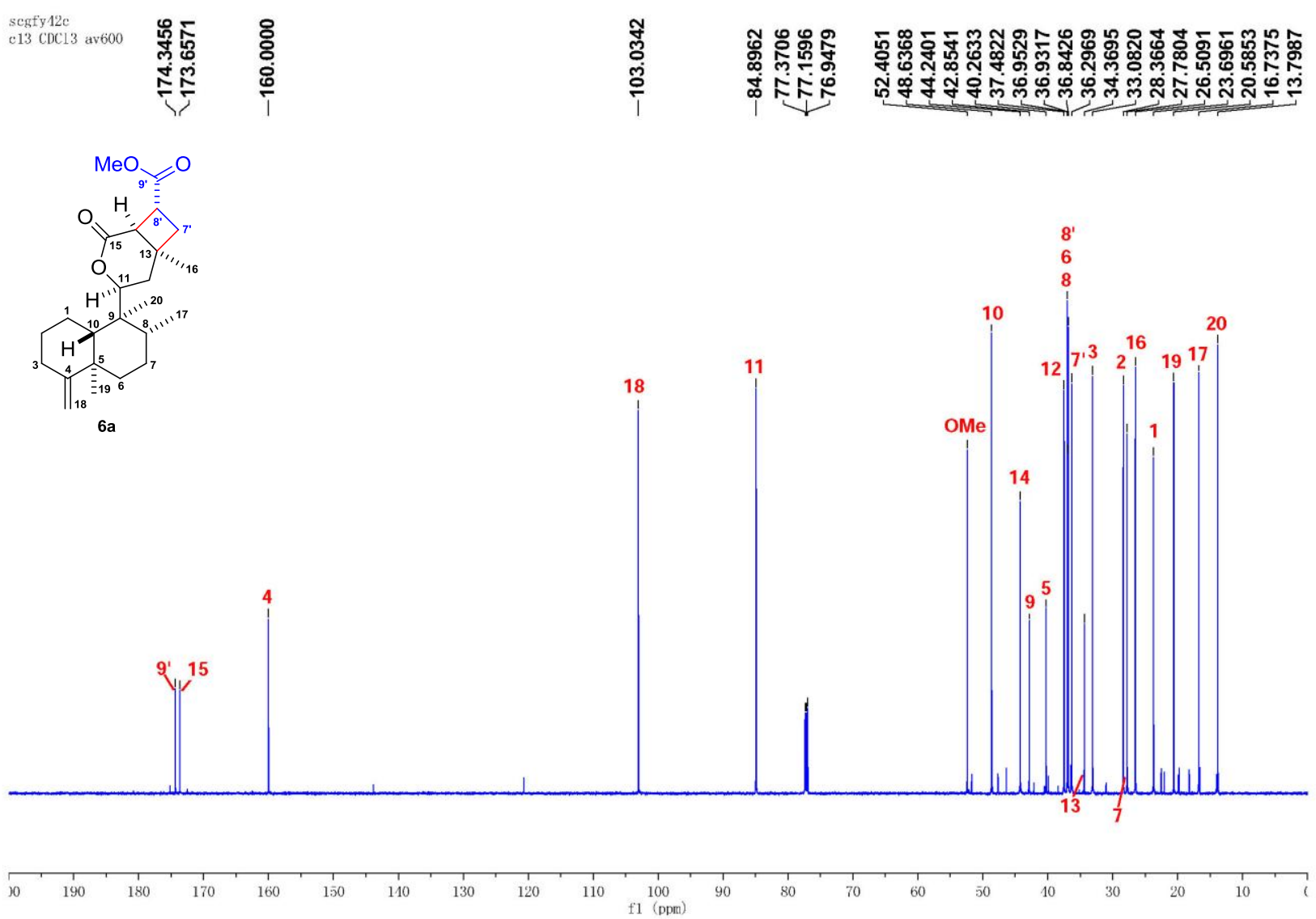
Figure S26. HSQC spectrum of $\mathbf{6 a}$

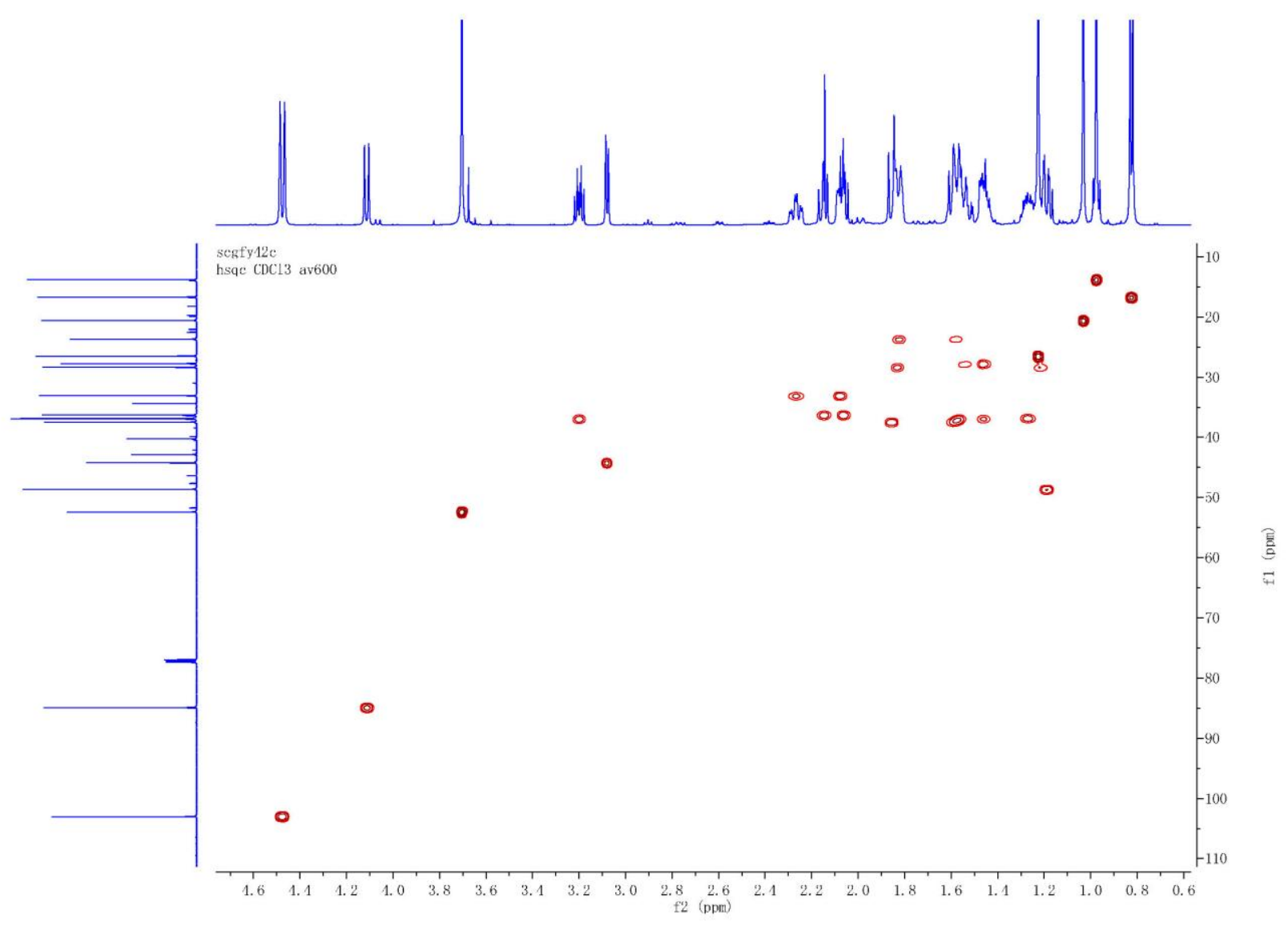

Figure S27. HMBC spectrum of $\mathbf{6 a}$

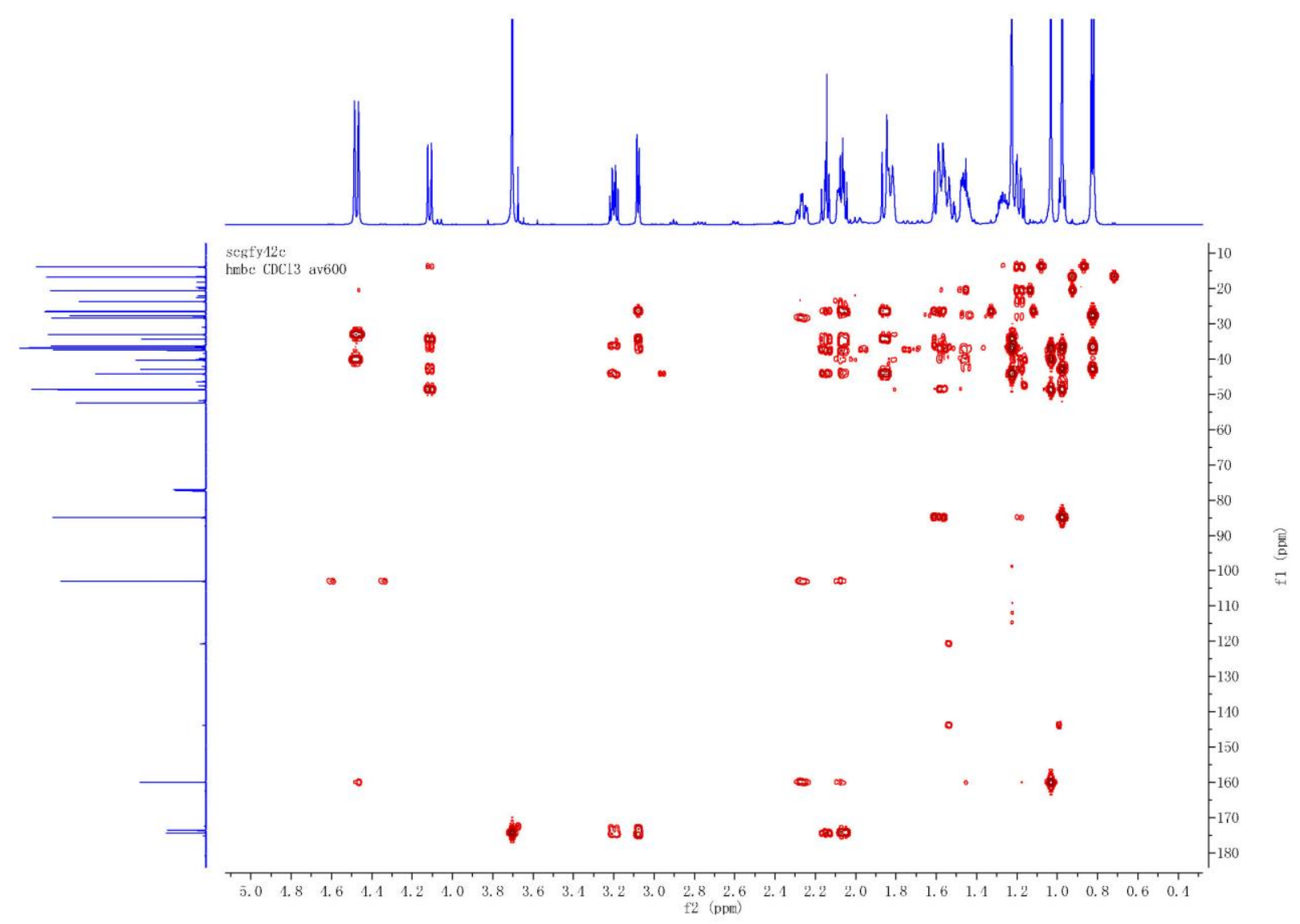


Figure S28. ${ }^{1} \mathrm{H}^{-1} \mathrm{H}$ COSY spectrum of $\mathbf{6 a}$

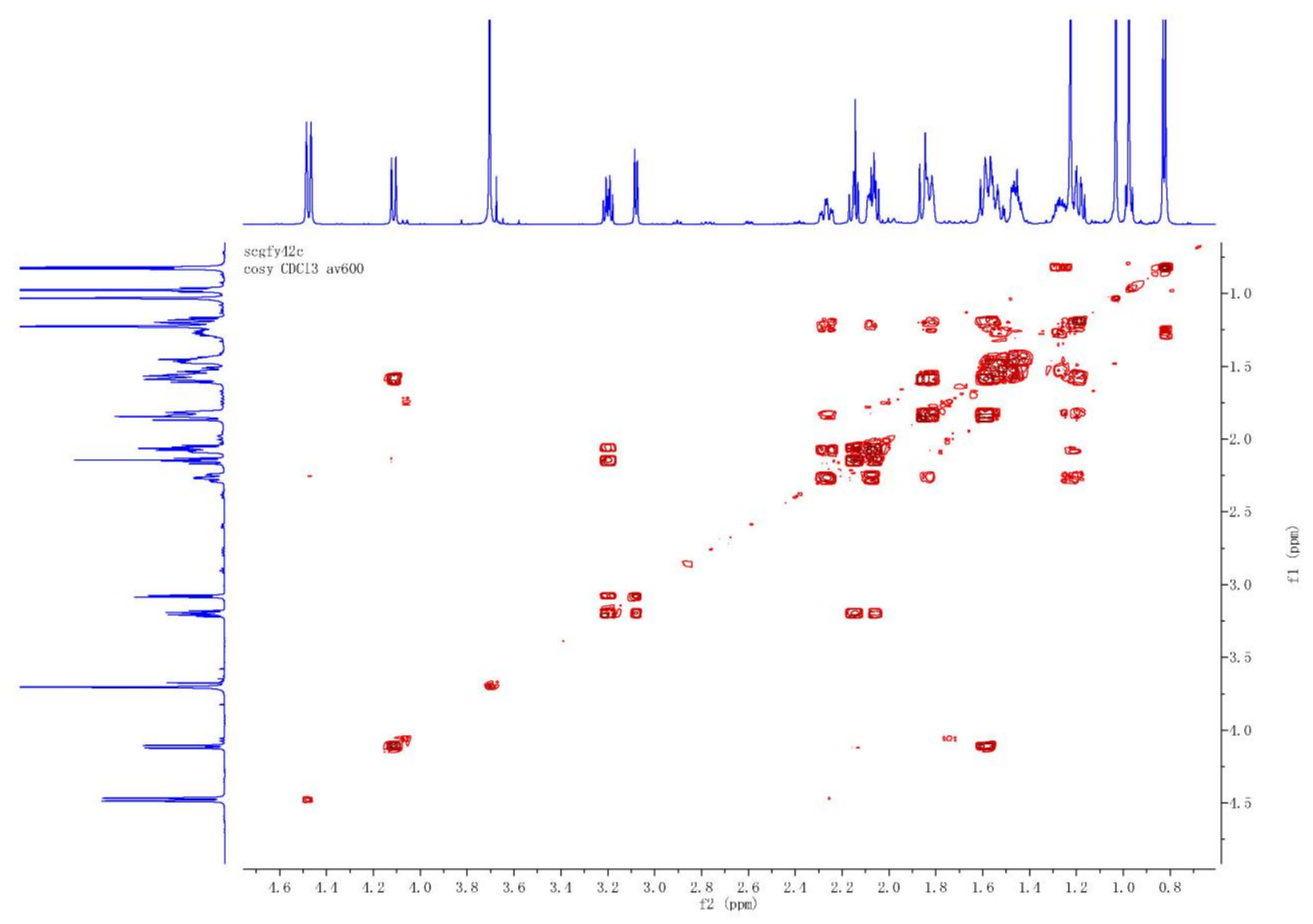

Figure S29. ROESY spectrum of $\mathbf{6 a}$

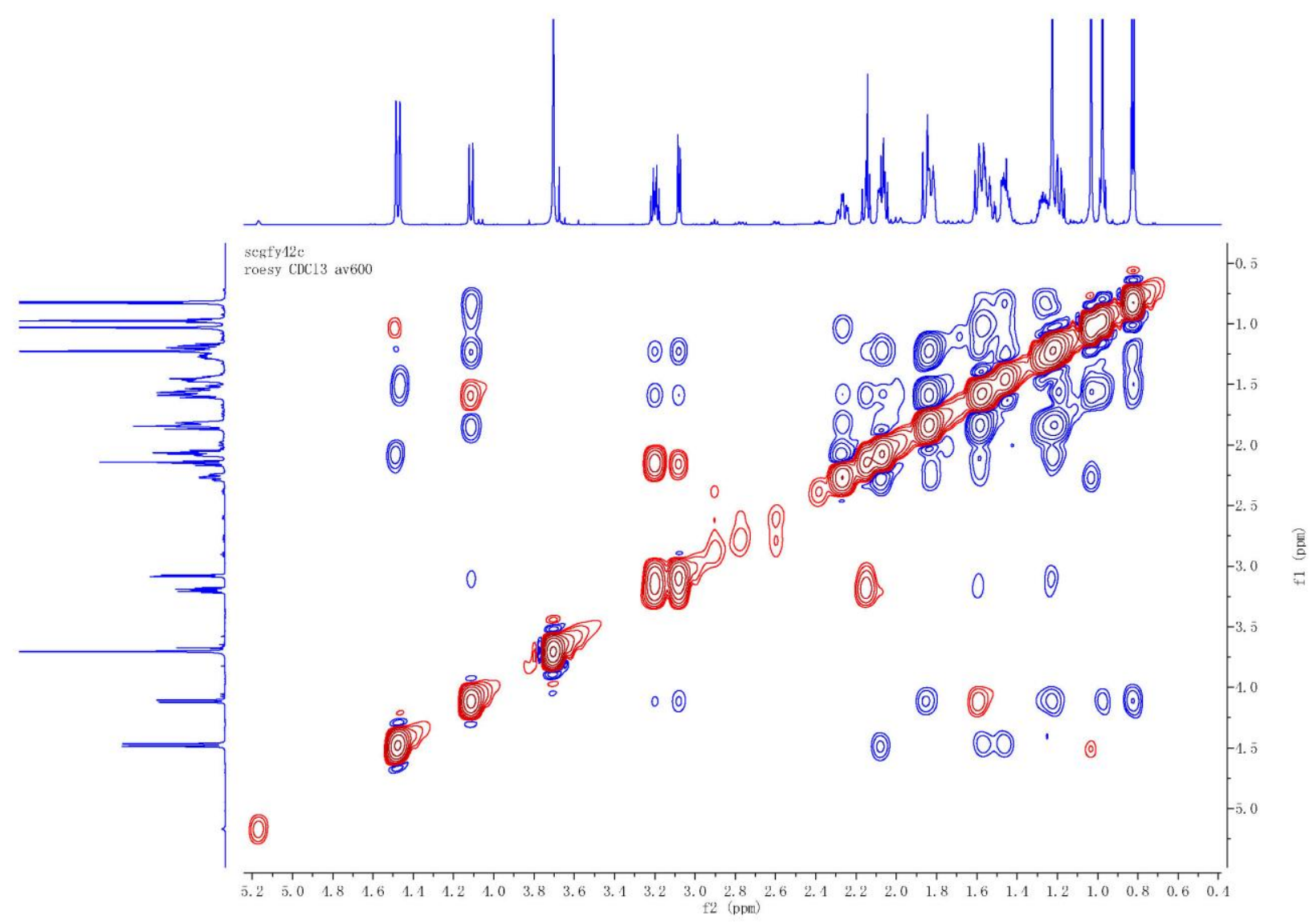


Figure S30. X-ray structure of $6 \mathbf{a}$
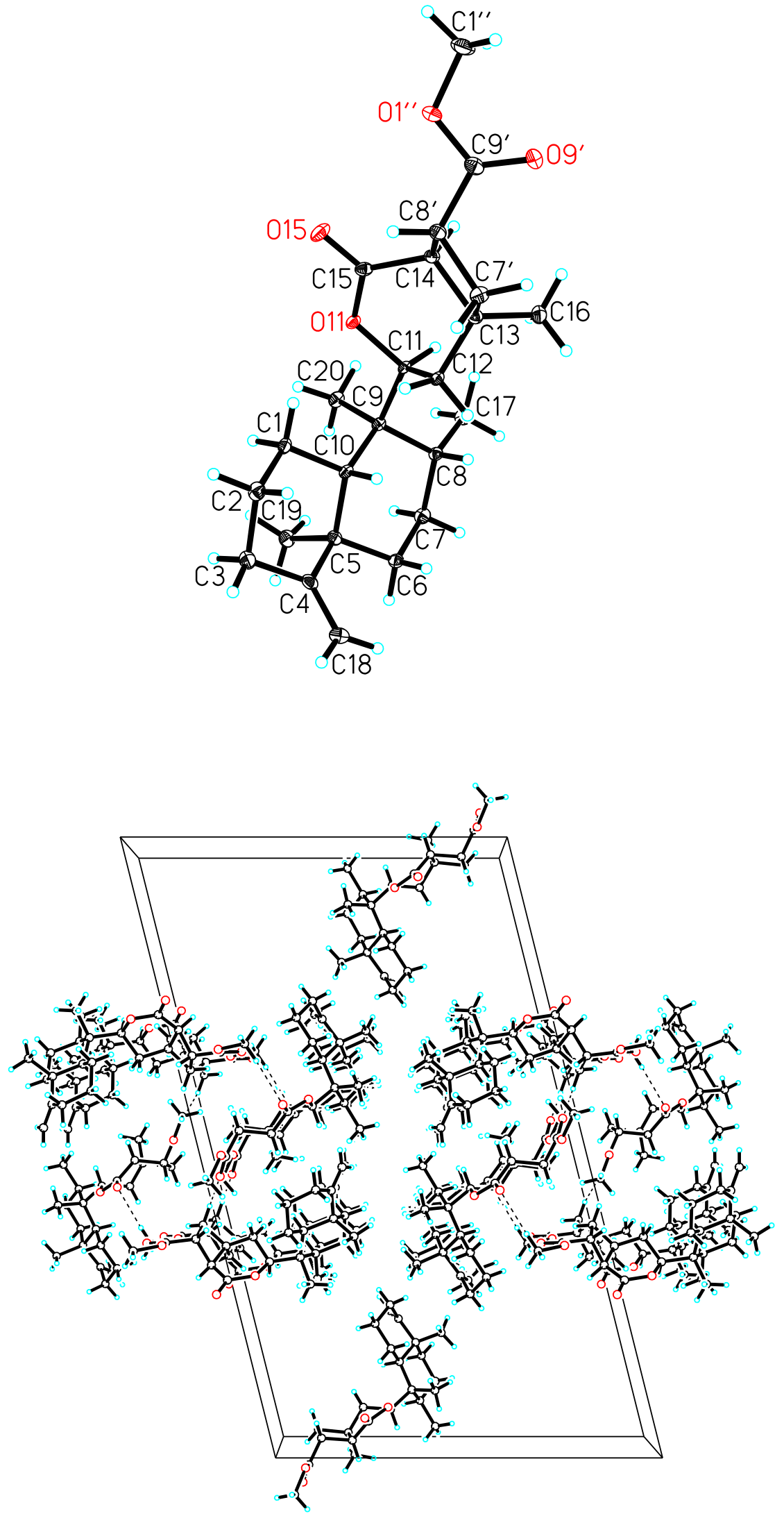
Figure S31. ${ }^{1} \mathrm{H}$ NMR spectrum of $\mathbf{6 b}$

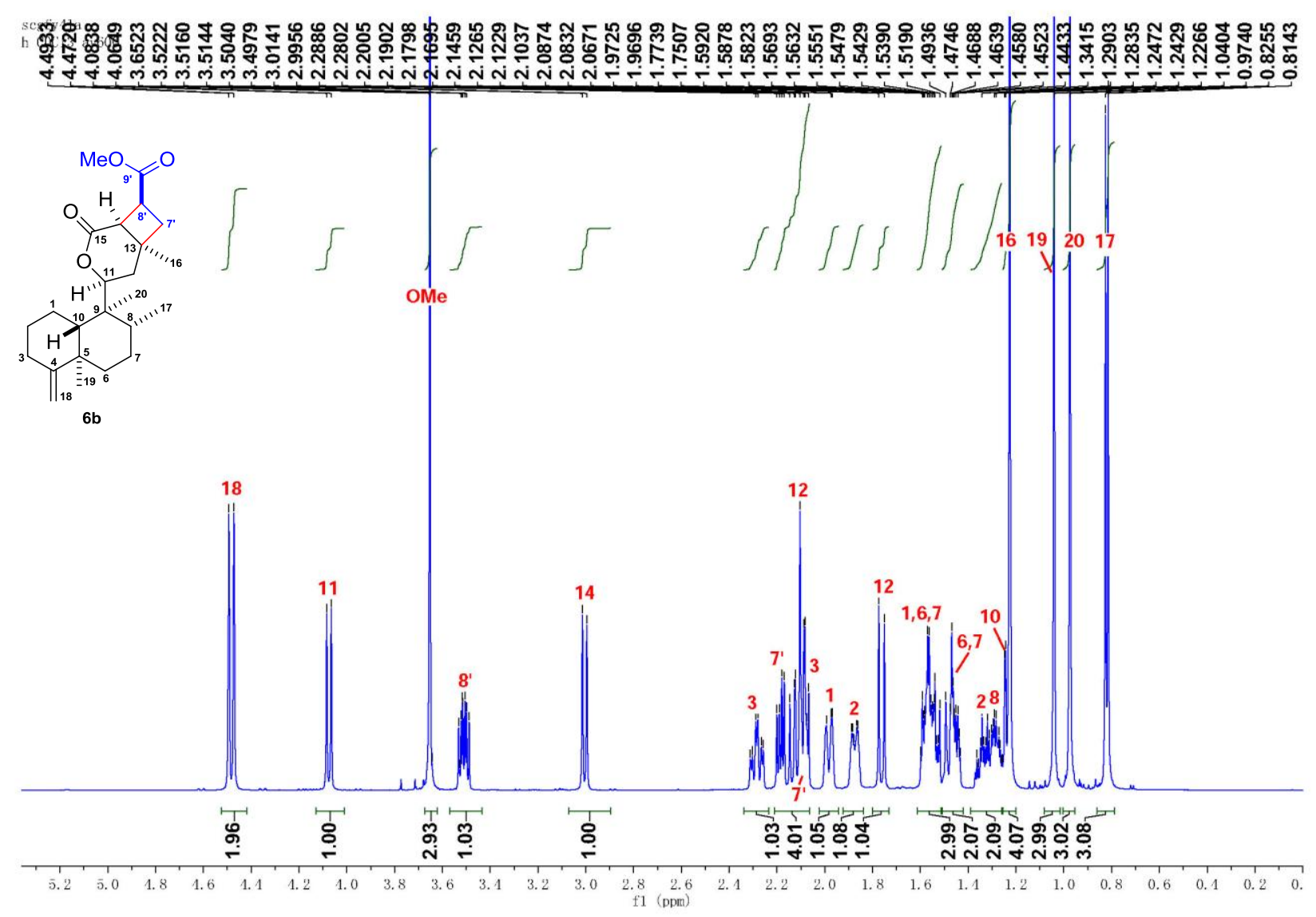

Figure S32. ${ }^{13} \mathrm{C}$ NMR spectrum of $\mathbf{6 b}$
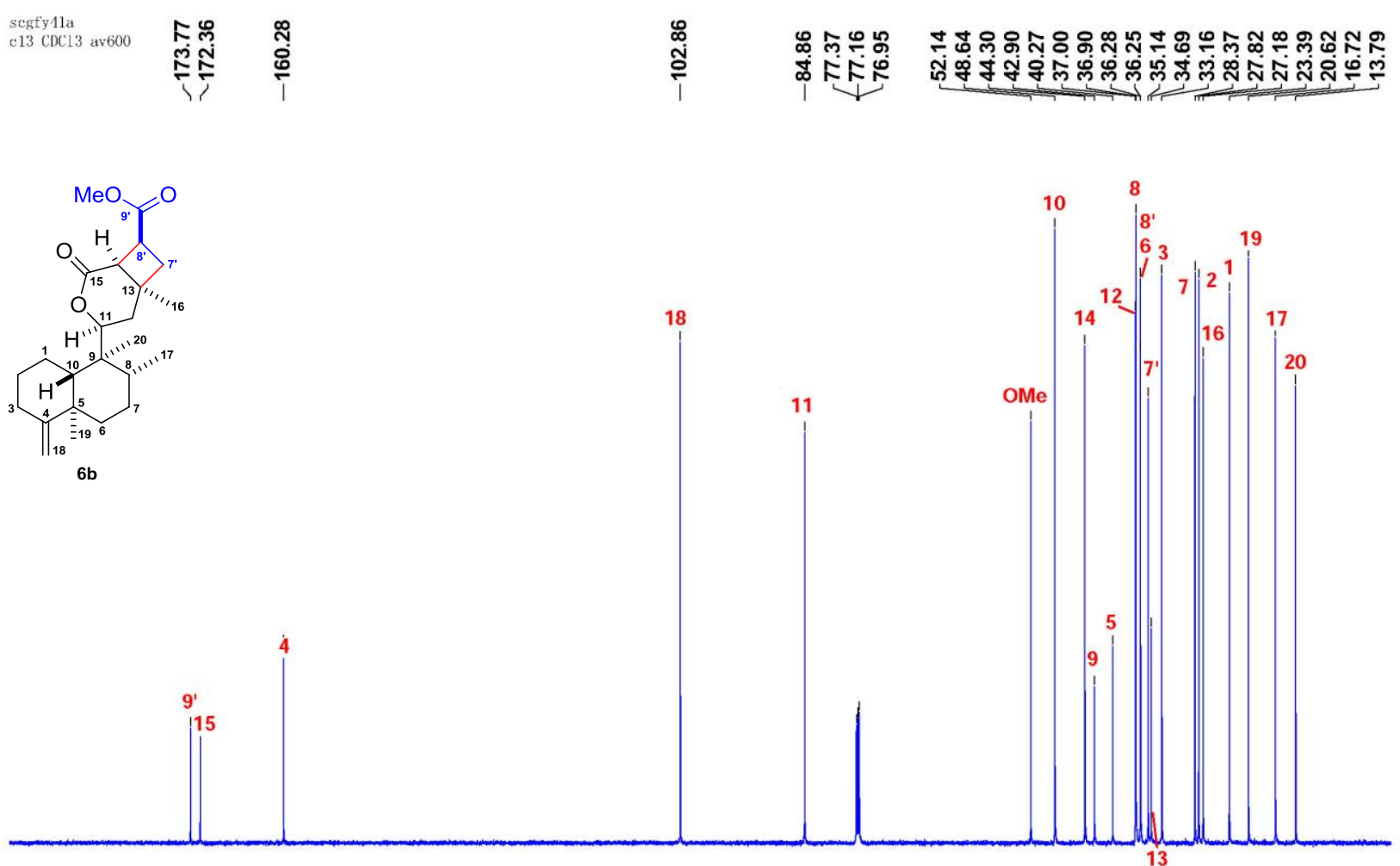

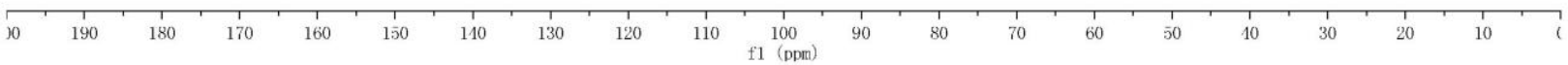


Figure S33. HSQC spectrum of $\mathbf{6 b}$

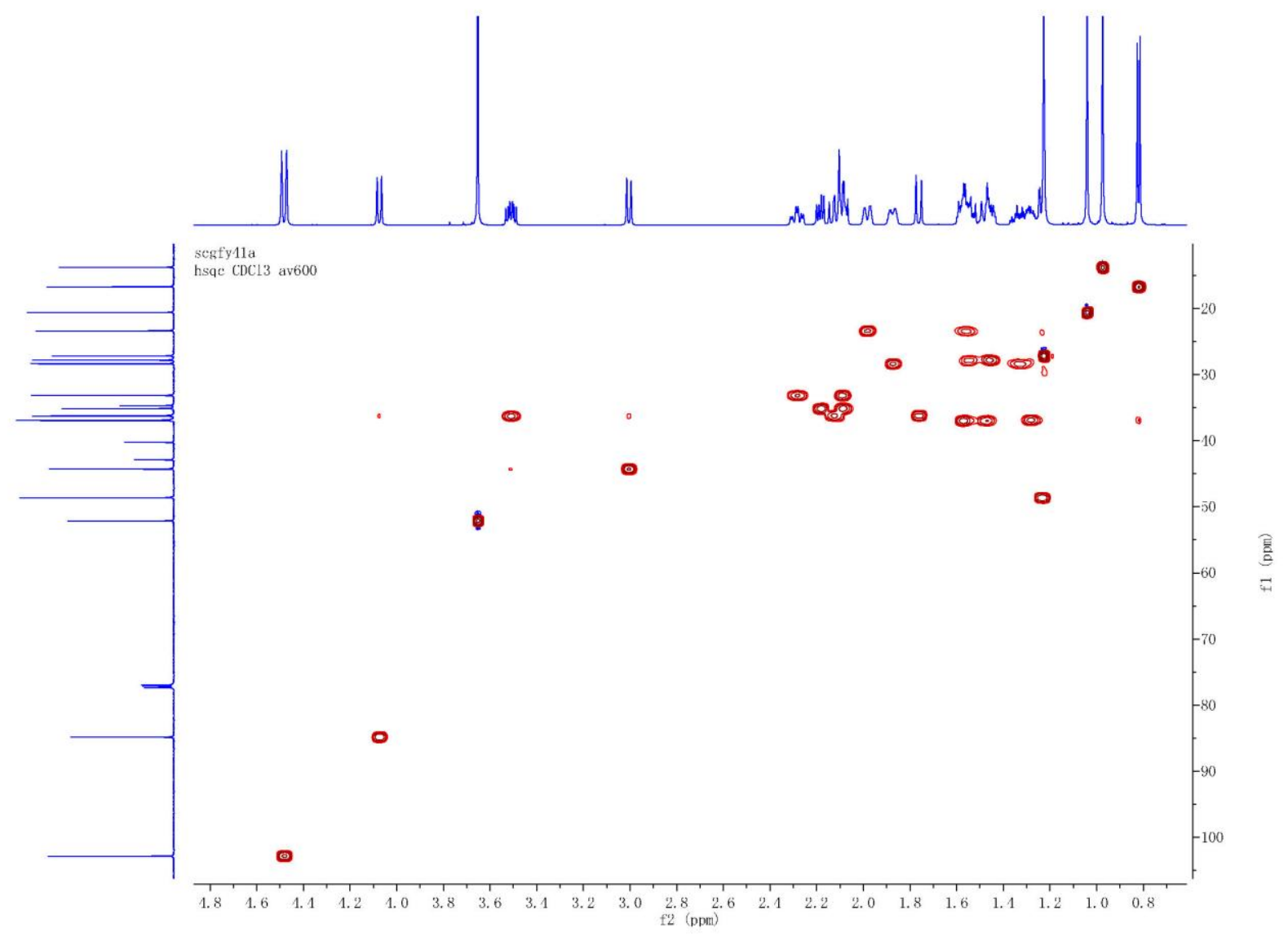

Figure S34. HMBC spectrum of $\mathbf{6 b}$

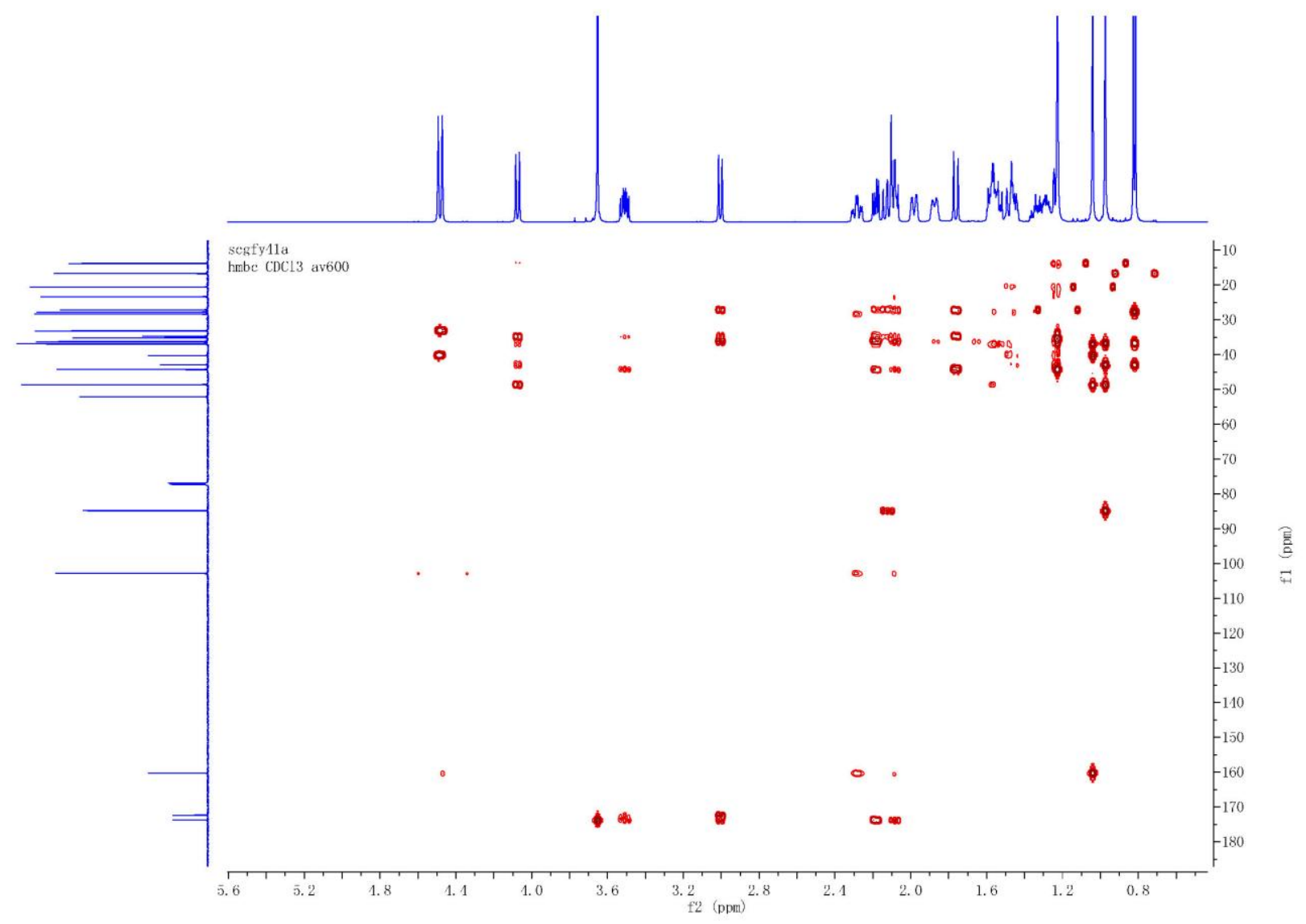


Figure $\mathrm{S} 35 .{ }^{1} \mathrm{H}-{ }^{1} \mathrm{H}$ COSY spectrum of $\mathbf{6 b}$

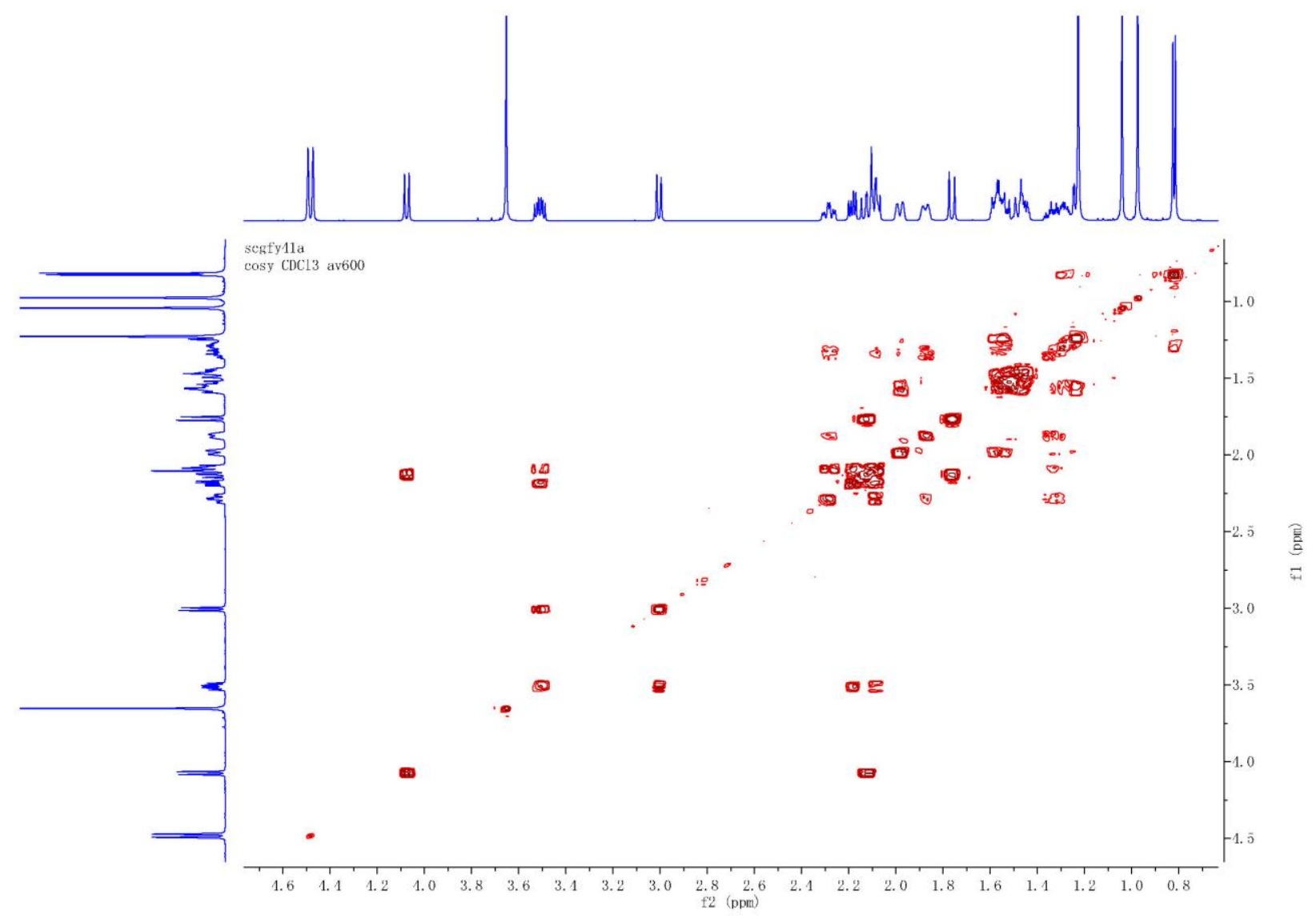

Figure S36. ROESY spectrum of $\mathbf{6 b}$

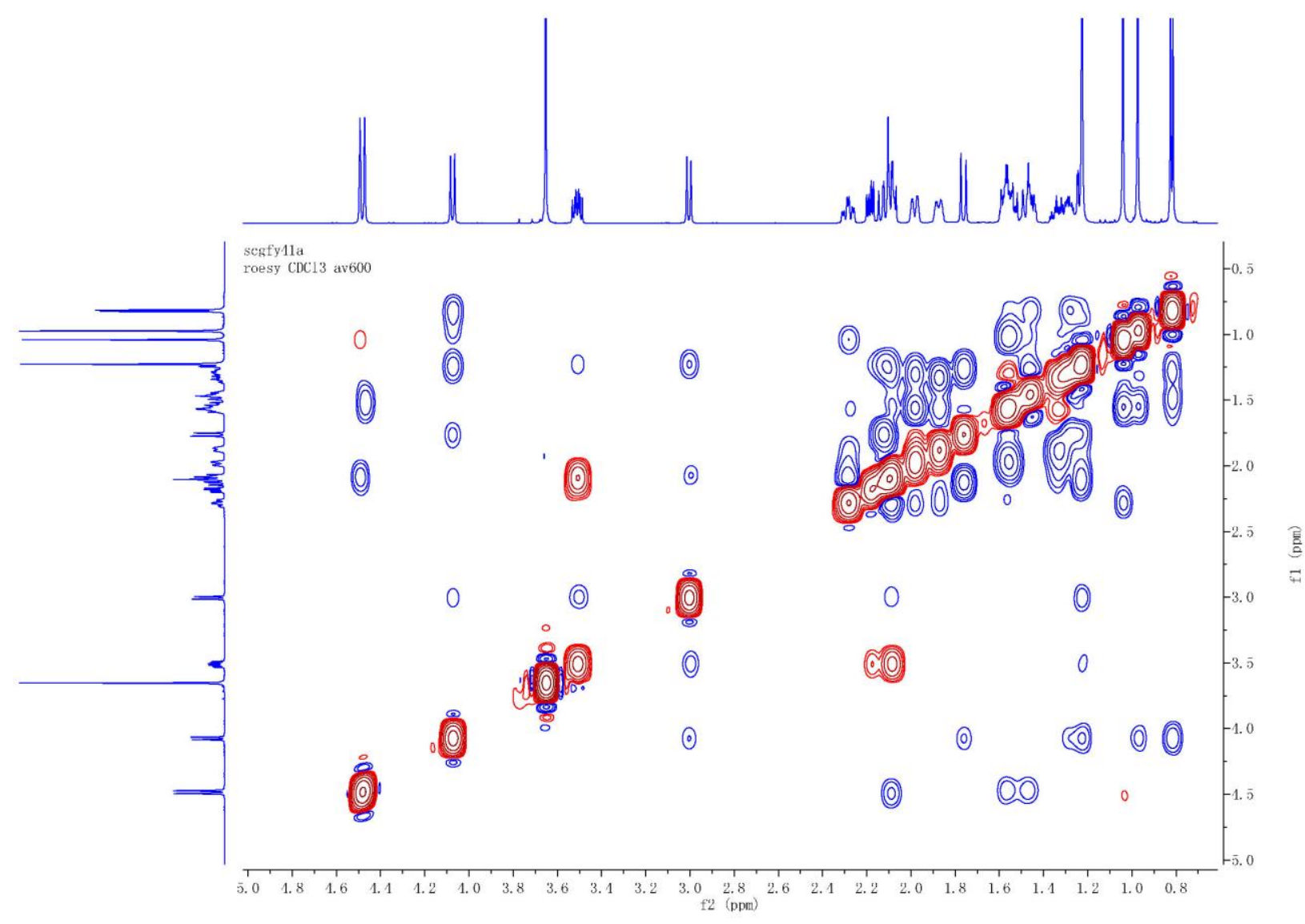


Figure S37. X-ray structure of $\mathbf{6 b}$
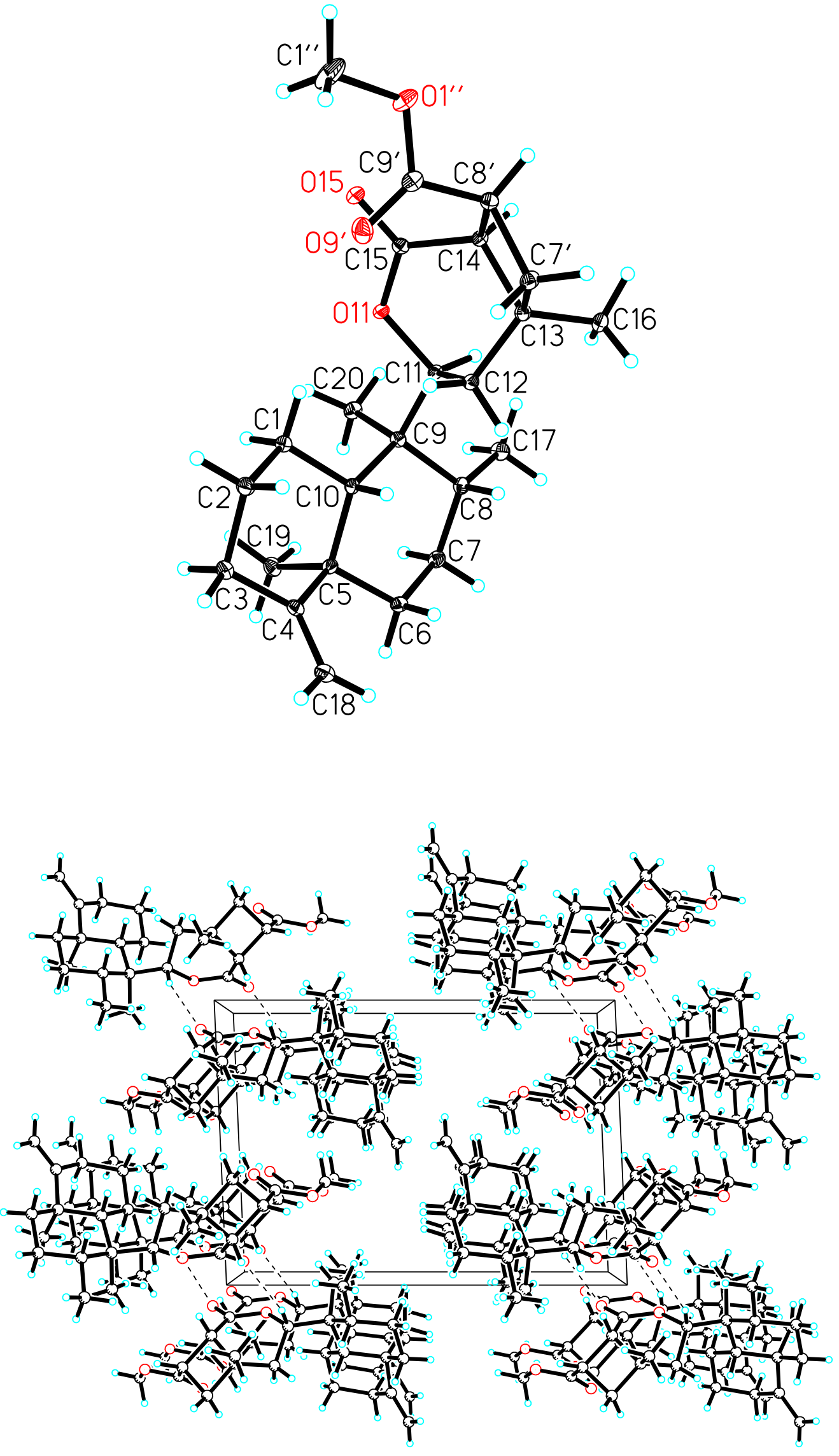
Figure S38. ${ }^{1} \mathrm{H}$ NMR spectrum of $\mathbf{6 c}$

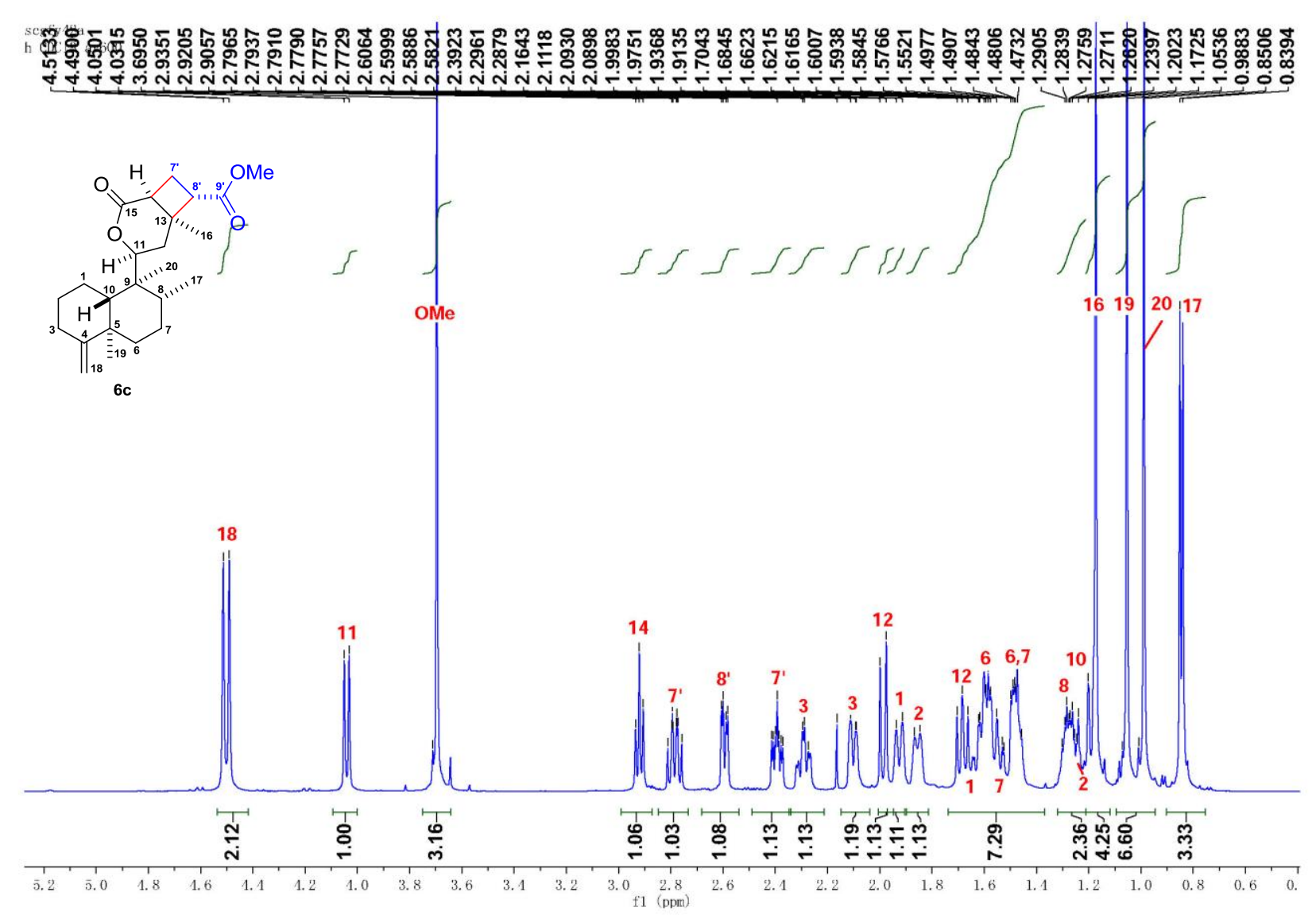

Figure S39. ${ }^{13} \mathrm{C}$ NMR spectrum of $\mathbf{6 c}$
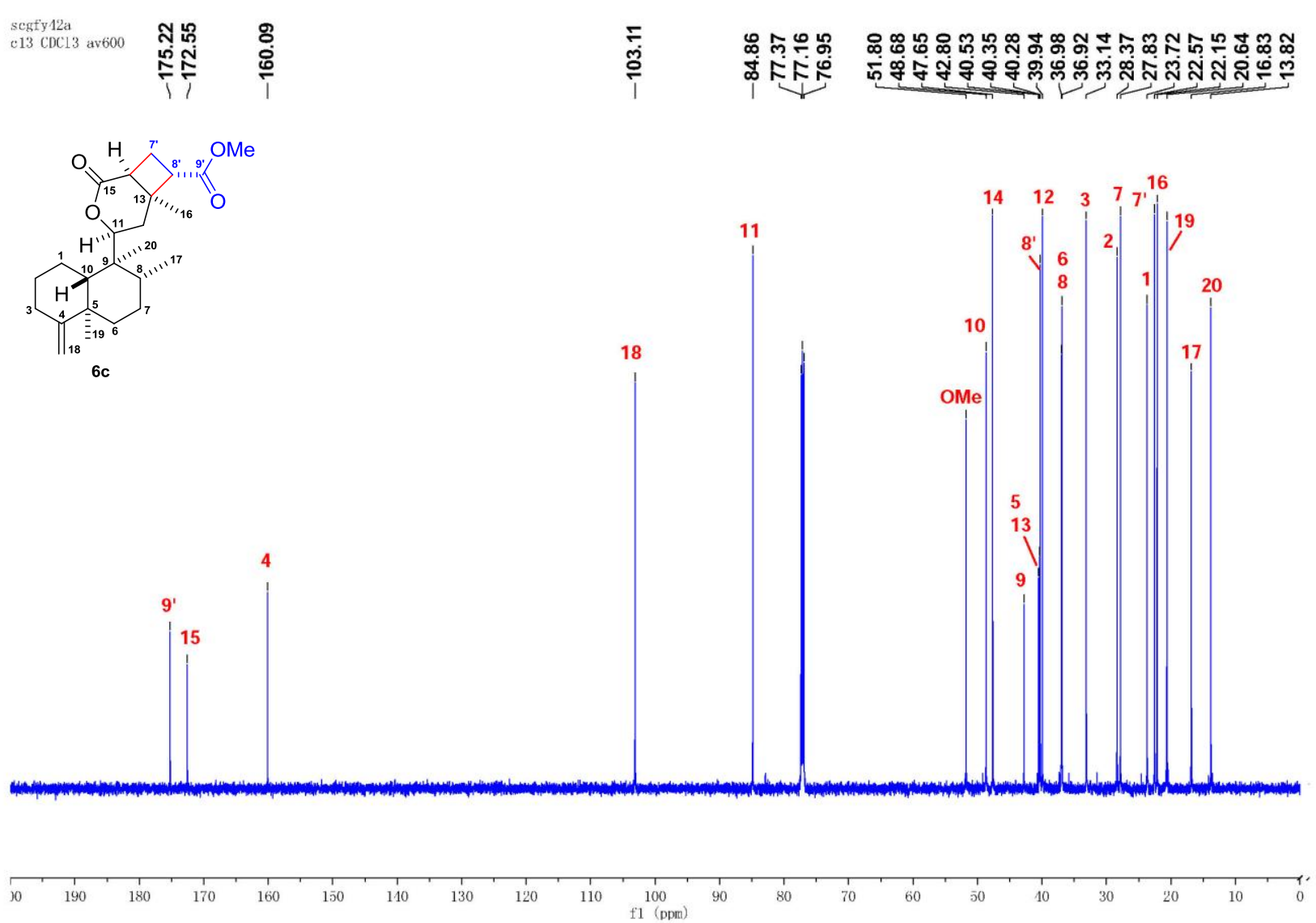
Figure S40. HSQC spectrum of $\mathbf{6 c}$

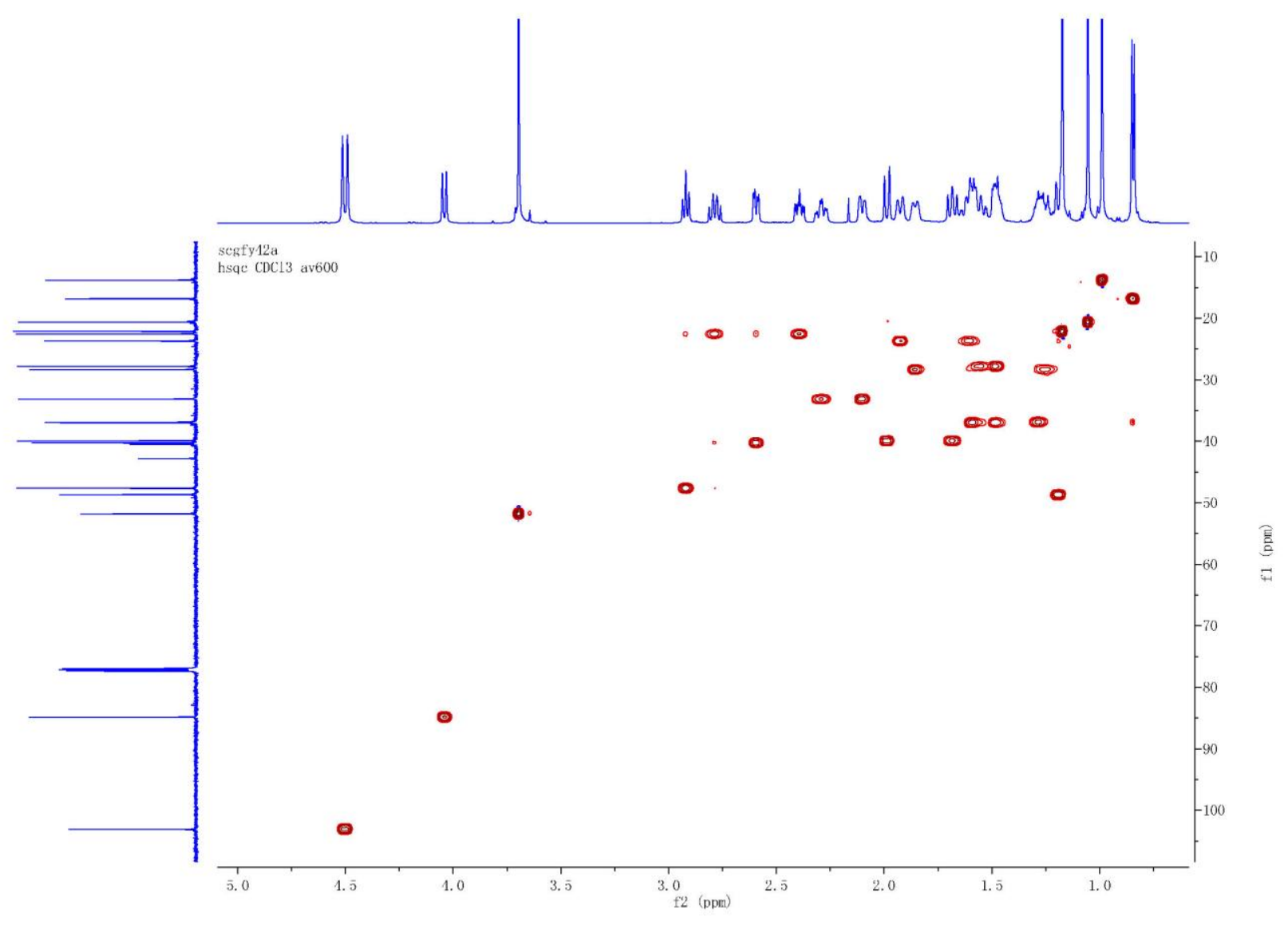

Figure S41. HMBC spectrum of $\mathbf{6 c}$

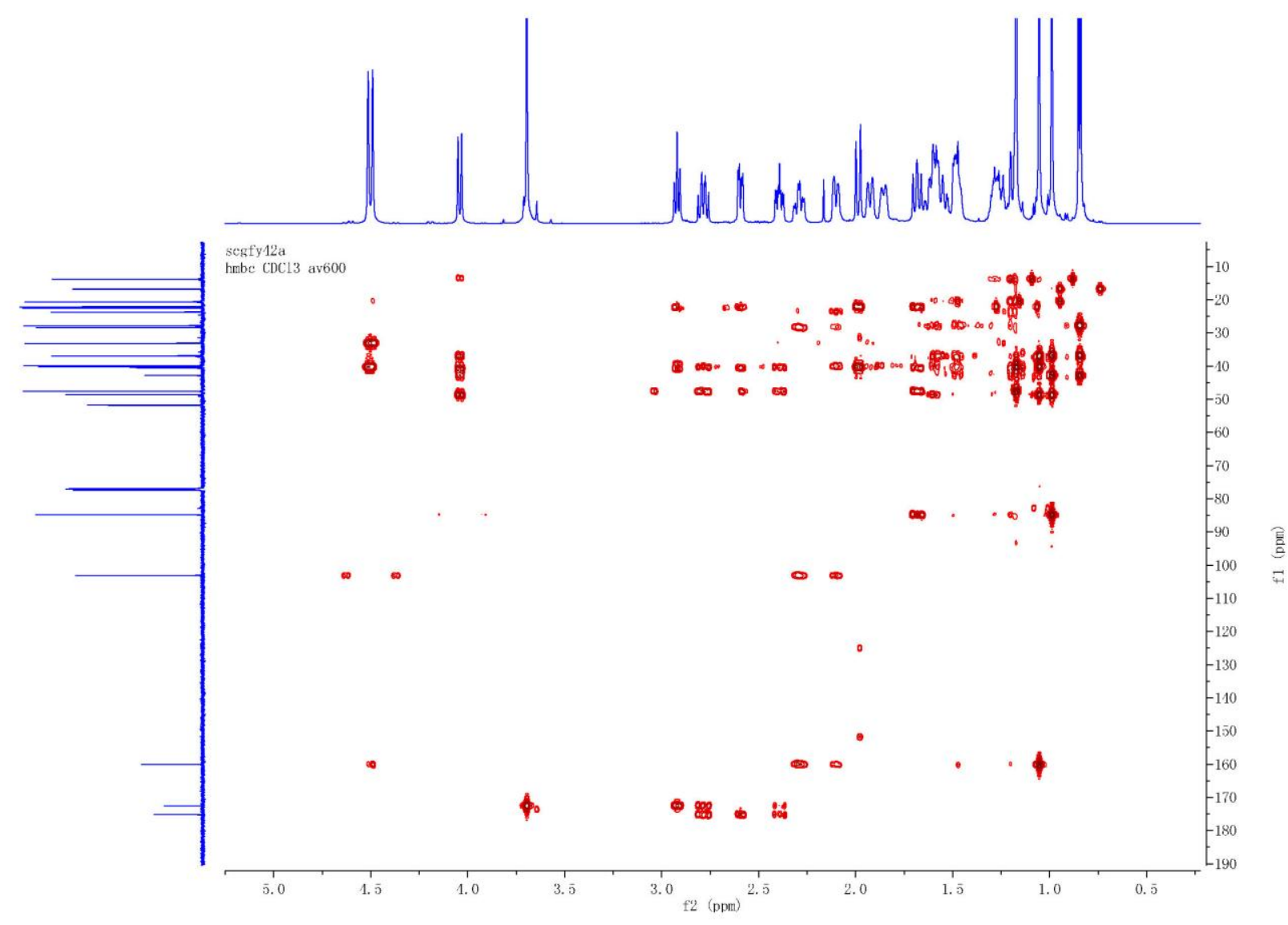


Figure S42. ${ }^{1} \mathrm{H}-{ }^{1} \mathrm{H}$ COSY spectrum of $\mathbf{6 c}$

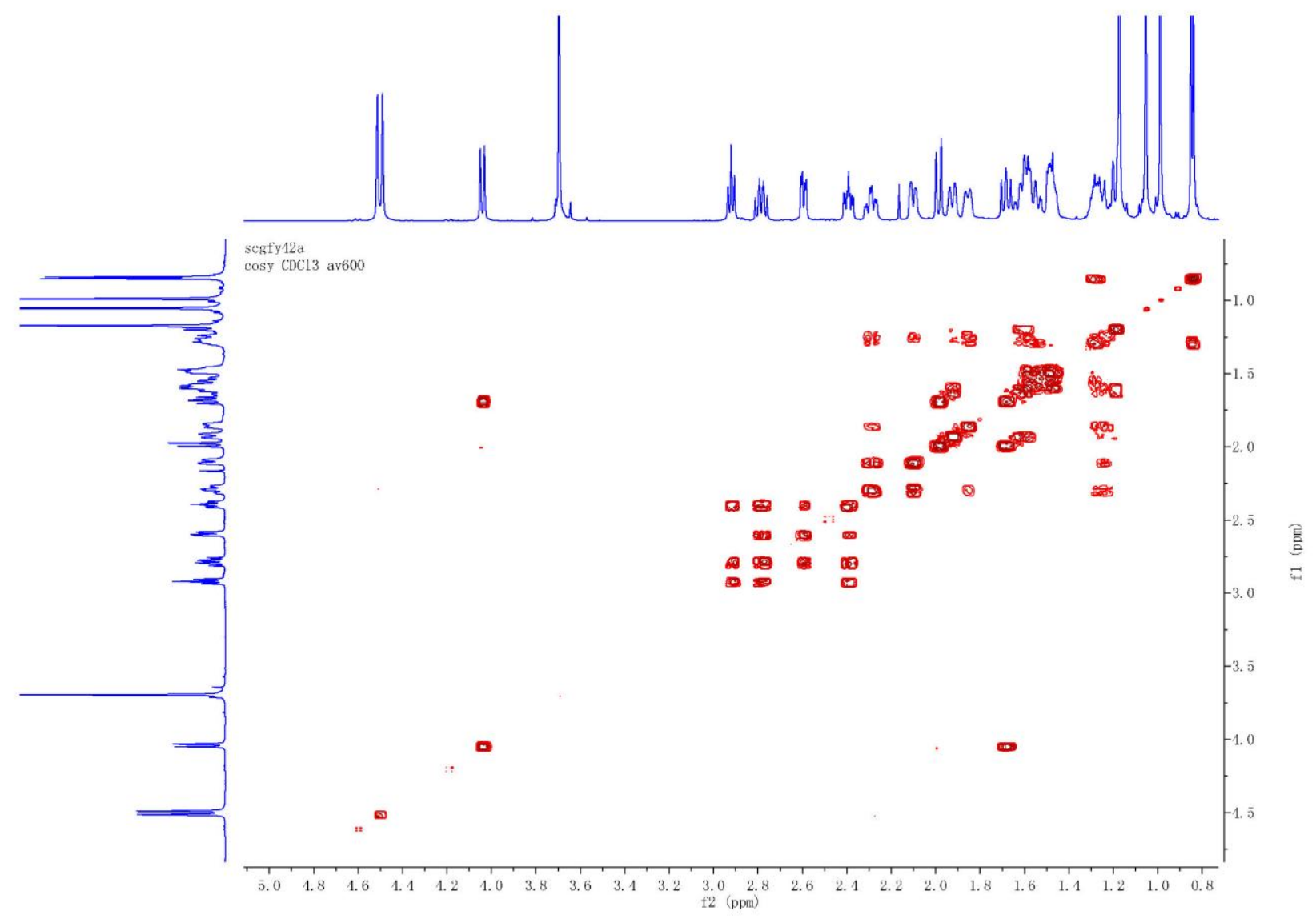

Figure S43. ROESY spectrum of $\mathbf{6 c}$

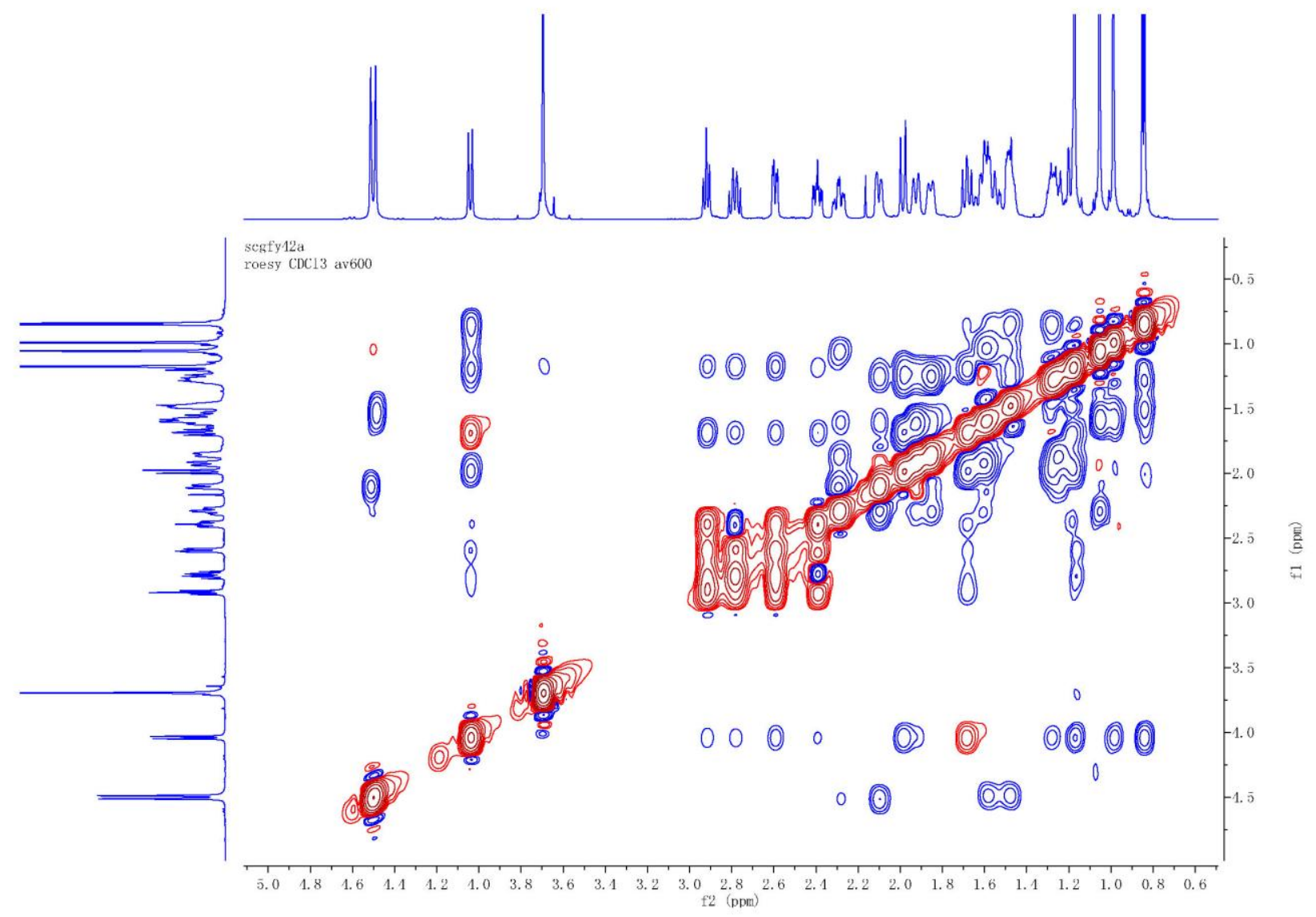


Figure S44. X-ray structure of $\mathbf{6 c}$
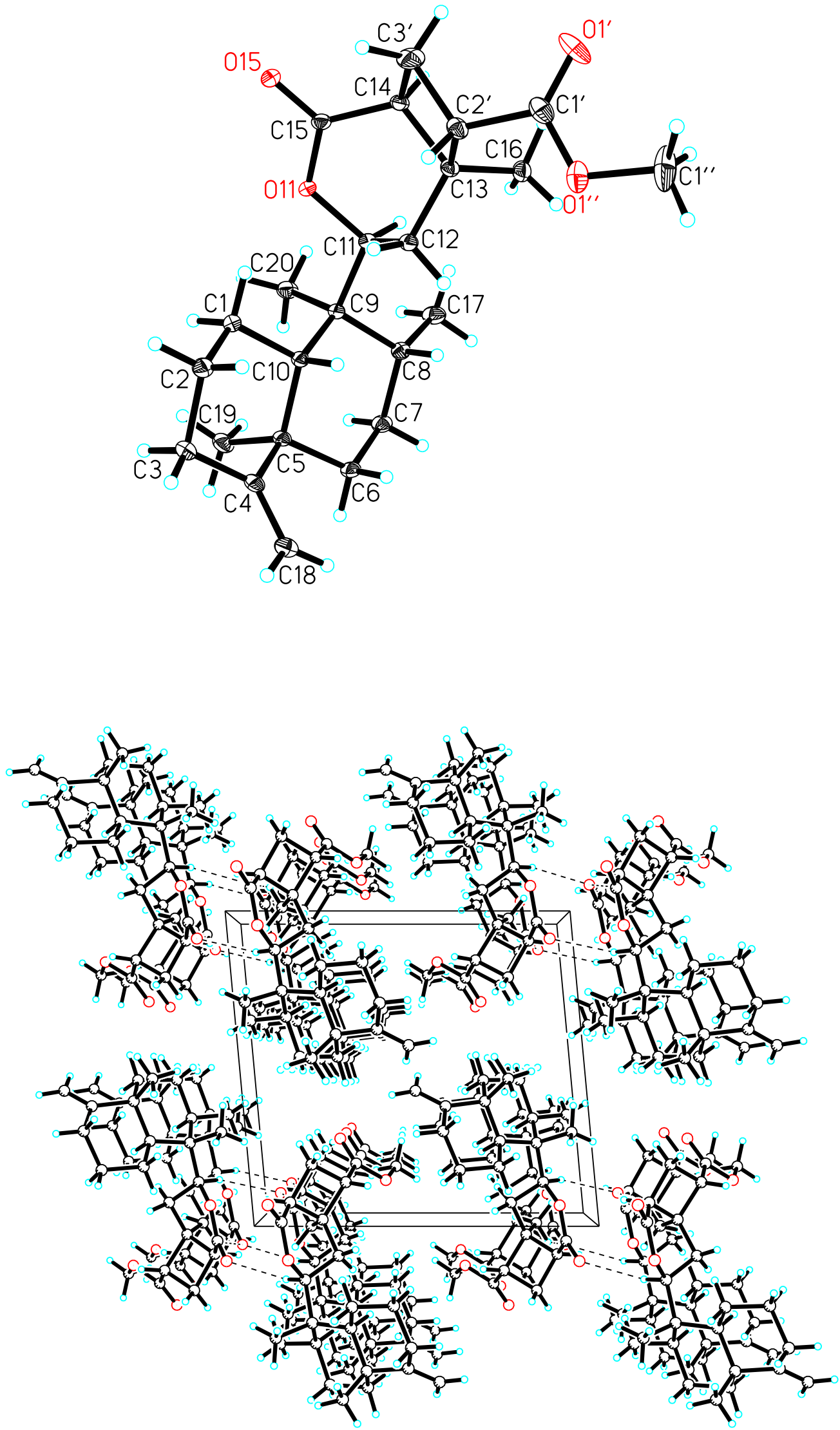
Figure S45. ${ }^{1} \mathrm{H}$ NMR spectrum of $\mathbf{6 d}$

segfy 73
h $\operatorname{CDC} 13$ av600

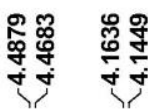

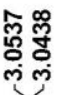

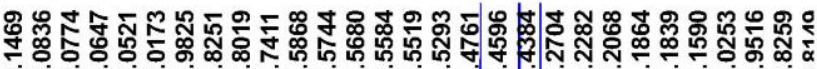

N N
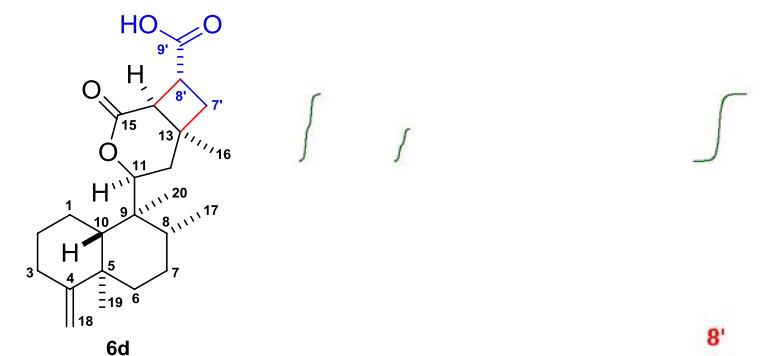

$\int_{S}$
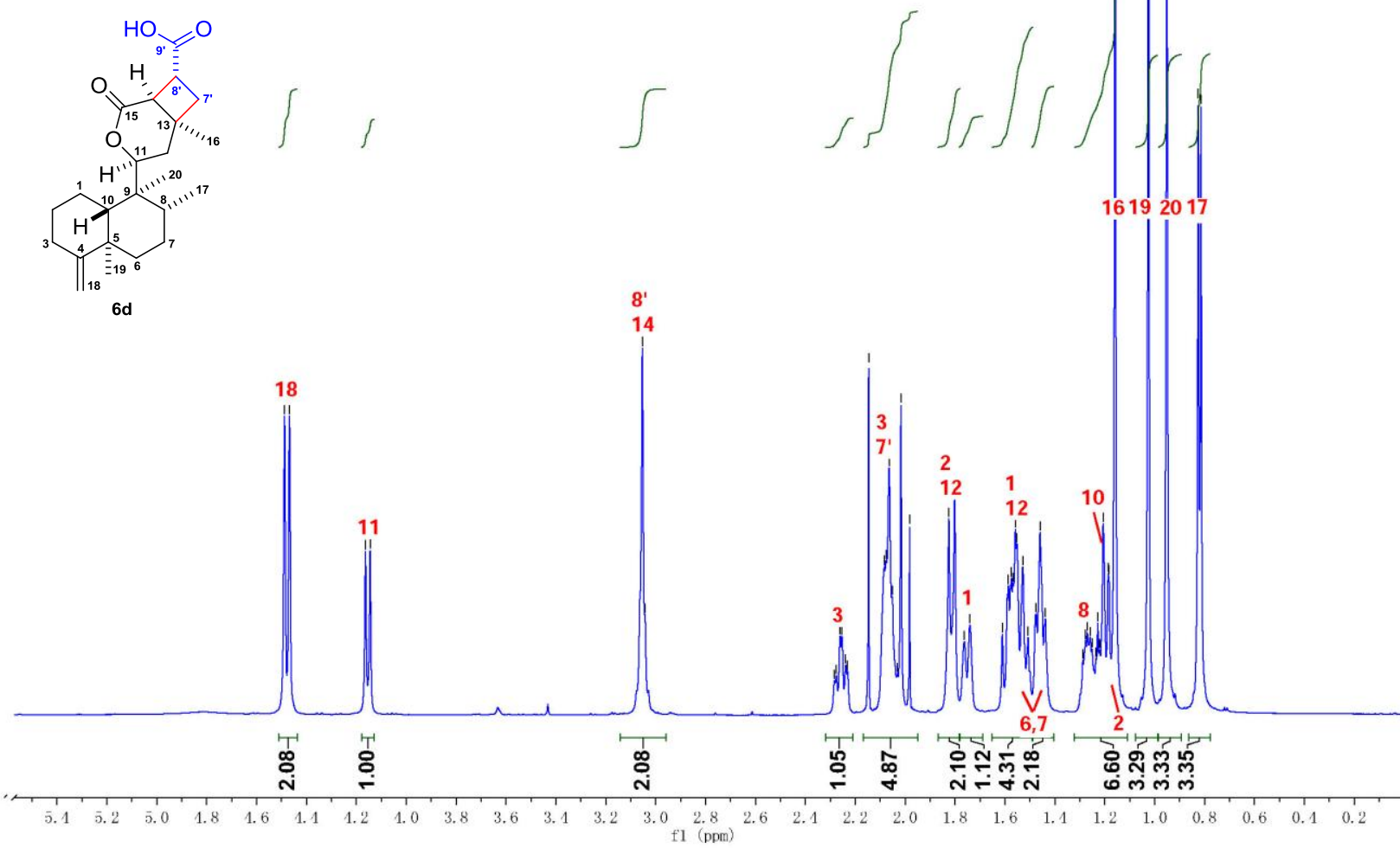

Figure S46. ${ }^{13} \mathrm{C}$ NMR spectrum of $\mathbf{6 d}$
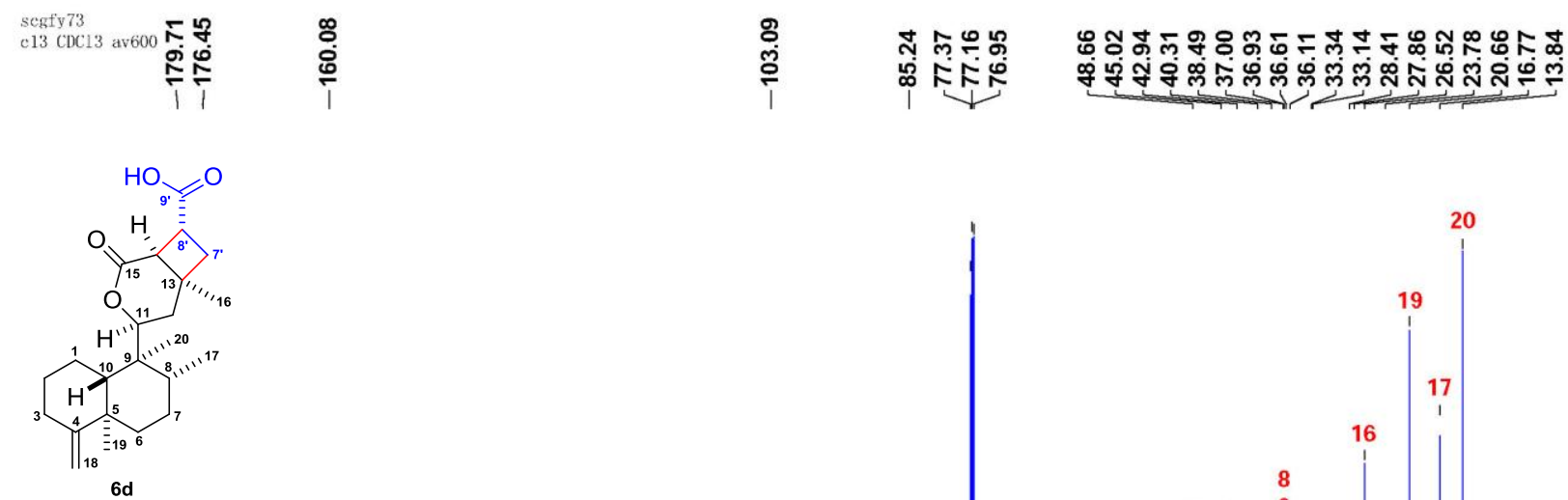

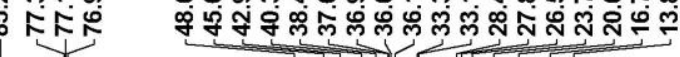

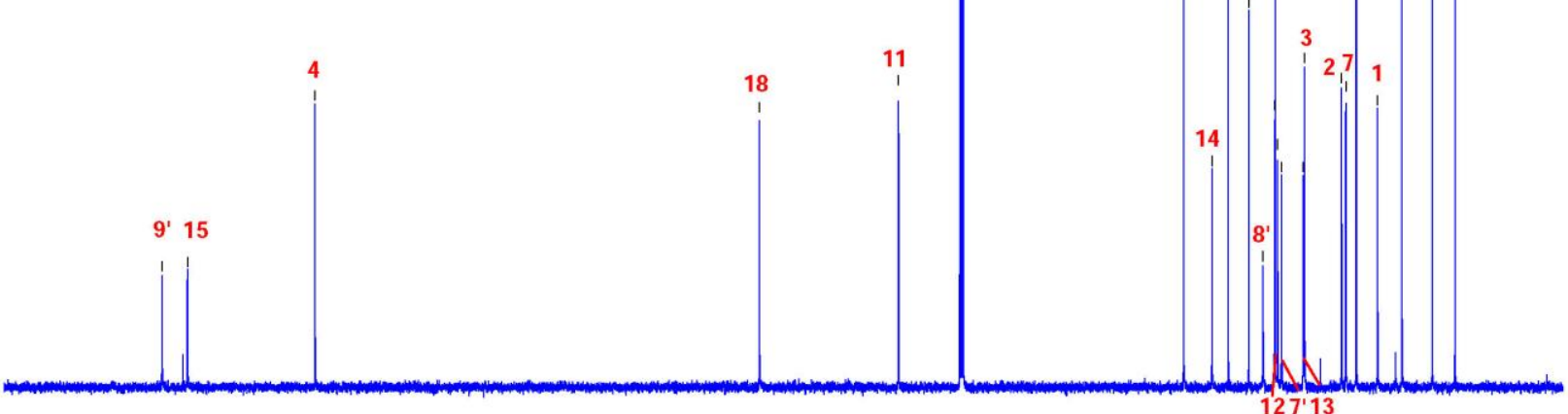

$127^{\prime} 13$

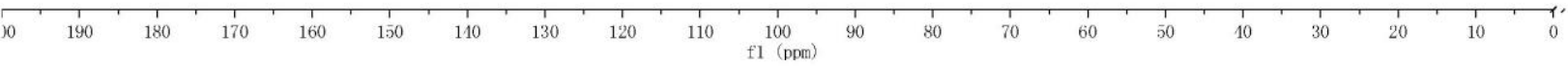


Figure S47. HSQC spectrum of $\mathbf{6 d}$

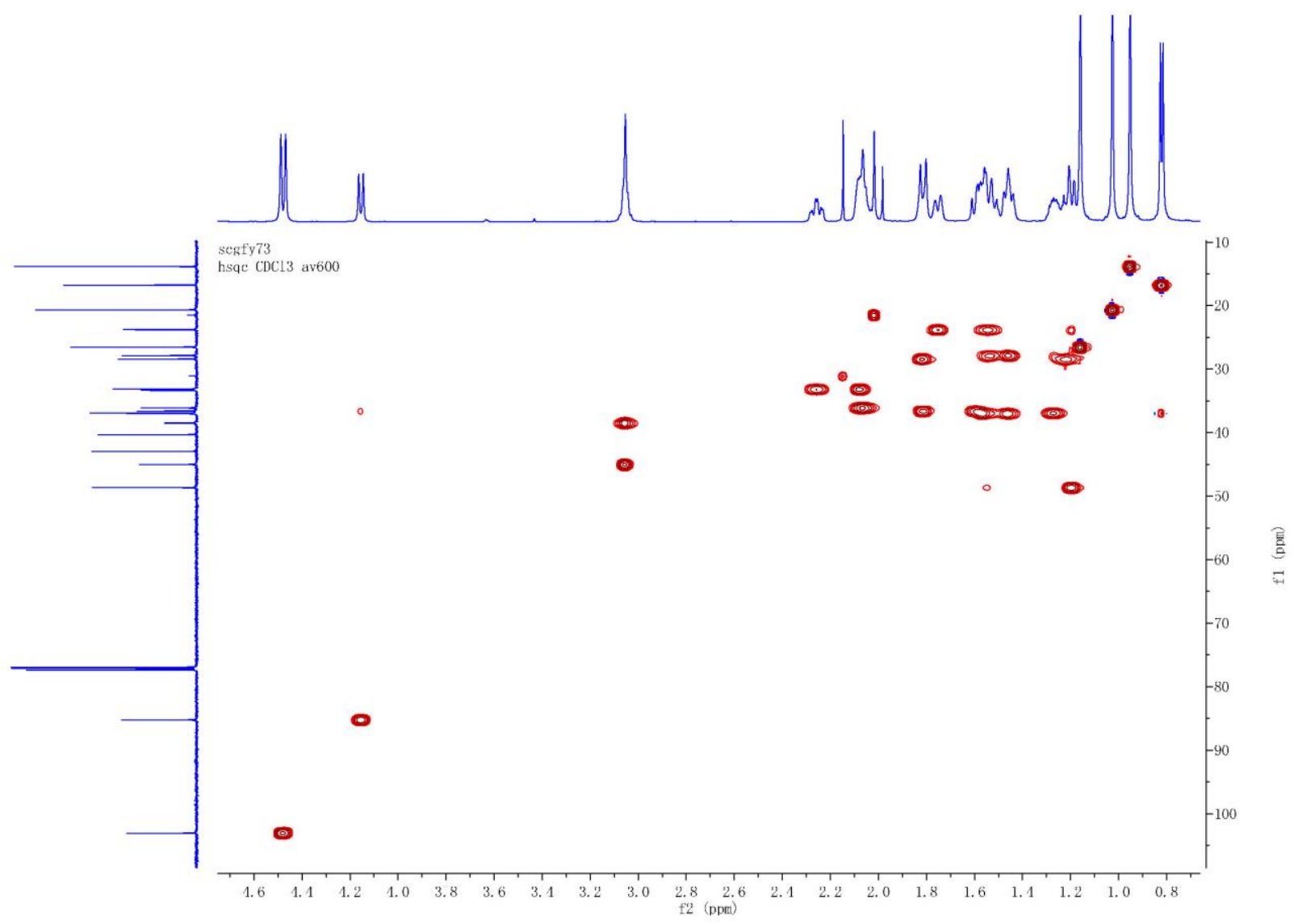

Figure S48. HMBC spectrum of $\mathbf{6 d}$

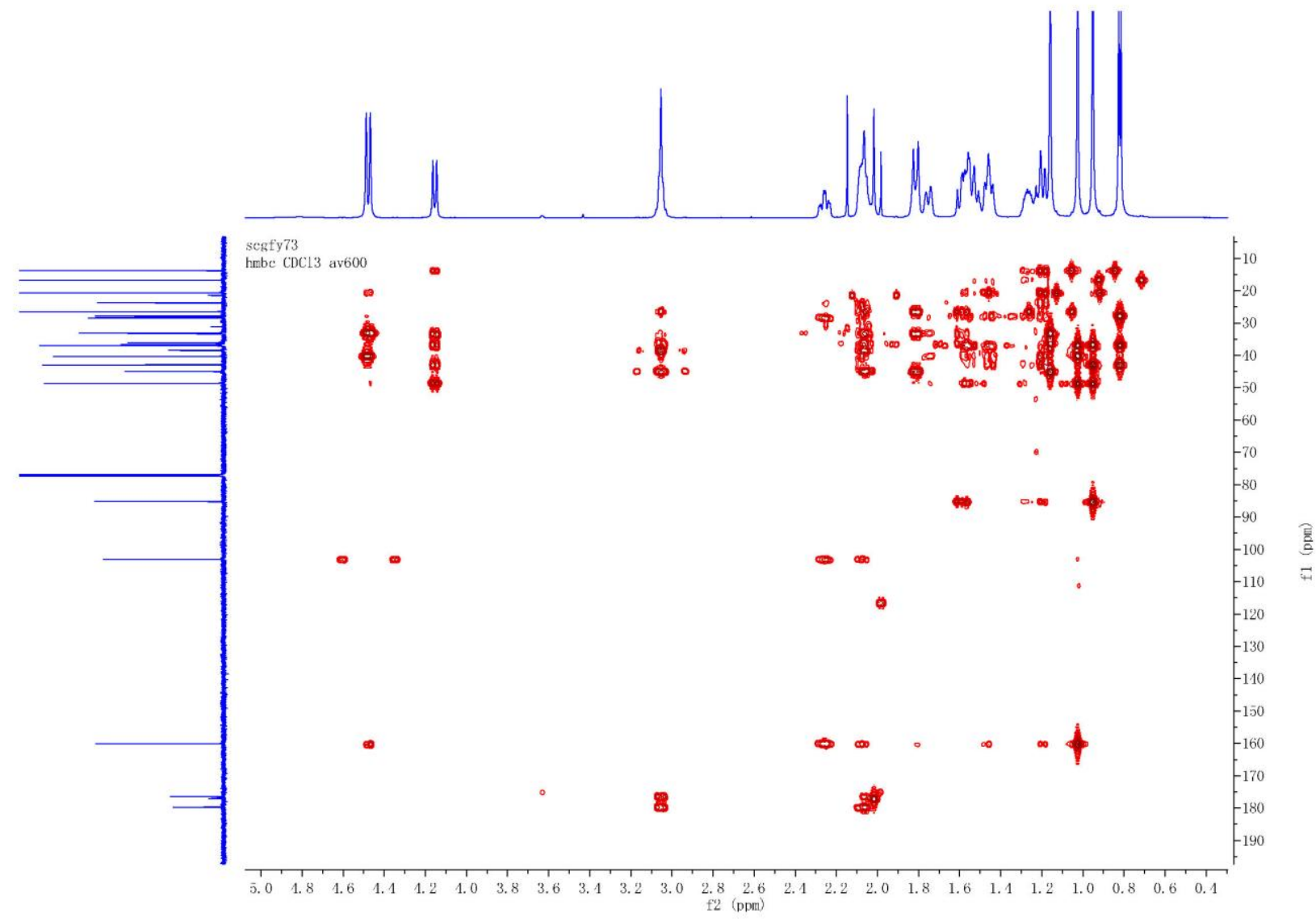


Figure S49. ${ }^{1} \mathrm{H}-{ }^{1} \mathrm{H}$ COSY spectrum of $\mathbf{6 d}$

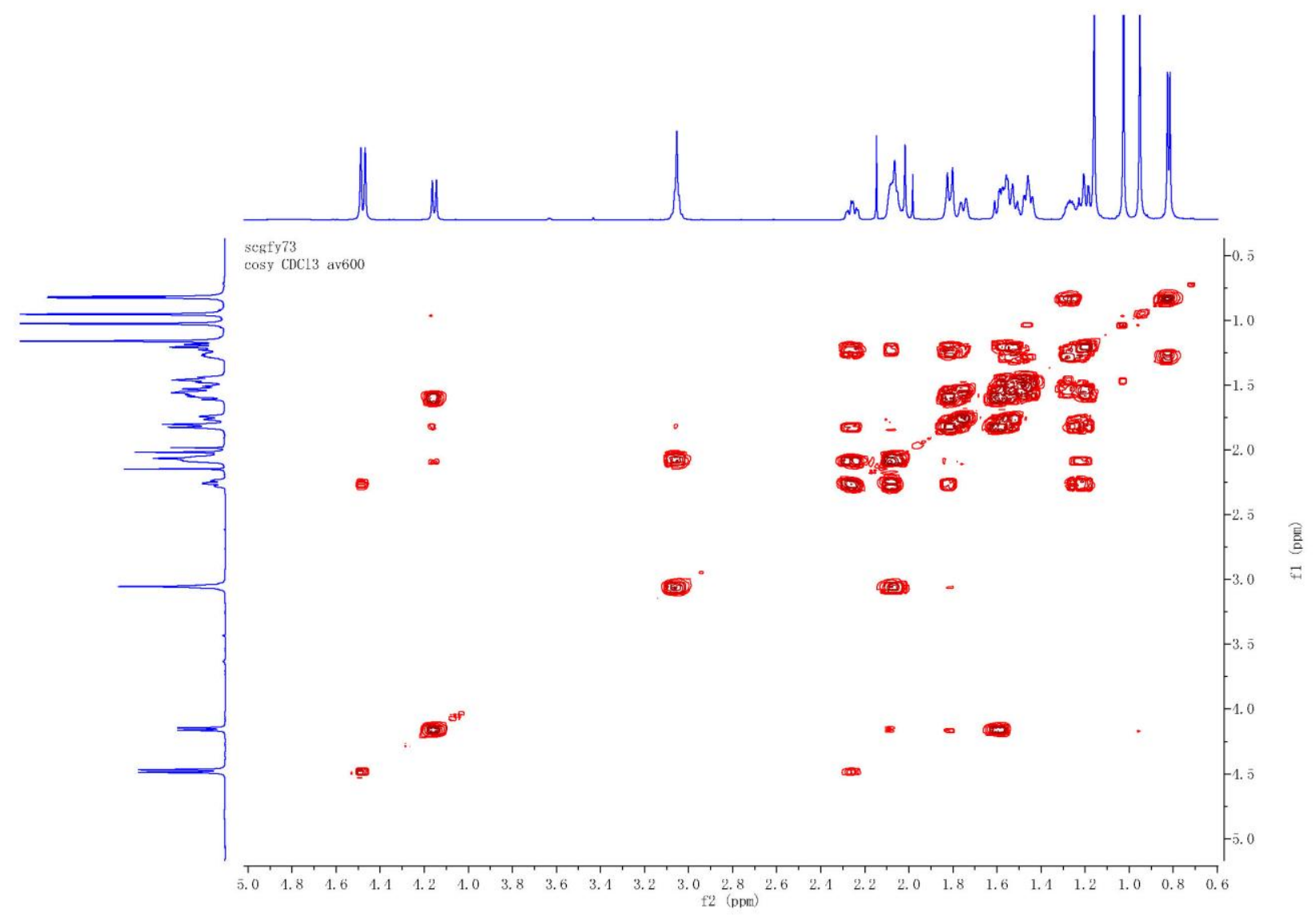

Figure S50. ROESY spectrum of $\mathbf{6 d}$

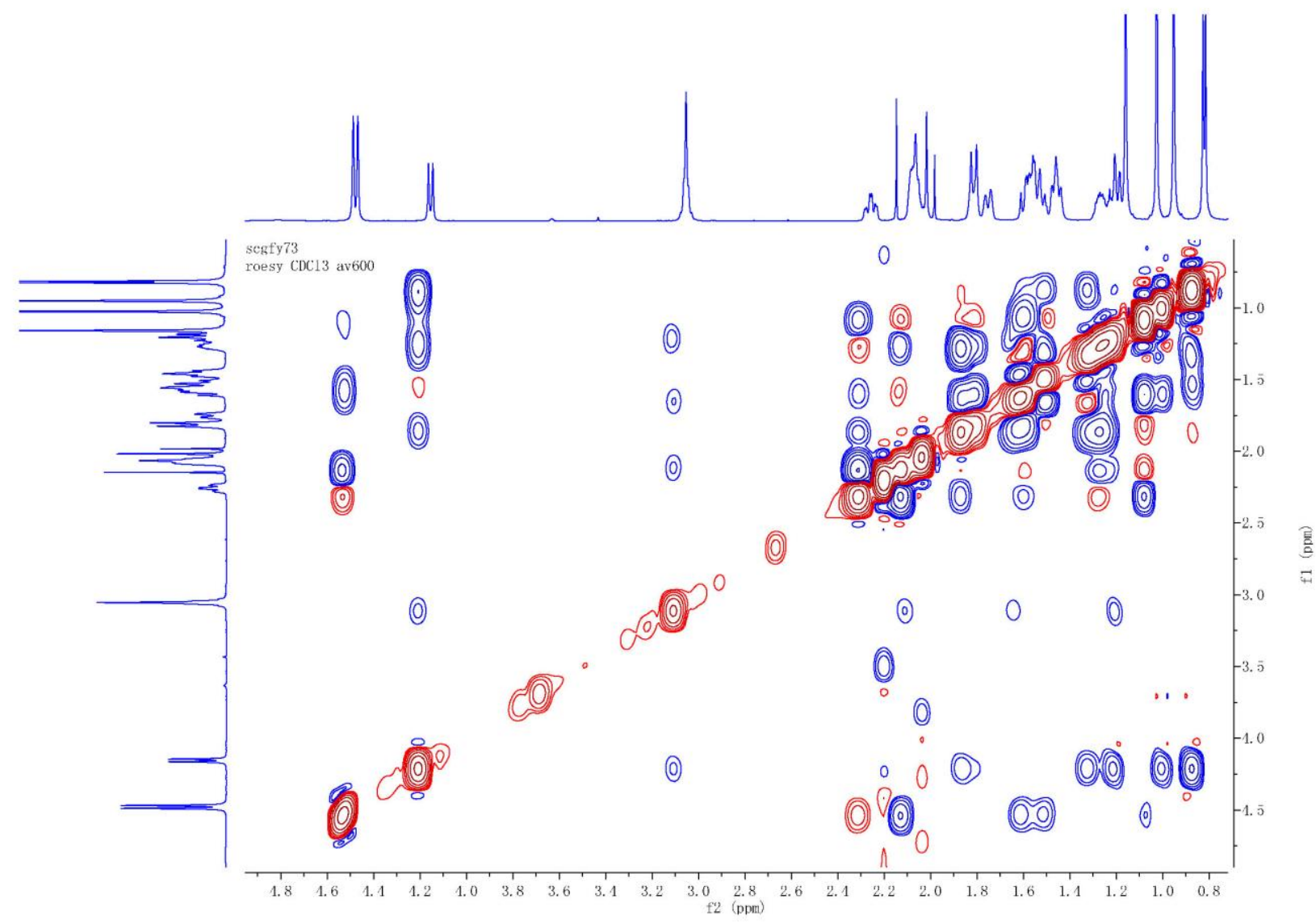


Figure S51. ${ }^{1} \mathrm{H}$ NMR spectrum of $\mathbf{6 e}$

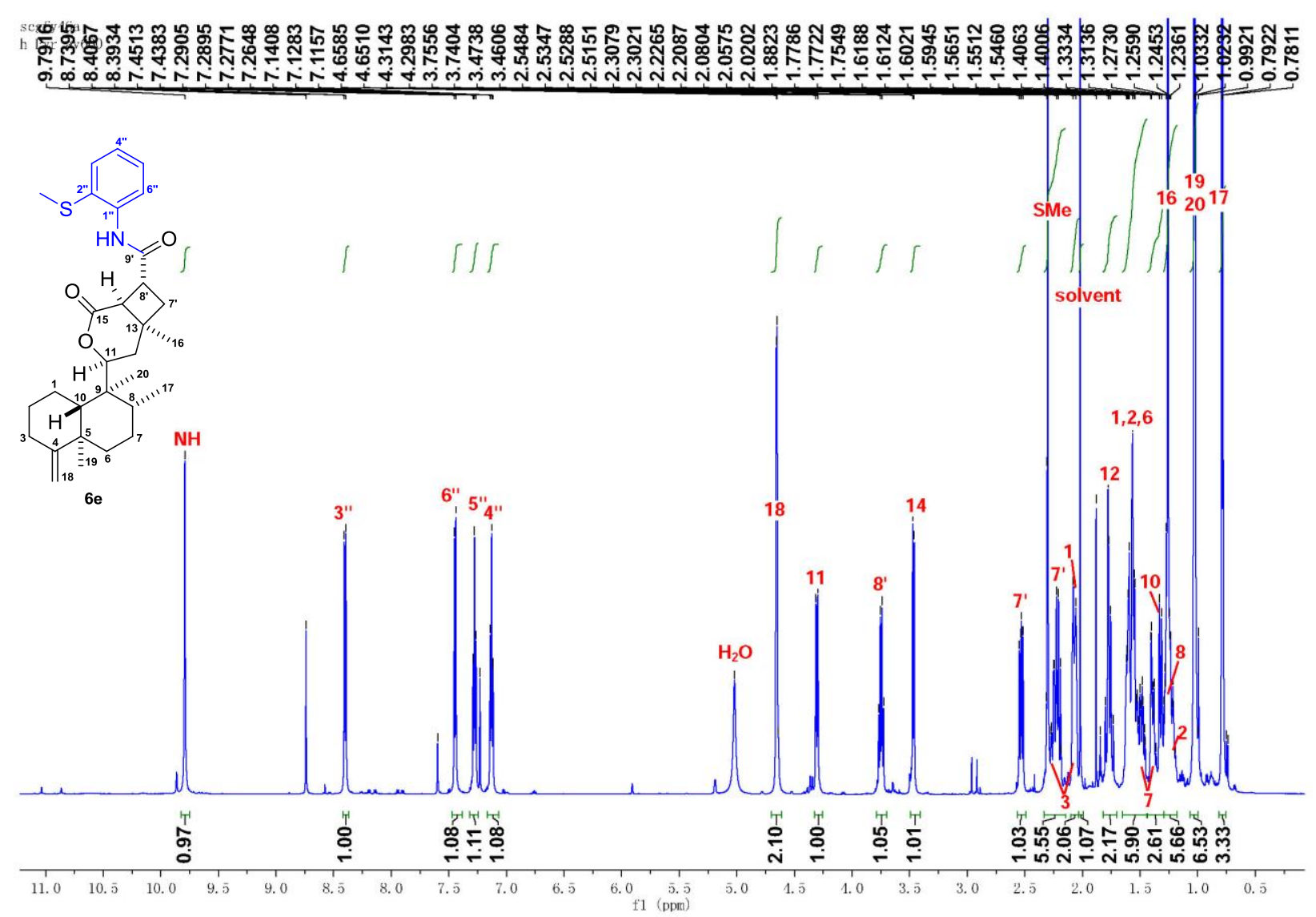

Figure S52. ${ }^{13} \mathrm{C}$ NMR spectrum of $6 \mathbf{e}$
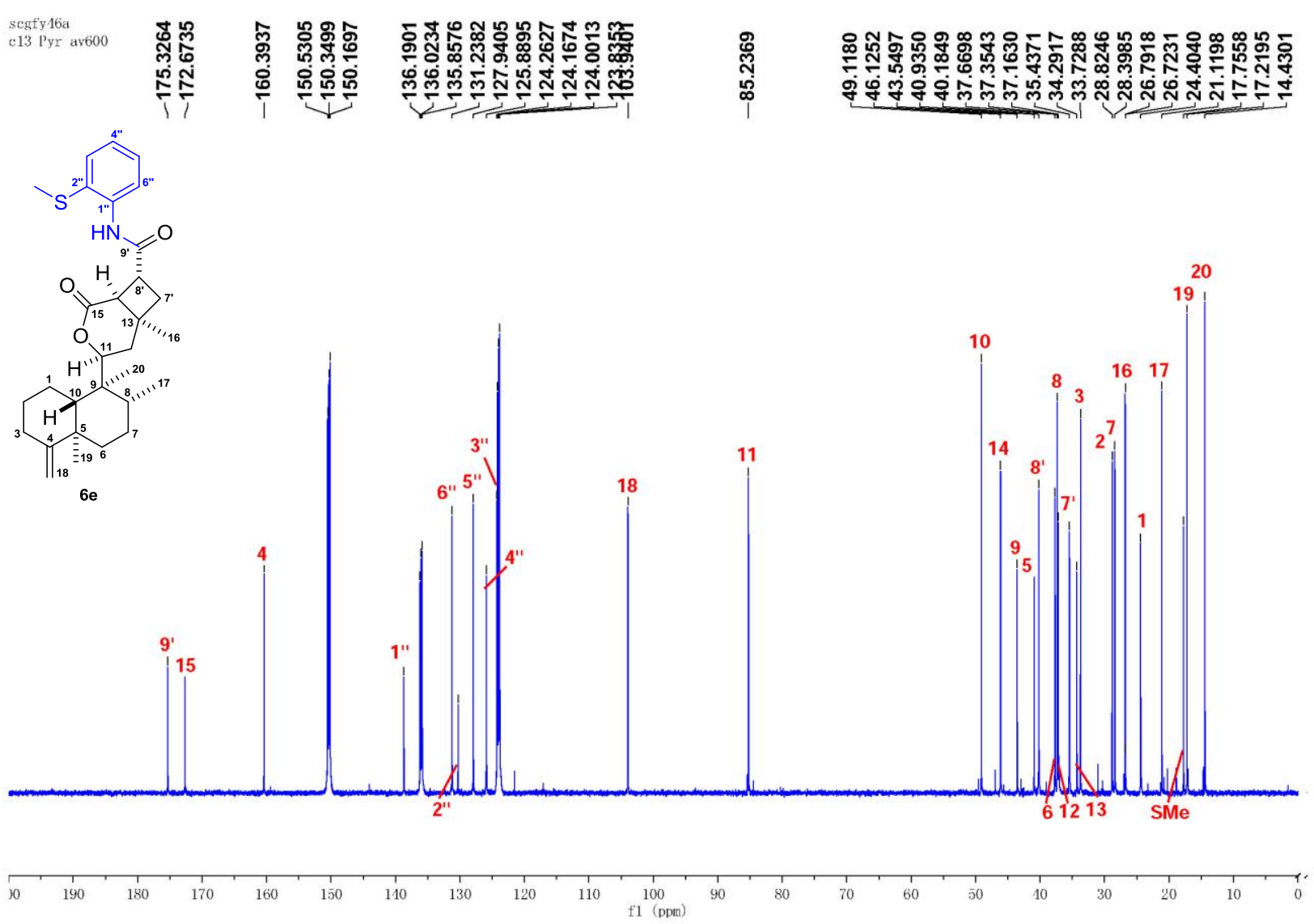
Figure S53. HSQC spectrum of $\mathbf{6 e}$

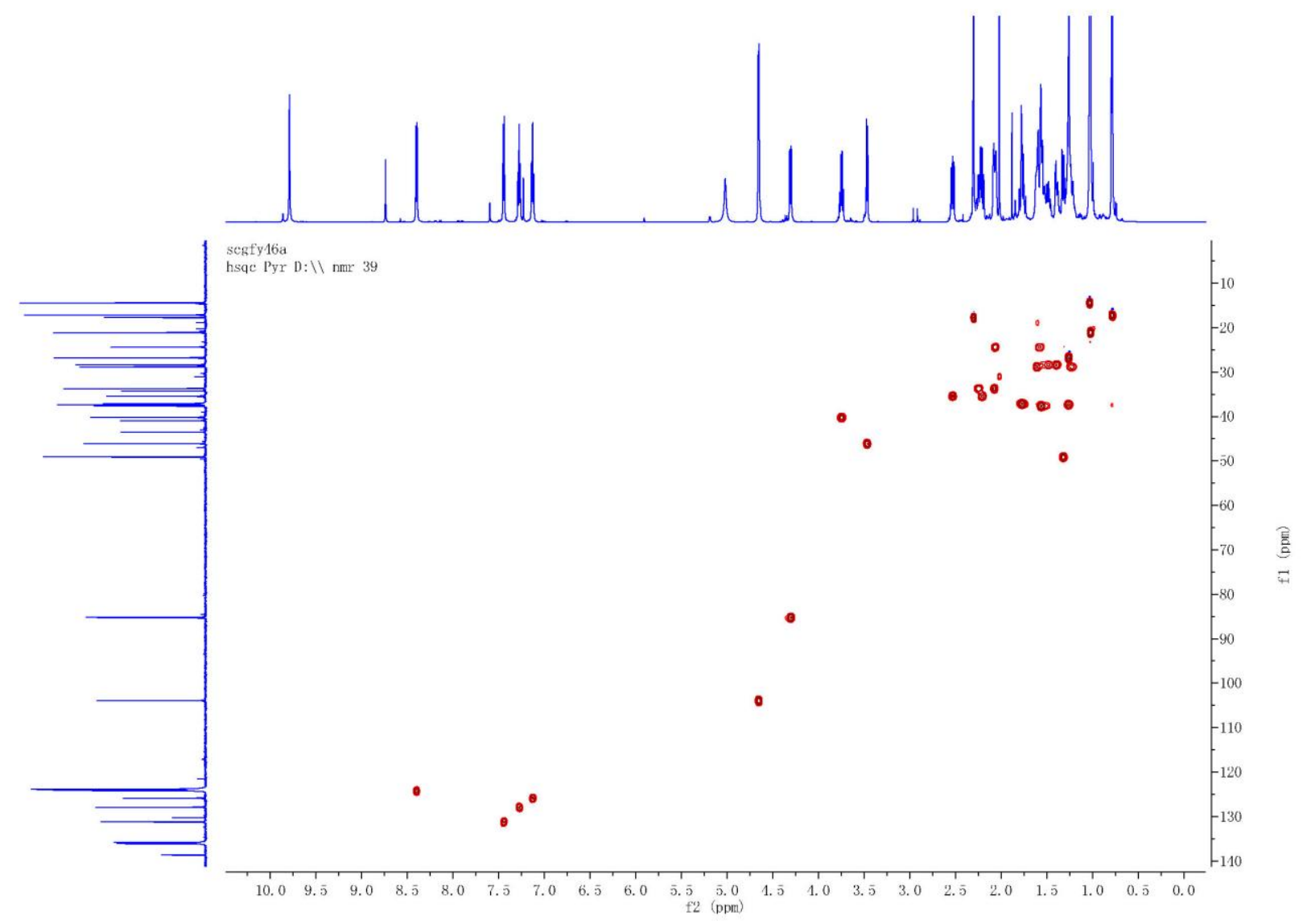

Figure S54. HMBC spectrum of $\mathbf{6 e}$

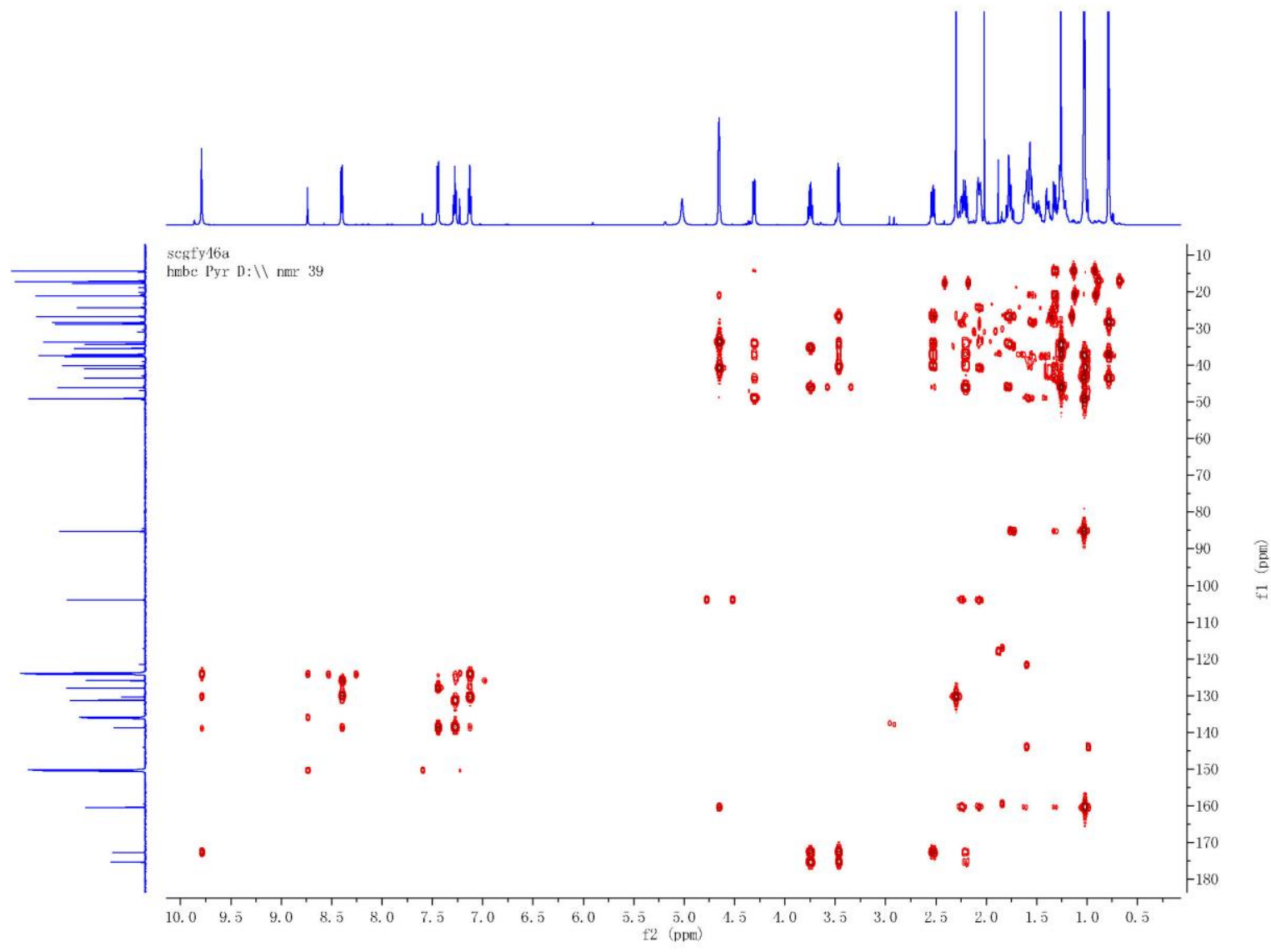


Figure S55. ${ }^{1} \mathrm{H}-{ }^{1} \mathrm{H}$ COSY spectrum of $\mathbf{6 e}$

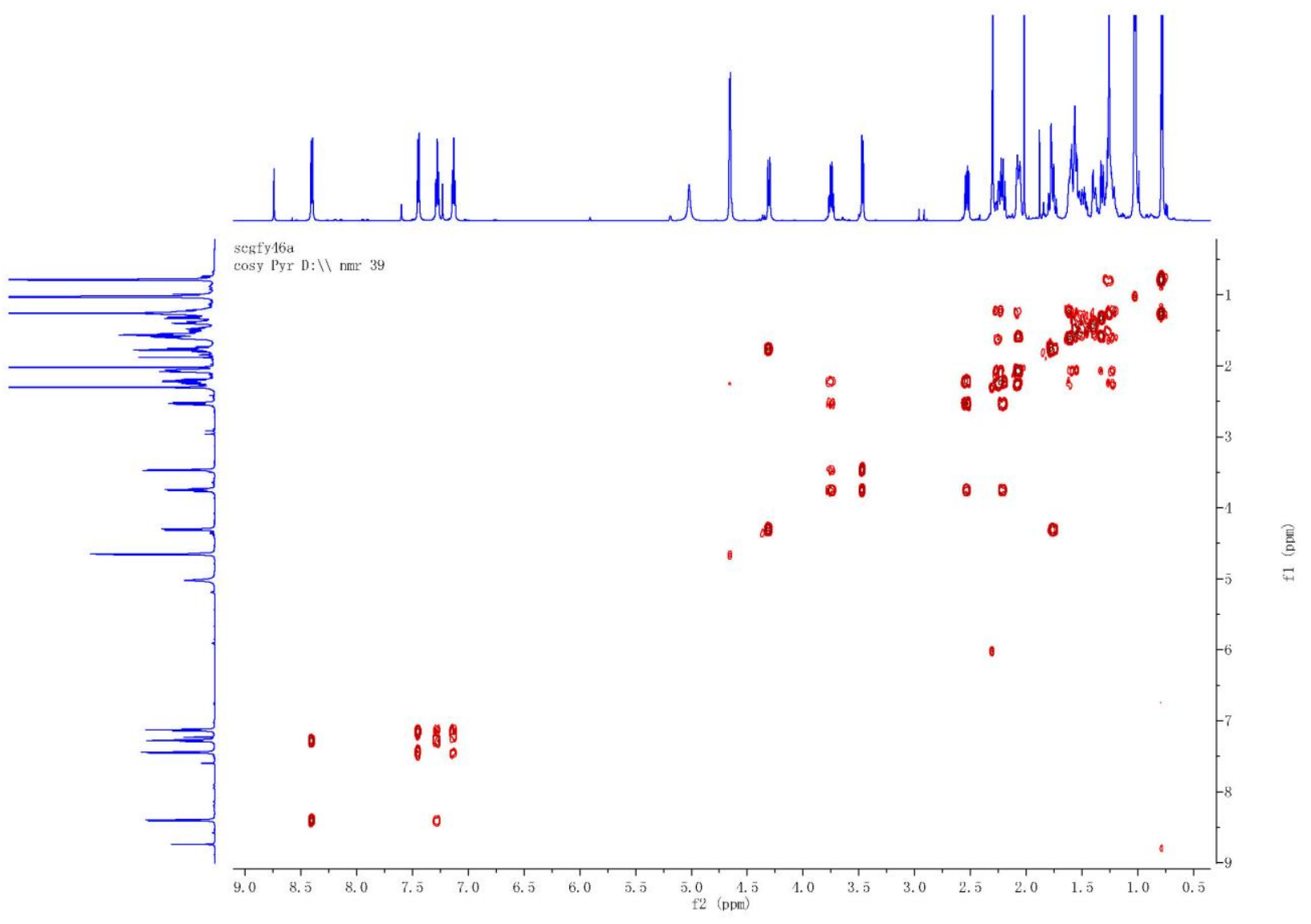

Figure S56. ROESY spectrum of $\mathbf{6 e}$

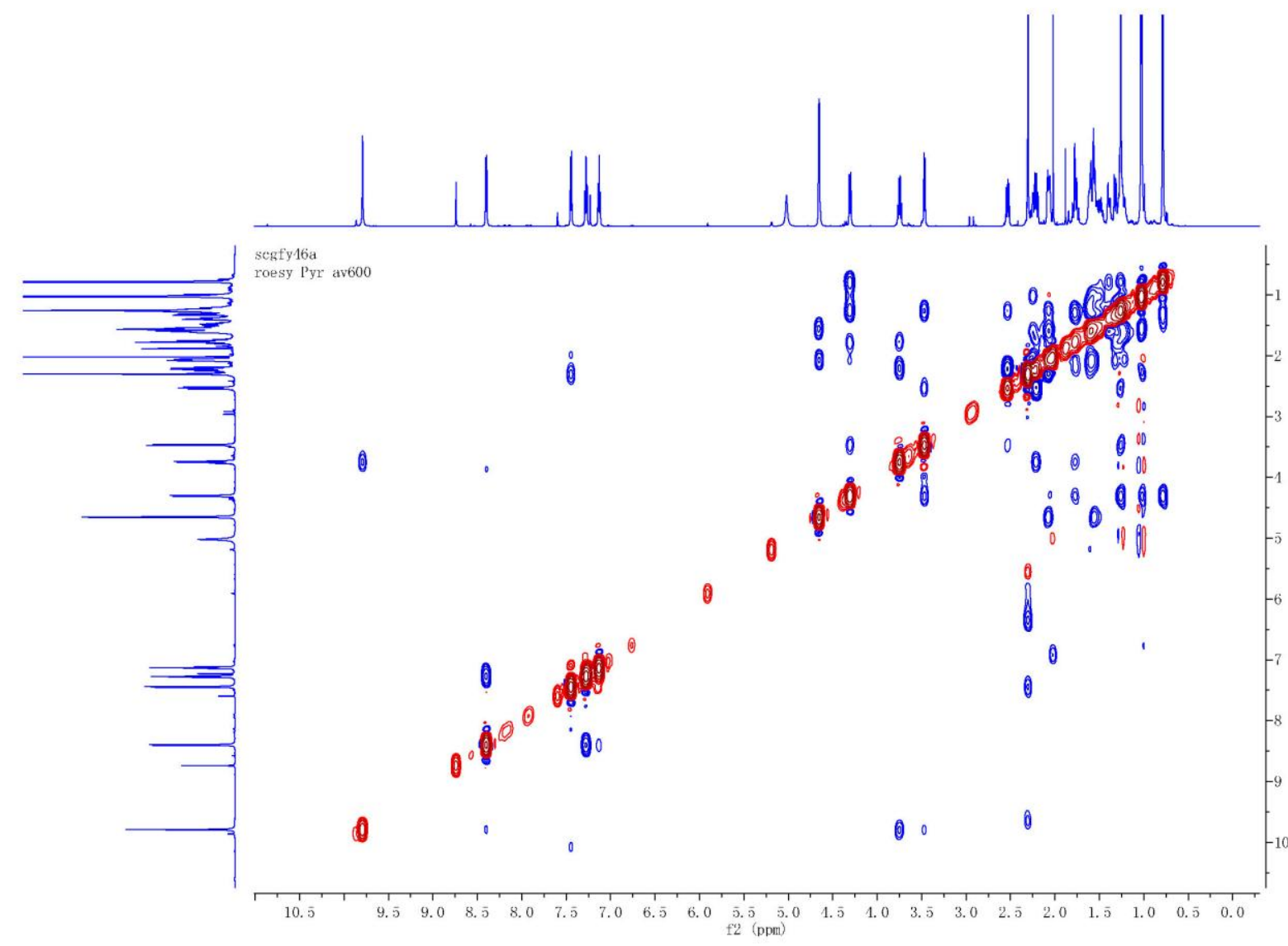


Figure S57. ${ }^{1} \mathrm{H}$ NMR spectrum of $\mathbf{7 a}$

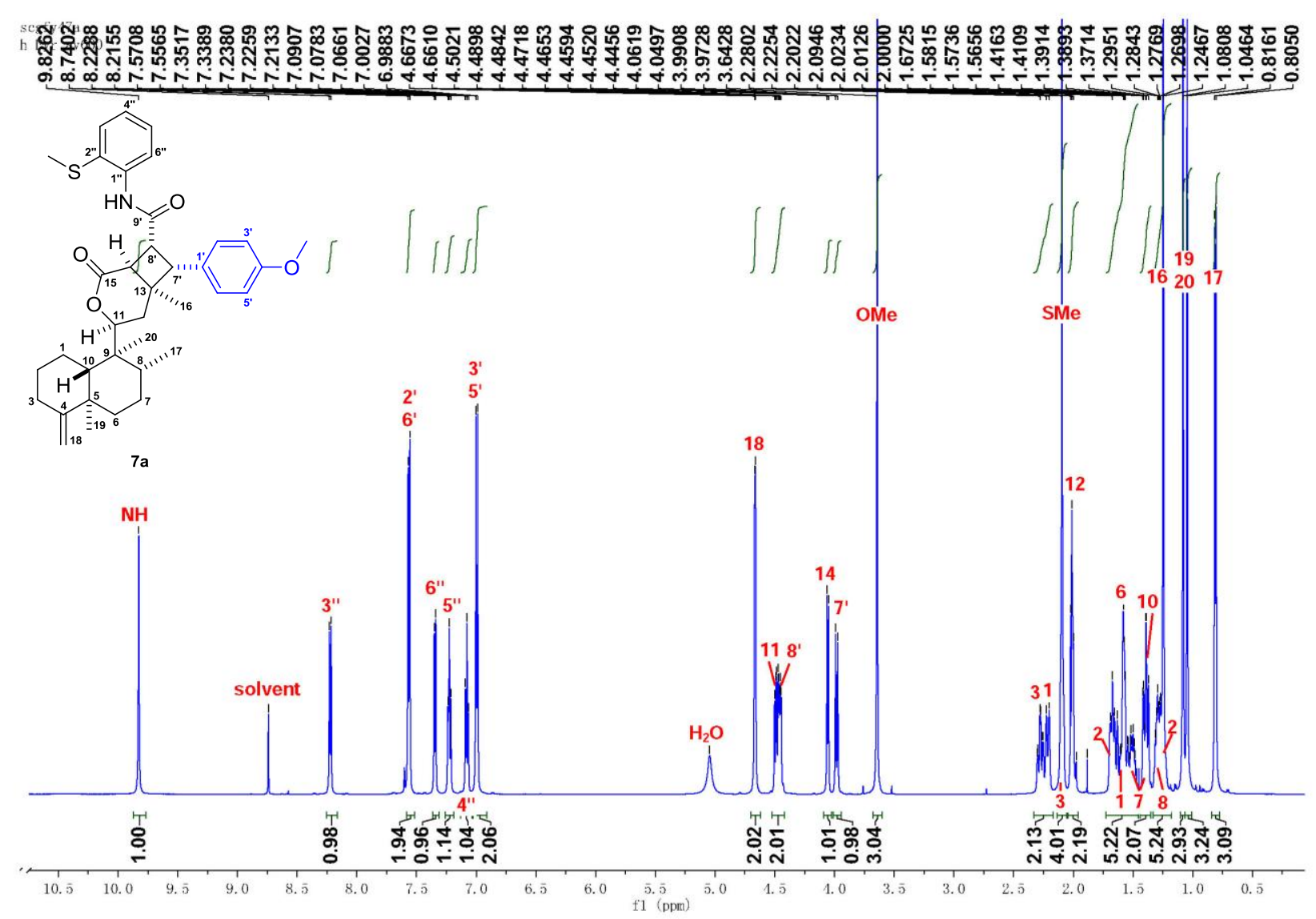

Figure S58. ${ }^{13} \mathrm{C}$ NMR spectrum of $7 \mathbf{a}$

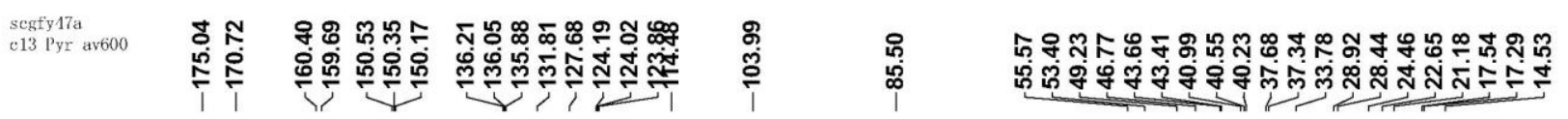
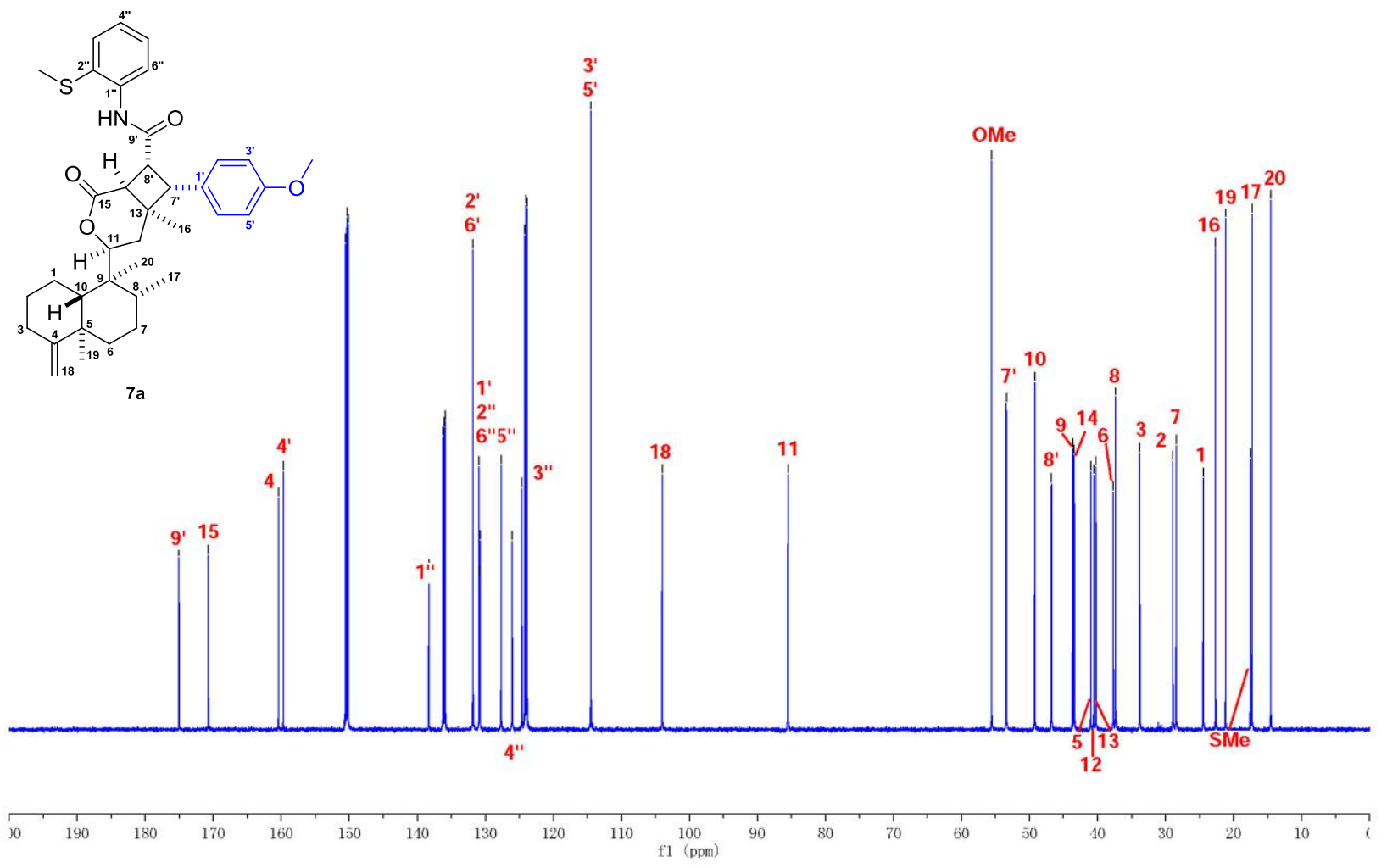
Figure S59. HSQC spectrum of 7a

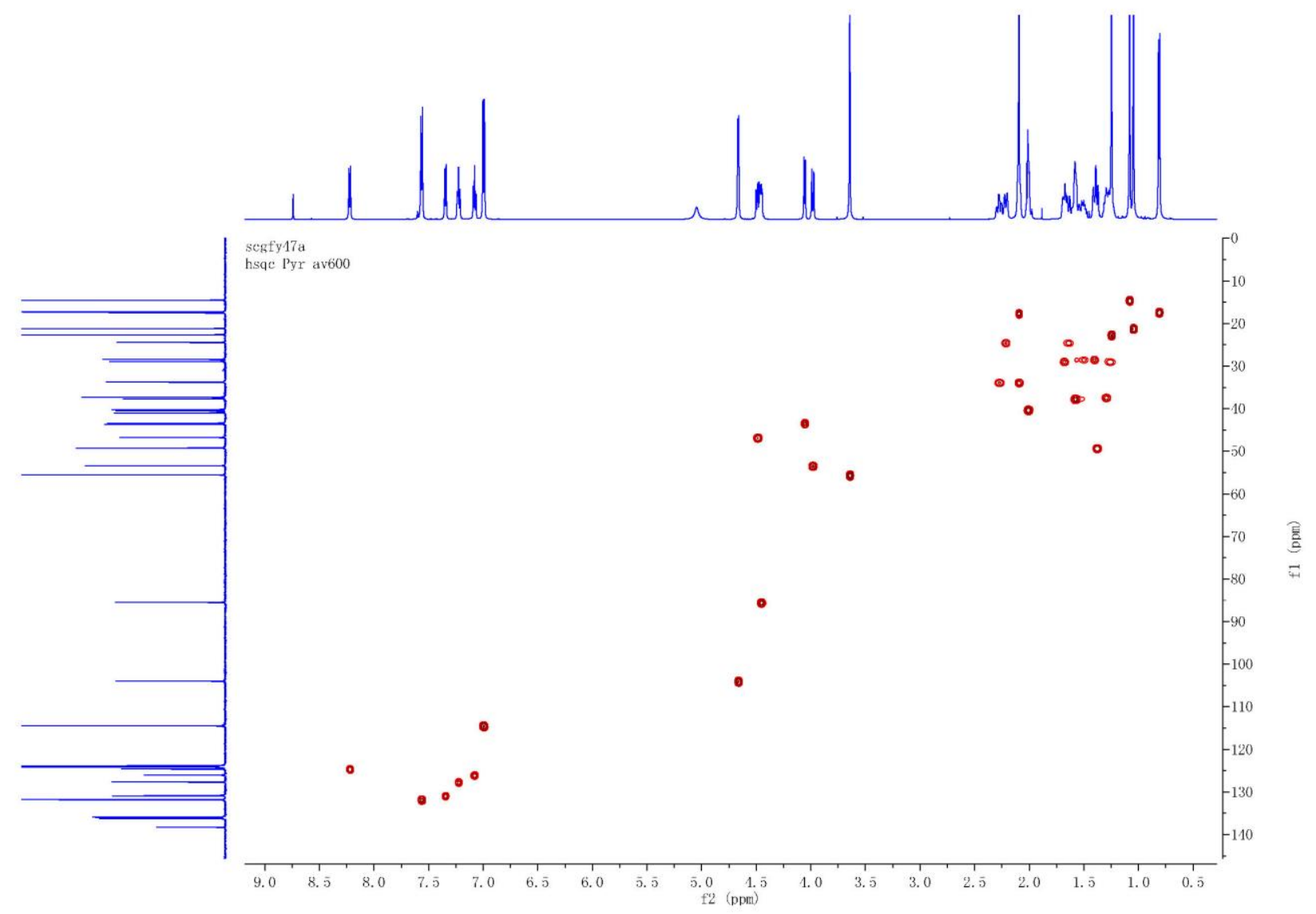

Figure S60. HMBC spectrum of 7a

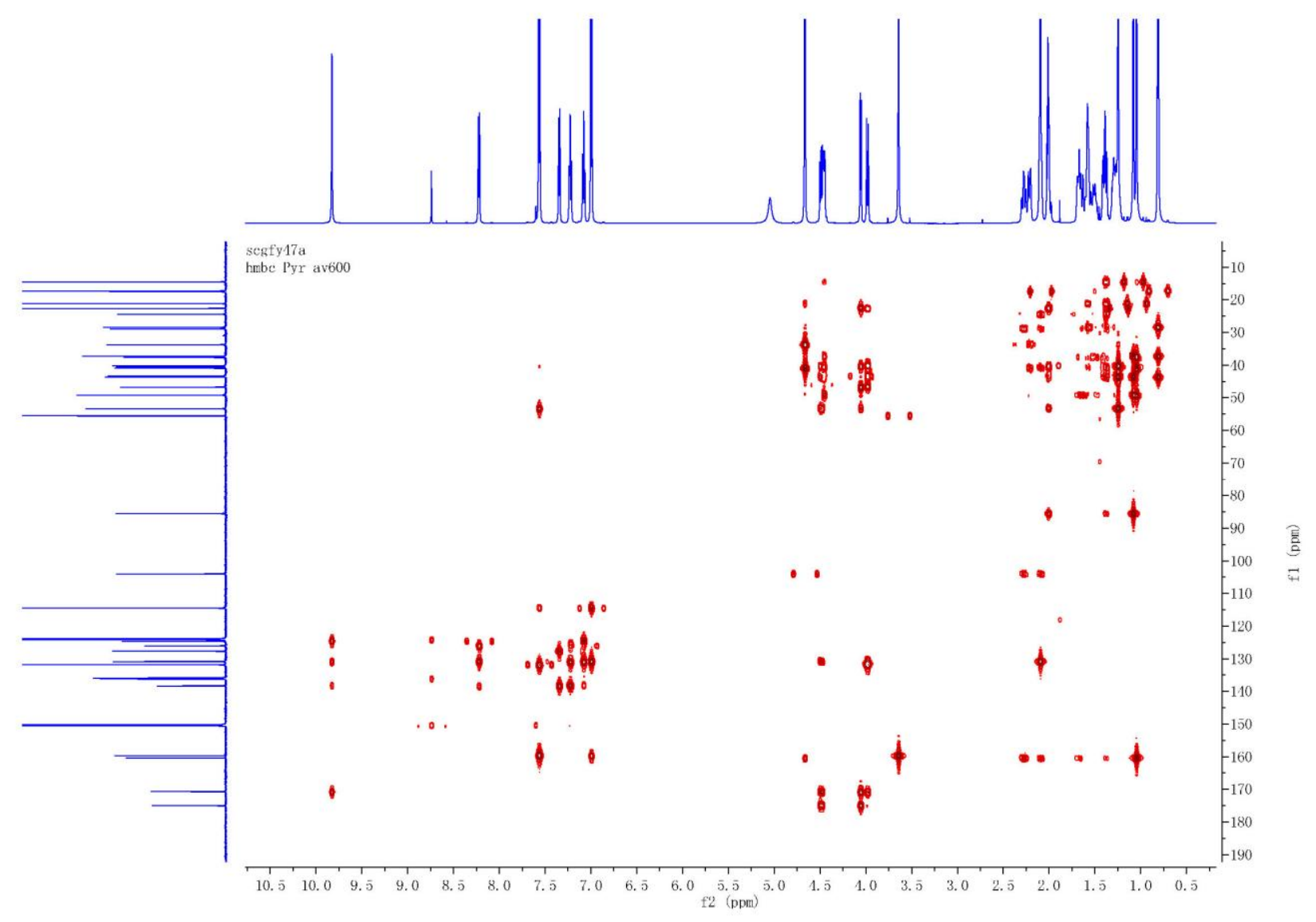


Figure S61. ${ }^{1} \mathrm{H}-{ }^{1} \mathrm{H}$ COSY spectrum of $7 \mathbf{a}$

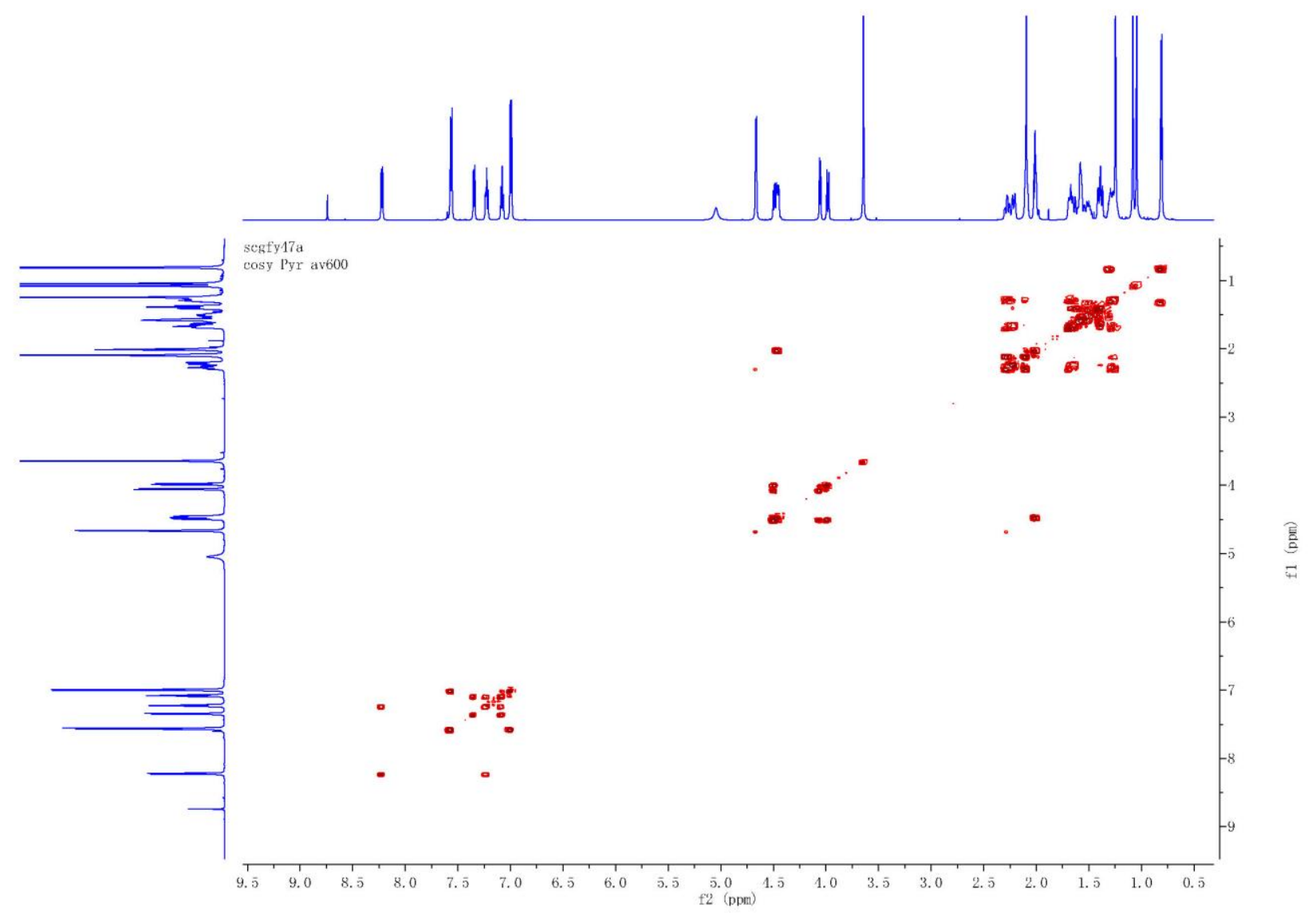

Figure S62. ROESY spectrum of 7a

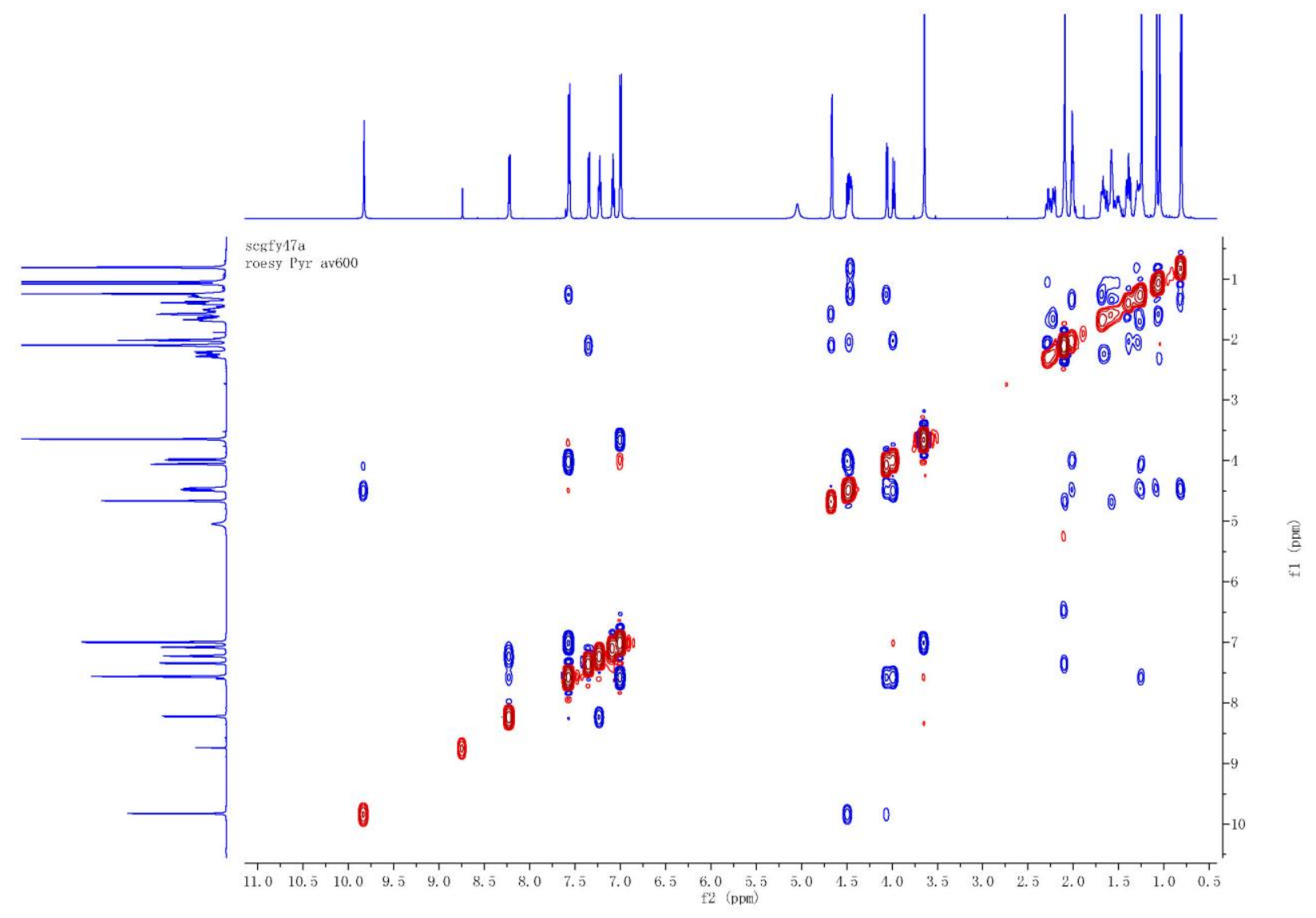


Figure S63. ${ }^{1}$ H NMR spectrum of 8

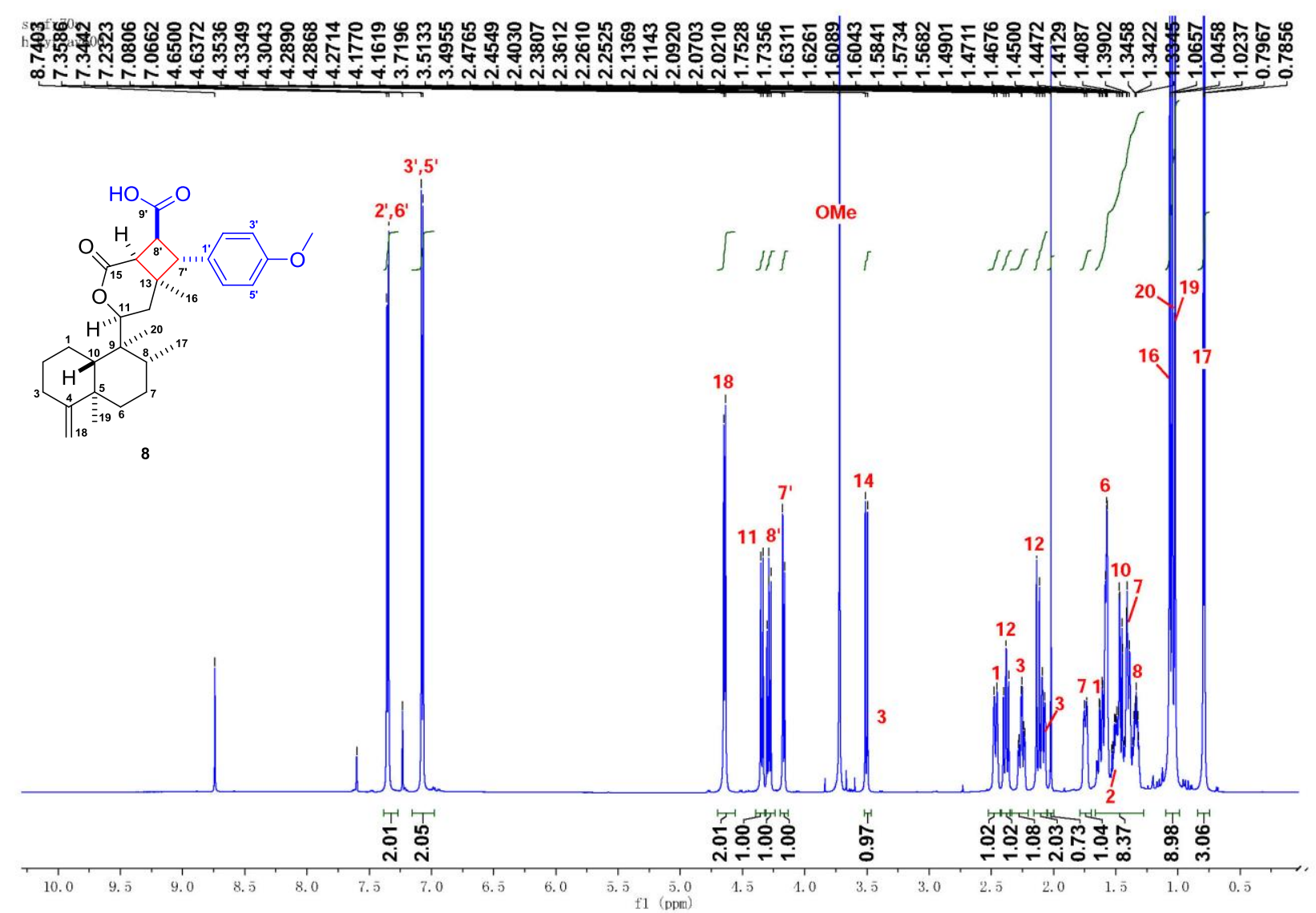

Figure S64. ${ }^{13} \mathrm{C}$ NMR spectrum of 8
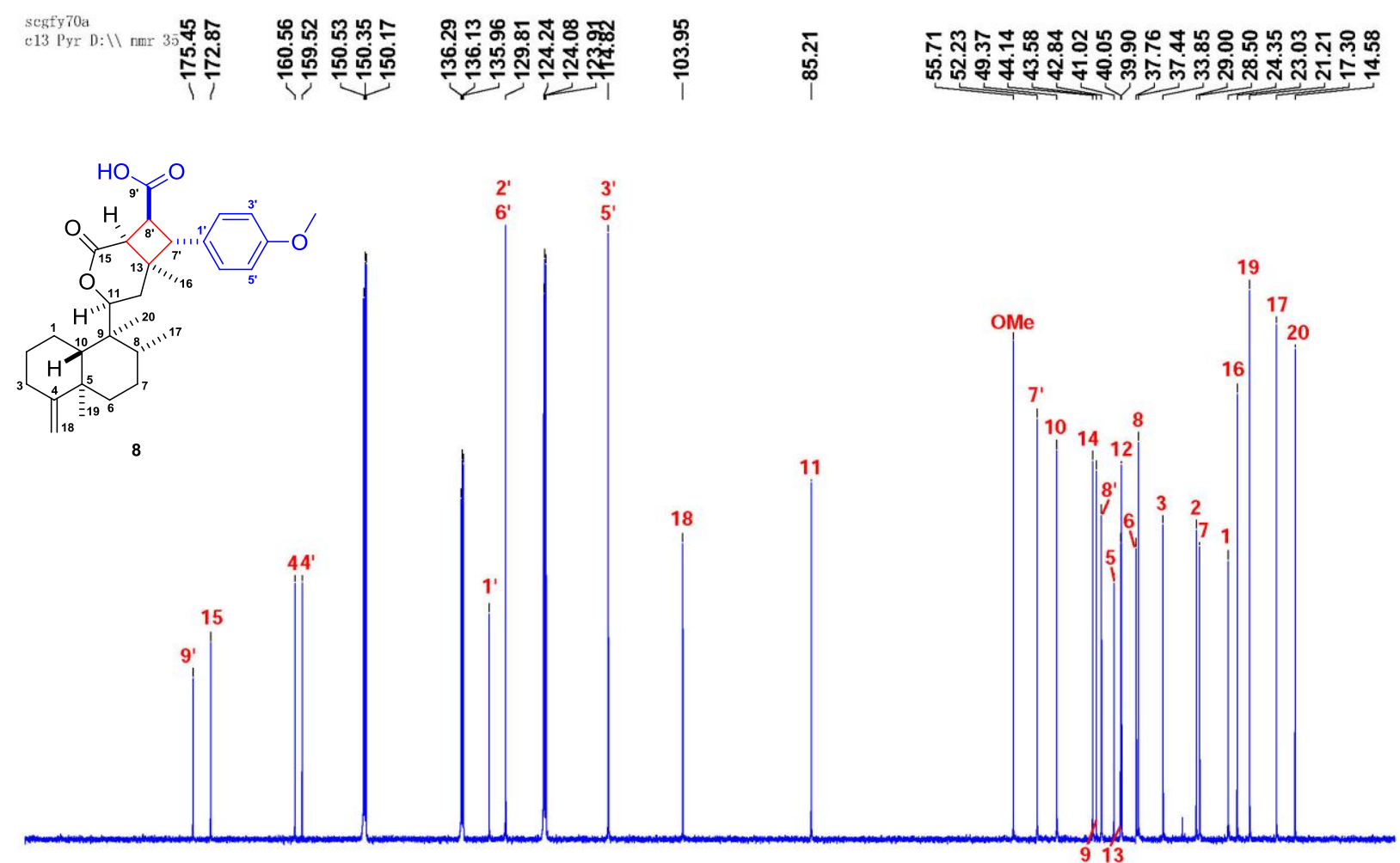
Figure S65. HSQC spectrum of $\mathbf{8}$

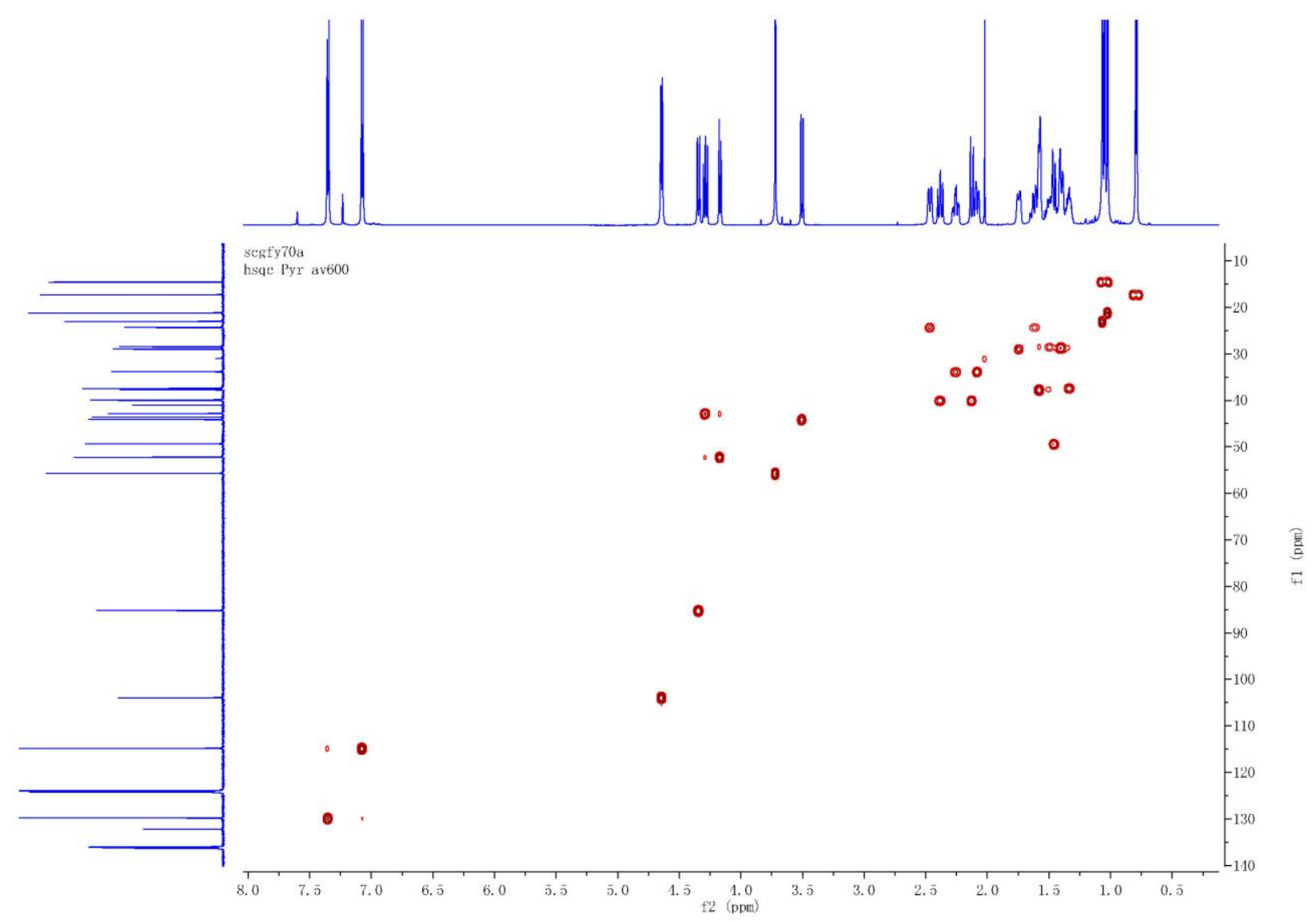

Figure S66. HMBC spectrum of $\mathbf{8}$

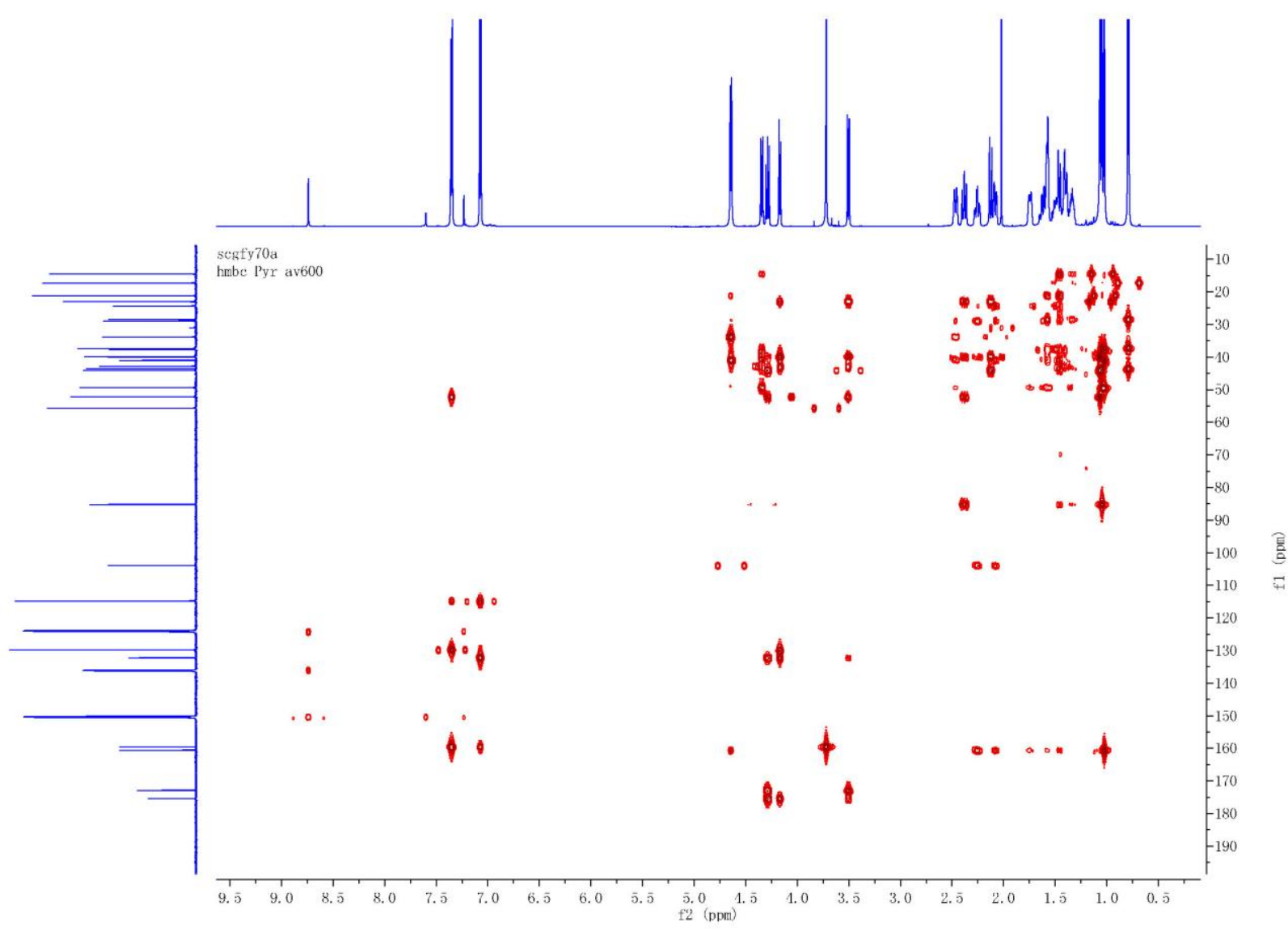


Figure S67. ${ }^{1} \mathrm{H}-{ }^{1} \mathrm{H}$ COSY spectrum of 8

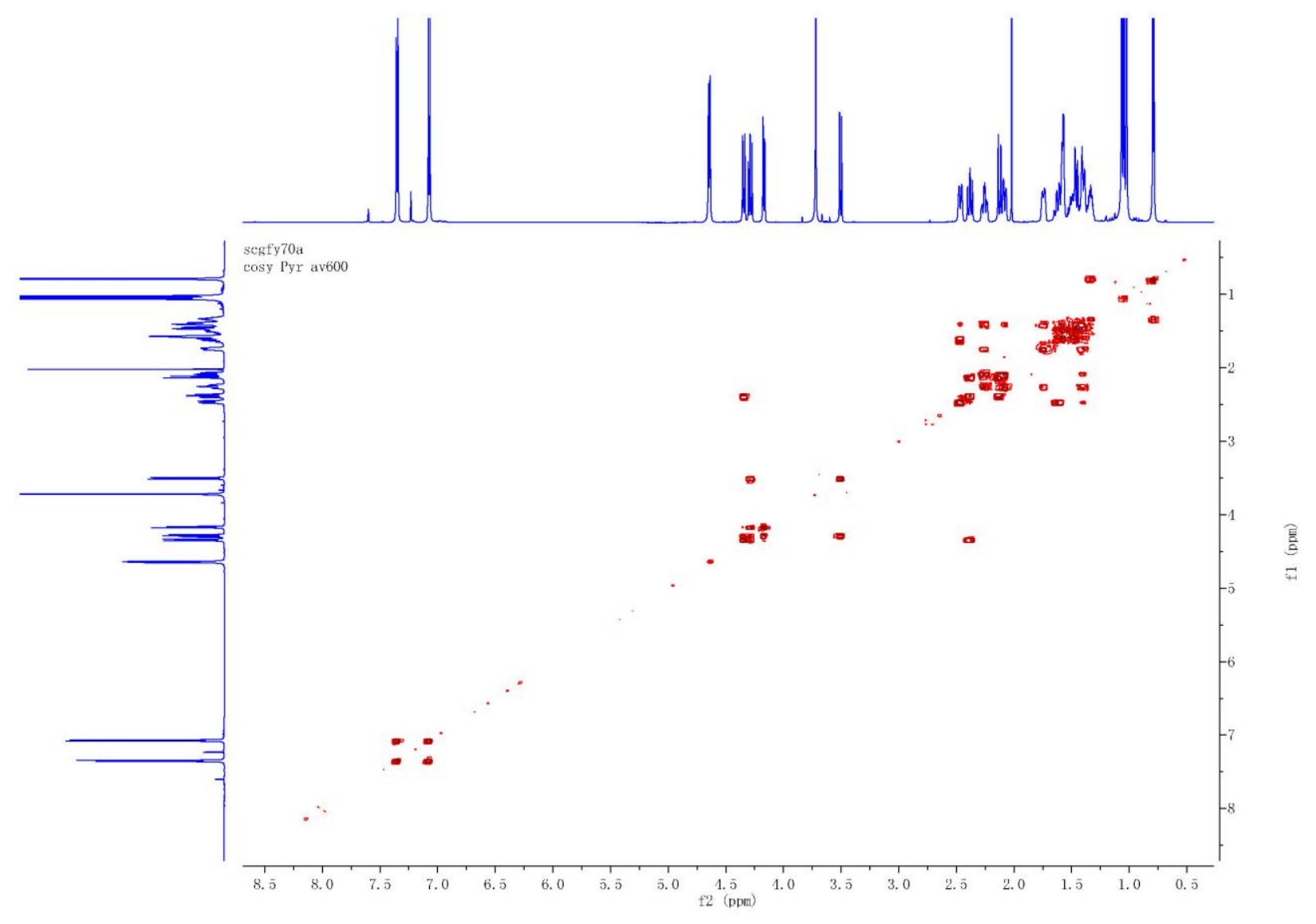

Figure S68 ROESY spectrum of 8

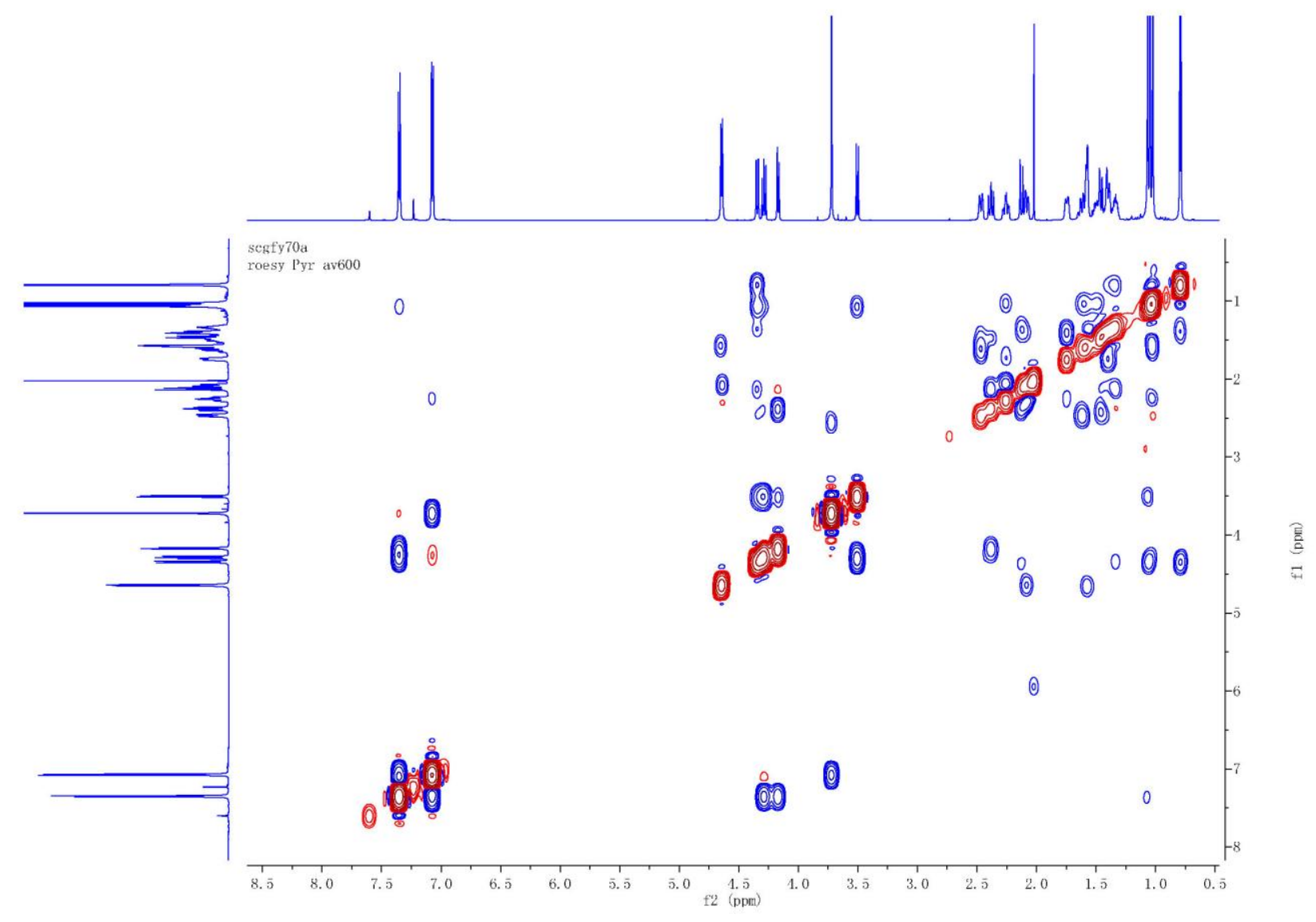


Figure S69. ${ }^{1} \mathrm{H}$ NMR spectrum of $\mathbf{1}$ (synthetic compound)

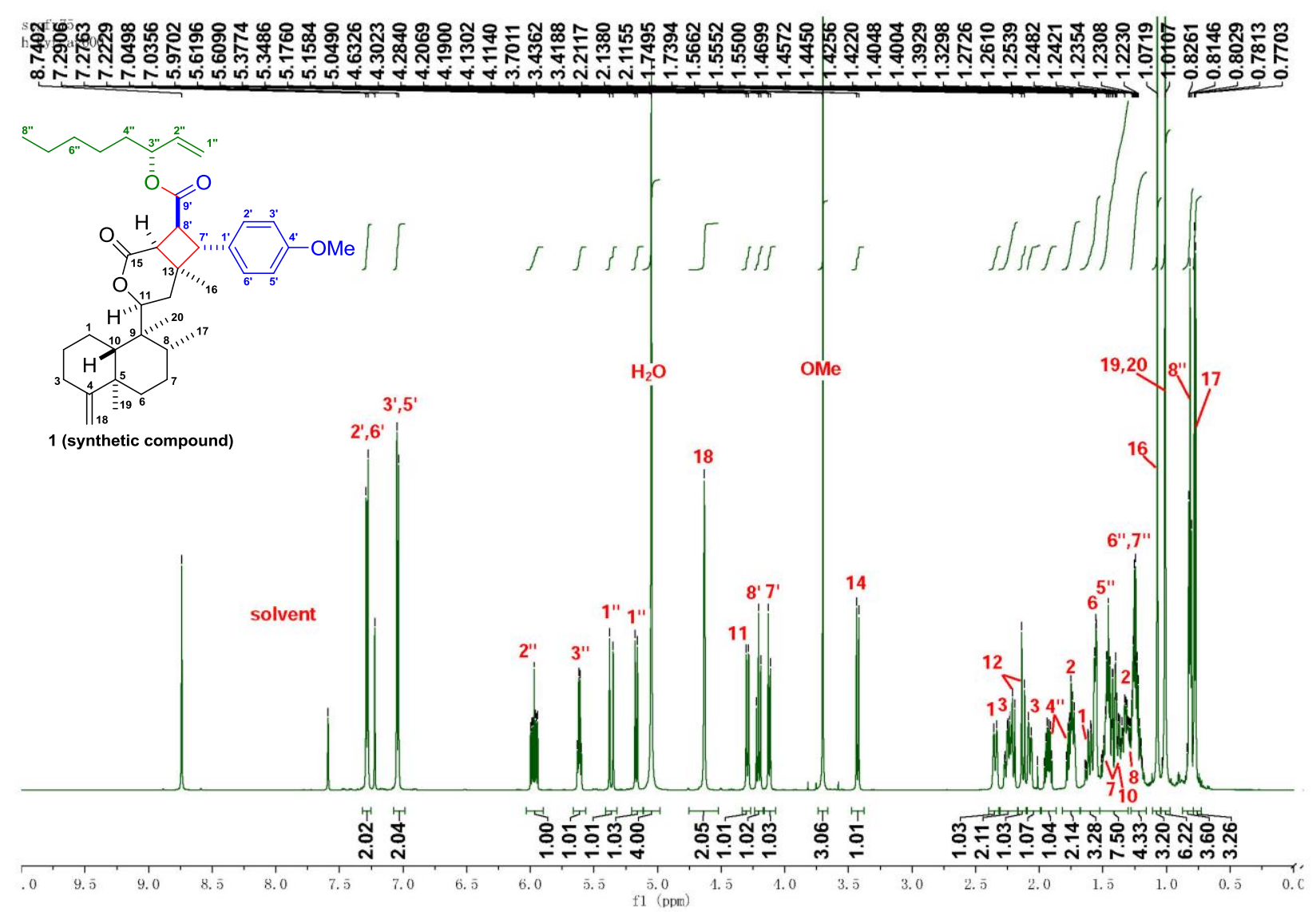

Figure S70. ${ }^{13} \mathrm{C}$ NMR spectrum of $\mathbf{1}$ (synthetic compound)
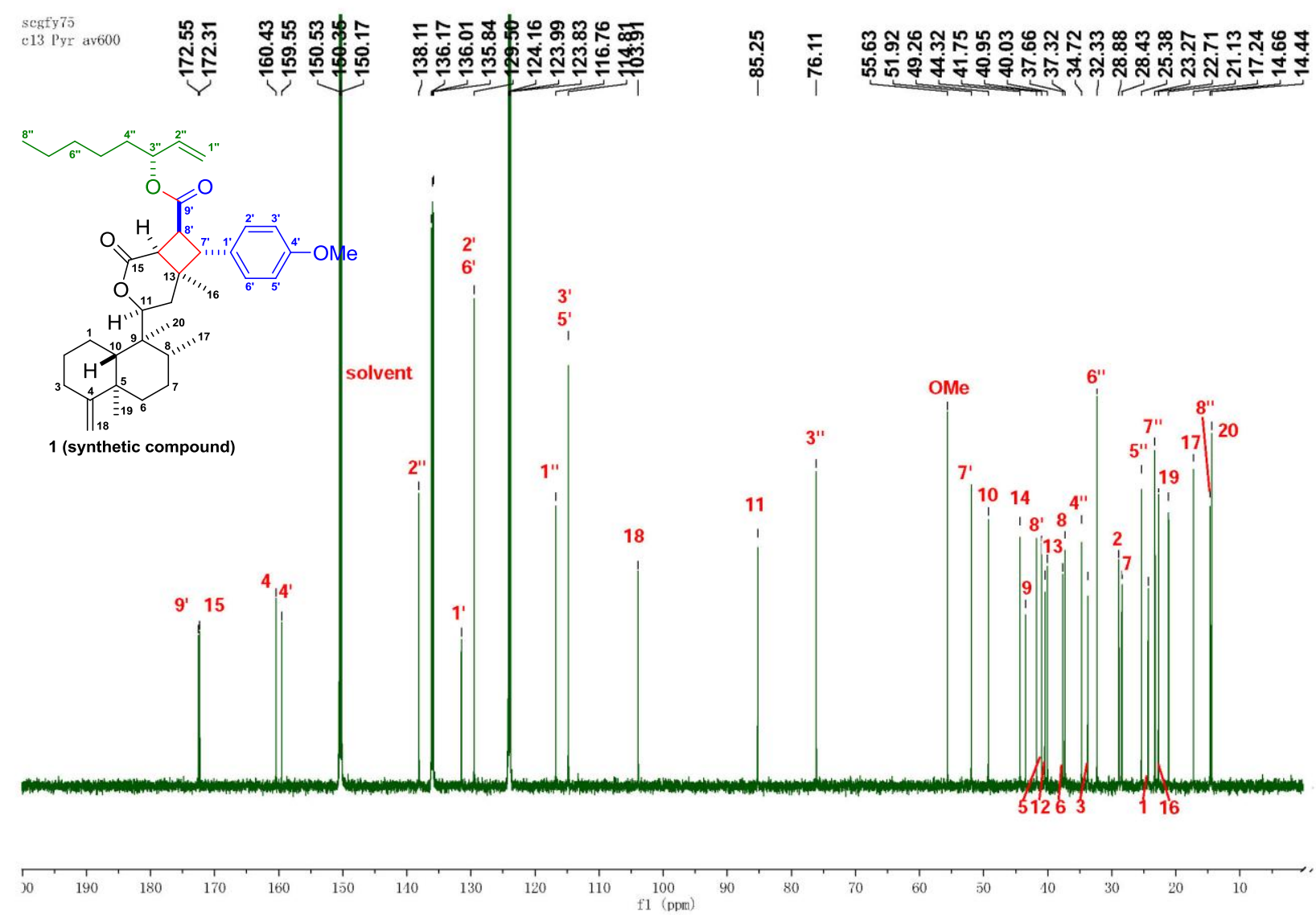
Figure S71. ${ }^{1} \mathrm{H}$ NMR spectrum of $\mathbf{1 0}$

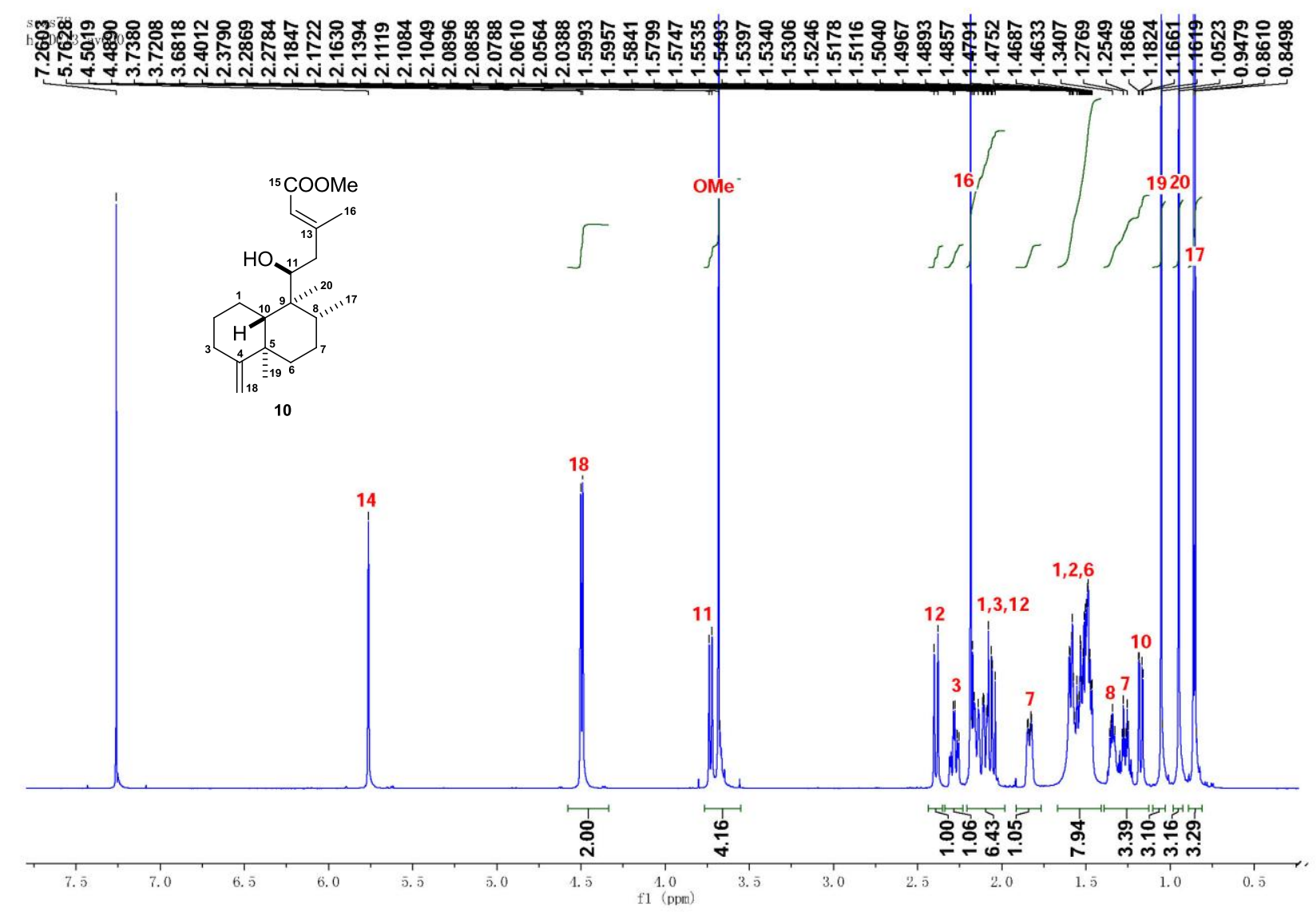

Figure S72. ${ }^{13} \mathrm{C}$ NMR spectrum of $\mathbf{1 0}$
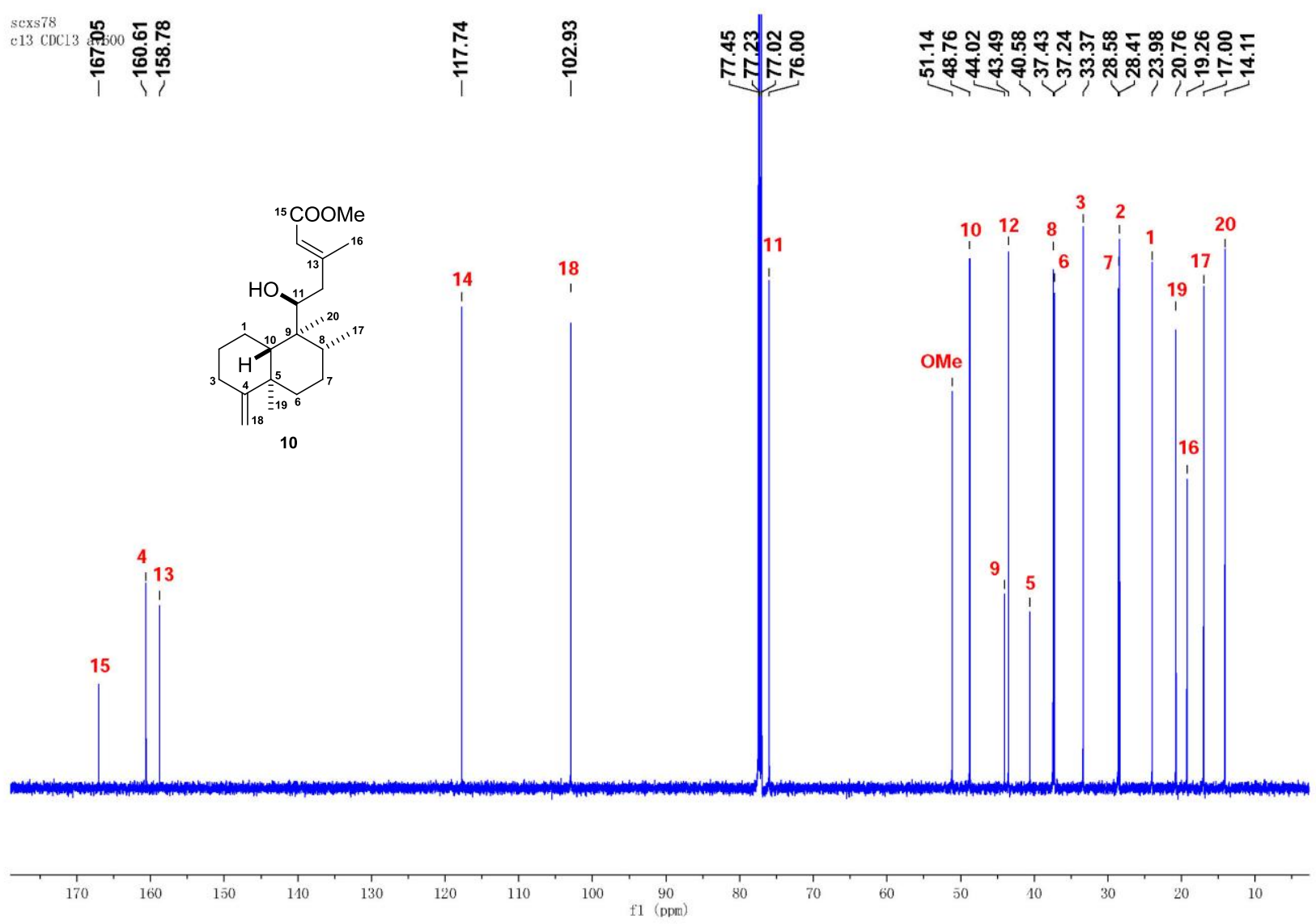
Figure S73. ${ }^{1} \mathrm{H}$ NMR spectrum of $\mathbf{1 2}$

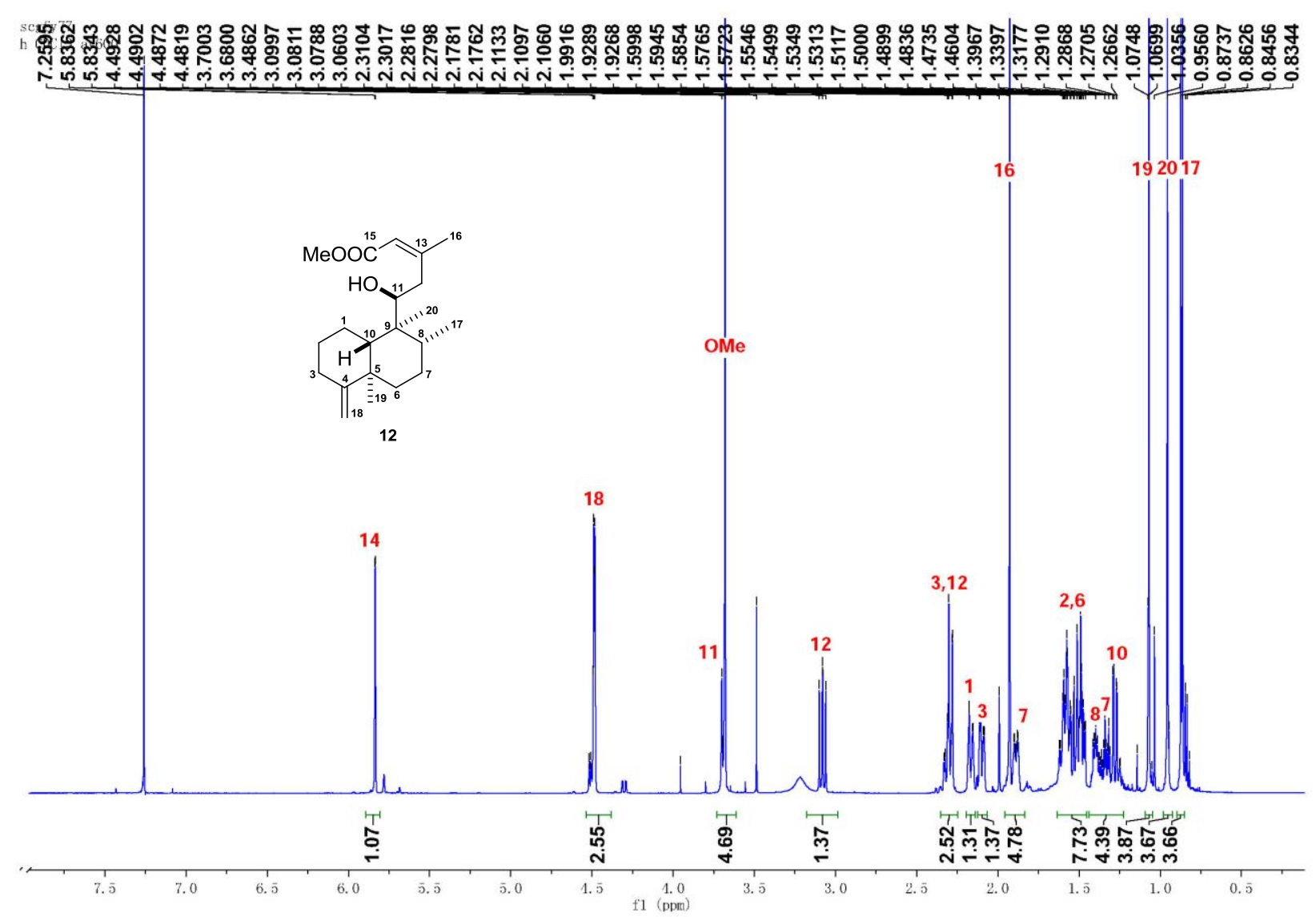

Figure S74. ${ }^{13} \mathrm{C}$ NMR spectrum of $\mathbf{1 2}$
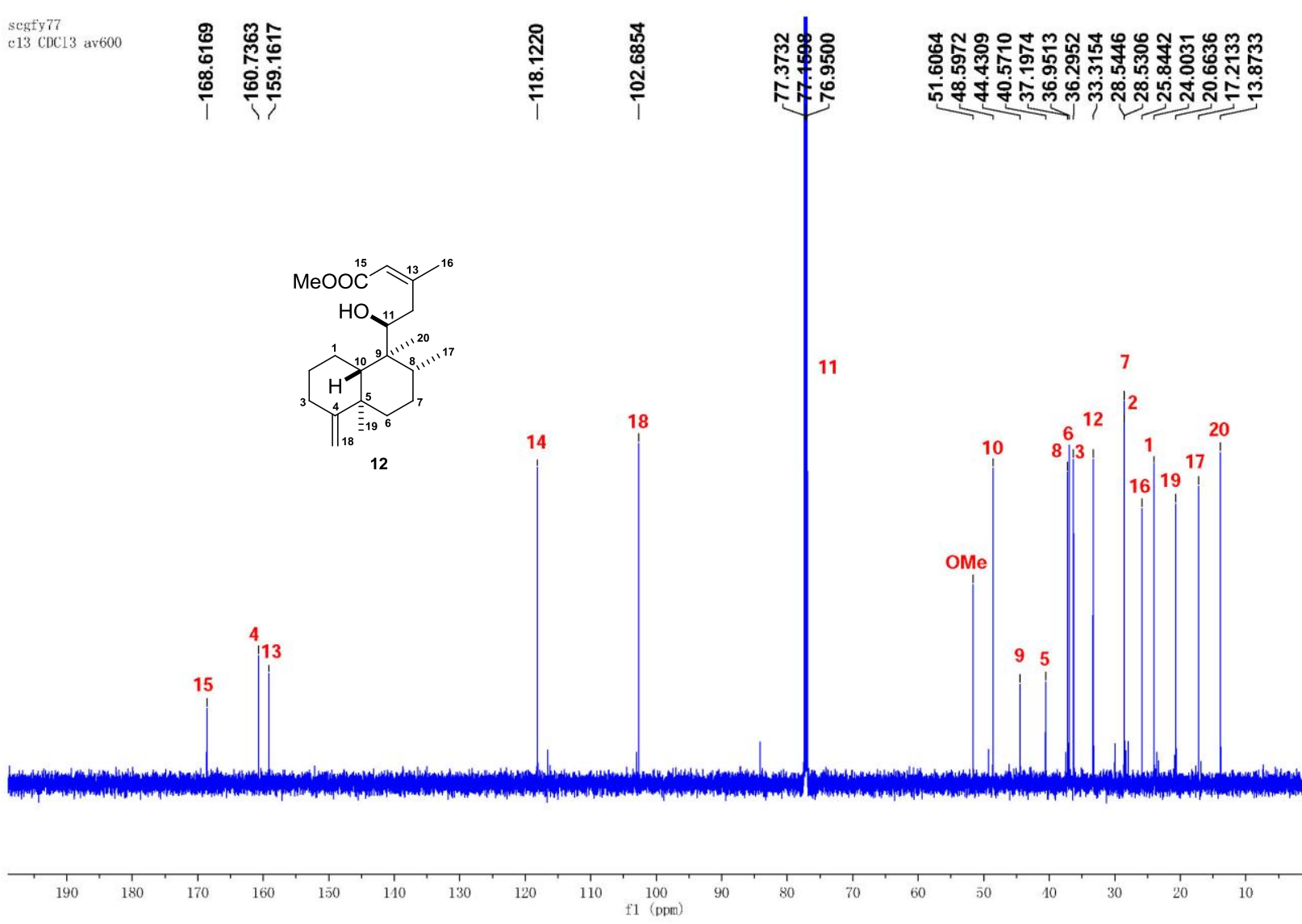
Figure S75. ${ }^{1} \mathrm{H}$ NMR spectrum of $\mathbf{1 5}$

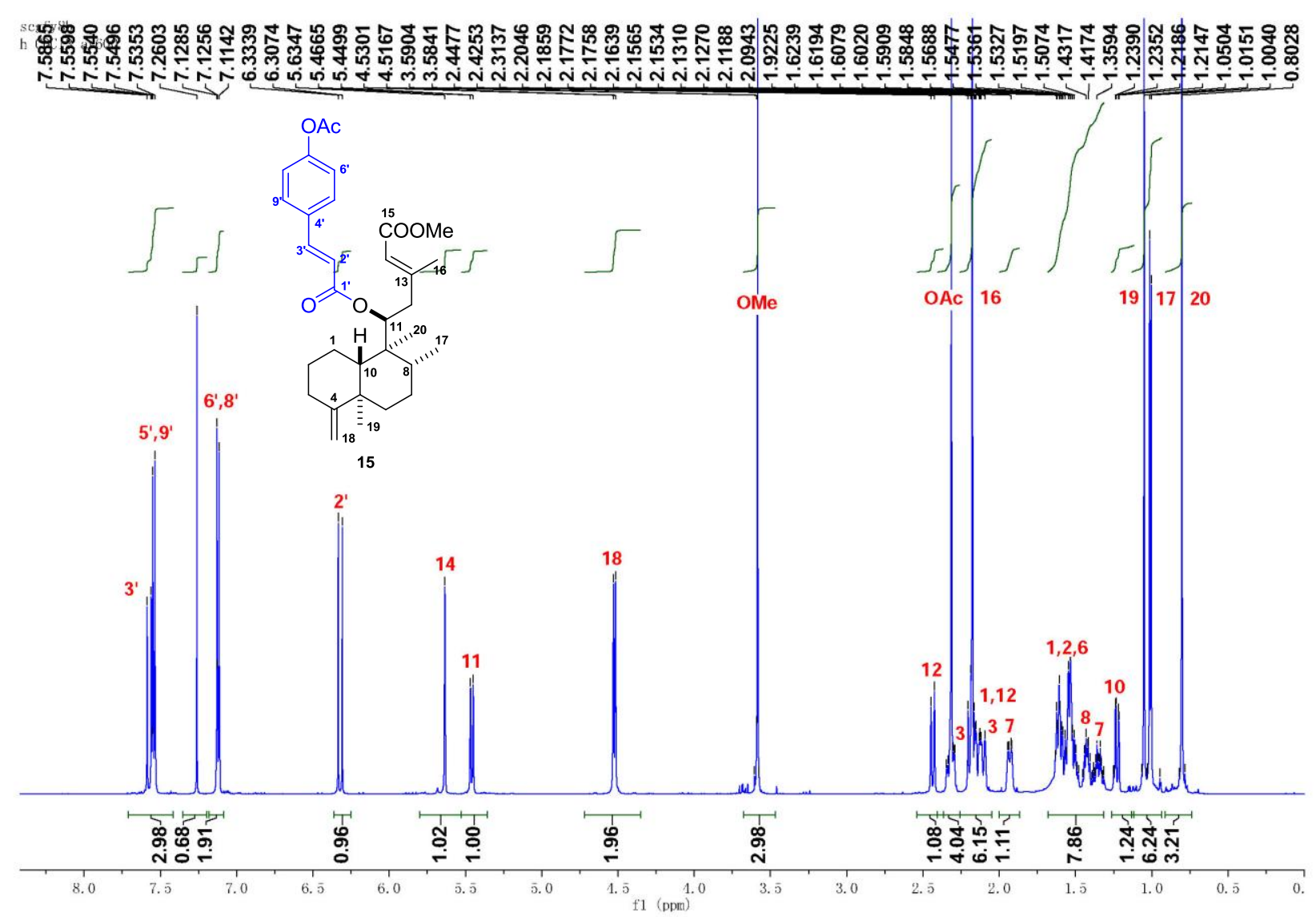

Figure S76. ${ }^{13} \mathrm{C}$ NMR spectrum of $\mathbf{1 5}$

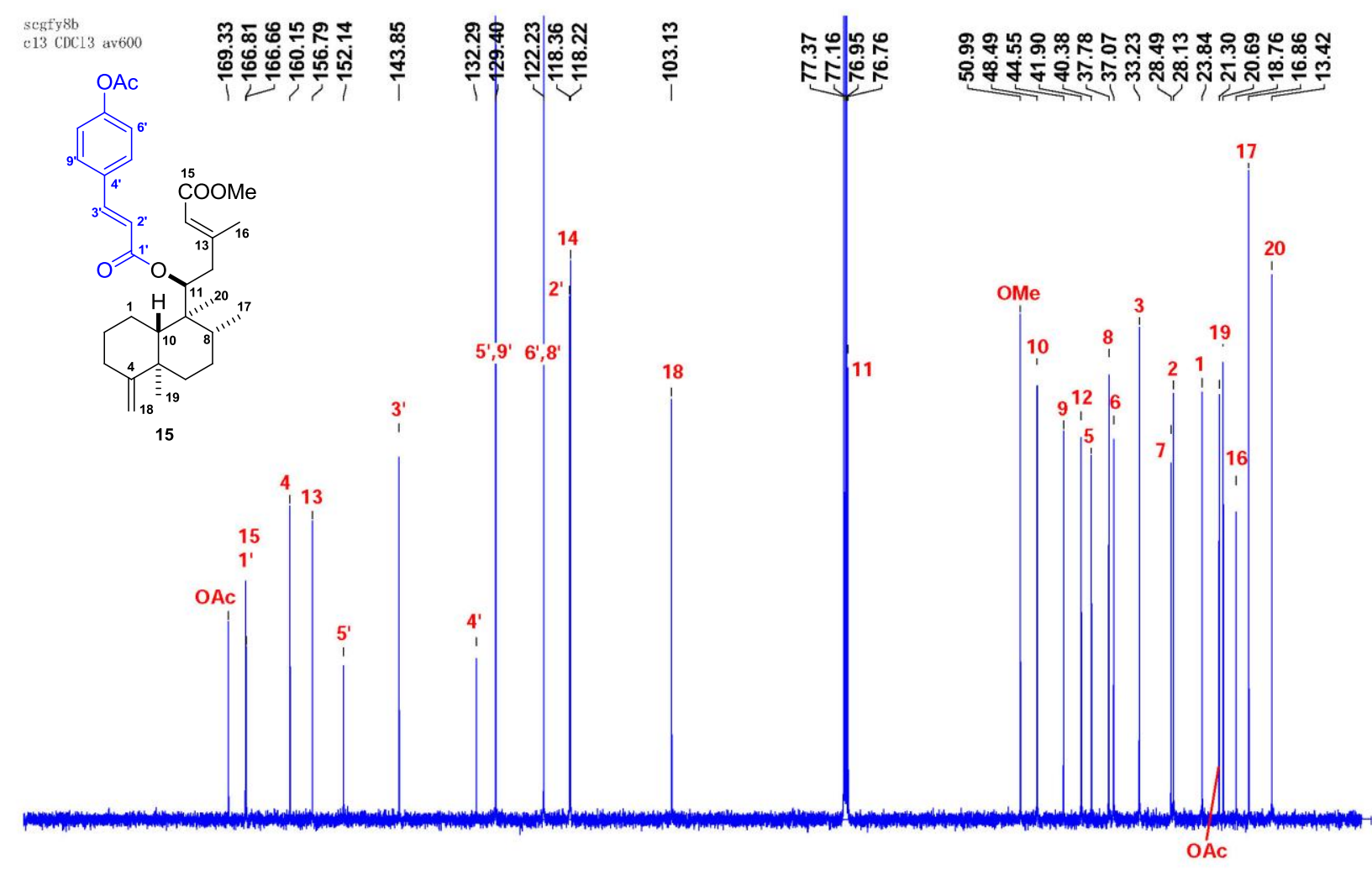

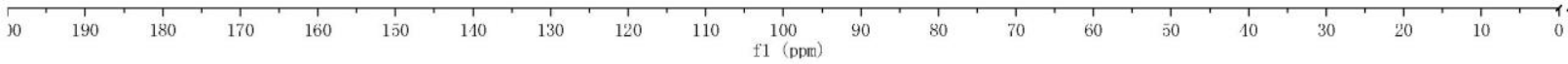


Figure S77. HSQC spectrum of $\mathbf{1 5}$

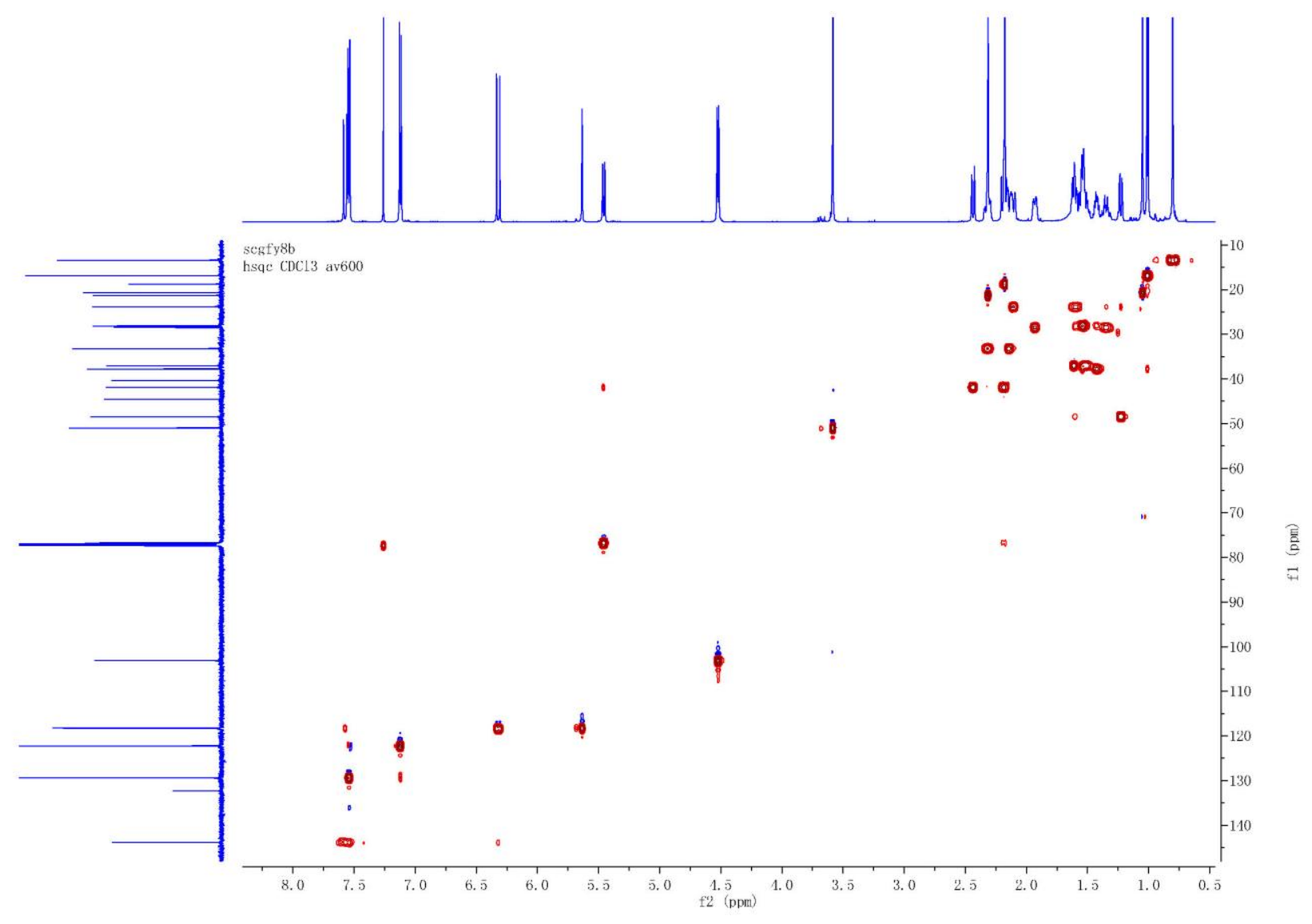

Figure S78. HMBC spectrum of $\mathbf{1 5}$

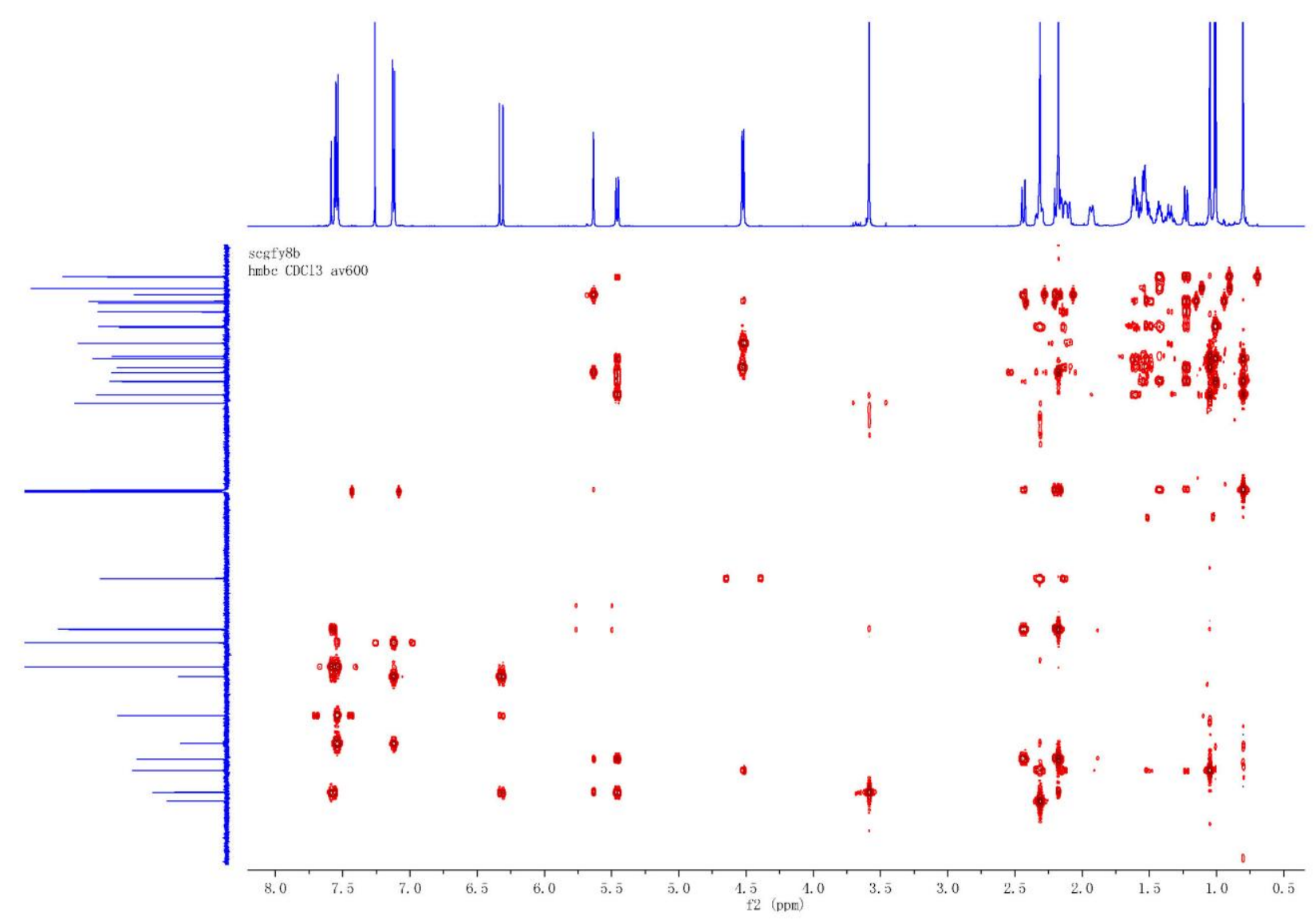


Figure S79. ${ }^{1} \mathrm{H}-{ }^{1} \mathrm{H}$ COSY spectrum of $\mathbf{1 5}$

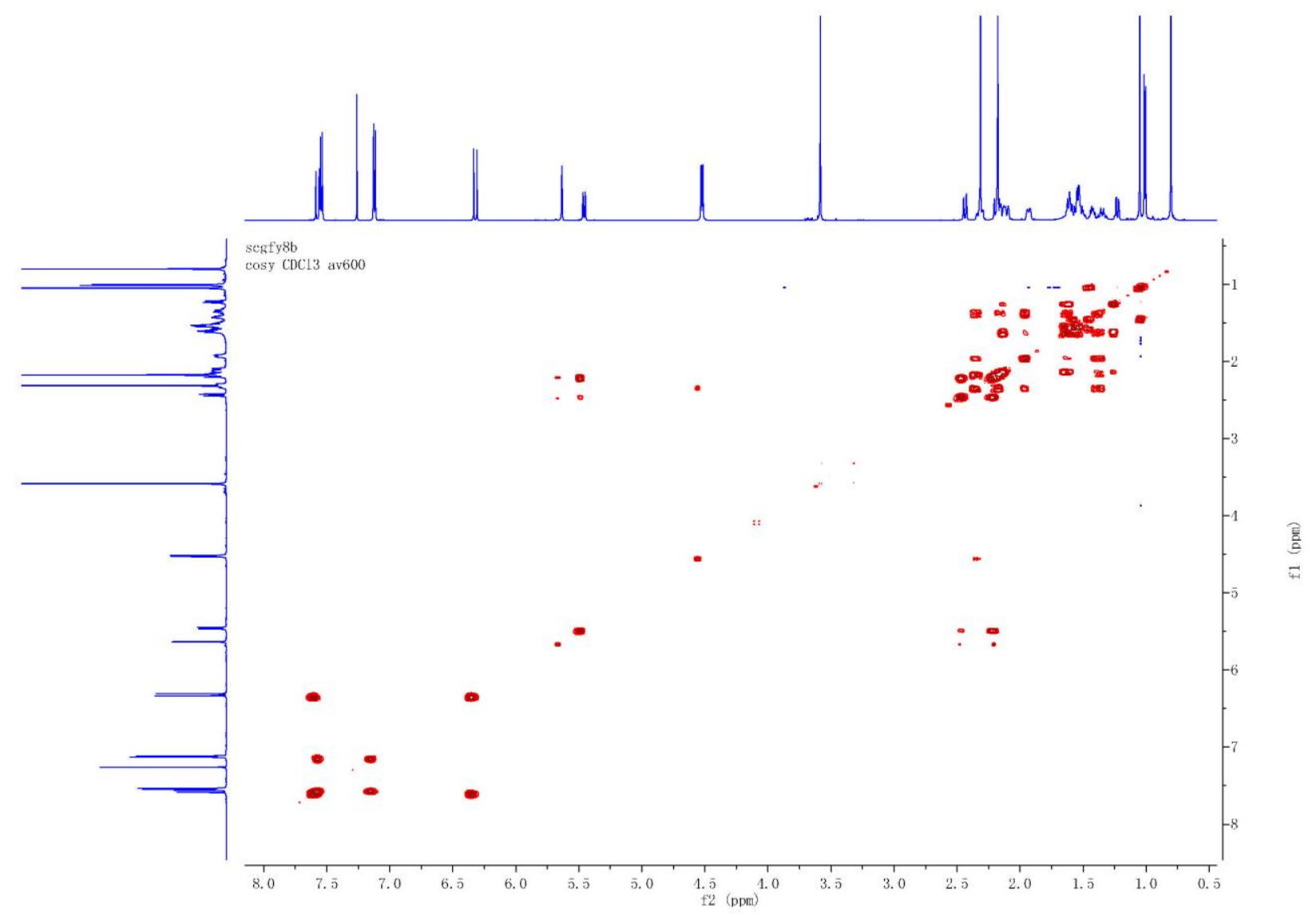

Figure S80. ROESY spectrum of $\mathbf{1 5}$

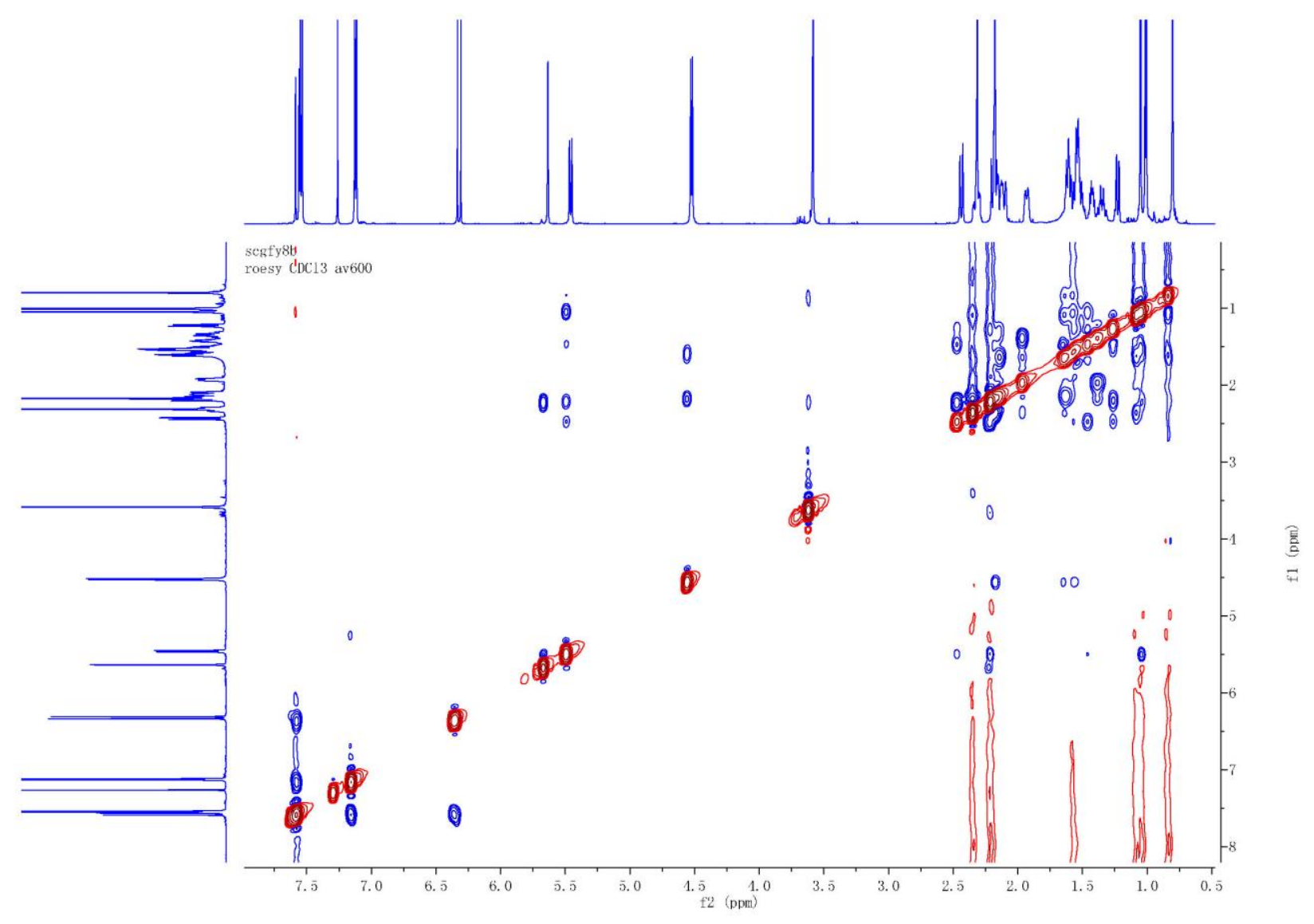


Figure S81. ${ }^{1} \mathrm{H}$ NMR spectrum of $\mathbf{1 6}$

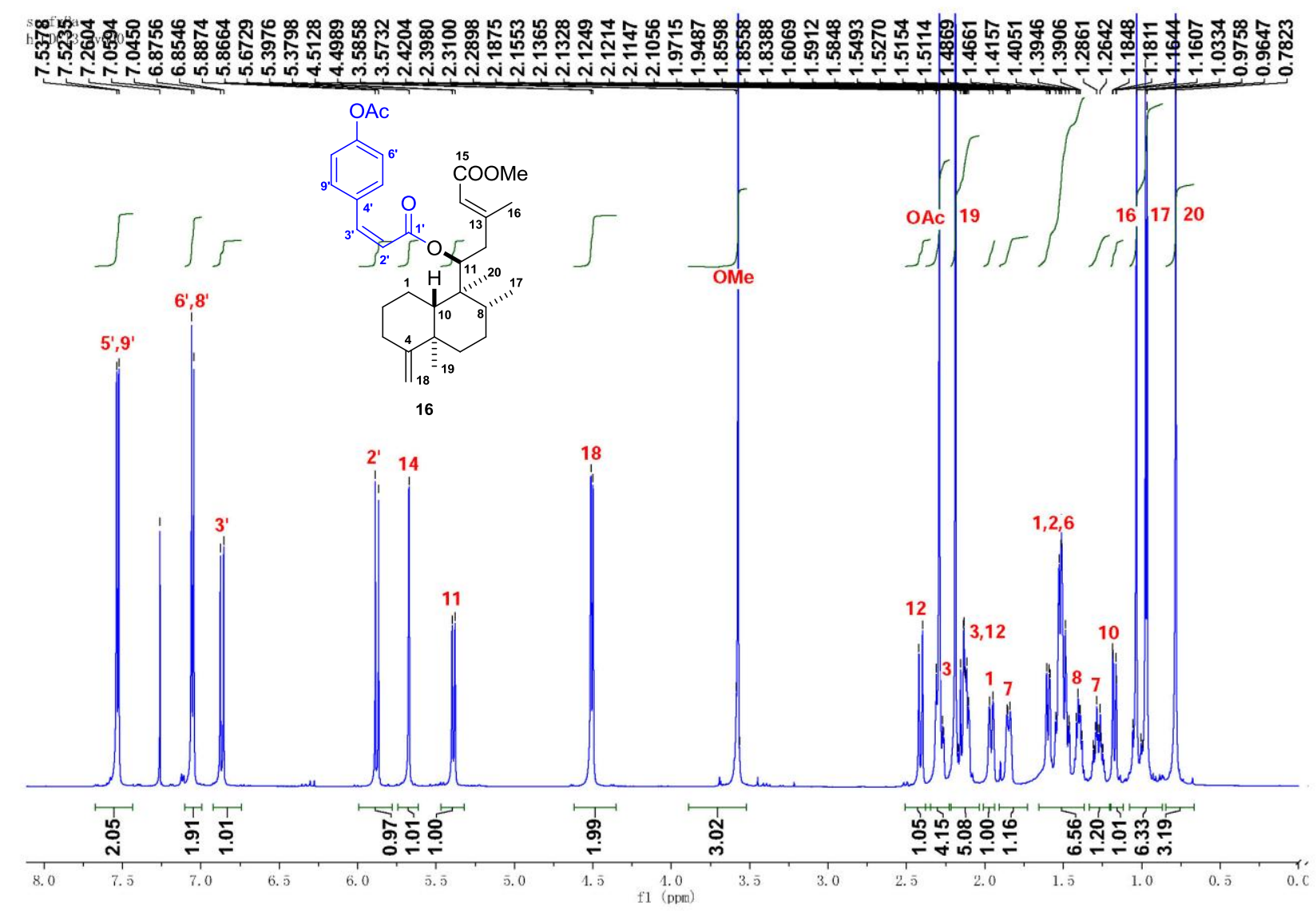

Figure S82. ${ }^{13} \mathrm{C}$ NMR spectrum of $\mathbf{1 6}$

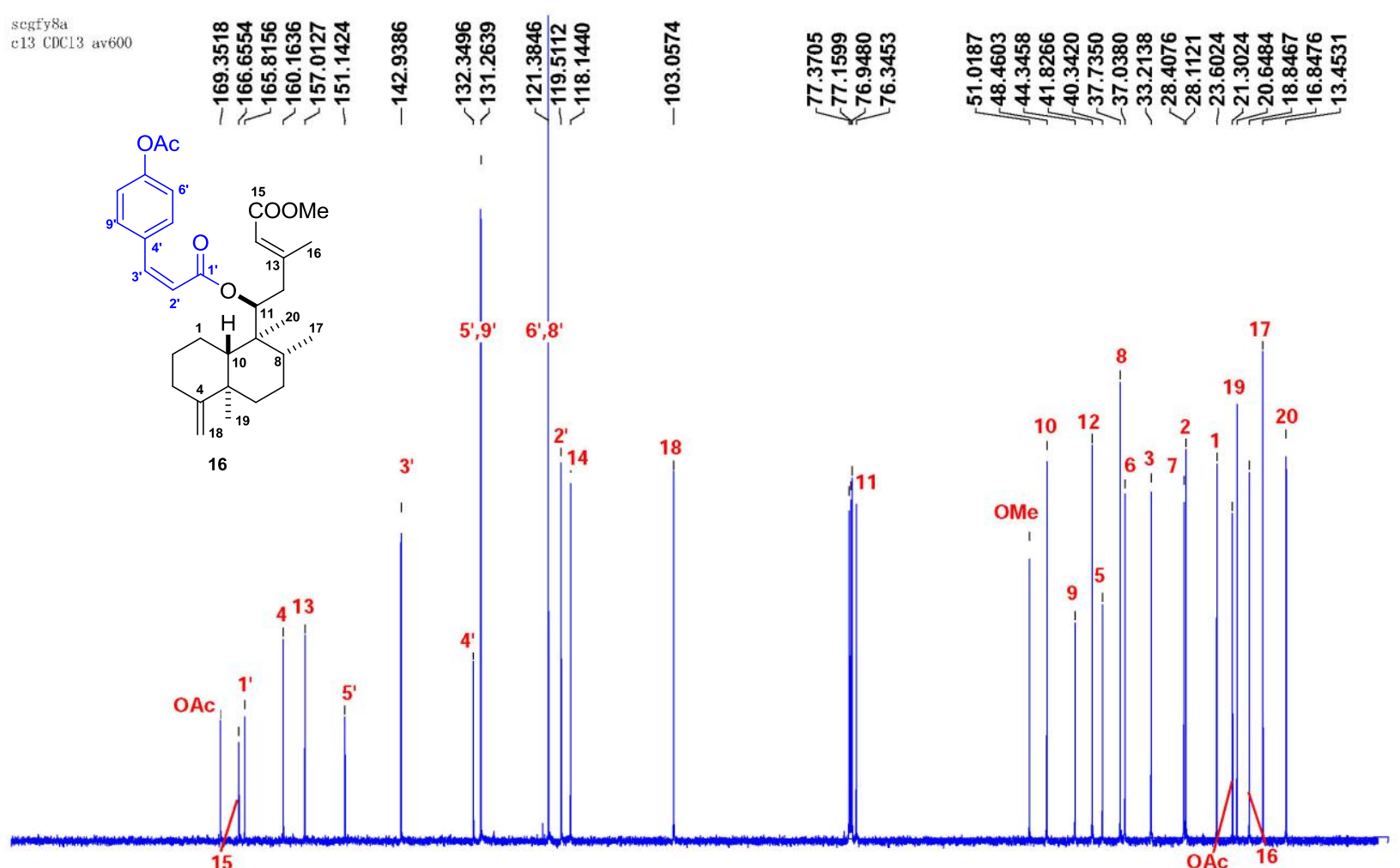

15

OAC 16

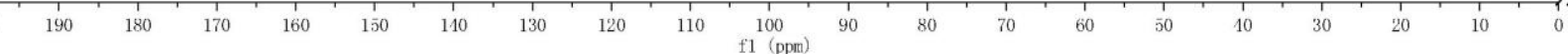


Figure S83. HSQC spectrum of $\mathbf{1 6}$

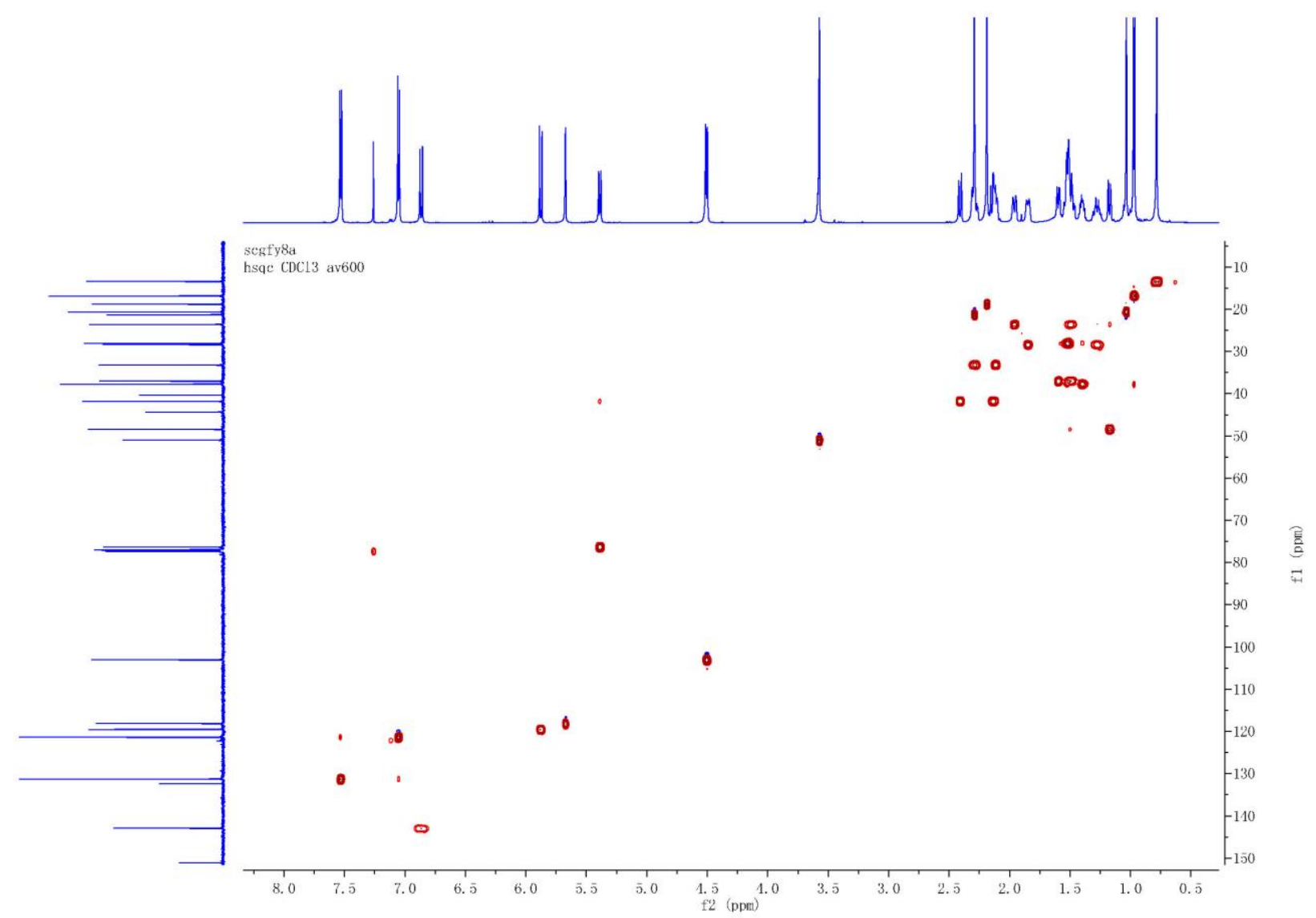

Figure S84. HMBC spectrum of $\mathbf{1 6}$

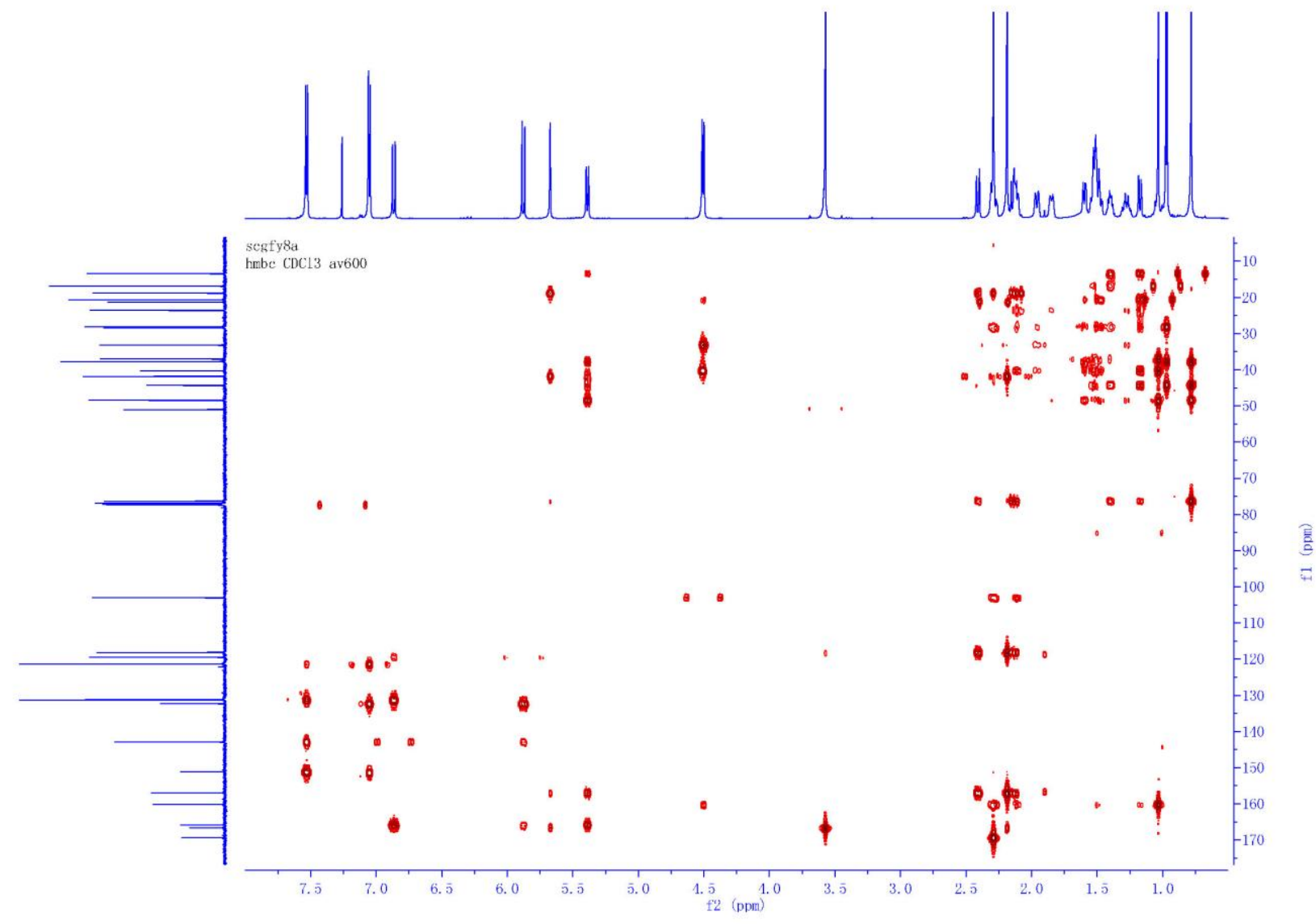


Figure S85. ${ }^{1} \mathrm{H}-{ }^{1} \mathrm{H}$ COSY spectrum of $\mathbf{1 6}$

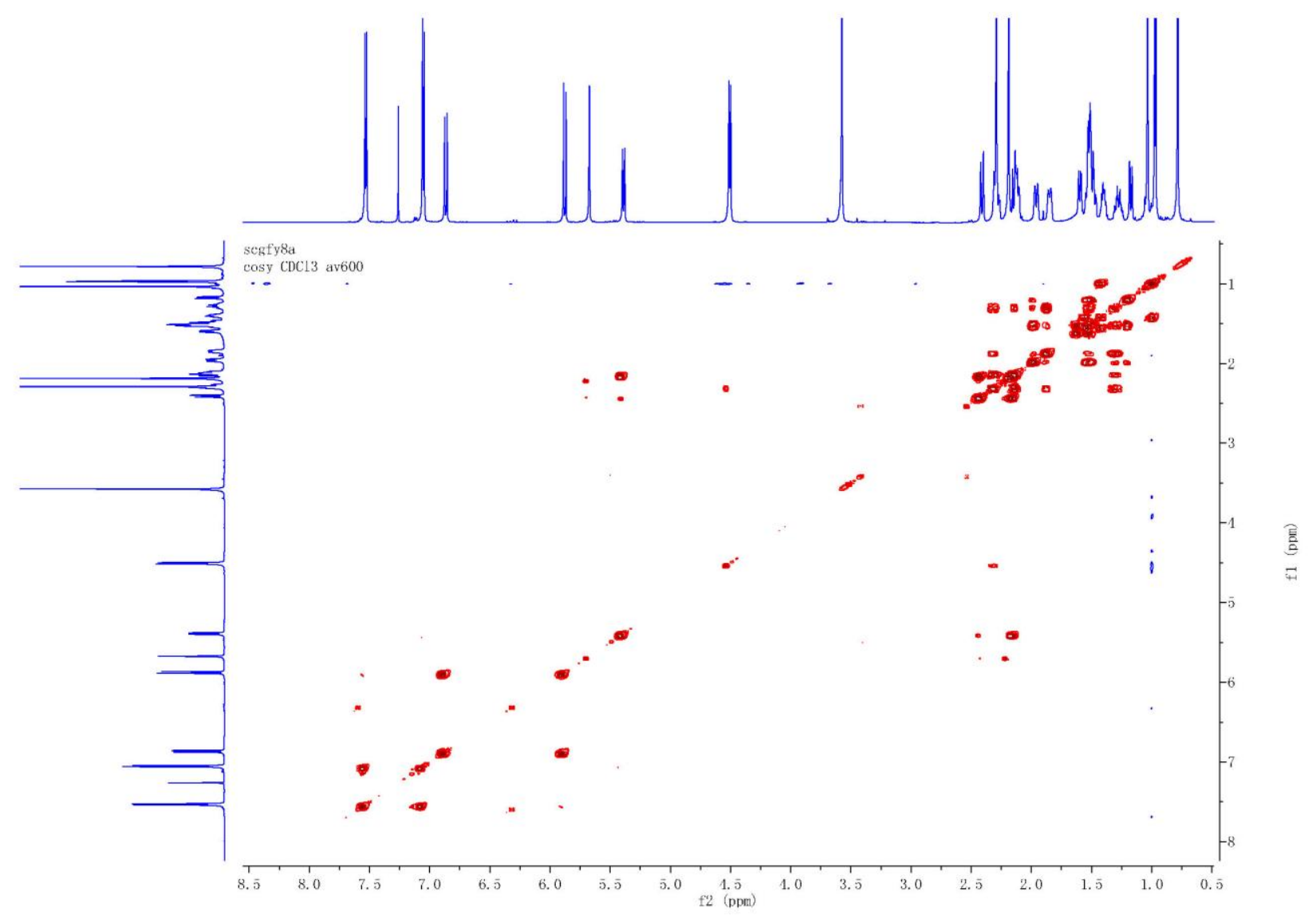

Figure S86. ROESY spectrum of $\mathbf{1 6}$

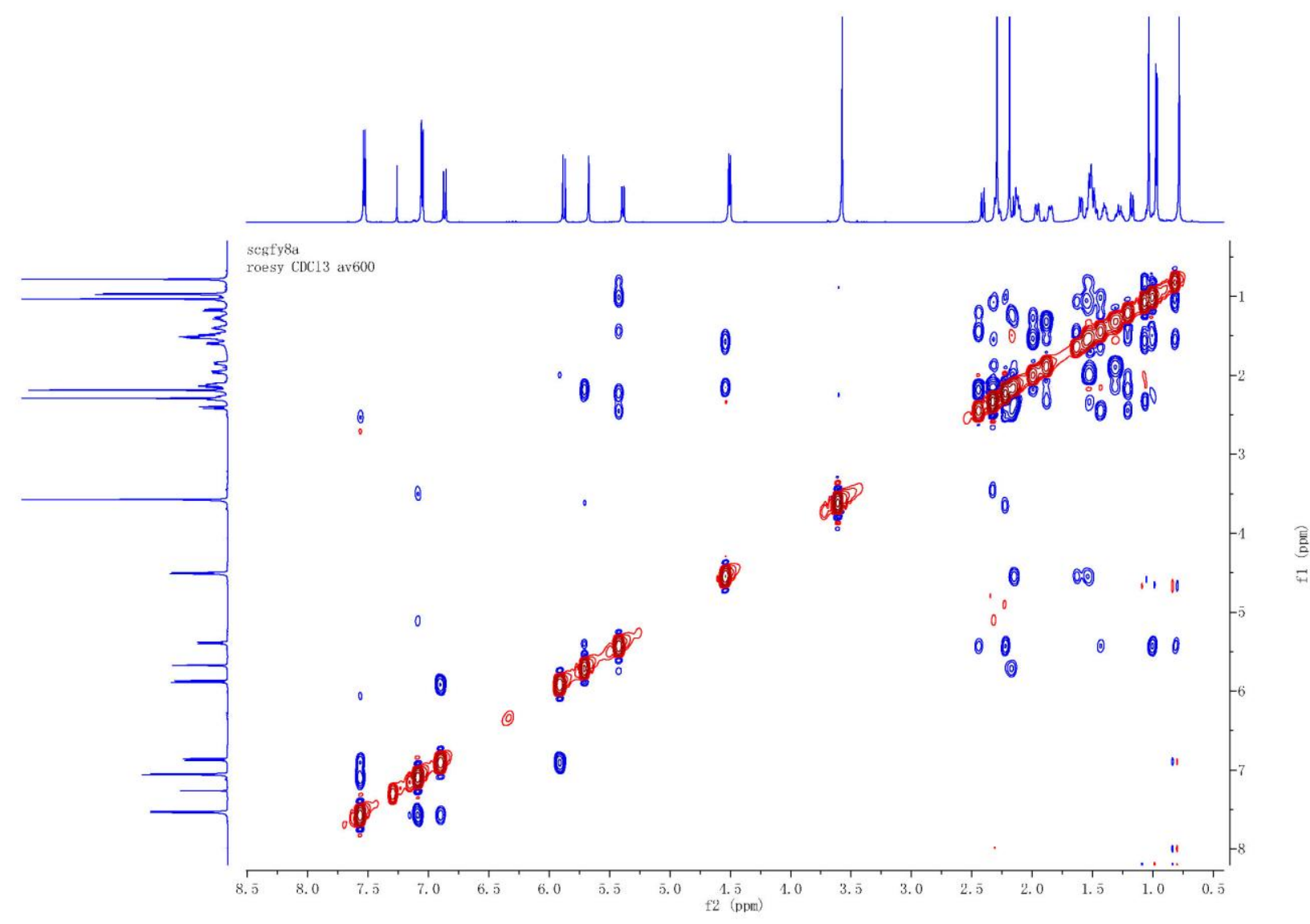


Figure S87. ${ }^{1} \mathrm{H}$ NMR spectrum of 21

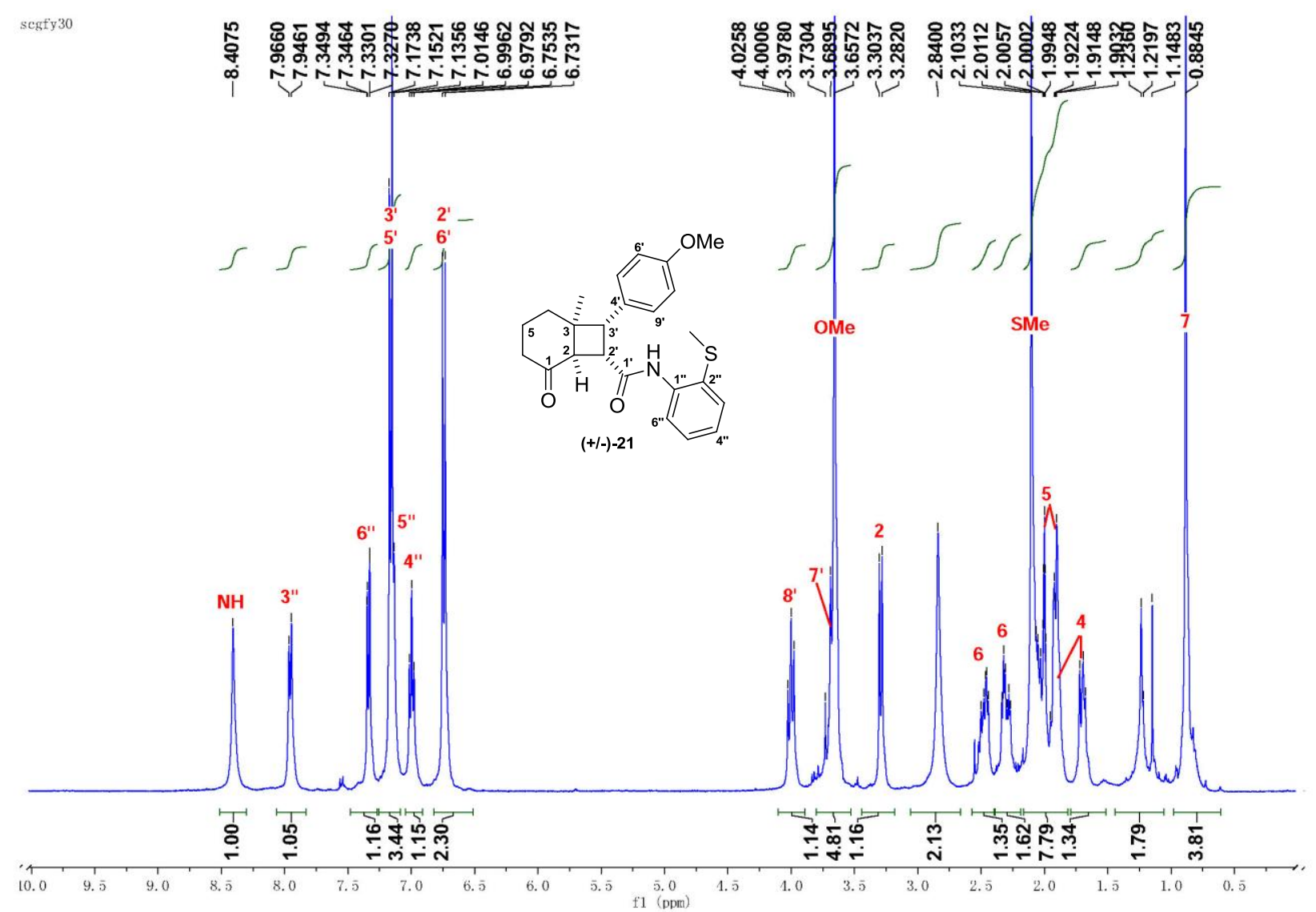

Figure S88. ${ }^{13} \mathrm{C}$ NMR spectrum of $\mathbf{2 1}$

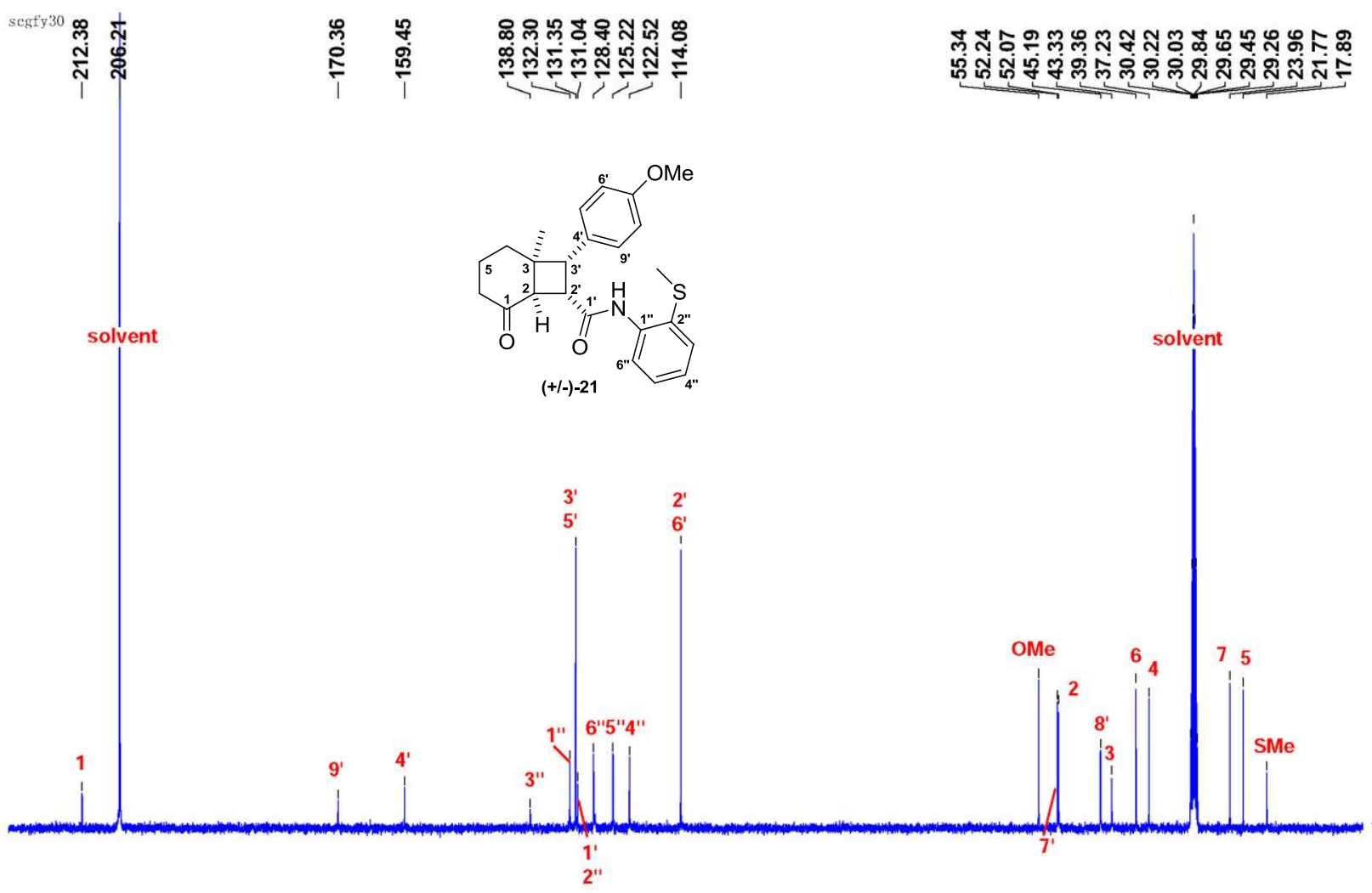

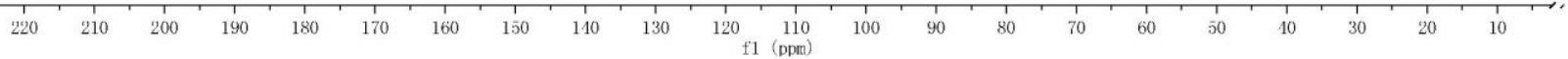


Figure S89. ${ }^{1} \mathrm{H}$ NMR spectrum of 22

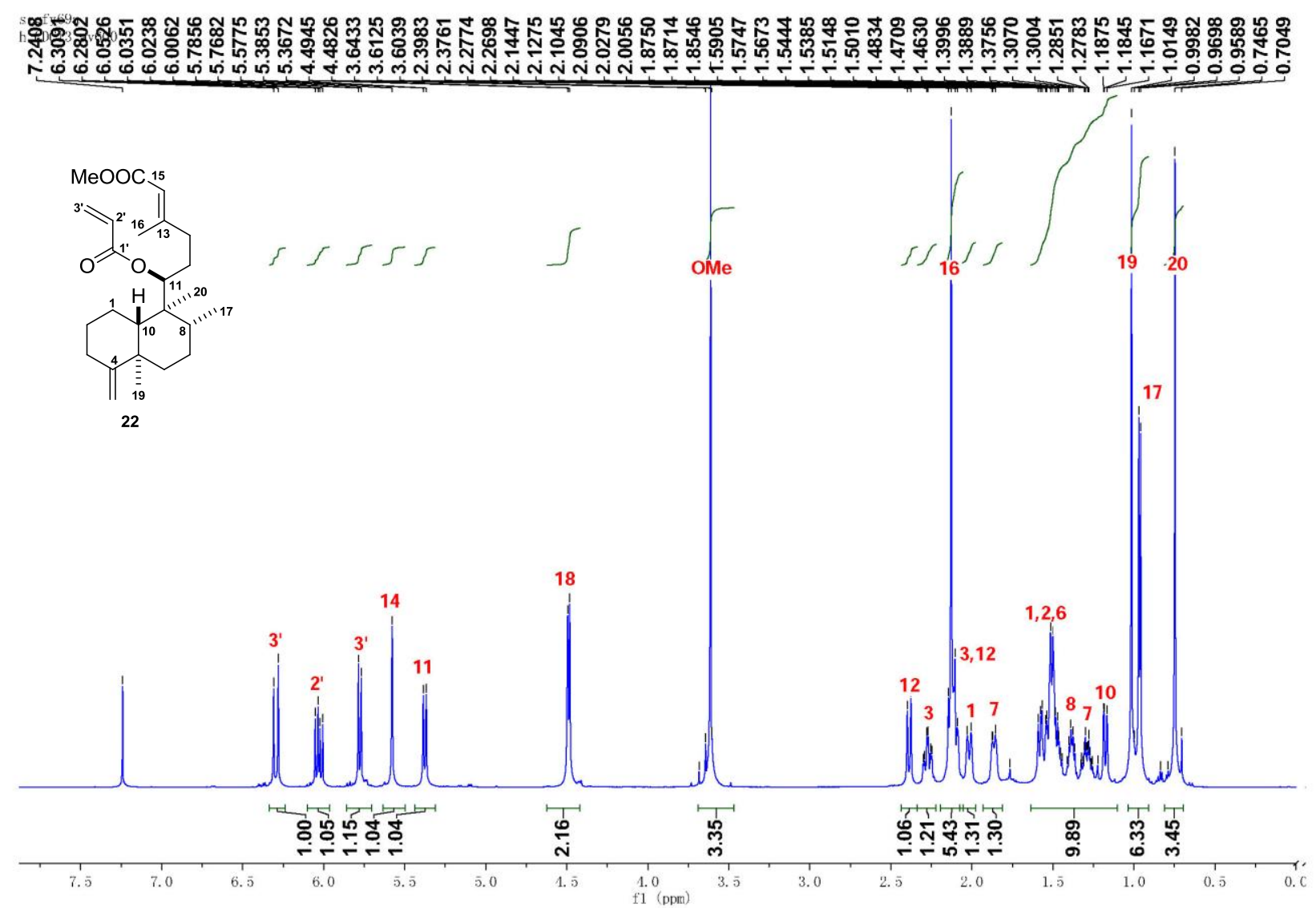

Figure S90. ${ }^{13} \mathrm{C}$ NMR spectrum of $\mathbf{2 2}$

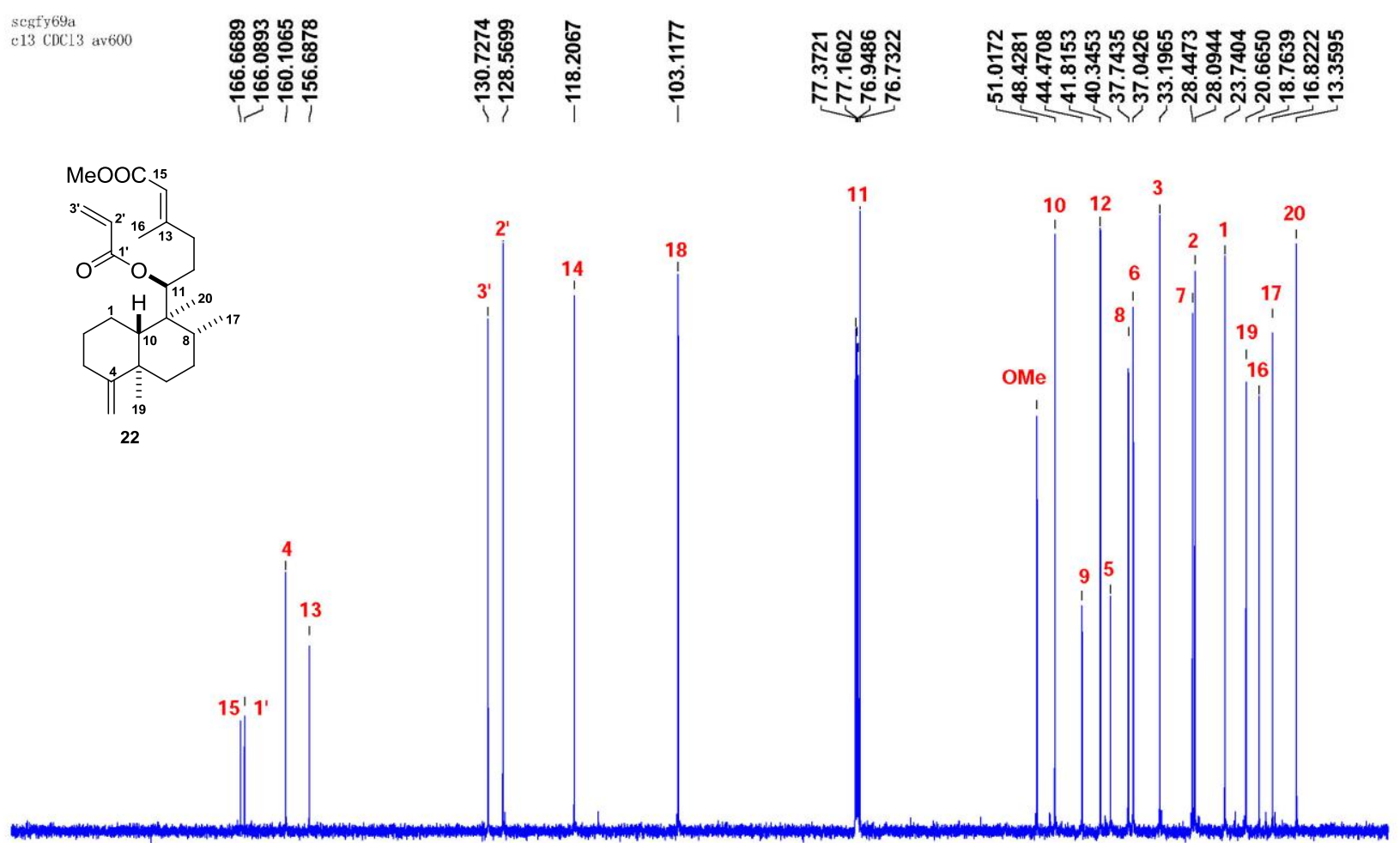

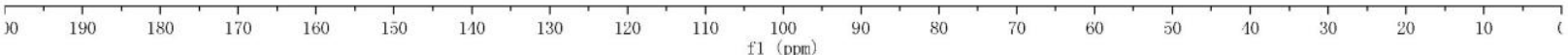


Figure S91. HSQC spectrum of $\mathbf{2 2}$

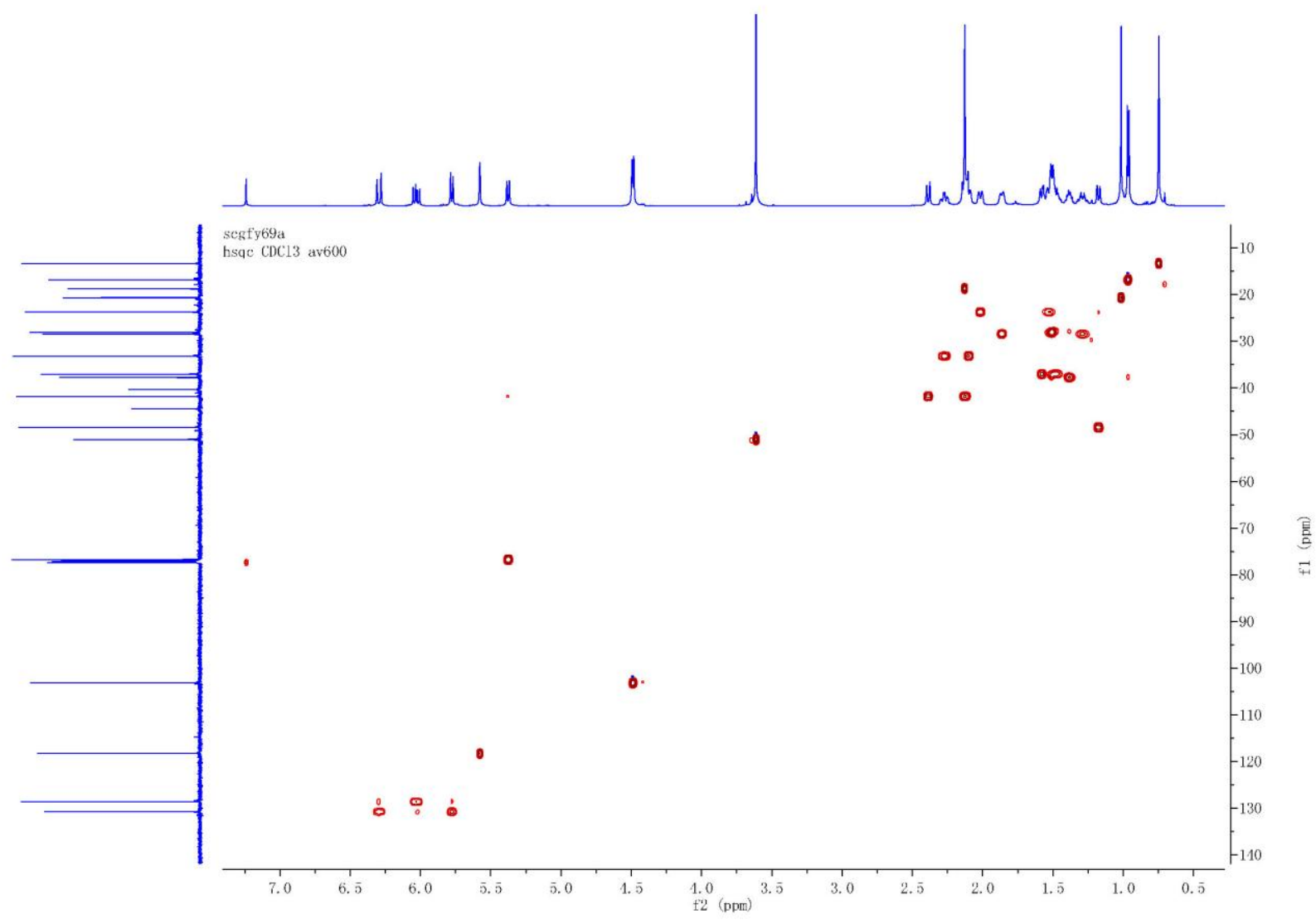

Figure S92. HMBC spectrum of $\mathbf{2 2}$

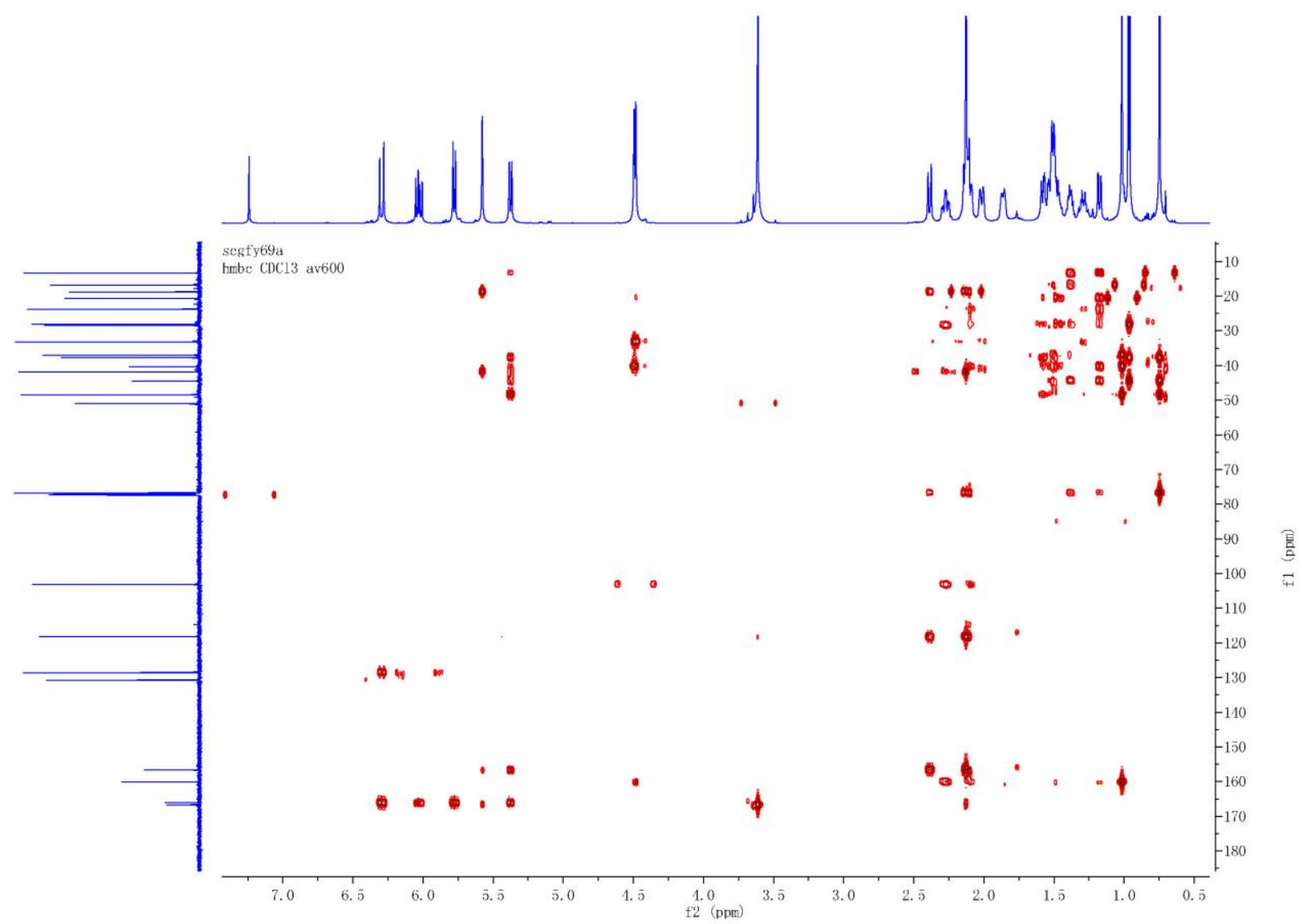


Figure S93. ${ }^{1} \mathrm{H}-{ }^{1} \mathrm{H}$ COSY spectrum of $\mathbf{2 2}$

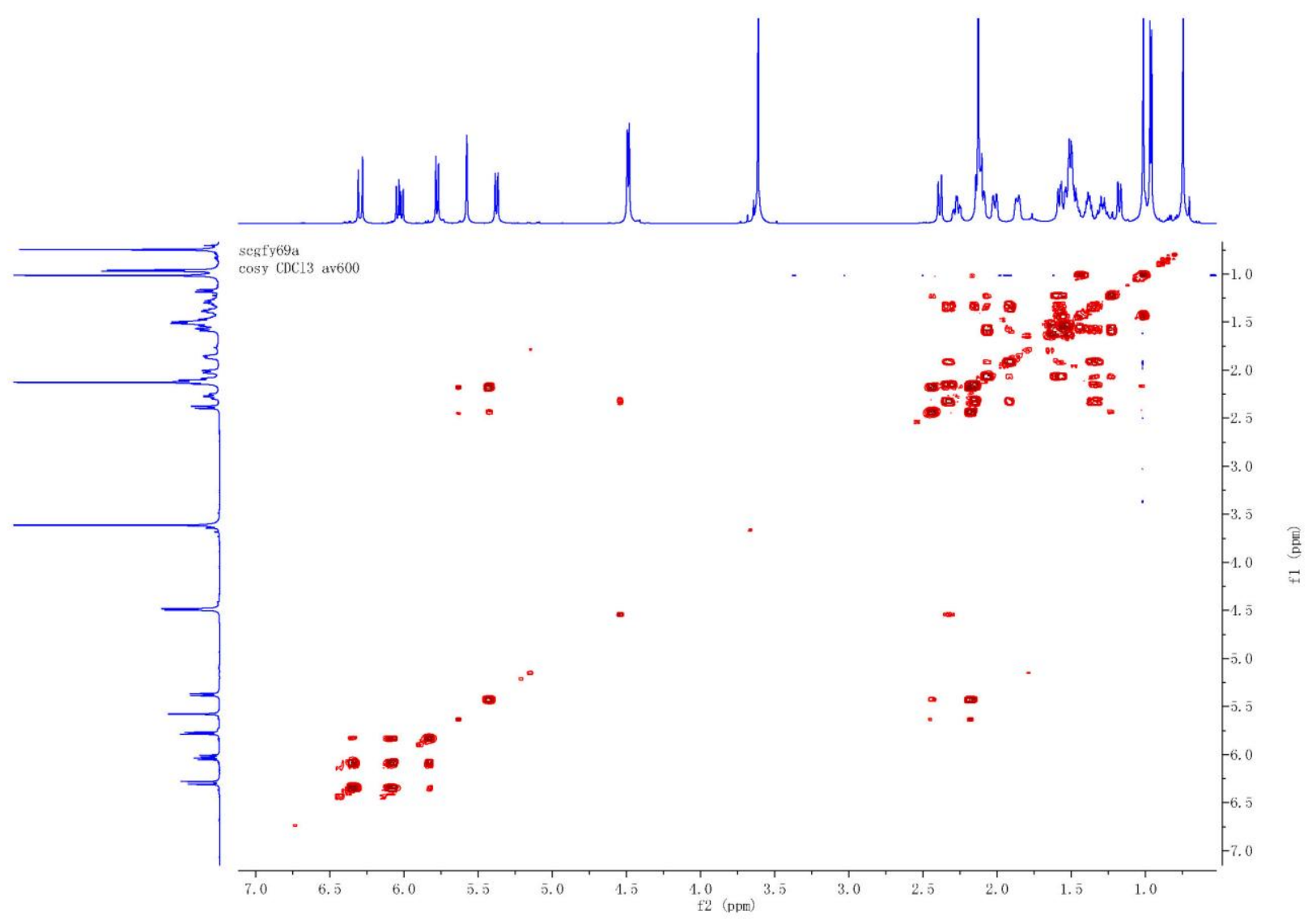

Figure S94. ROESY spectrum of $\mathbf{2 2}$

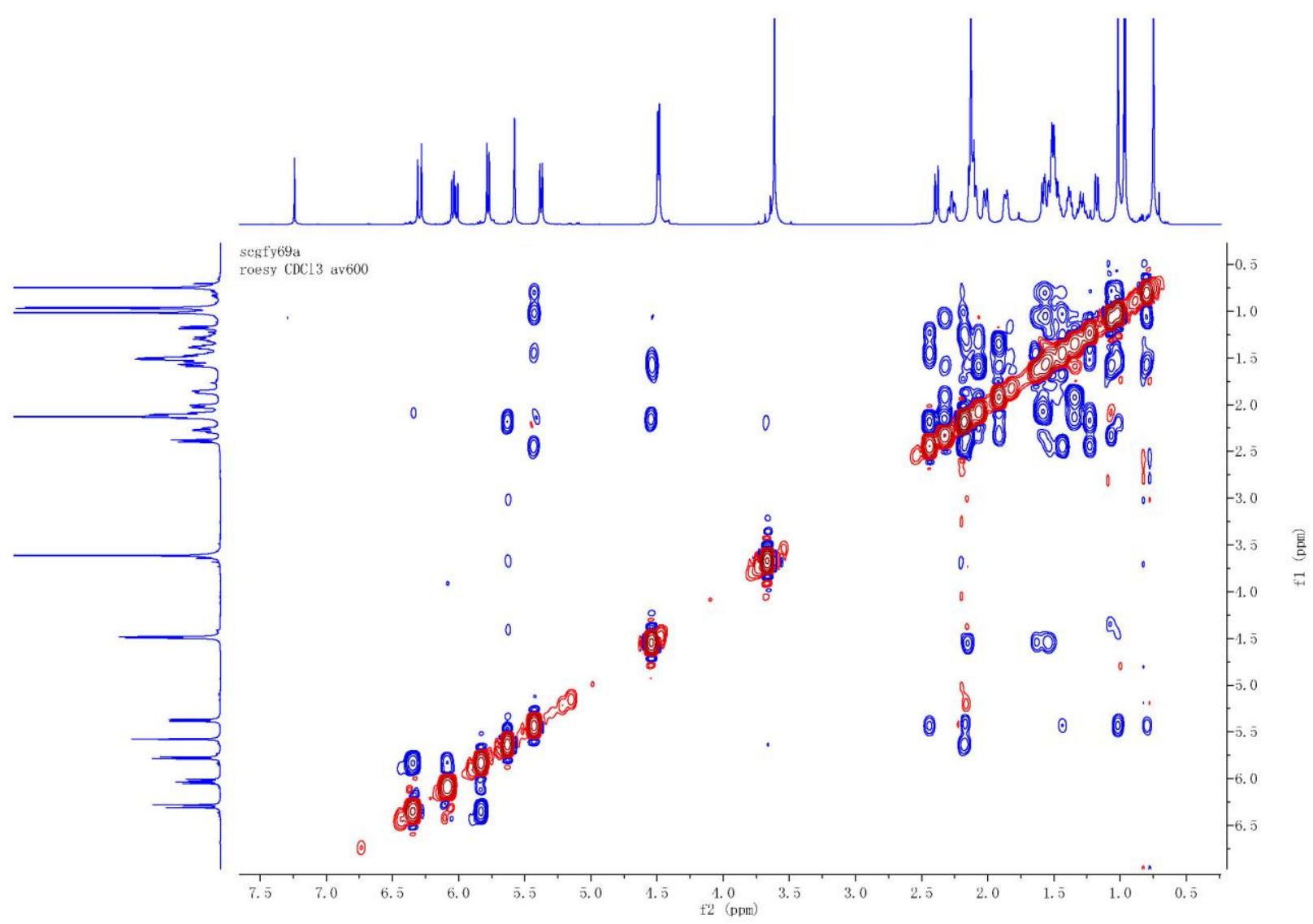


Figure S95. ${ }^{1} \mathrm{H}$ NMR spectrum of $\mathbf{2 3}$

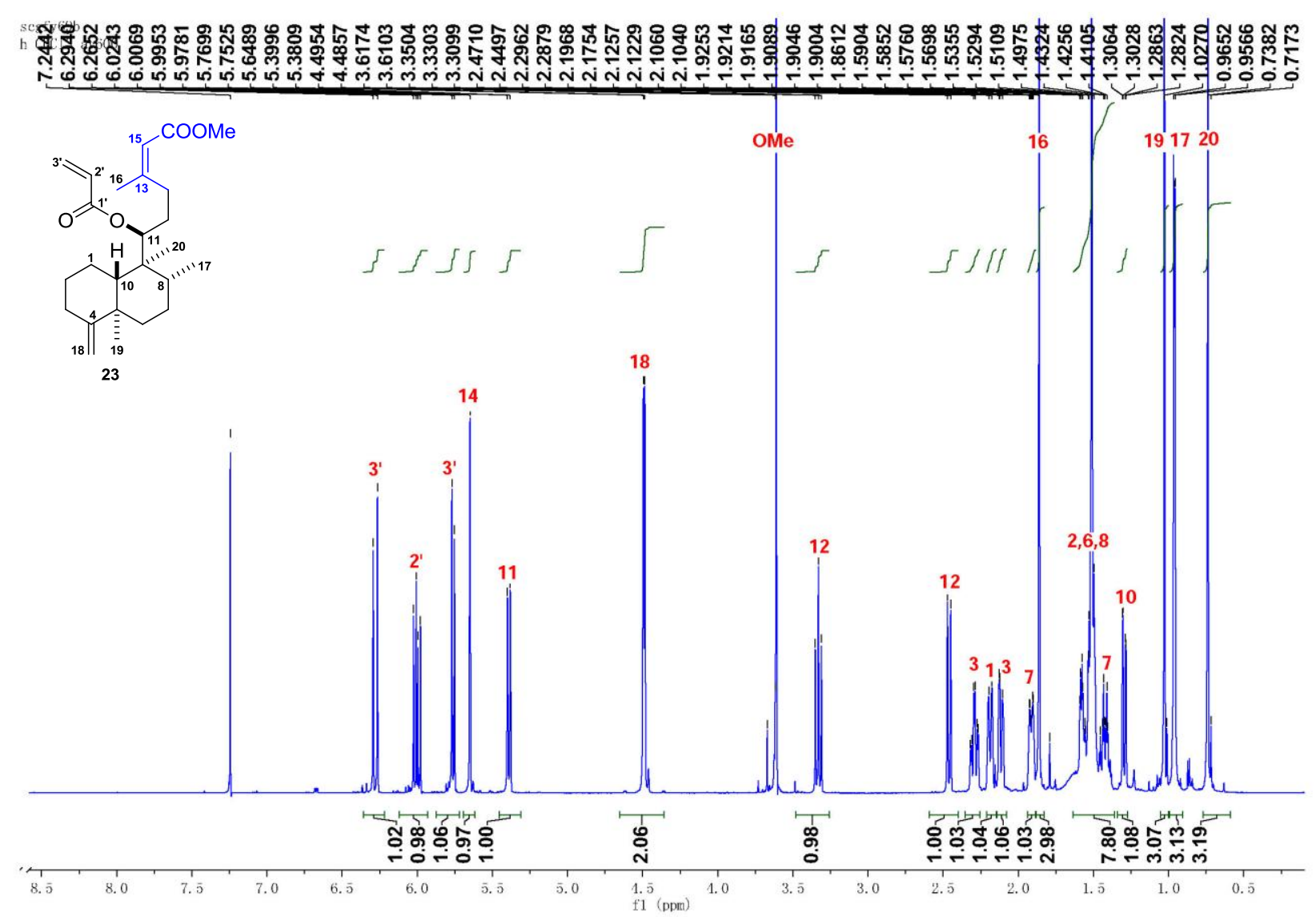

Figure S96. ${ }^{13} \mathrm{C}$ NMR spectrum of $\mathbf{2 3}$
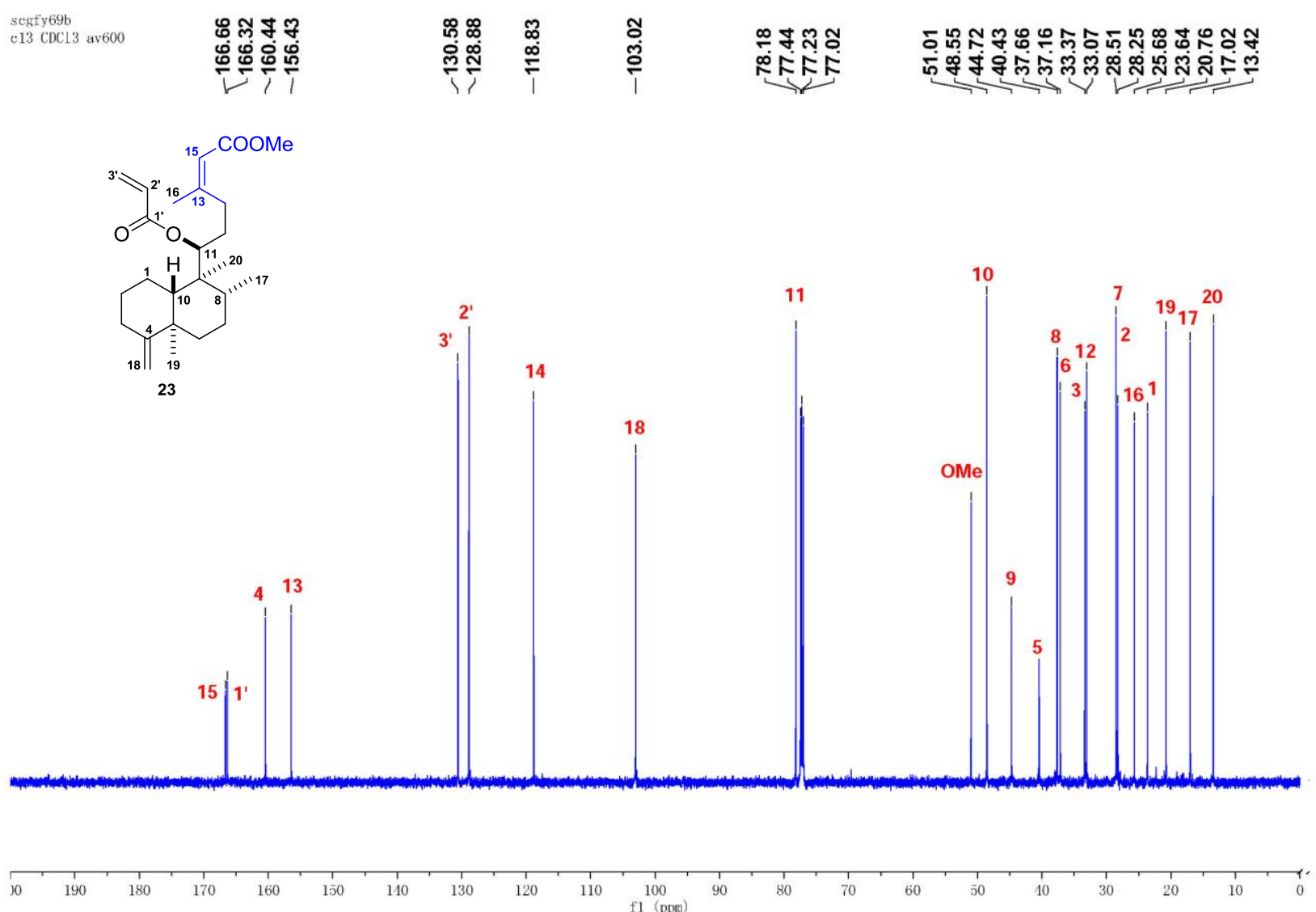
Figure S97. HSQC spectrum of $\mathbf{2 3}$

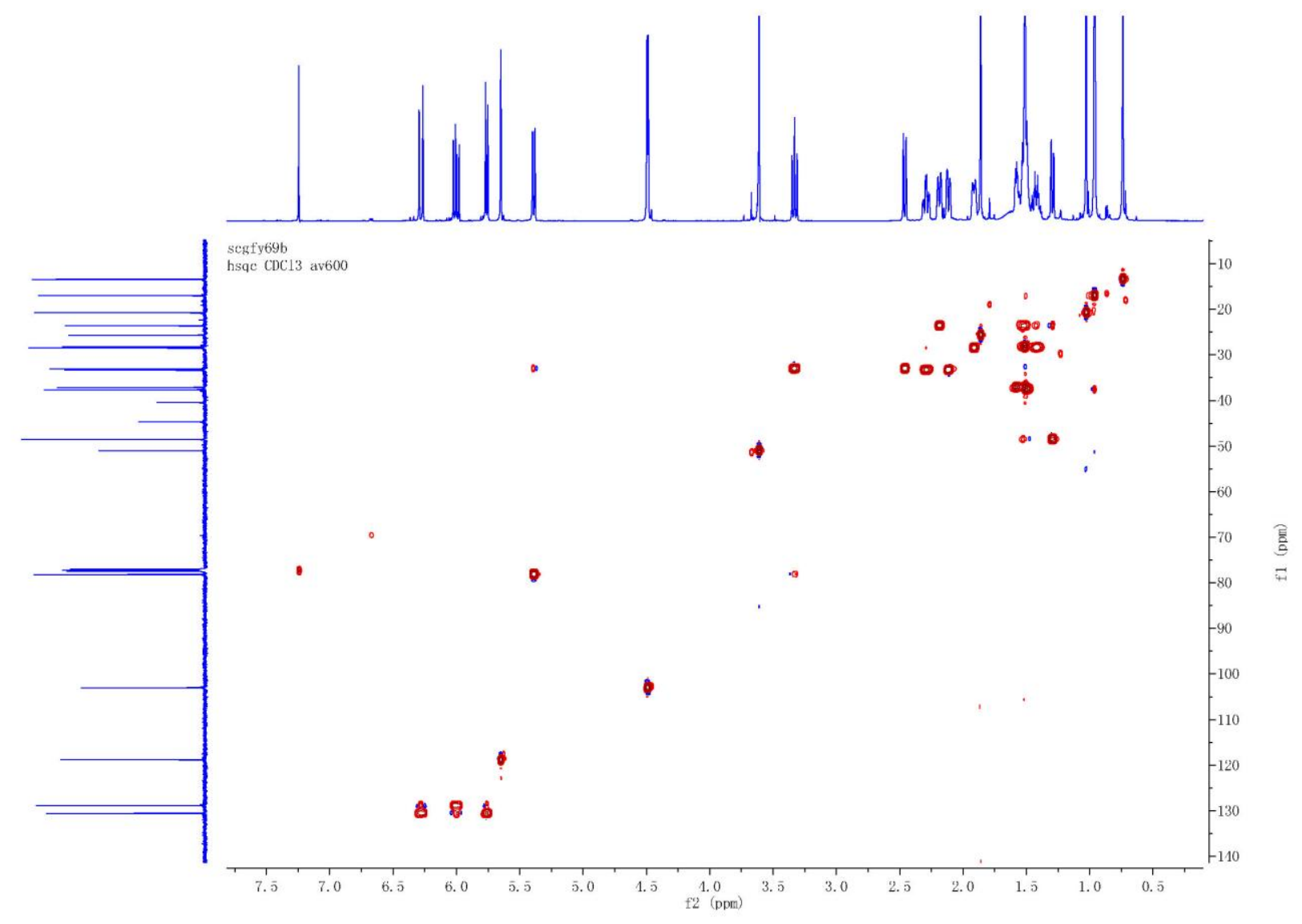

Figure S98. HMBC spectrum of $\mathbf{2 3}$

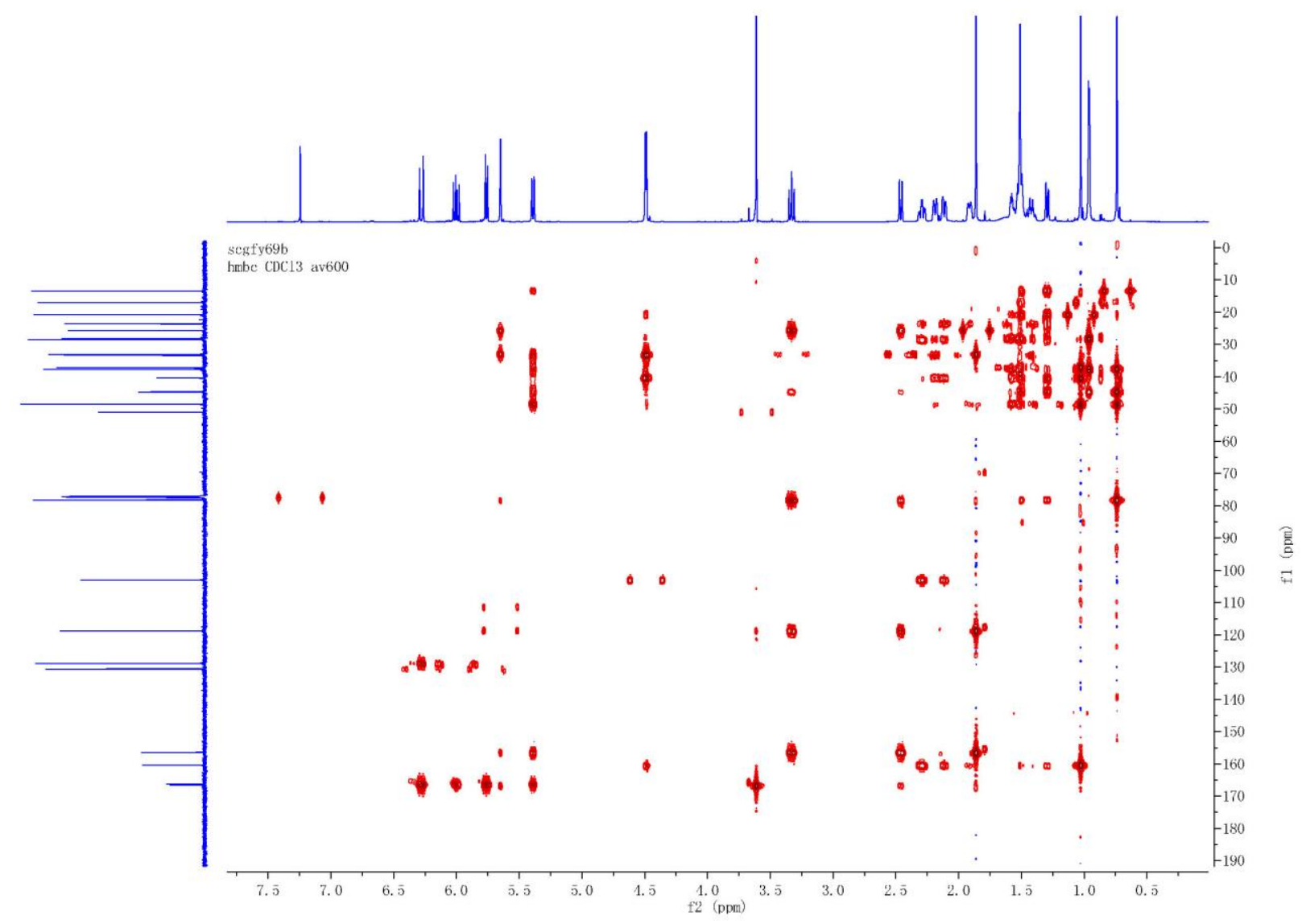


Figure $\mathrm{S} 99 .{ }^{1} \mathrm{H}-{ }^{1} \mathrm{H}$ COSY spectrum of $\mathbf{2 3}$

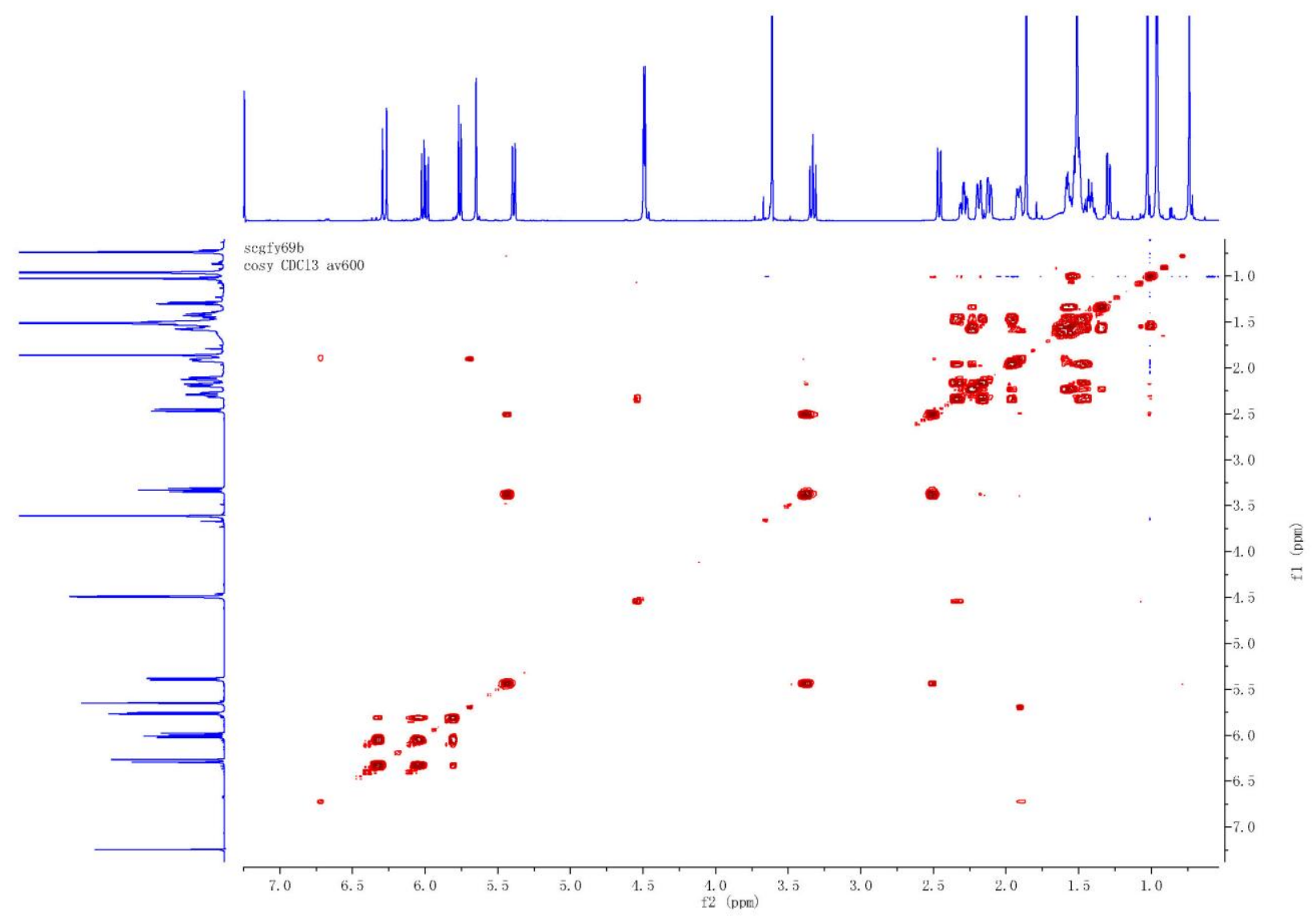

Figure S100. ROESY spectrum of $\mathbf{2 3}$

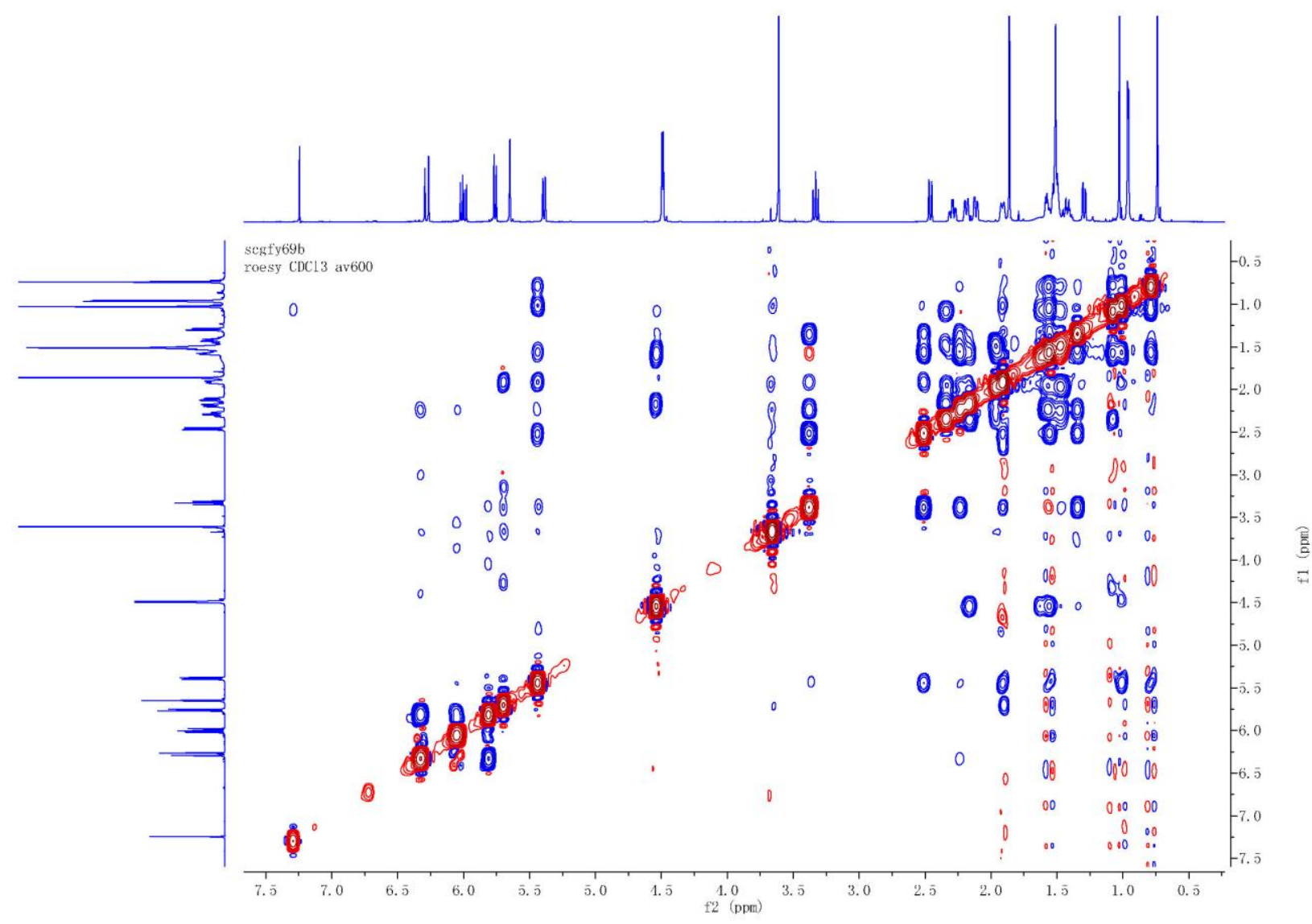


Figure S101. ${ }^{1} \mathrm{H}$ NMR spectrum of $\mathbf{7 b}$

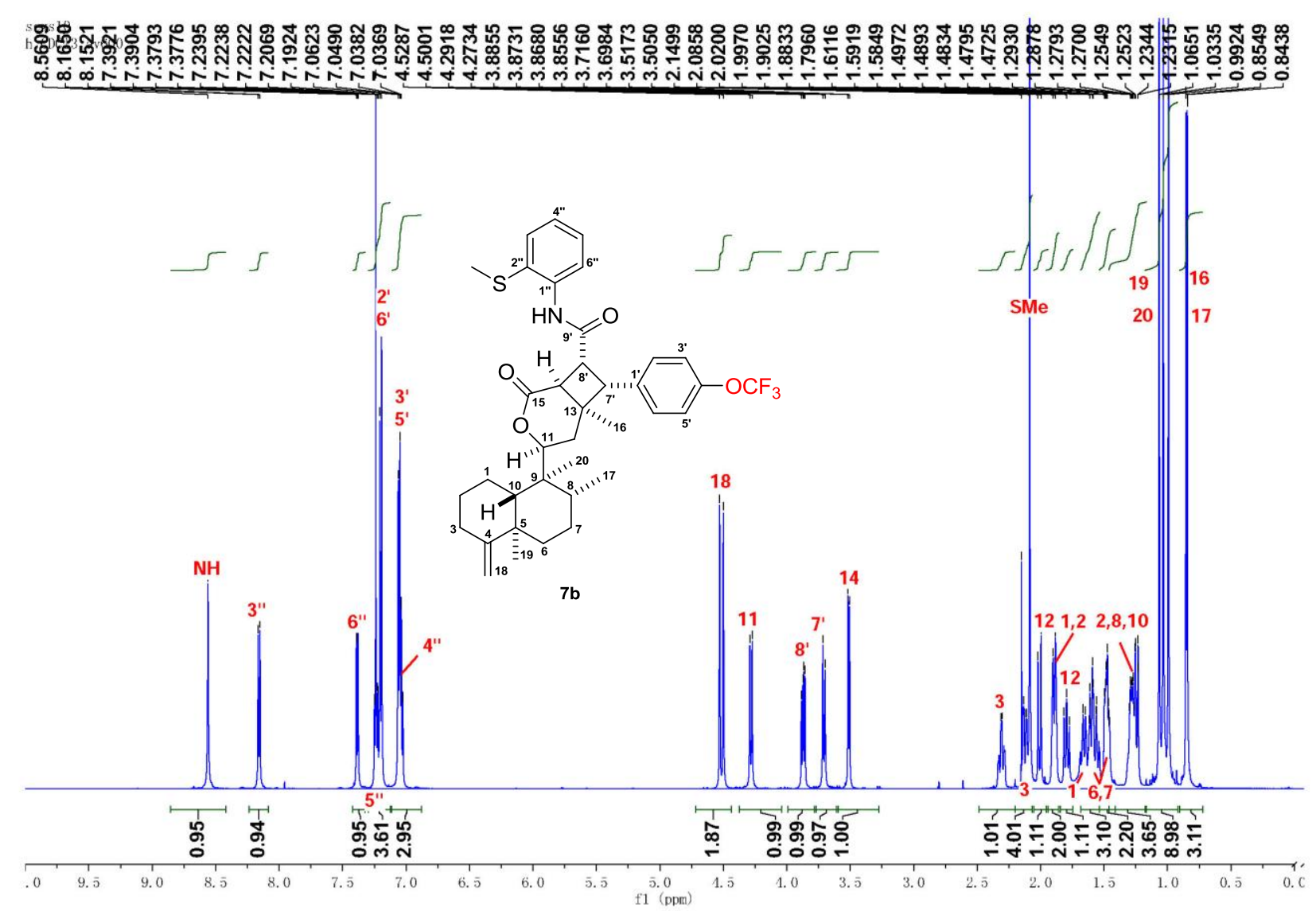

Figure S102. ${ }^{13} \mathrm{C}$ NMR spectrum of $\mathbf{7 b}$
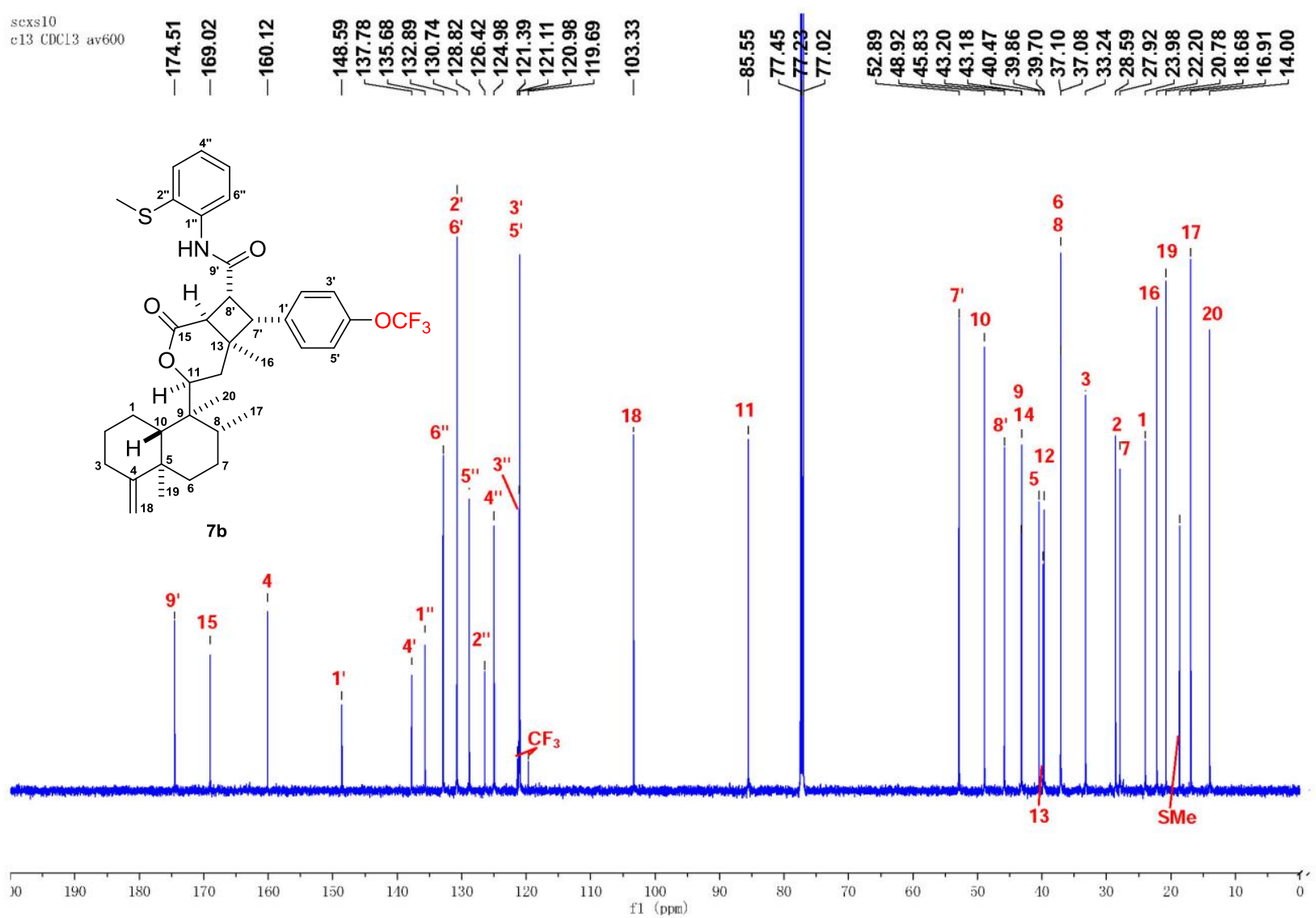
Figure S103. HSQC spectrum of 7b

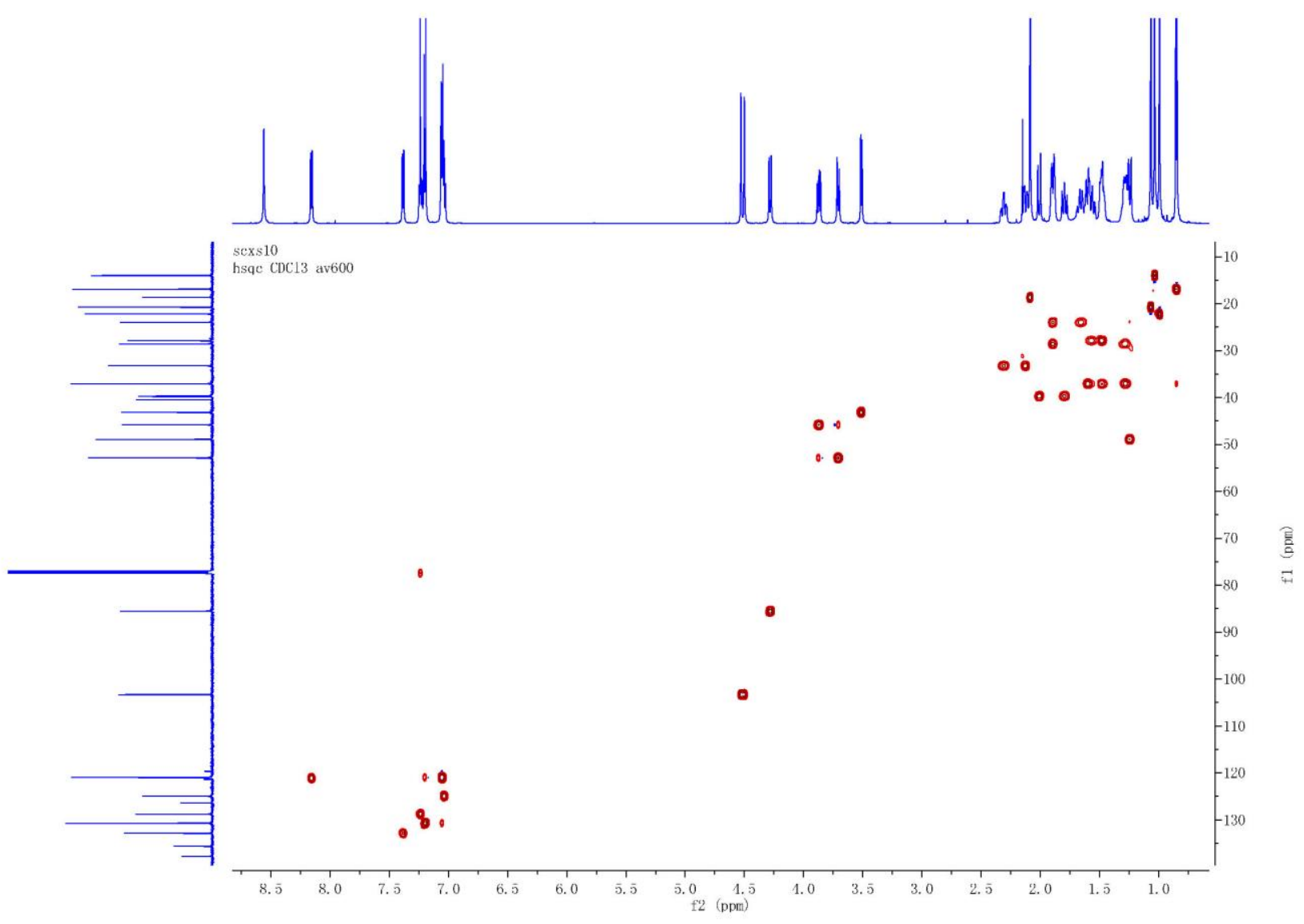

Figure S104. HMBC spectrum of 7b

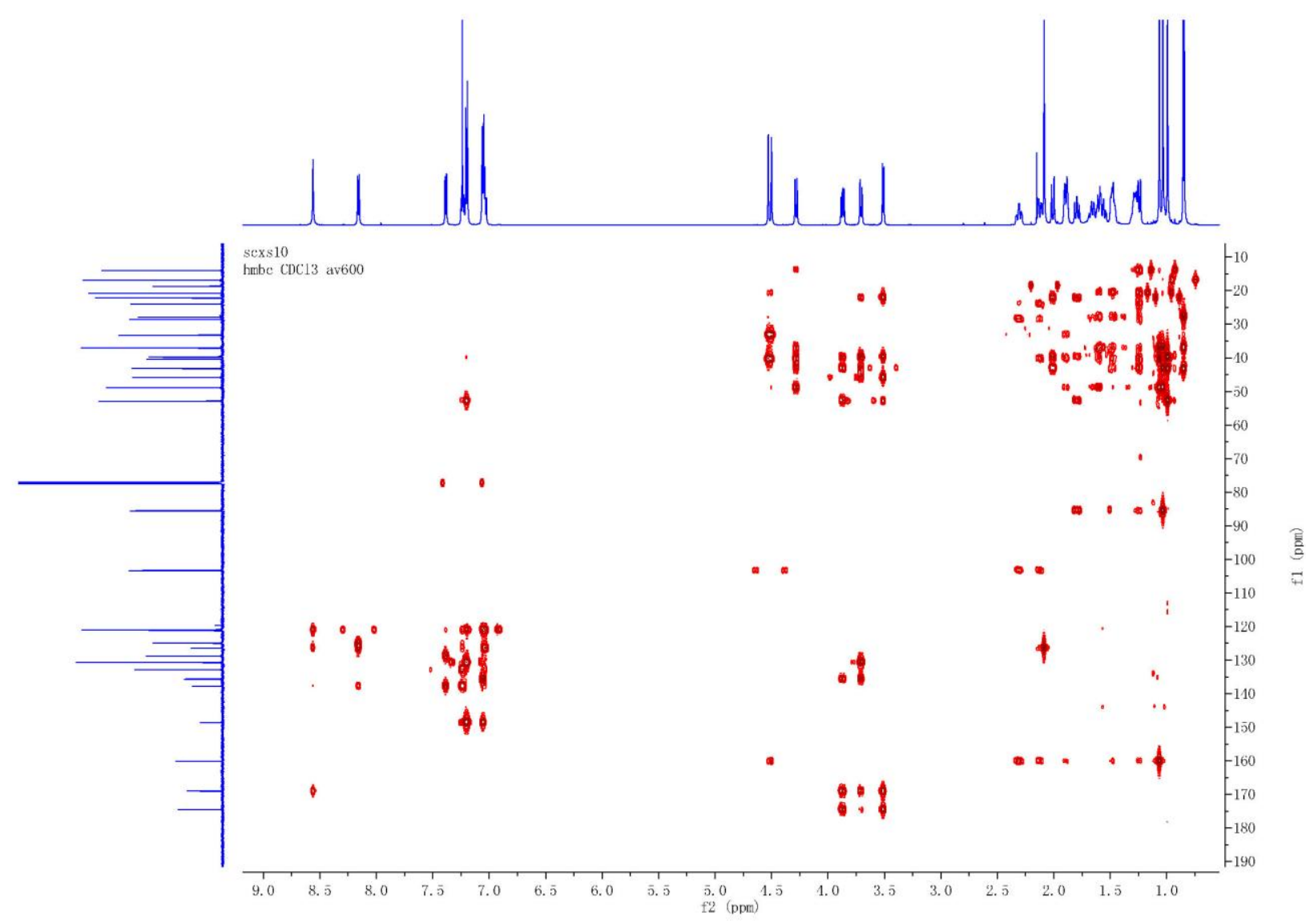


Figure S105. ${ }^{1} \mathrm{H}-{ }^{1} \mathrm{H}$ COSY spectrum of $\mathbf{7 b}$

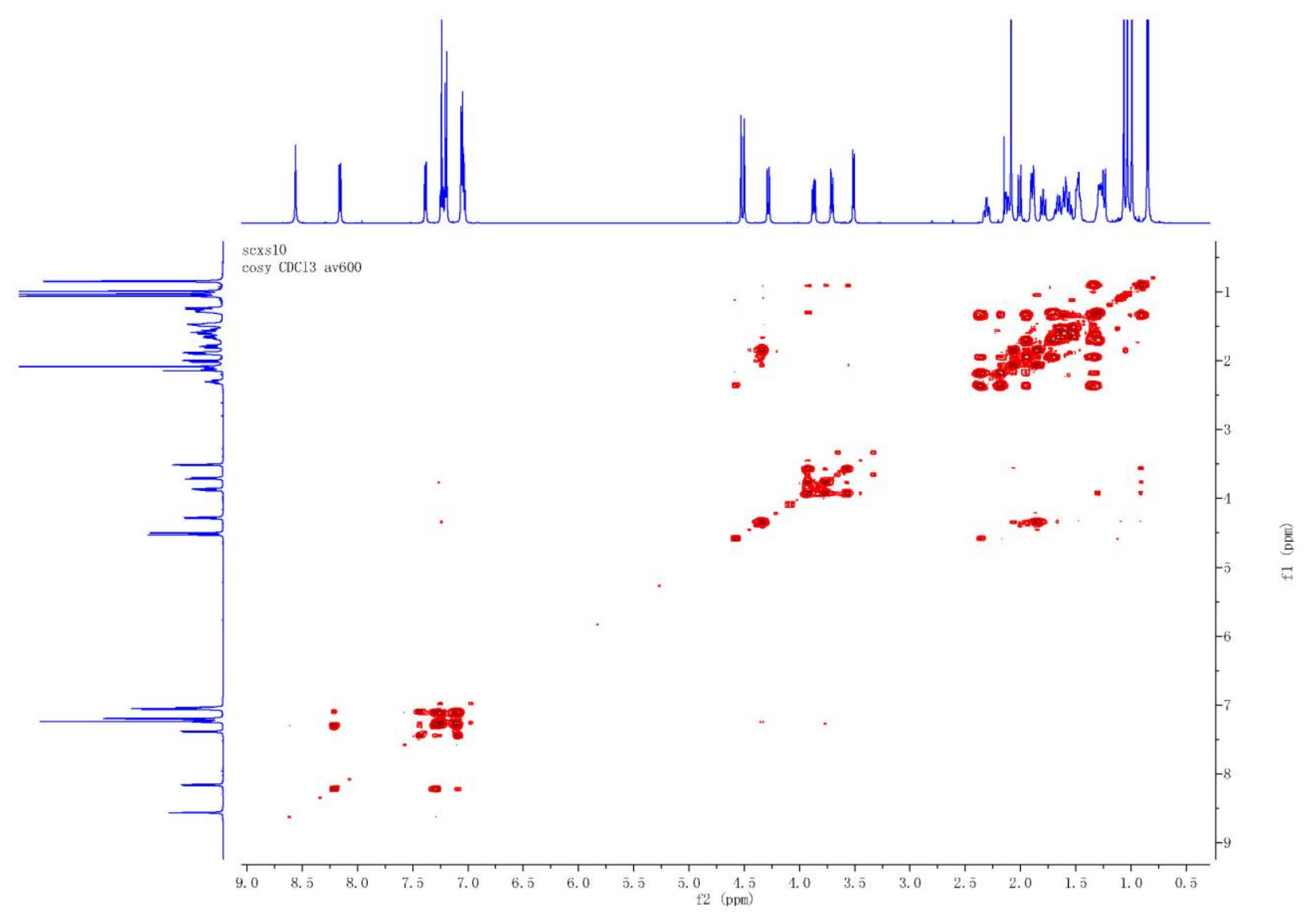

Figure S106. ROESY spectrum of $\mathbf{7 b}$

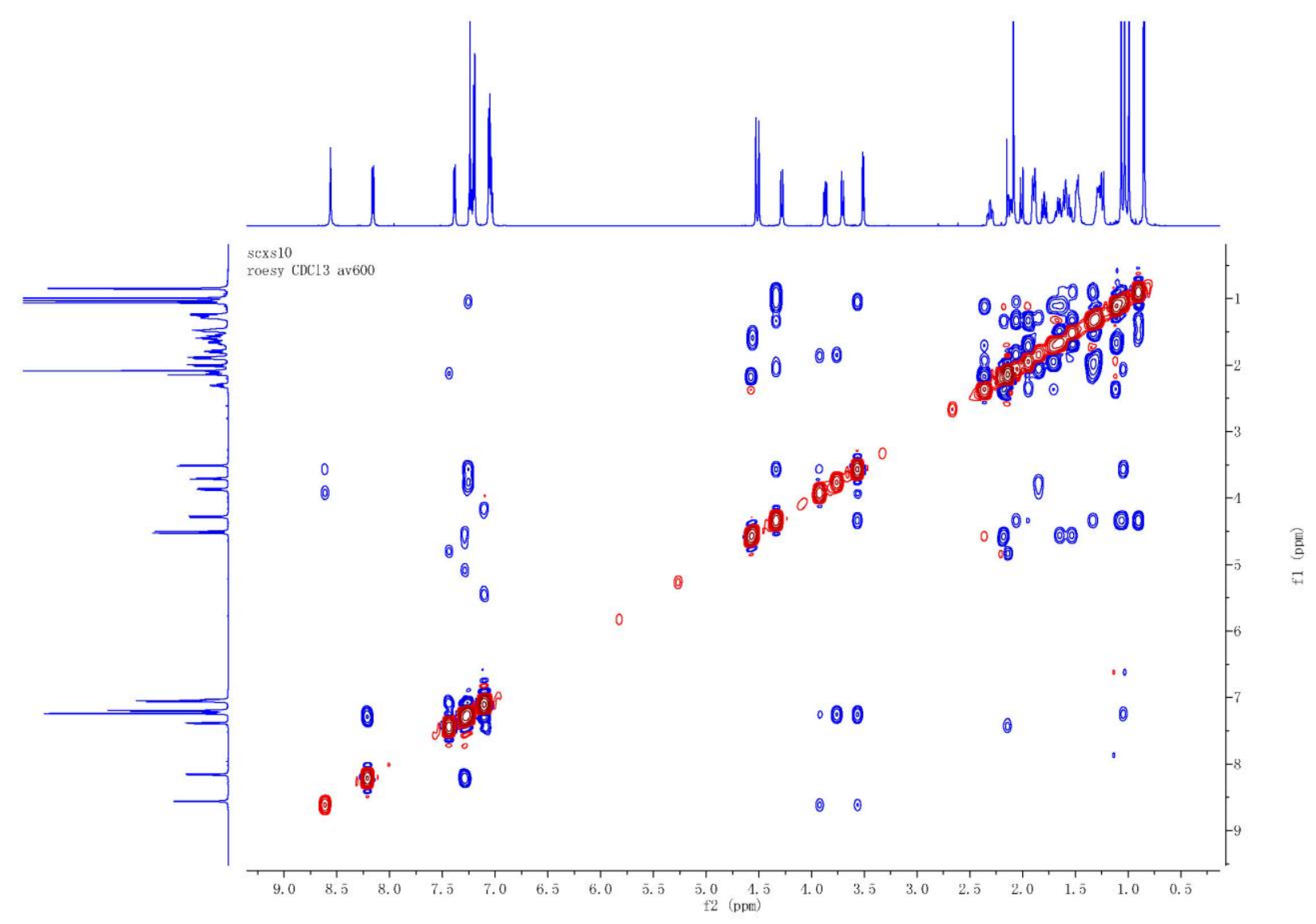


Figure S107. ${ }^{1} \mathrm{H}$ NMR spectrum of $7 \mathbf{c}$

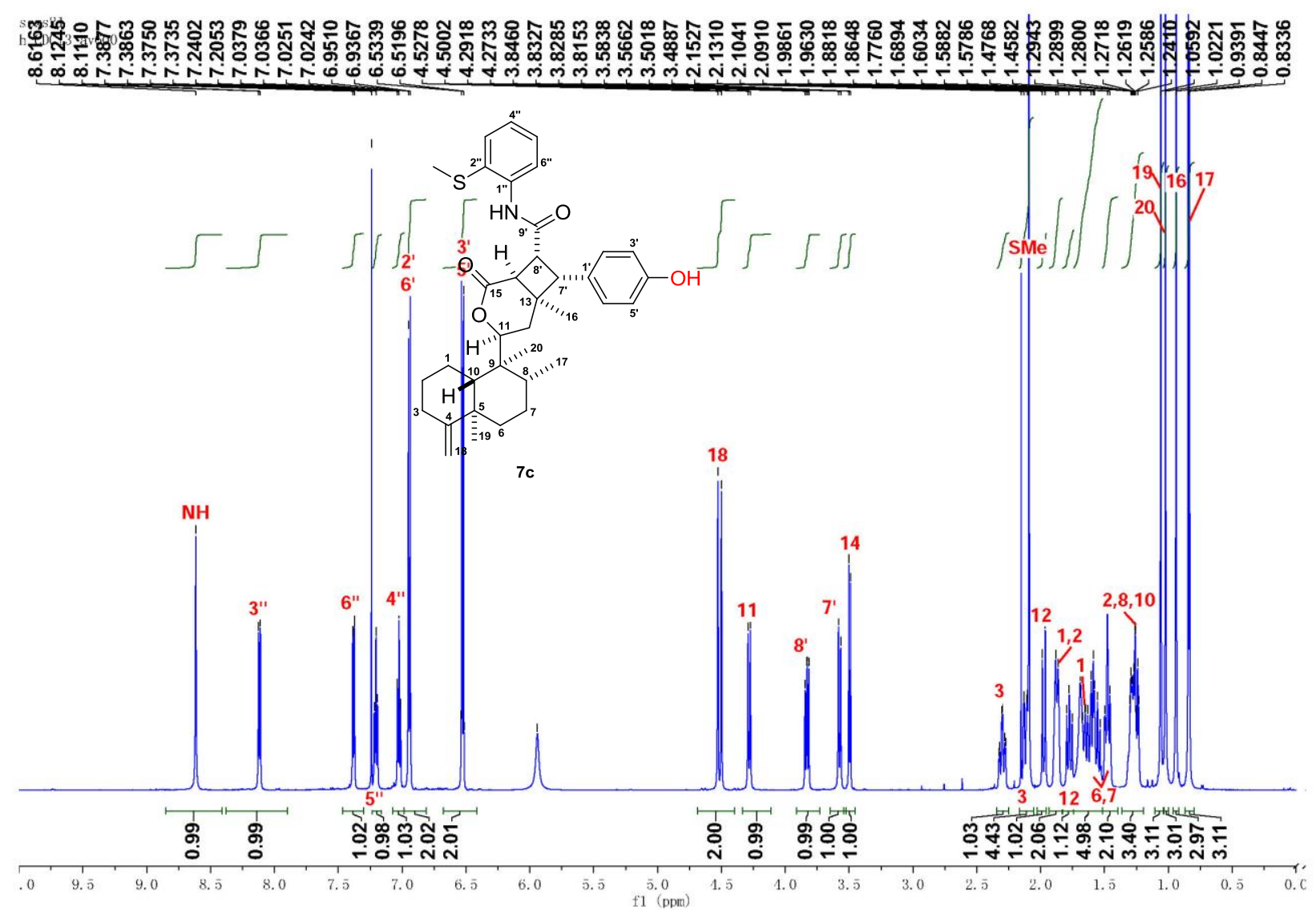

Figure S108. ${ }^{13} \mathrm{C}$ NMR spectrum of $\mathbf{7 c}$

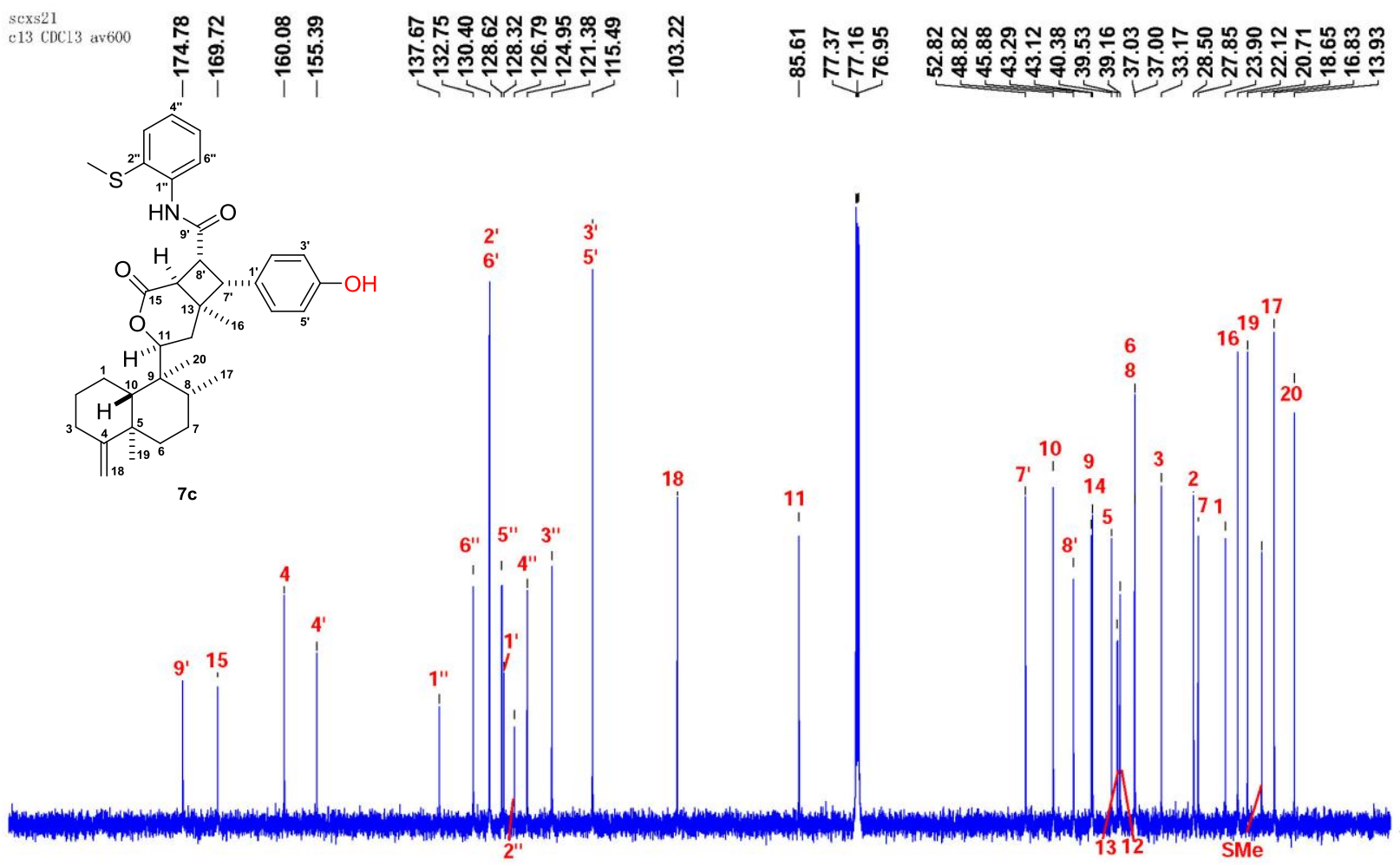

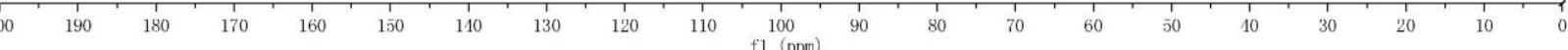


Figure S109. HSQC spectrum of 7c

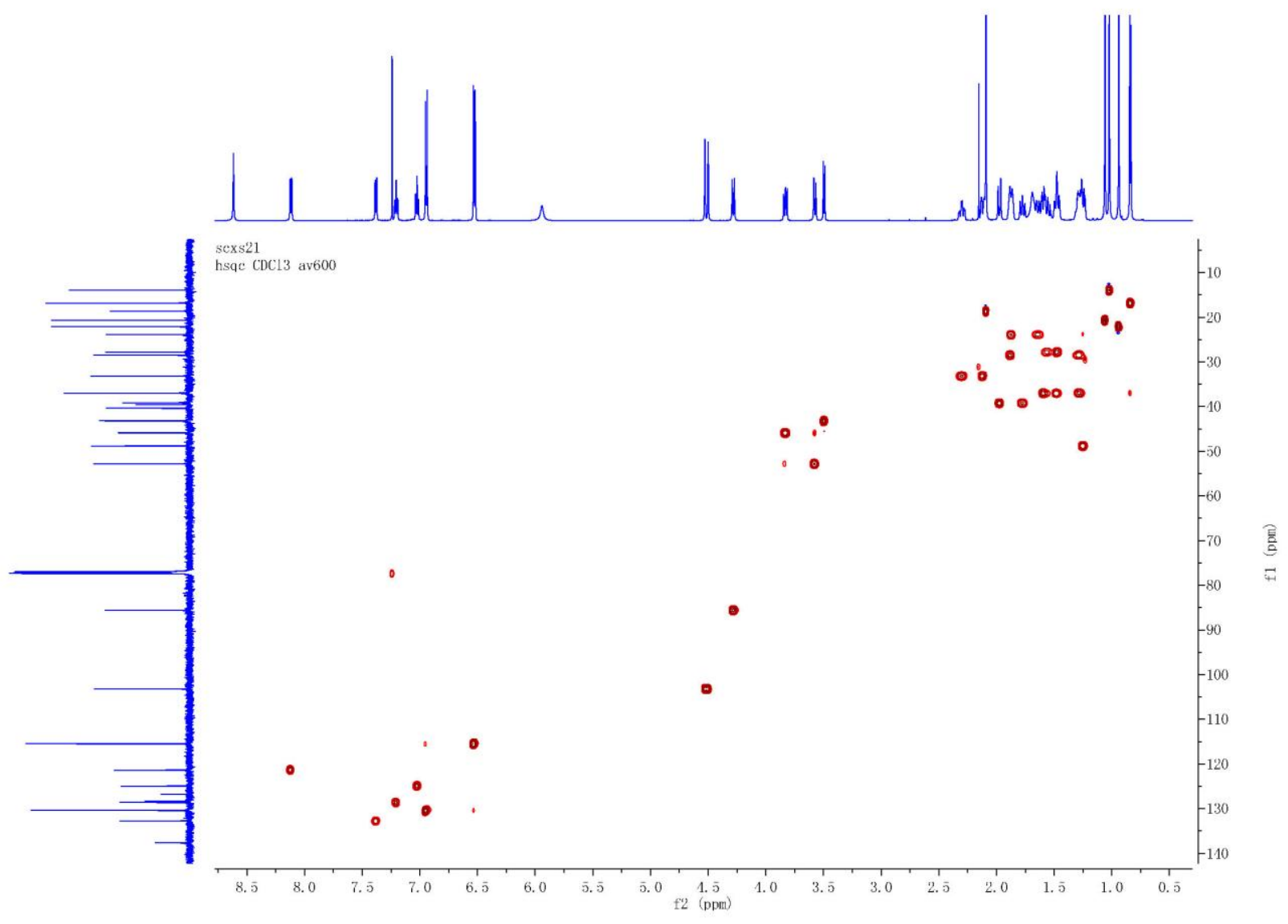

Figure S110. HMBC spectrum of 7

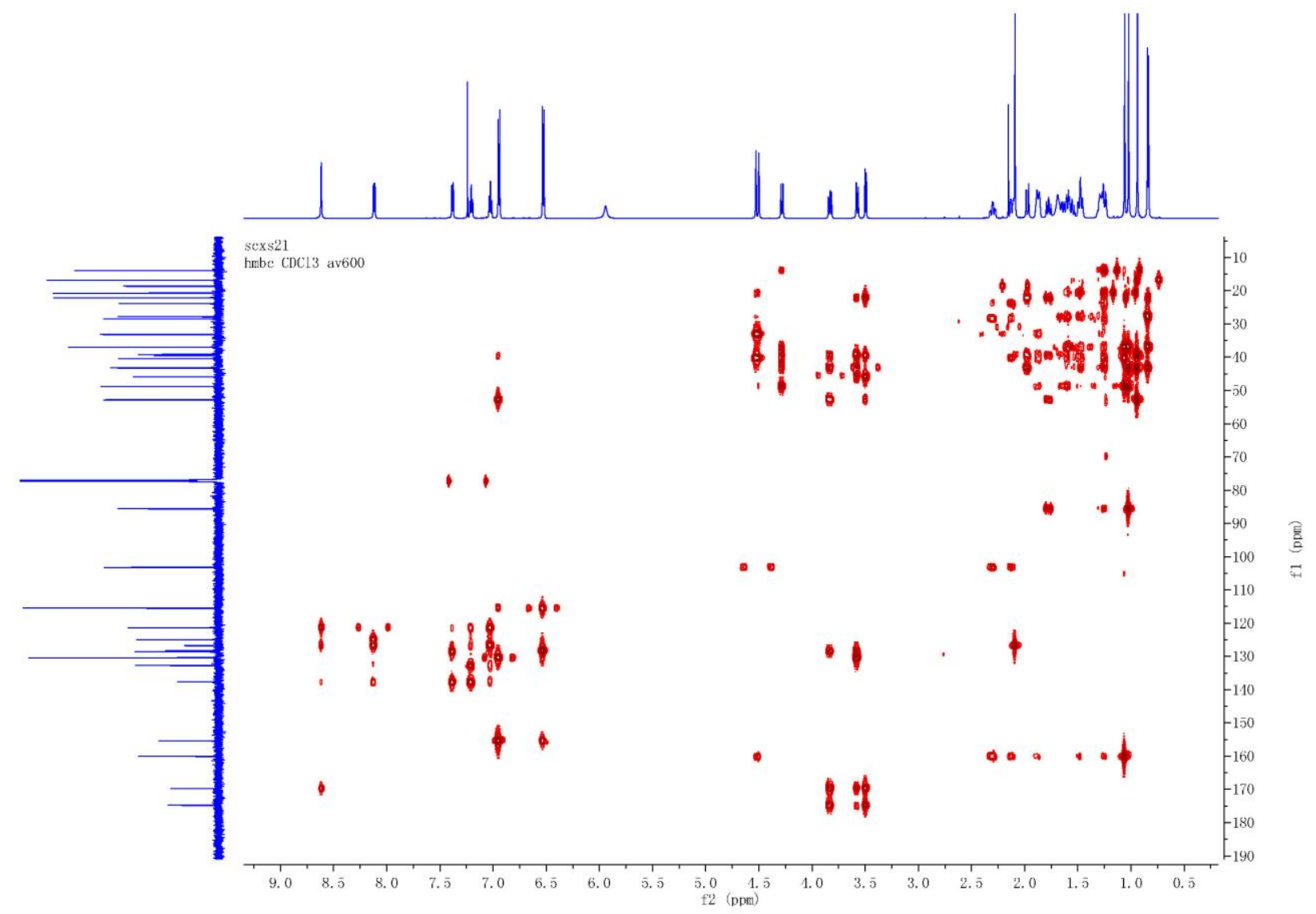


Figure S111. ${ }^{1} \mathrm{H}-{ }^{1} \mathrm{H}$ COSY spectrum of $\mathbf{7 c}$

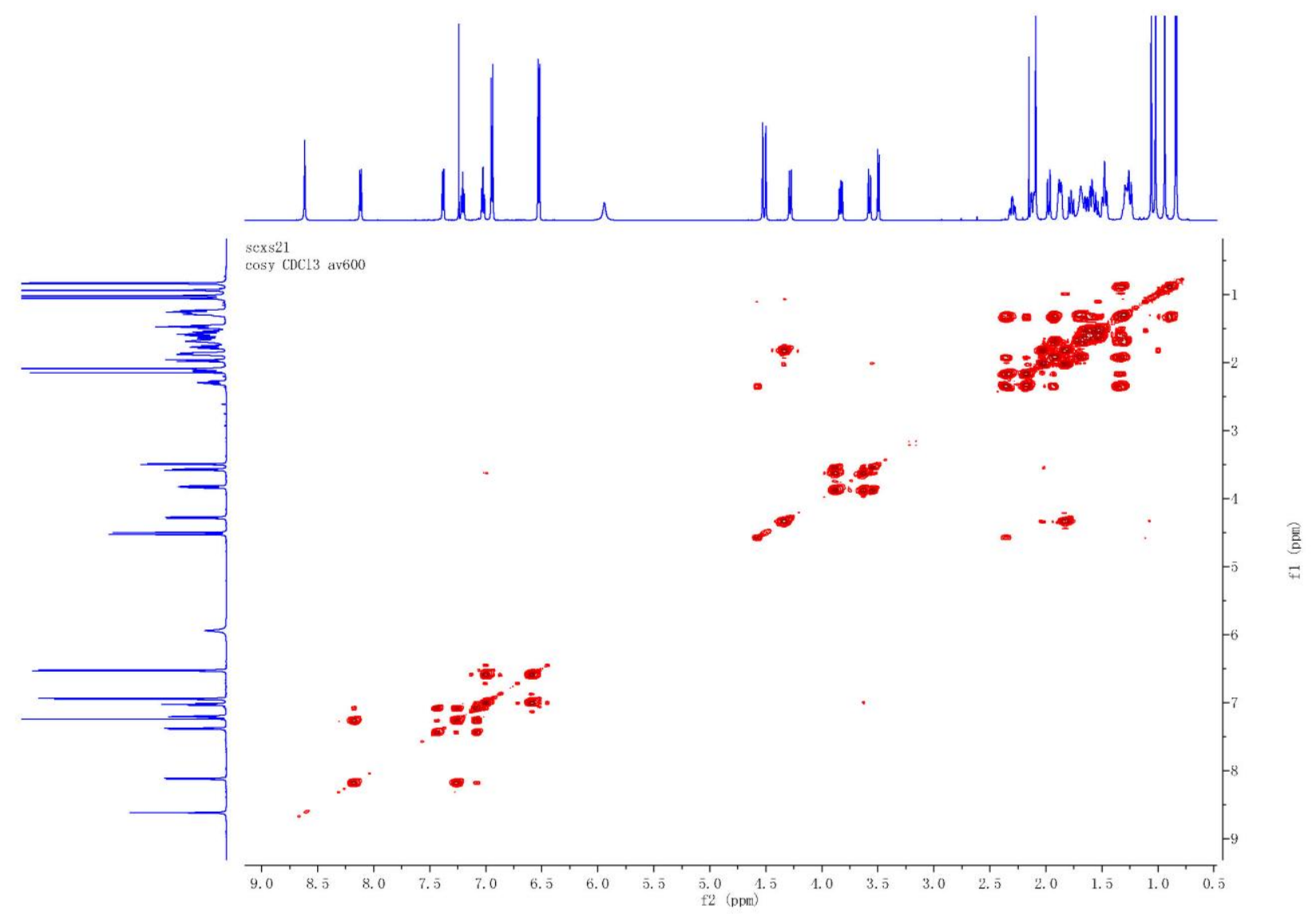

Figure S112. ROESY spectrum of $\mathbf{7 c}$

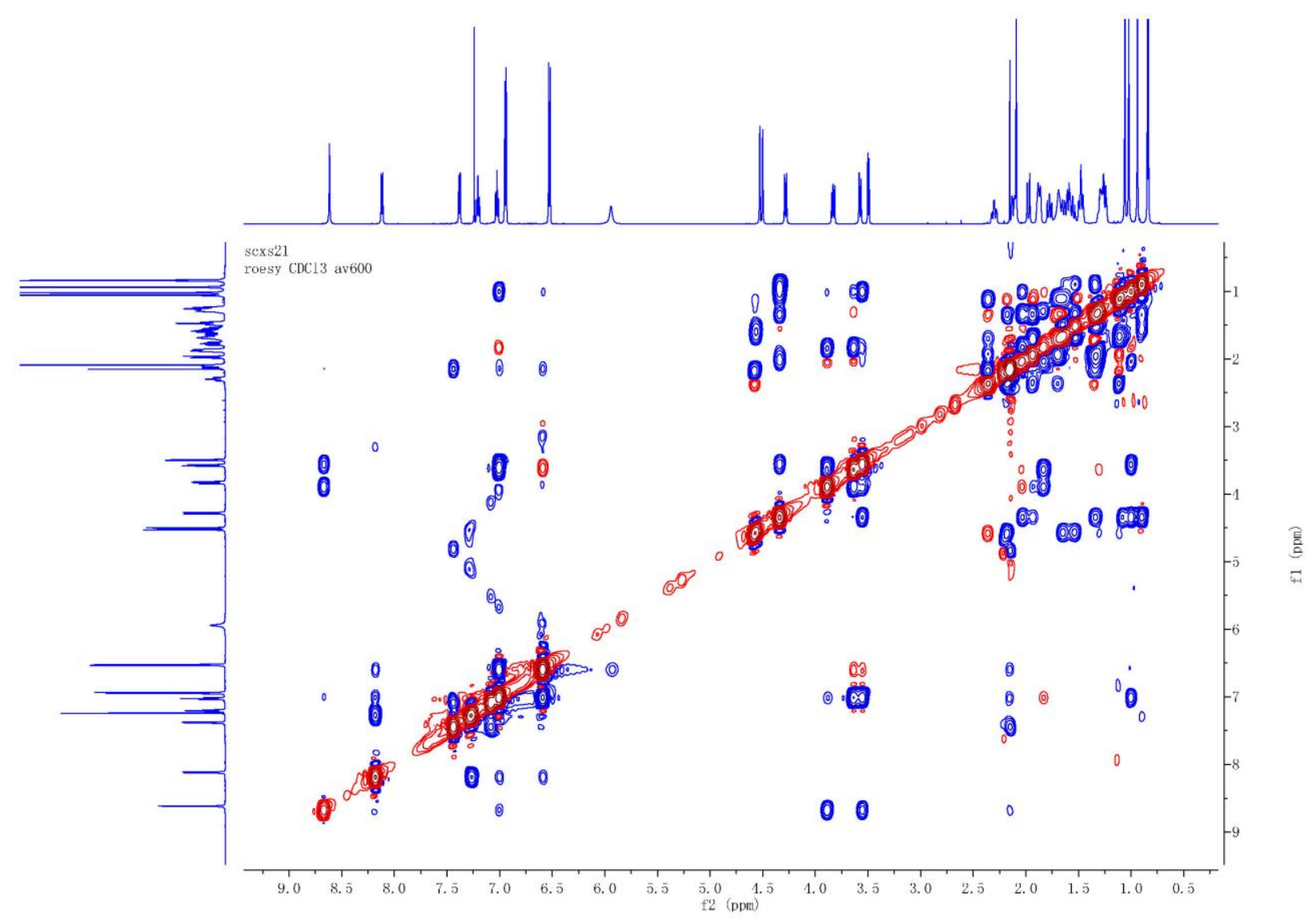


Figure S113. ${ }^{1} \mathrm{H}$ NMR spectrum of $\mathbf{7 d}$

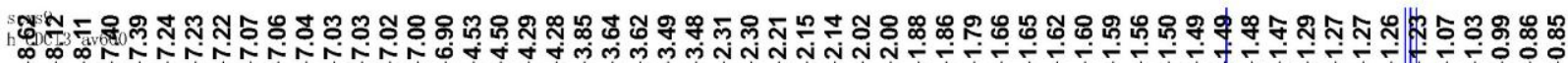

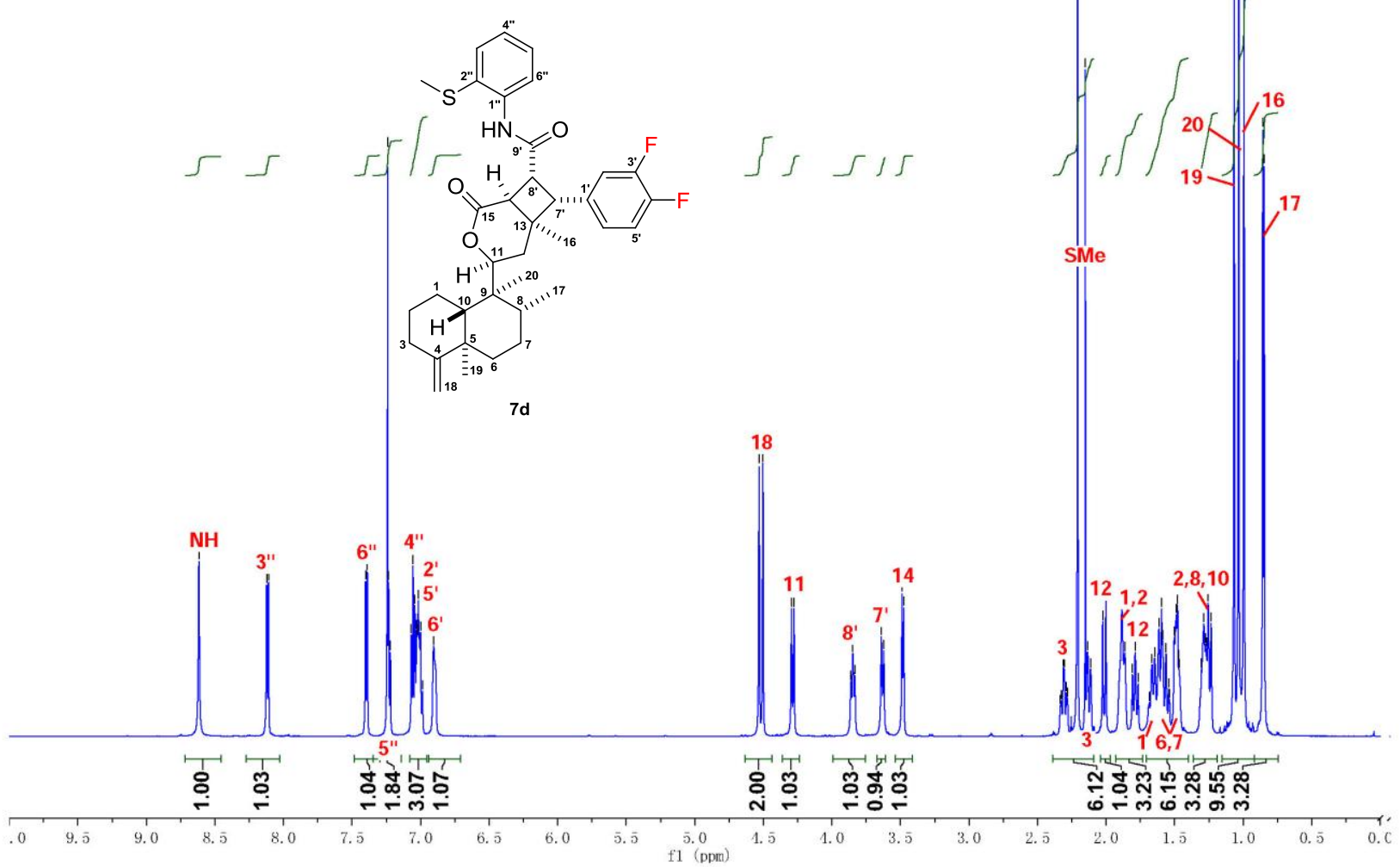

Figure S114. ${ }^{13} \mathrm{C}$ NMR spectrum of $7 \mathbf{d}$
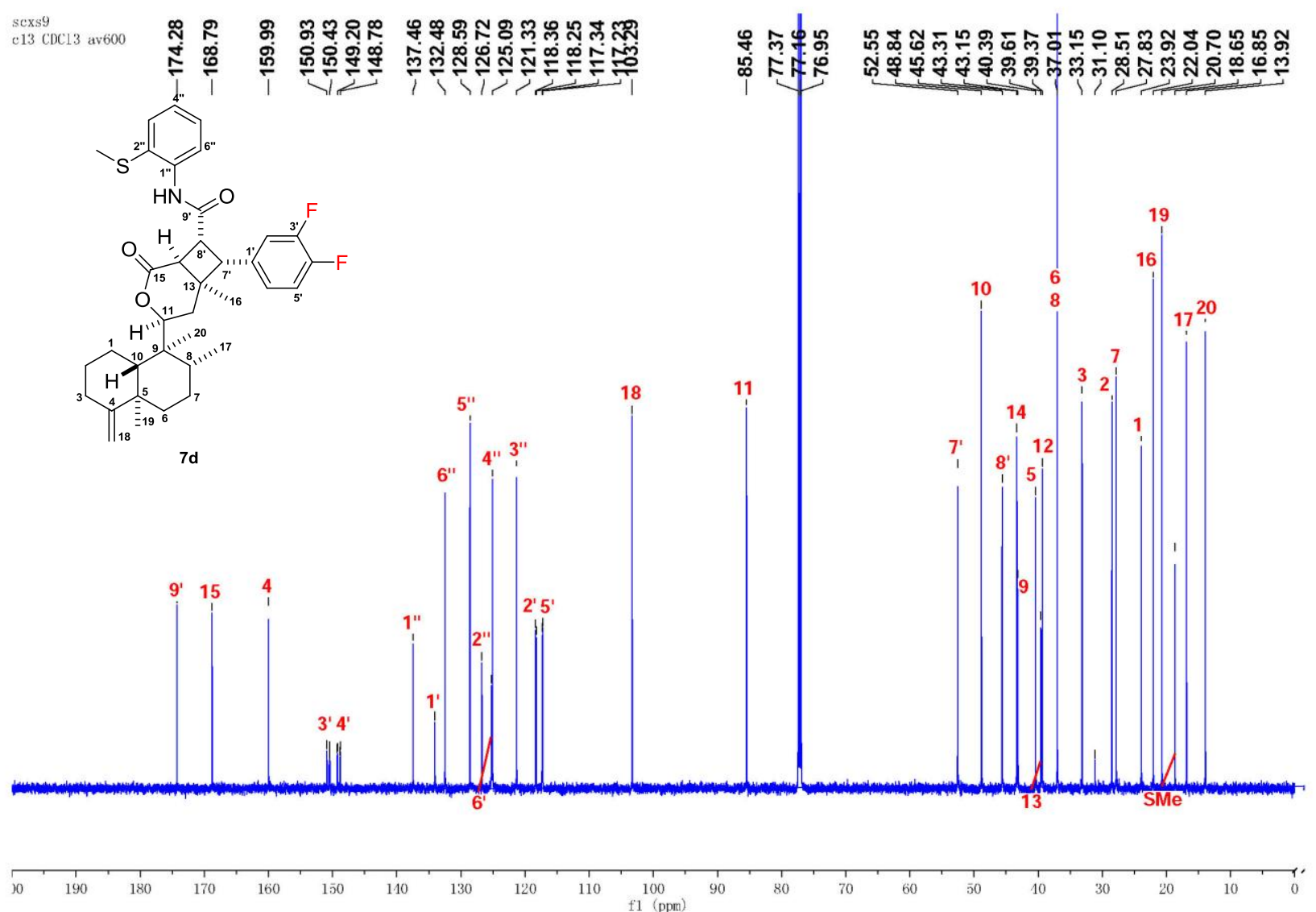
Figure S115. HSQC spectrum of 7d

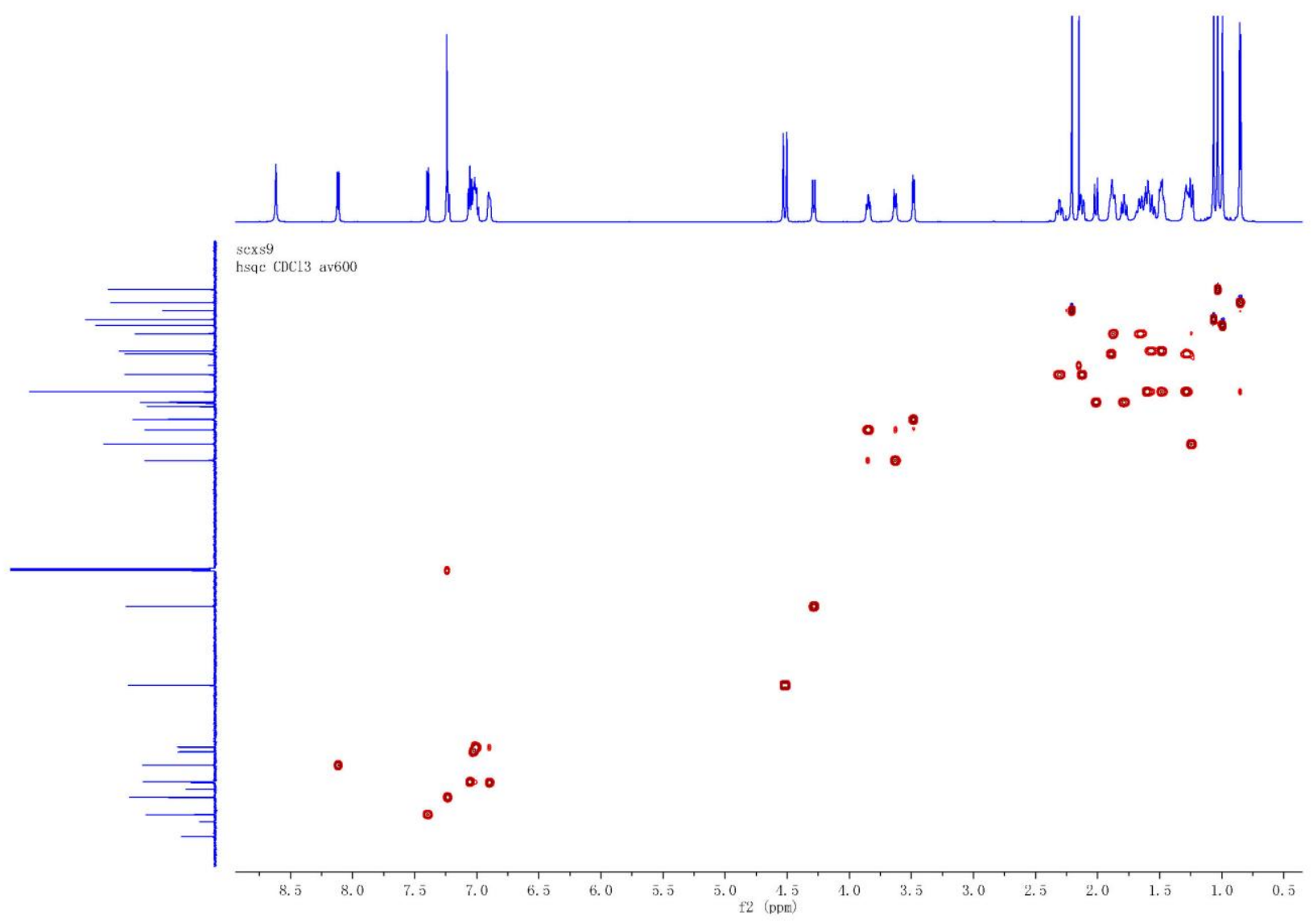

Figure S116. HMBC spectrum of 7d

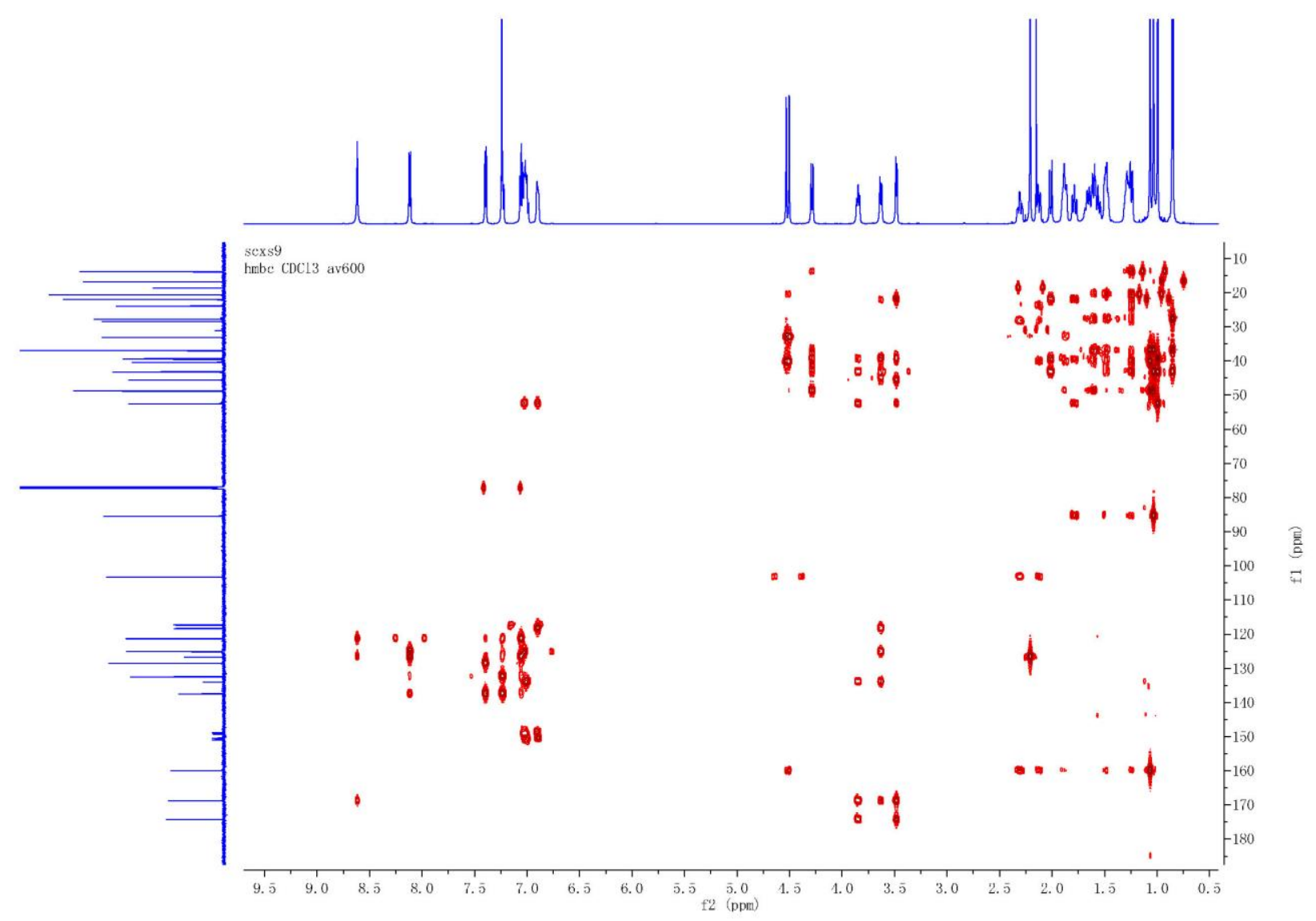


Figure S117. ${ }^{1} \mathrm{H}-{ }^{1} \mathrm{H}$ COSY spectrum of $\mathbf{7 d}$

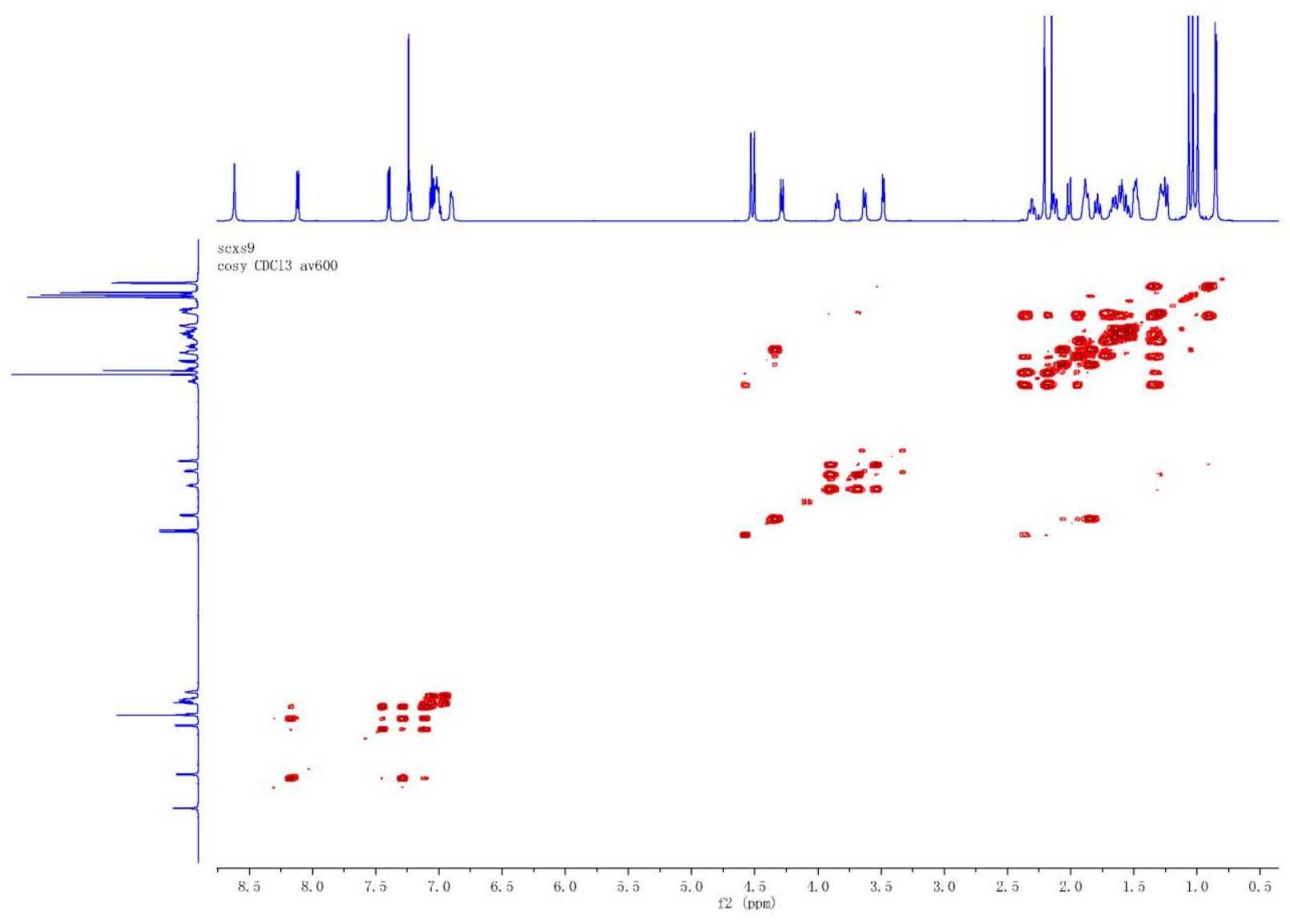

Figure S118. ROESY spectrum of 7d

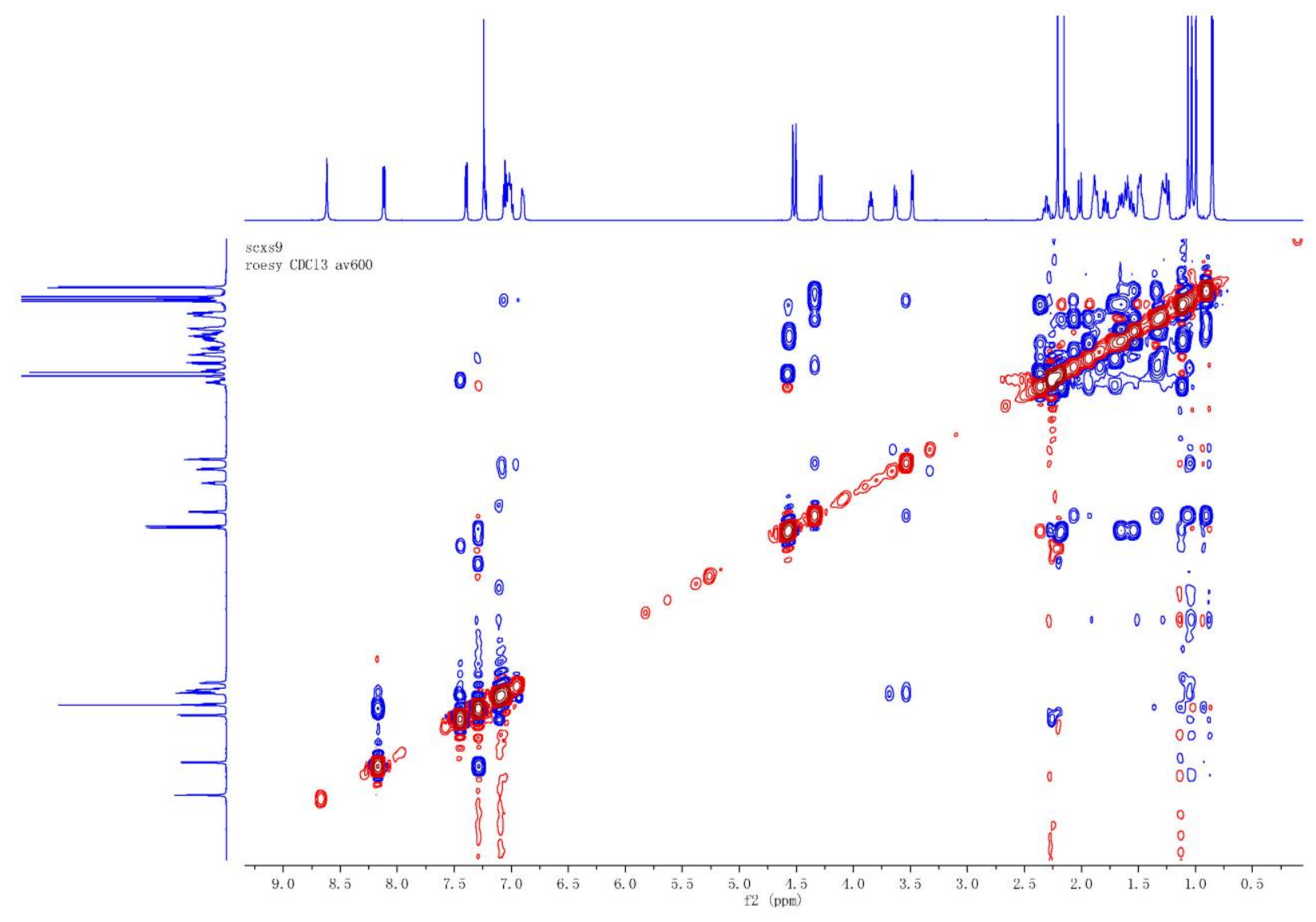


Figure S119. ${ }^{1} \mathrm{H}$ NMR spectrum of $7 \mathbf{e}$

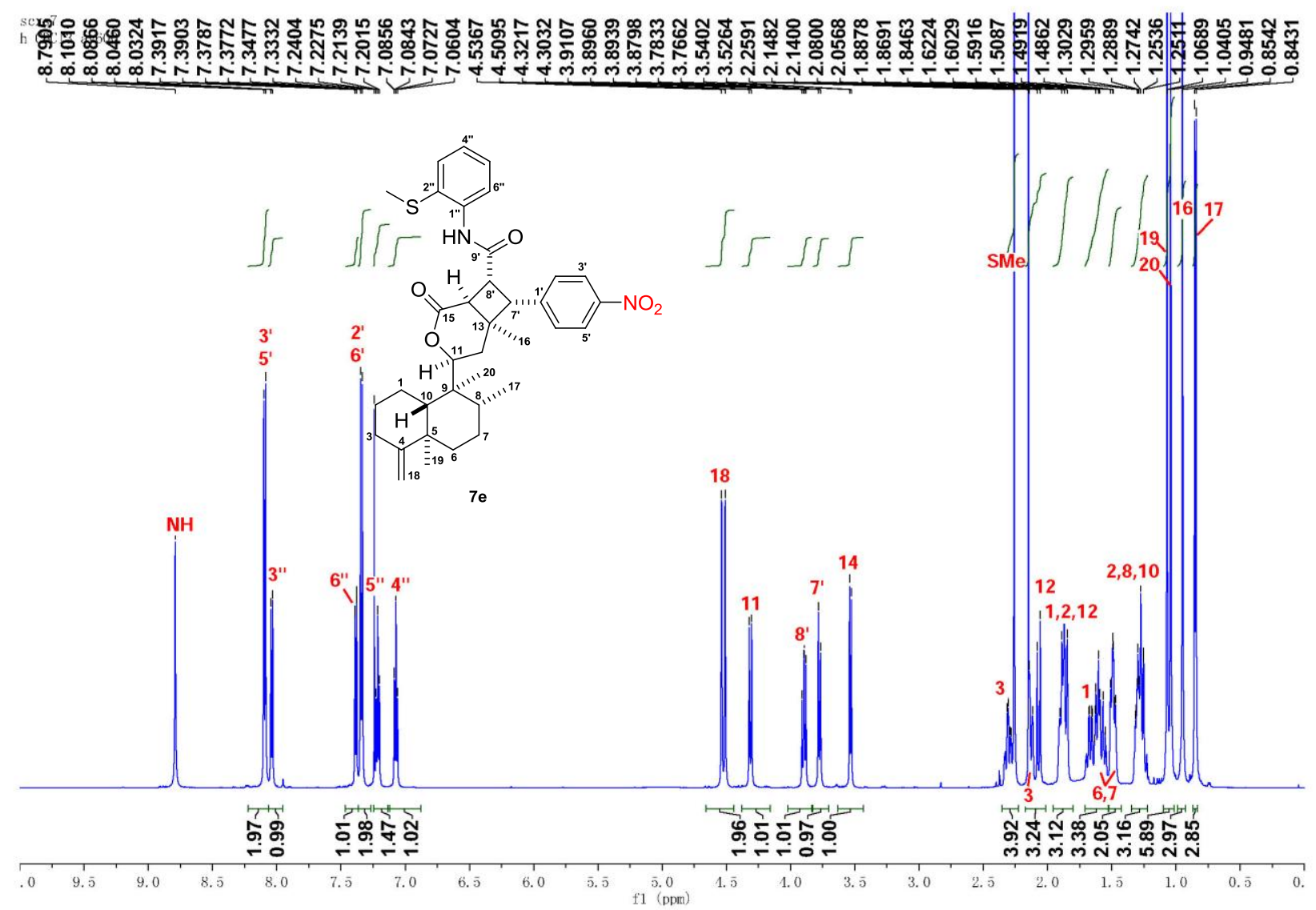

Figure S120. ${ }^{13} \mathrm{C}$ NMR spectrum of $\mathbf{7 e}$

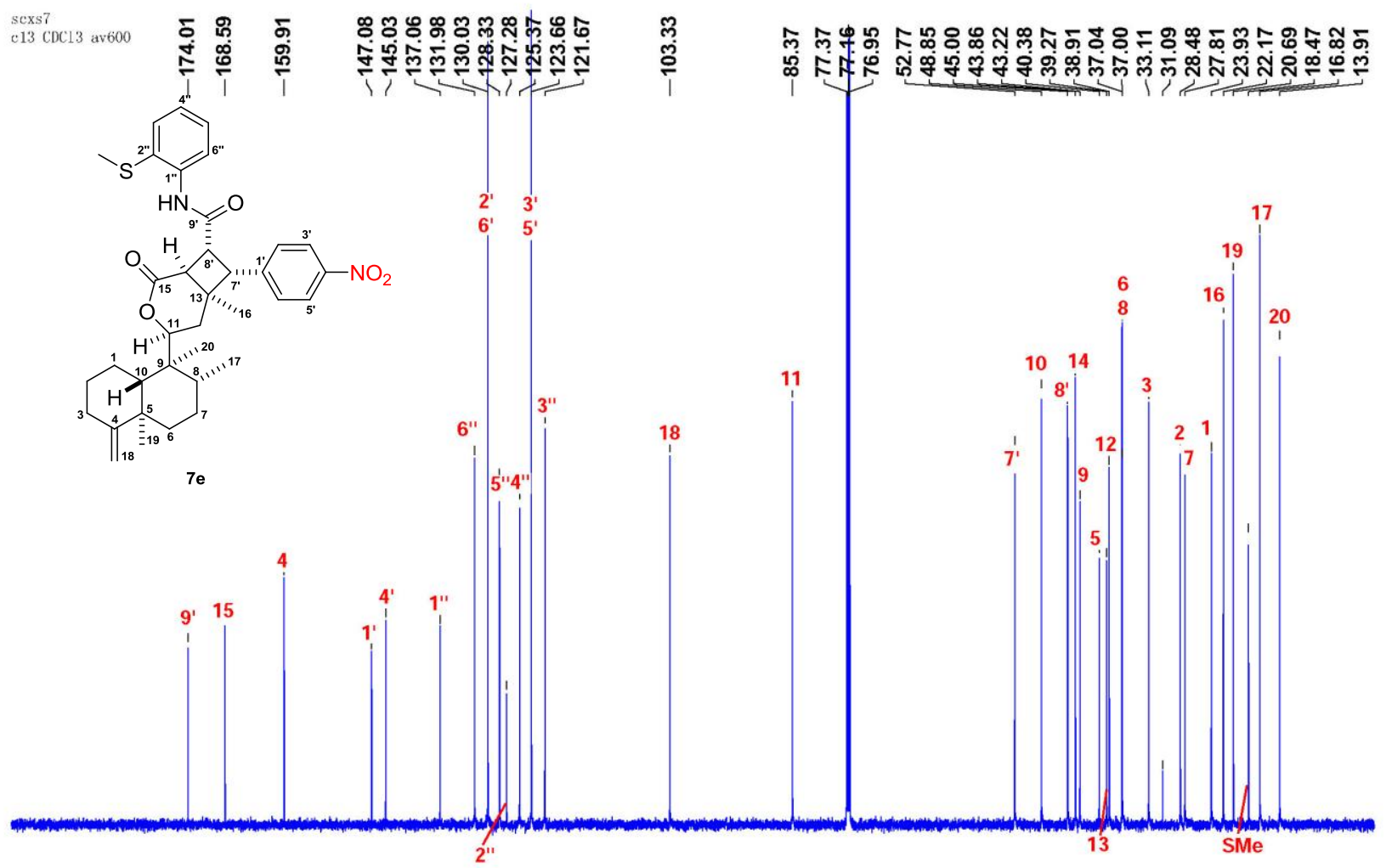

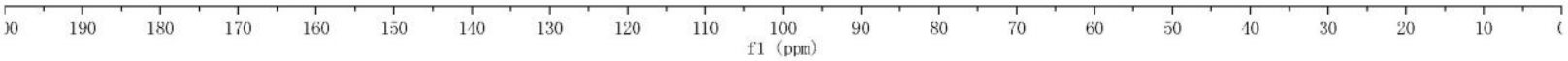


Figure S121. HSQC spectrum of 7e

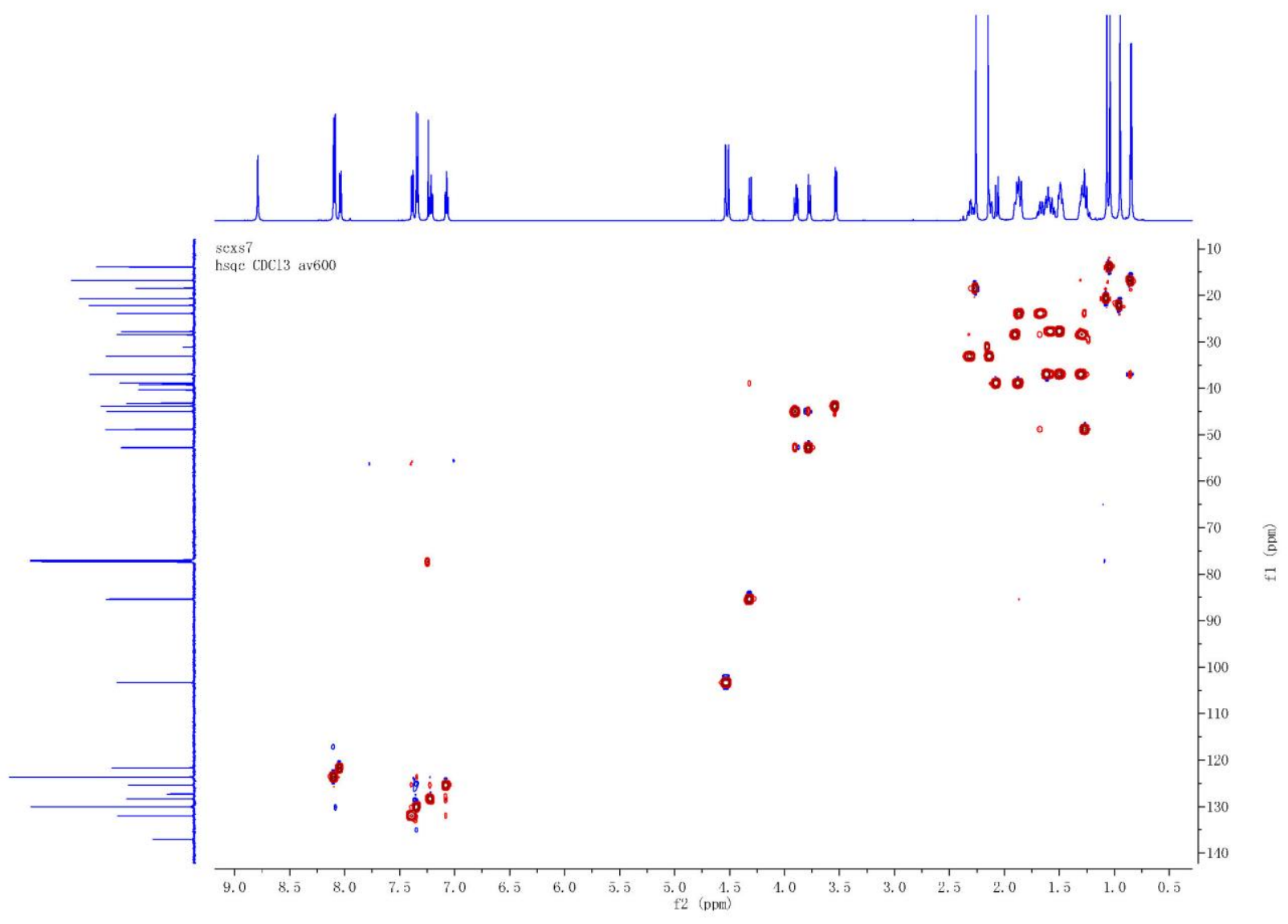

Figure S122. HMBC spectrum of 7e

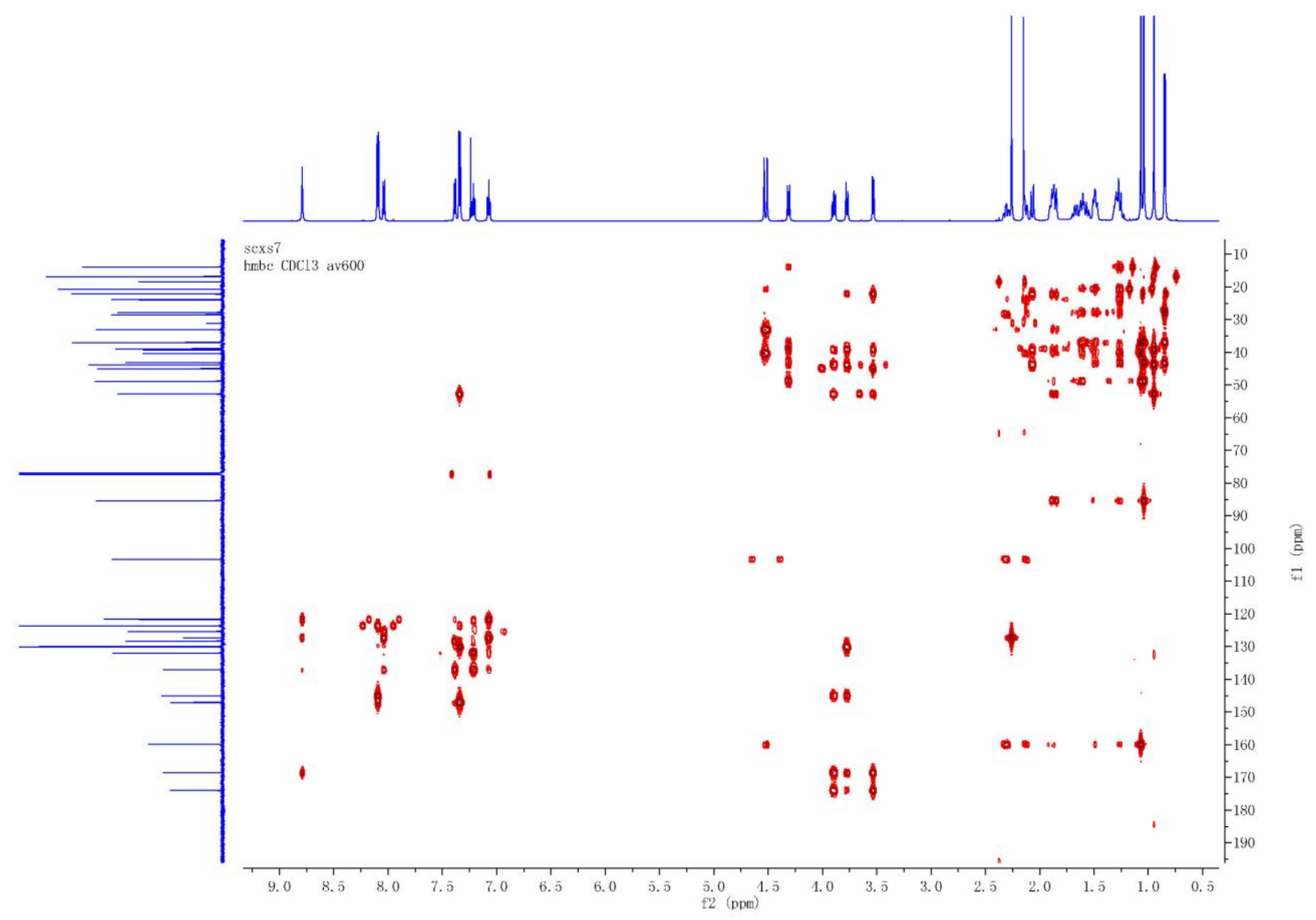


Figure S123. ${ }^{1} \mathrm{H}-{ }^{1} \mathrm{H}$ COSY spectrum of $\mathbf{7 e}$

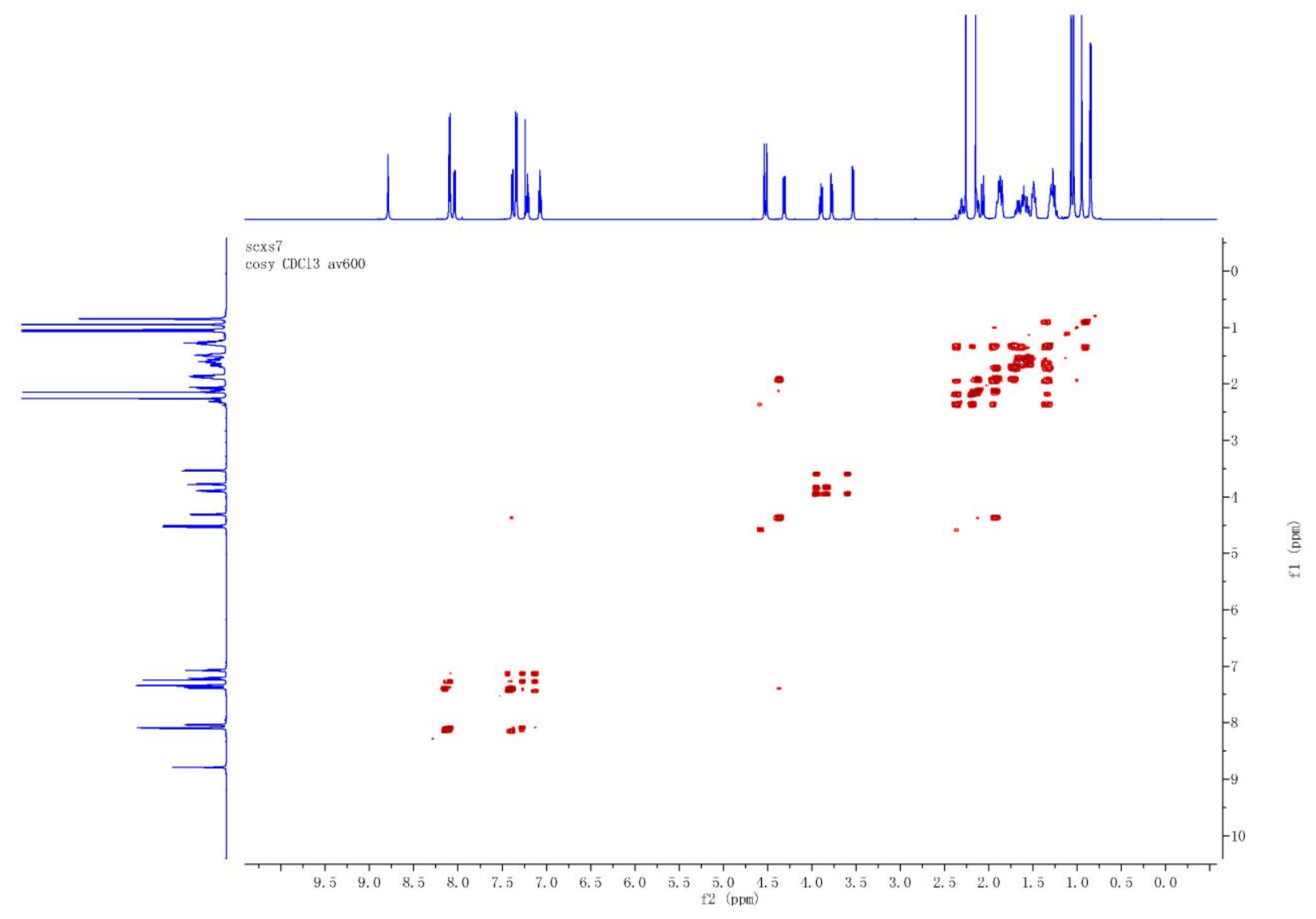

Figure S124. ROESY spectrum of 7e

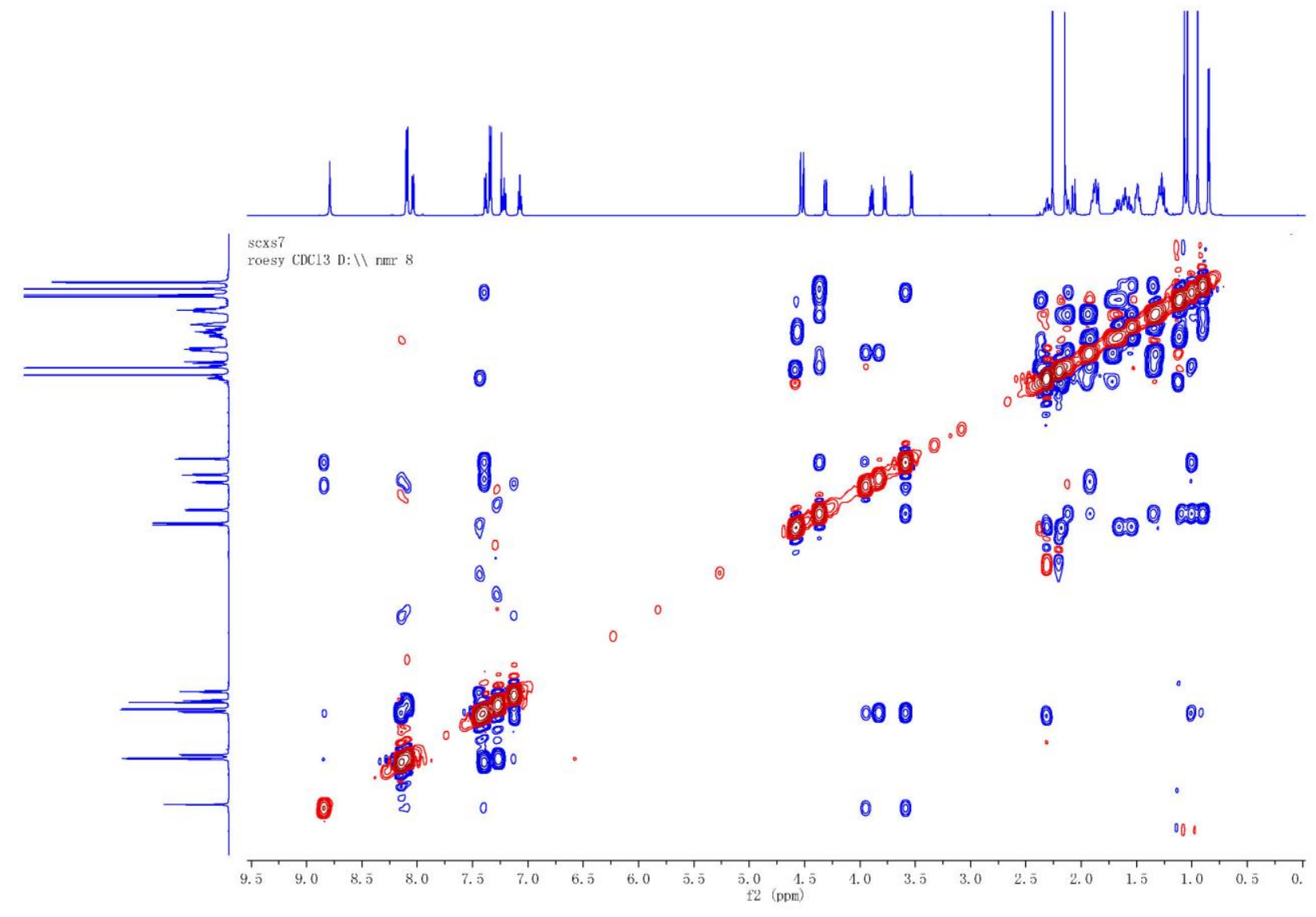




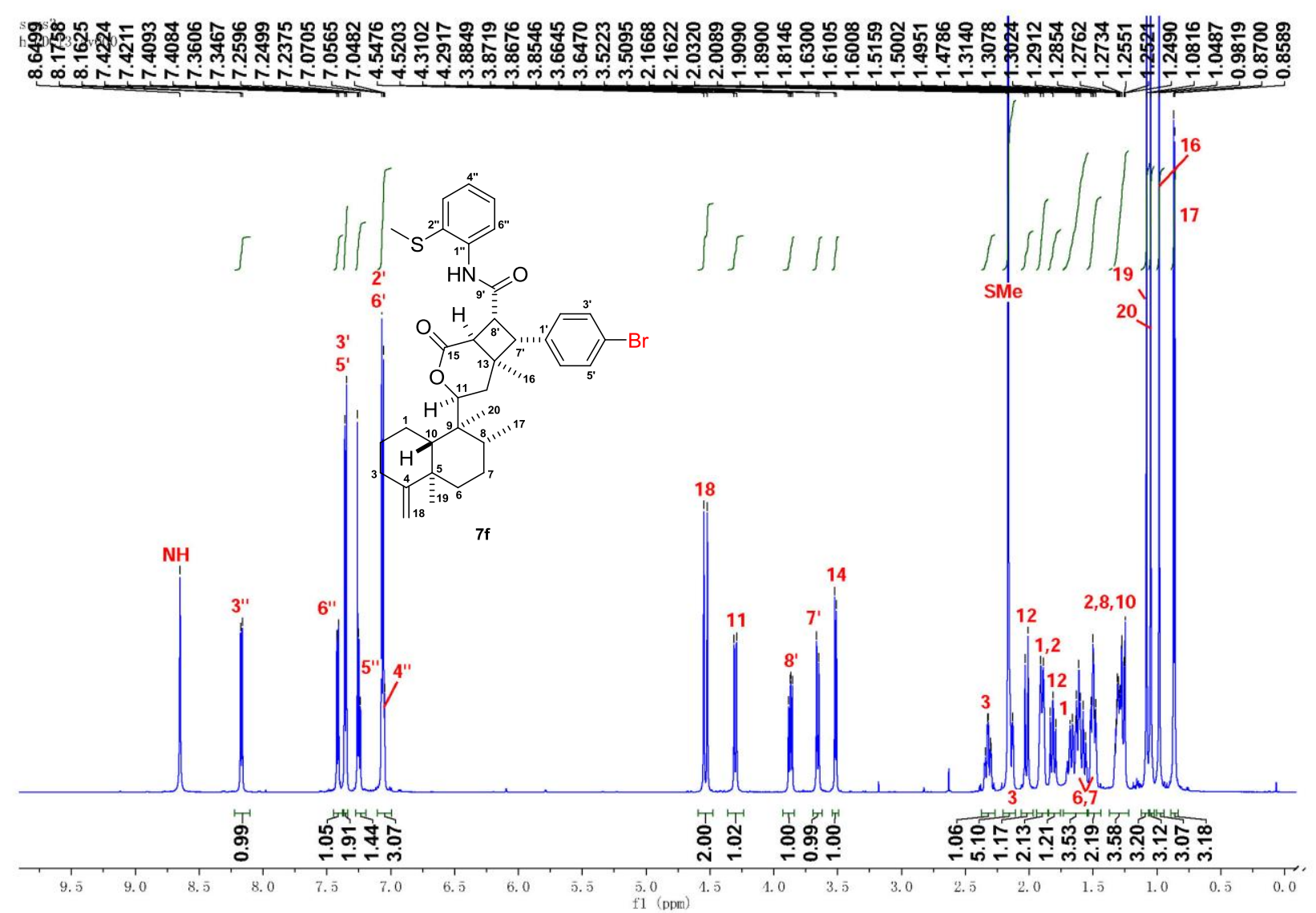

Figure S126. ${ }^{13} \mathrm{C}$ NMR spectrum of $\mathbf{7 f}$
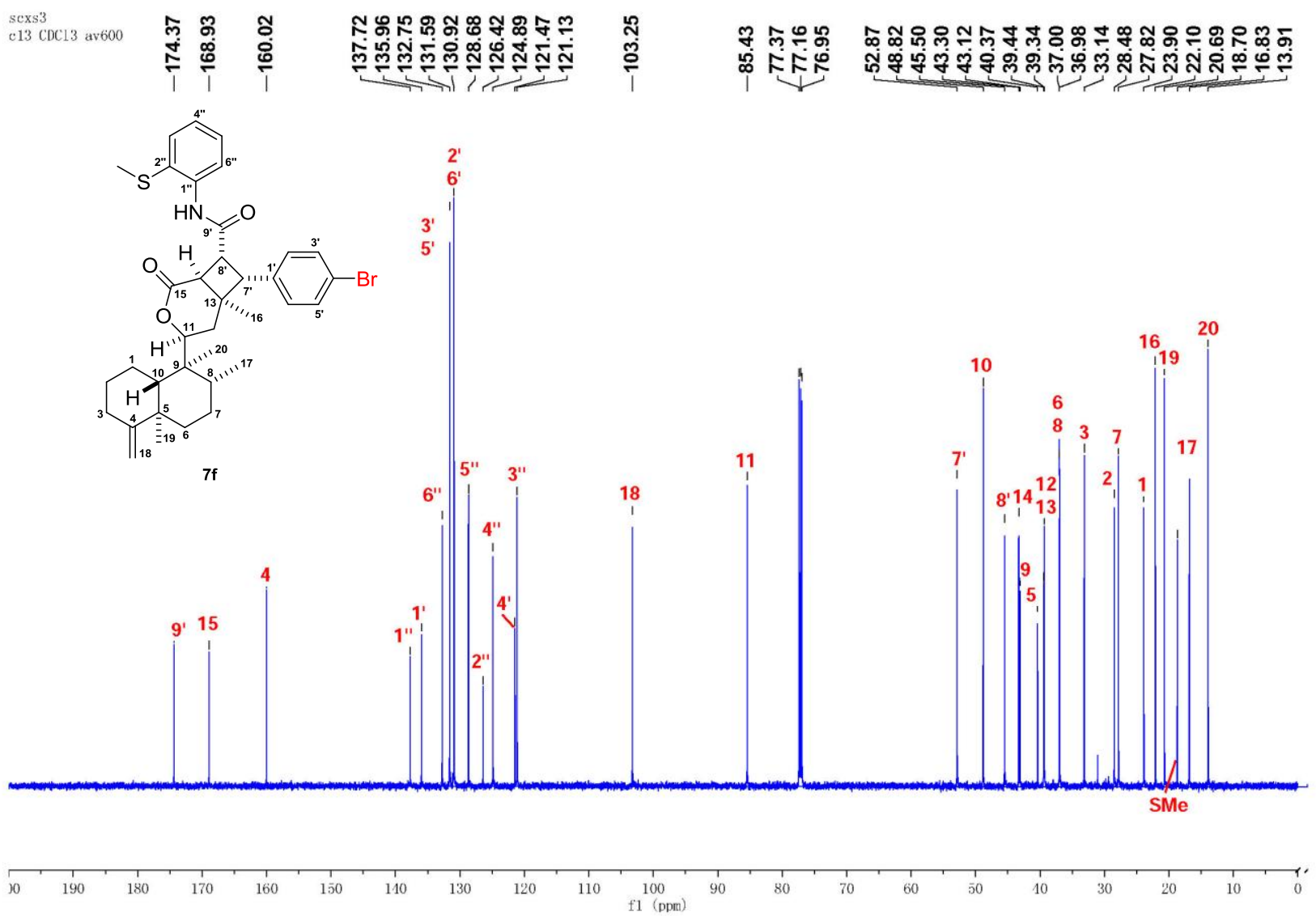
Figure S127. HSQC spectrum of $7 \mathbf{f}$

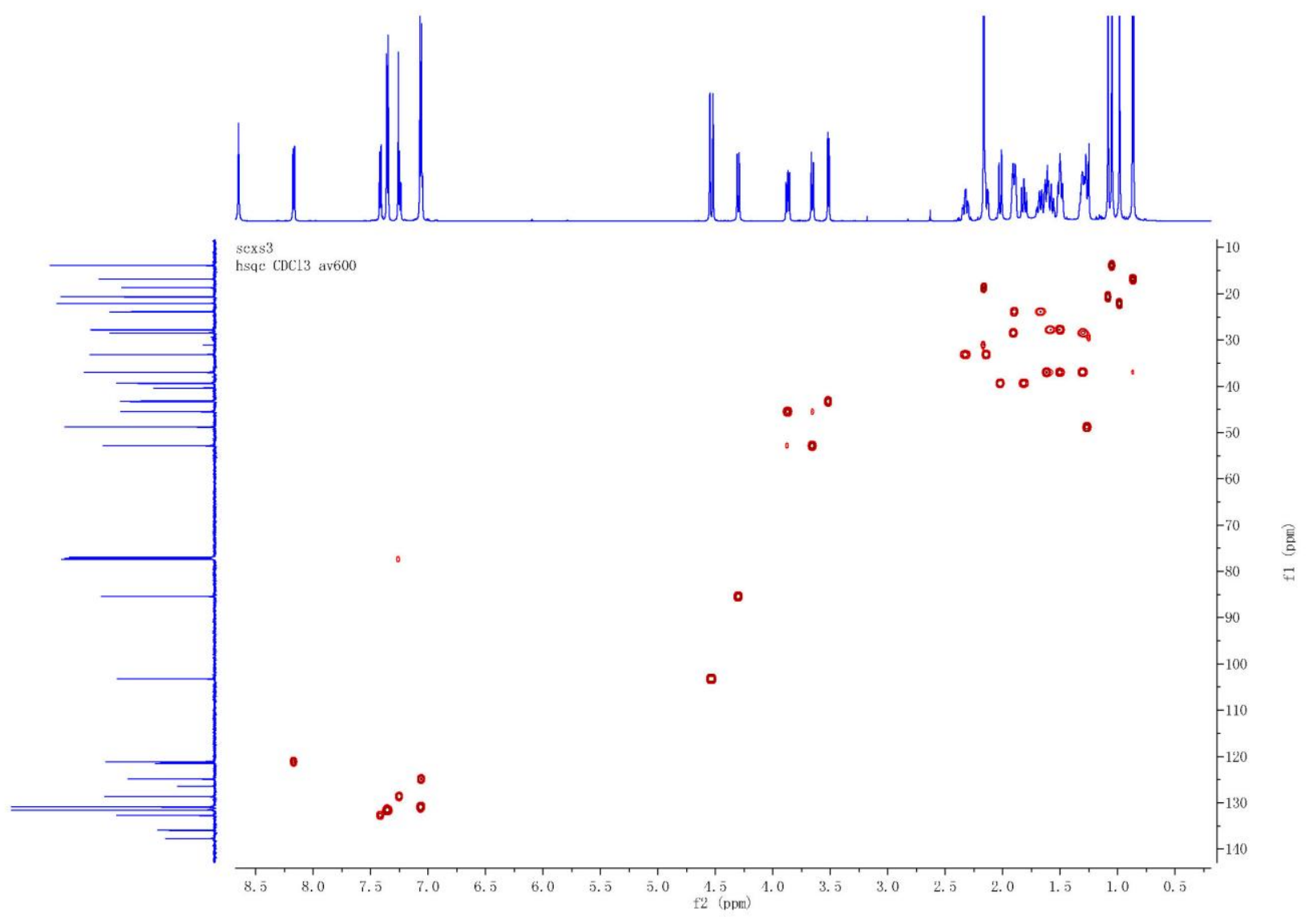

Figure S128. HMBC spectrum of $\mathbf{7 f}$

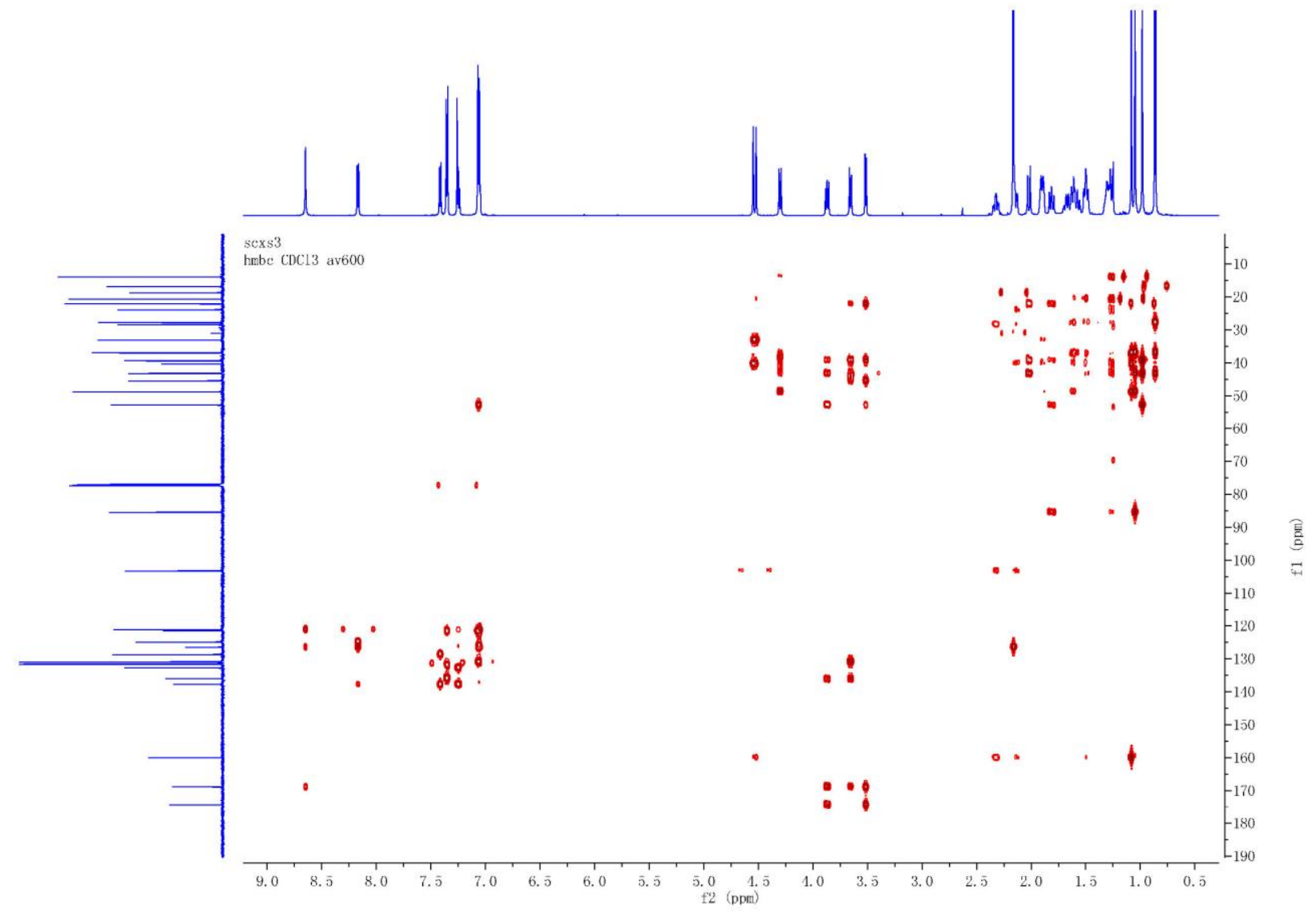


Figure S129. ${ }^{1} \mathrm{H}-{ }^{1} \mathrm{H}$ COSY spectrum of $\mathbf{7 f}$

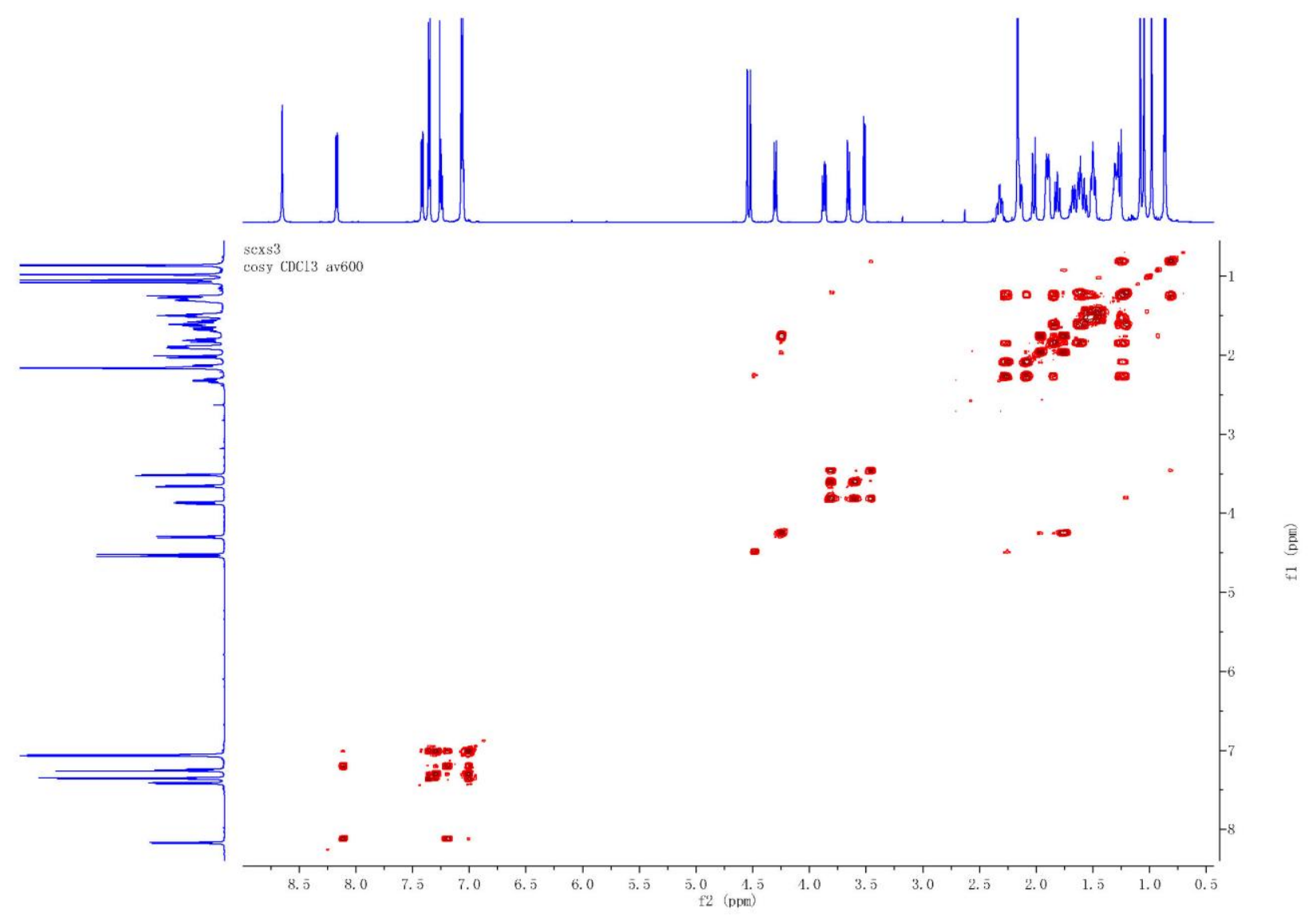

Figure S130. ROESY spectrum of $7 \mathbf{f}$

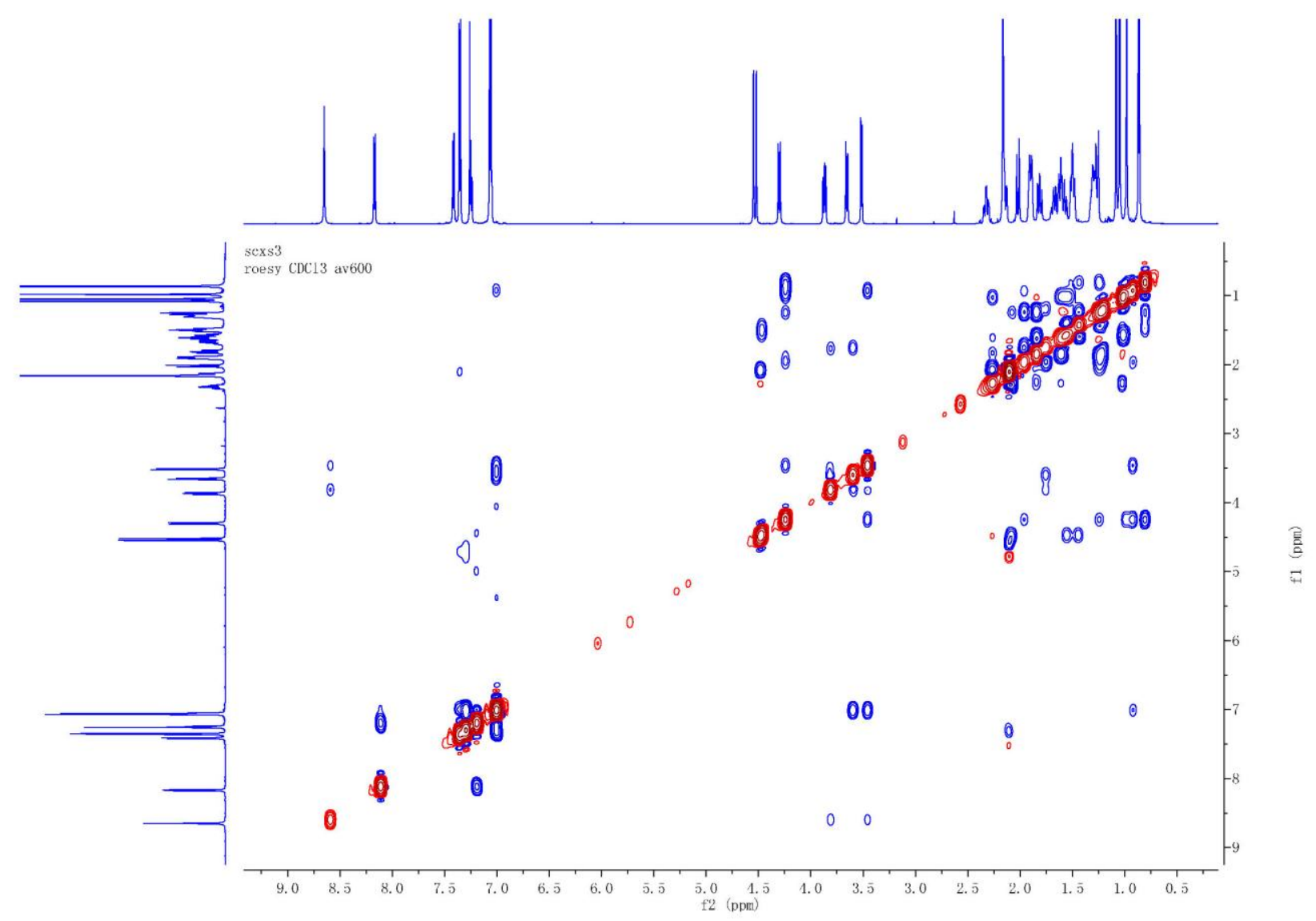


Figure S131. ${ }^{1} \mathrm{H}$ NMR spectrum of $\mathbf{7 g}$

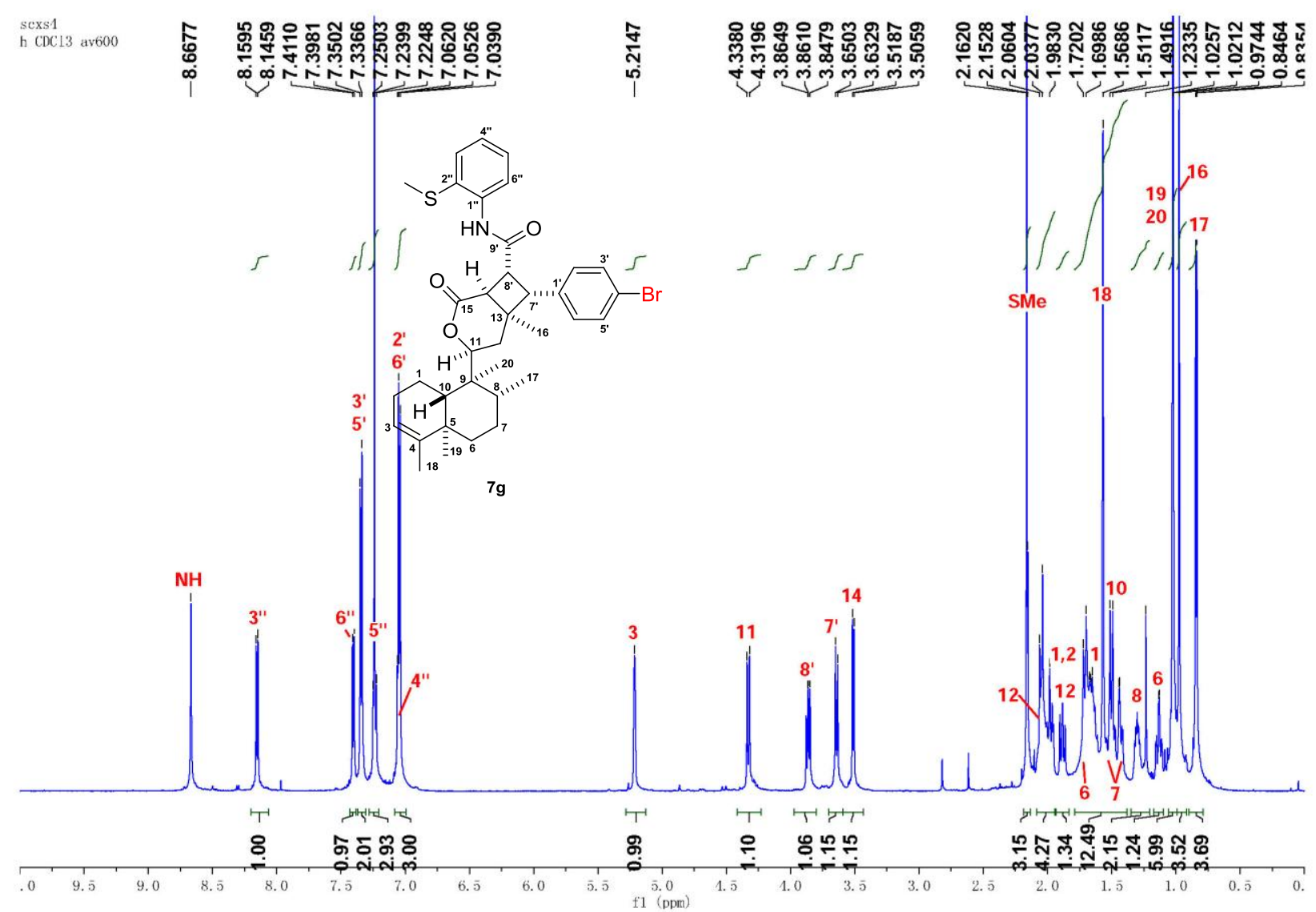

Figure S132. ${ }^{13} \mathrm{C}$ NMR spectrum of $\mathbf{7 g}$
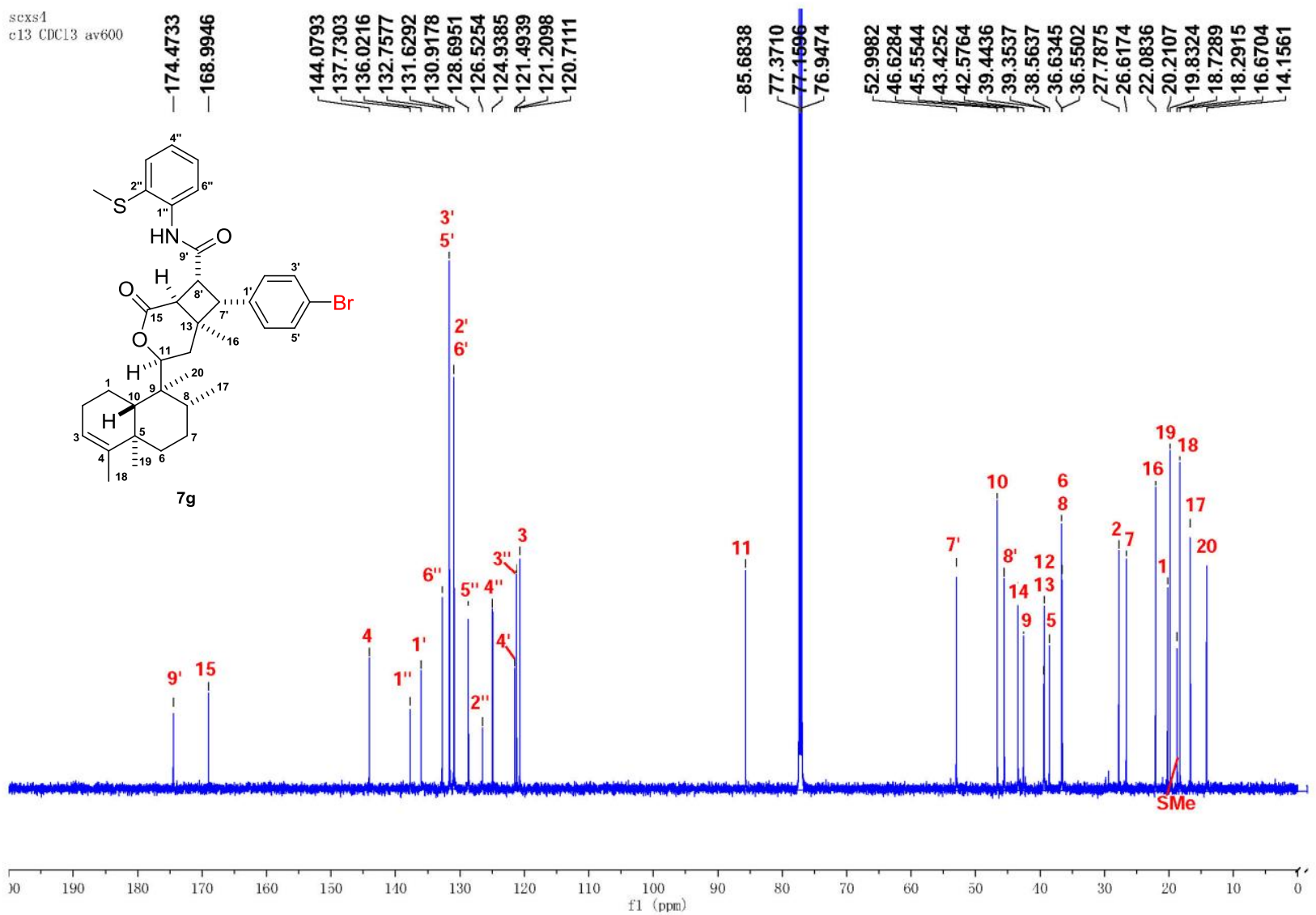
Figure S133. HSQC spectrum of $\mathbf{7 g}$

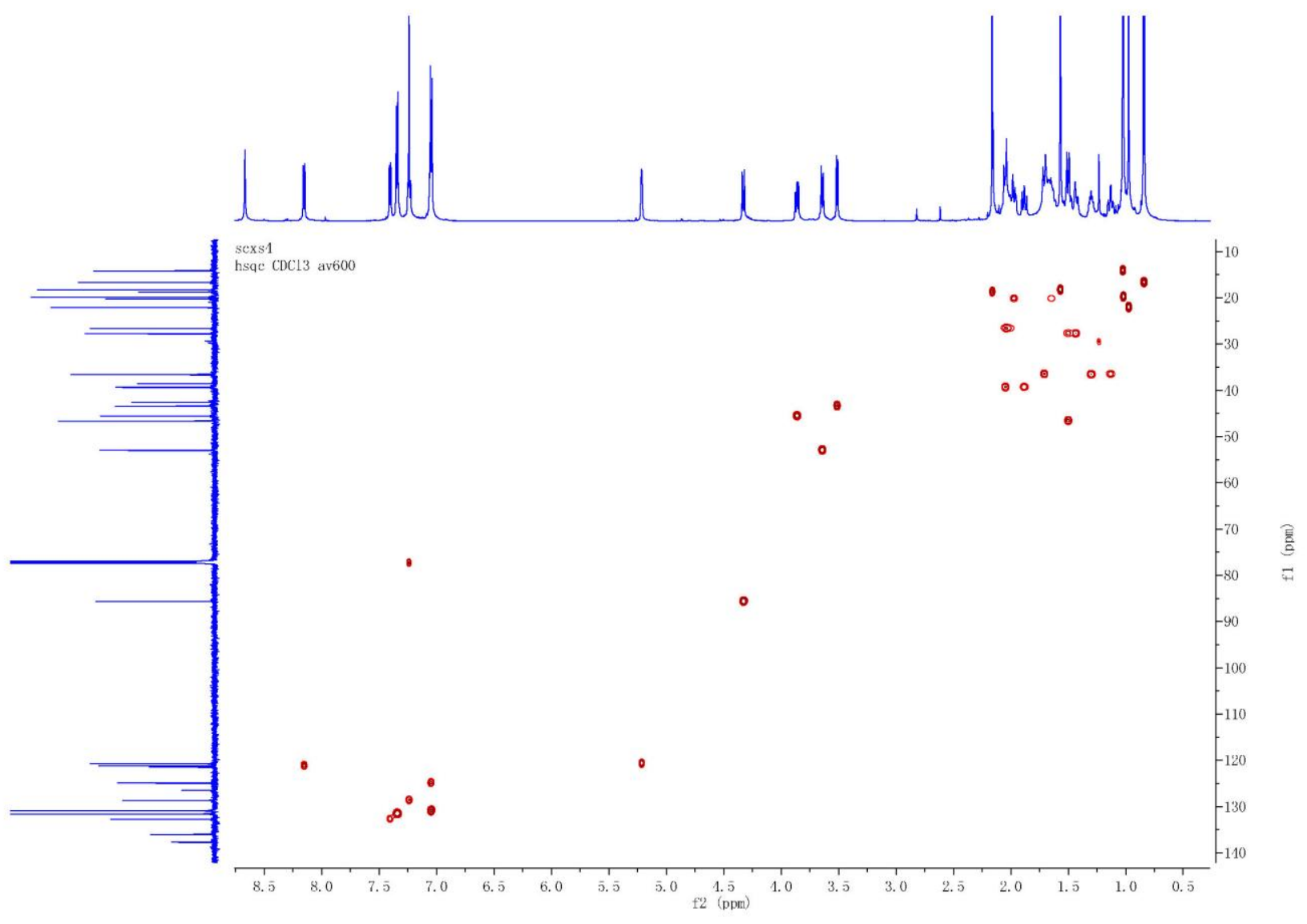

Figure S134. HMBC spectrum of $\mathbf{7 g}$

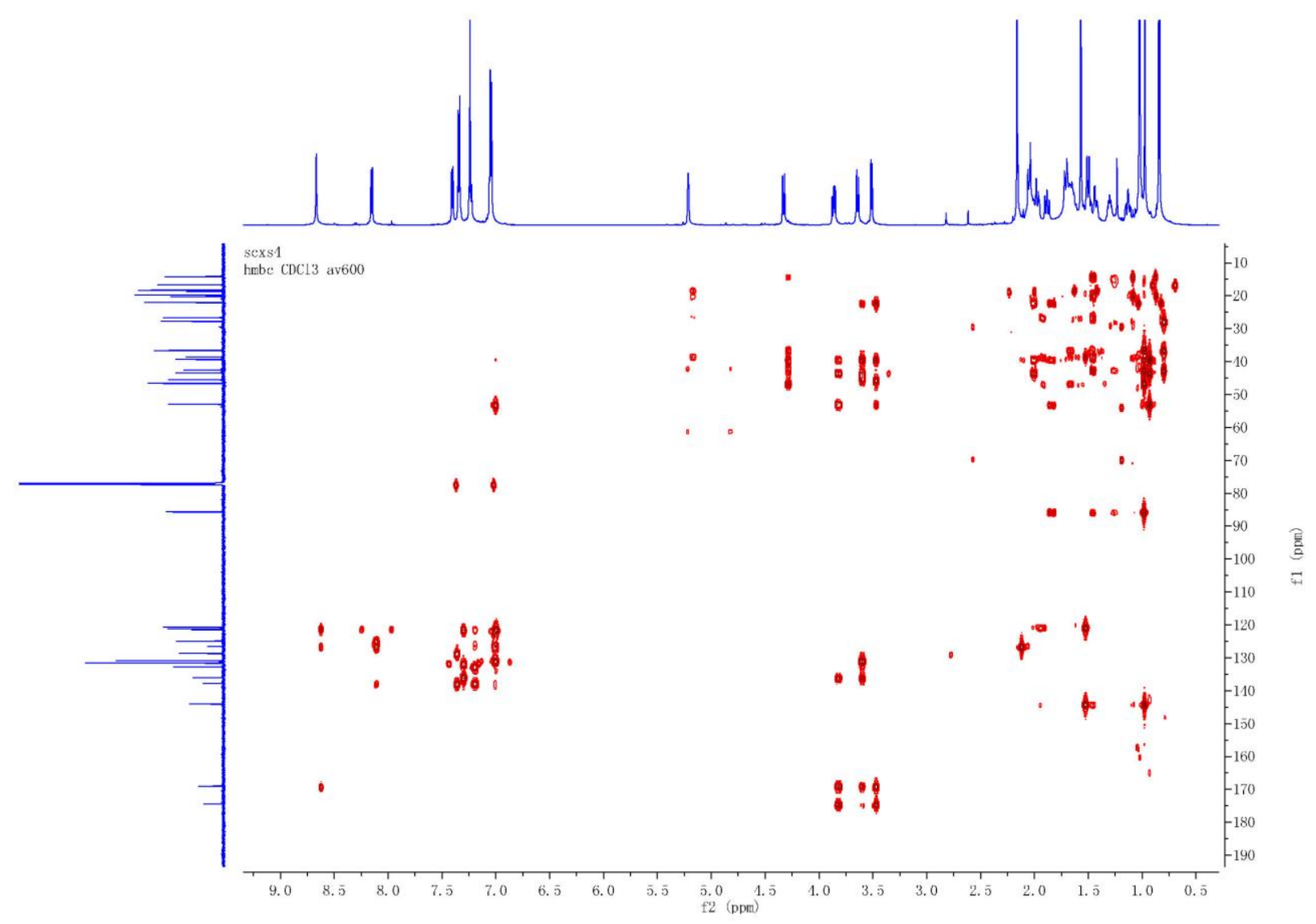


Figure S135. ${ }^{1} \mathrm{H}-{ }^{1} \mathrm{H}$ COSY spectrum of $\mathbf{7 g}$

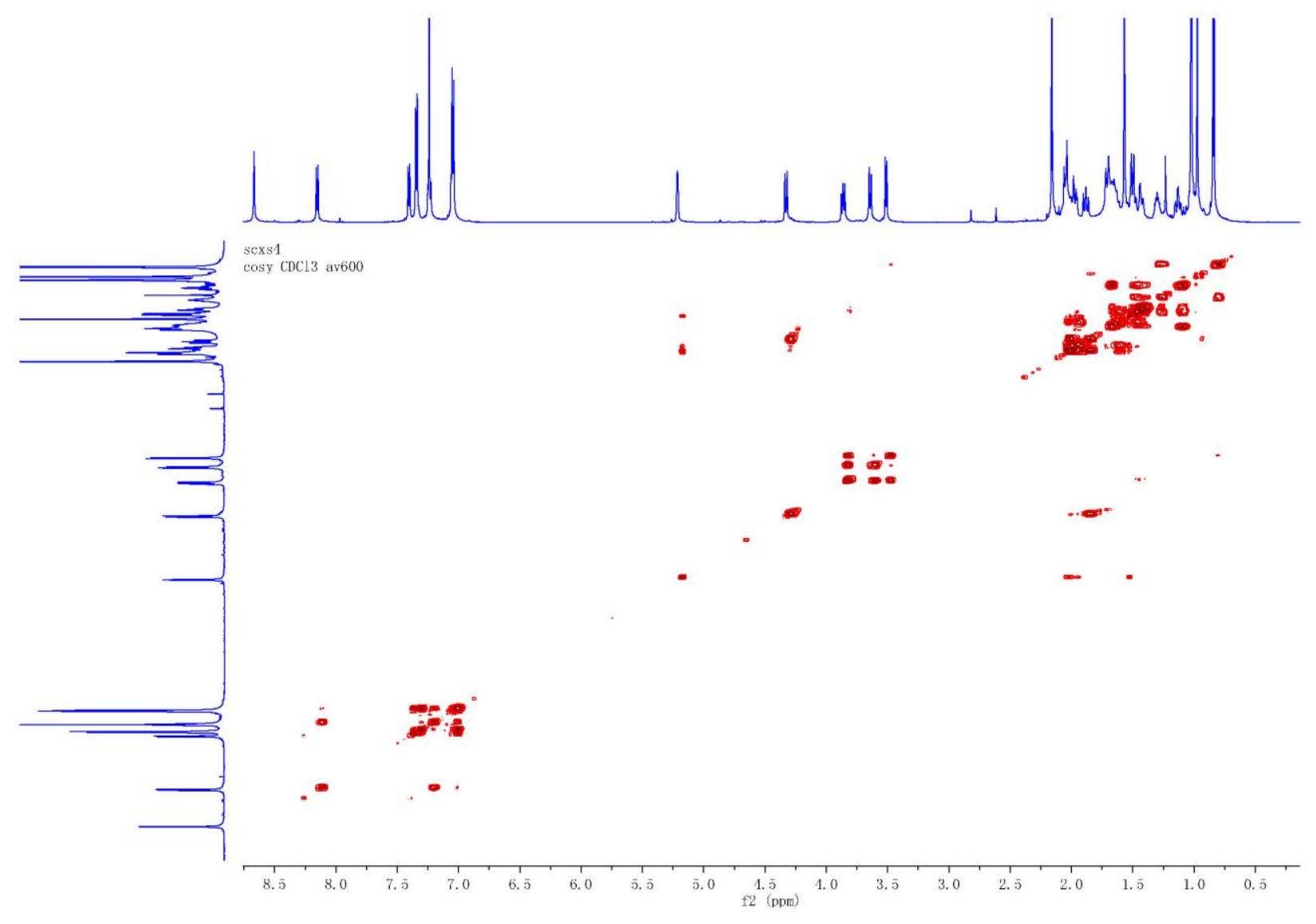

Figure S136. ROESY spectrum of $\mathbf{7 g}$

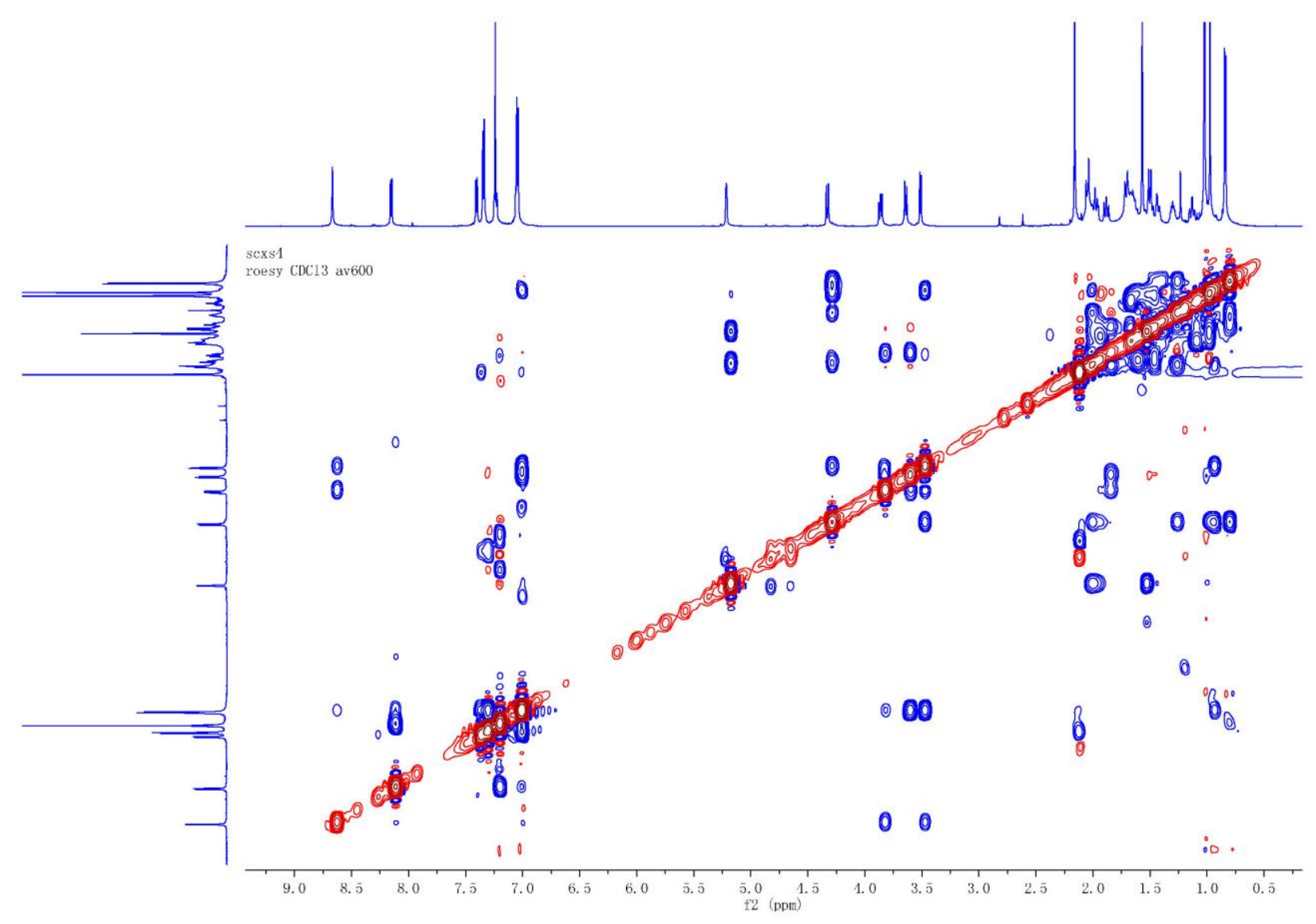


Figure S137. ${ }^{1} \mathrm{H}$ NMR spectrum of $\mathbf{7 h}$

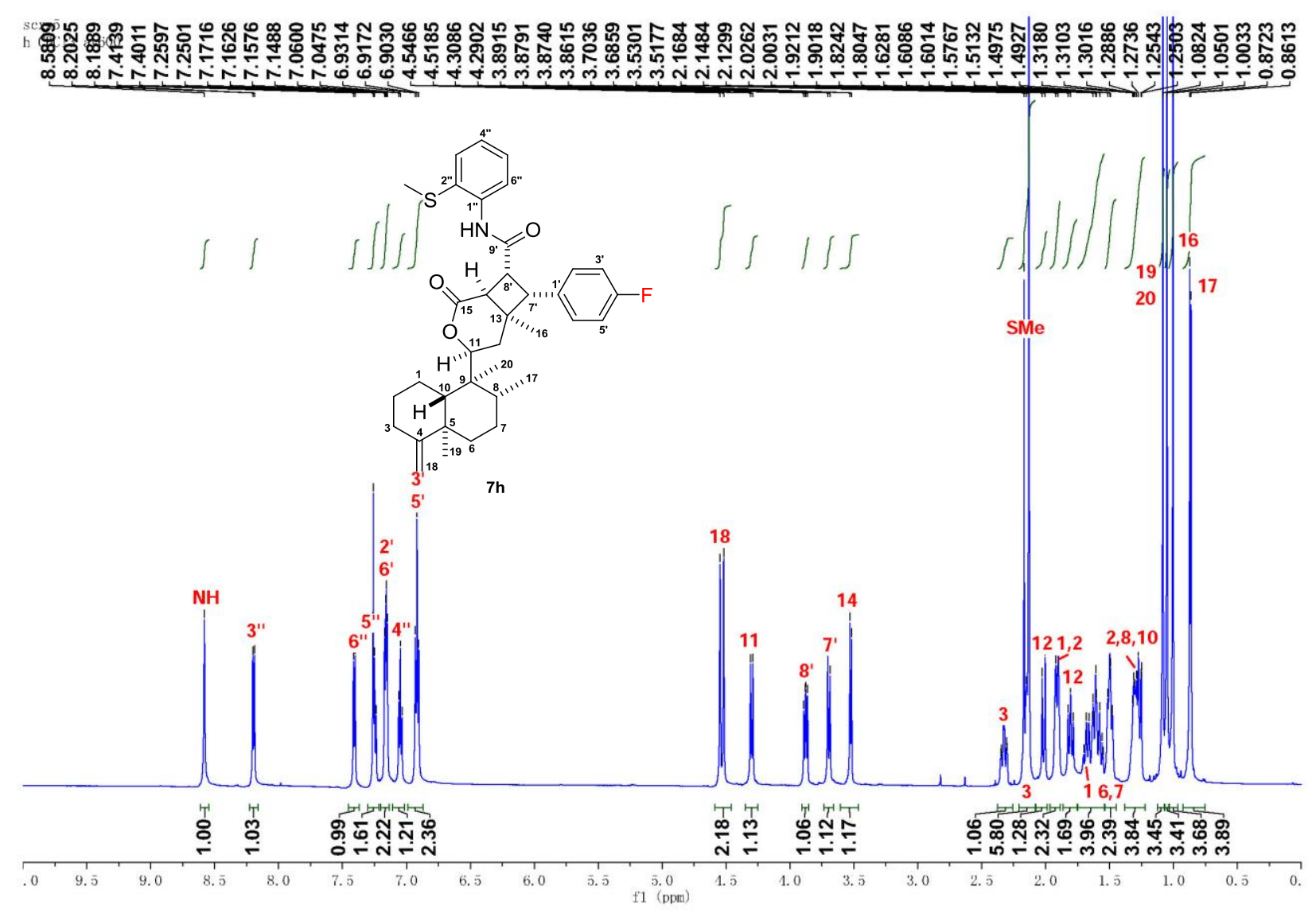

Figure S138. ${ }^{13} \mathrm{C}$ NMR spectrum of $7 \mathbf{h}$

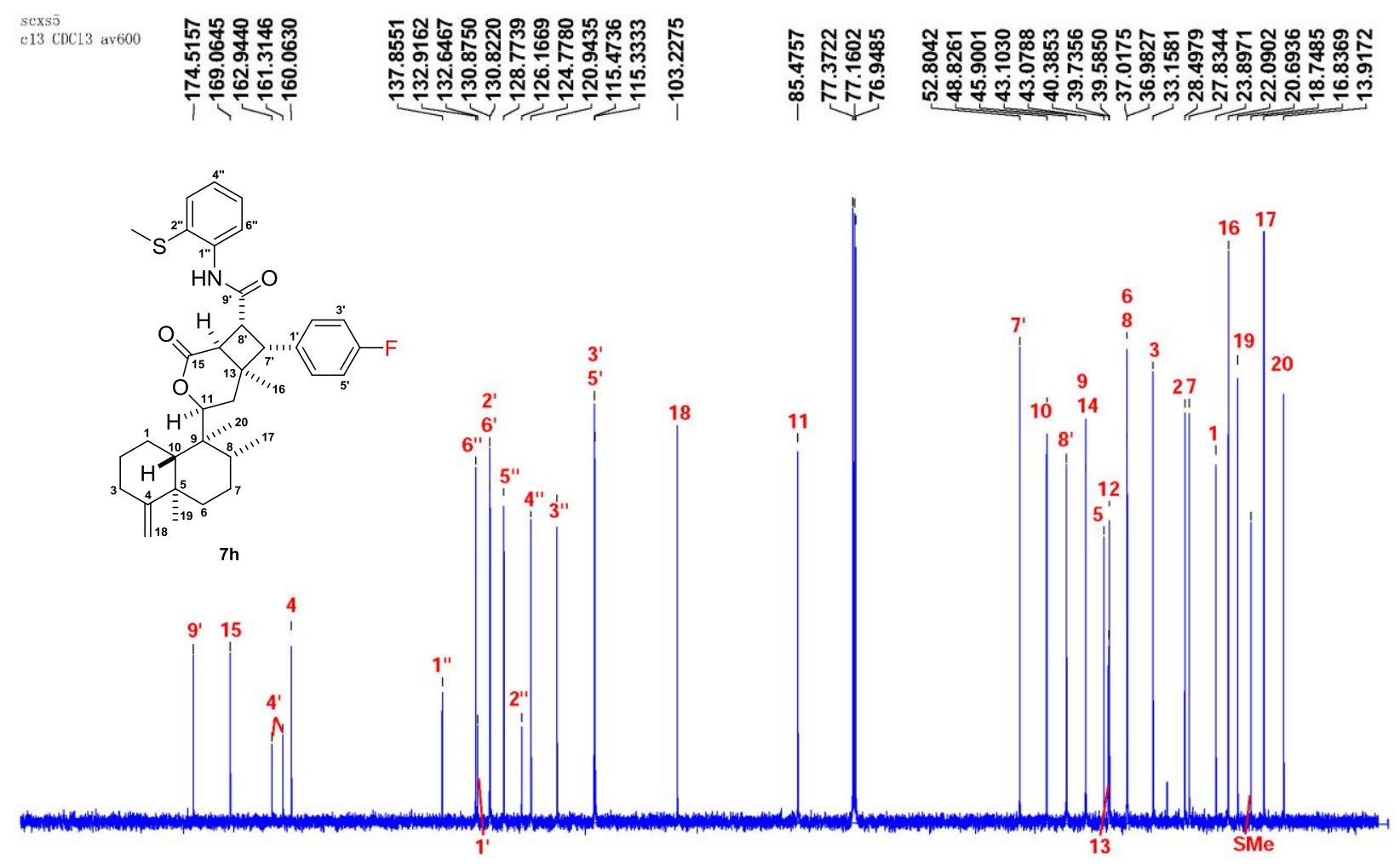

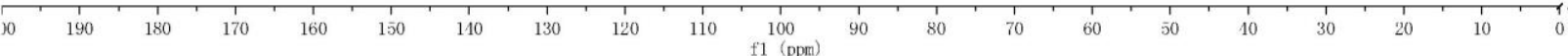


Figure S139. HSQC spectrum of $\mathbf{7 h}$

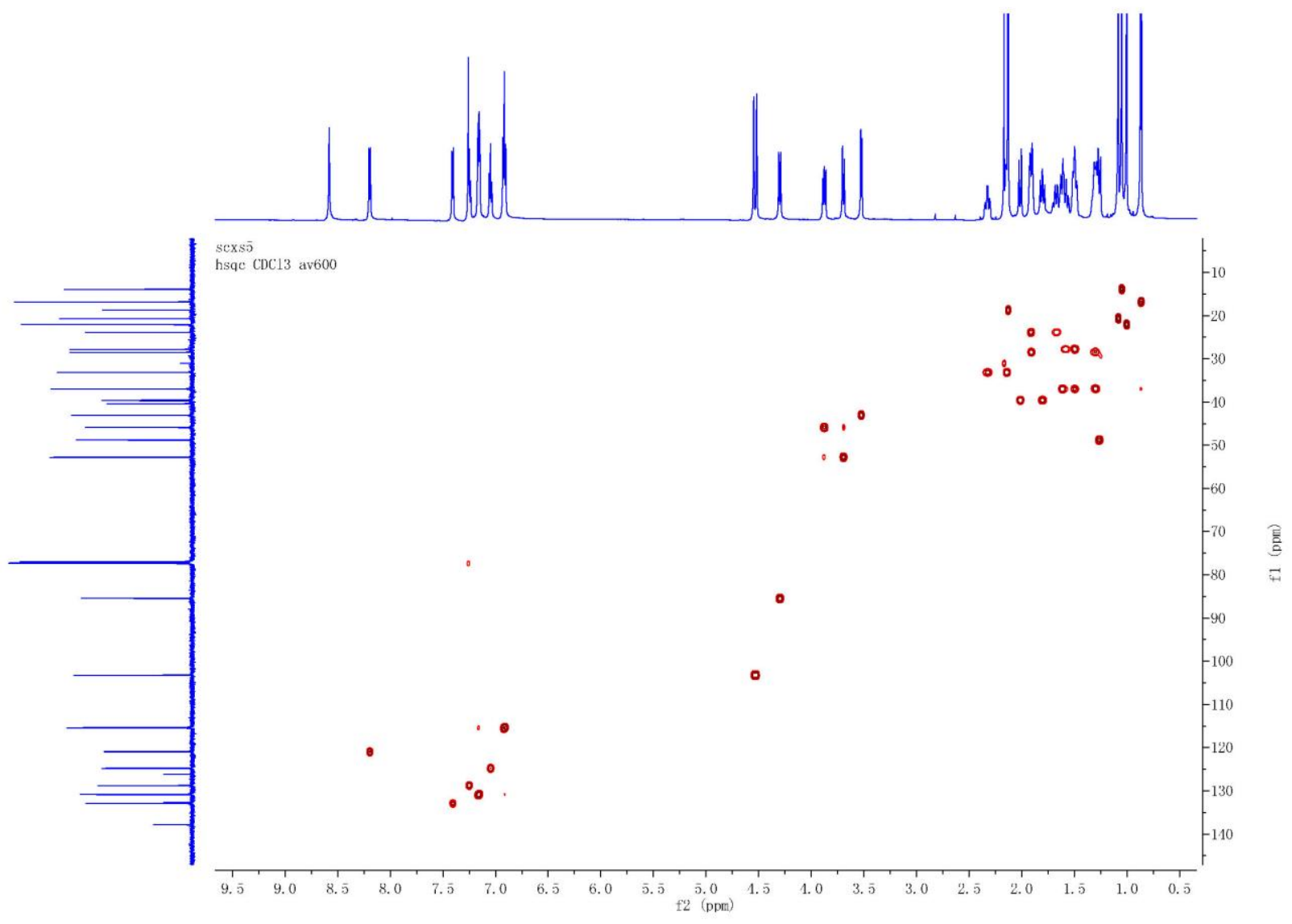

Figure S140. HMBC spectrum of $\mathbf{7 h}$

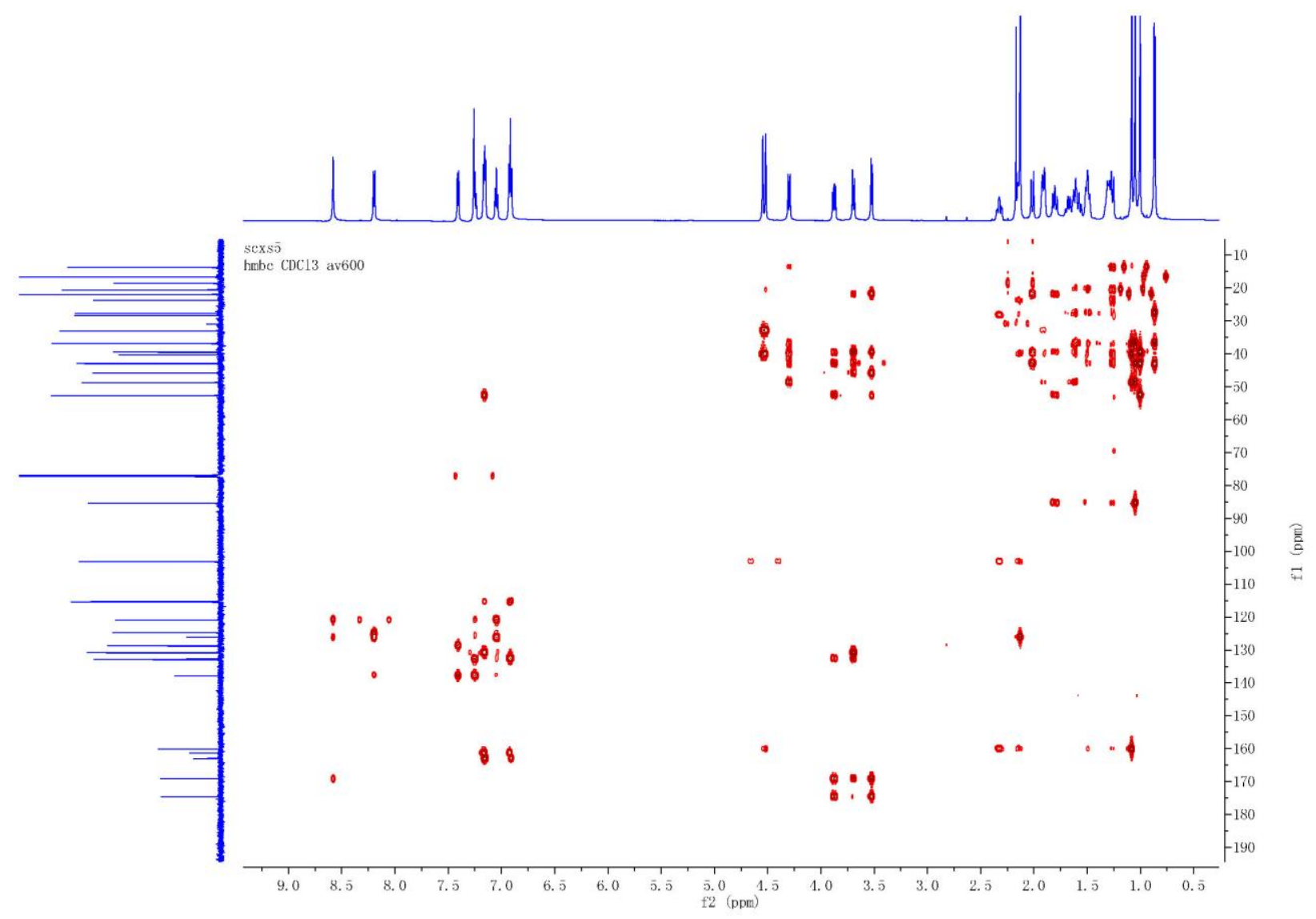


Figure S141. ${ }^{1} \mathrm{H}-{ }^{1} \mathrm{H}$ COSY spectrum of $\mathbf{7 h}$

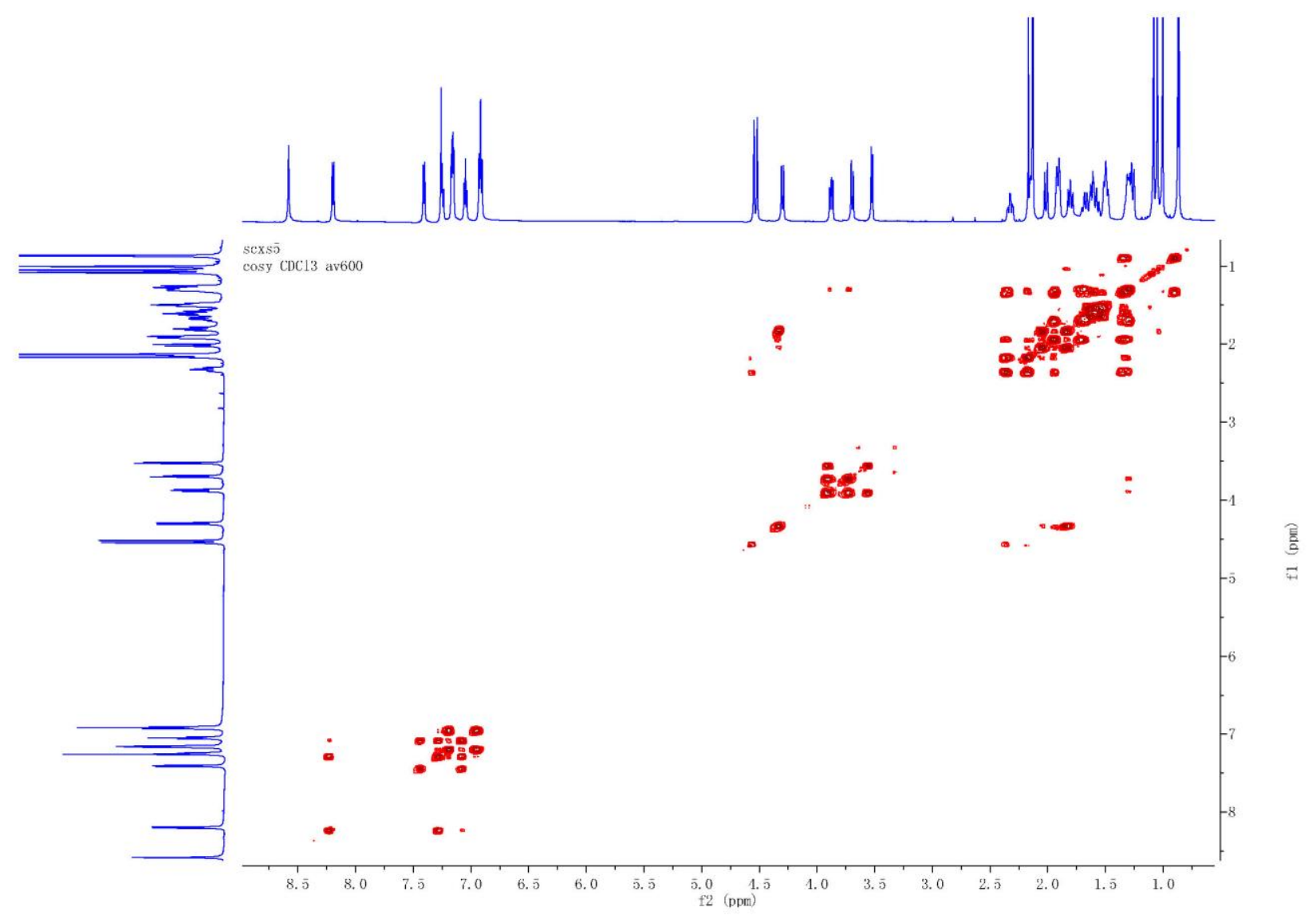

Figure S142. ROESY spectrum of $\mathbf{7 h}$

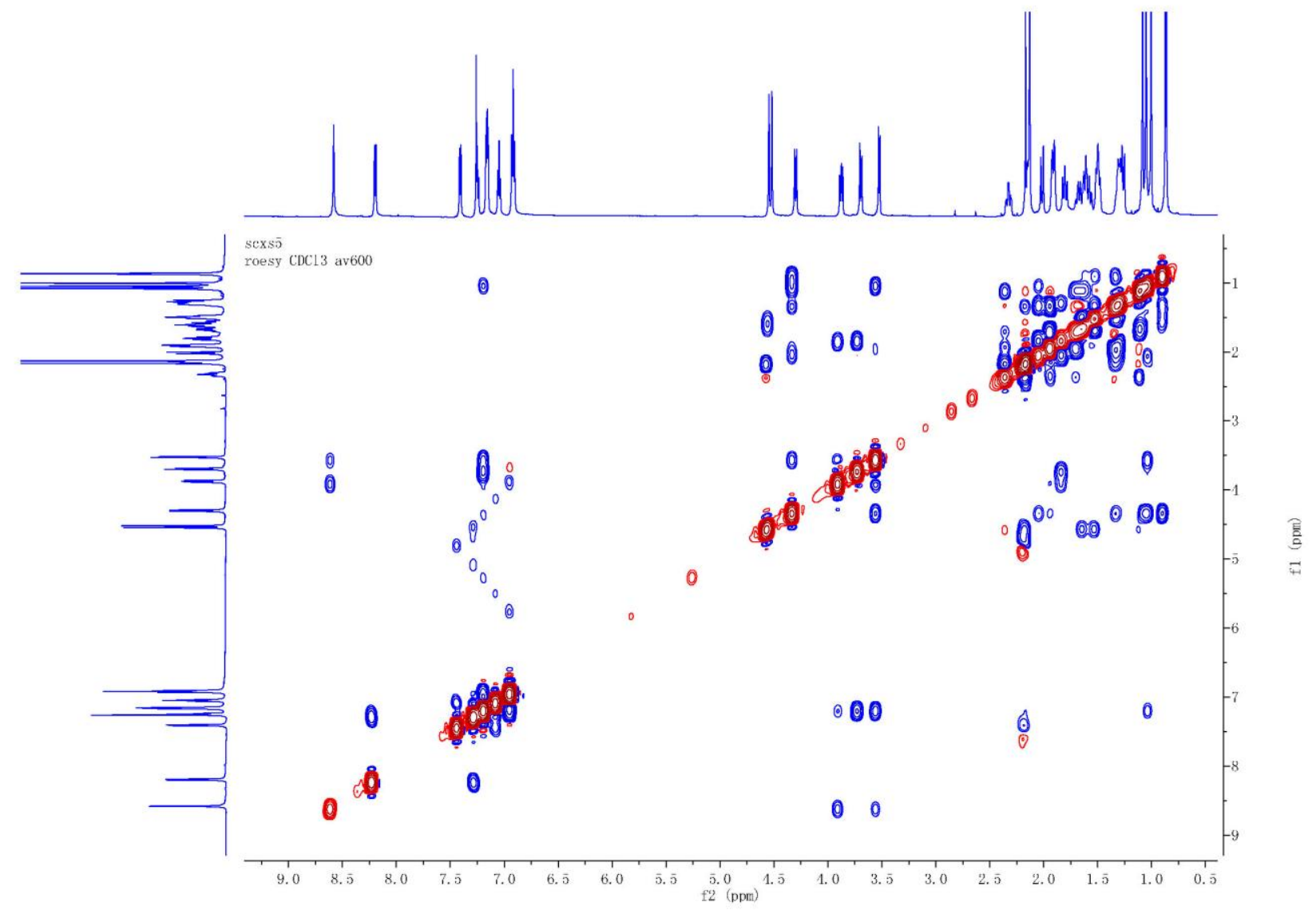


Figure S143. ${ }^{1} \mathrm{H}$ NMR spectrum of $7 \mathbf{i}$

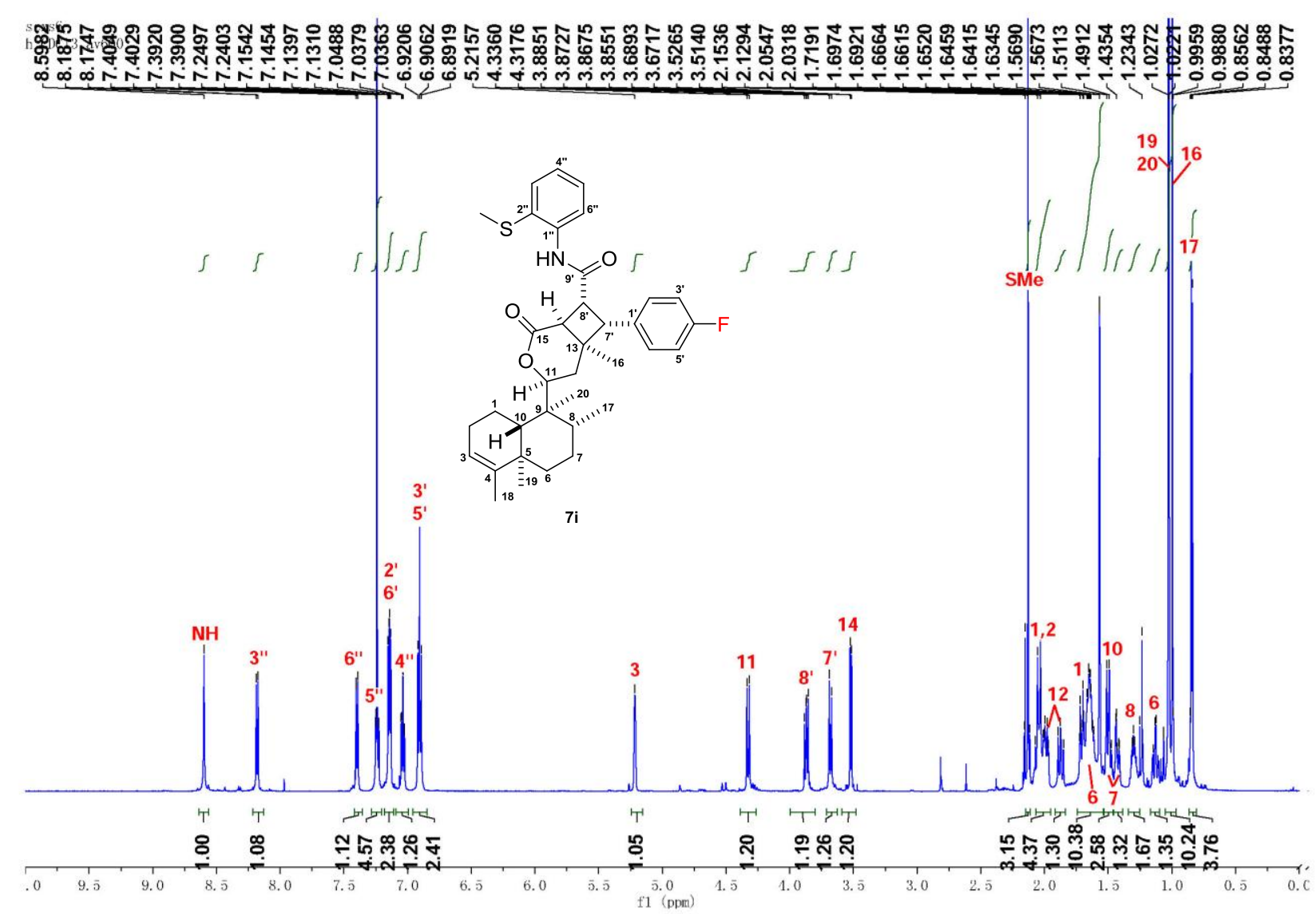

Figure S144. ${ }^{13} \mathrm{C}$ NMR spectrum of $7 \mathbf{i}$

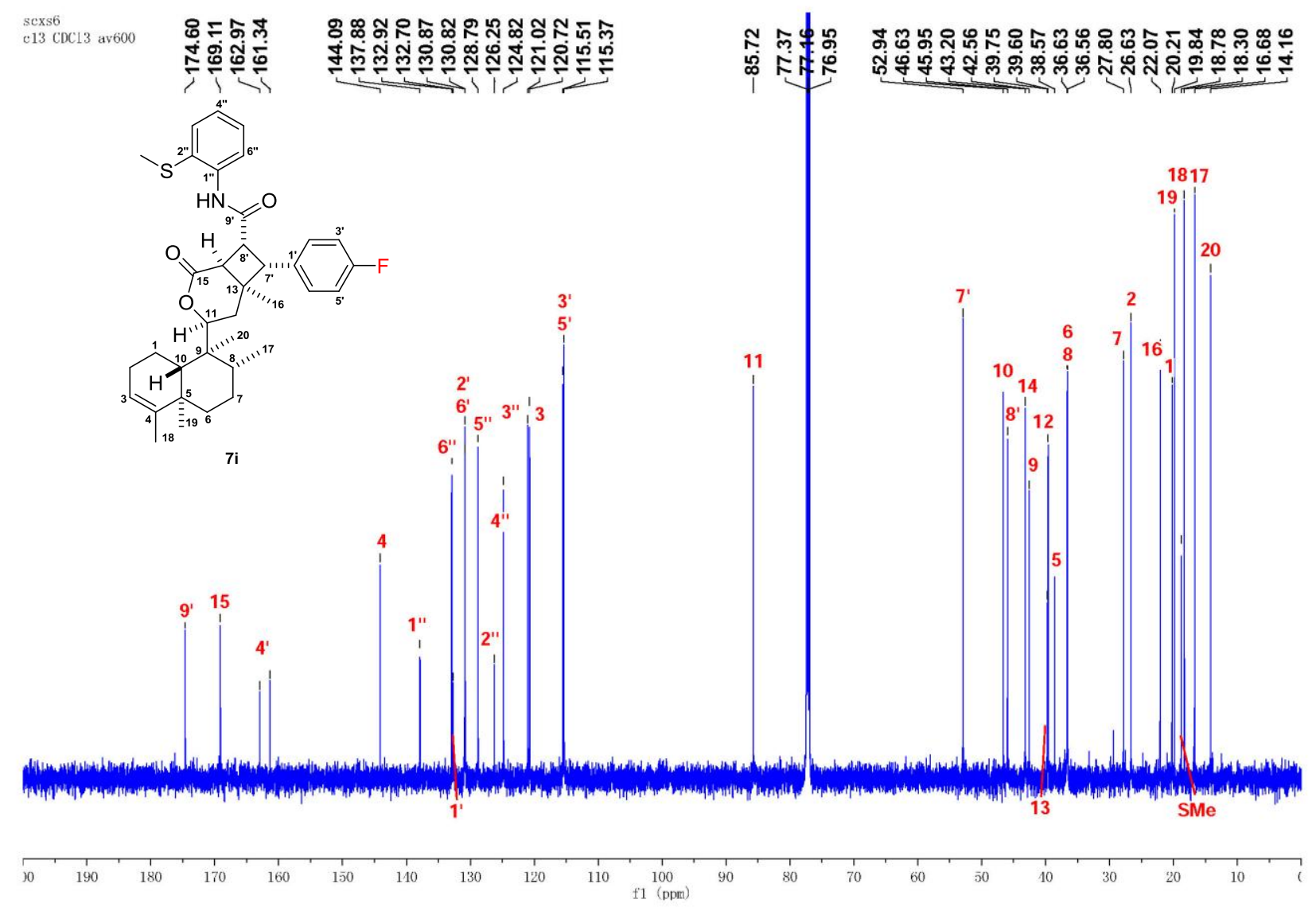


Figure S145. HSQC spectrum of 7i

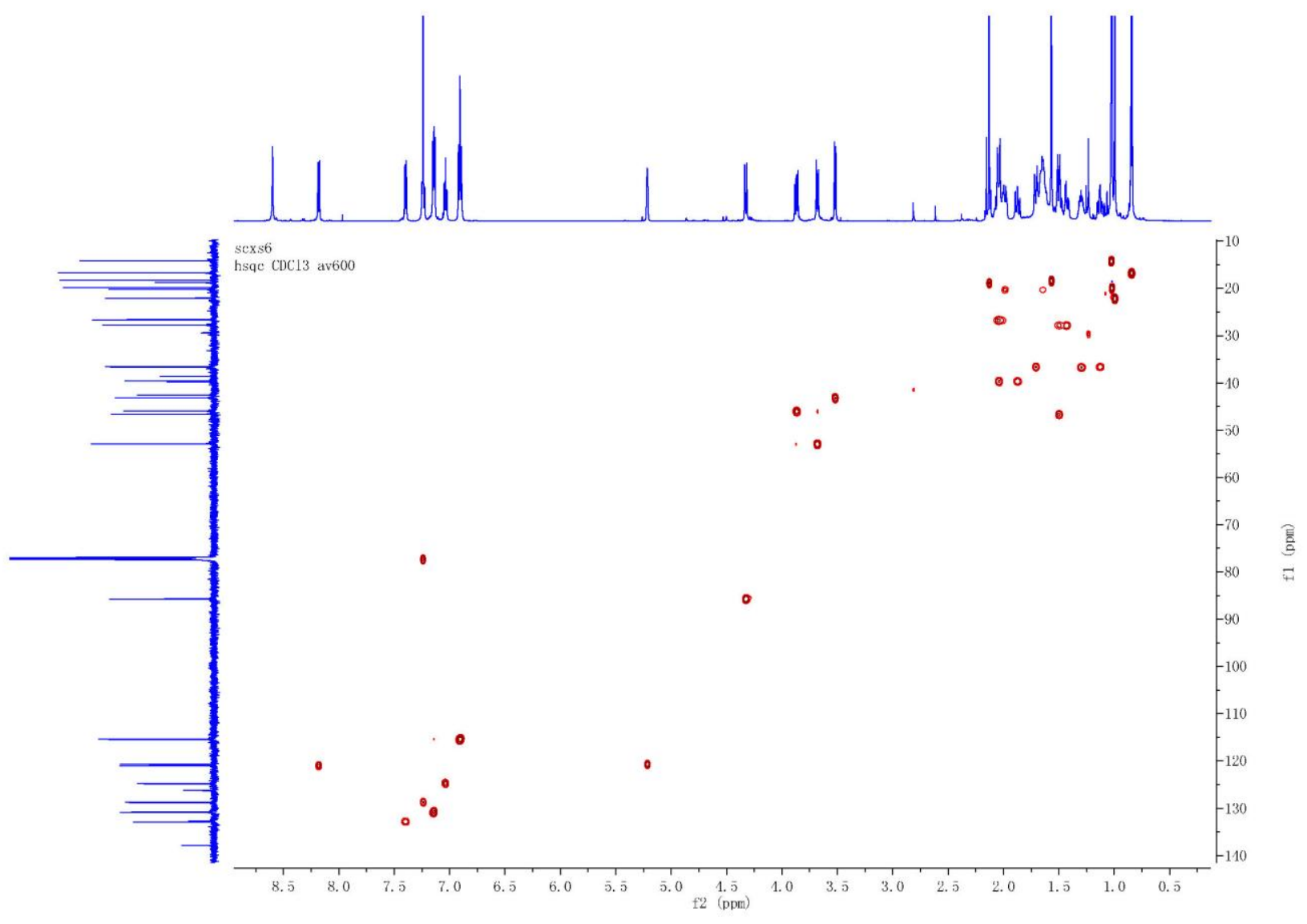

Figure S146. HMBC spectrum of $7 \mathbf{i}$

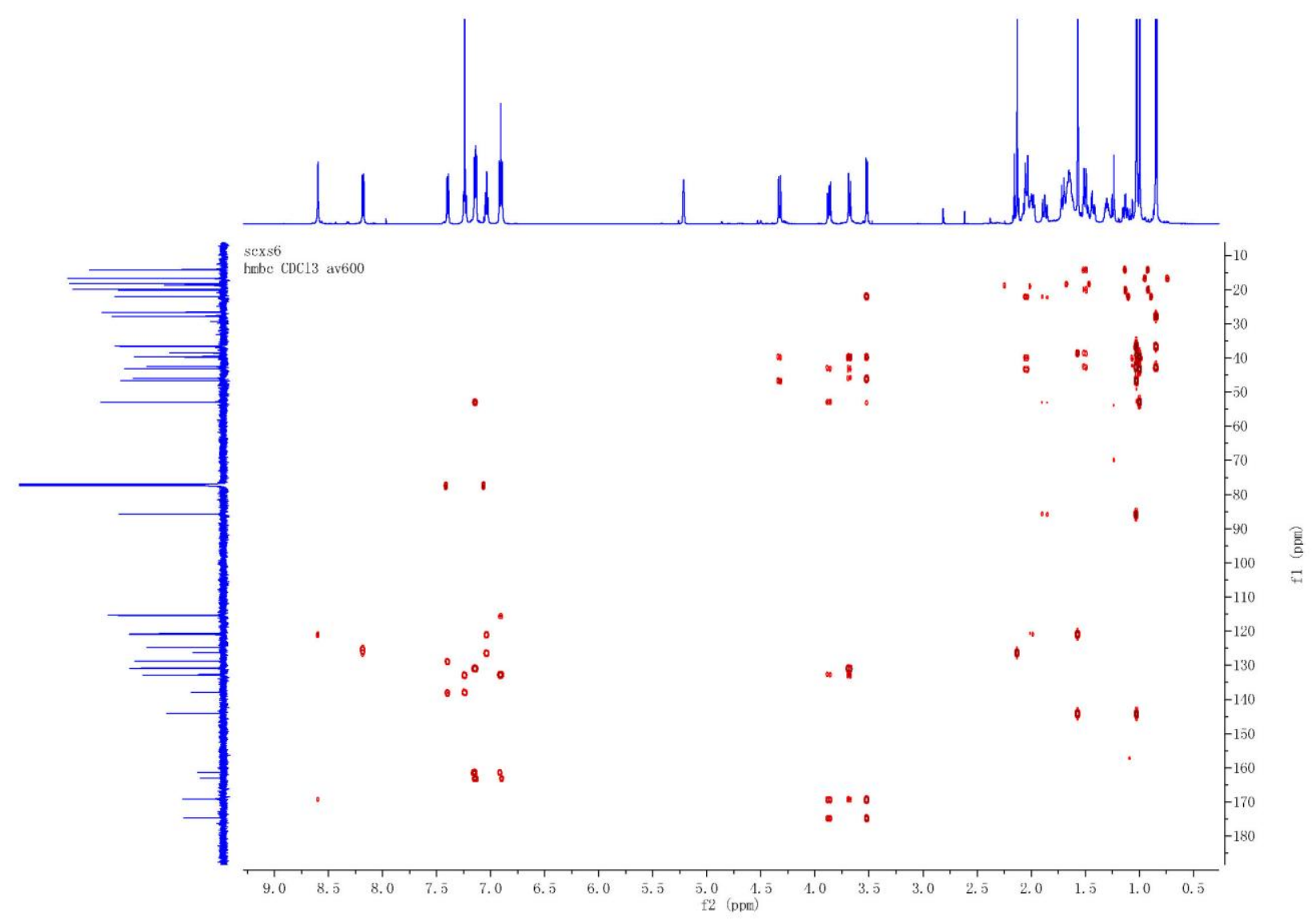


Figure S147. ${ }^{1} \mathrm{H}-{ }^{1} \mathrm{H}$ COSY spectrum of $7 \mathbf{i}$

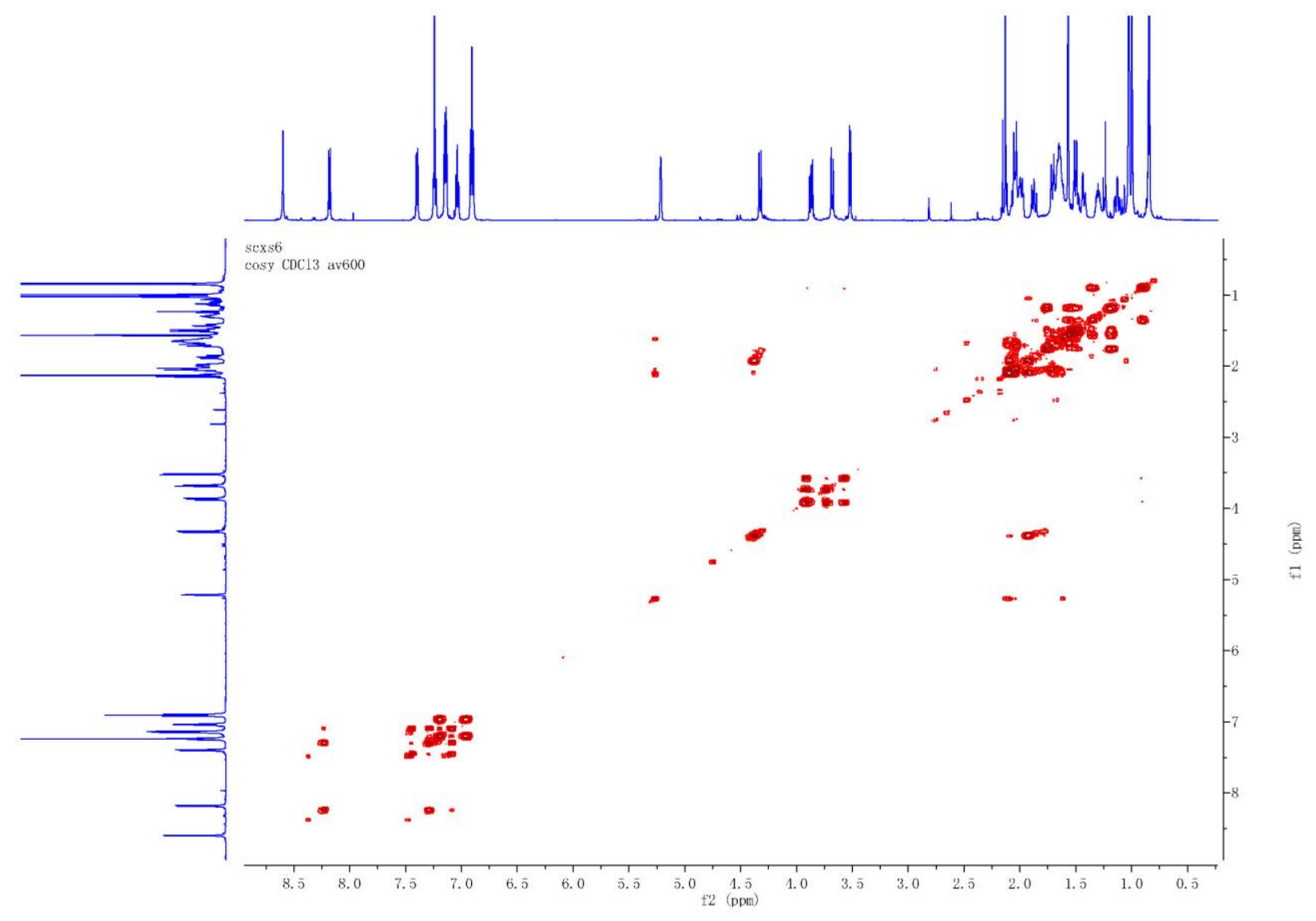

Figure S148. ROESY spectrum of 7i

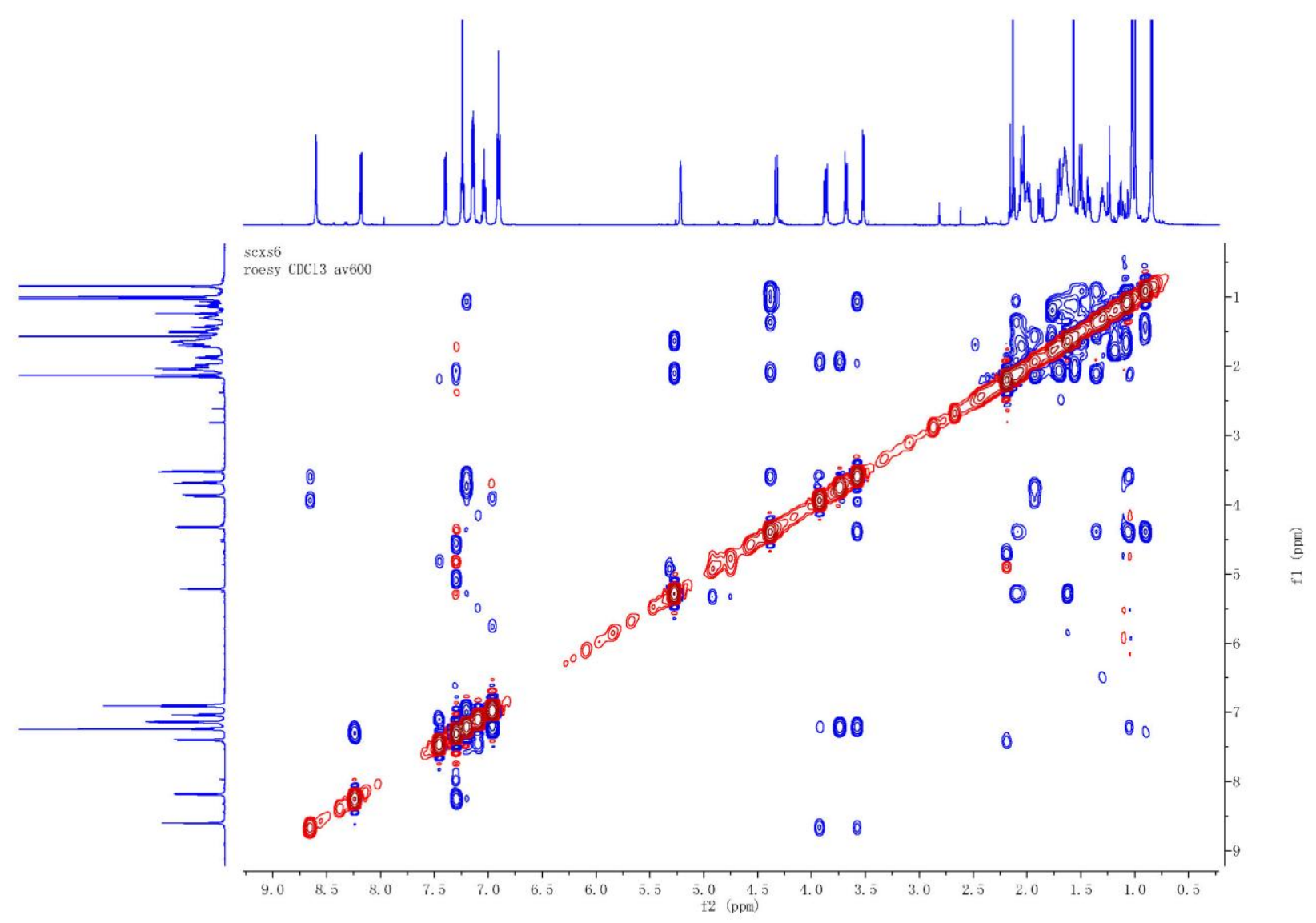


Figure S49. ${ }^{1} \mathrm{H}$ NMR spectrum of $\mathbf{7 b}$

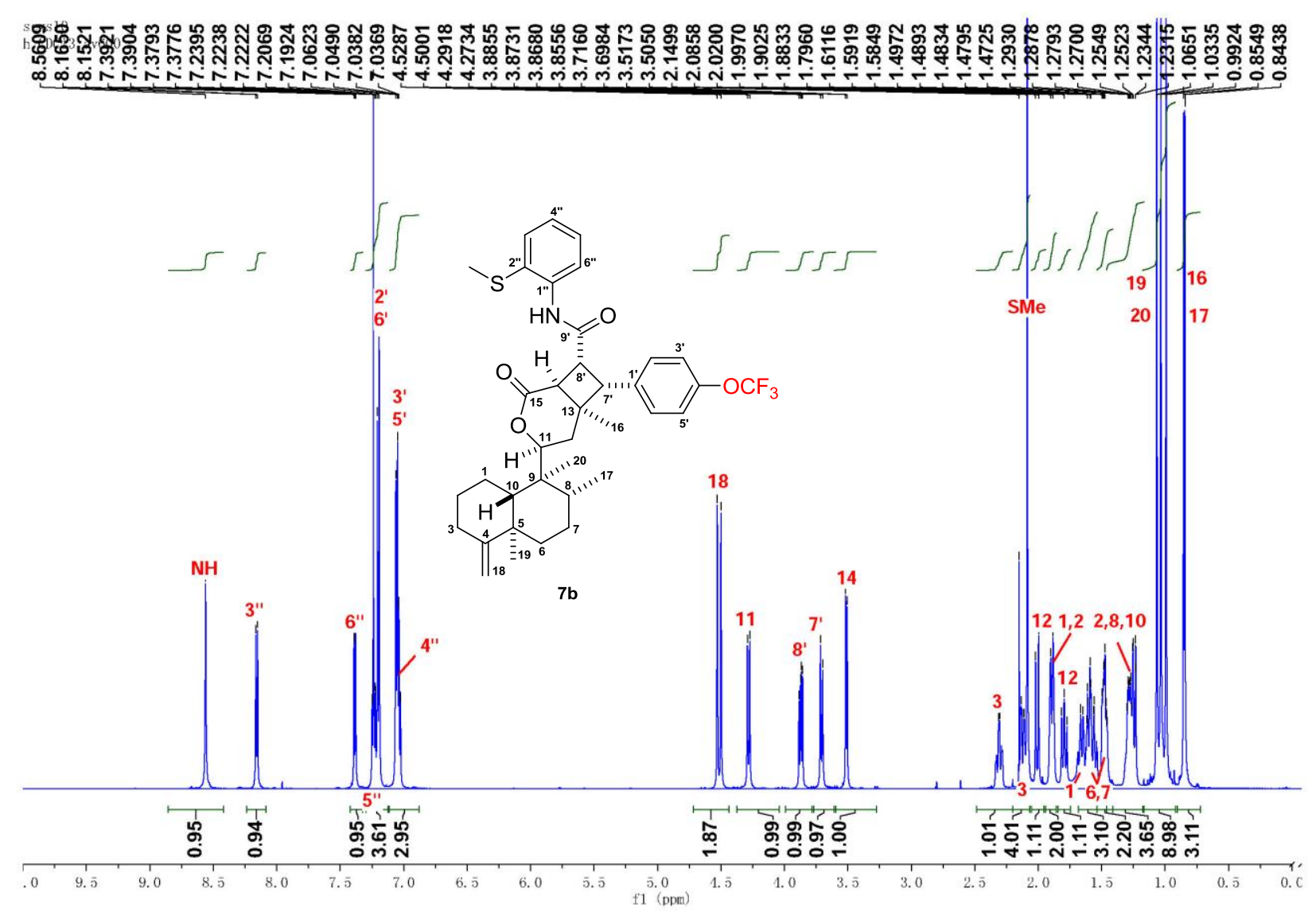

Figure S150. ${ }^{13} \mathrm{C}$ NMR spectrum of $7 \mathbf{b}$
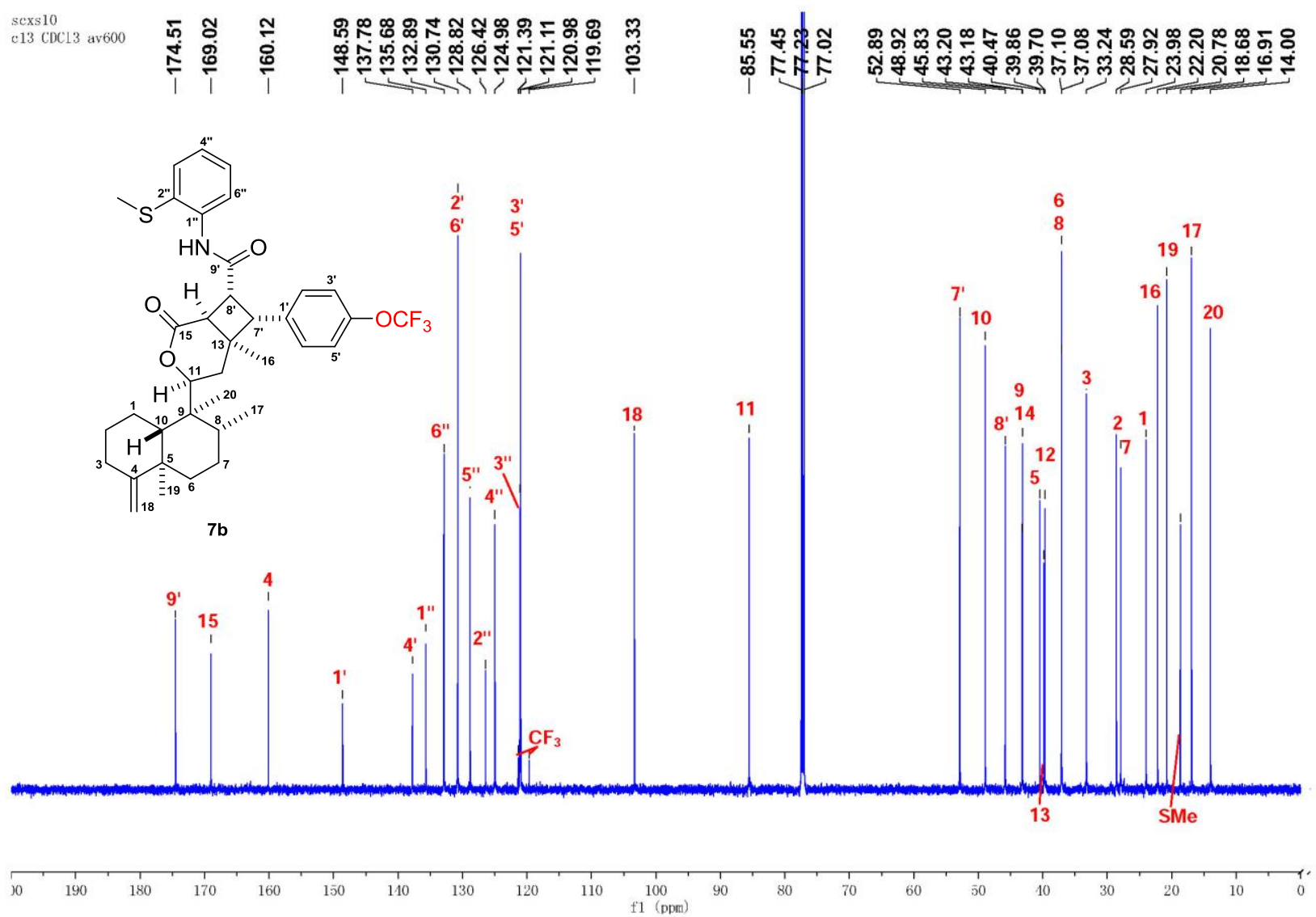
Figure S151. HSQC spectrum of 7b

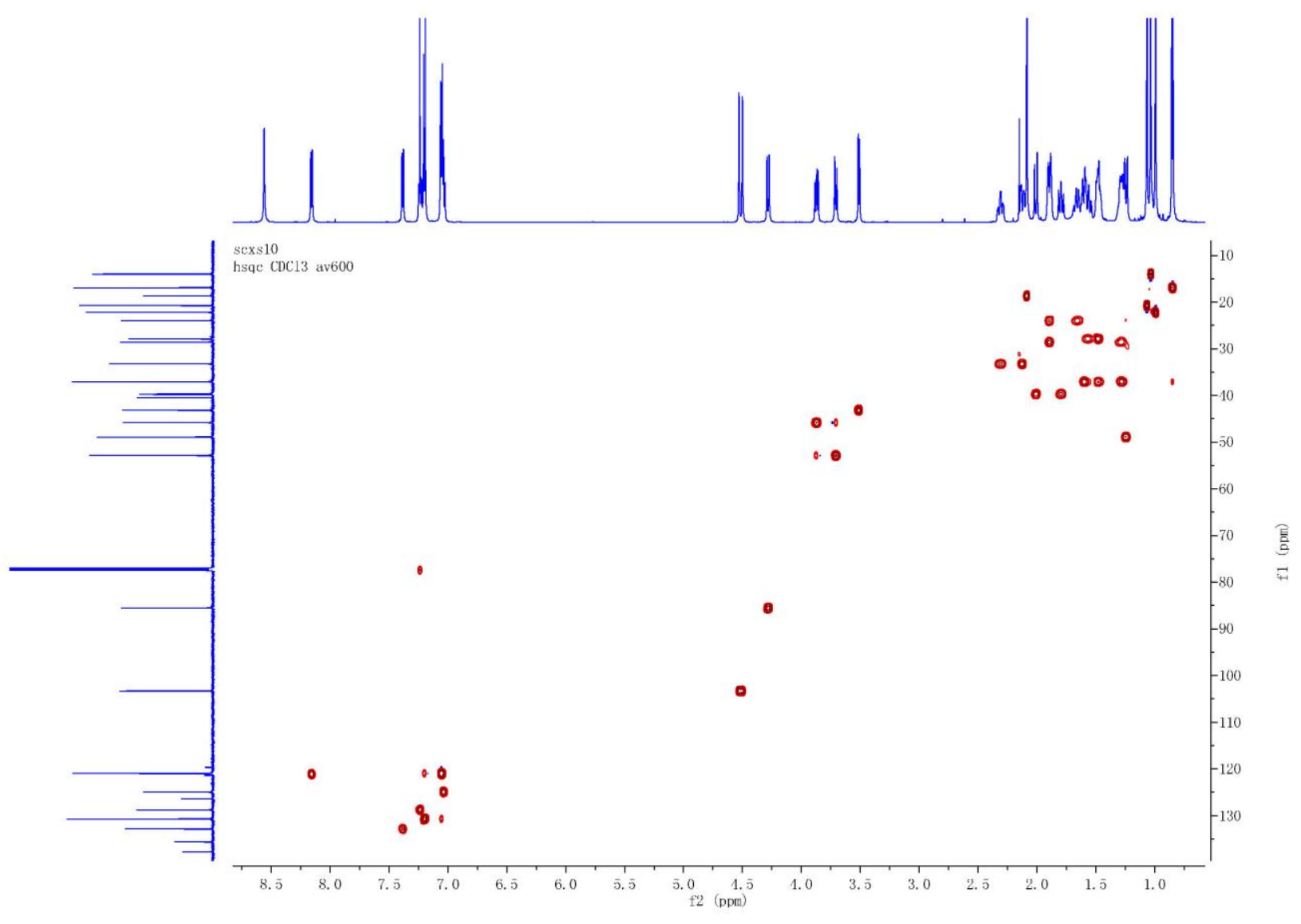

Figure S152. HMBC spectrum of $7 \mathbf{b}$

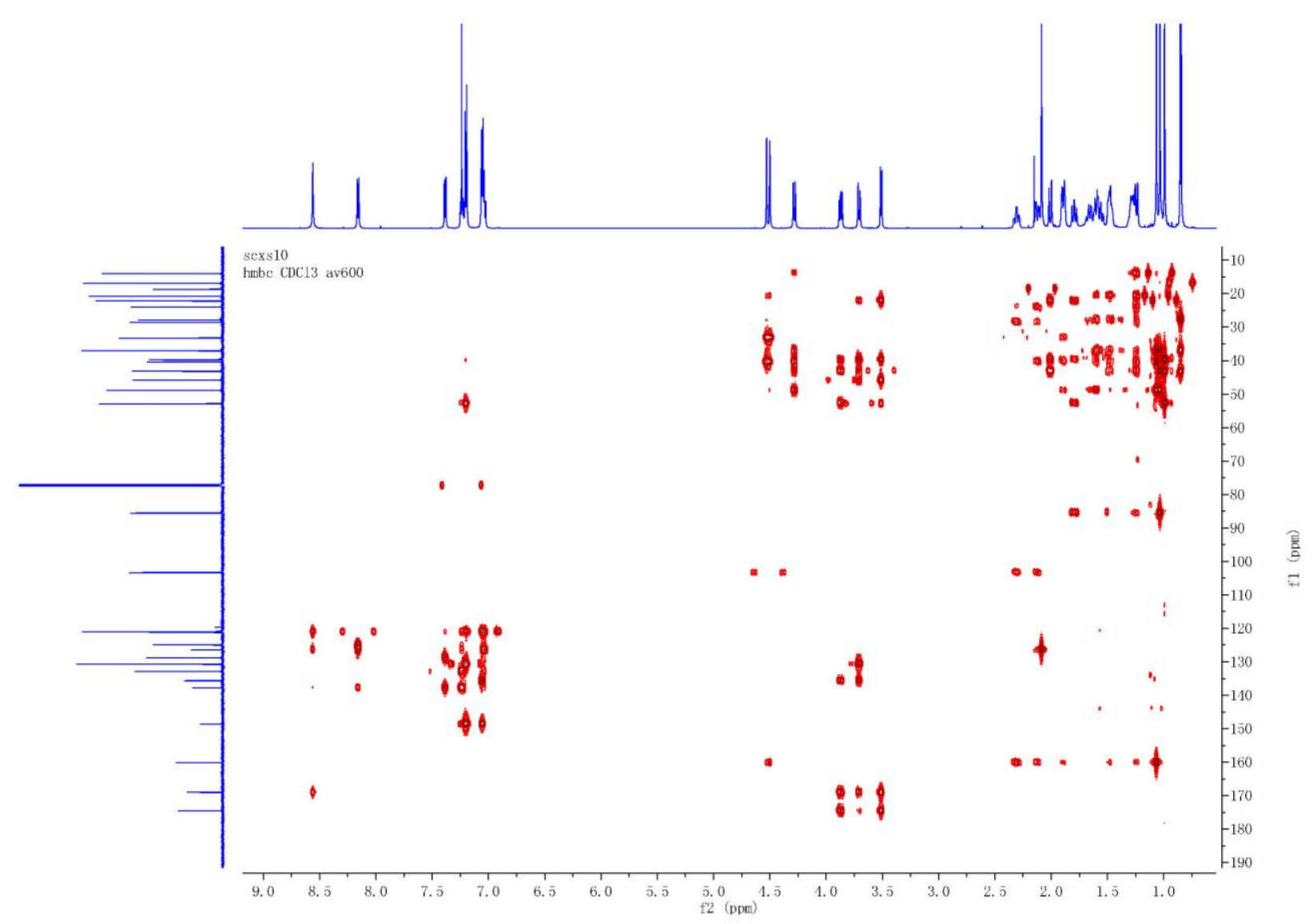


Figure S153. ${ }^{1} \mathrm{H}-{ }^{1} \mathrm{H}$ COSY spectrum of $\mathbf{7 b}$

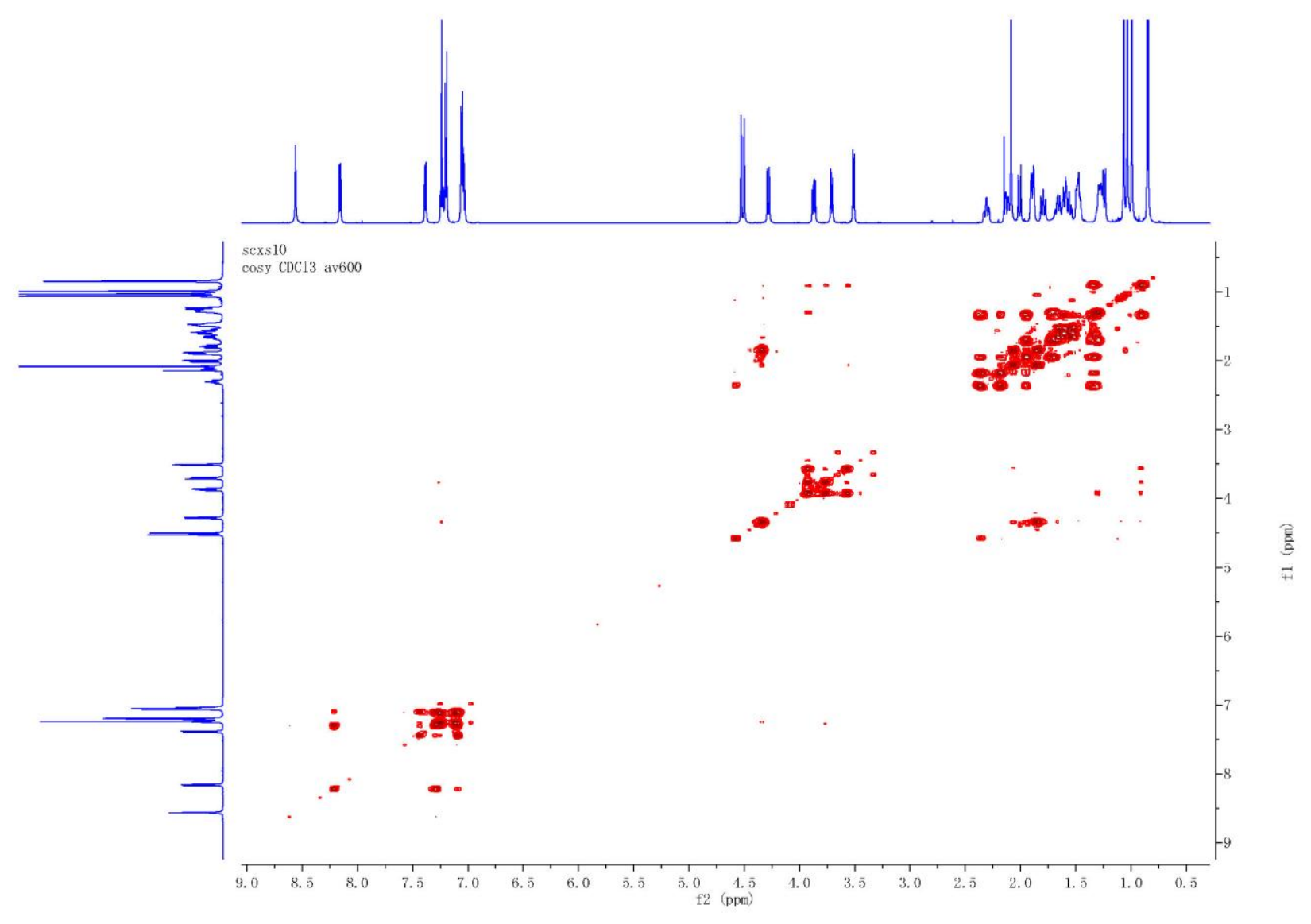

Figure S154. ROESY spectrum of $\mathbf{7 b}$

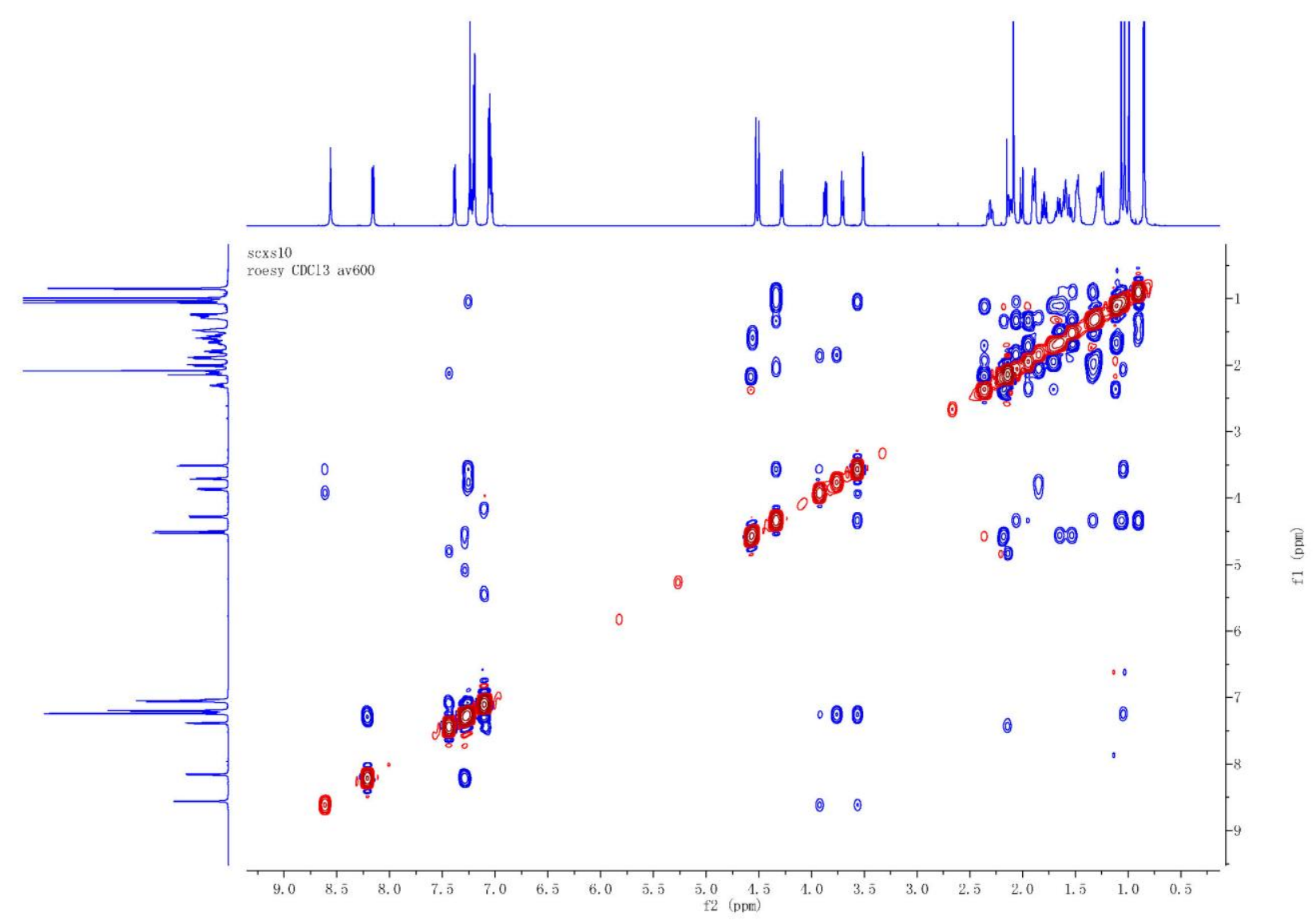


Figure S155. ${ }^{1} \mathrm{H}$ NMR spectrum of $\mathbf{7 k}$

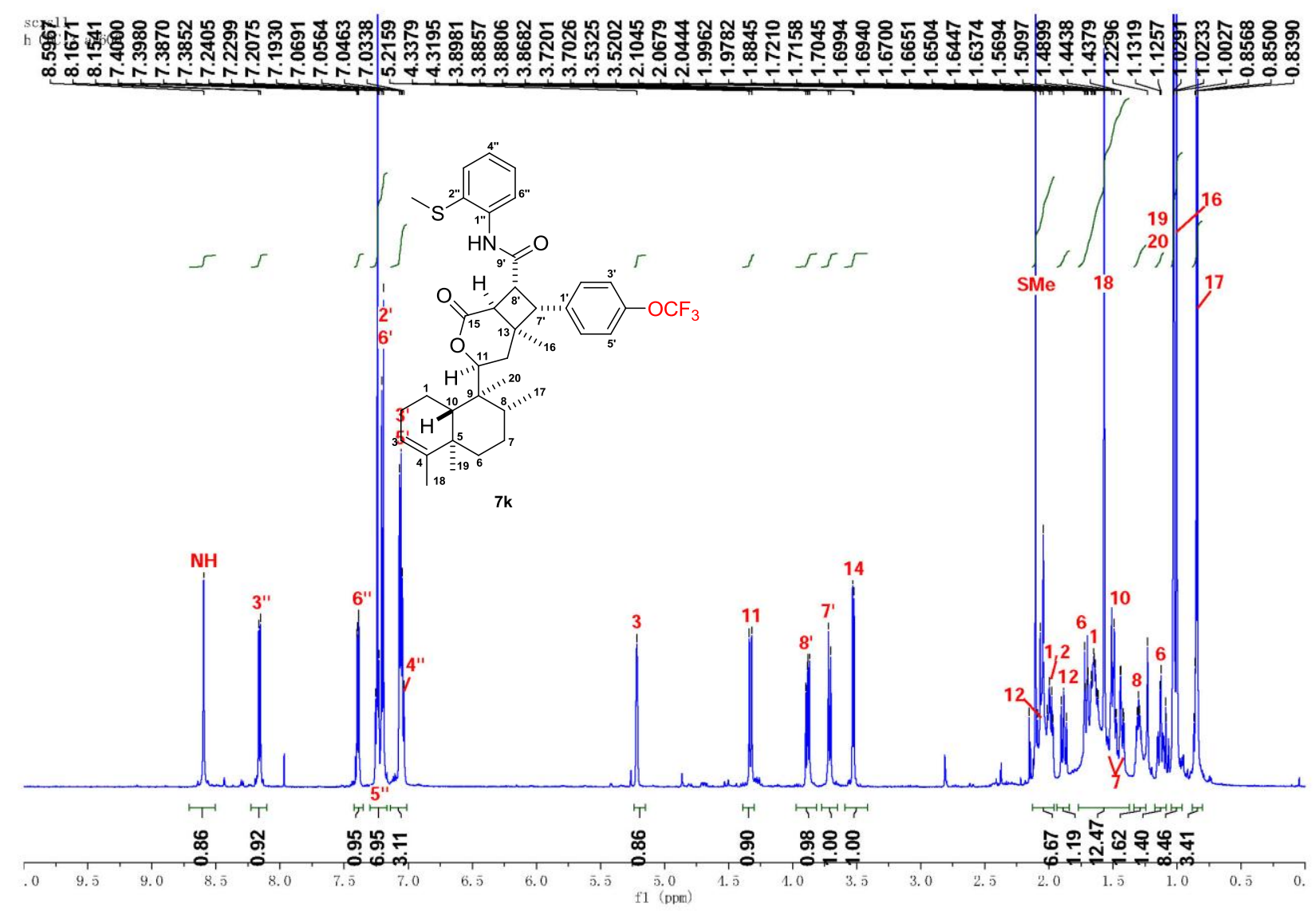

Figure S156. ${ }^{13} \mathrm{C}$ NMR spectrum of $7 \mathbf{k}$

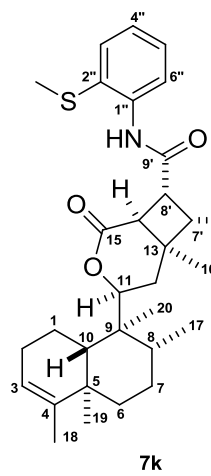

$\mathrm{CF}_{3}$

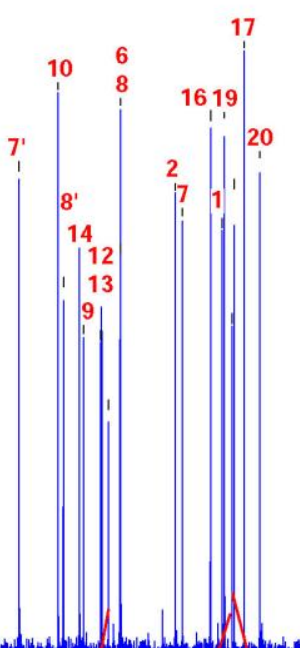


Figure S157. HSQC spectrum of 7k

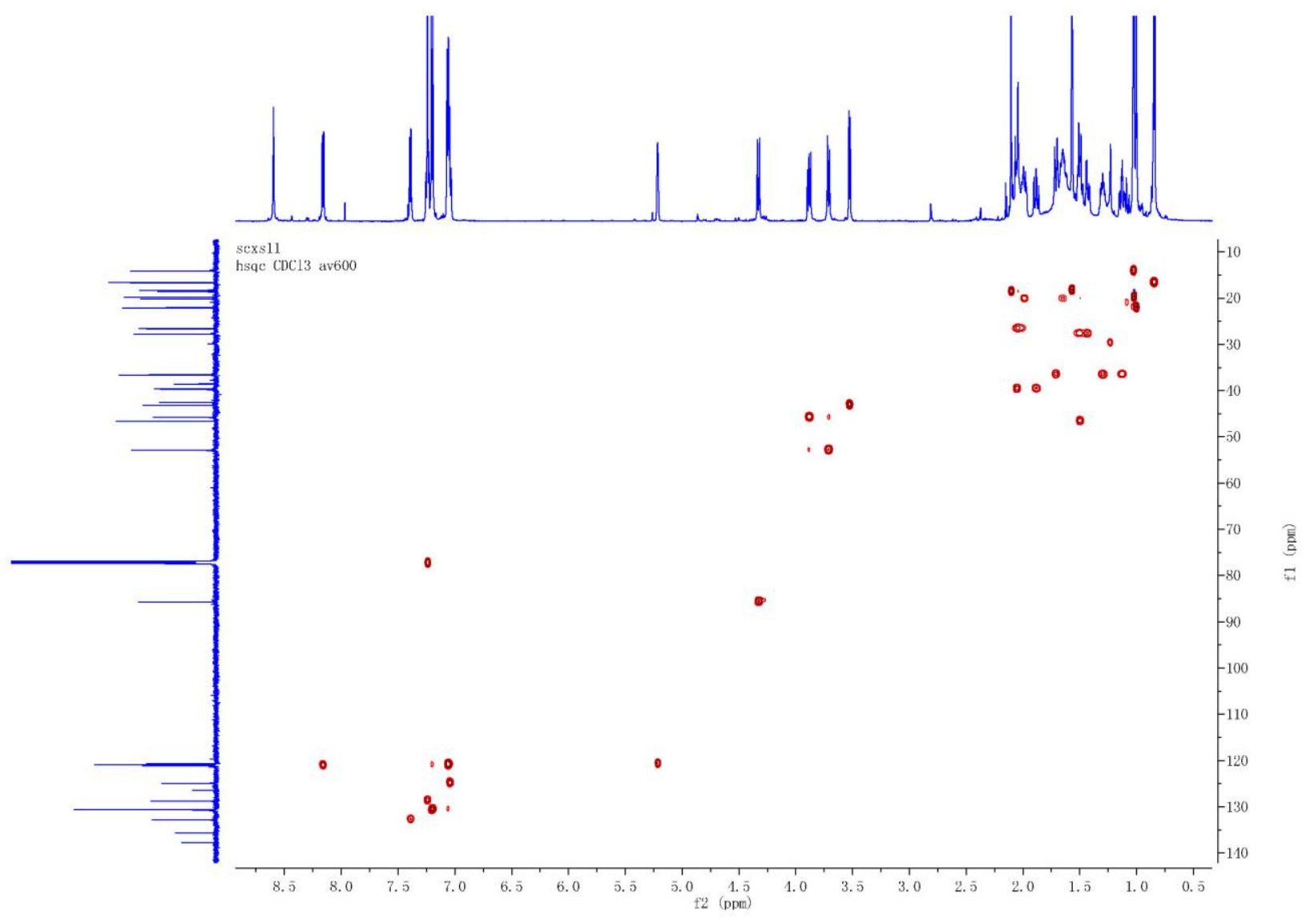

Figure S158. HMBC spectrum of 7k

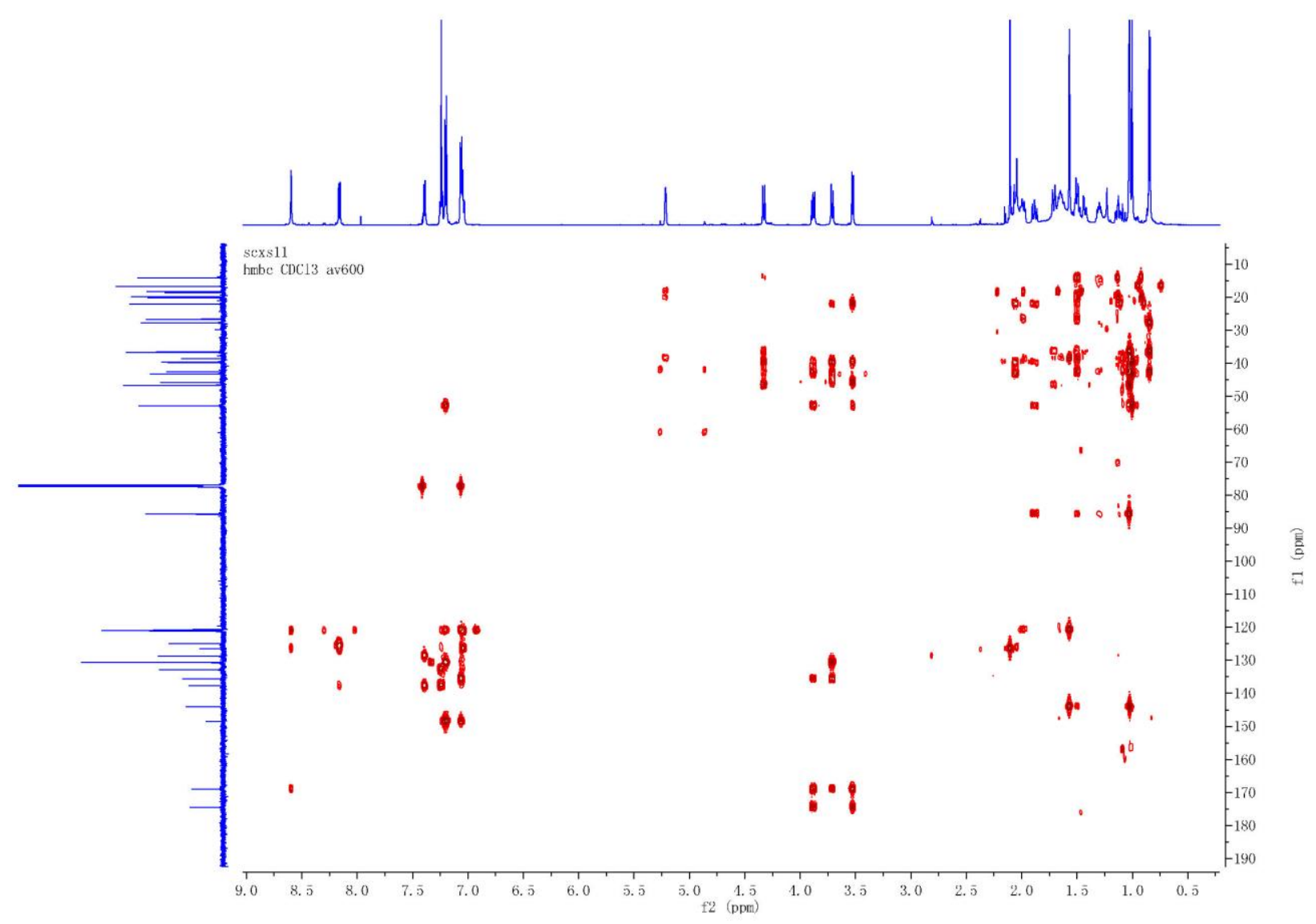


Figure S159. ${ }^{1} \mathrm{H}-{ }^{1} \mathrm{H}$ COSY spectrum of $7 \mathbf{k}$

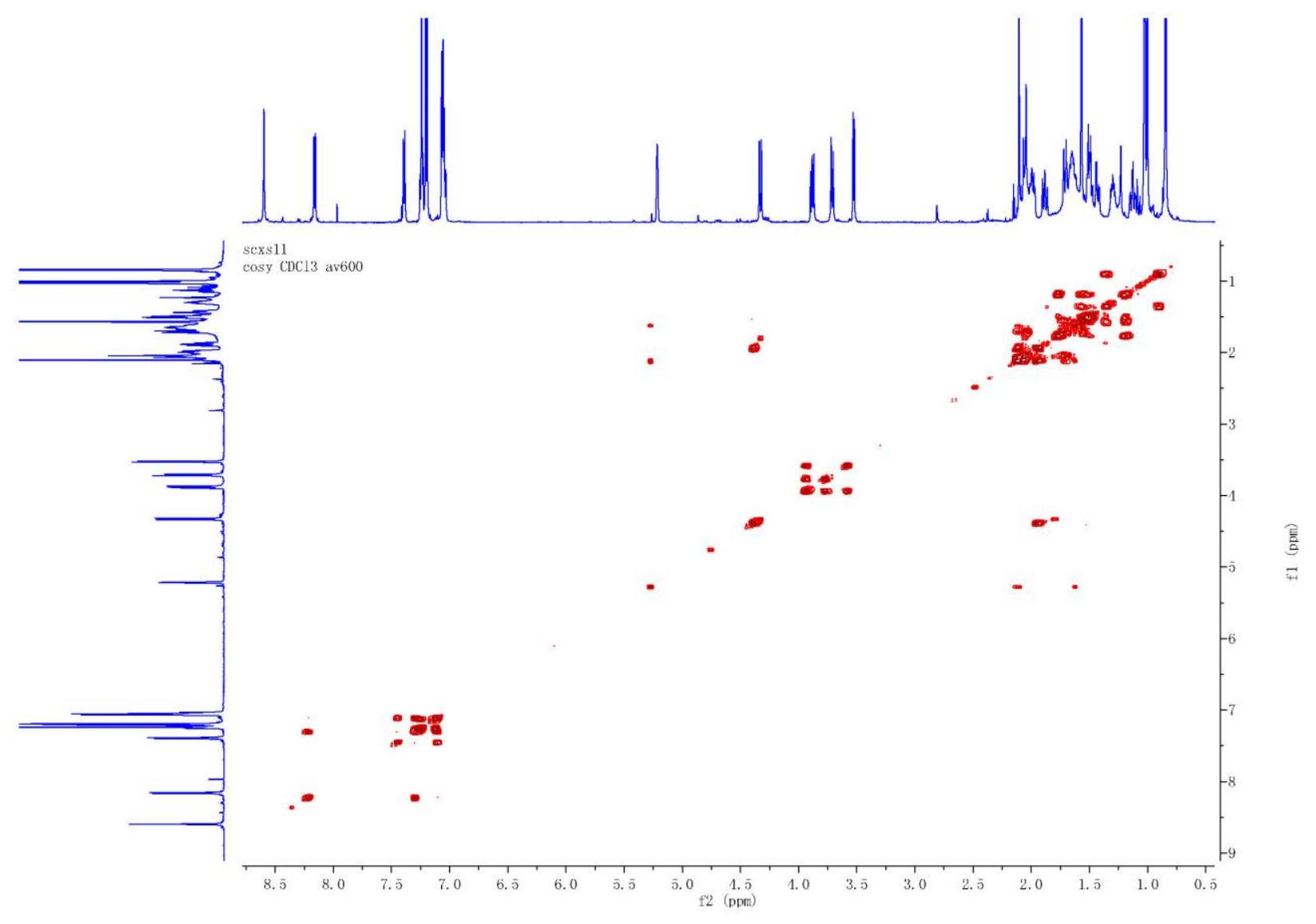

Figure S160. ROESY spectrum of 7k

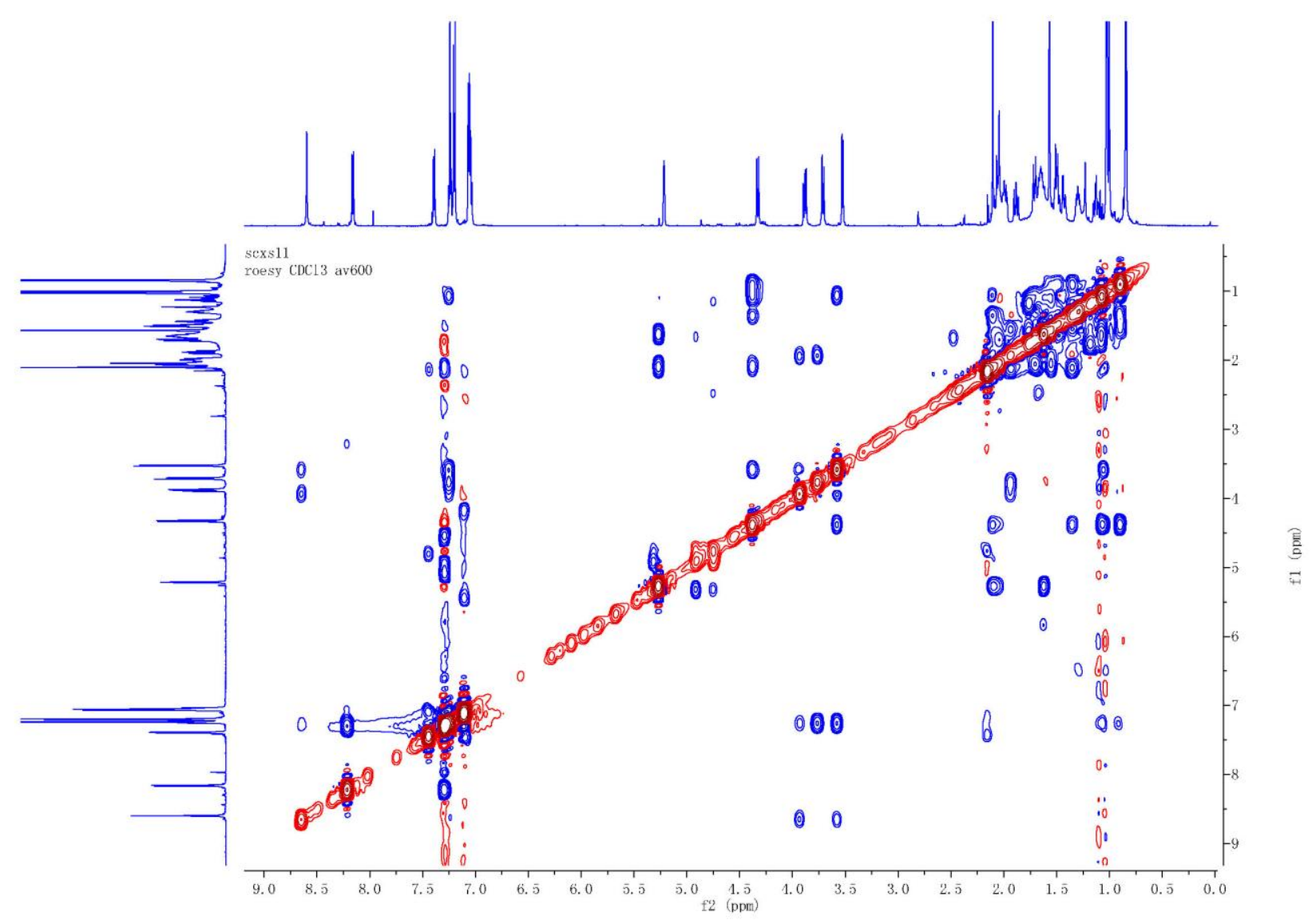


Figure S161. ${ }^{1} \mathrm{H}$ NMR spectrum of 10a

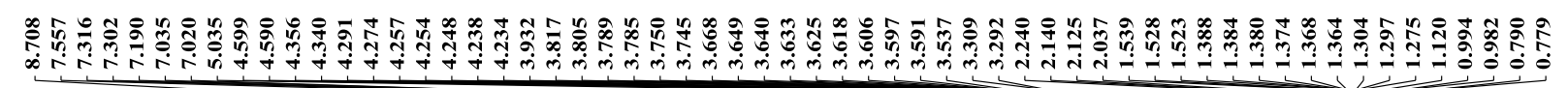

slxr046

PROTON-sxhuo Pyr D: $\backslash \backslash$ root 10
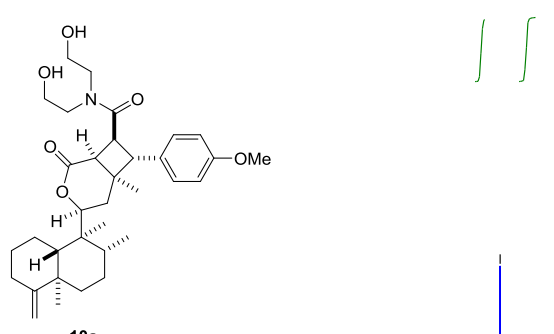

10a

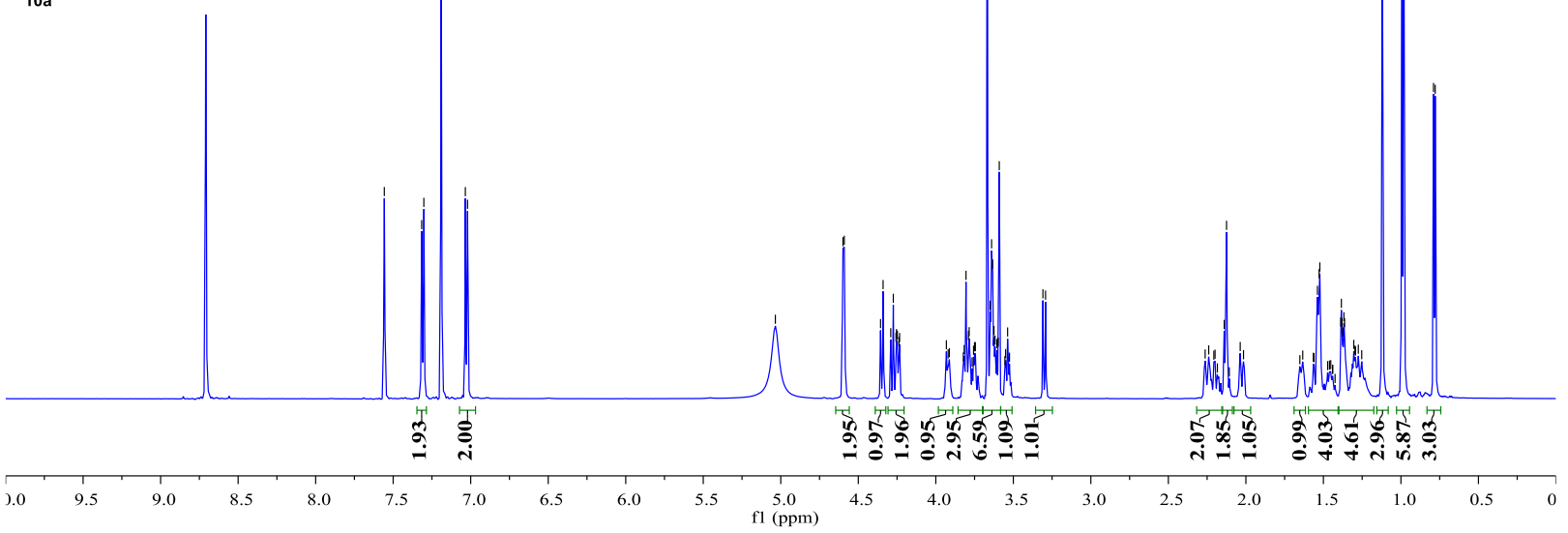

Figure S162. ${ }^{13} \mathrm{C}$ NMR spectrum of 10a

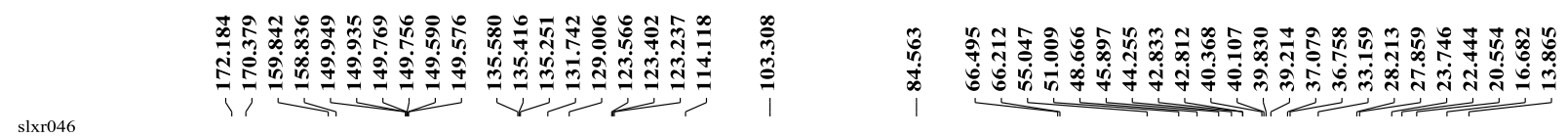

slxr046

ZGDC-sxhuo Pyr D: $\backslash \backslash$ root 10
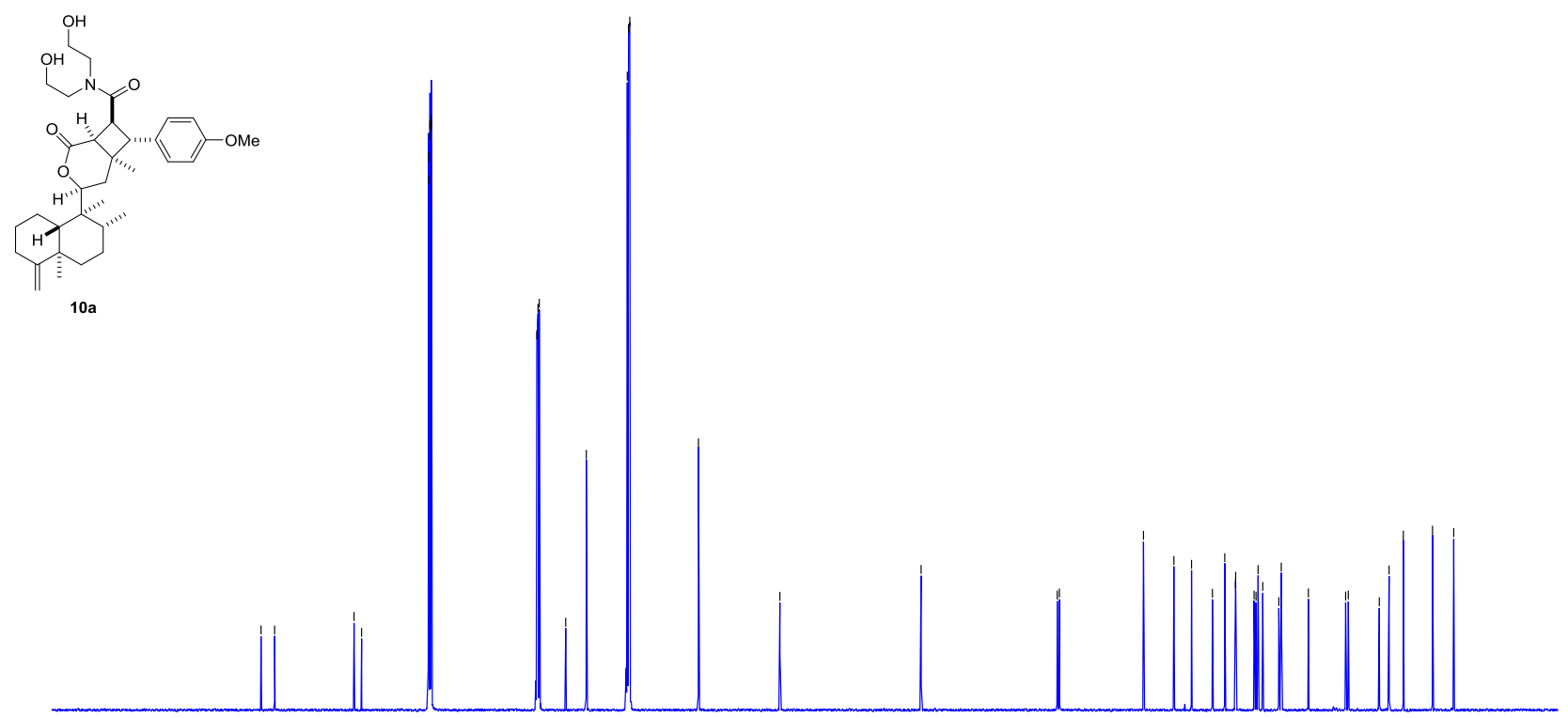

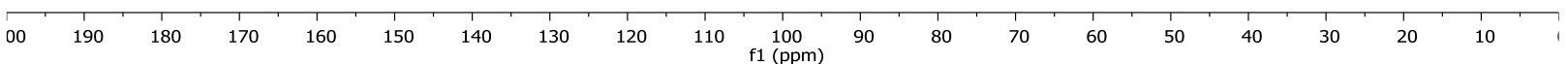


Figure S163. ${ }^{1} \mathrm{H}$ NMR spectrum of $\mathbf{1 0 b}$

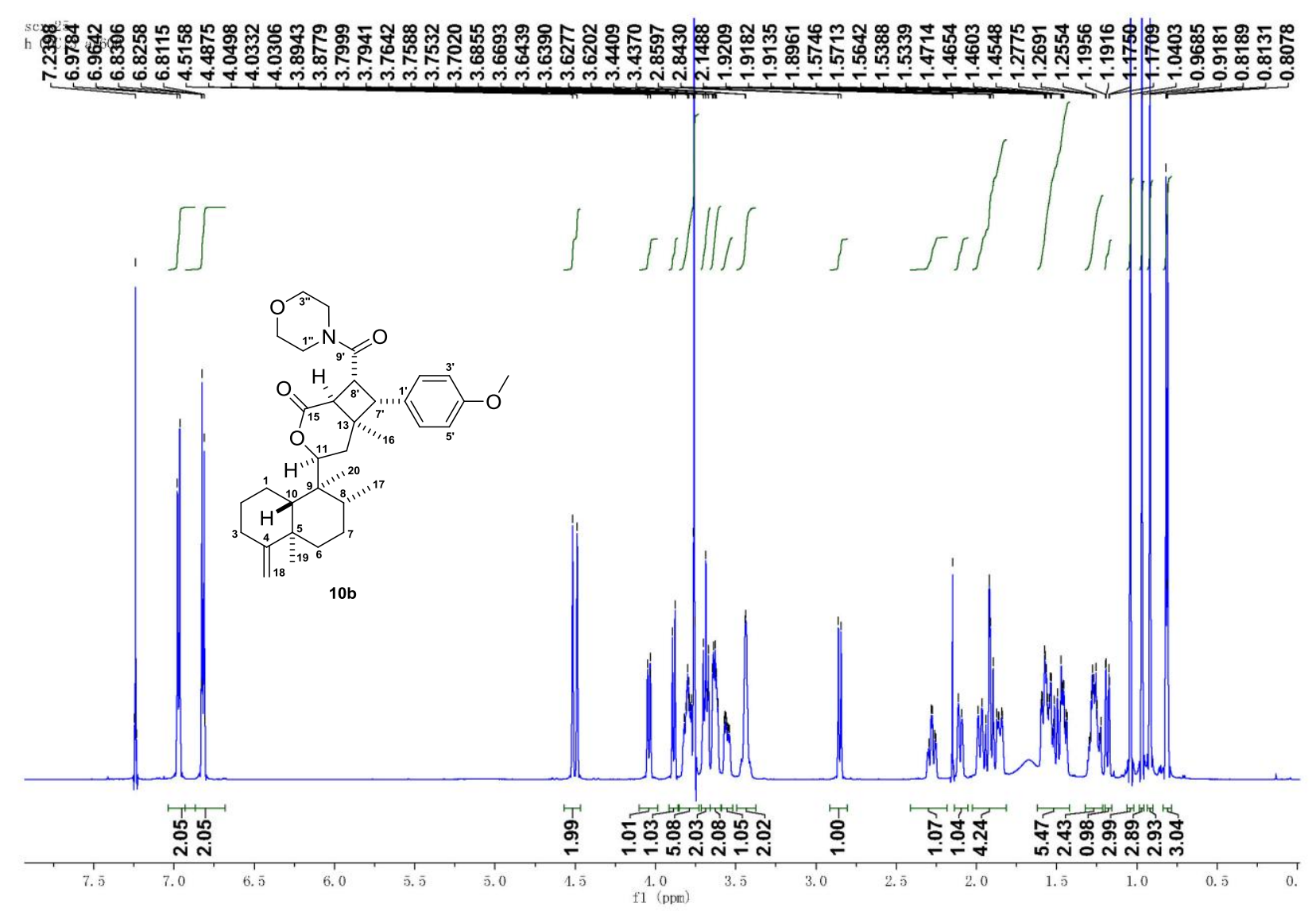

Figure S164. ${ }^{13} \mathrm{C}$ NMR spectrum of $\mathbf{1 0 b}$
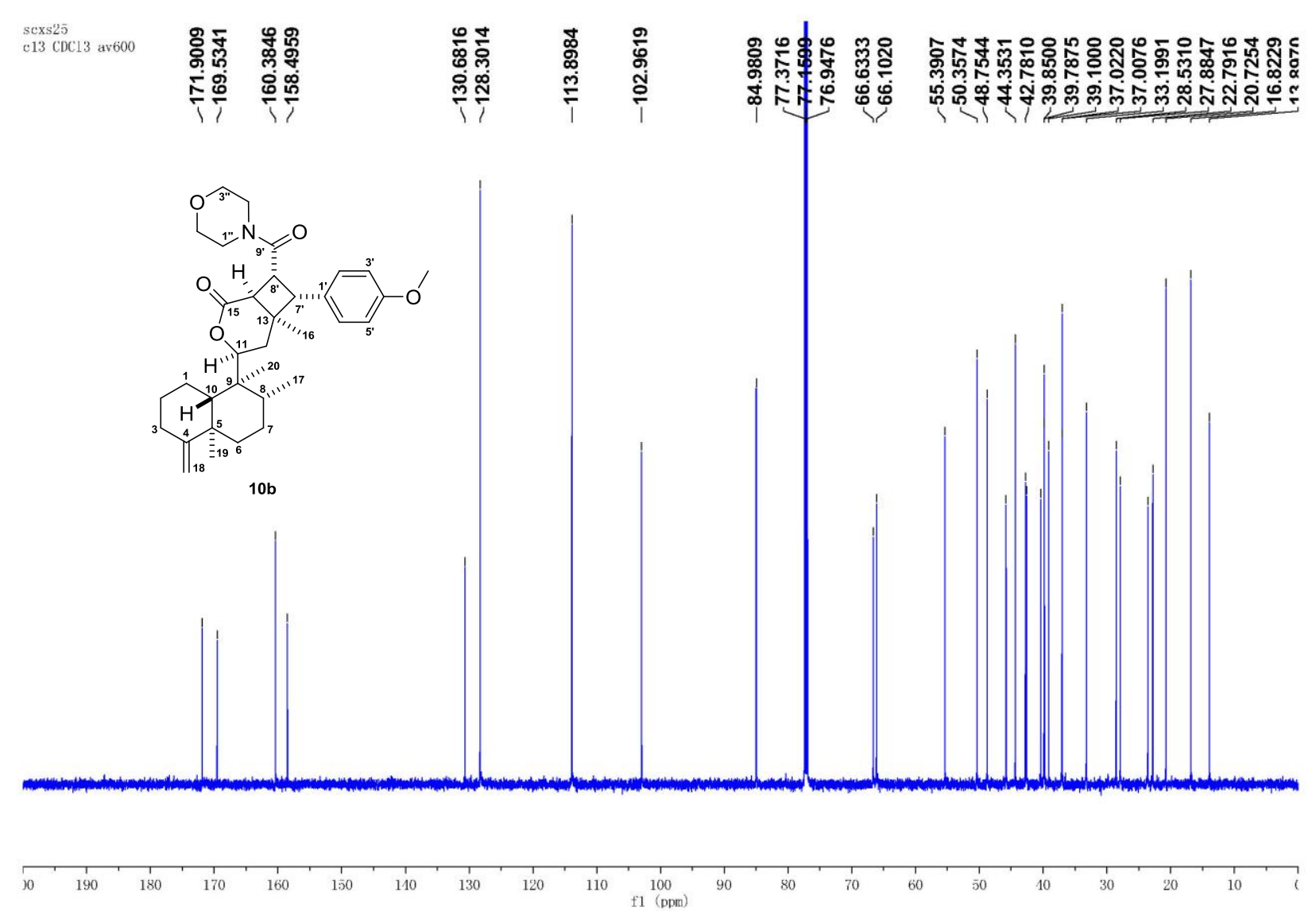
Figure S165. ${ }^{1} \mathrm{H}$ NMR spectrum of 10c

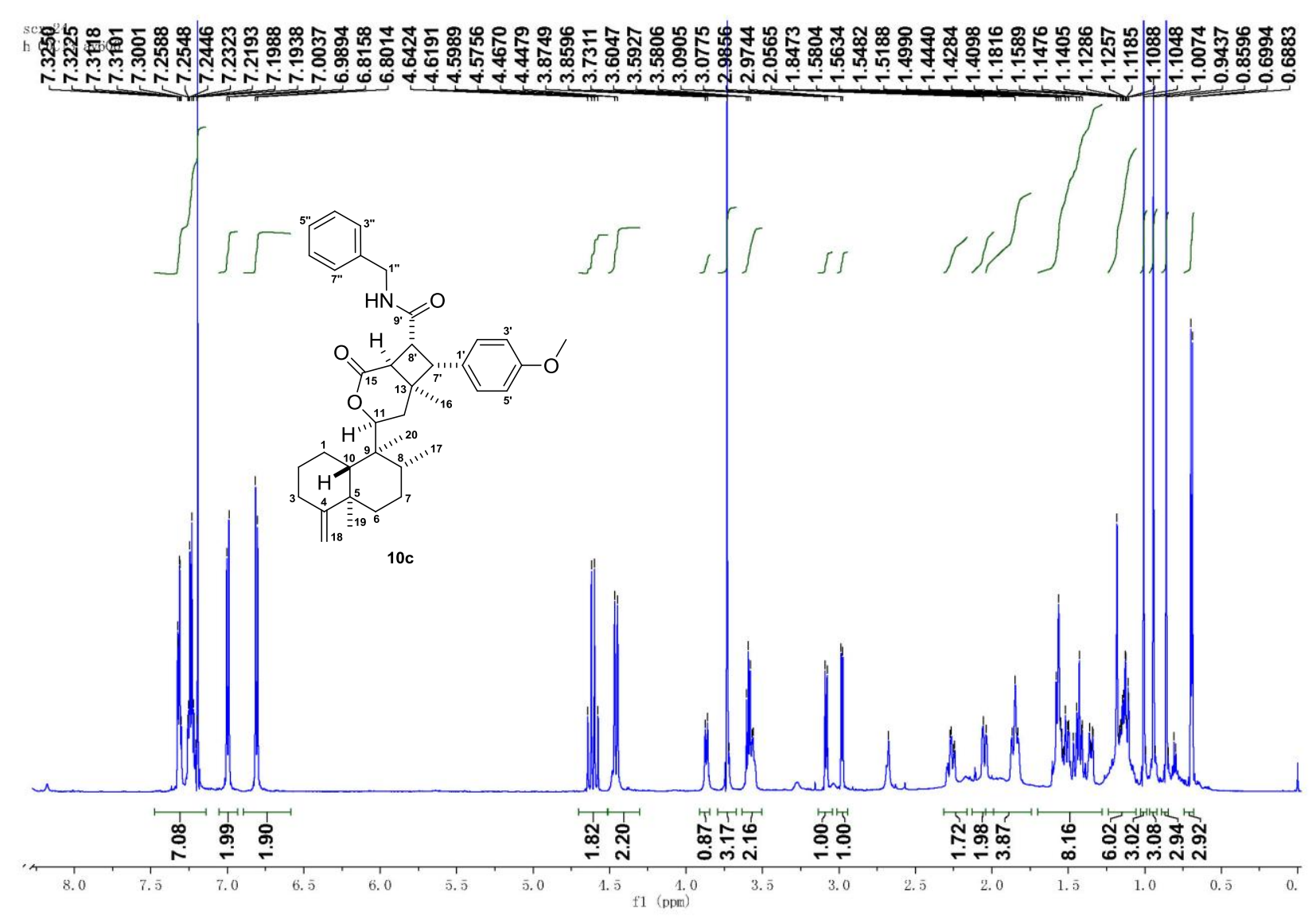

Figure S166. ${ }^{13} \mathrm{C}$ NMR spectrum of 10c

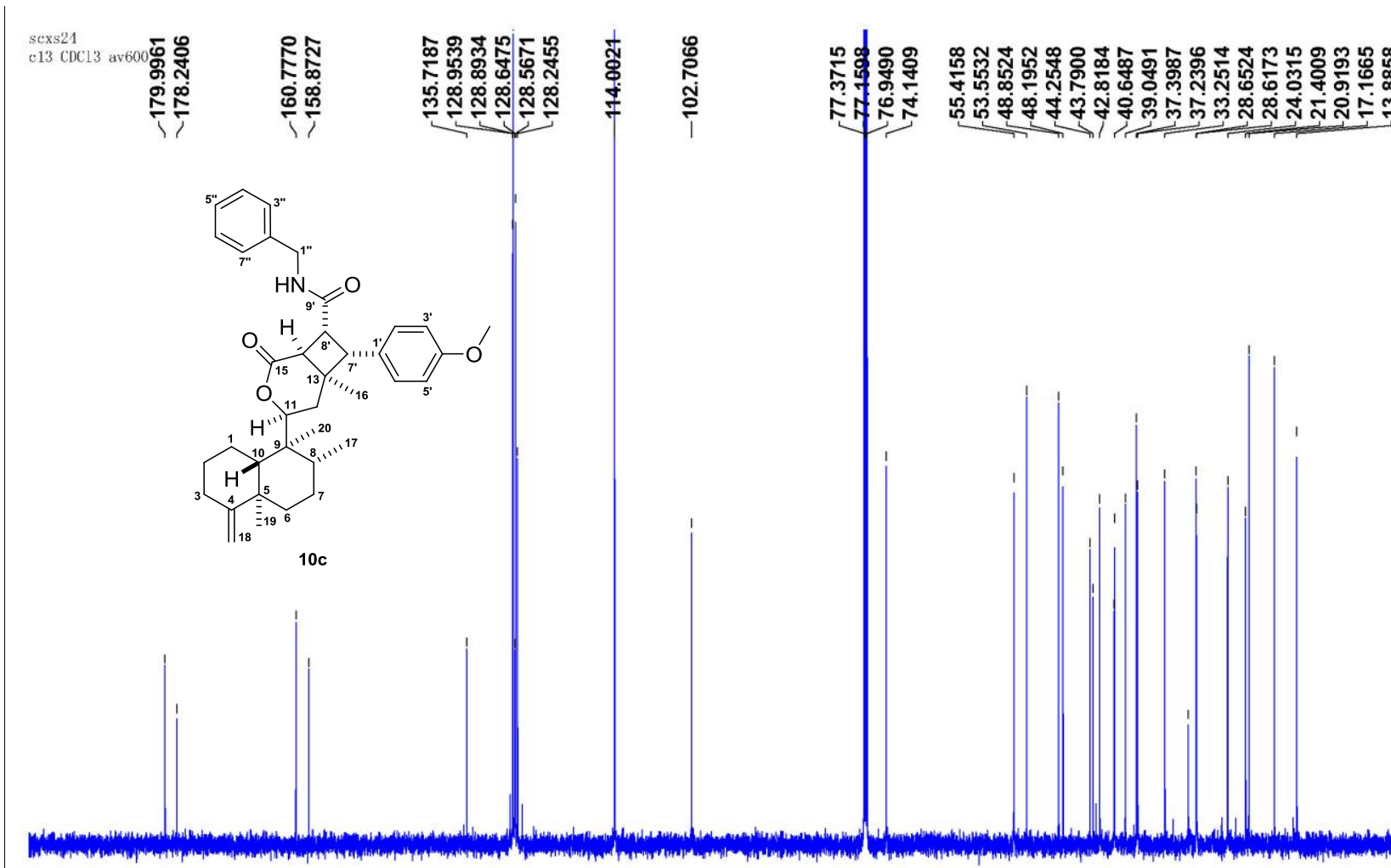

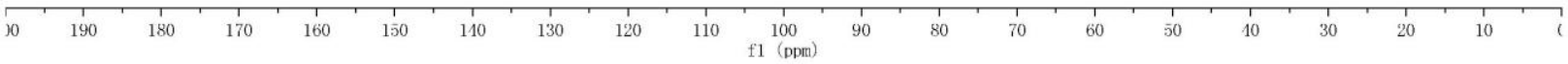


Figure S167. ${ }^{1} \mathrm{H}$ NMR spectrum of 10d

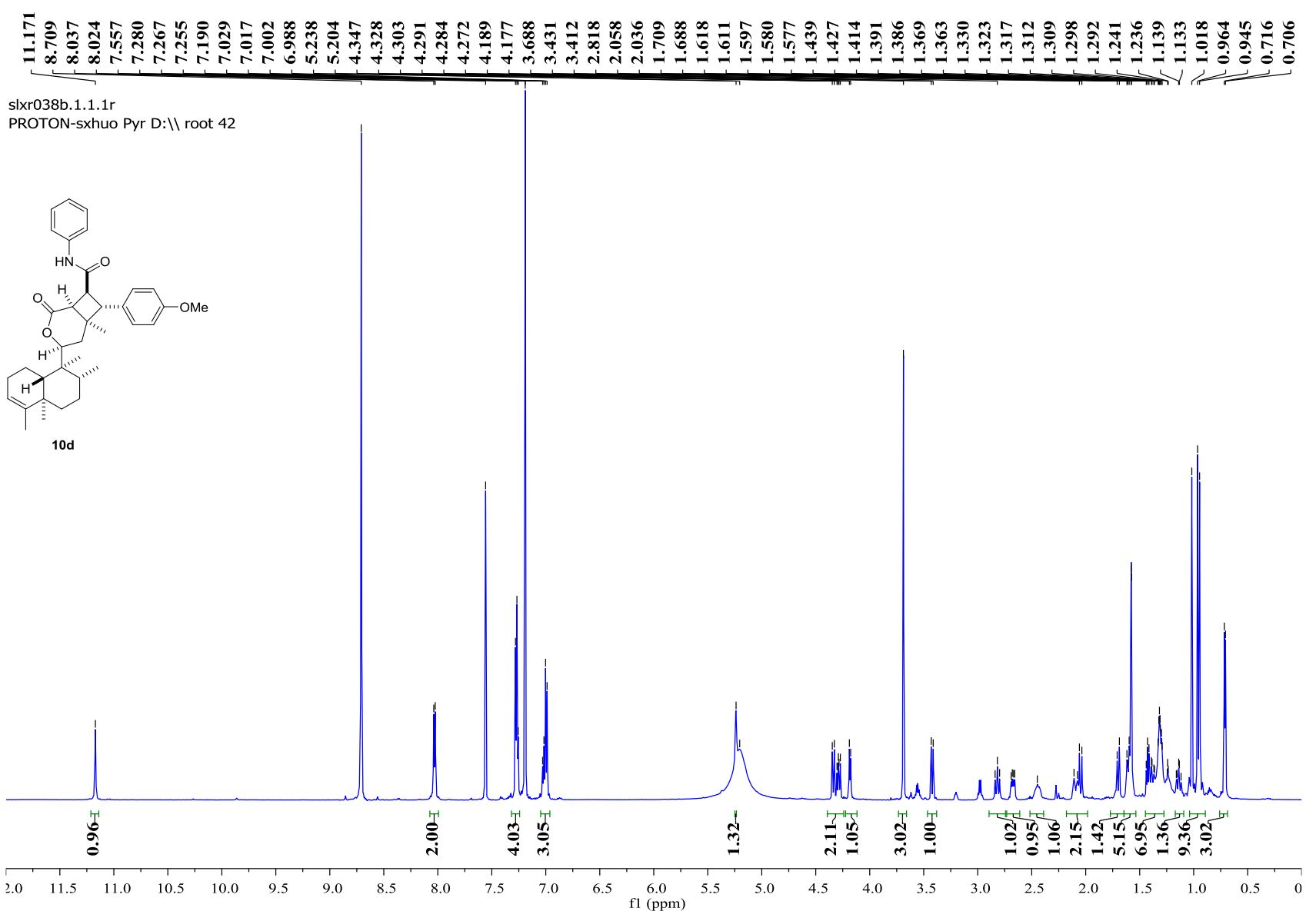

Figure S168. ${ }^{13} \mathrm{C}$ NMR spectrum of 10d

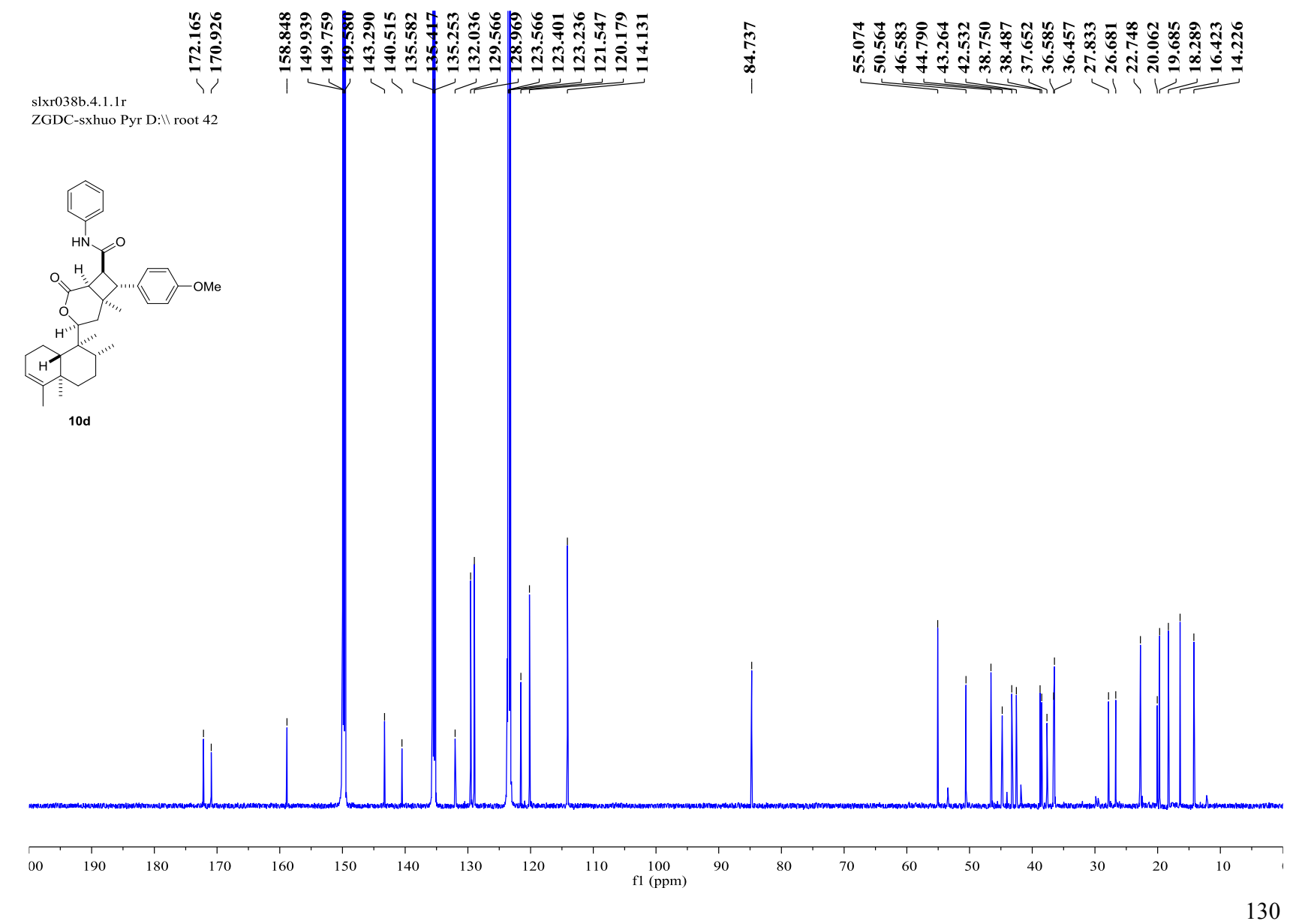


Figure S169. ${ }^{1} \mathrm{H}$ NMR spectrum of $\mathbf{1 0 e}$

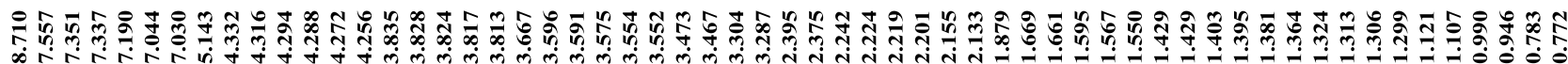

slxr037b.1.1.1r

PROTON-sxhuo Pyr D: $\backslash \backslash$ root 28
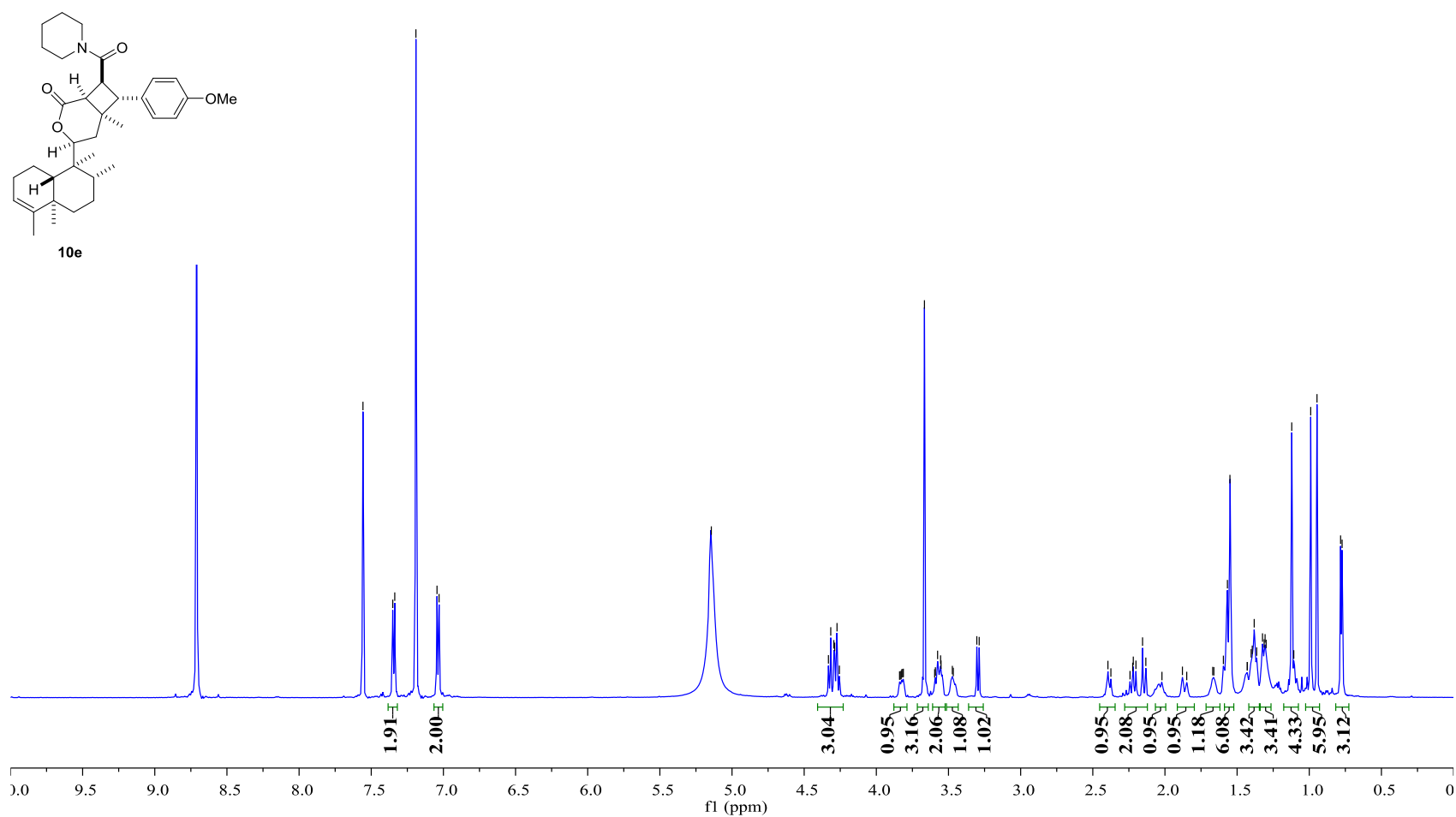

Figure S170. ${ }^{13} \mathrm{C}$ NMR spectrum of $\mathbf{1 0 e}$

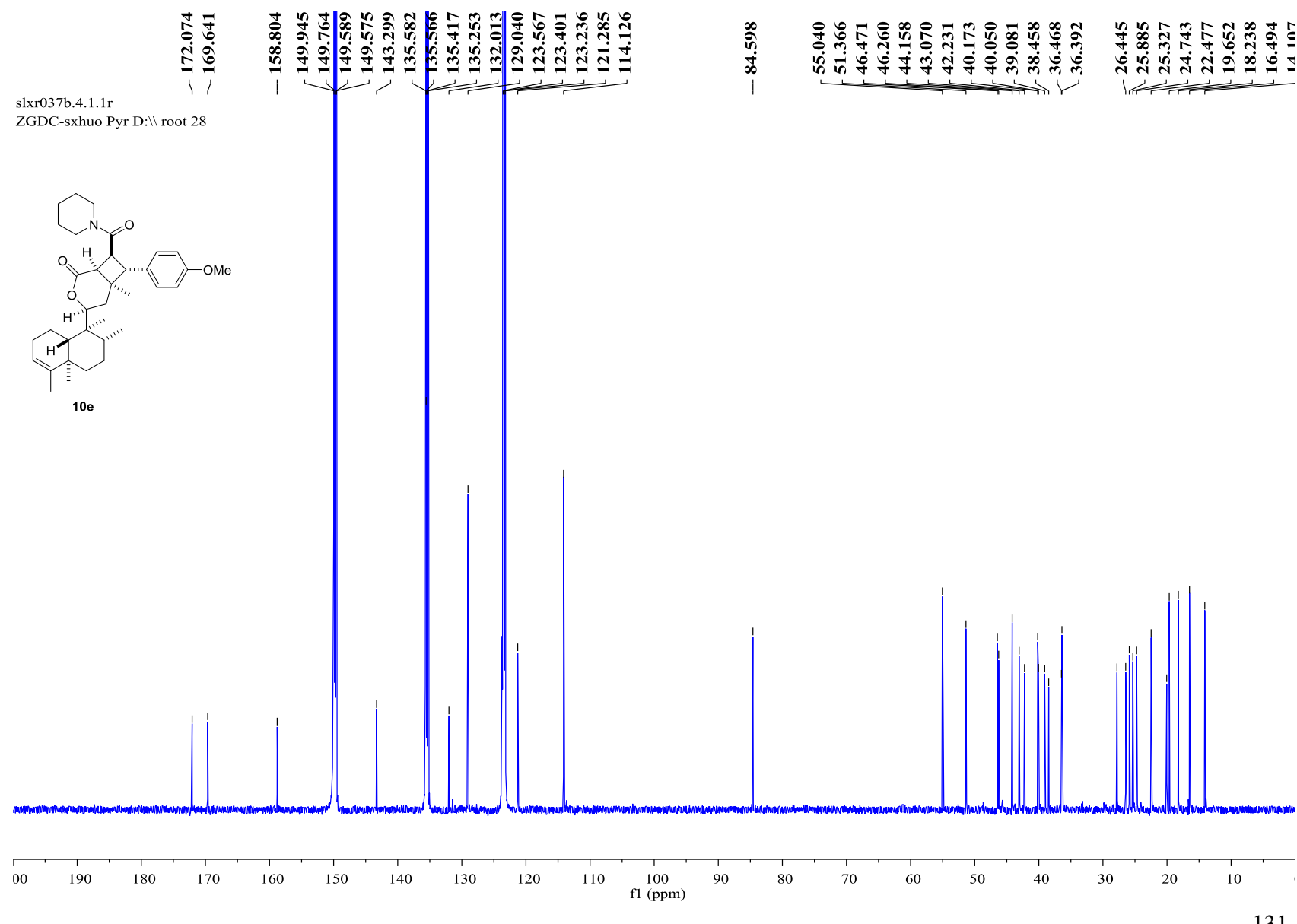


Figure S171. ${ }^{1} \mathrm{H}$ NMR spectrum of $\mathbf{1 0 f}$

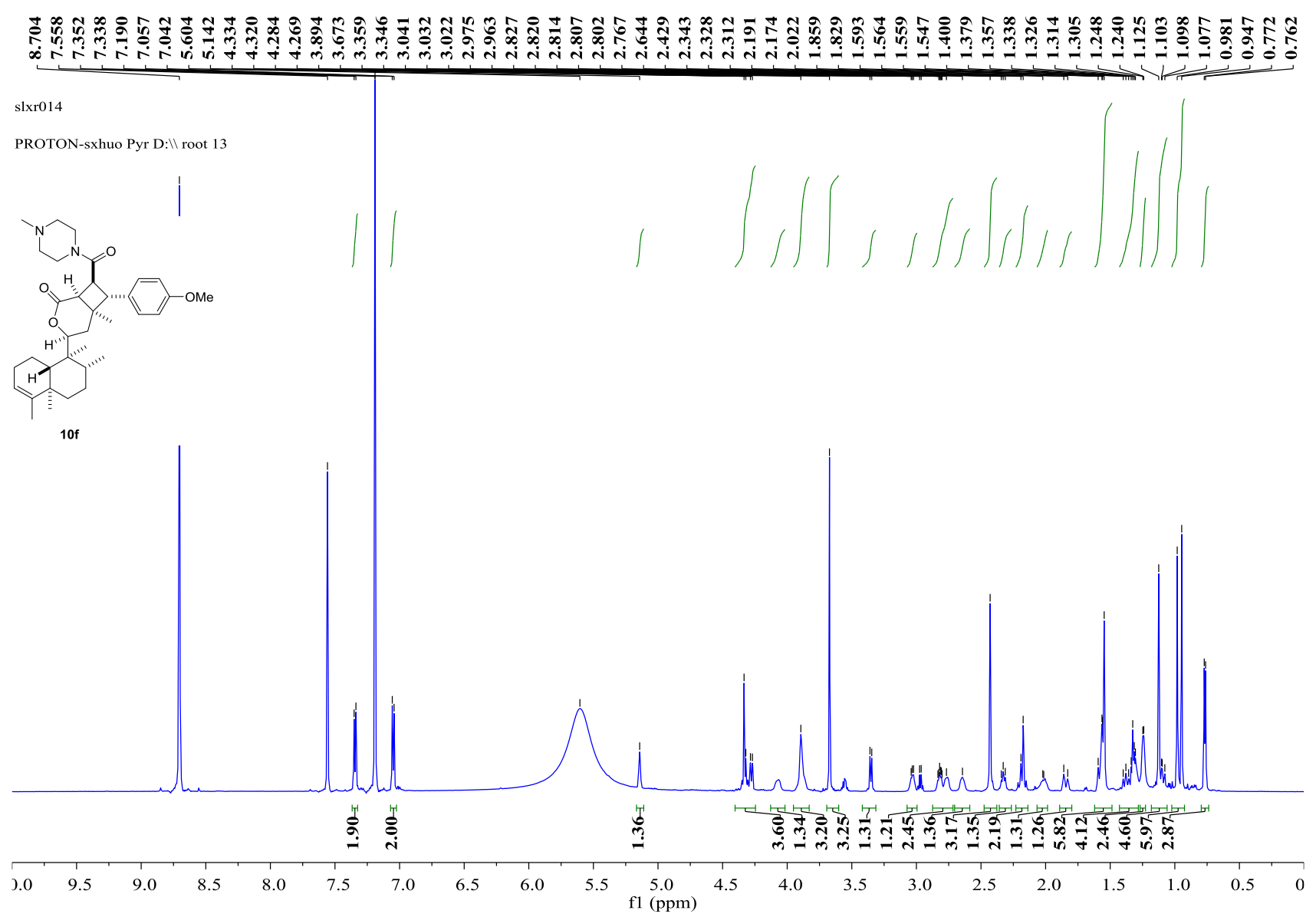

Figure S172. ${ }^{13} \mathrm{C}$ NMR spectrum of $\mathbf{1 0 f}$

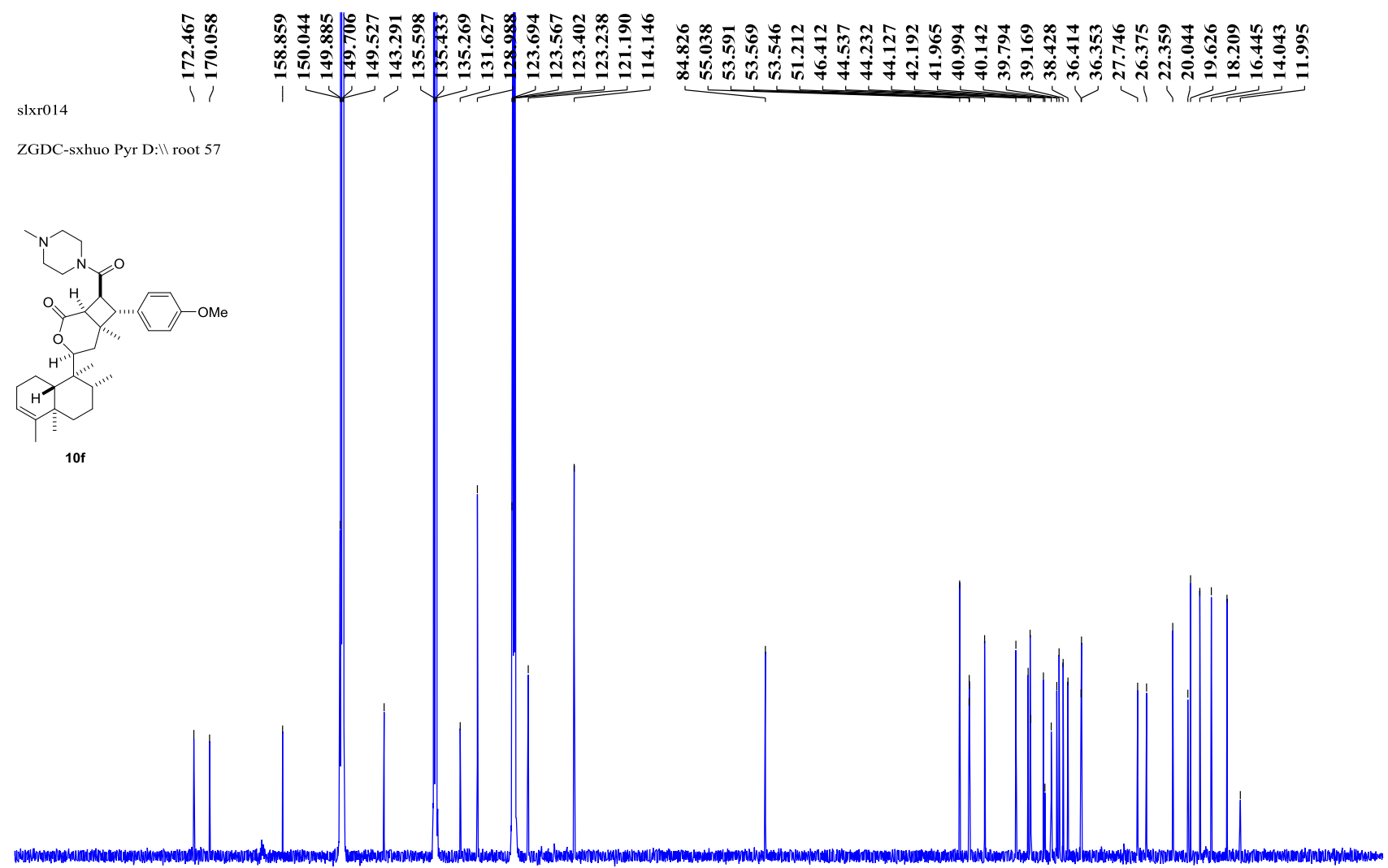

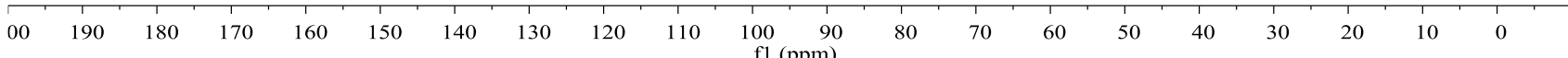


Figure S173. ${ }^{1} \mathrm{H}$ NMR spectrum of $\mathbf{1 0 g}$

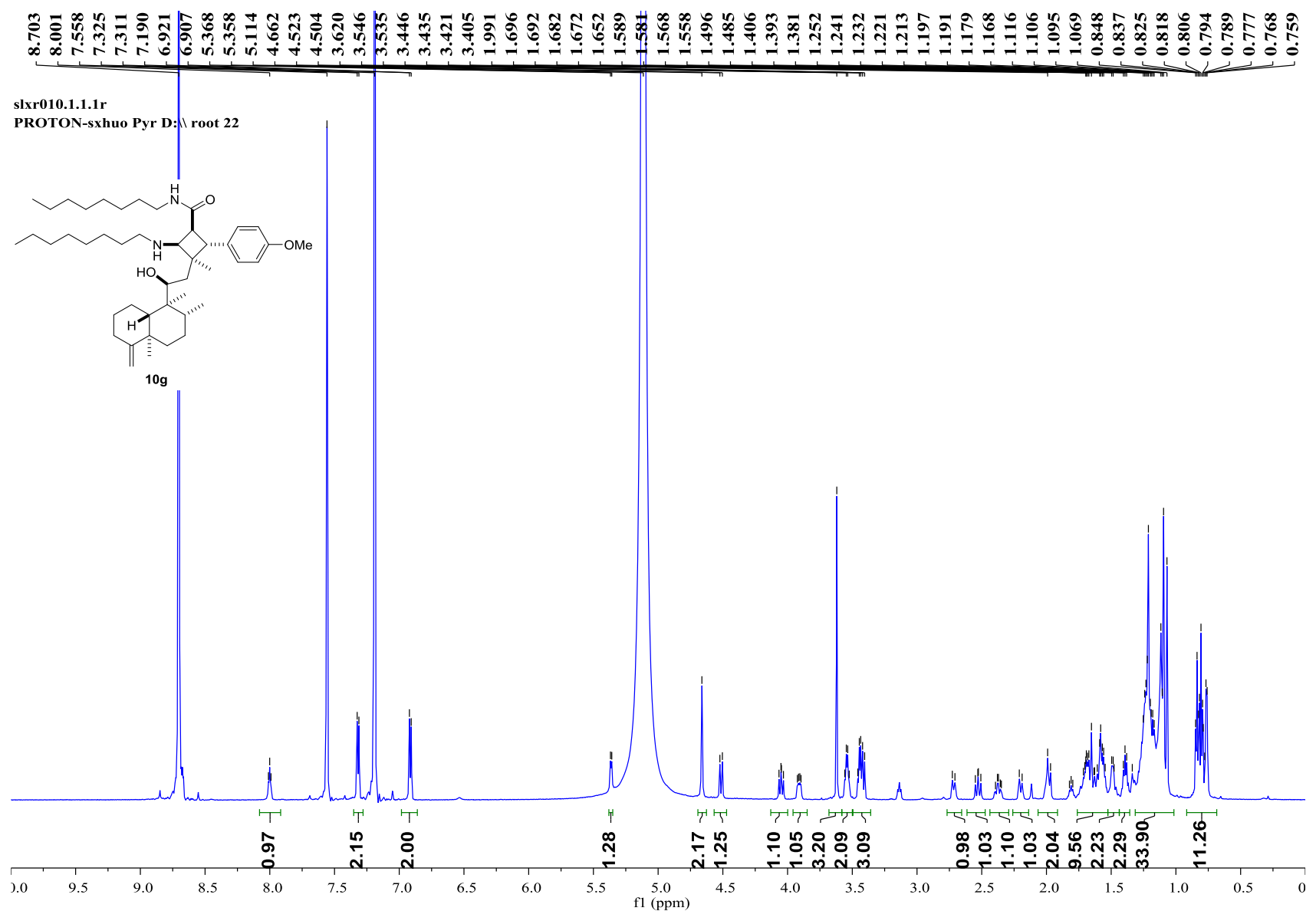

Figure S174. ${ }^{13} \mathrm{C}$ NMR spectrum of $10 \mathrm{~g}$

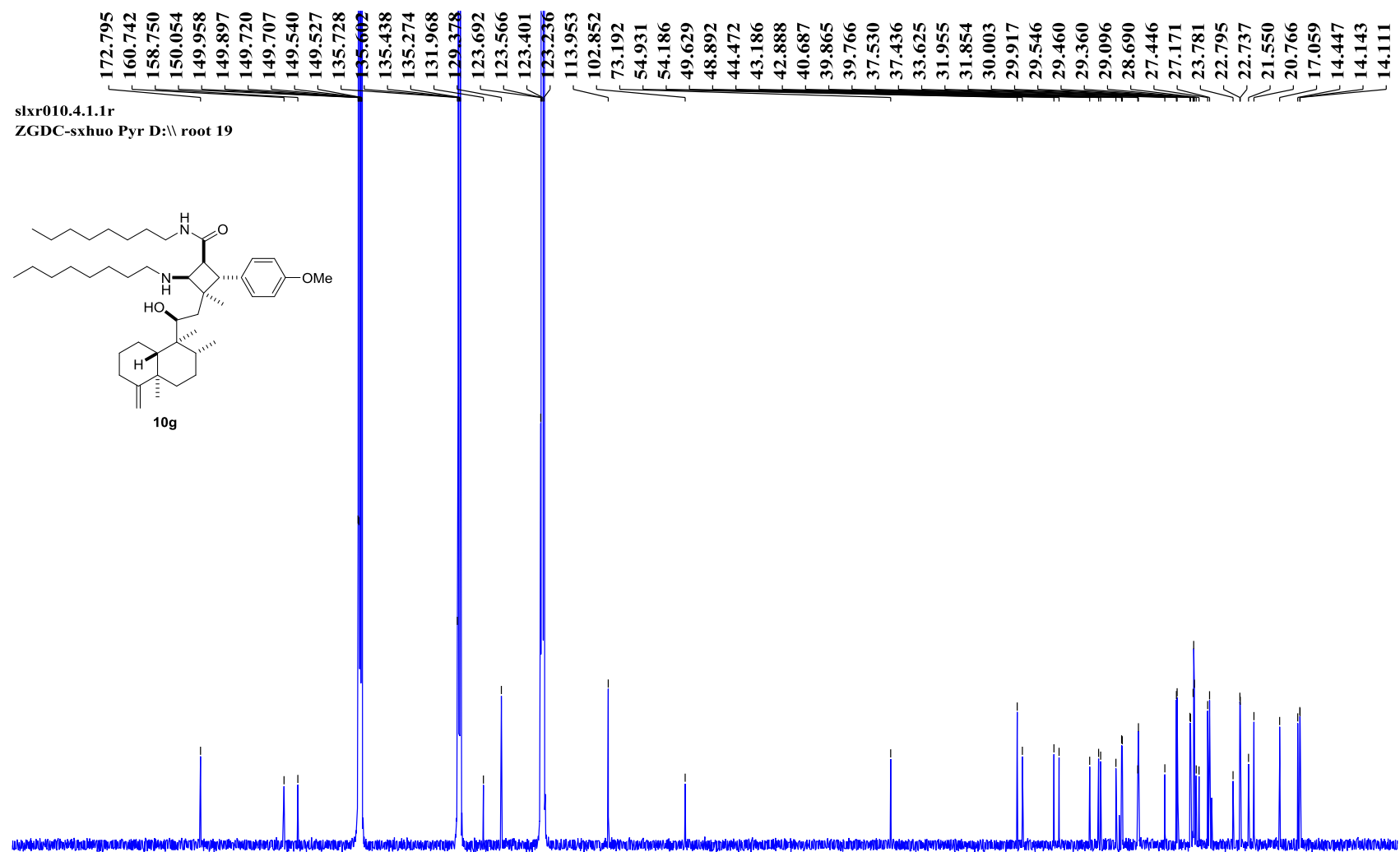

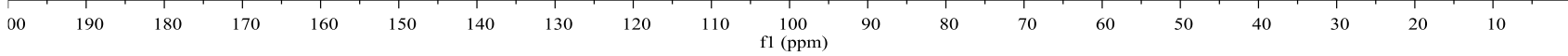


Figure S175. ${ }^{1} \mathrm{H}$ NMR spectrum of $\mathbf{1 0 h}$

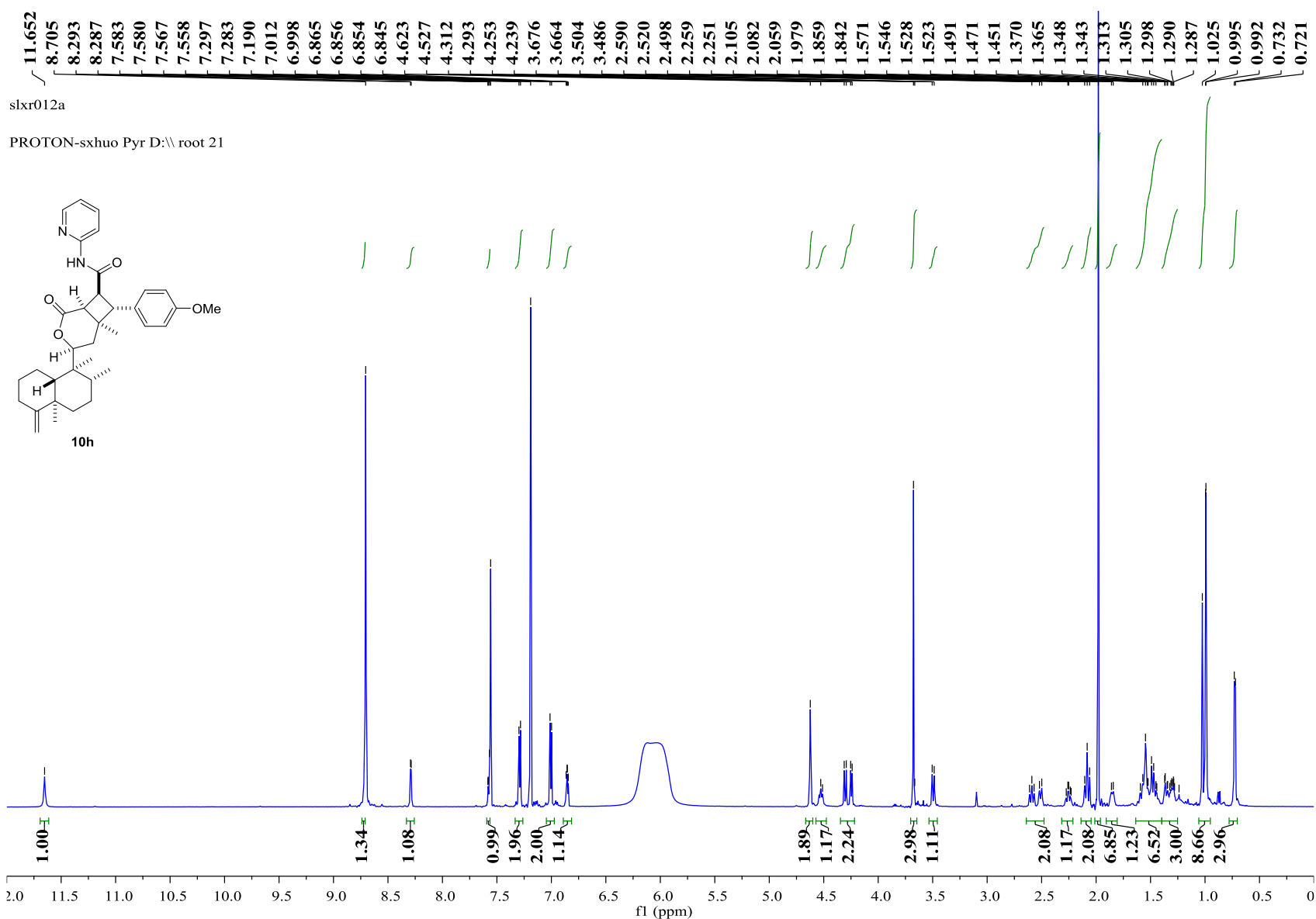

Figure S176. ${ }^{13} \mathrm{C}$ NMR spectrum of $\mathbf{1 0 h}$

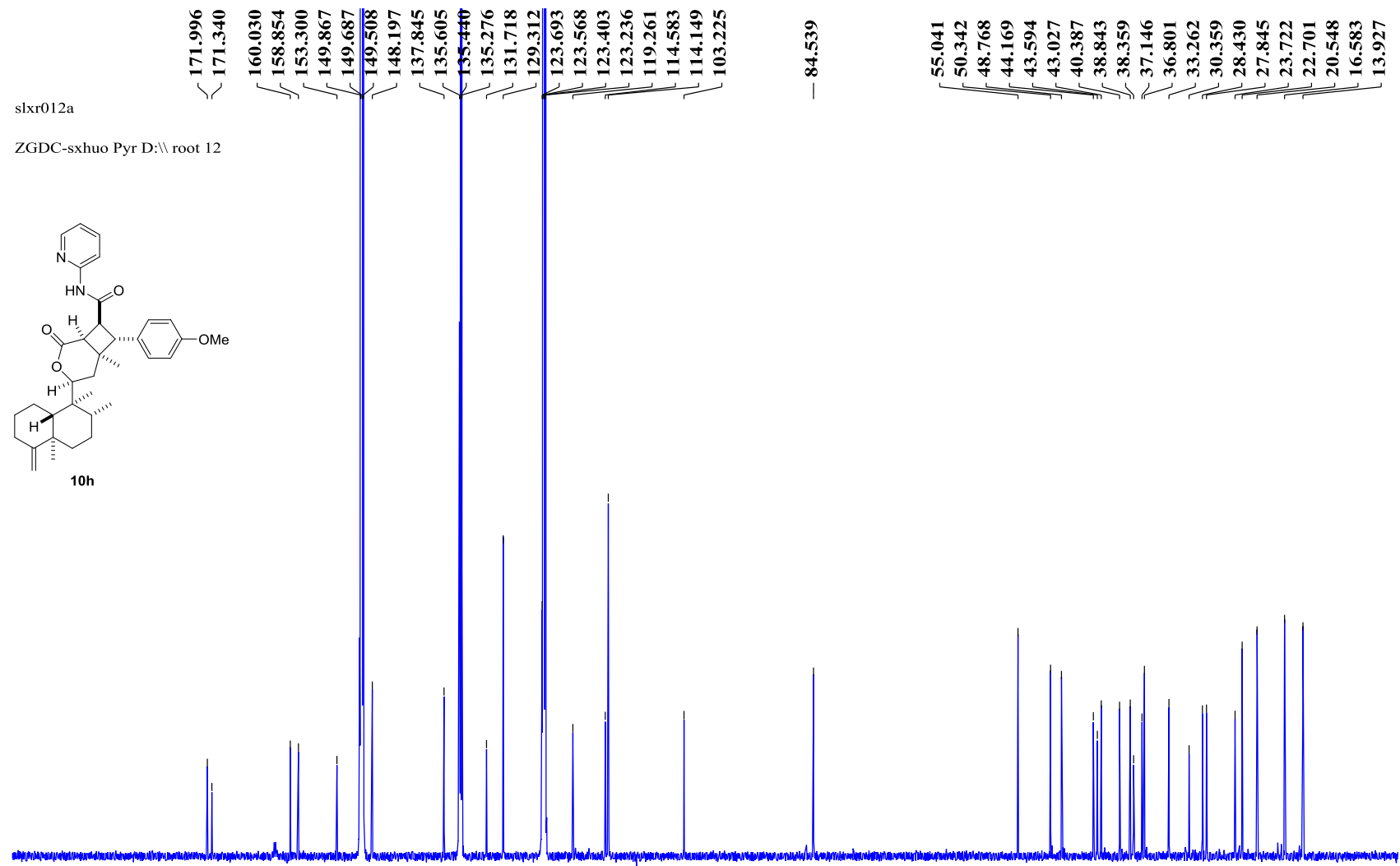

\begin{tabular}{lllllllllllllllllllll}
\hline 00 & 190 & 180 & 170 & 160 & 150 & 140 & 130 & 120 & 110 & $\begin{array}{c}100 \\
\mathrm{fl}(\mathrm{ppm})\end{array}$ & 90 & 80 & 70 & 60 & 50 & 40 & 30 & 20 & 10 & 10
\end{tabular} 
Figure S177. ${ }^{1} \mathrm{H}$ NMR spectrum of $\mathbf{1 0 i}$

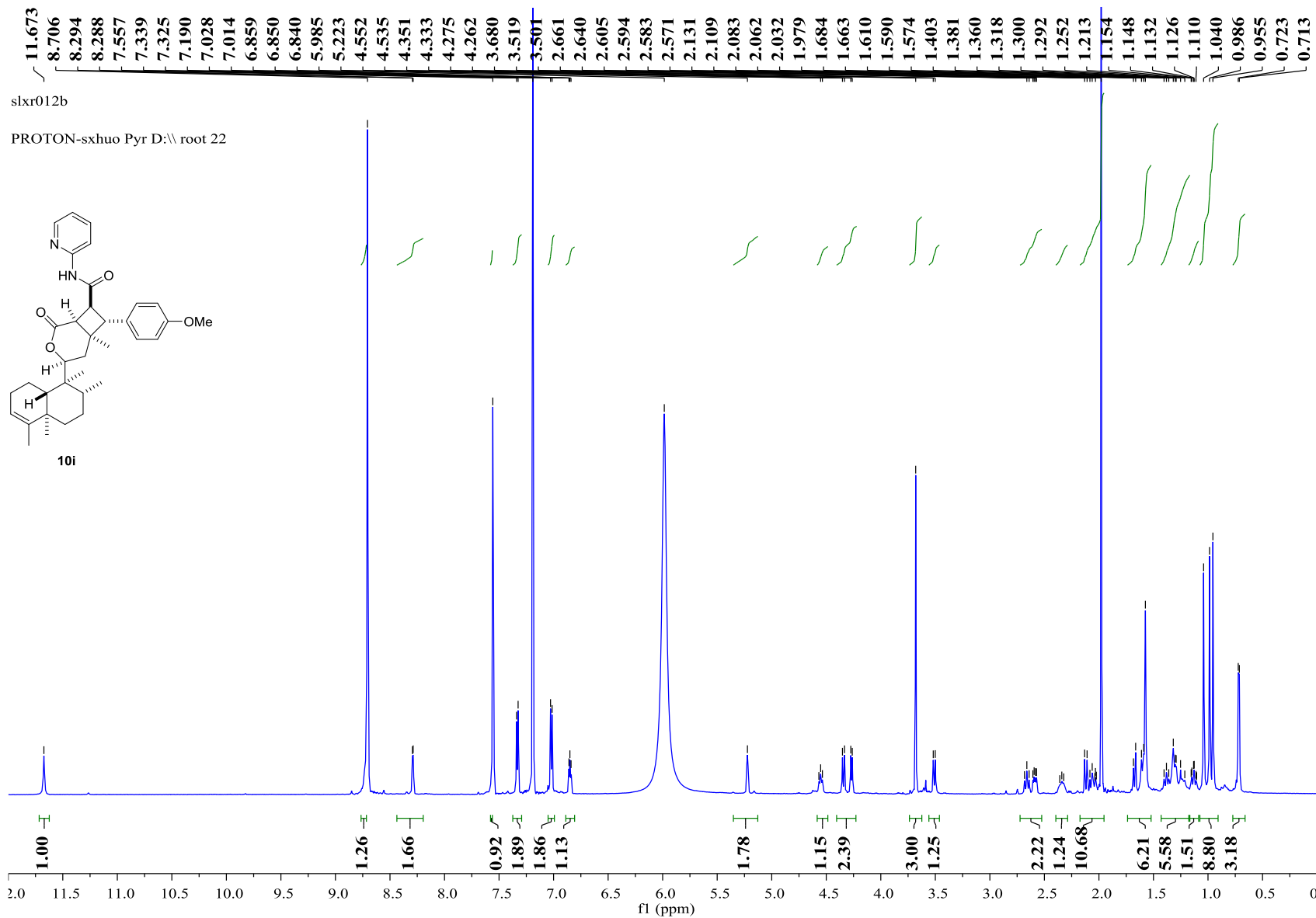

Figure S178. ${ }^{13} \mathrm{C}$ NMR spectrum of $\mathbf{1 0 i}$

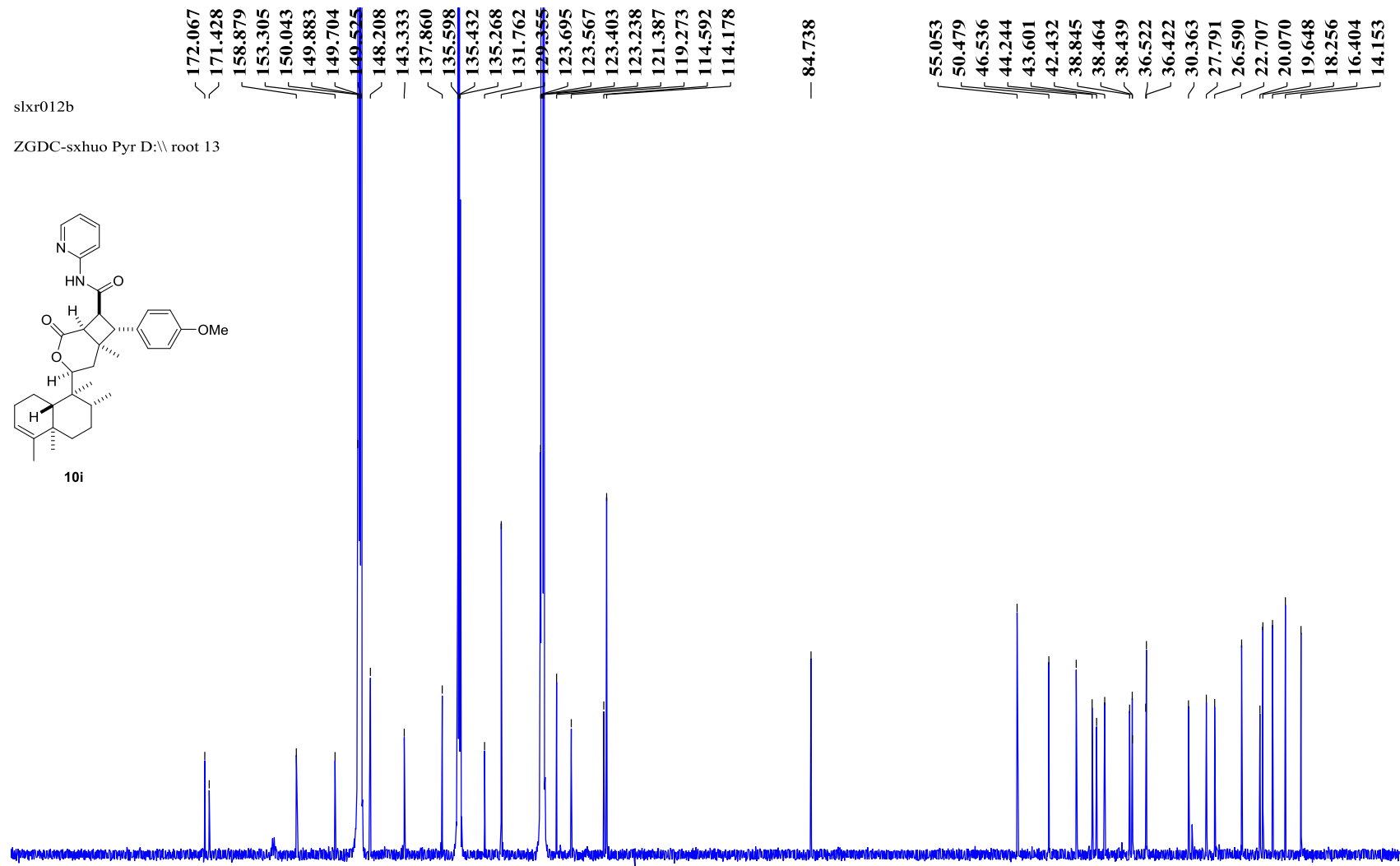

$\begin{array}{llllllllllllllllllll}00 & 190 & 180 & 170 & 160 & 150 & 140 & 130 & 120 & 110 & \begin{array}{c}100 \\ \mathrm{f} 1(\mathrm{ppm})\end{array} & 90 & 80 & 70 & 60 & 50 & 40 & 30 & 20 & 10\end{array}$ 
Figure S179. ${ }^{1} \mathrm{H}$ NMR spectrum of $\mathbf{1 0 j}$

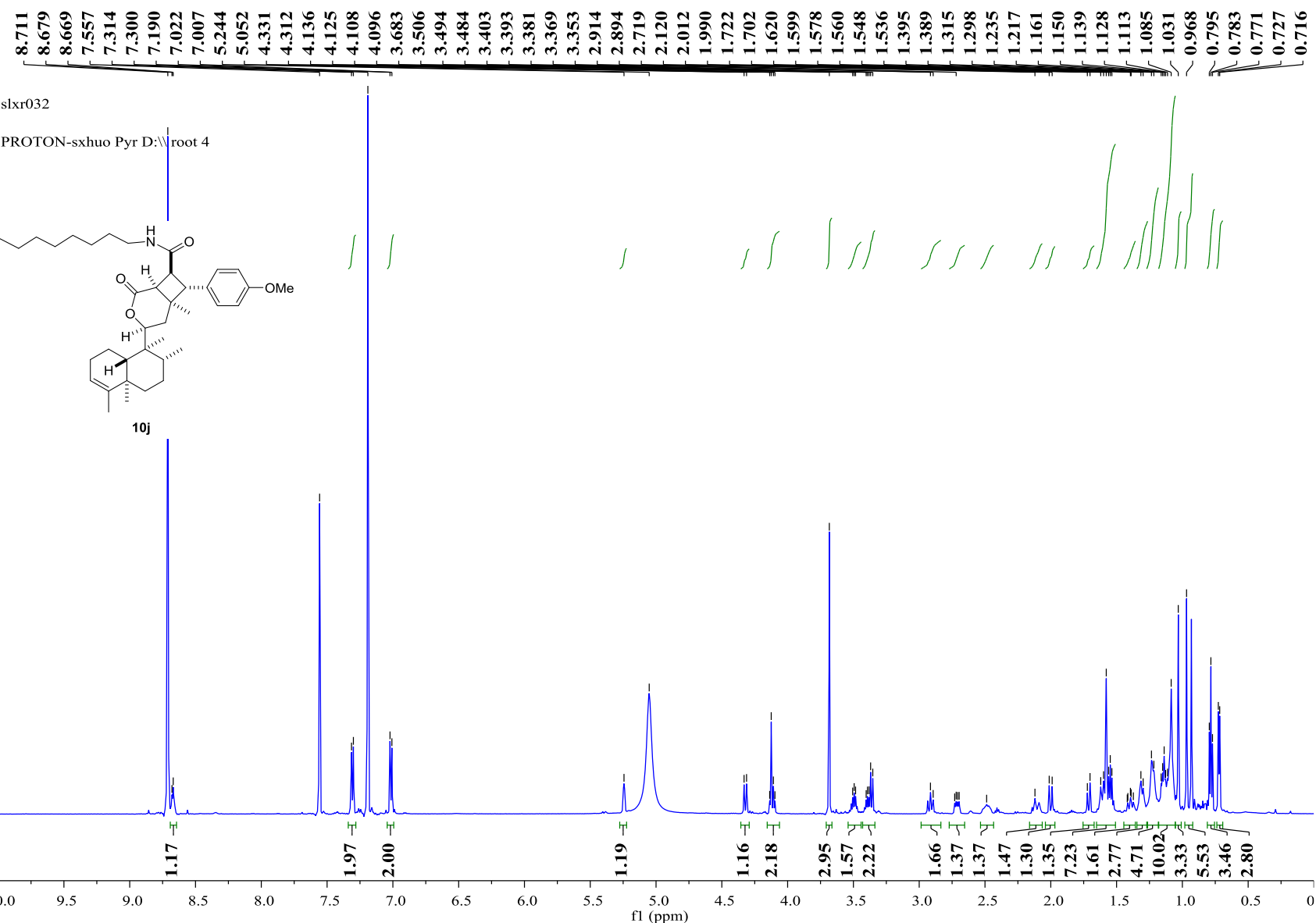

Figure S180. ${ }^{13} \mathrm{C}$ NMR spectrum of $\mathbf{1 0 j}$

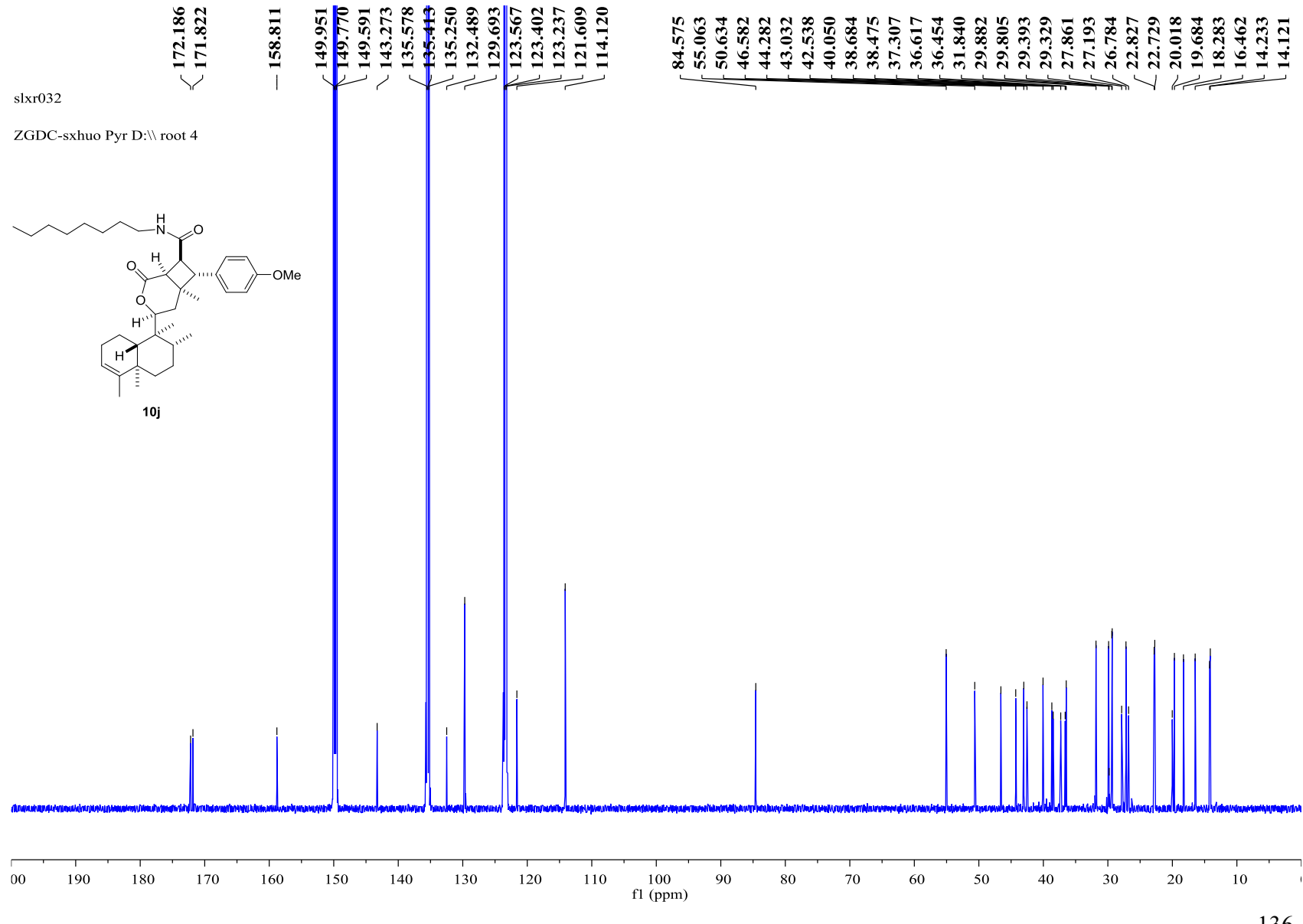




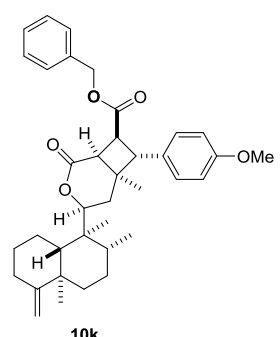

$10 \mathrm{k}$

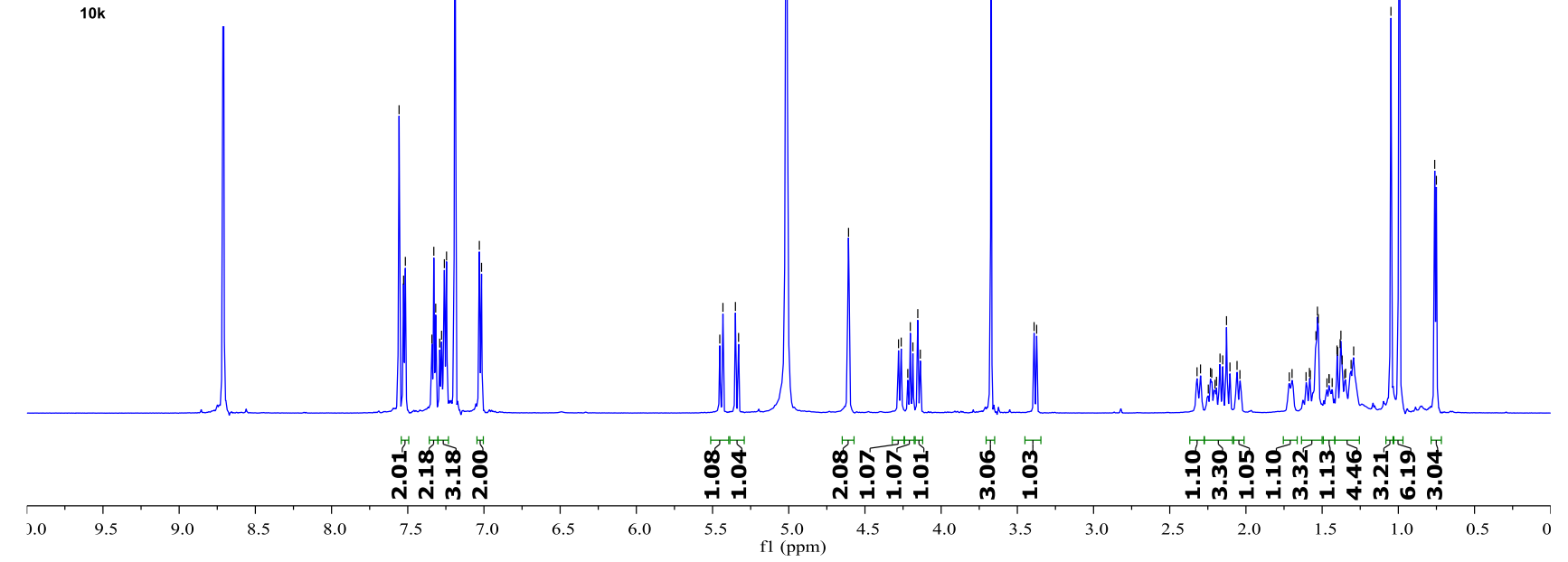

Figure S182. ${ }^{13} \mathrm{C}$ NMR spectrum of $\mathbf{1 0 k}$

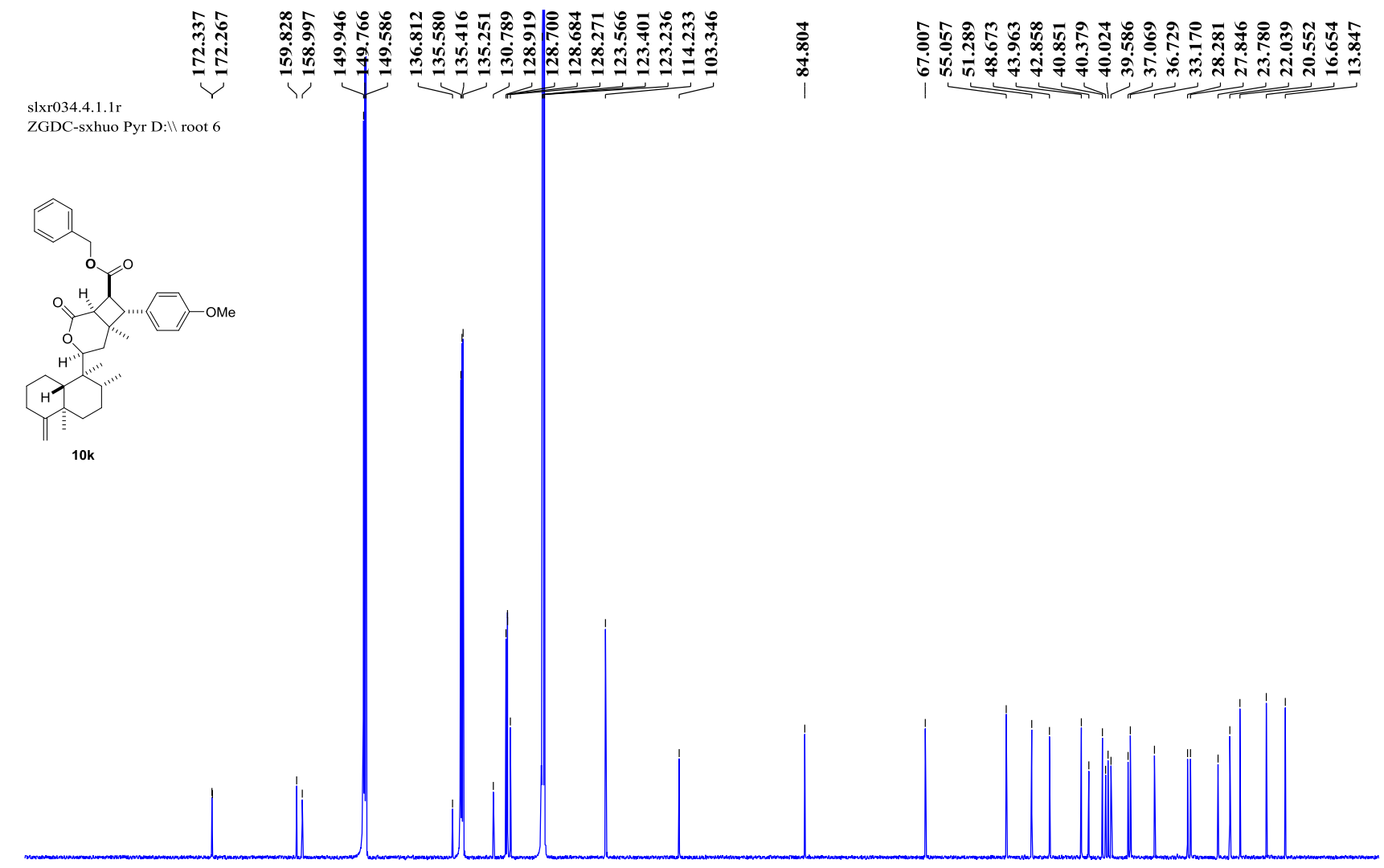

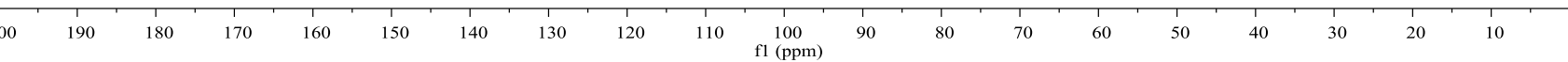


Figure S183. ${ }^{1} \mathrm{H}$ NMR spectrum of $\mathbf{1 0 l}$

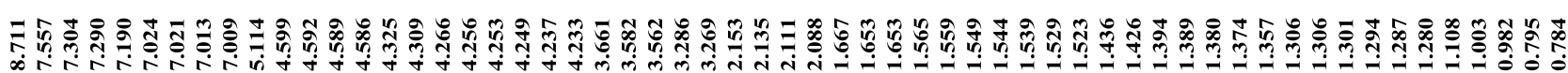

slxr037a.1.1.1

PROTON-sxhuo Pyr D: $\mid$ root 43

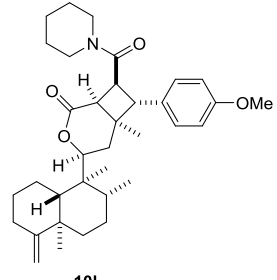

101

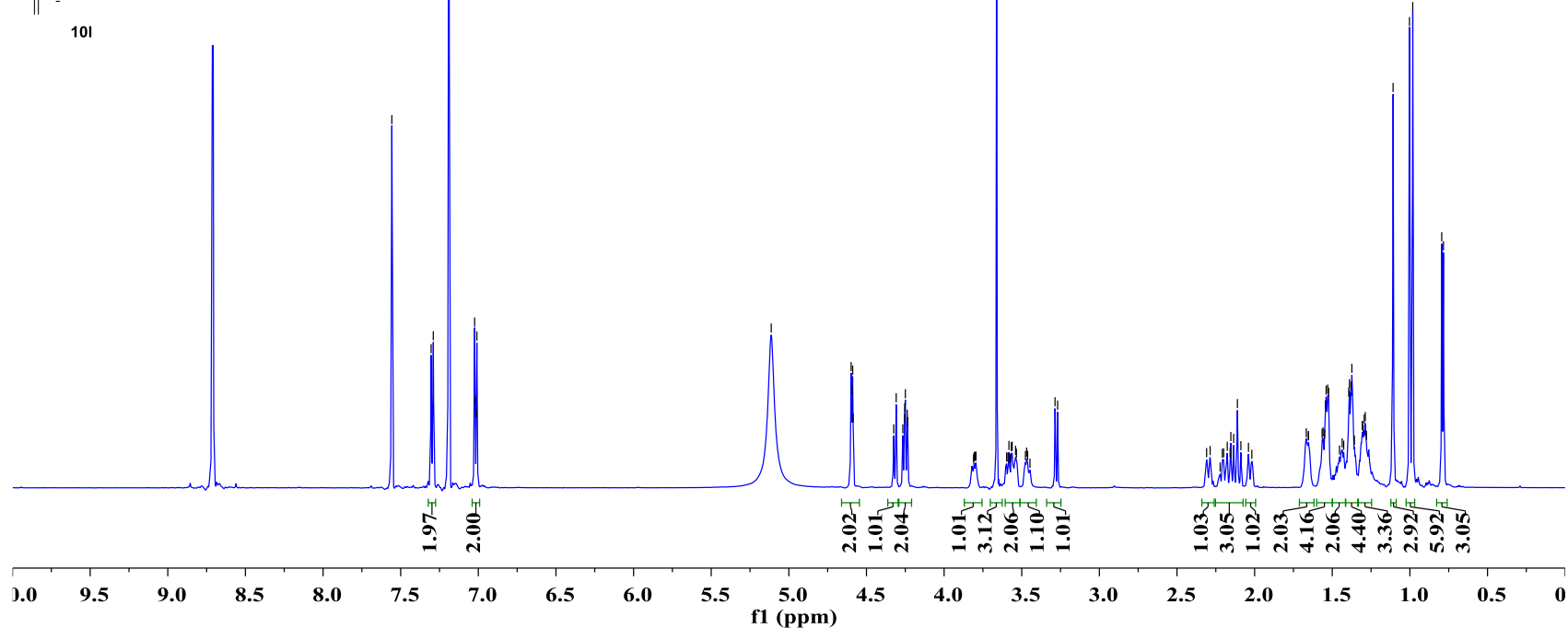

Figure S184. ${ }^{13} \mathrm{C}$ NMR spectrum of $\mathbf{1 0 1}$

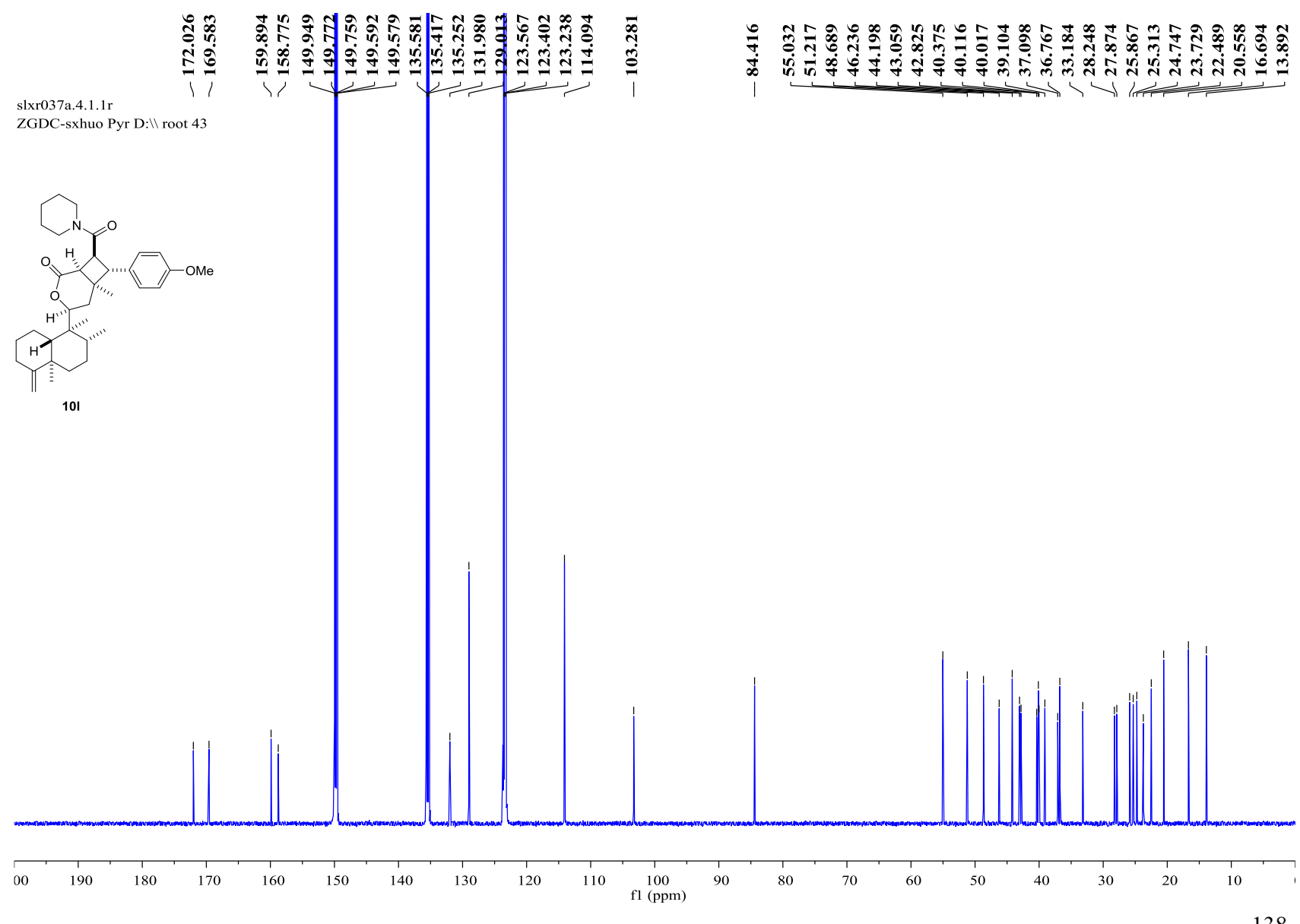


Figure S185. ${ }^{1} \mathrm{H}$ NMR spectrum of $\mathbf{1 0 m}$

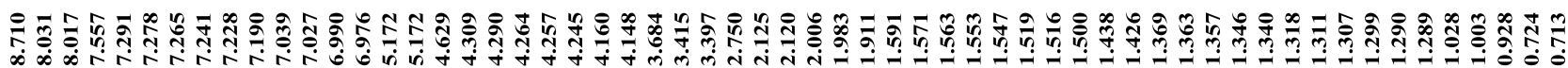

slxr038a.1.1.1

PROTON-sxhuo Pyr D: \\ root 7
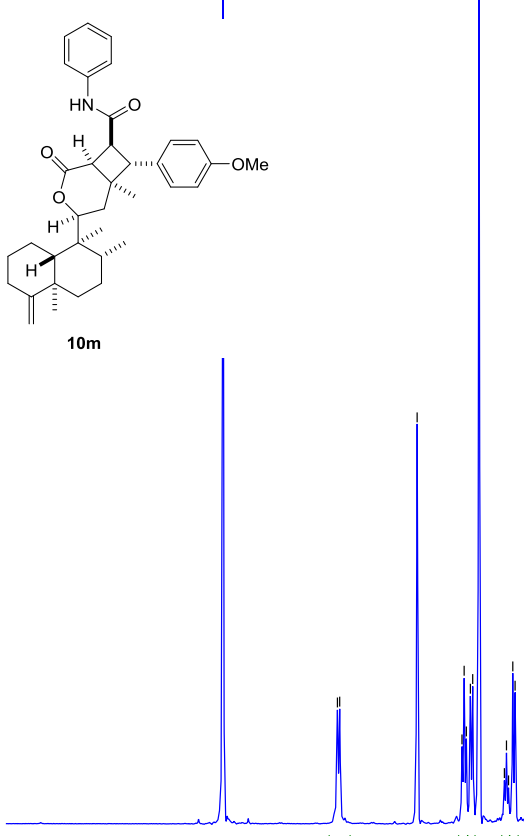

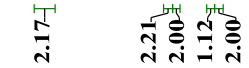

Figure S186. ${ }^{13} \mathrm{C}$ NMR spectrum of $\mathbf{1 0 m}$

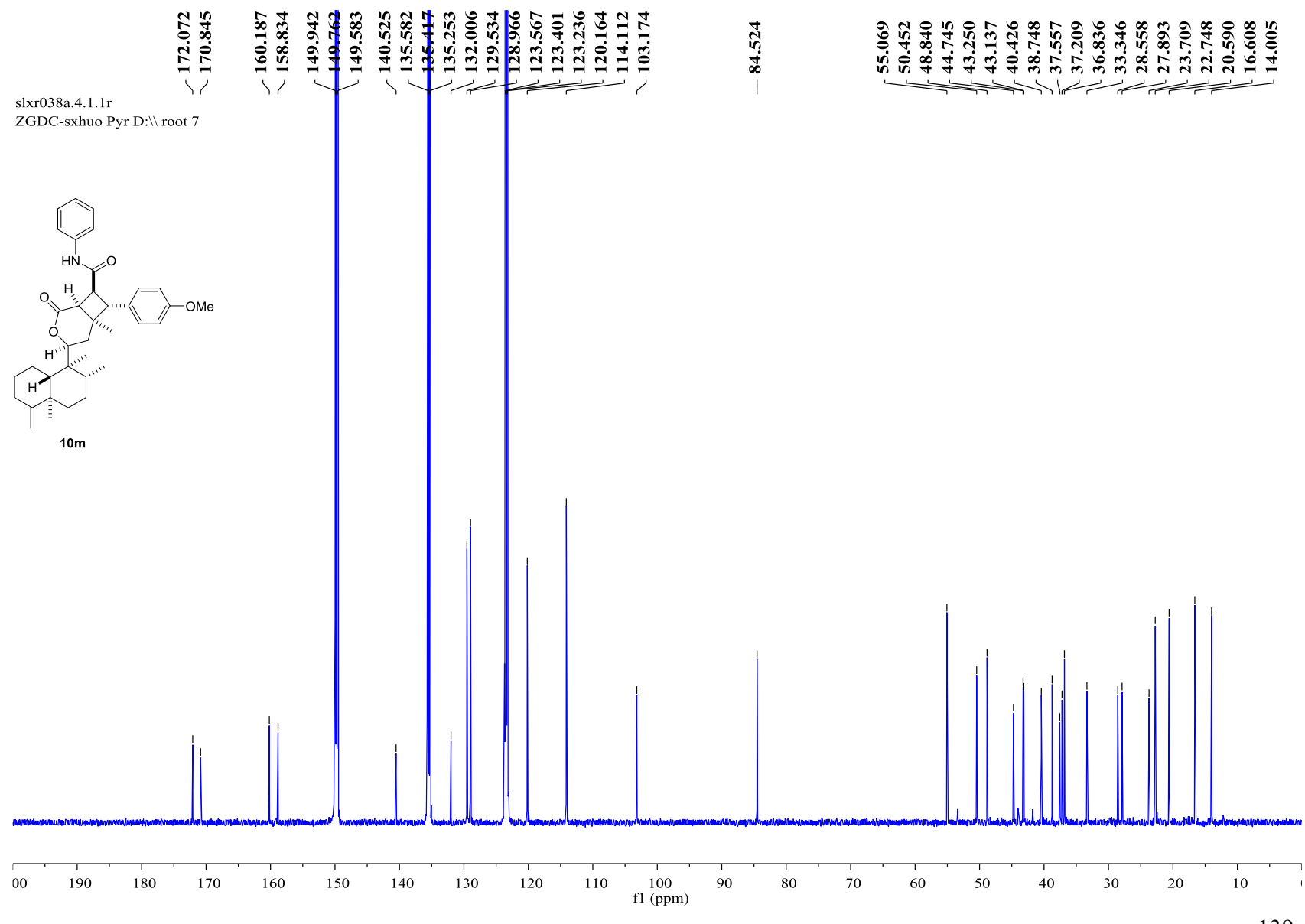



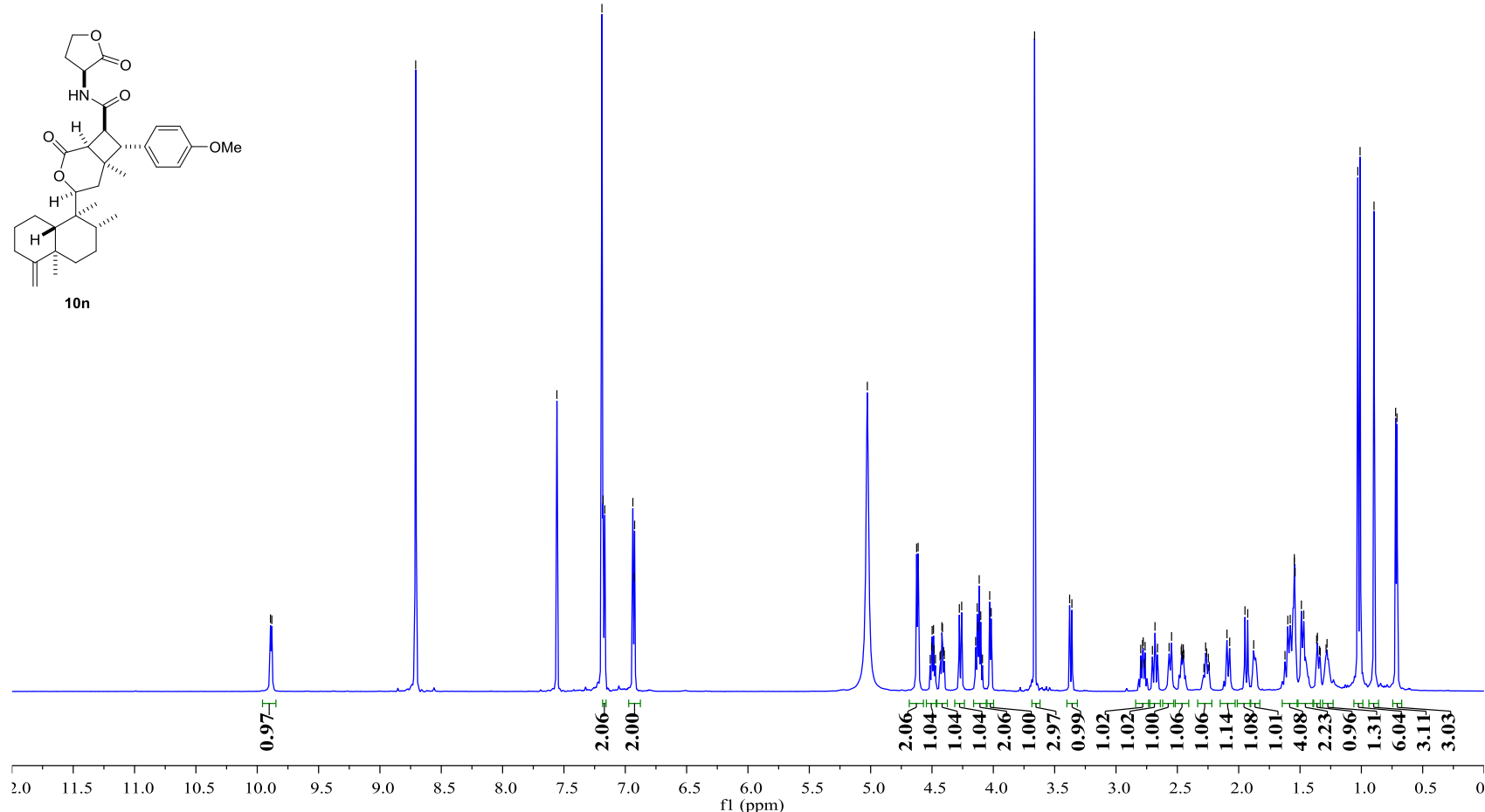

Figure S188. ${ }^{13} \mathrm{C}$ NMR spectrum of 10n

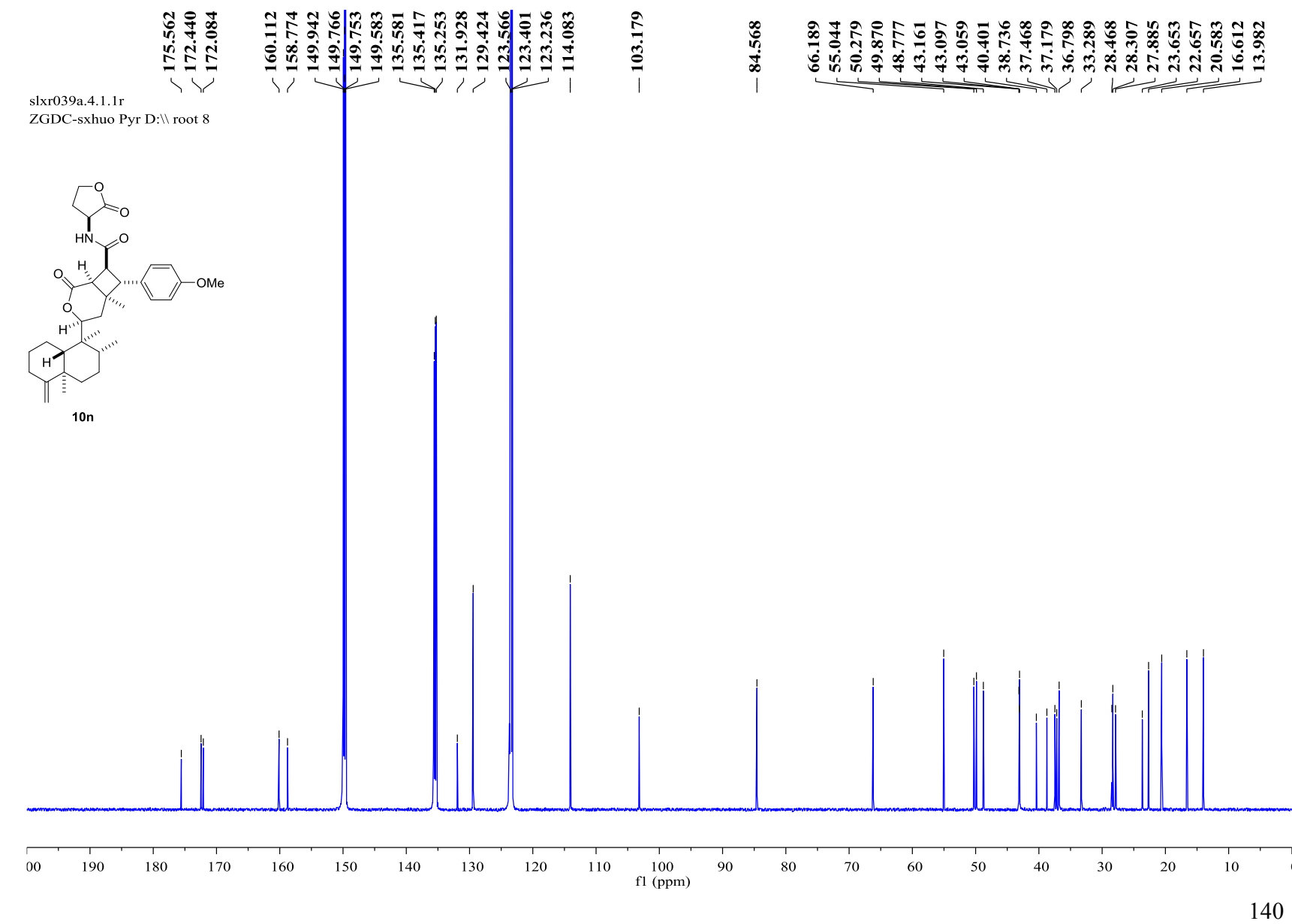


Figure S189. ${ }^{1} \mathrm{H}$ NMR spectrum of $\mathbf{1 0 0}$

ลั.

slxr039b.1.1.1r

PROTON-sxhuo Pyr D: $\backslash \backslash$ root 9

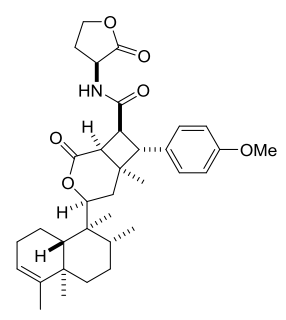

100

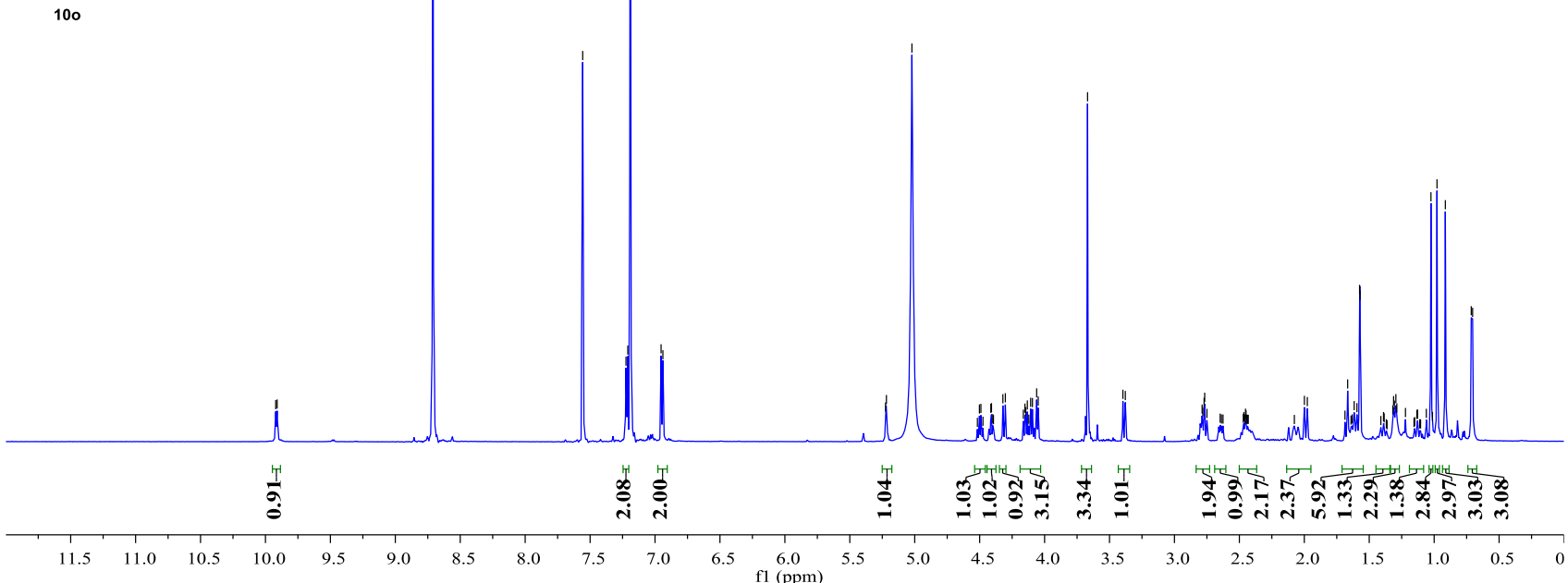

Figure S190. ${ }^{13} \mathrm{C}$ NMR spectrum of $\mathbf{1 0 0}$

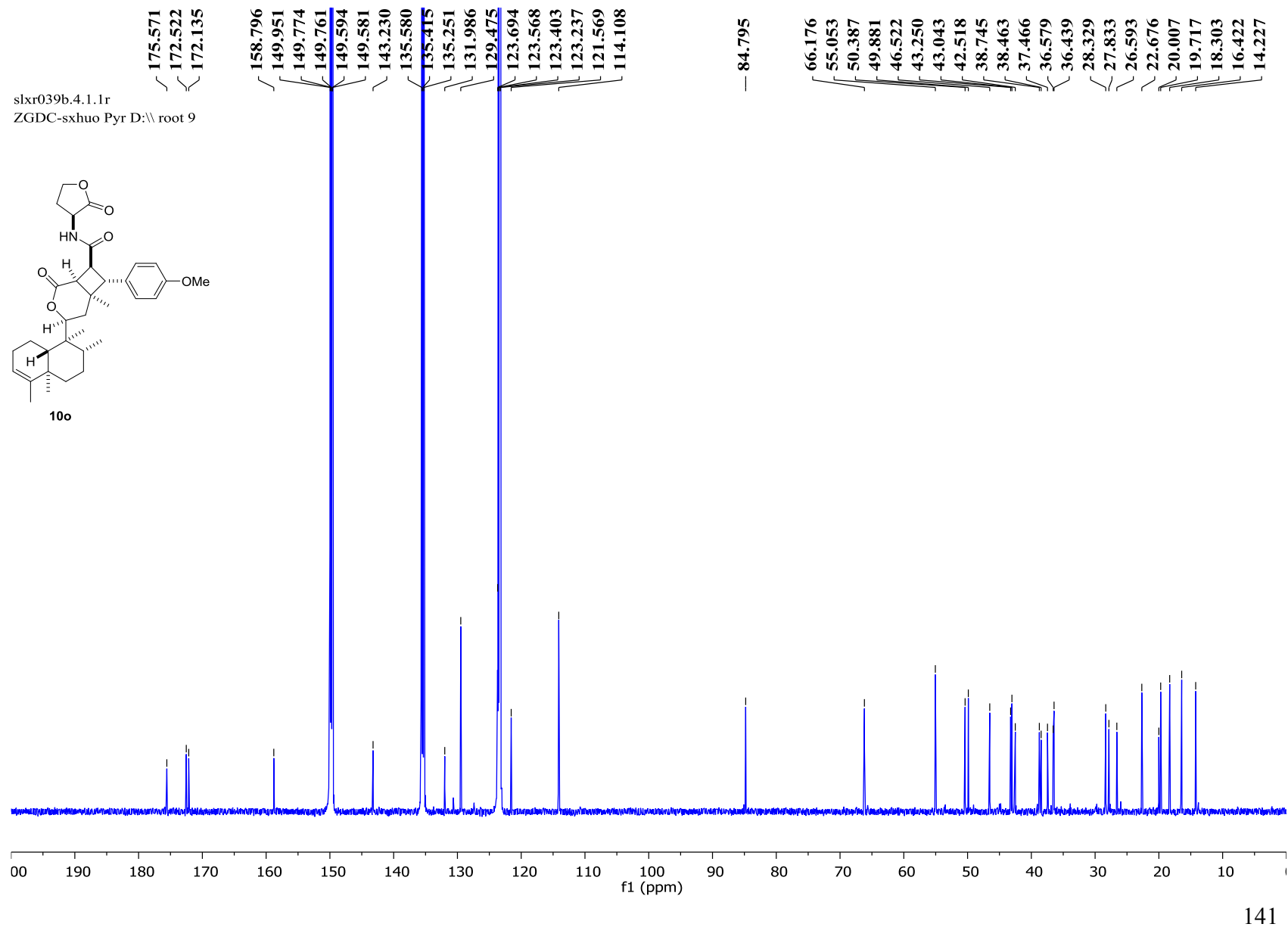


Figure S191. ${ }^{1} \mathrm{H}$ NMR spectrum of 10p

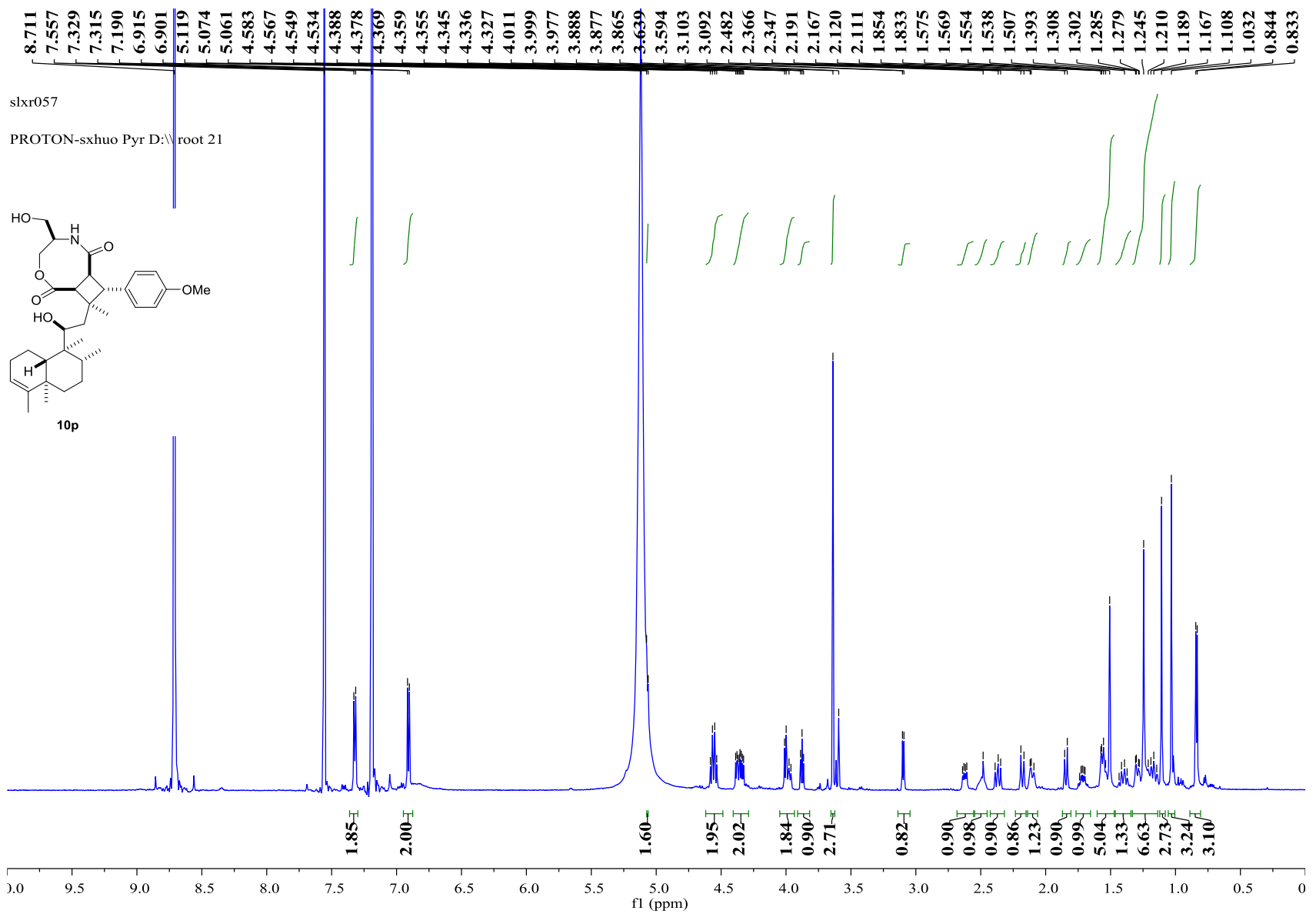

Figure S192. ${ }^{13} \mathrm{C}$ NMR spectrum of 10p
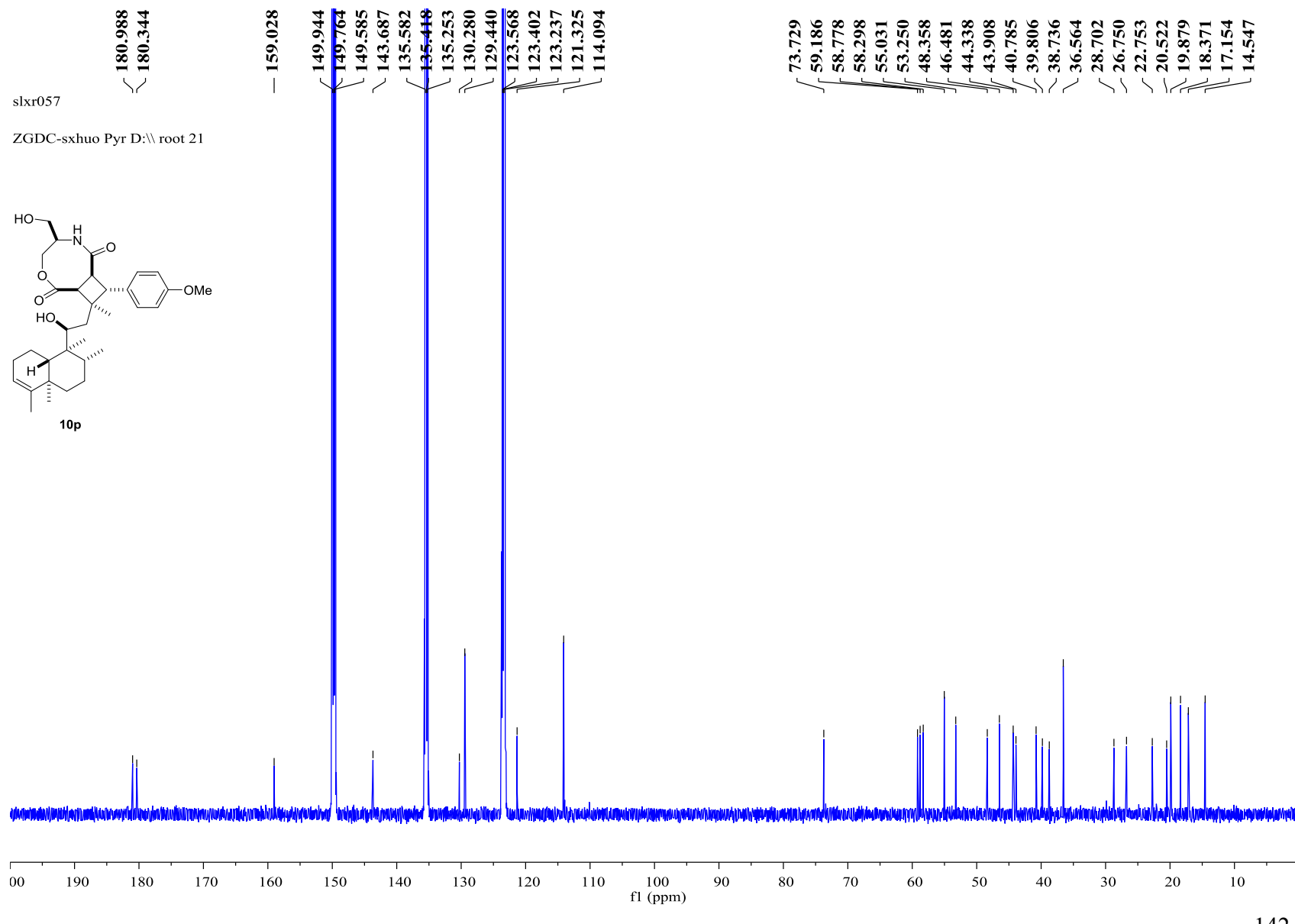
Figure S193. ${ }^{1} \mathrm{H}$ NMR spectrum of $\mathbf{1 0 q}$

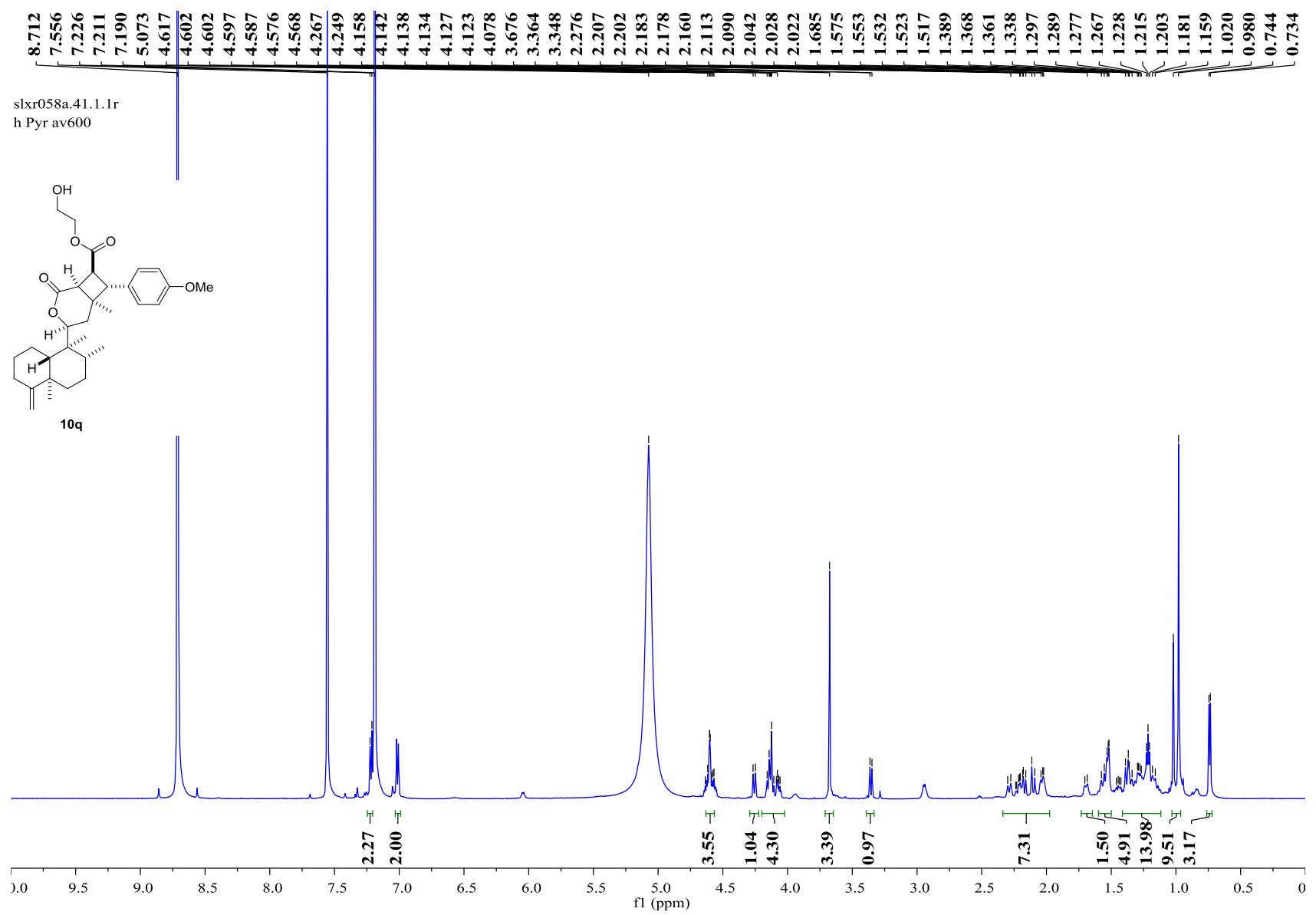

Figure S194. ${ }^{13} \mathrm{C}$ NMR spectrum of $\mathbf{1 0 q}$

slxr058a.44.1.1r

$$
\text { 㘳 }
$$

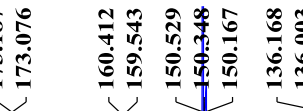

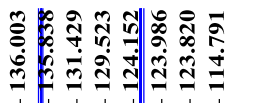
$\stackrel{m}{\frac{m}{2}}$

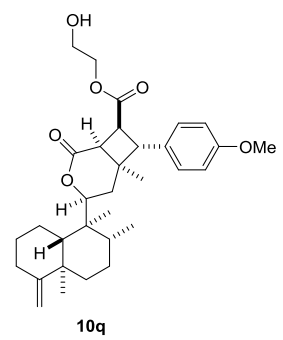

10q

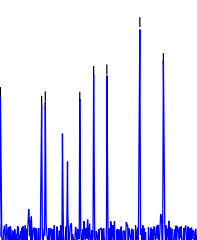


Figure S195. ${ }^{1} \mathrm{H}$ NMR spectrum of 10r

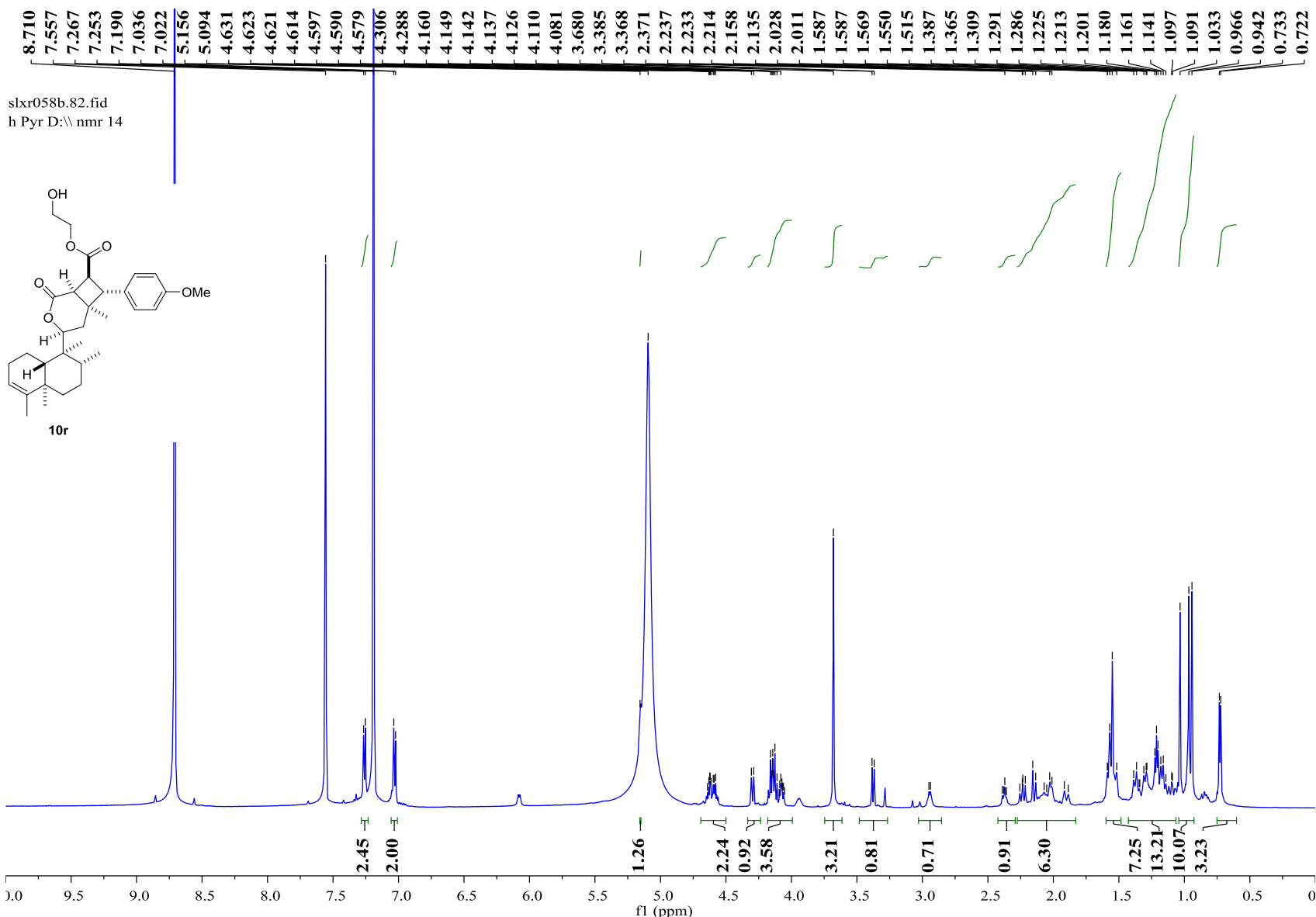

Figure S196. ${ }^{13} \mathrm{C}$ NMR spectrum of $\mathbf{1 0 r}$
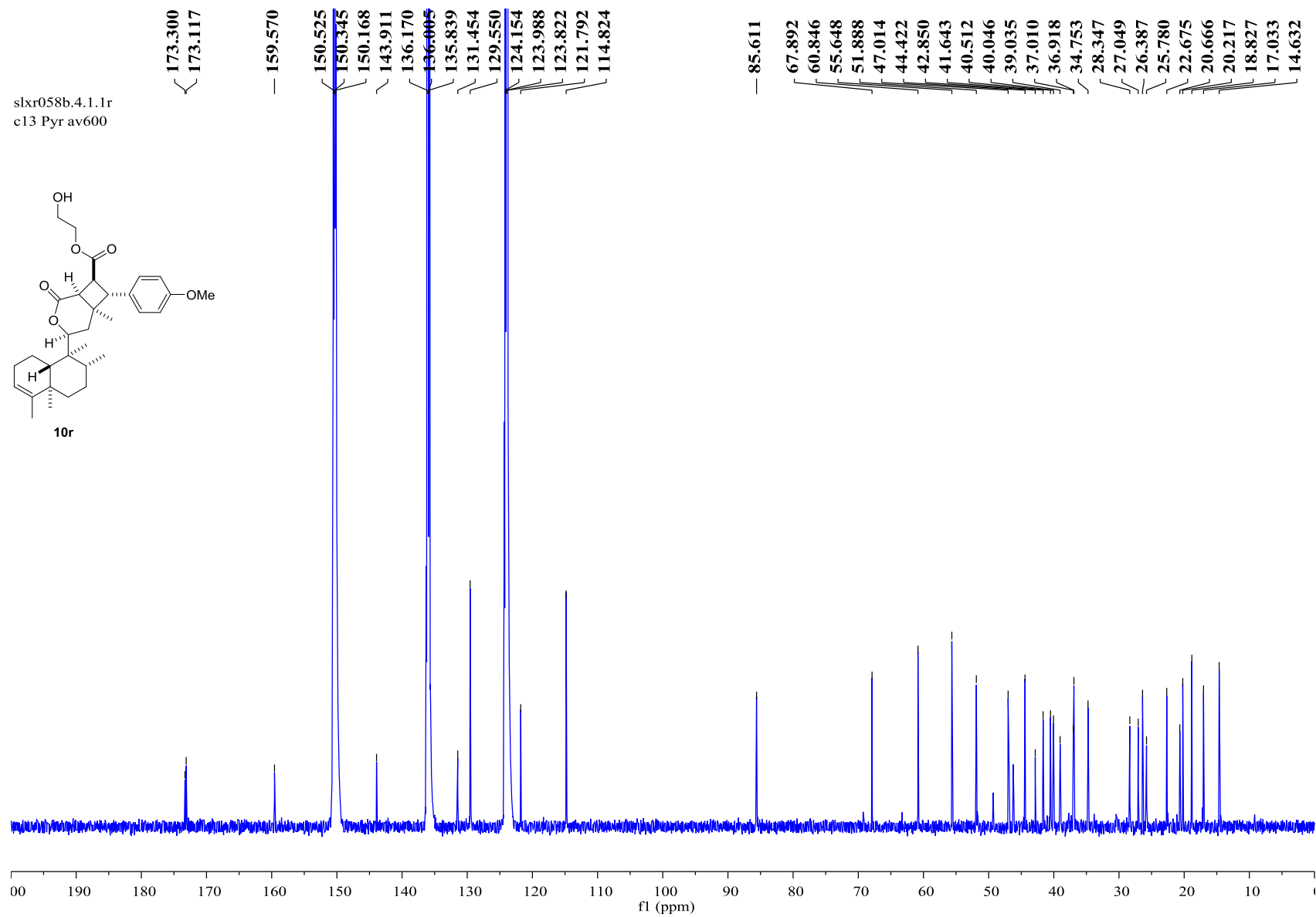
Figure S197. ${ }^{1} \mathrm{H}$ NMR spectrum of 10s

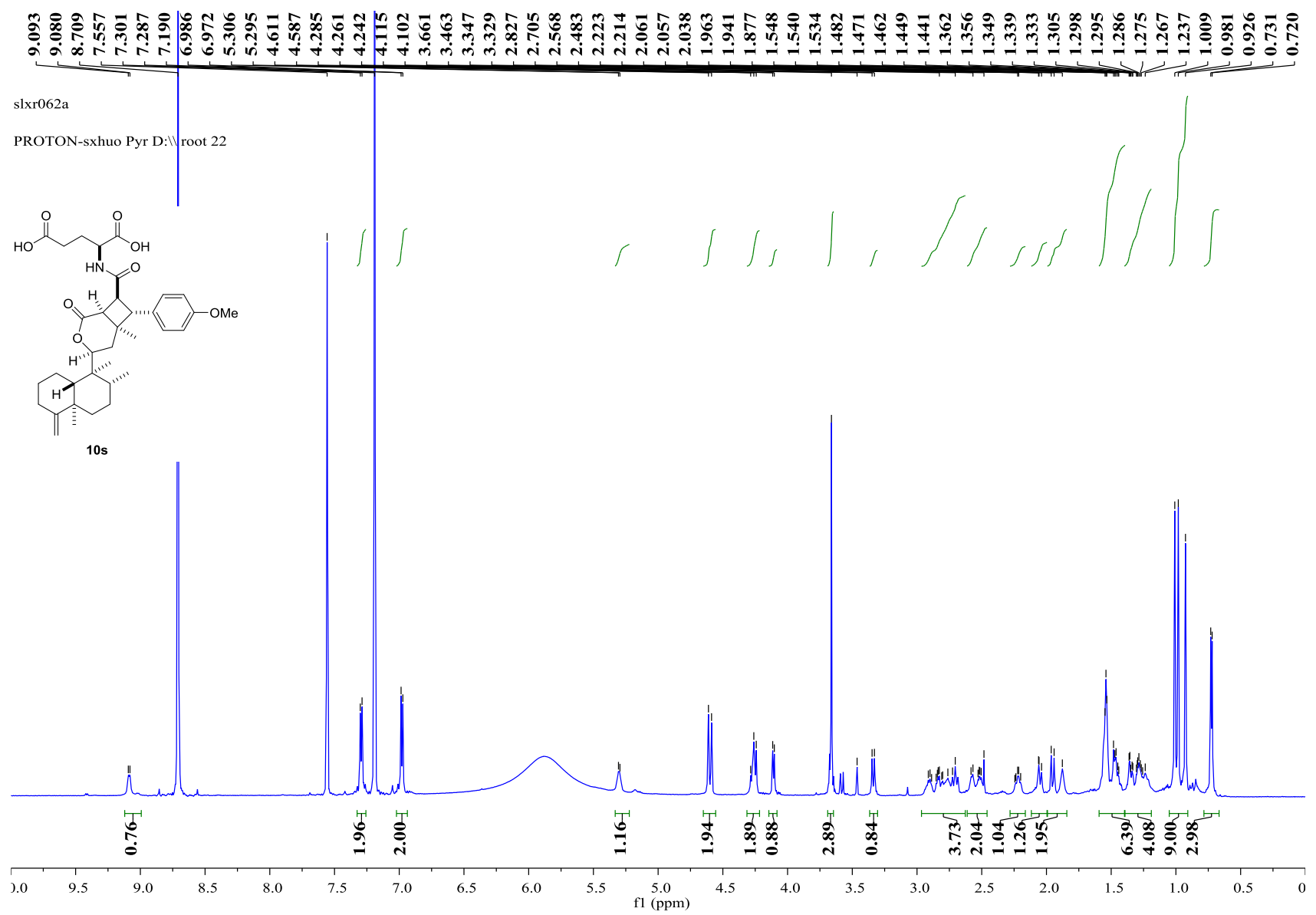

Figure S198. ${ }^{13} \mathrm{C}$ NMR spectrum of $\mathbf{1 0 s}$
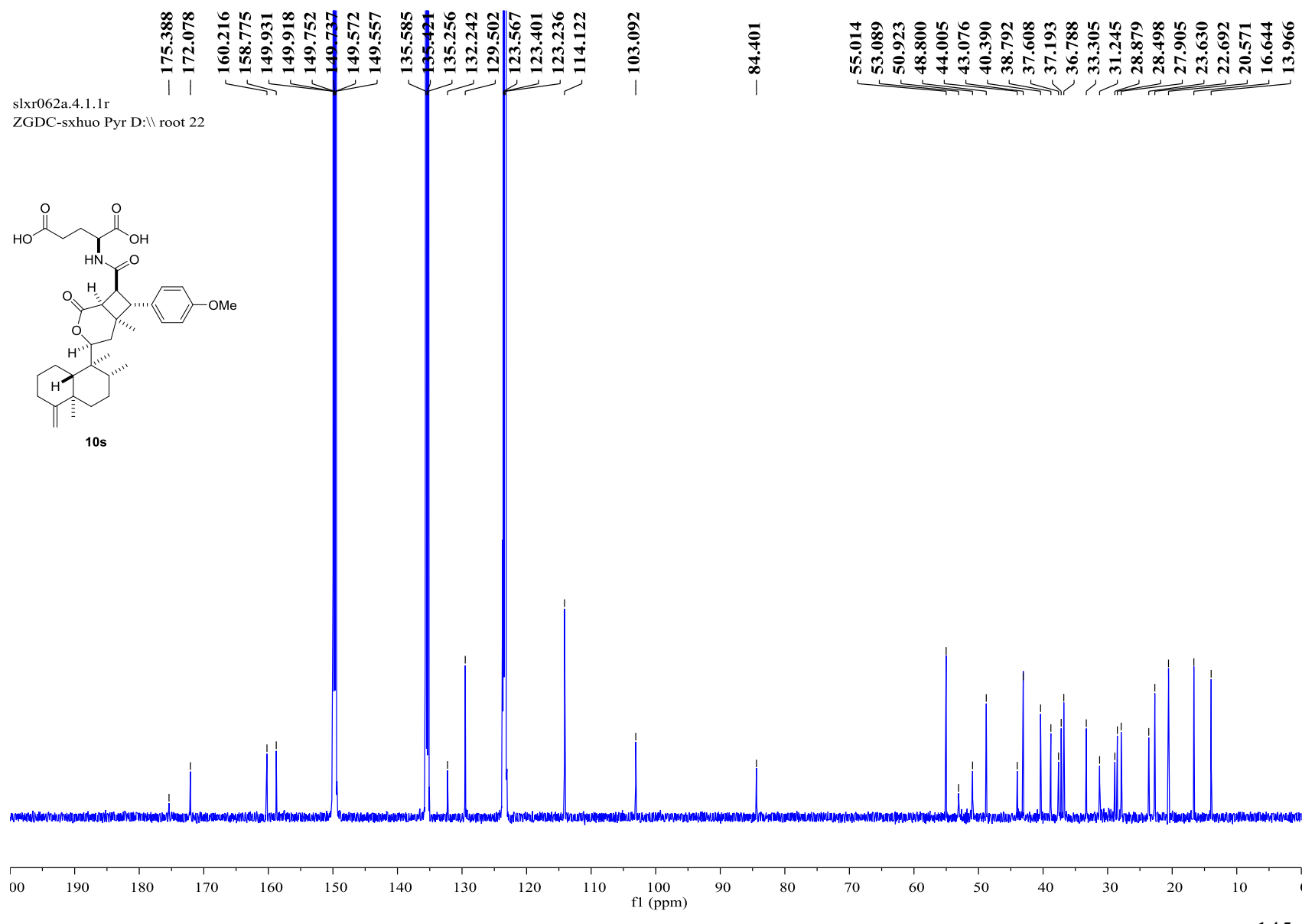
Figure S199. ${ }^{1} \mathrm{H}$ NMR spectrum of $\mathbf{1 0 t}$

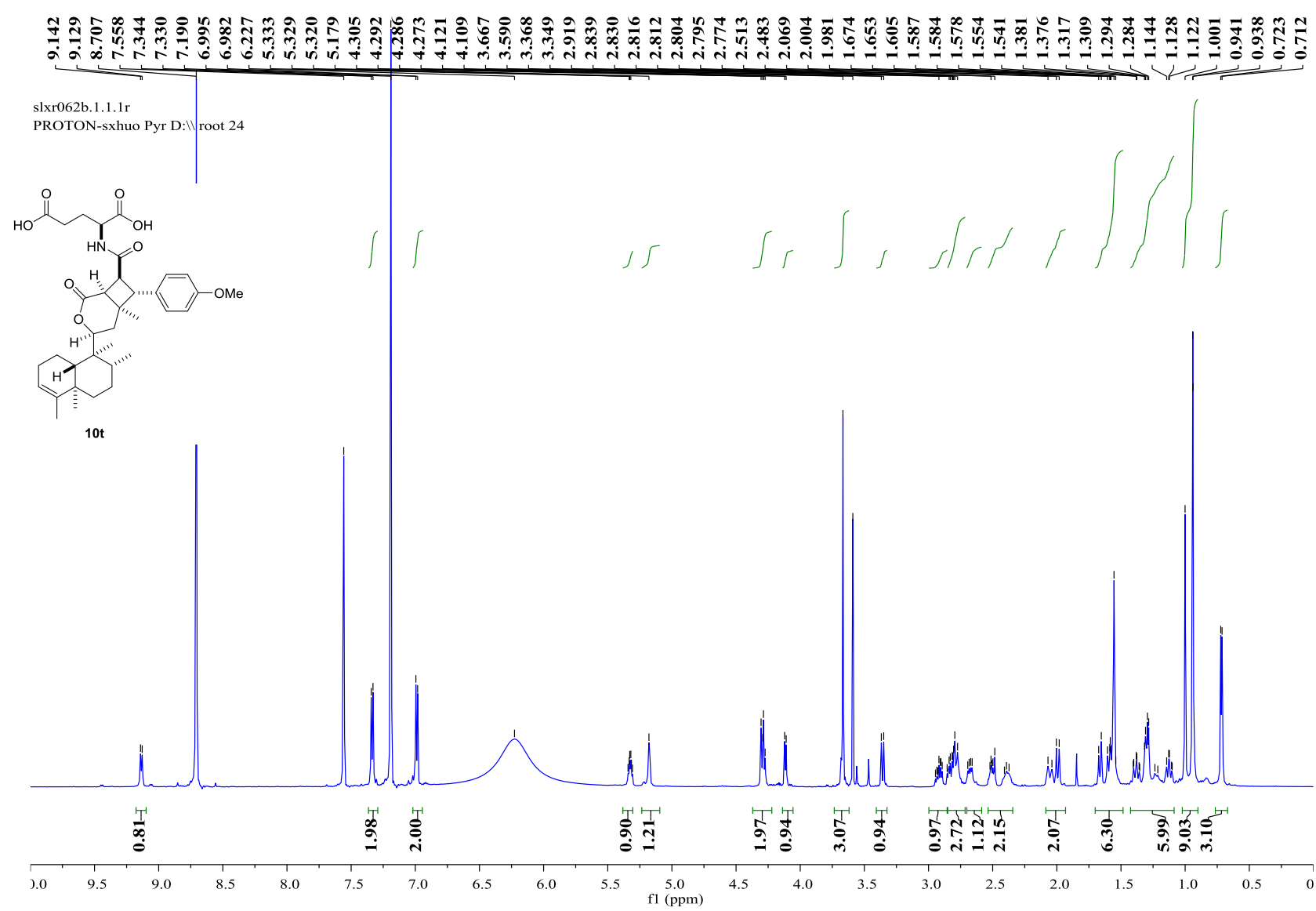

Figure S200. ${ }^{13} \mathrm{C}$ NMR spectrum of $\mathbf{1 0 t}$

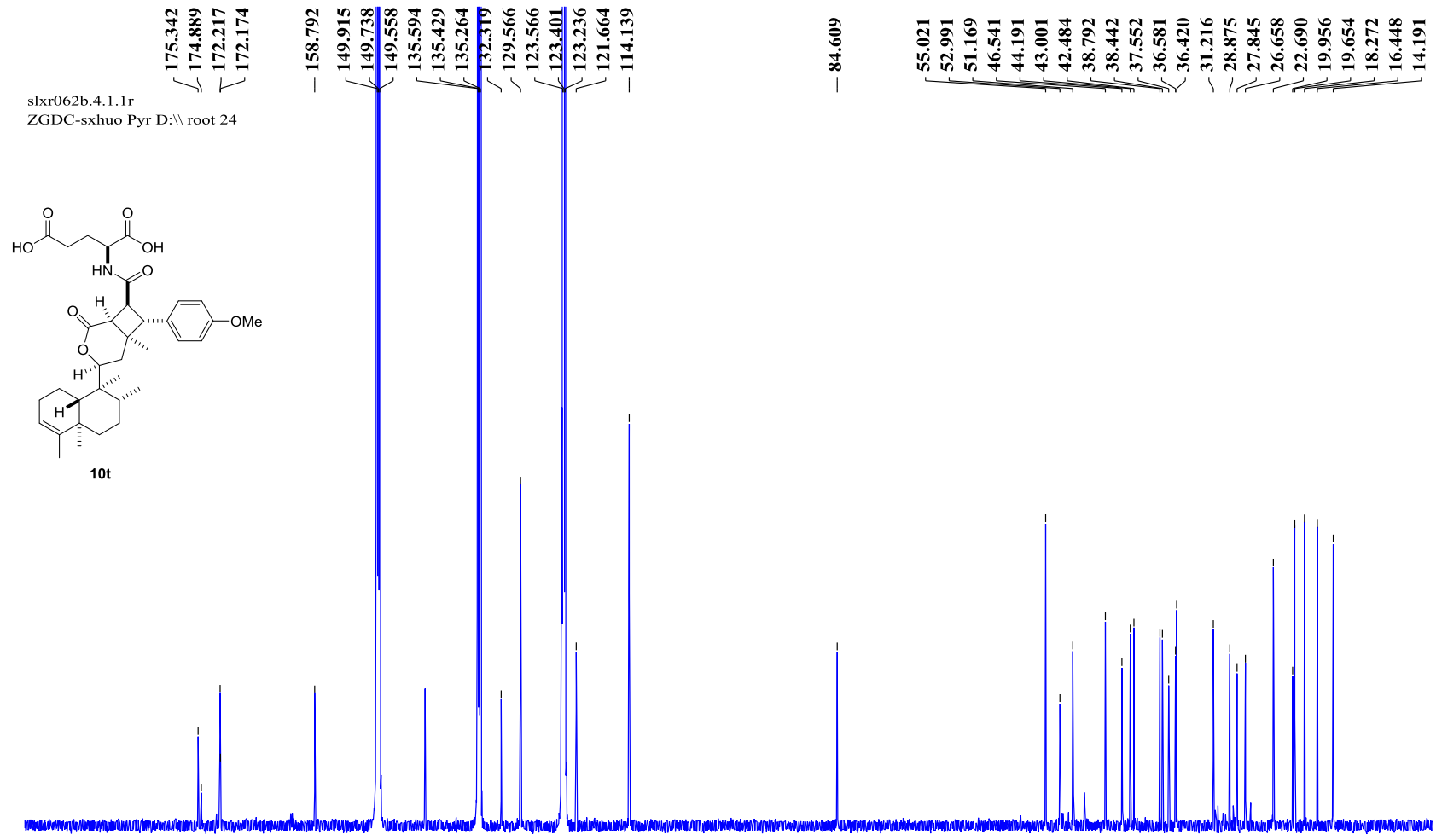

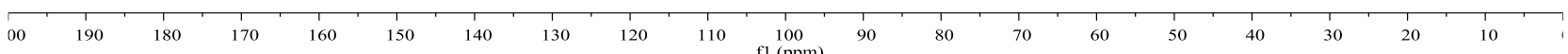


Figure S201. ${ }^{1} \mathrm{H}$ NMR spectrum of $\mathbf{1 0 u}$

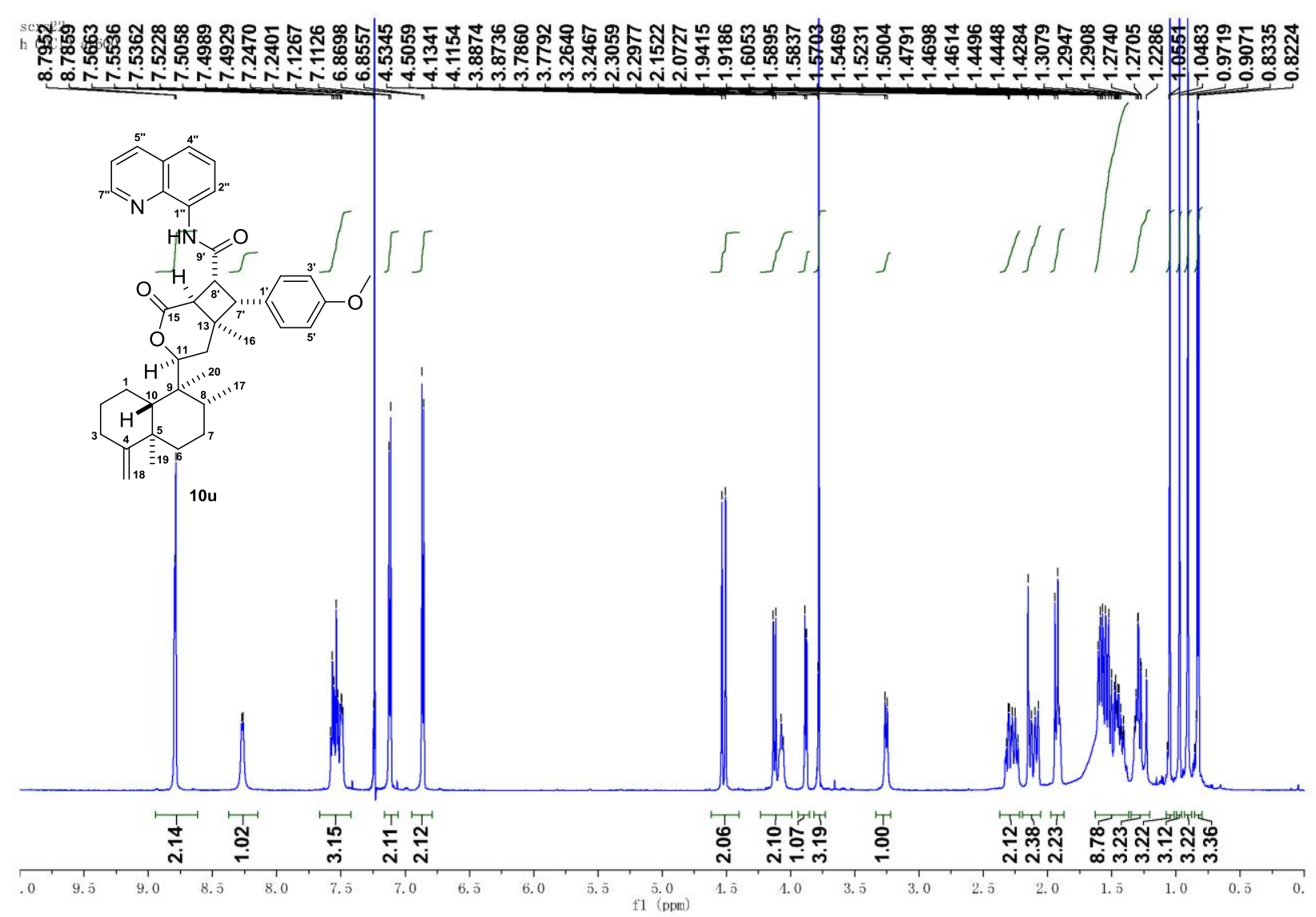

Figure S202. ${ }^{13} \mathrm{C}$ NMR spectrum of $\mathbf{1 0 u}$
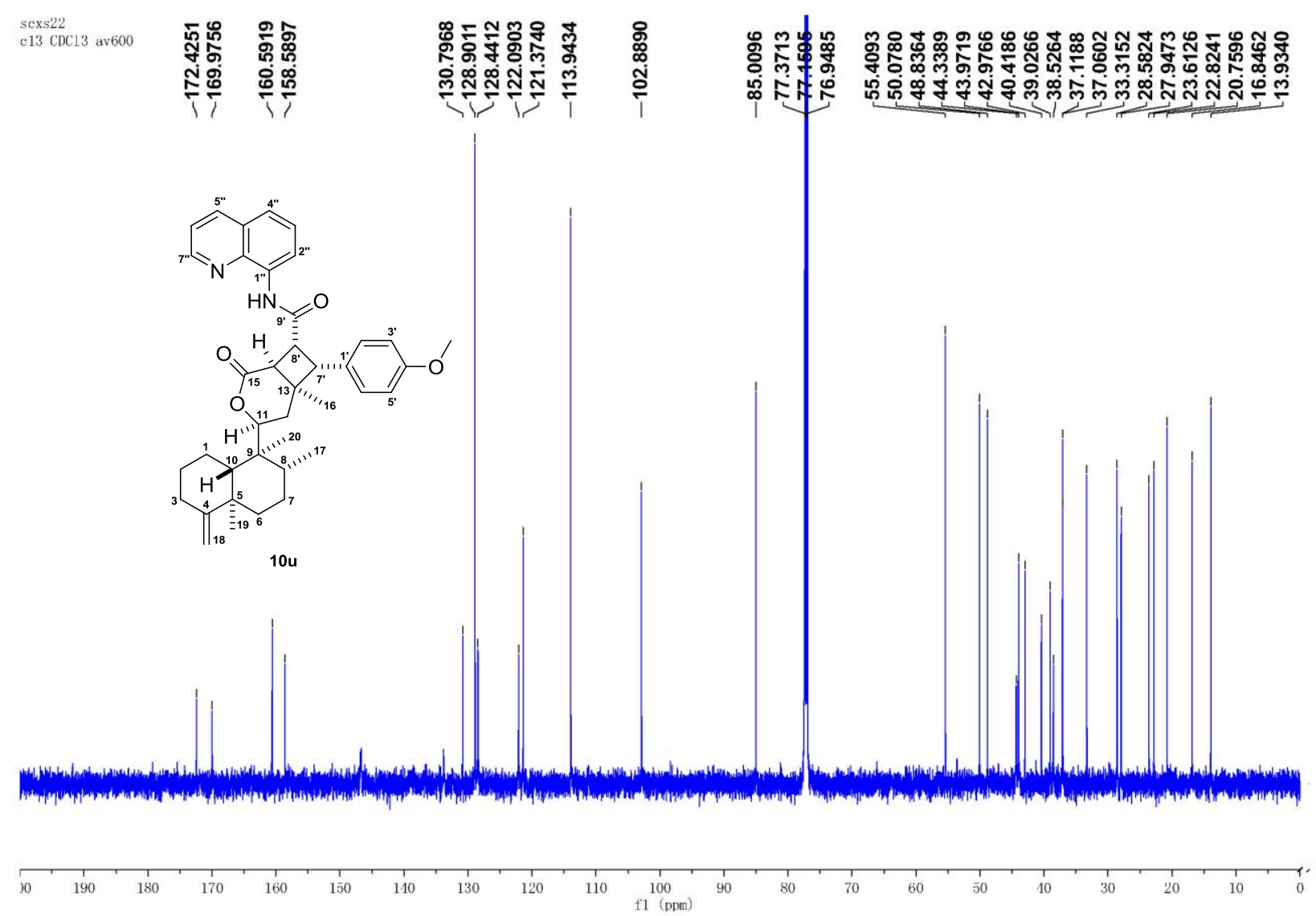
Figure S203. ${ }^{1} \mathrm{H}$ NMR spectrum of $\mathbf{1 0 v}$

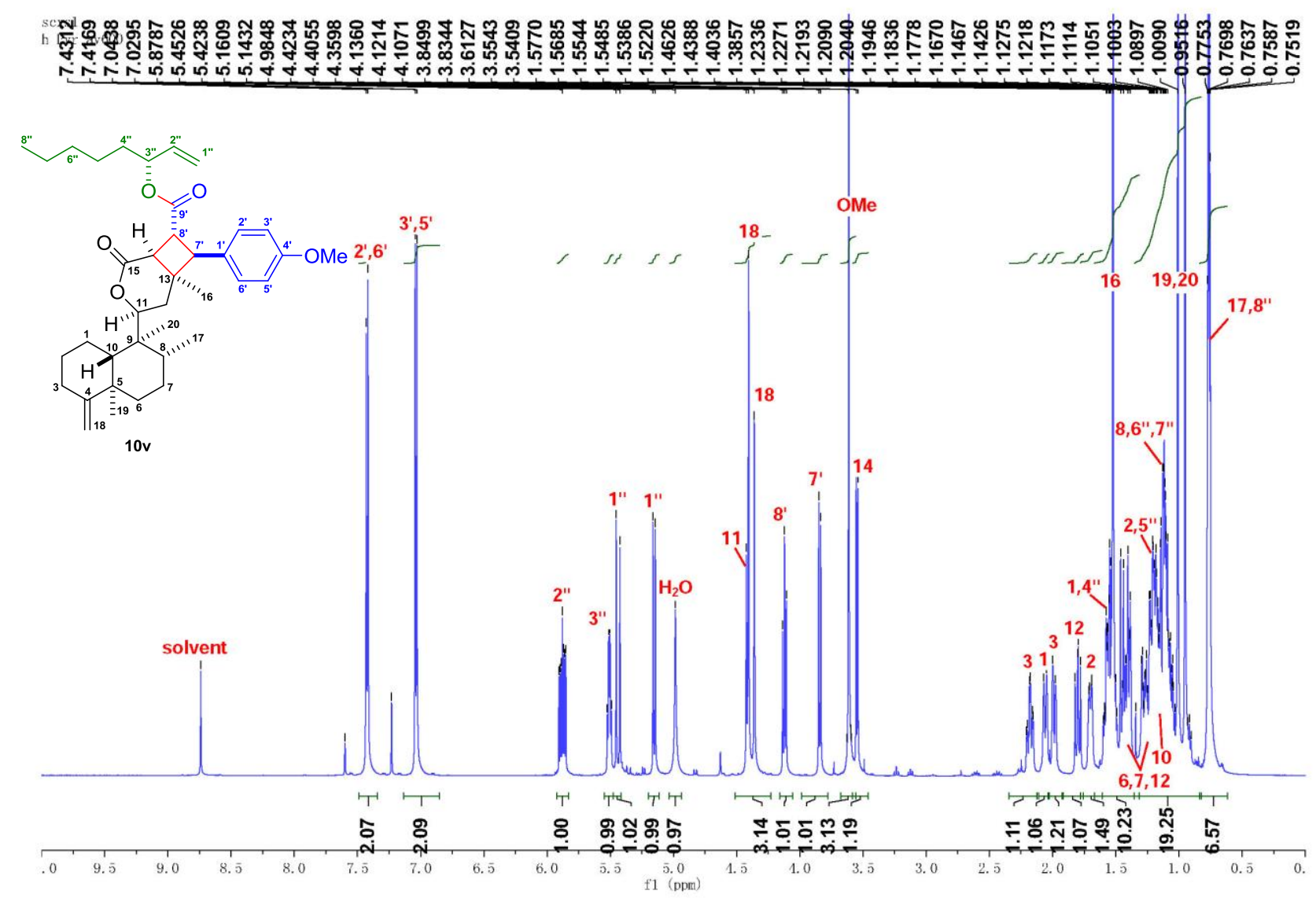

Figure S204. ${ }^{13} \mathrm{C}$ NMR spectrum of $\mathbf{1 0 v}$

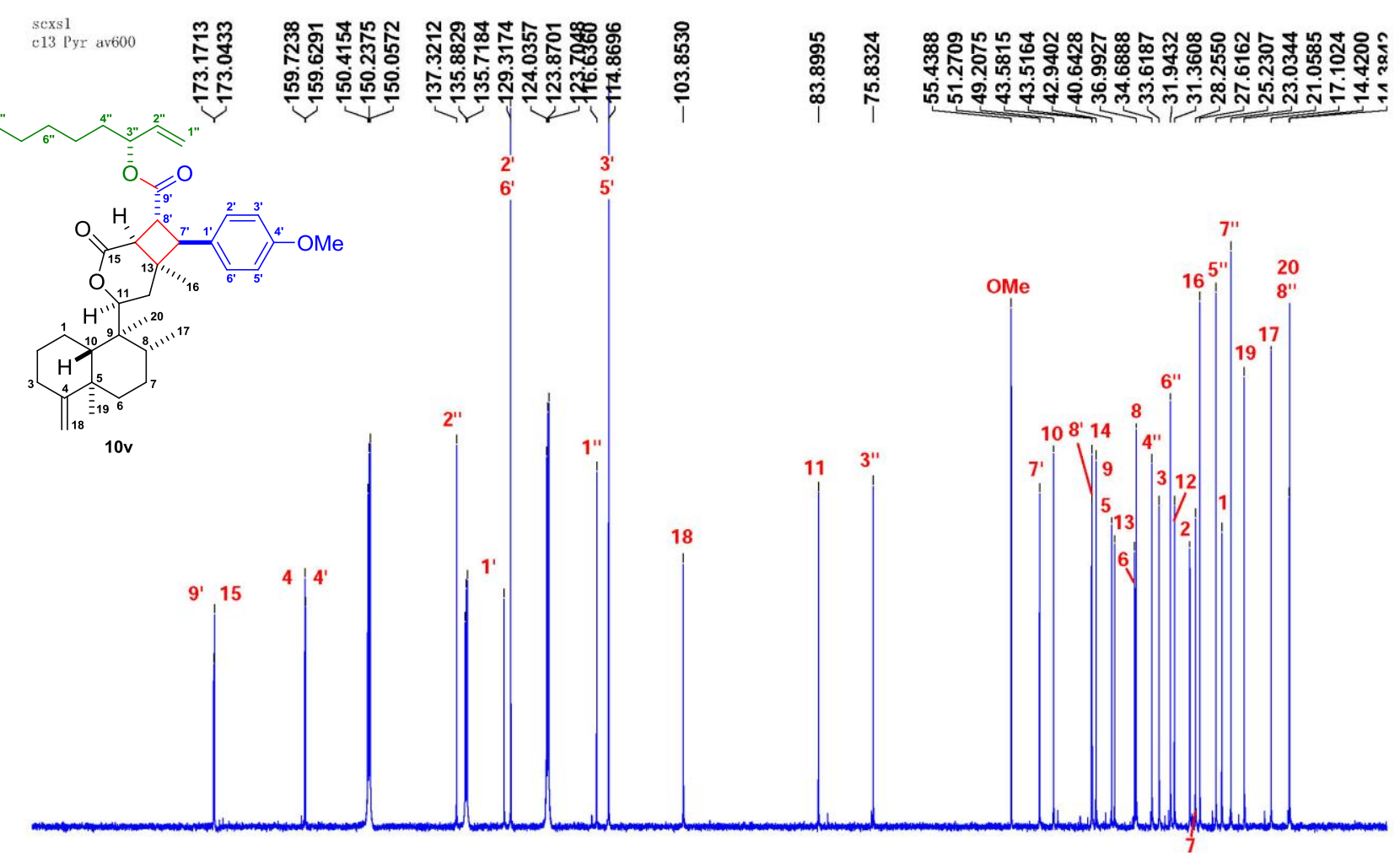

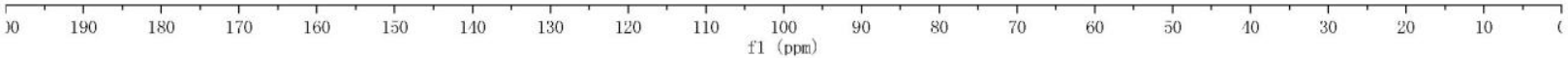


Figure S205. HSQC spectrum of $\mathbf{1 0 v}$

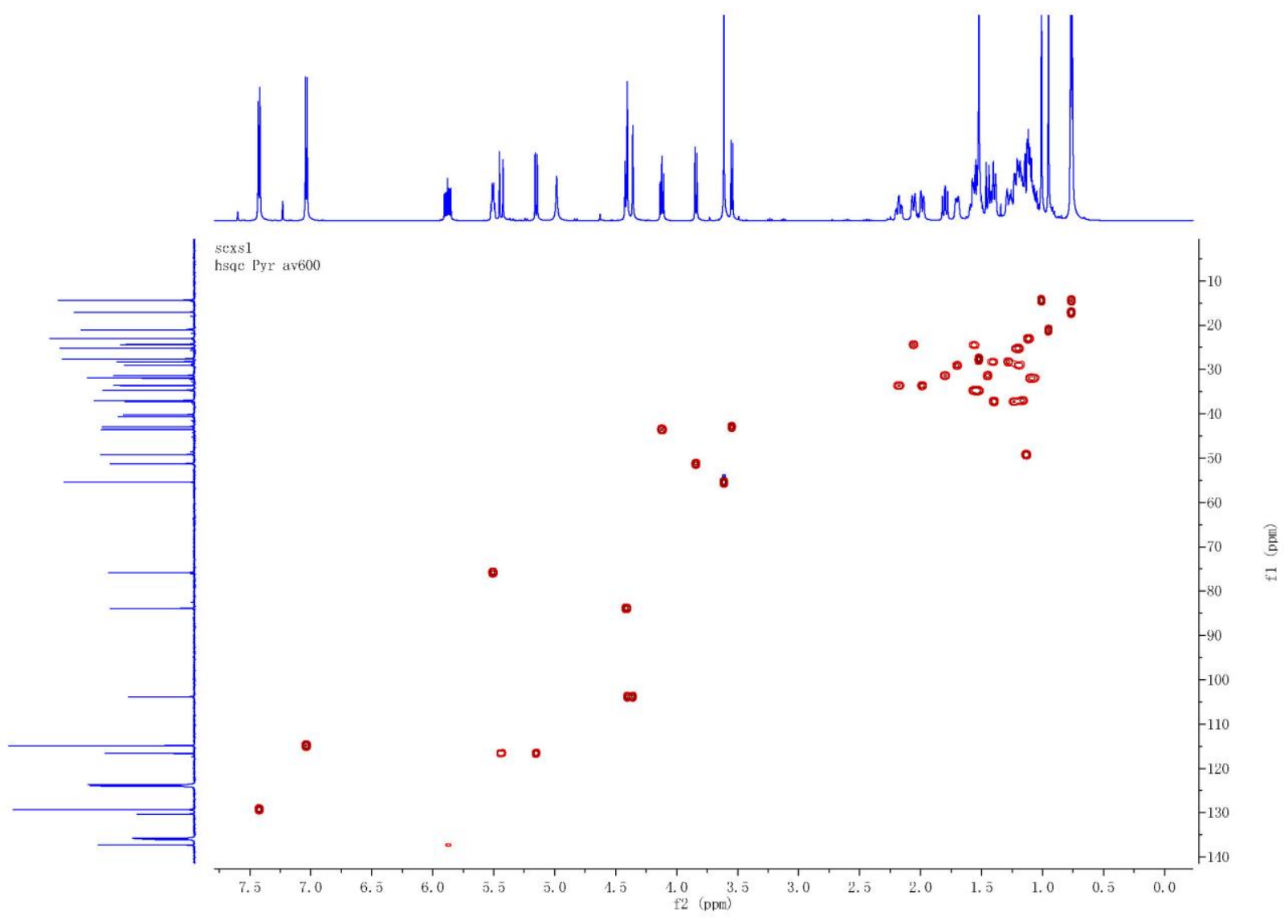

Figure S206. HMBC spectrum of 10v

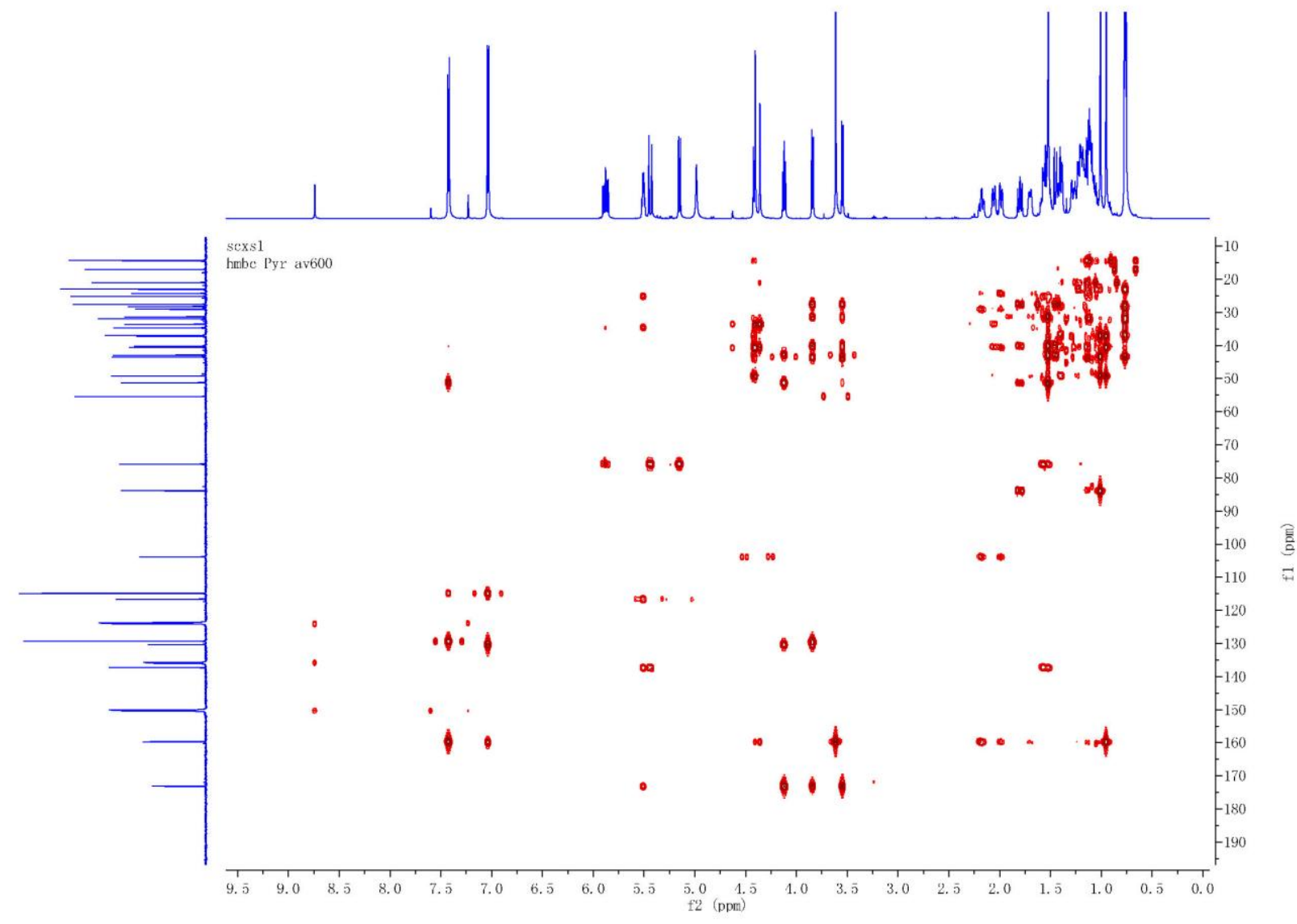


Figure S207. ${ }^{1} \mathrm{H}-{ }^{1} \mathrm{H}$ COSY spectrum of $\mathbf{1 0 v}$

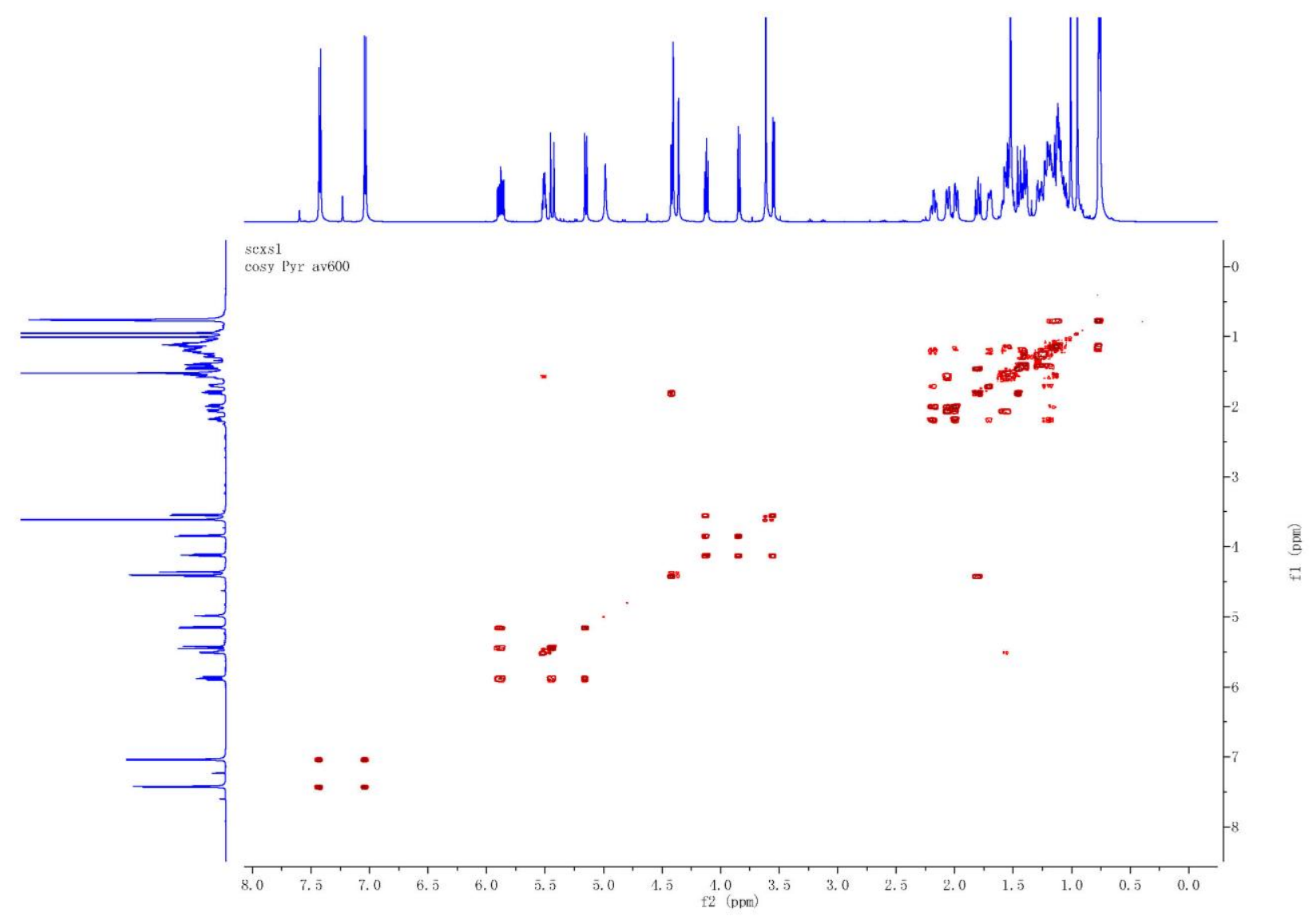

Figure S208. ROESY spectrum of $\mathbf{1 0 v}$

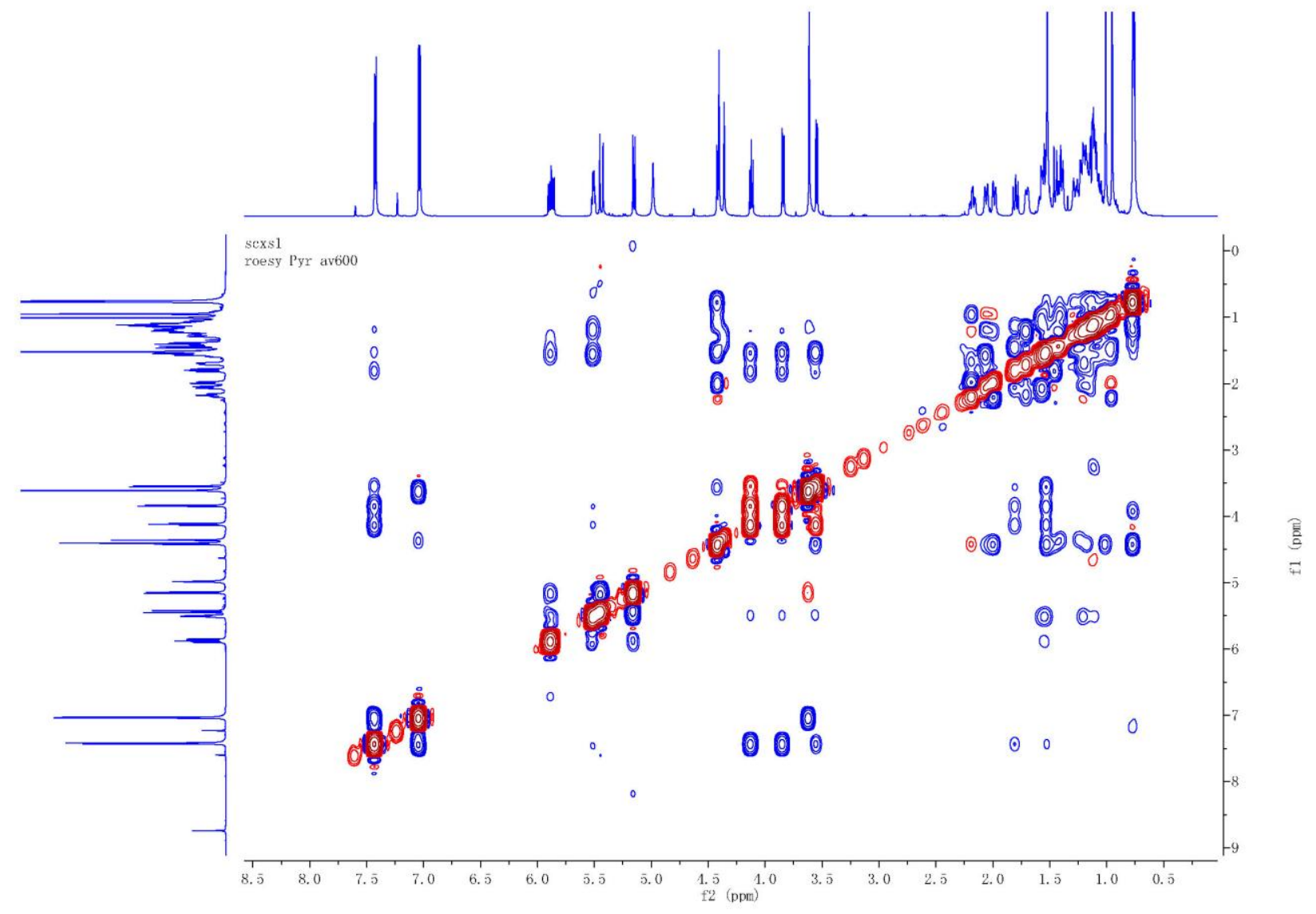


Figure S209. ${ }^{1} \mathrm{H}$ NMR spectrum of $\mathbf{1 0 w}$

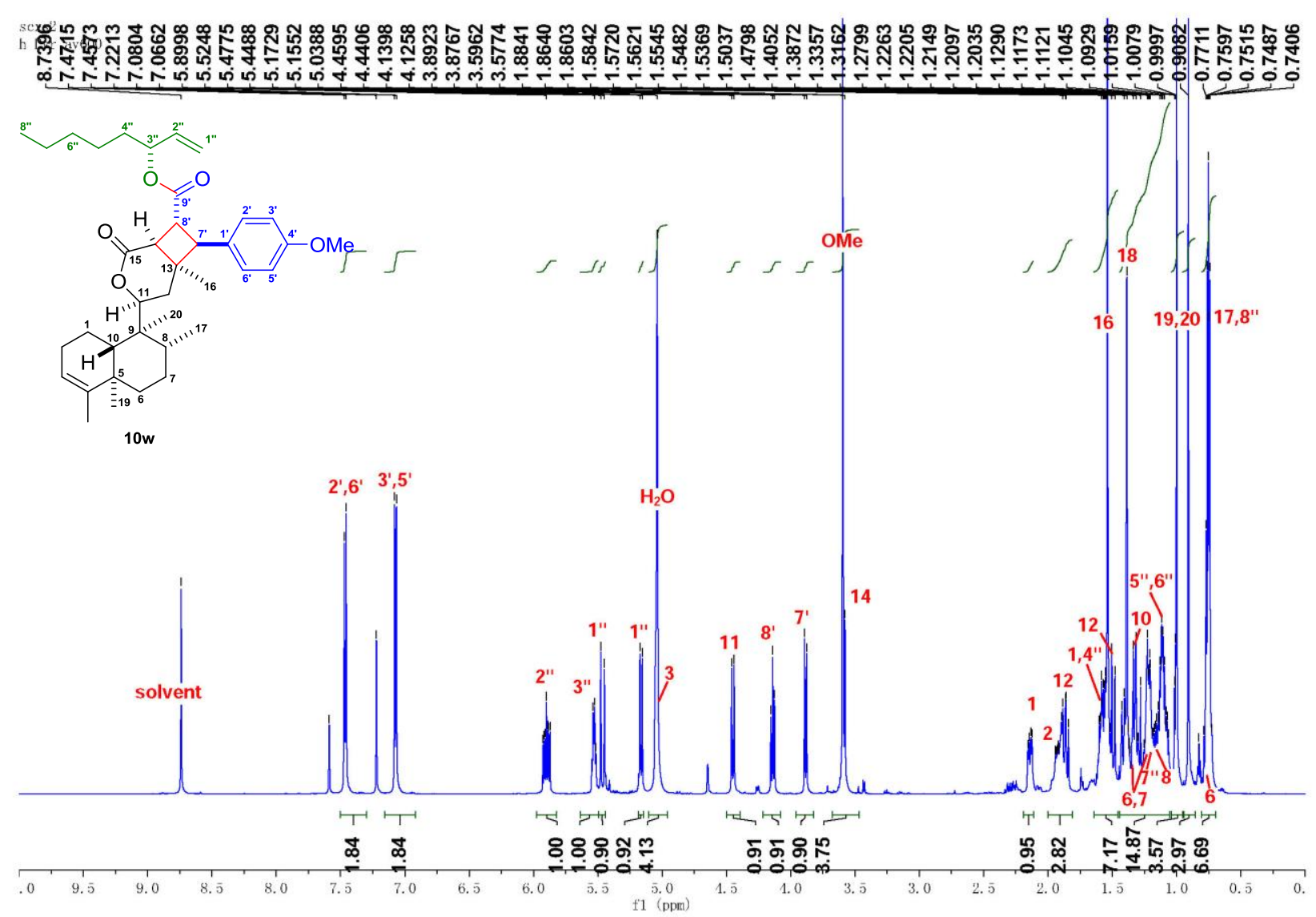

Figure S210. ${ }^{13} \mathrm{C}$ NMR spectrum of $\mathbf{1 0 w}$
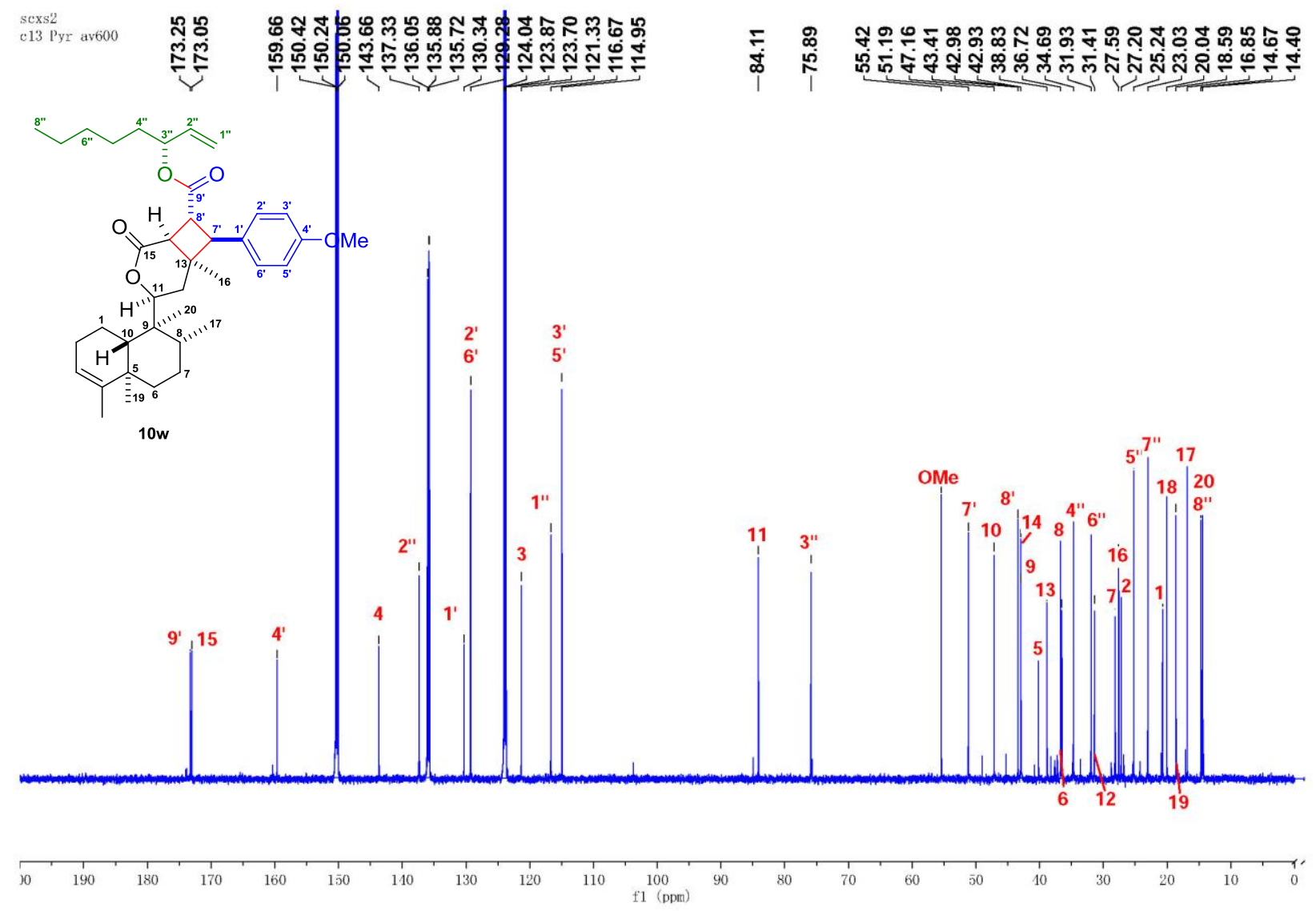
Figure S211. HSQC spectrum of $\mathbf{1 0 w}$

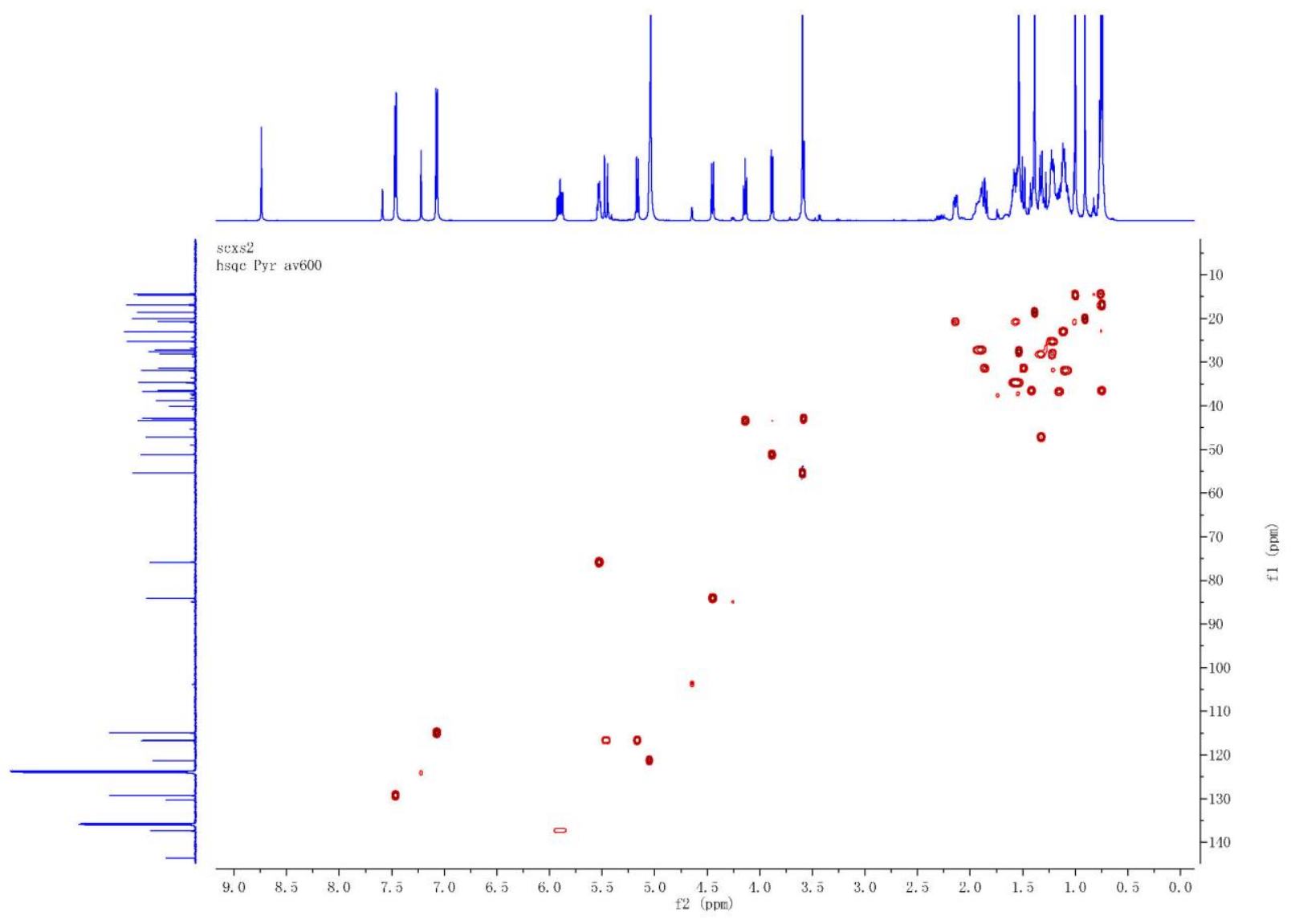

Figure S212. HMBC spectrum of $\mathbf{1 0 w}$

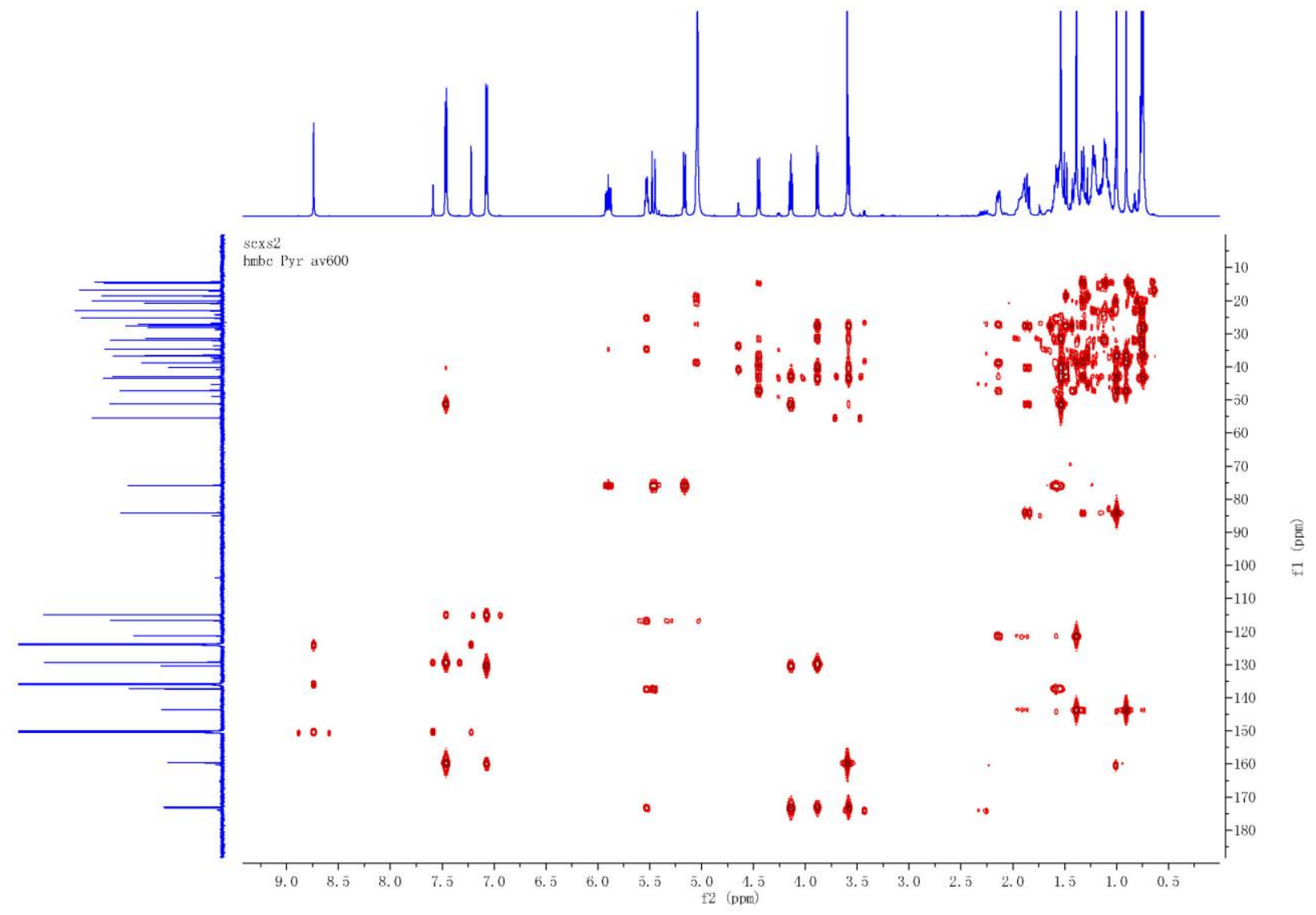


Figure S213. ${ }^{1} \mathrm{H}-{ }^{1} \mathrm{H}$ COSY spectrum of $\mathbf{1 0 w}$

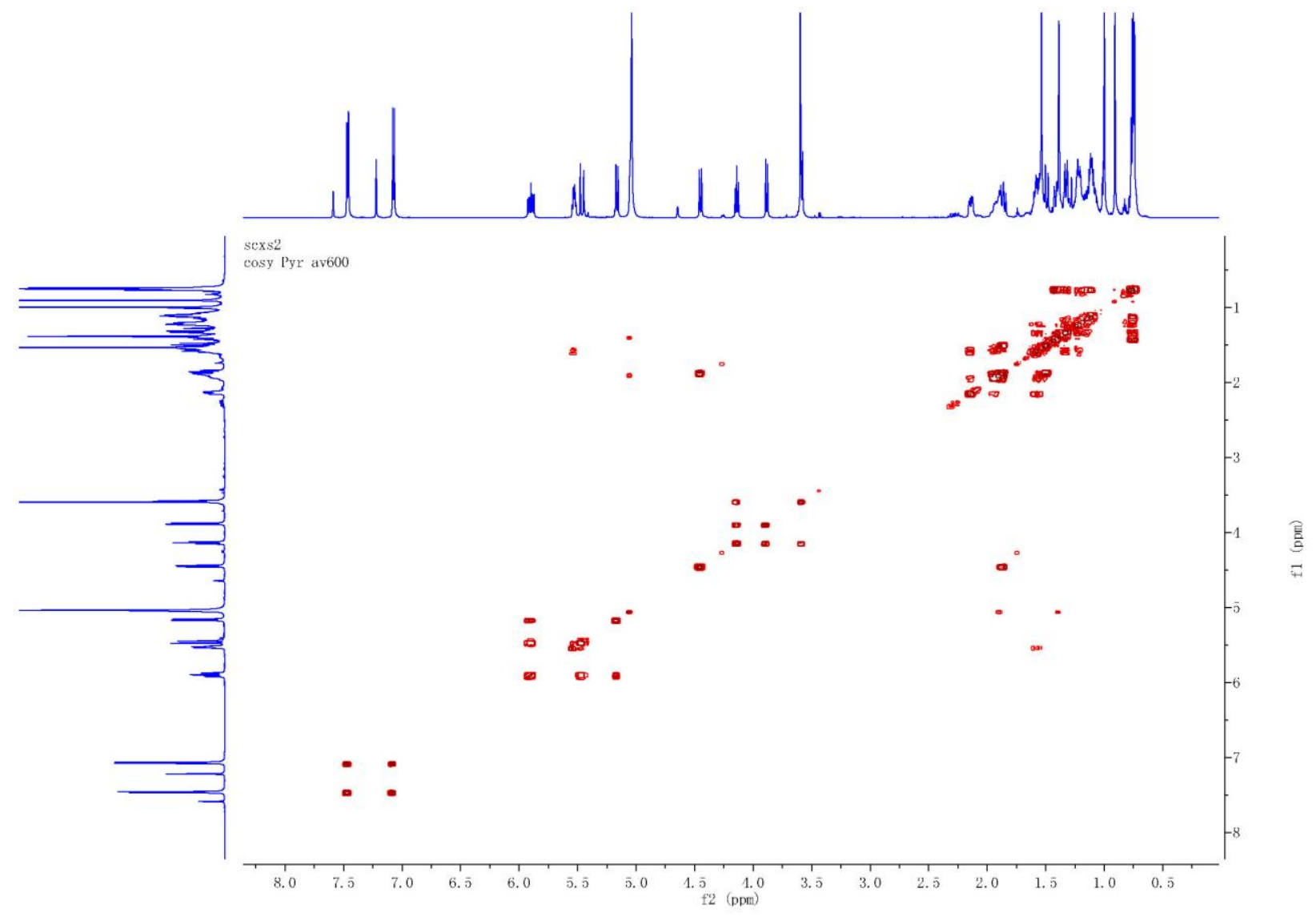

Figure S214. ROESY spectrum of 10w

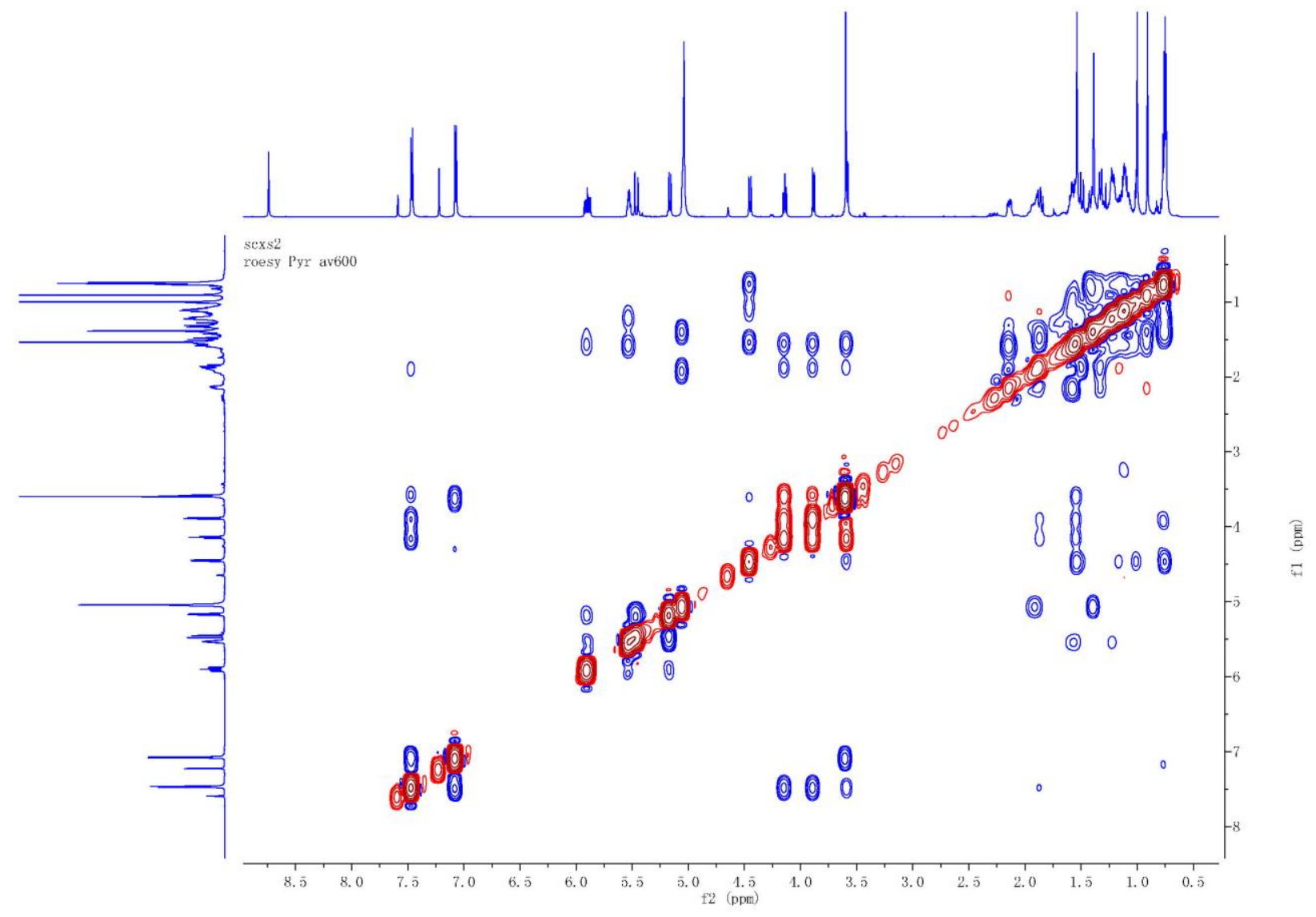


Figure S215. ${ }^{1} \mathrm{H}$ NMR spectrum of $\mathbf{6 f}$

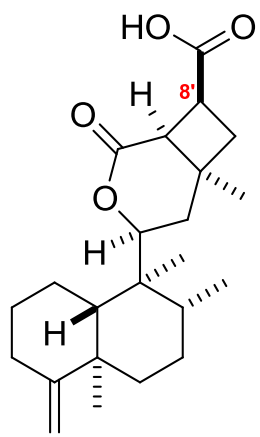

6f

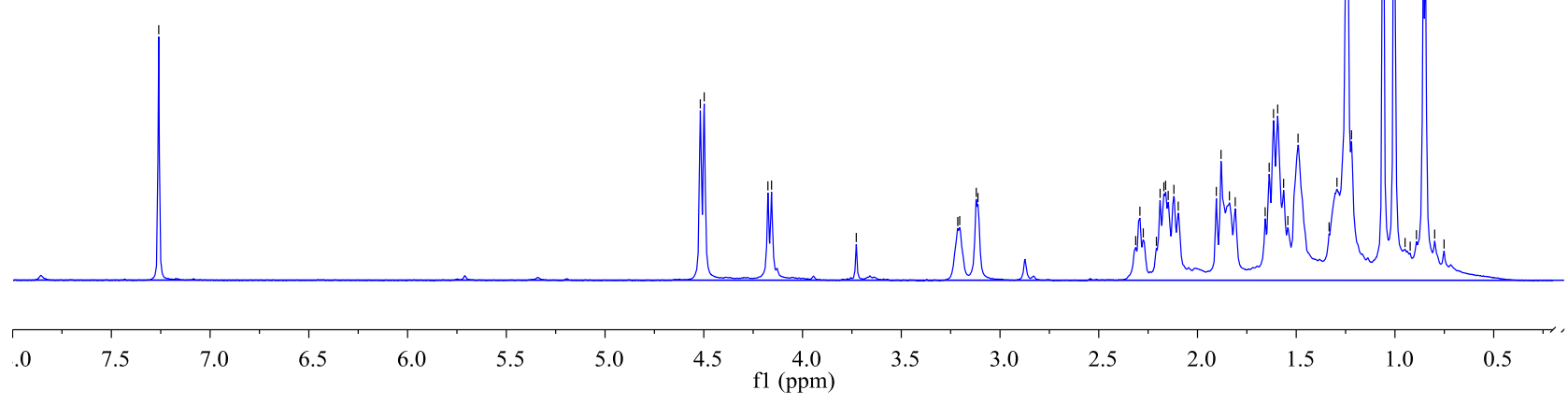

Figure S216. ${ }^{13} \mathrm{C}$ NMR spectrum of $\mathbf{6} \mathbf{f}$

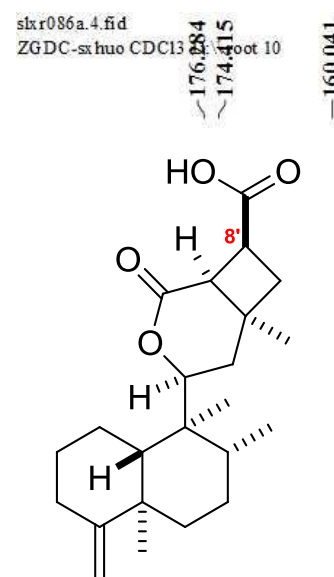

$6 f$

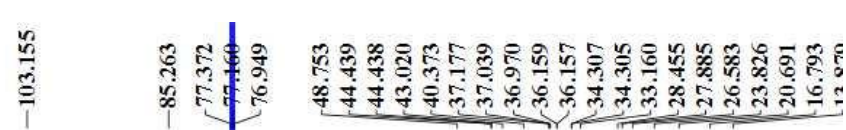

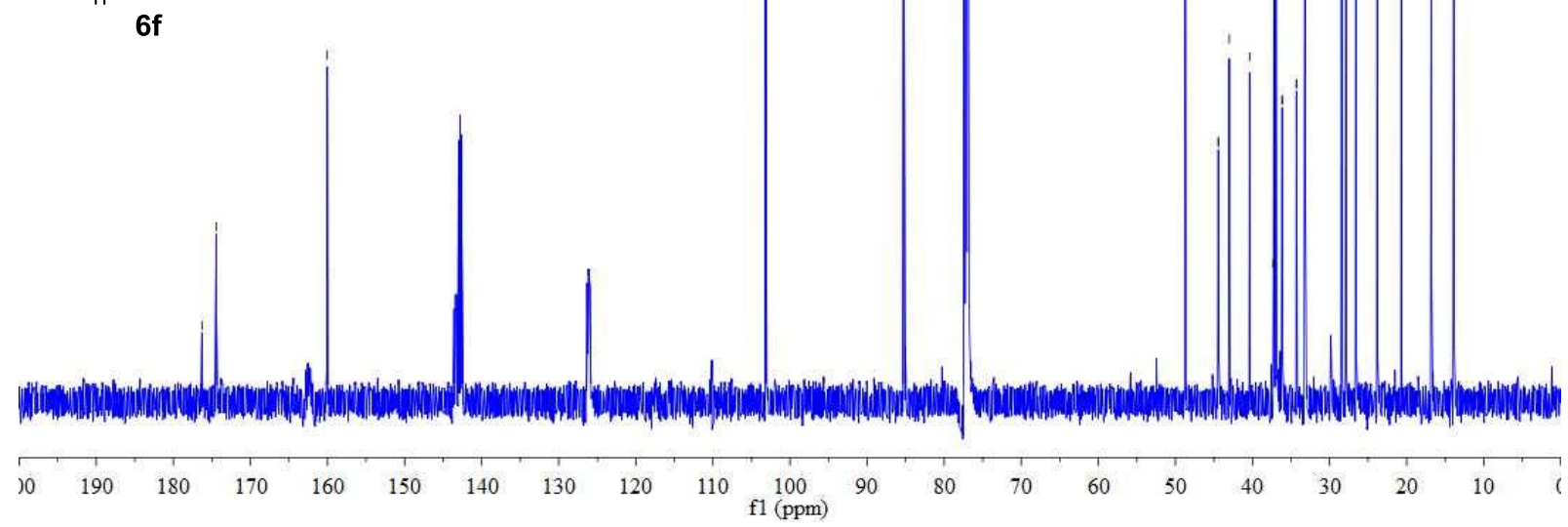

